\title{
COMPILATION OF SELECTED DATA FOR THERMAL-WATER WELLS AND SPRINGS IN IDAHO, 1921 THROUGH 1991
}

By D.J. Parliman and H.W. Young

U.S. Geological Survey

Open-File Report 92-175

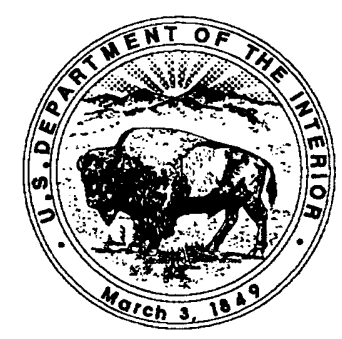

Boise, Idaho

1992 


\section{U.S. DEPARTMENT OF THE INTERIOR MANUEL LUJAN, Jr., Secretary}

\section{U.S. GEOLOGICAL SURVEY}

Dallas L. Peck, Director

For additional information write to:

District Chief

U.S. Geological Survey

230 Collins Road

Boise, ID 83702
Copies of this report can be purchased from:

U.S. Geological Survey

Books and Open-File Reports Section

Federal Center, Box 25425

Denver, CO 80225 


\section{CONTENTS}

Abstract 1

Well- and spring-numbering system 1

Introduction 1

\section{FIGURES}

1. Diagram showing well- and spring-numbering system 2

2-7. Maps showing:

2. Locations of thermal-water sites in north-central Idaho 3

3. Locations of thermal-water sites in east-central Idaho 4

4. Locations of thermal-water sites in west-central Idaho 5

5. Locations of thermal-water sites in southwest Idaho 6

6. Locations of thermal-water sites in south-central Idaho 7

7. Locations of thermal water sites in southeast Idaho 8

\section{TABLES}

1. Statistical summary of selected water-chemistry data 9

2. Well depth and and names of selected springs $\mathbf{1 1}$

3. Chemical and isotopic analyses of water from selected thermal-water wells and springs 26

\section{CONVERSION FACTORS}

\section{Multiply}

acre

foot

mile
By

4,047

0.3048

1.609

\section{To obtain}

square meter

meter

kilometer

Temperature in ${ }^{\circ} \mathrm{C}$ (degrees Celsius) can be converted to ${ }^{\circ} \mathrm{F}$ (degrees Fahrenheit) as follows:

$$
{ }^{\circ} \mathrm{F}=(1.8)\left({ }^{\circ} \mathrm{C}\right)+32 .
$$

All water temperatures are reported to the nearest $0.5^{\circ} \mathrm{C}$. 


\title{
COMPILATION OF SELECTED DATA FOR THERMAL- WATER WELLS AND SPRINGS IN IDAHO, 1921 THROUGH 1991
}

\author{
By D.J. Parliman and H.W. Young
}

\begin{abstract}
Selected well-inventory and water-chemistry data for 718 thermal-water wells and springs in Idaho were compiled. A total of 1,319 chemical analyses are presented. Analyses were performed at U.S. Geological Survey laboratories from 1921 through 1991. Most data are for sites in southwest and south-central parts of the State.
\end{abstract}

\section{WELL- AND SPRING-NUMBERING SYSTEM}

The well- and spring-numbering system used by the U.S. Geological Survey in Idaho indicates the location of wells and springs within the official rectangular subdivision of public land, with reference to the Boise base line and Meridian. The first two segments of the number designate the township (north or south) and range (east or west). The third segment gives the section number; four letters, which indicate the $1 / 4$ section $(160$-acre tract), 1/4-1/4 section (40-acre tract), 1/4-1/4-1/4 section (10-acre tract), and serial number of the well or spring within the tract. Some locations also include a 1/4-1/4-1/4-1/4 section (2 1/2-acre tract) letter within the section number. Quarter sections are designated by the letters A, B, C, and $D$ in counterclockwise order from the northeast quarter of each section. Forty-acre, 10 -acre, and $21 / 2$-acre tracts within each quarter section are lettered in the same manner. Well 3S-6E-27DDD1 (fig. 1) is in the SE1/4 SE1/4SE1/4 sec. 27, T. 3 S., R. 6 E., and was the first well inventoried in that tract. Springs are designated by the letter " $S$ " following the last numeral; for example 21N-1E-23ABA1S.

\section{INTRODUCTION}

Selected well-inventory and waterchemistry data for 718 thermal-water (greater than $19.5^{\circ} \mathrm{C}$ ) wells and springs in Idaho were compiled from the U.S. Geological Survey, Water Resources Division, QWDATA data base. Locations of these wells and springs are shown in figures 2 through 7. Most data are for sites in southwest and south-central Idaho. A statistical summary of the most frequently analyzed water-chemistry characteristics is shown in table 1. Total well depth and spring name information is shown in table 2 . Chemical analyses for each well or spring are presented in table 3 and include selected physical properties, major ions, nutrients, trace elements, radiochemicals, and stable isotopes. Analyses are listed by county, well or spring location, and sample dates. A total of 1,319 analyses are presented. Analyses were performed at U.S. Geological Survey laboratories from 1921 through 1991. 


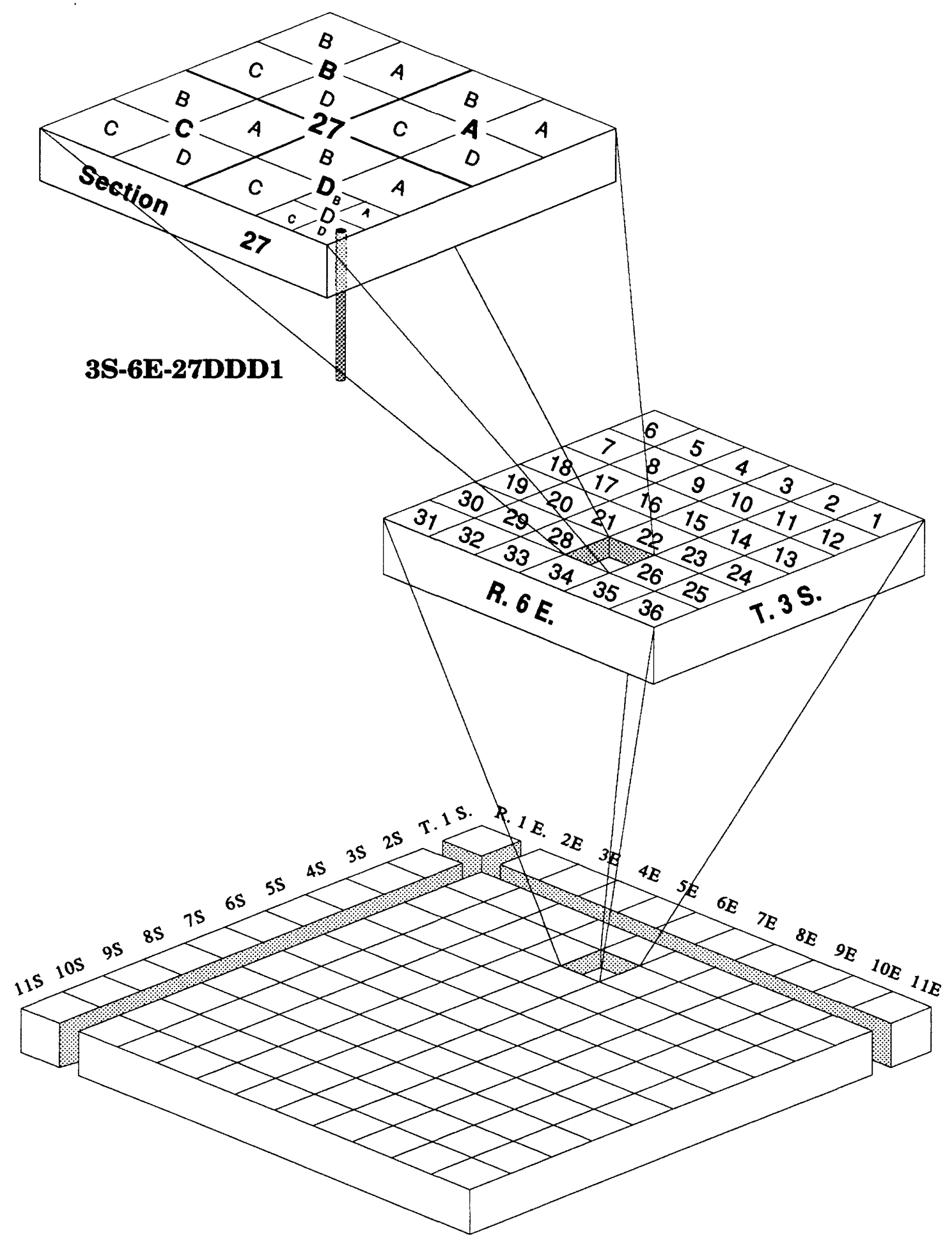

Figure 1.-Well- and spring-numbering system. 


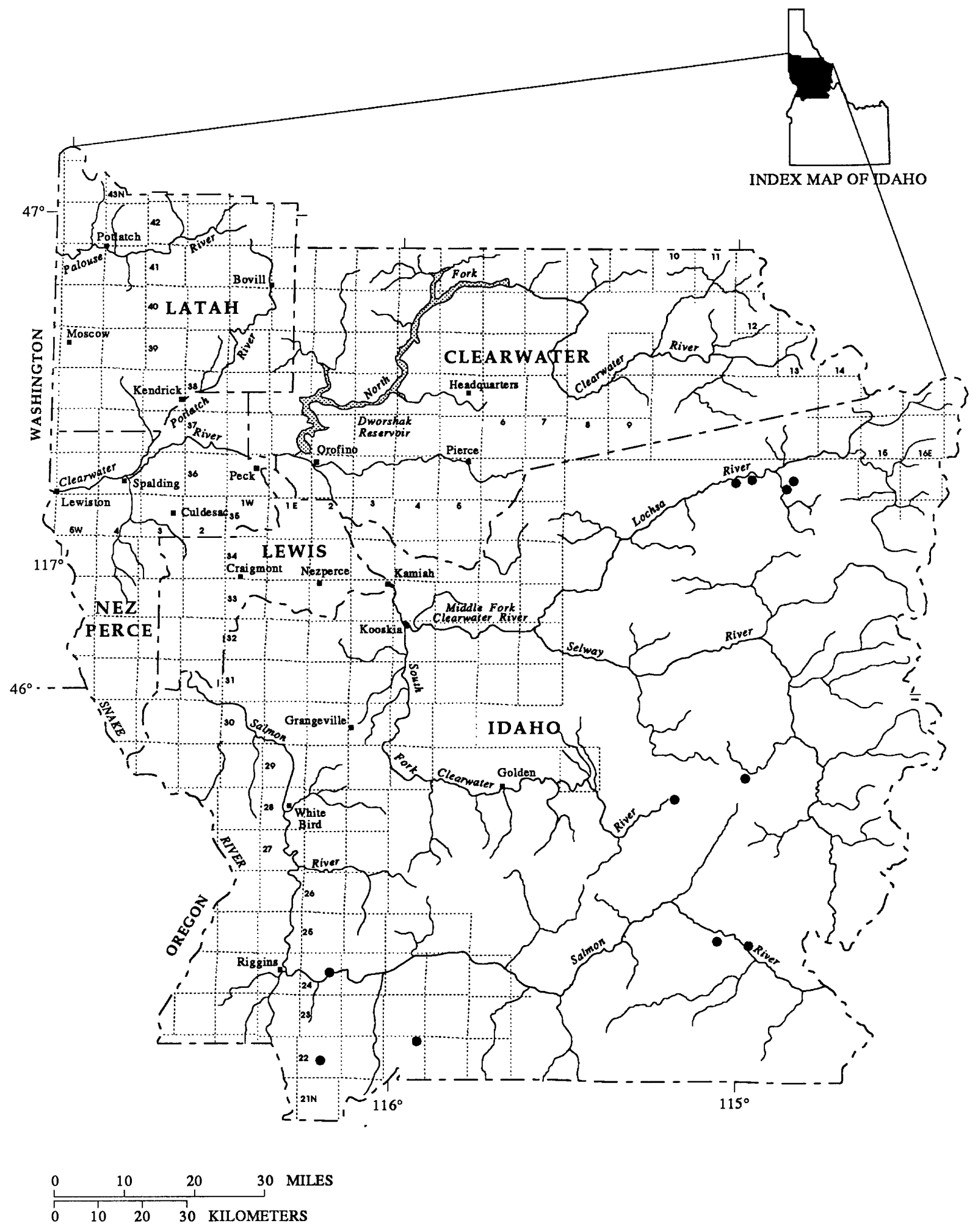

EXPLANATION

- Thermal-water well or spring

Figure 2.-Locations of thermal-water sites in north-central Idaho. 


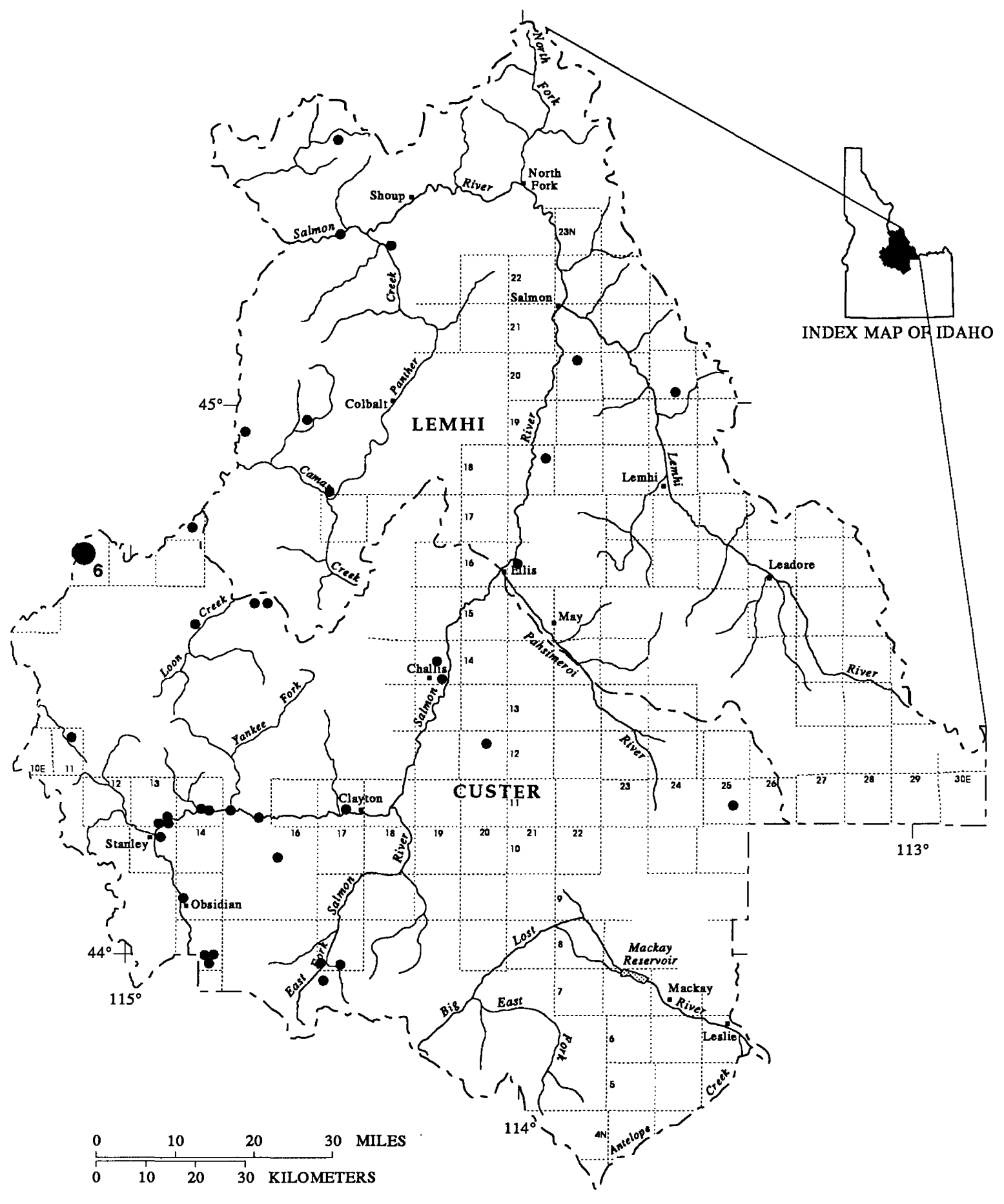

\section{EXPLANATION}

- Thermal-water well or spring

6 More than five thermal-water sites per township and number of sites

Figure 3.-Locations of thermal-water sites in east-central Idaho. 


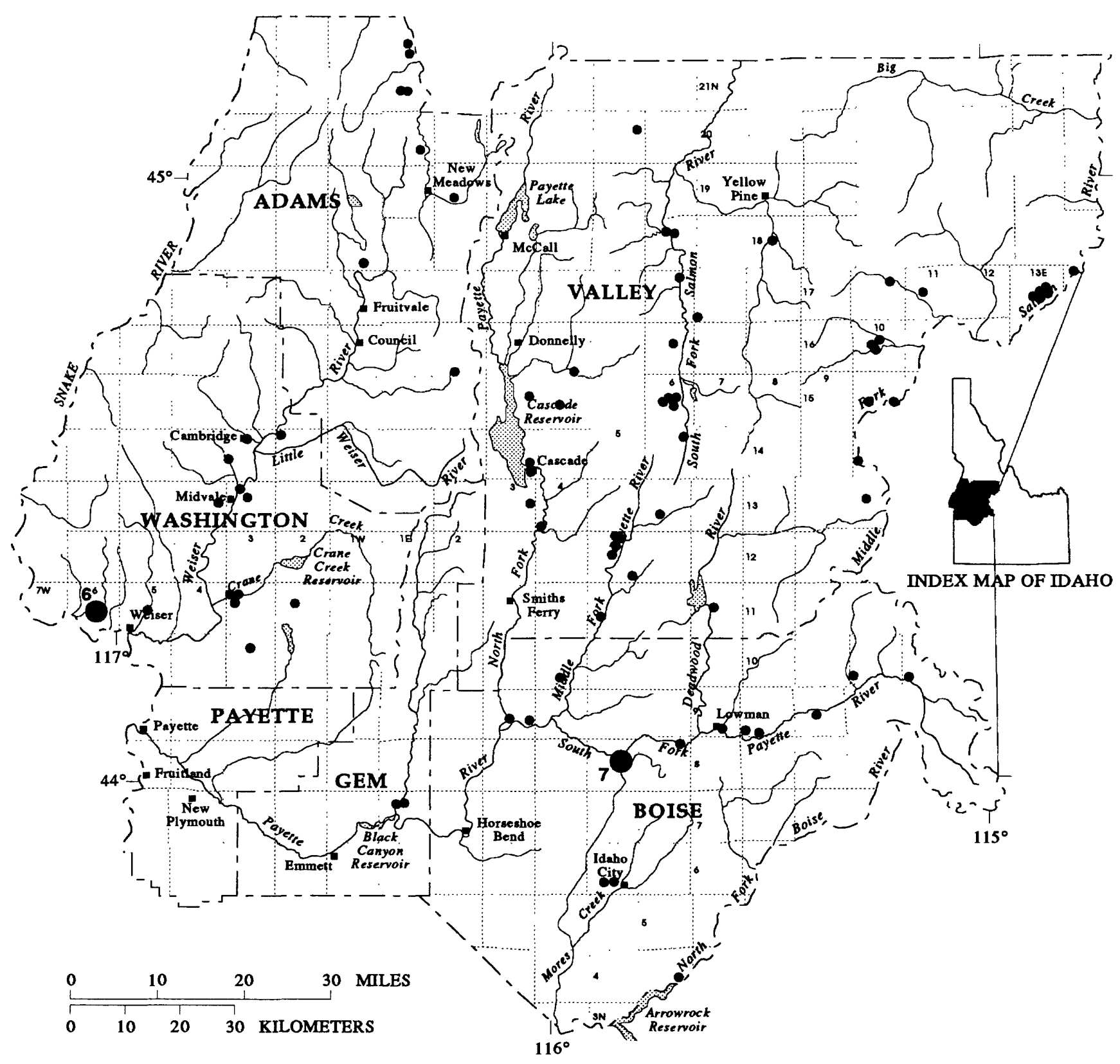

\section{EXPLANATION}

- Thermal-water well or spring

6 More than five thermal-water sites per township and number of sites

Figure 4.-Locations of thermal-water sites in west-central Idaho. 


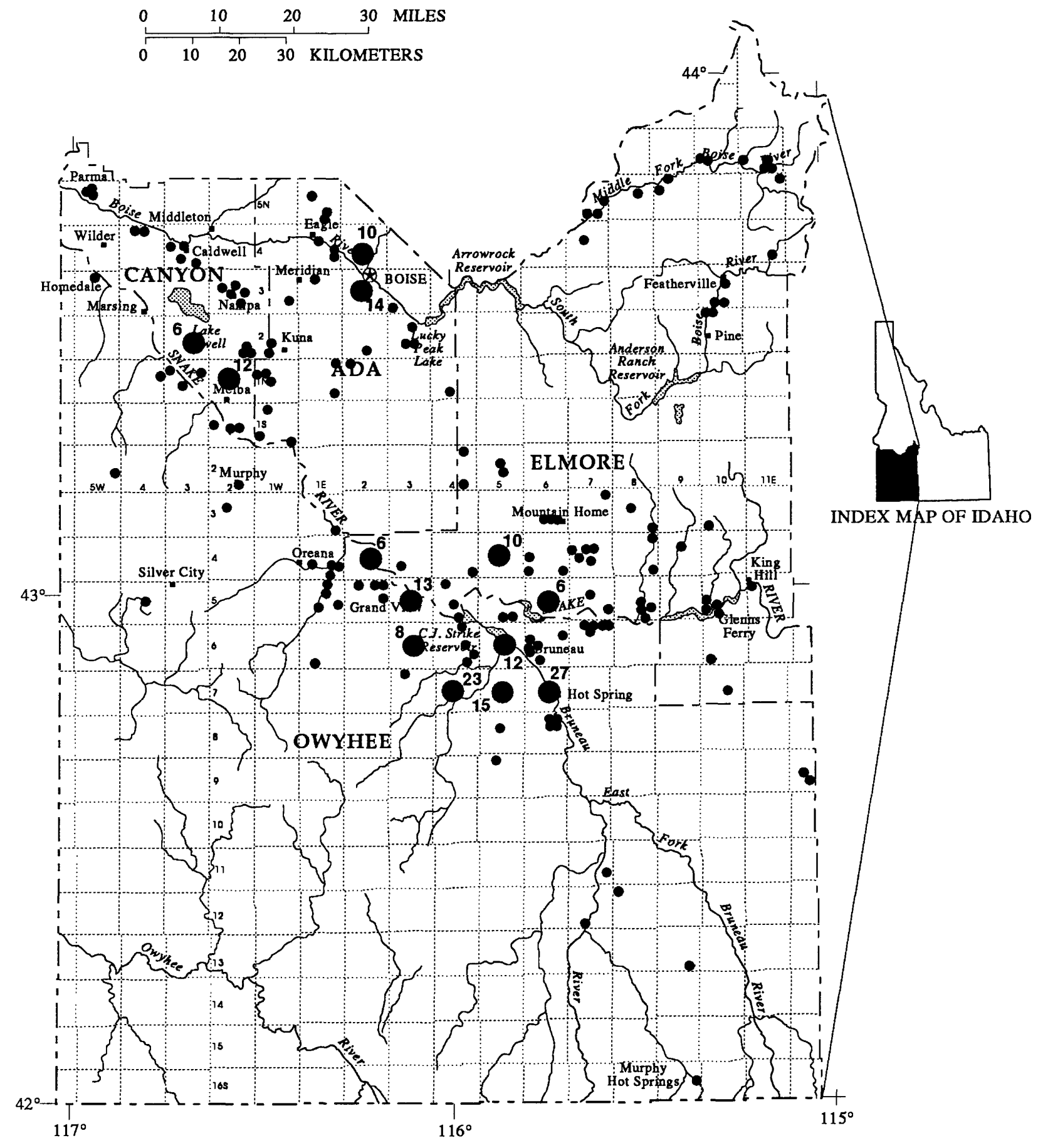

\section{EXPLANATION}

- Thermal-water well or spring

6 More than five thermal-water sites per township and number of sites

Figure 5.-Locations of thermal-water sites in southwest Idaho. 


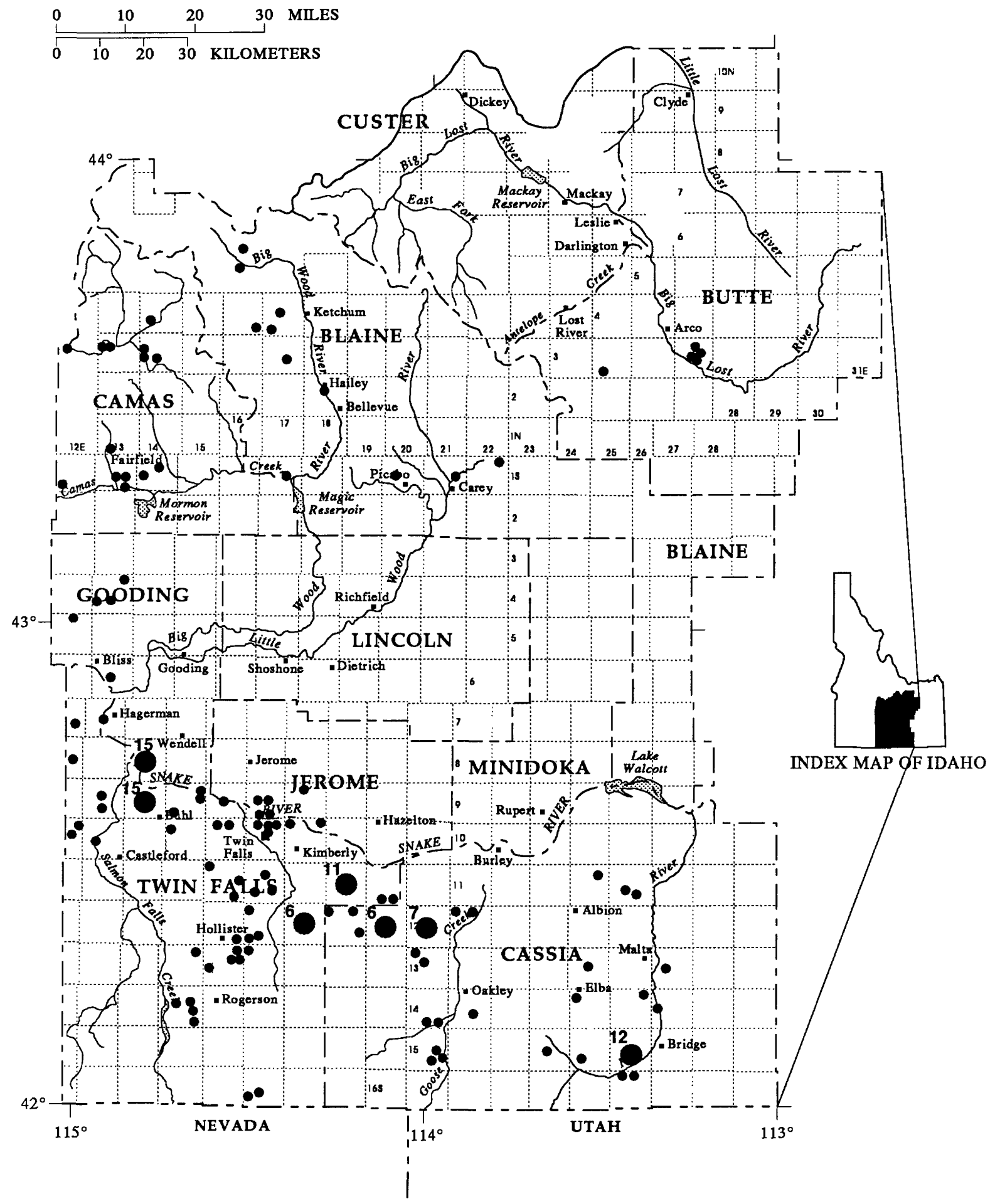

\section{EXPLANATION}

- Thermal-water well or spring

6 More than five thermal-water sites per township and number of sites

Figure 6.-Locations of thermal-water sites in south-central Idaho. 


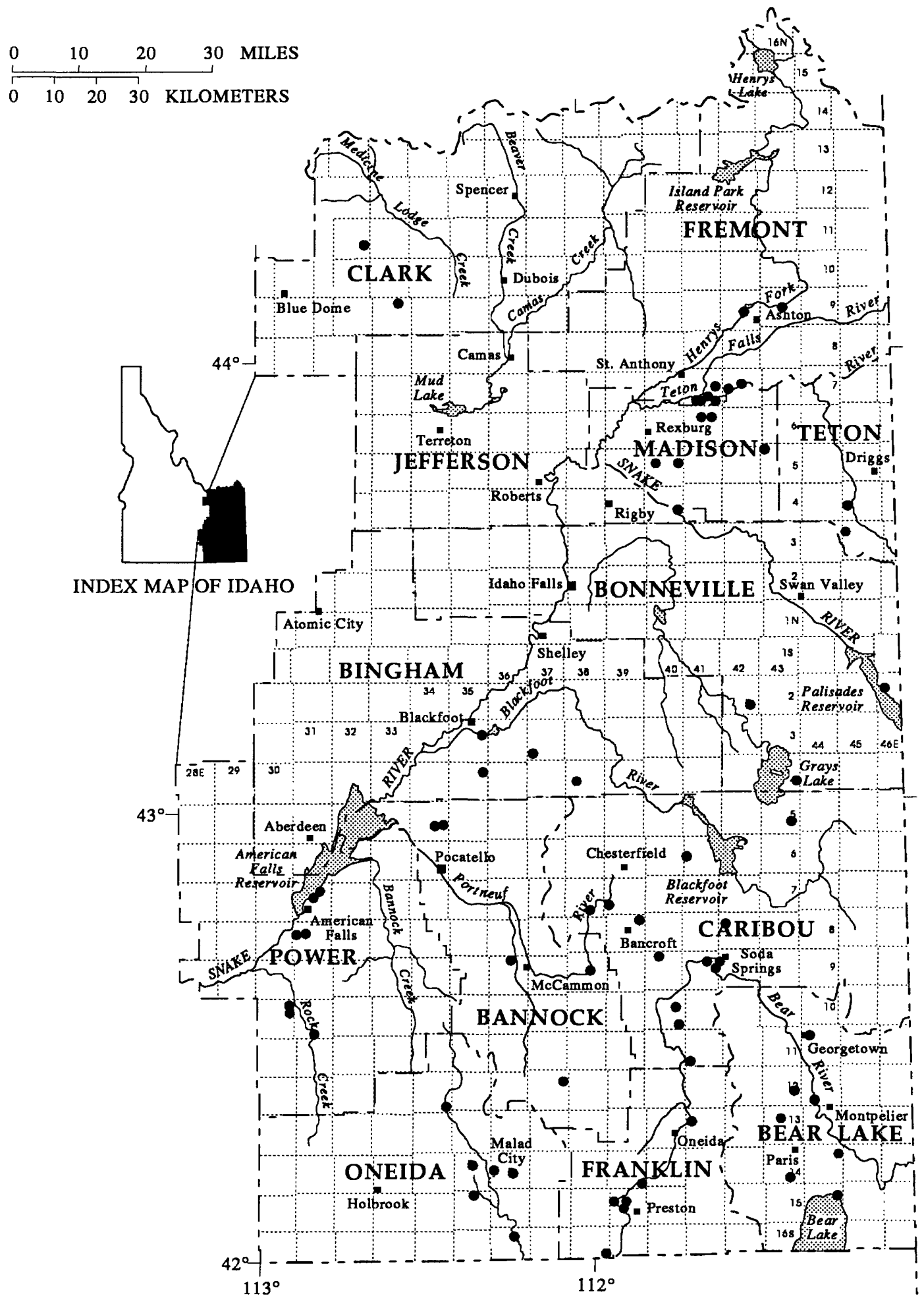

EXPLANATION

- Thermal-water well or spring

Figure 7.-Locations of thermal-water sites in southeast Idaho. 


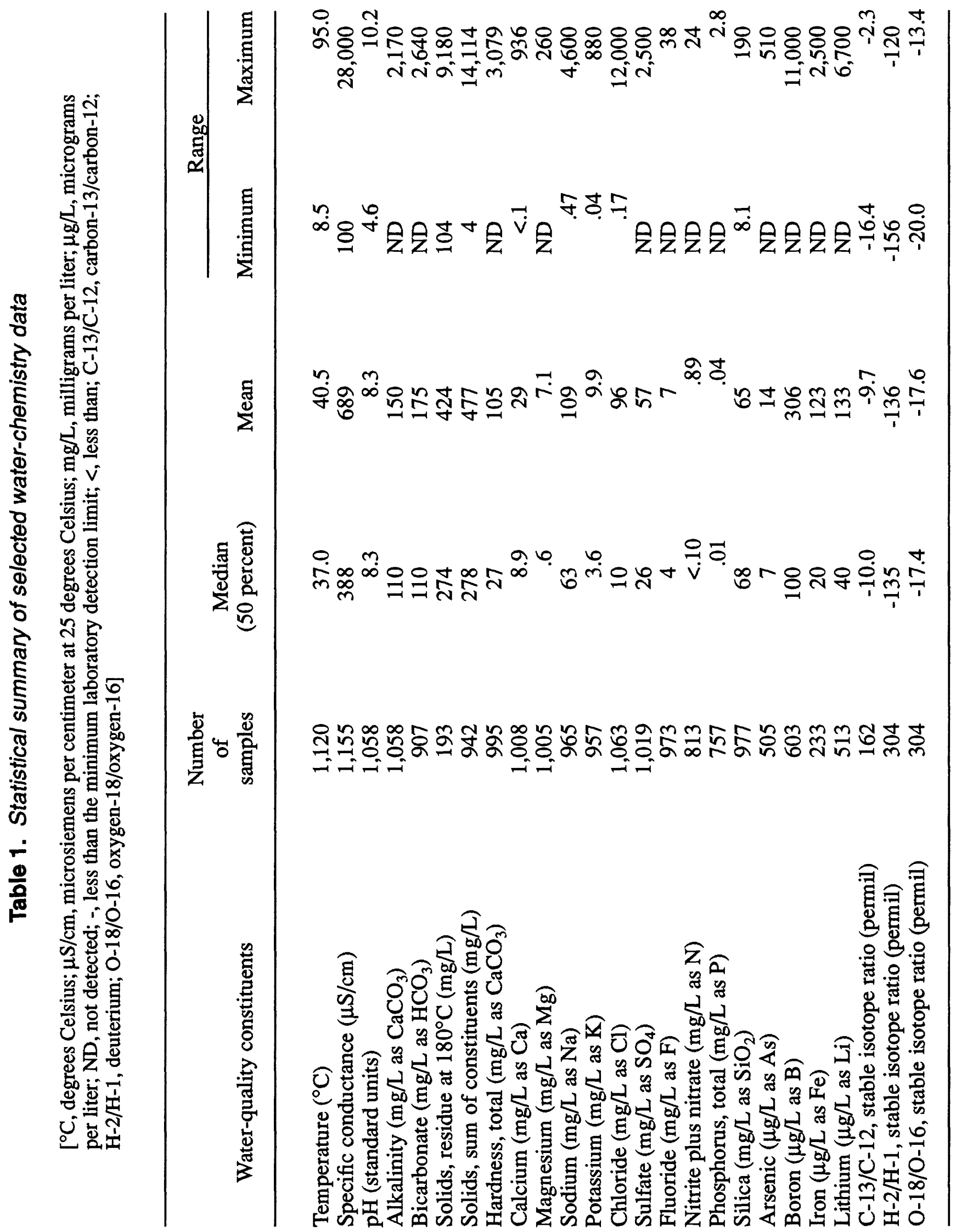


Table 2. Well depth and names of selected springs

\section{Headnotes}

WELL OR SPRING LOCATION: Well or spring location in township, range, section (fig. 1)

COUNTY:
001-Ada
003-Adams
005-Bannock
007-Bear Lake
011-Bingham
013-Blaine
015-Boise
019-Bonneville
023-Butte
025-Camas
027-Canyon

029-Caribou
031-Cassia
033-Clark
037-Custer
039-Elmore
041-Franklin
043-Fremont
045-Gem
047-Gooding
049-Idaho
051-Jefferson

053-Jerome

059-Lemhi

065-Madison

071-Oneida

073-Owyhee

077-Power

081-Teton

083-Twin Falls

085-Valley

087-Washington

SITE:

GW ground water

SP spring

\section{DEPTH OF WELL:}

- data not available

NAME OF SPRING:

$\begin{array}{ll}\text { HS } & \text { hot spring(s) } \\ \text { SP } & \text { spring } \\ \text { nr } & \text { near } \\ \text { USGS } & \text { U.S. Geological Survey } \\ \text { SPS } & \text { springs } \\ \text { Mtn } & \text { mountain } \\ \text { Ck } & \text { creek } \\ \text { USFS } & \text { U.S. Forest Service } \\ \text { BSA } & \text { Boy Scouts of America } \\ \text { BLM } & \text { U.S. Bureau of Land Management }\end{array}$




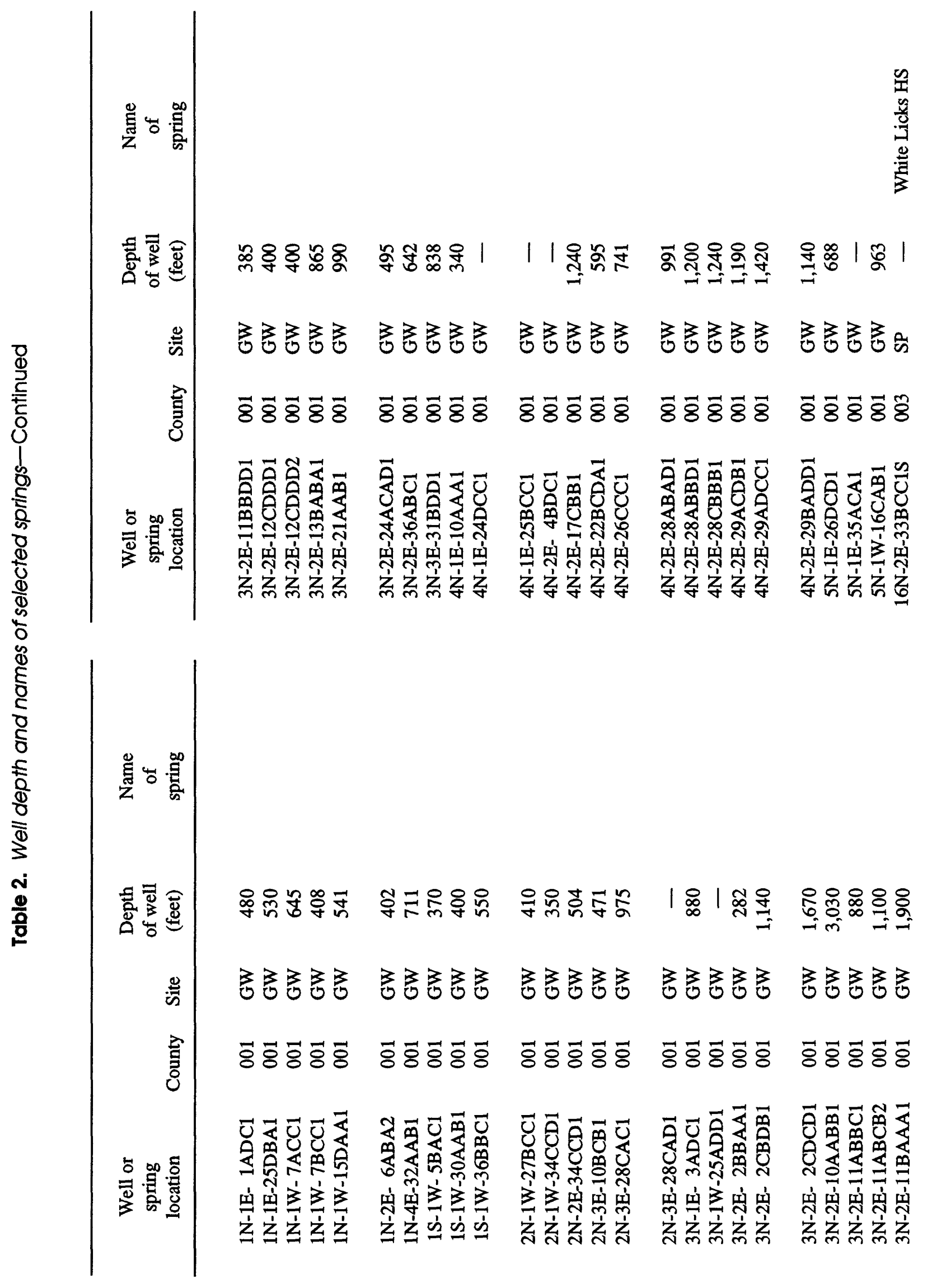




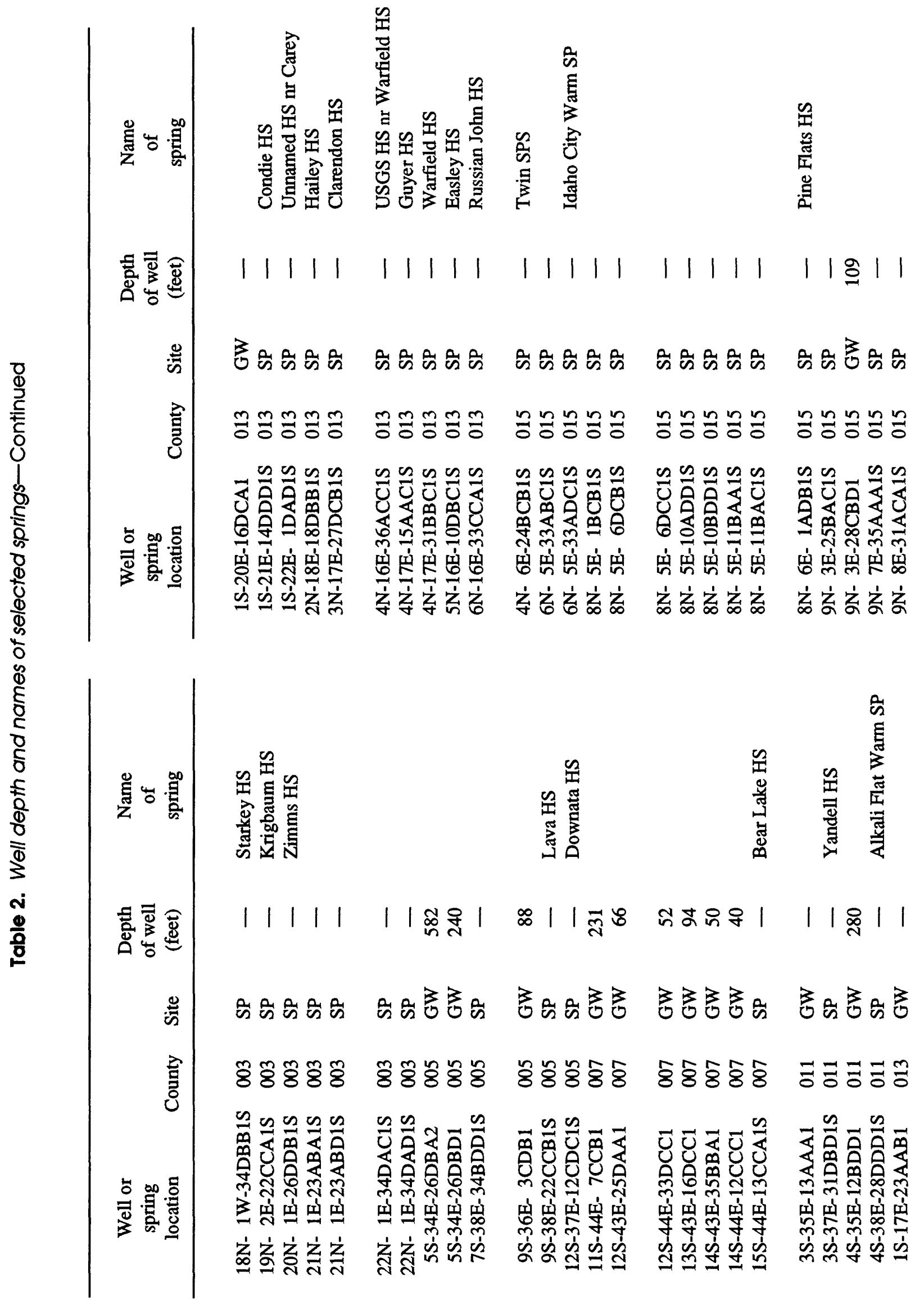




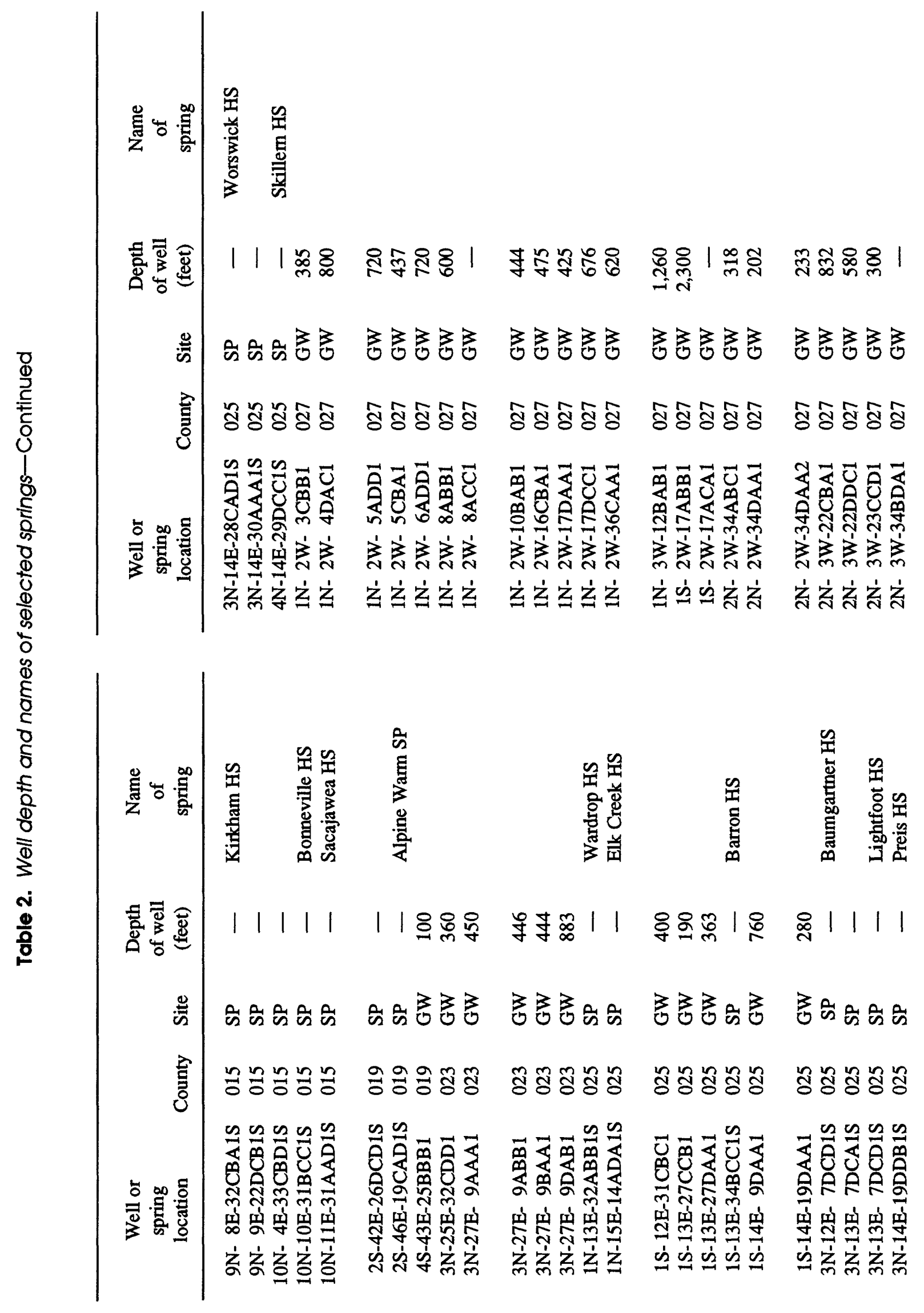




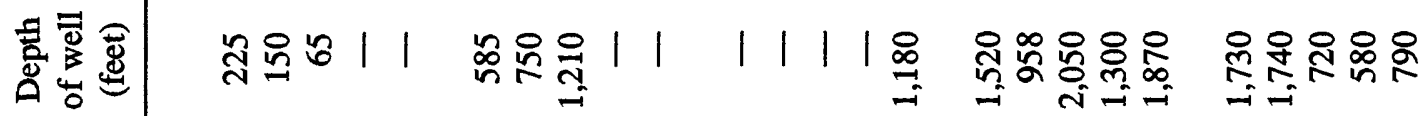

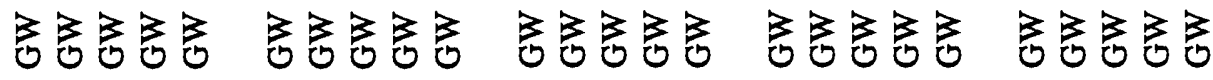

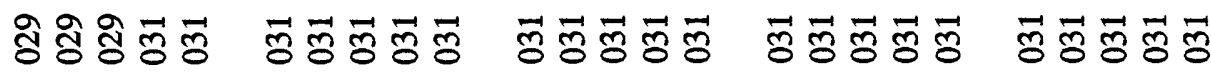

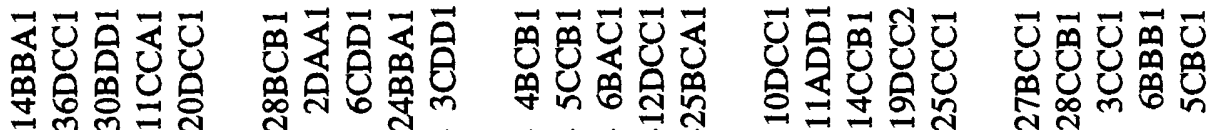

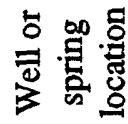

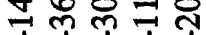

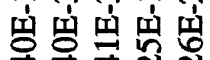
के

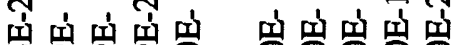

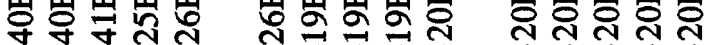
응을

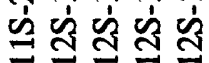
$\rightarrow \rightarrow \rightarrow$

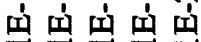
त)

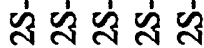

N

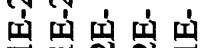
สี สิ สิ

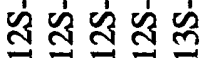

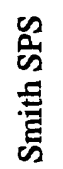

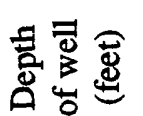

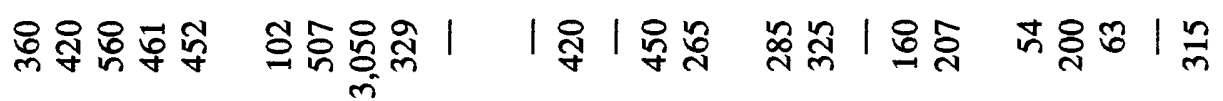

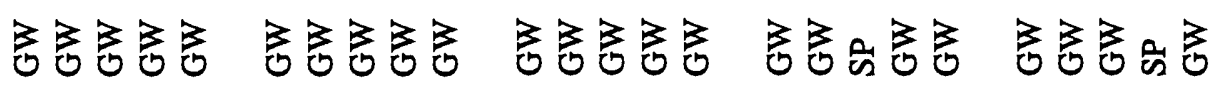

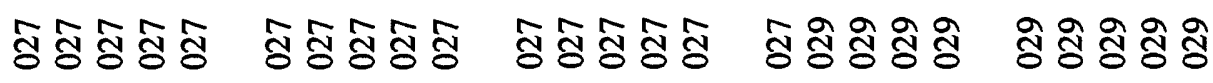

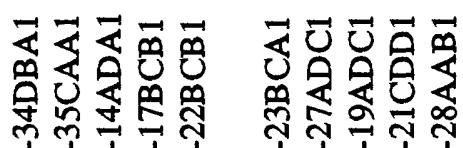

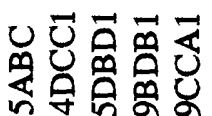
यै官字

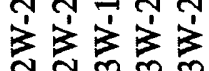

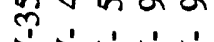

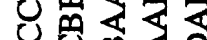
on

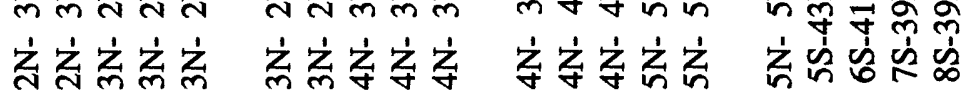

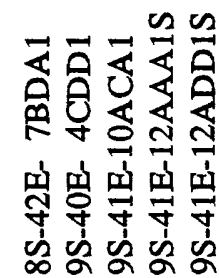




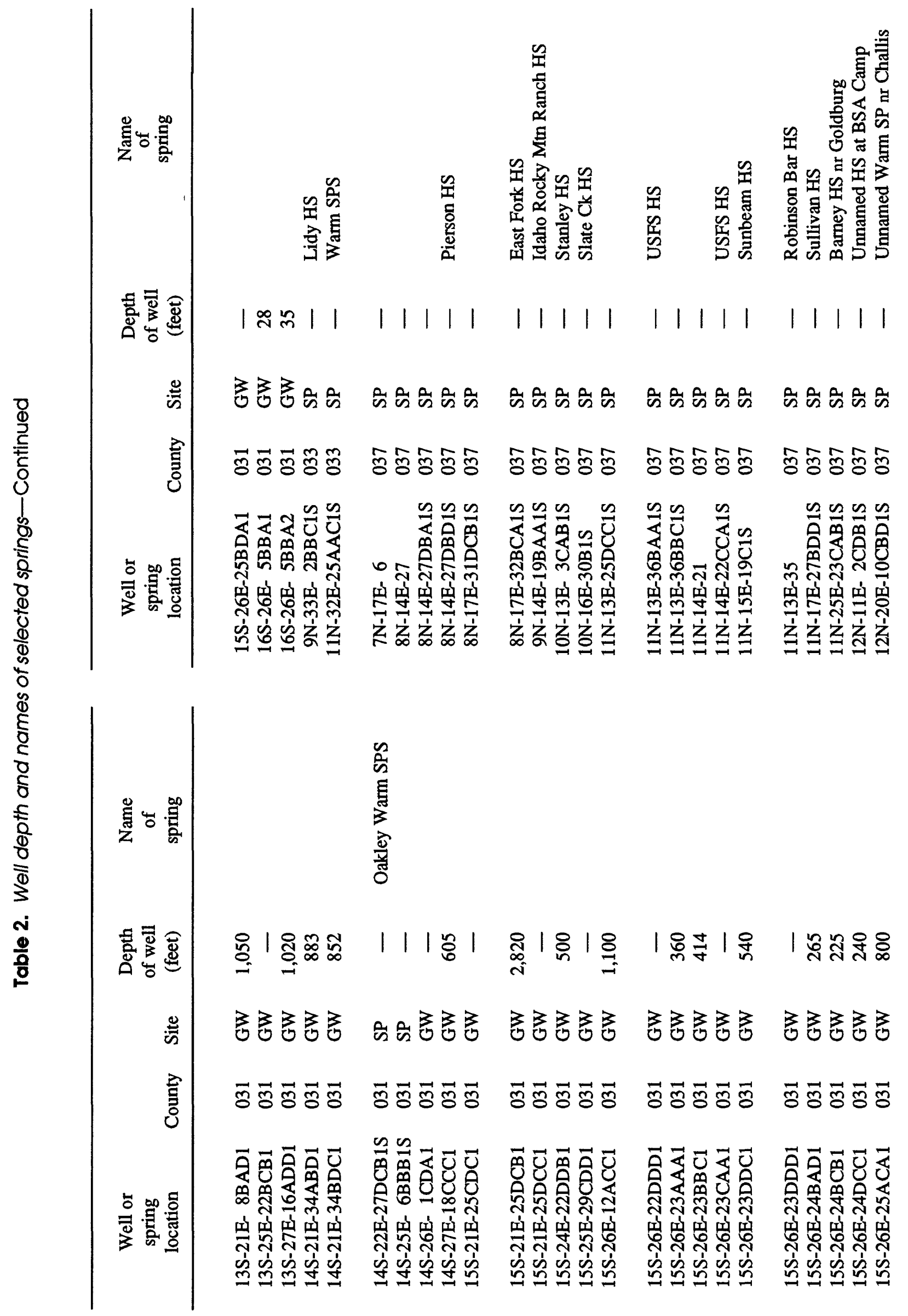




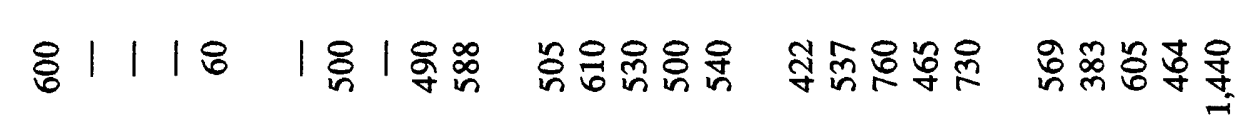

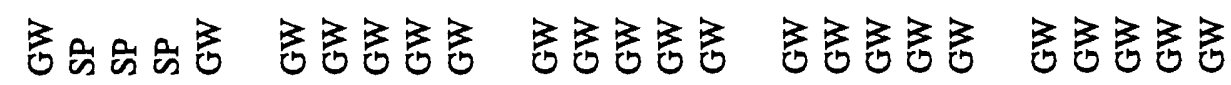

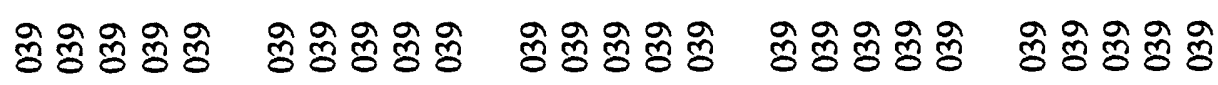

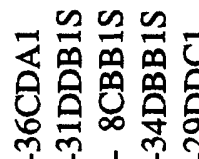

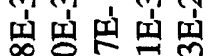
率究安守

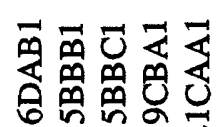
它弌它 สิ่

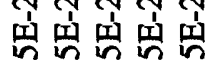
岁息崖出出 鸽导解导

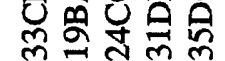

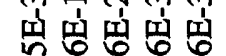
的部范

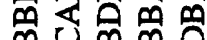
용옹

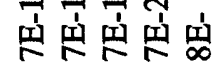

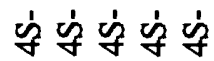
字字字字字 字字字字字 字字字字字

壳芉葛

产

资

盗留

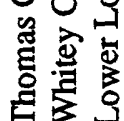

里急

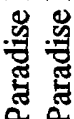

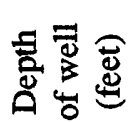

|

$1|1| 1$

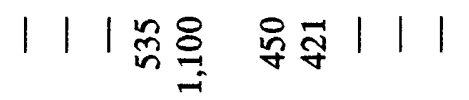

윰흐은

离

芯完零

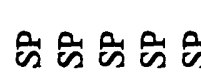

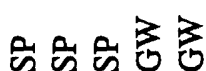

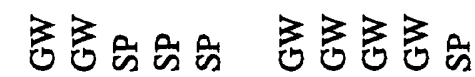

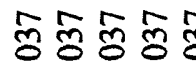

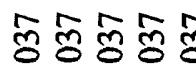

ิํㅇㅎㅀㅇ్ㅇ용

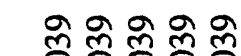

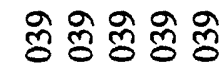

会苔它号

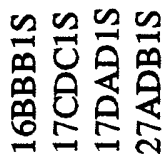

명면면

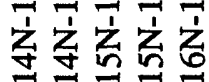

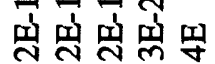

字宗宗宗

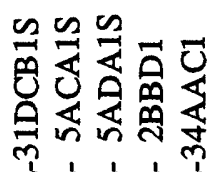

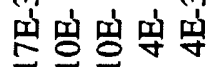

安芸灾岕

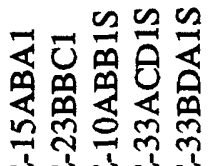

तิ m

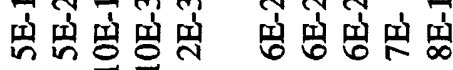

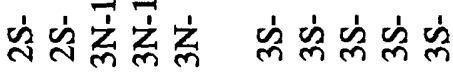




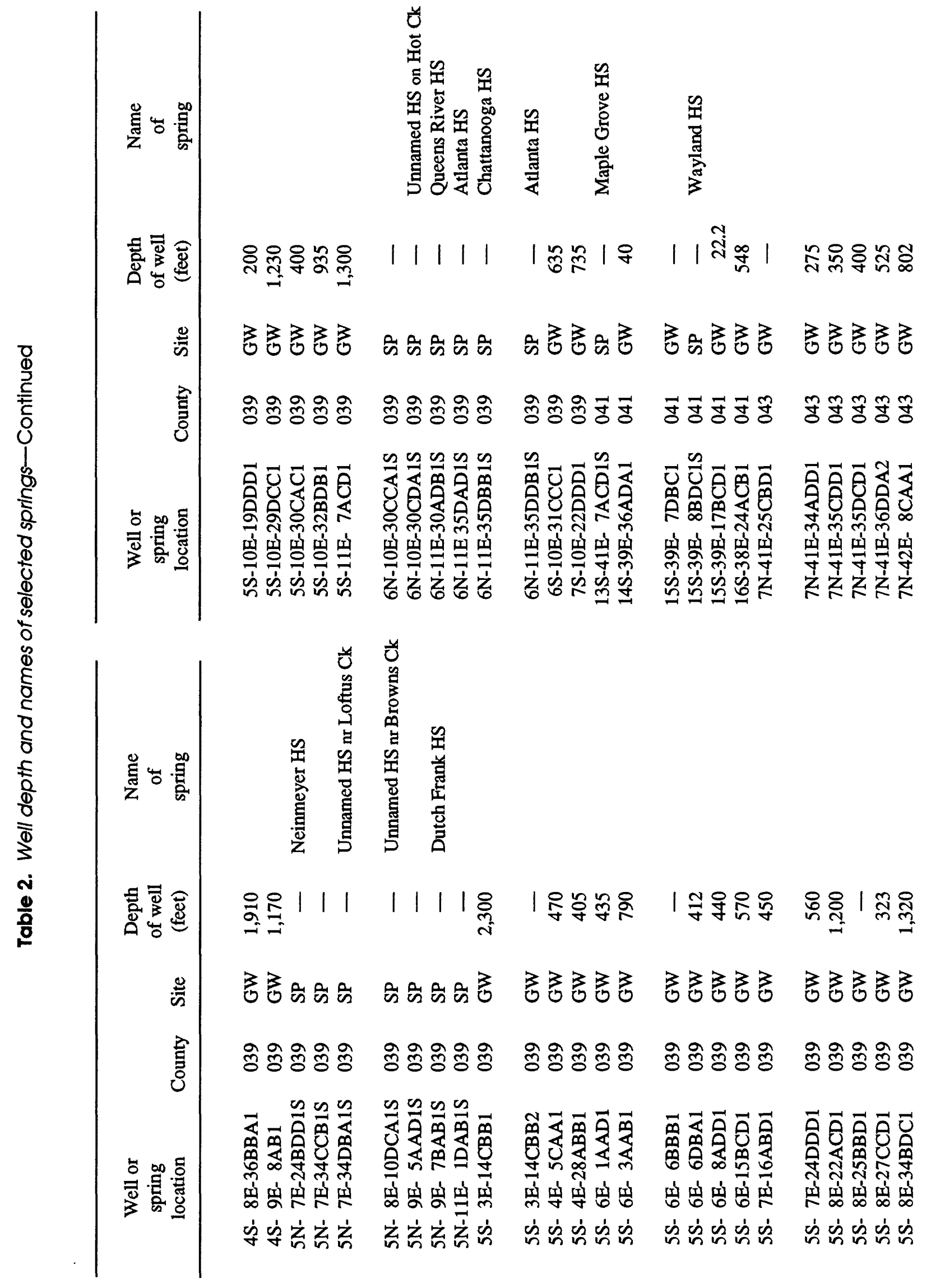




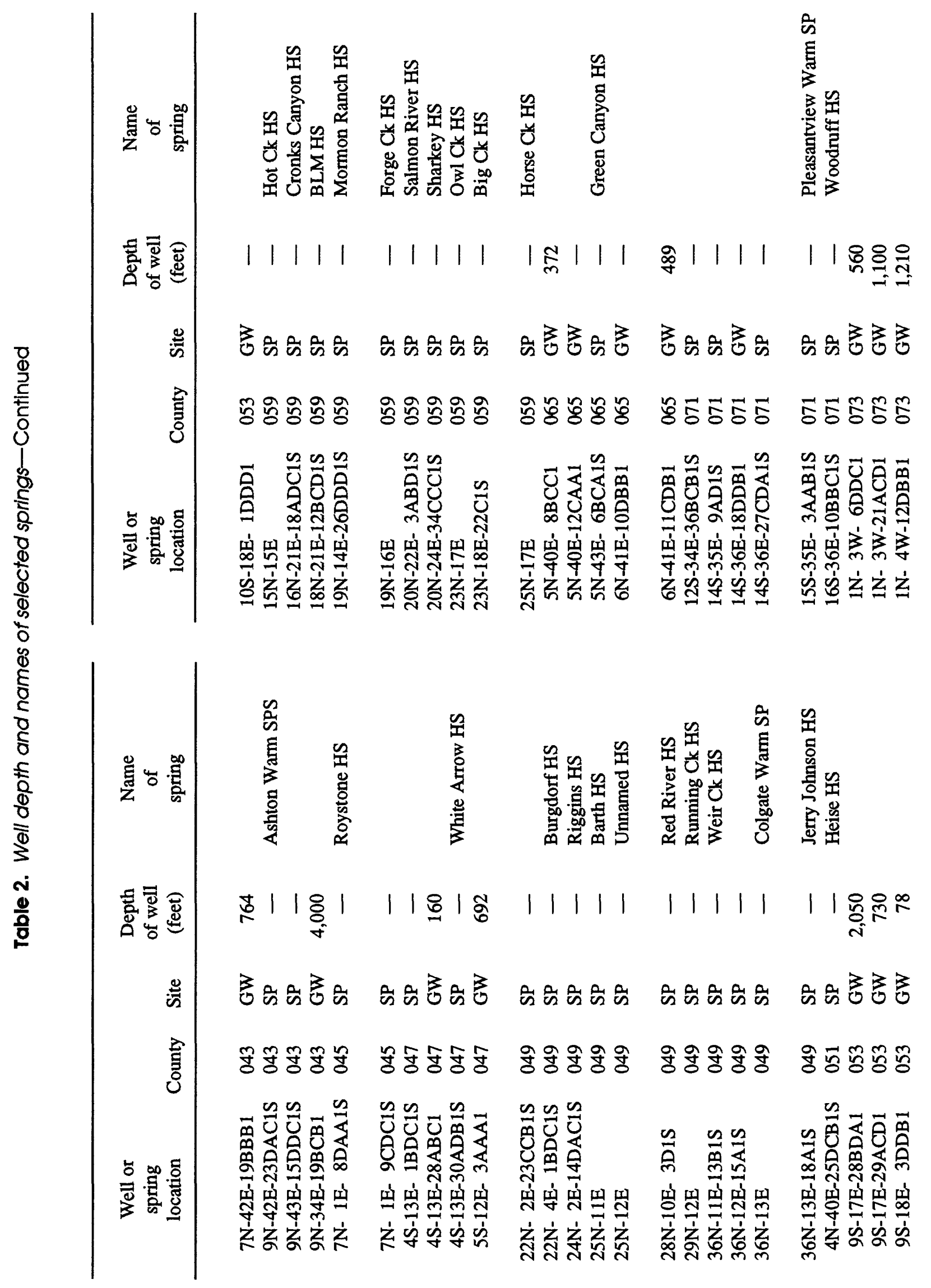




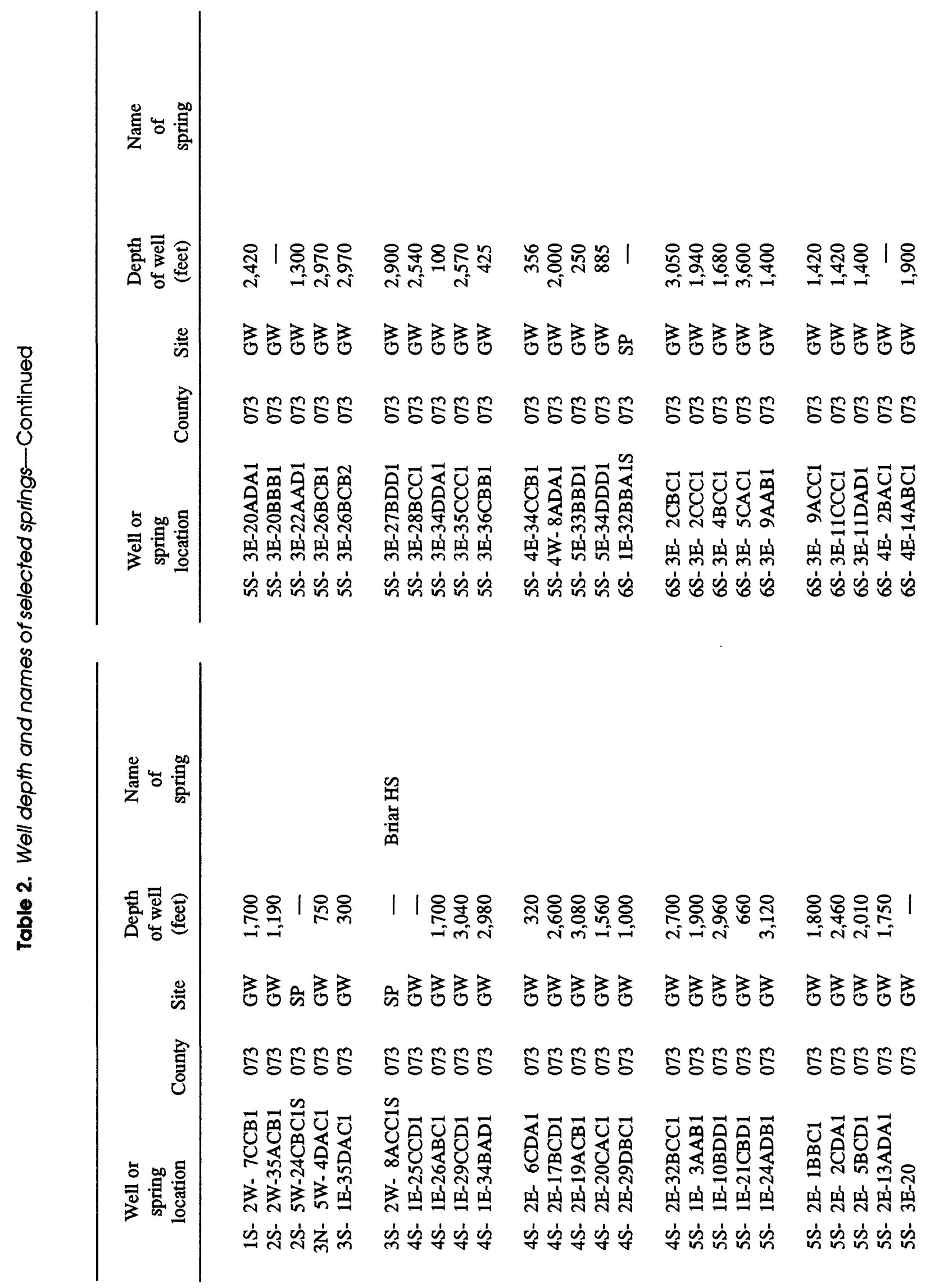




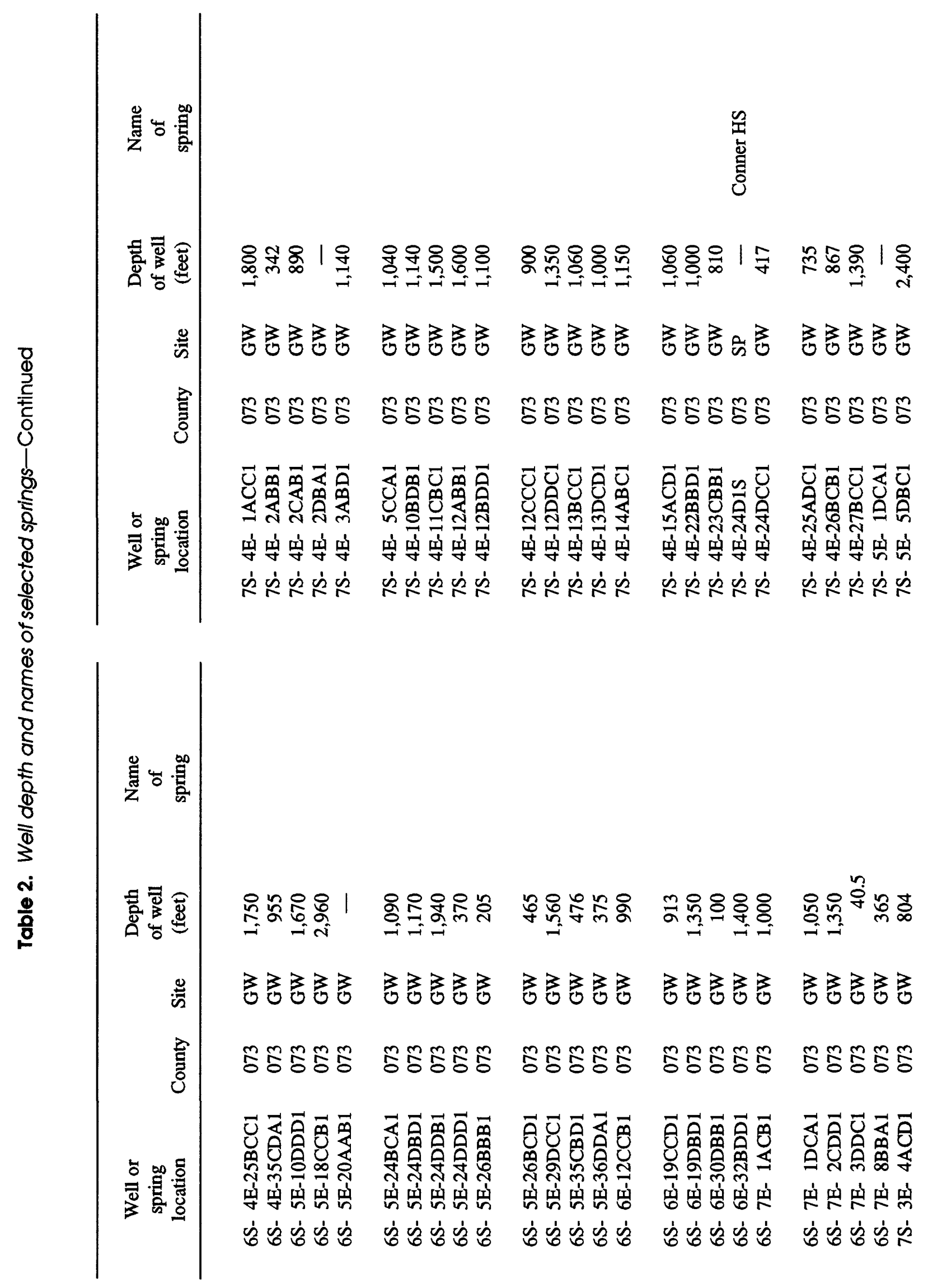














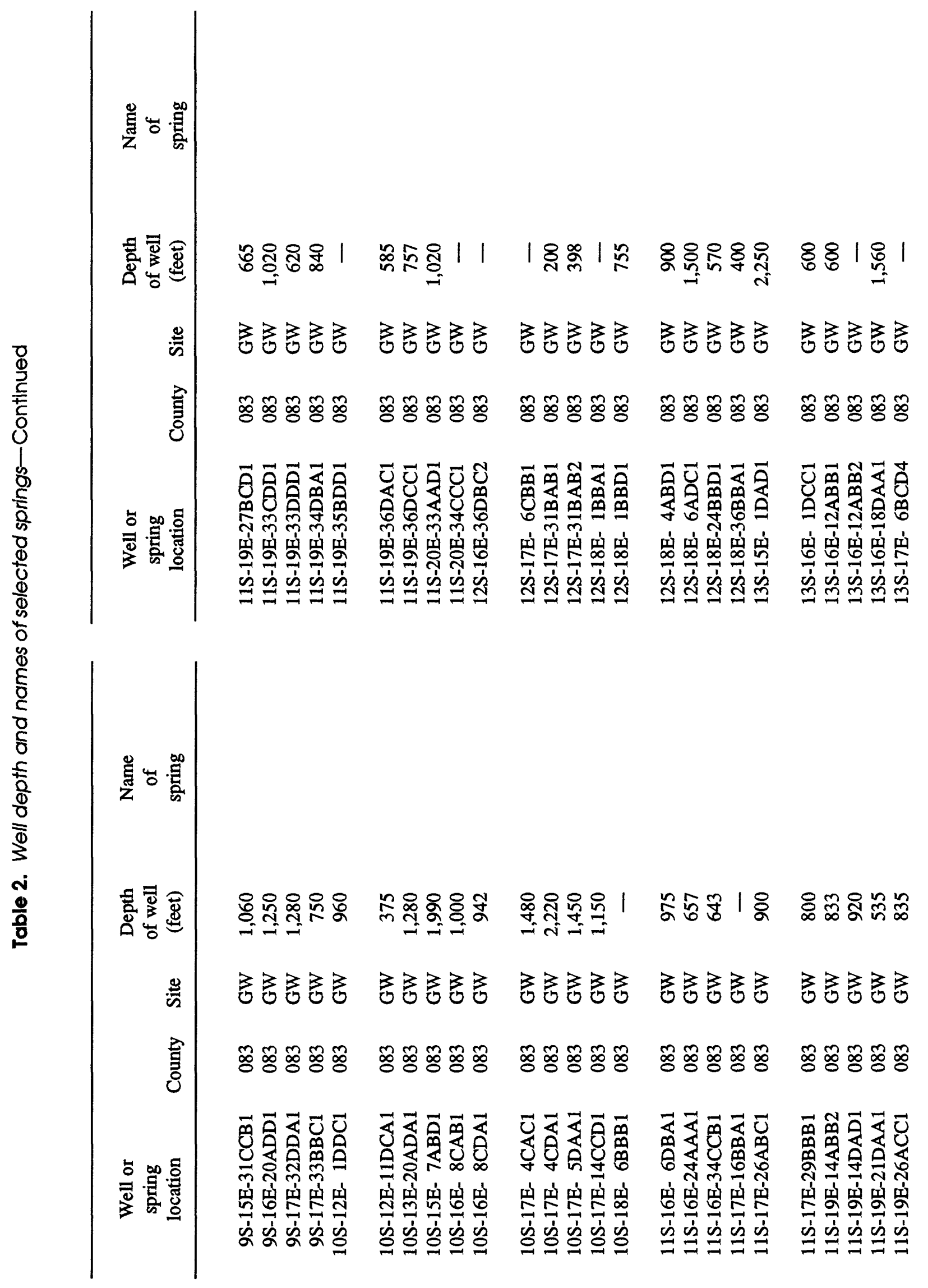




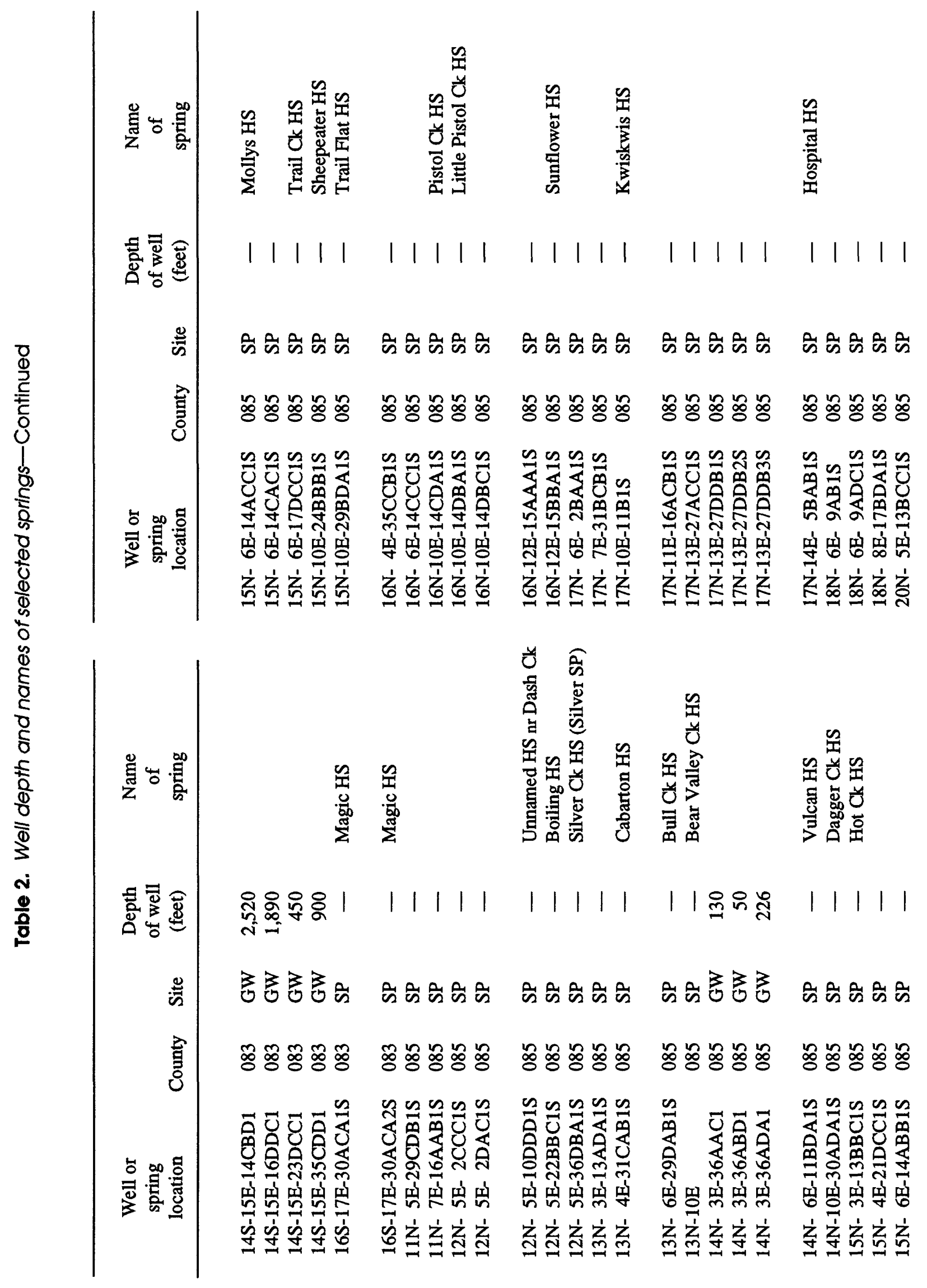




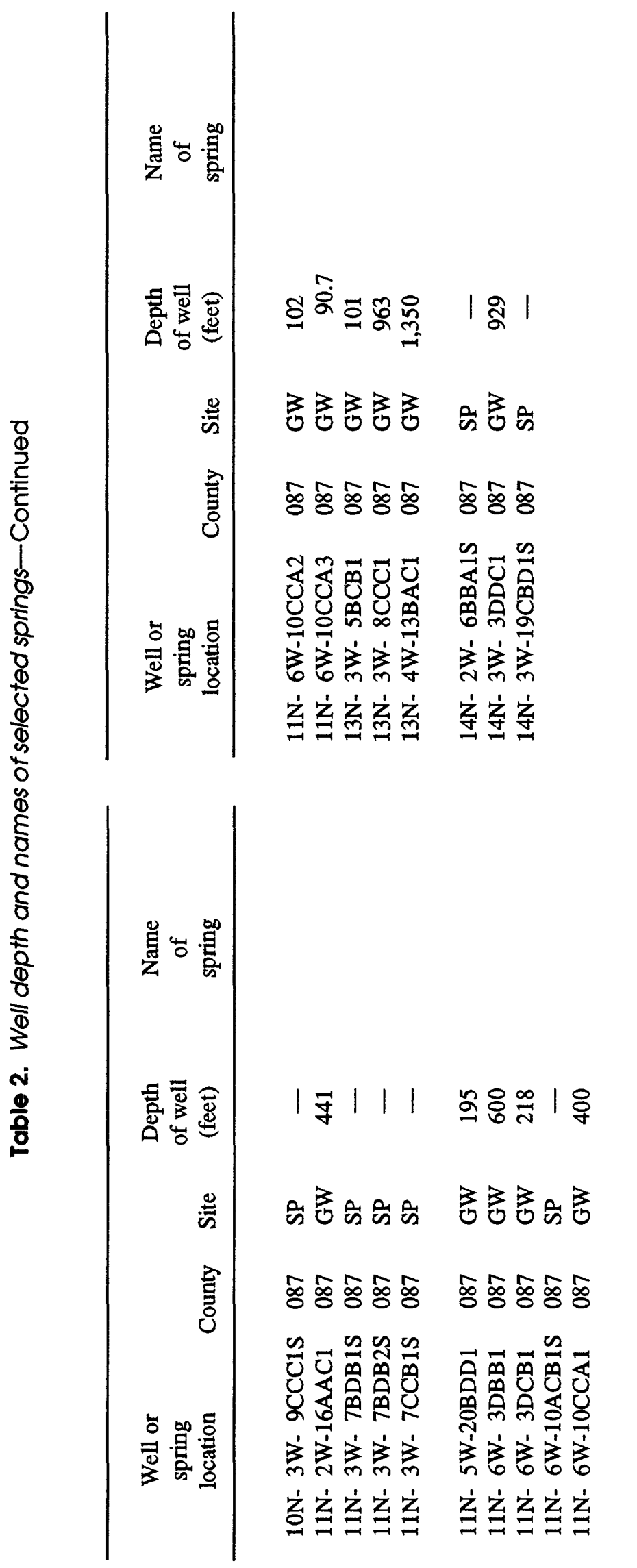


Table 3. Chemical and isotopic analyses of water from selected thermal-water wells and springs

\section{Headnotes}

WELL OR SPRING LOCATION: Well or spring location in township, range, section (fig. 1)

COUNTY:

001-Ada
003-Adams
005-Bannock
007-Bear Lake
011-Bingham
013-Blaine
015-Boise
019-Bonneville
023-Butte
025-Camas
027-Canyon

029-Caribou
031-Cassia
033-Clark
037-Custer
039-Elmore
041-Franklin
043-Fremont
045-Gem
047-Gooding
049-Idaho
051-Jefferson

053-Jerome

059-Lemhi

065-Madison

071-Oneida

073-Owyhee

077-Power

081-Teton

083-Twin Falls

085-Valley

087-Washington

SITE:

GW

SP

ground water (well)

spring

DATE: date of sample

DEG C or DEG. C degrees Celsius

US/CM microsiemens per centimeter at 25 degrees Celsius

WAT water

WH whole

TOT total

FET fixed end-point titration

MG/L milligrams per liter

IT incremental titration

FLD field (onsite) determination

UG/L micrograms per liter

$\mathrm{PCI} / \mathrm{L}$ or $\mathrm{PC} / \mathrm{L} \quad$ picocuries per liter

$\mathrm{ND}$

C-13/C-12

$\mathrm{H}-2 / \mathrm{H}-1$

not detected (concentration less than the minimum laboratory detection limit)

O-18/0-16

S-34/S-32

RA-226

U-NAT

carbon-13/carbon-12

deuterium

oxygen-18/oxygen-16

sulfur-34/sulfur-32

radium-226

uranium, natural

SR/YT-90

CS-137

strontium/ytrium-90

cesium-137

A.A.S.

atomic absorption spectroscopy

$--$

data not available

$<$

less than

greater than

E estimated

* water sample obtained by Idaho Department of Water Resources personnel but analyzed by

USGS laboratory 


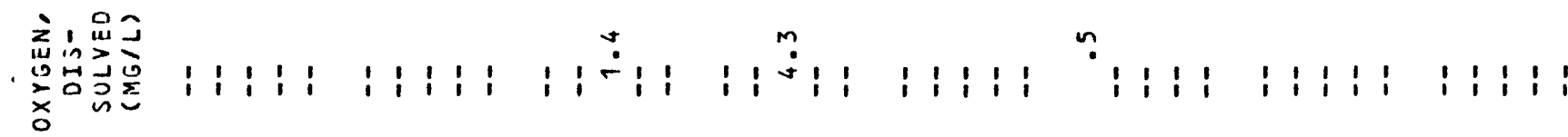

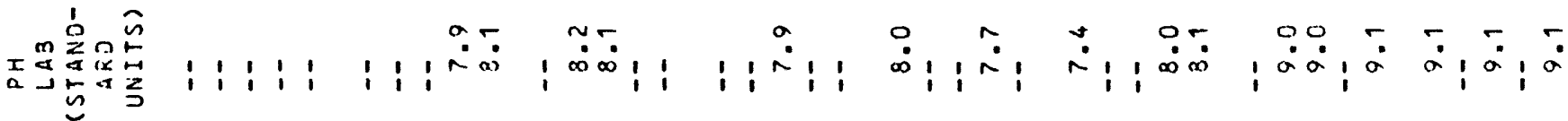

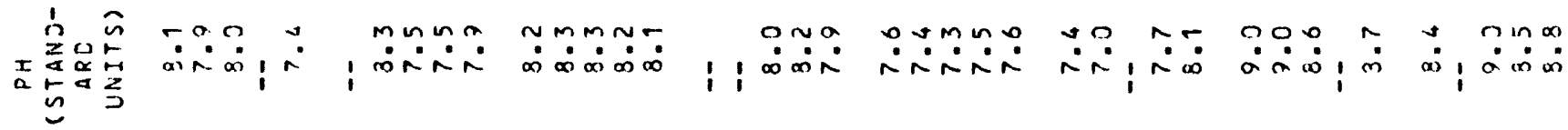

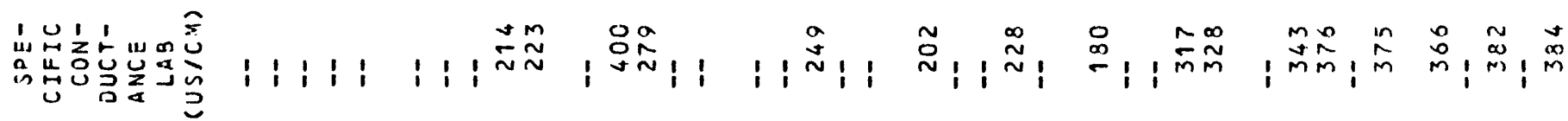

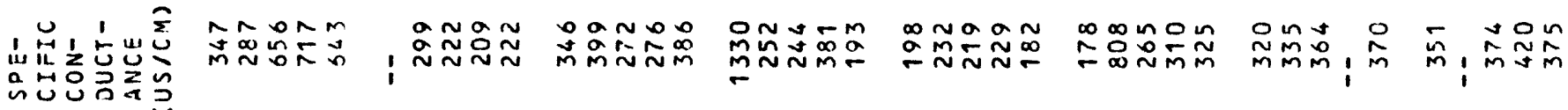
क्य

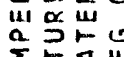

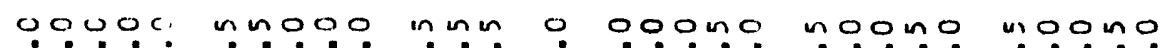

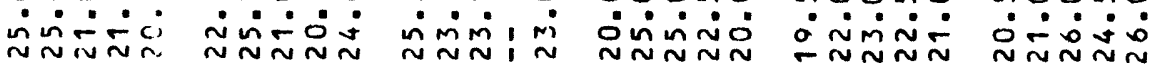
000 on un oom

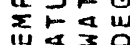
añis bisiog

\section{0
$\infty$}

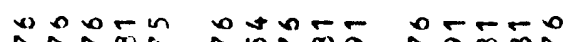

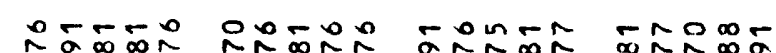

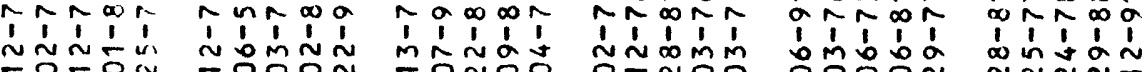

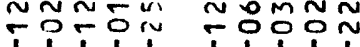

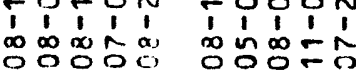
TO iา $\infty \infty$ $\hat{n}$

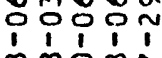

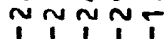

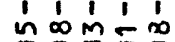
*

nom $n$

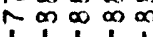

l $\begin{array}{lllll}1 & 1 & 1 & 1 & 1 \\ 0 & 1 & 1 & 0 & 0\end{array}$

Nㅜㅇㅛ

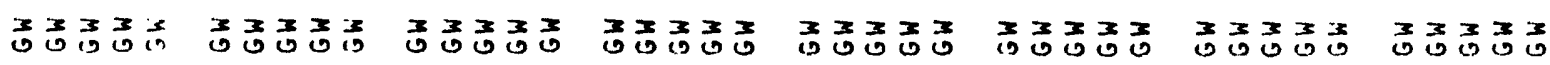

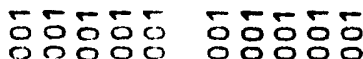

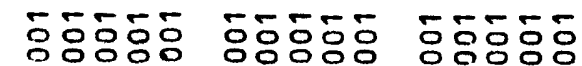

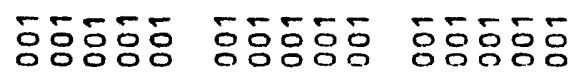

\begin{tabular}{|c|c|c|c|c|c|c|c|c|c|c|c|c|c|c|}
\hline 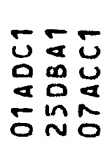 & $\begin{array}{l}\dot{y} \\
\dot{a} \\
a \\
c\end{array}$ & 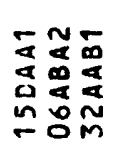 & $\begin{array}{l}5 \\
\vdots \\
\infty \\
n \\
0\end{array}$ & $\begin{array}{l}5 \\
0 \\
a \\
a \\
0 \\
m\end{array}$ & $\begin{array}{l}5 \\
0 \\
0 \\
0 \\
0 \\
m\end{array}$ & 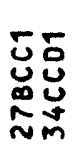 & 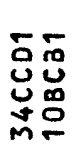 & 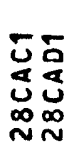 & $\begin{array}{l}5 \\
\vdots \\
\alpha \\
0 \\
0\end{array}$ & 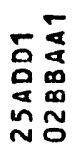 & $\begin{array}{l}\text { on } \\
0 \\
0 \\
u \\
\sim \\
0\end{array}$ & 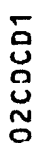 & $\begin{array}{l}5 \\
0 \\
0 \\
a \\
a \\
0 \\
\sigma\end{array}$ & 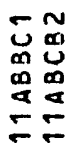 \\
\hline $\begin{array}{l}m x \\
505\end{array}$ & $\frac{5}{5}$ & 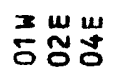 & $\frac{x}{5}$ & $\frac{1}{0}$ & $\frac{7}{5}$ & $\frac{x}{5}$ & 㟧㟧 & mm & $\frac{m}{0}$ & 풍 & $\underset{\sim}{\sim}$ & $\underset{\sim}{\sim}$ & $\underset{\sim}{\sim}$ & $\underset{\sim}{\sim} \underset{\sim}{\sim}$ \\
\hline $\begin{array}{l}2 z 2 \\
050\end{array}$ & $\frac{2}{0}$ & $\begin{array}{l}z z 2 \\
\frac{1}{0} 5\end{array}$ & $\frac{1}{0}$ & $\frac{1}{0}$ & $\frac{n}{0}$ & $\begin{array}{l}2 z \\
\tilde{0} \\
0 \%\end{array}$ & $\begin{array}{ll}z & z \\
\tilde{N} & 0\end{array}$ & $\begin{array}{l}z z \\
\mathbb{N} \tilde{N}\end{array}$ & $\stackrel{z}{m}$ & zz & $\stackrel{z}{m}$ & $\begin{array}{l}z \\
0\end{array}$ & $\begin{array}{l}z \\
0 \\
0\end{array}$ & $\begin{array}{l}z z \\
M \\
0\end{array}$ \\
\hline
\end{tabular}




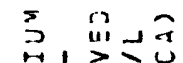

Si $>>15$

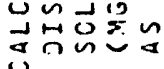

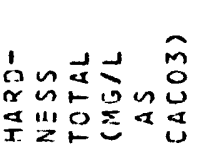

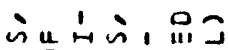

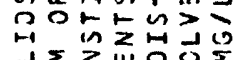

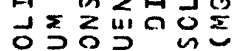

madom m nomno

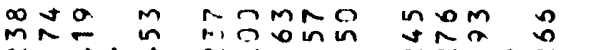
的心只

初出, 品正 둔ㅁำ

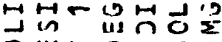
○แผ

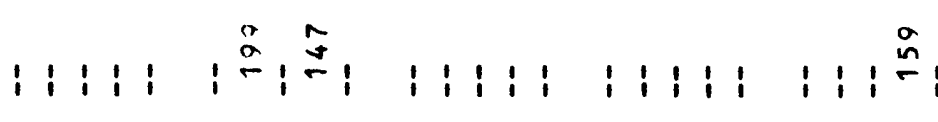

zํㅡ, ก,

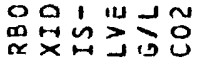

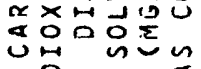

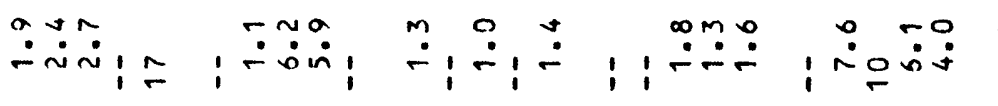

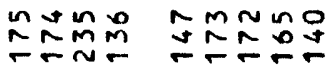

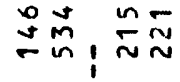

$\stackrel{2}{2} \stackrel{2}{\sim}$ $\stackrel{n}{n} ;: \stackrel{n}{n}, \stackrel{n}{n} \stackrel{n}{n}$ ! uI

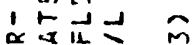

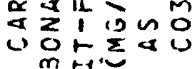

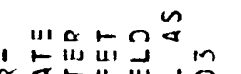
a

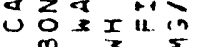

' "I"'

aㅁ

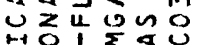

th

1 11111111

1:只: 1:品

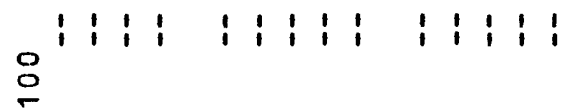

1 $w \propto ⿻ 0 \%$

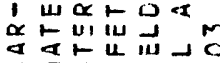

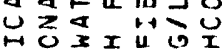
$m_{m}=14$

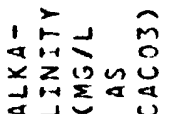

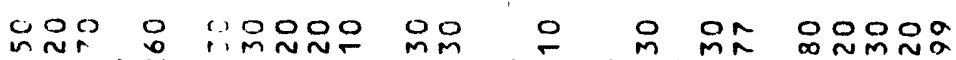

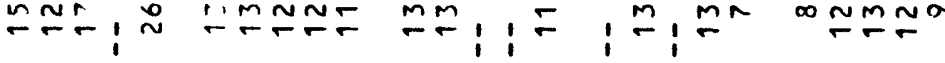

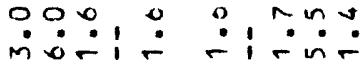

$\infty \stackrel{\infty}{\infty}, i$

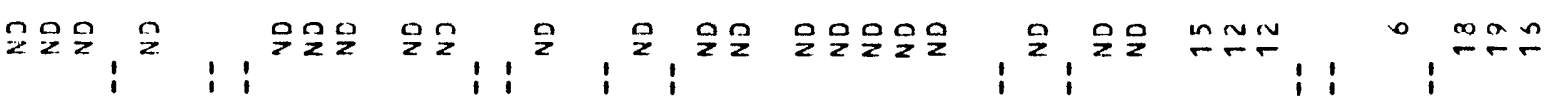

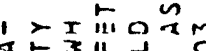

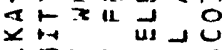

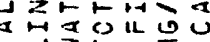

$\stackrel{\infty}{\sim} \underset{\sim}{\infty}, \stackrel{\circ}{\sim}$

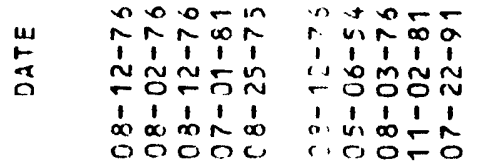

no-

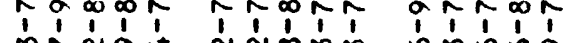

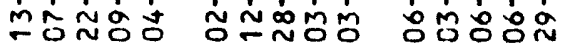

1111

icion

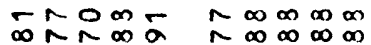

$\infty$

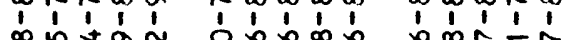

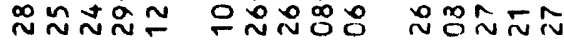
0000

111

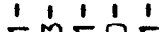

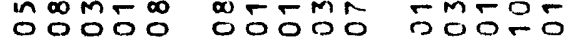




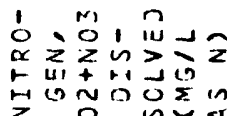

군,

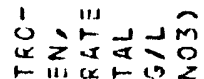

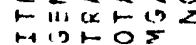

글을

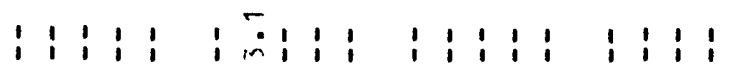

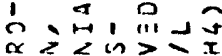

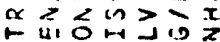

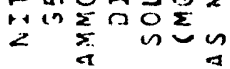

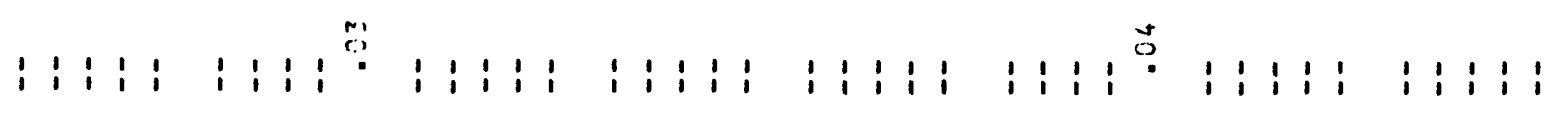

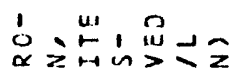

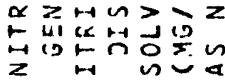

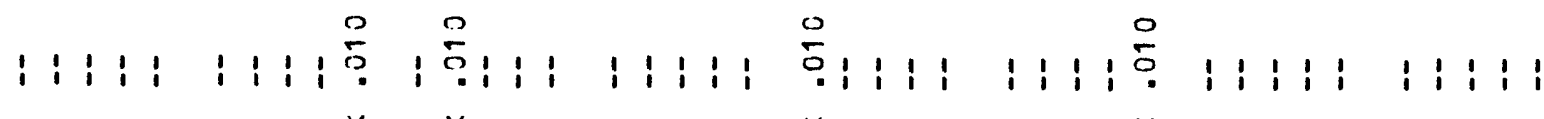

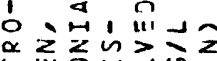

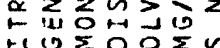

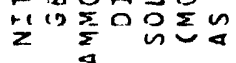

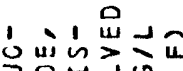

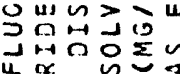

i i i i i i i i

$i \frac{0}{3} i$

$\checkmark$

$\checkmark$

$\frac{0}{0} 1: 1$

$i: i i_{0}^{\stackrel{0}{0}}$

$\checkmark$

$\checkmark$

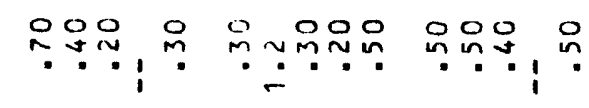

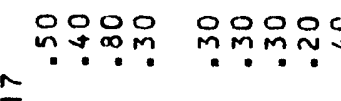

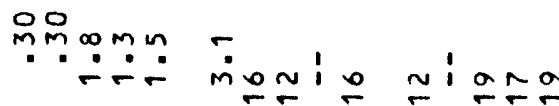

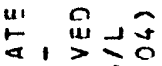

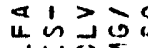

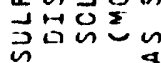

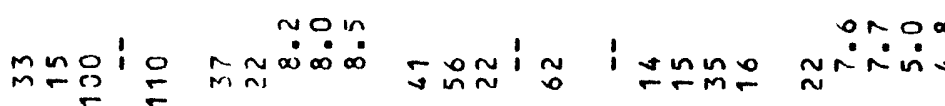

ioliza ñmiñ

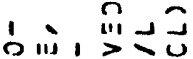

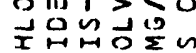

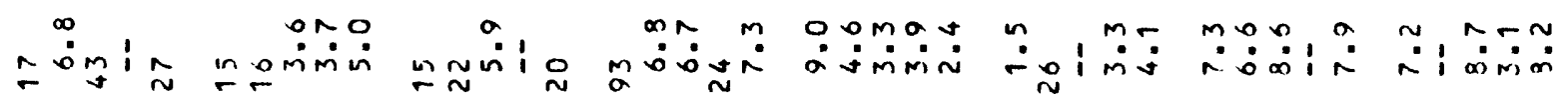

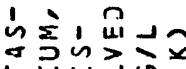

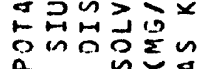

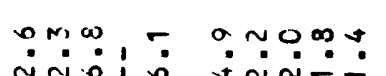

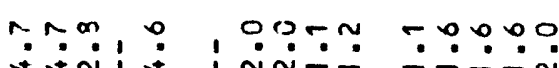

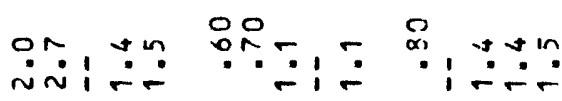

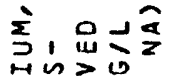

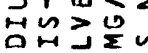

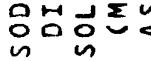

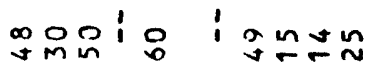

$\underset{y}{\infty} \operatorname{og} \operatorname{li}^{1}$ in

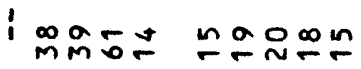

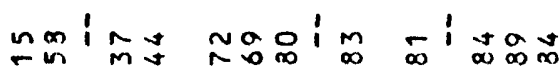

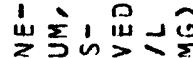

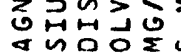

$\infty$

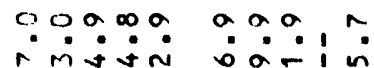

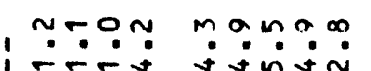

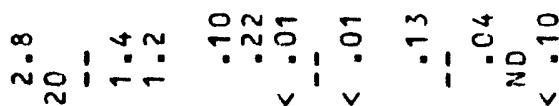

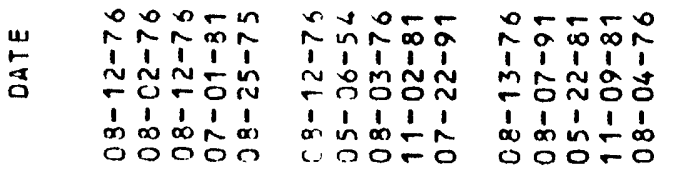

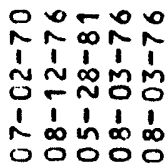

axinga

rnom-

$\sim \infty \infty$ a) $\infty$

1'Tิ1

응응웅

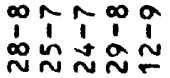

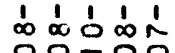

致的亩

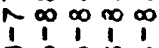

응

1́tín

$\infty \infty n$

$\infty, \infty \sim \infty$

00000

o ocó

1 0 政

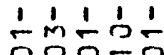




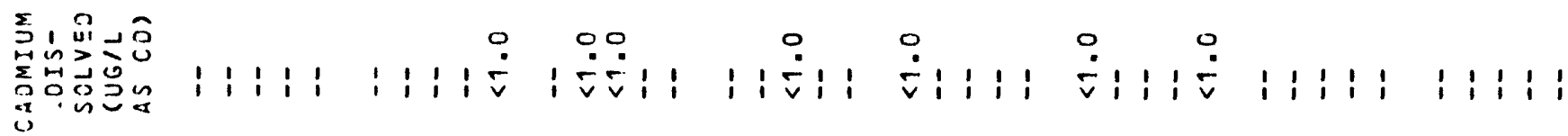

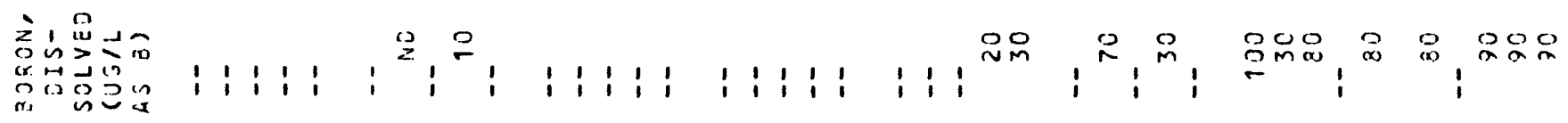

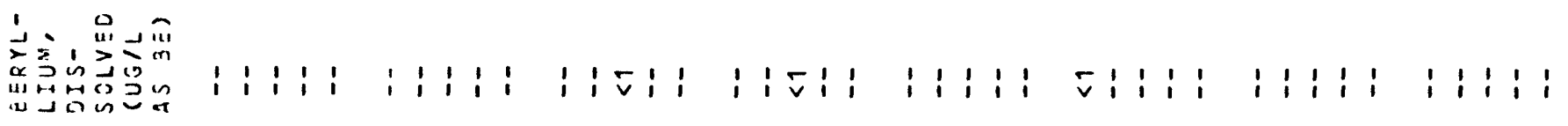

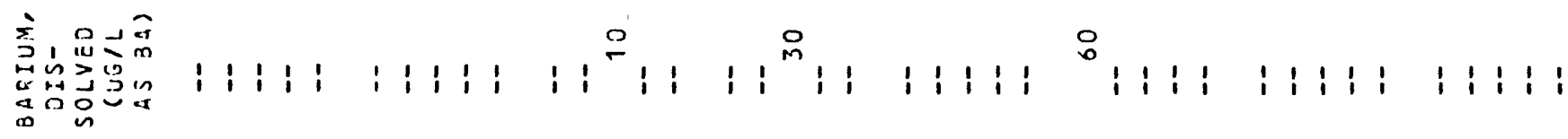

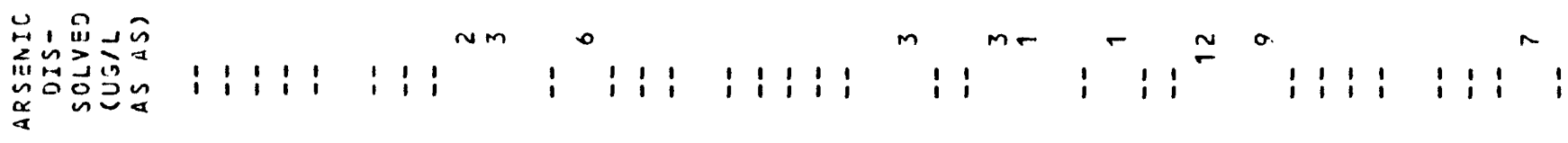

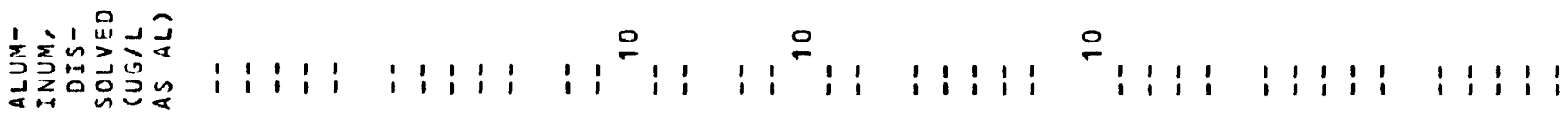

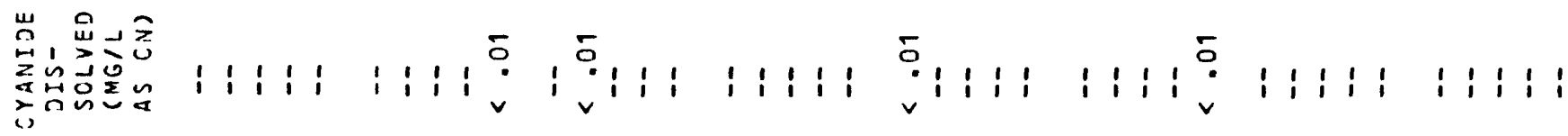

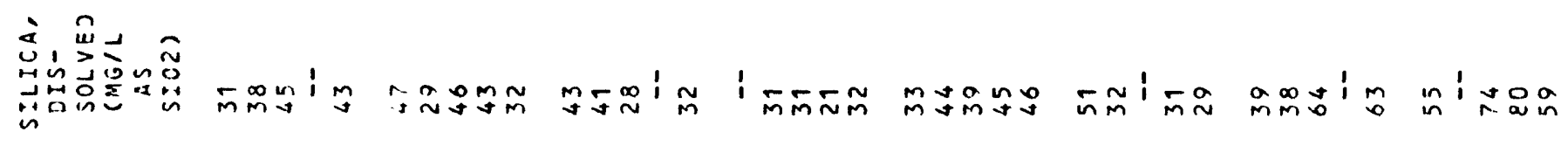

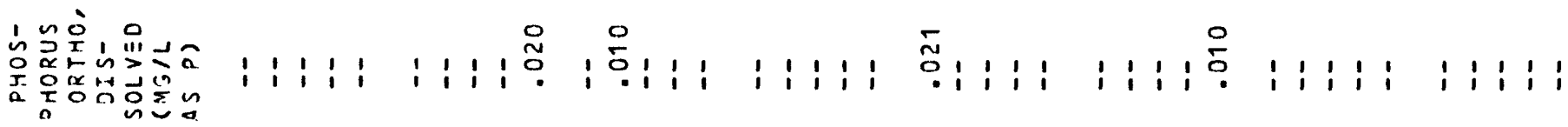

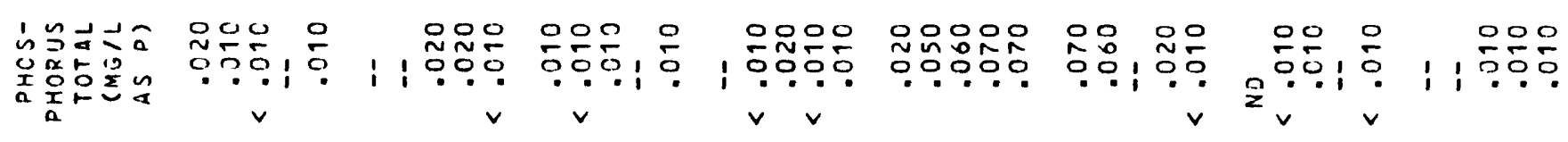

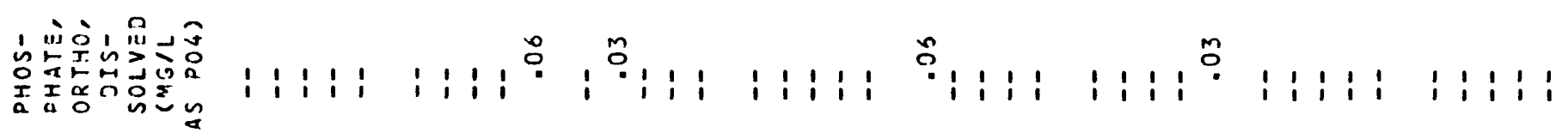

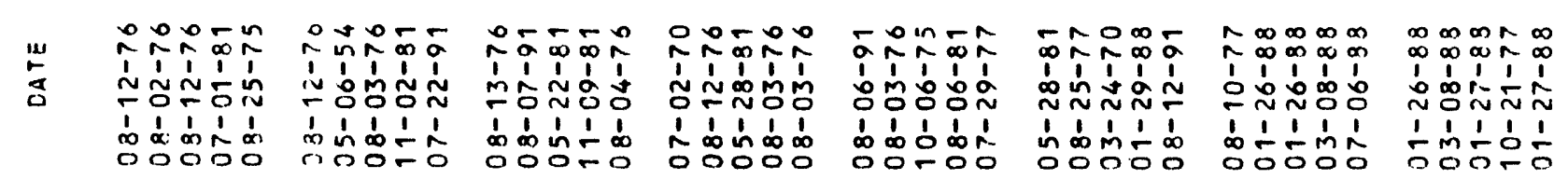




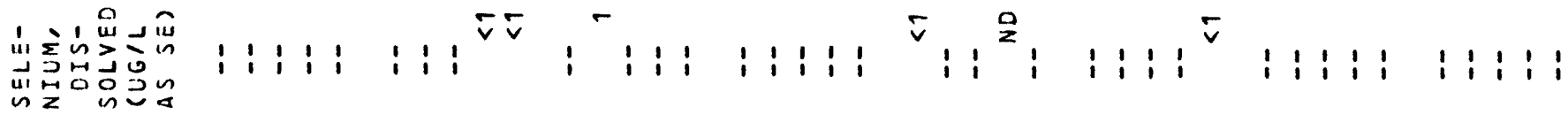

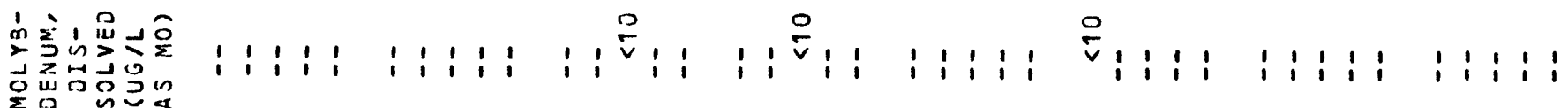

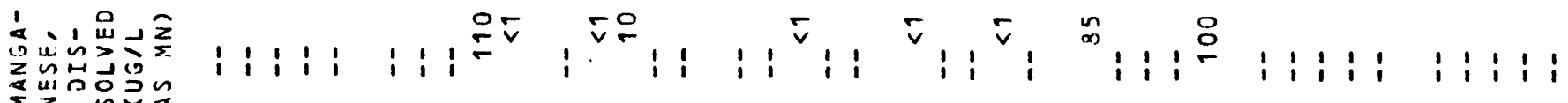

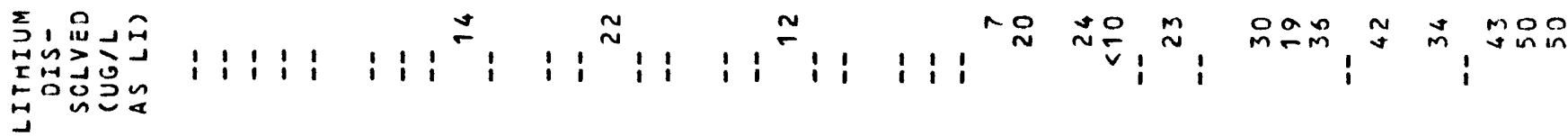

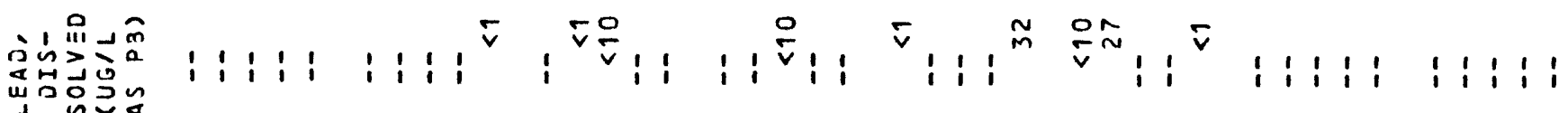

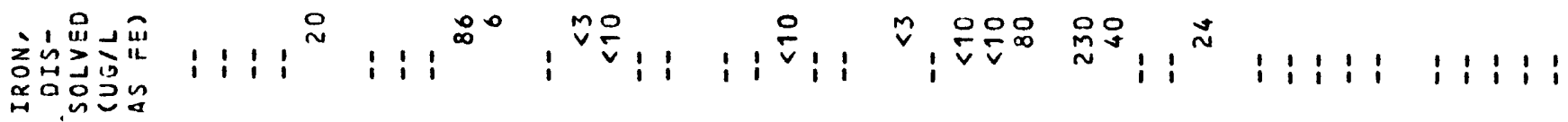

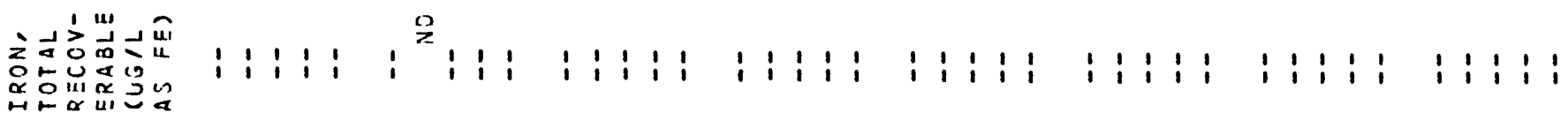

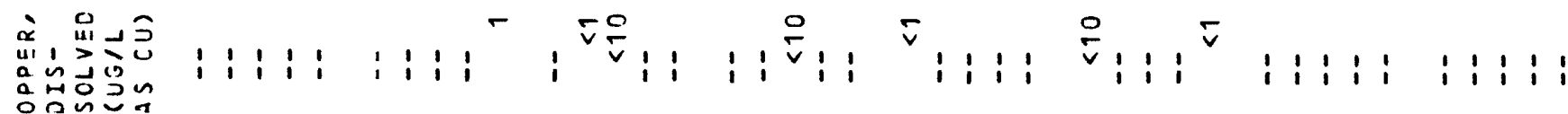

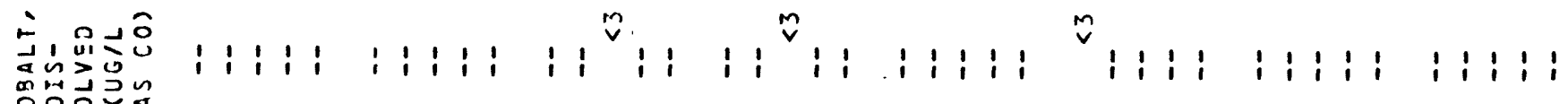

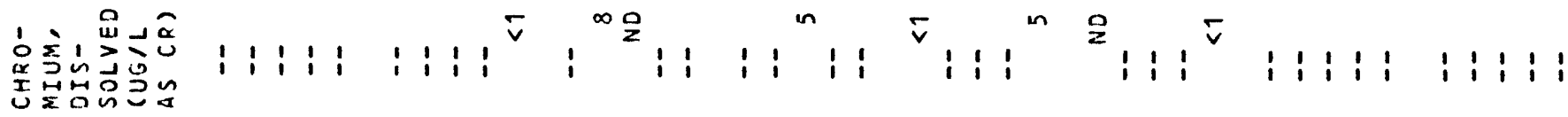

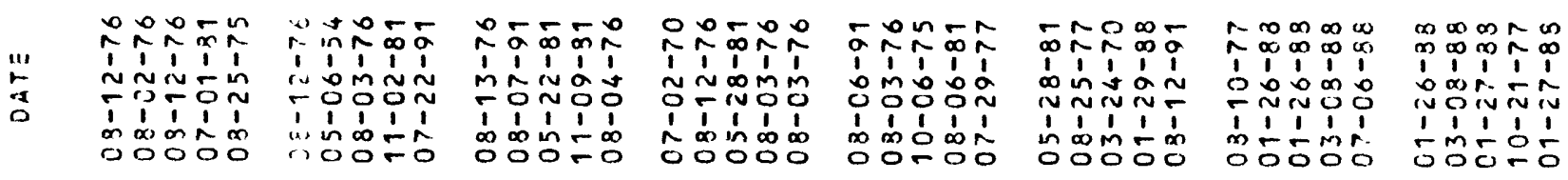




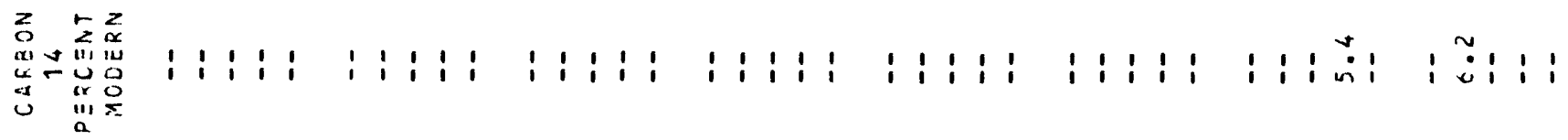

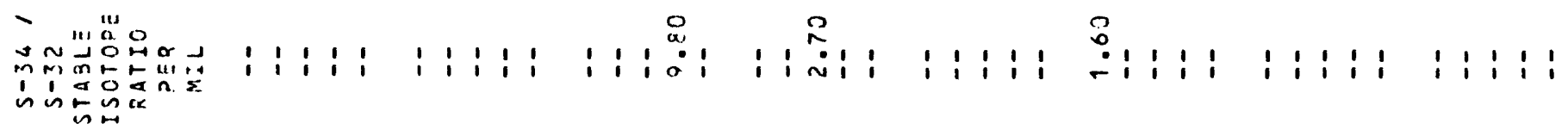

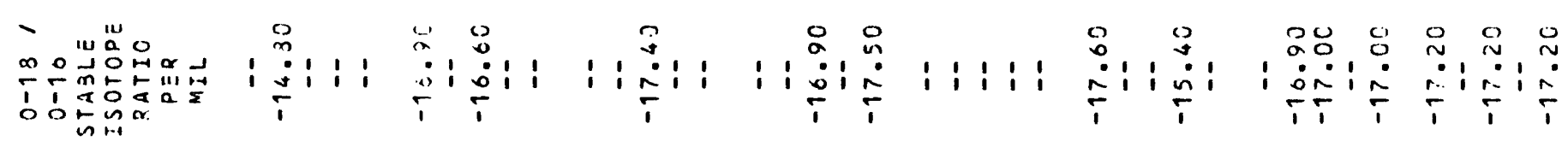

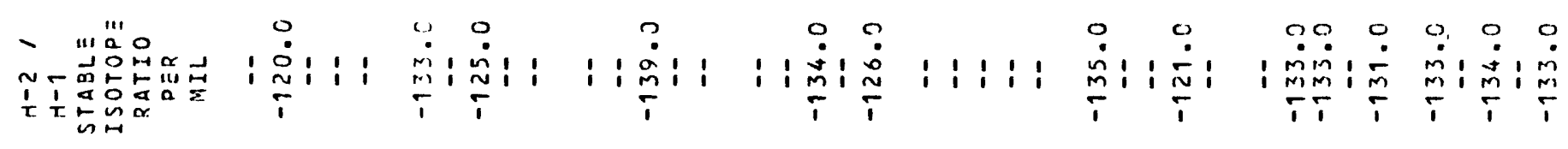

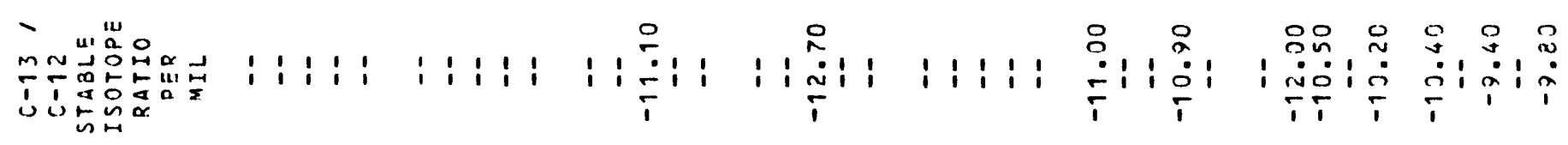

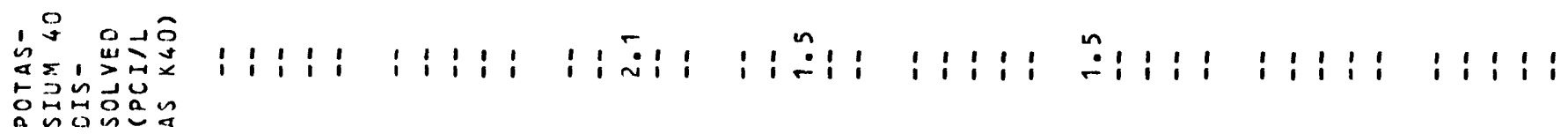

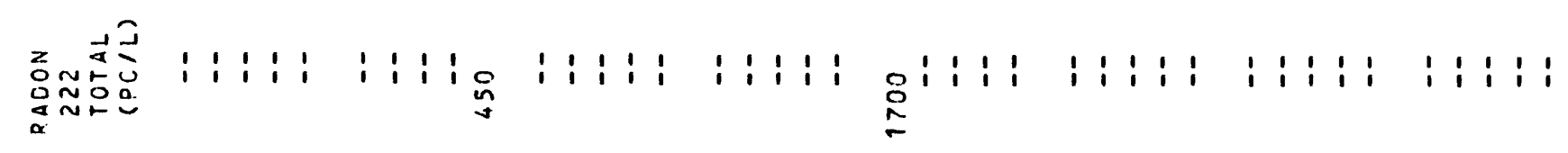

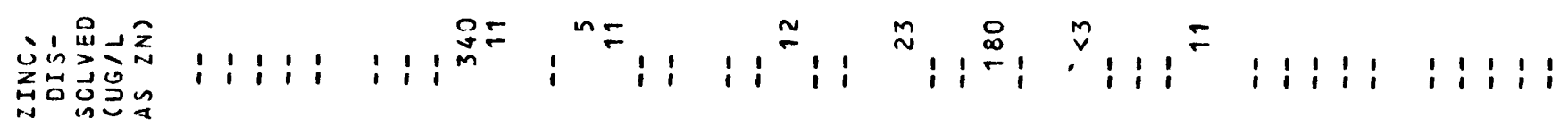

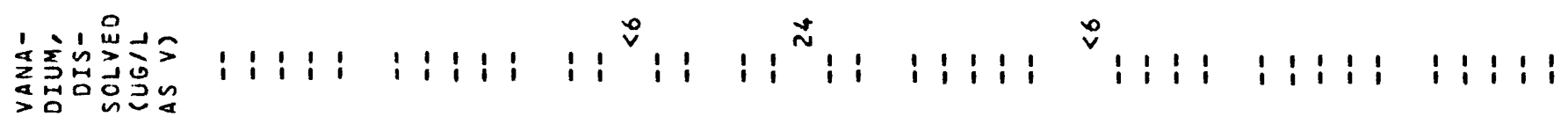

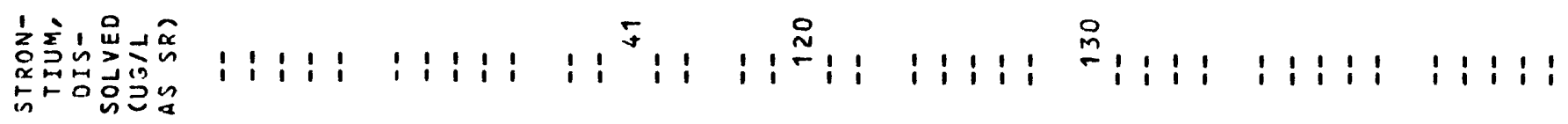

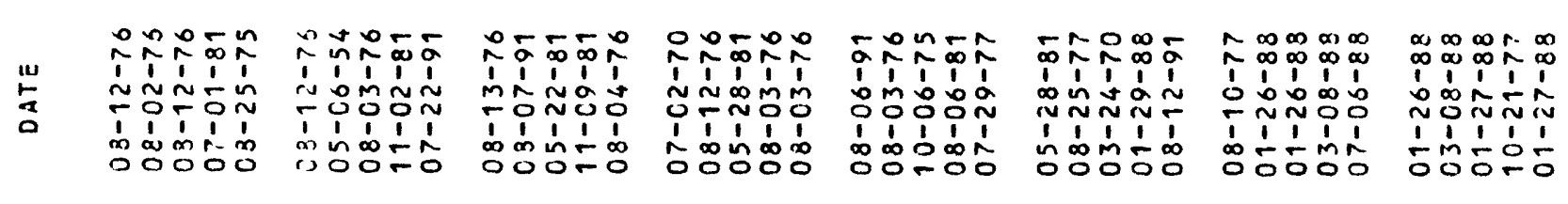




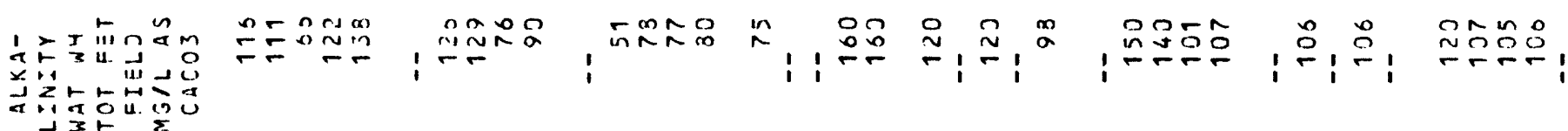

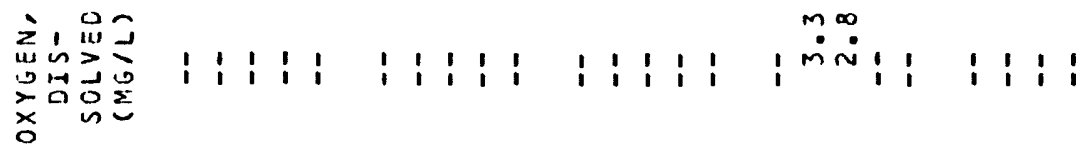

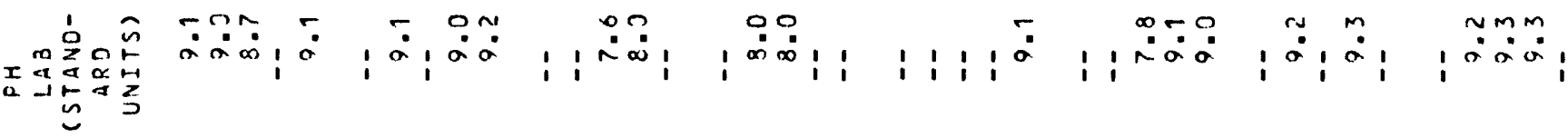

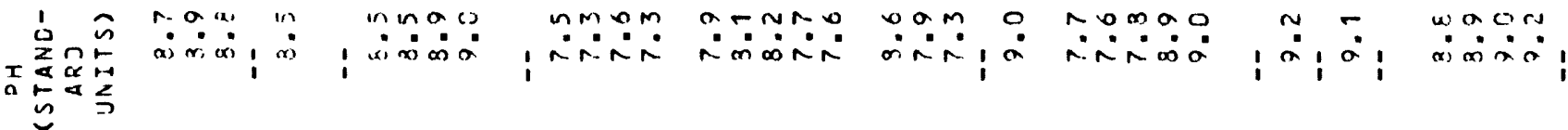

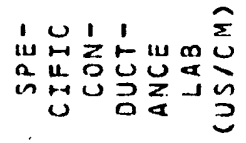

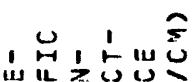

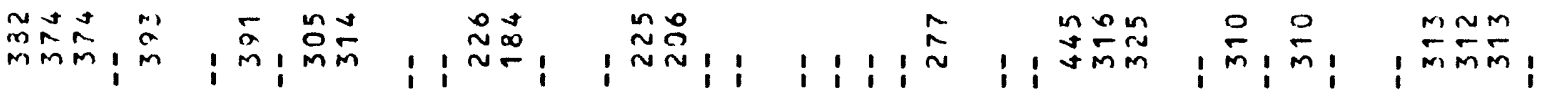

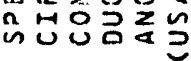

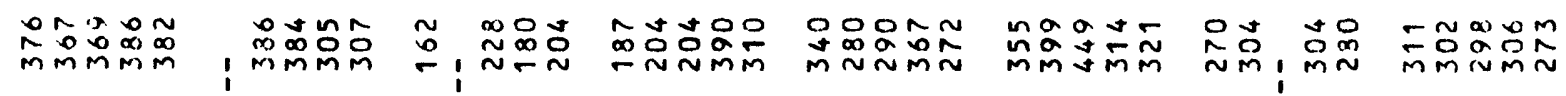

$\dot{\alpha} u \propto \hat{U}$

minom

ono in ? nn ? ?

0 in in in

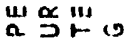

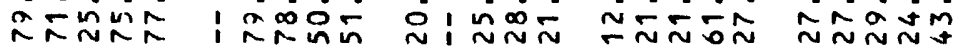

a $-0 \pm m$

in -1

ᄉ $\cos$

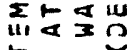

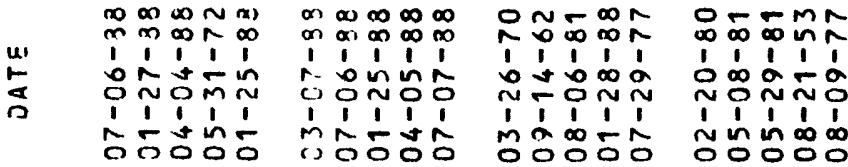

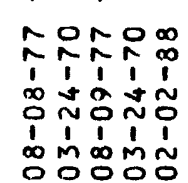

$0 \ln \infty \infty$ i $110 \infty$ mo 051

ว

$0 \infty \infty \infty 0$

$\sim \infty \infty \infty$

$N \infty \infty \infty 0$ 1 11 1 $10 \%$

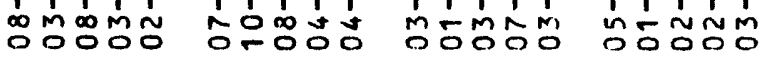

ज禹而芯

- $N$ N

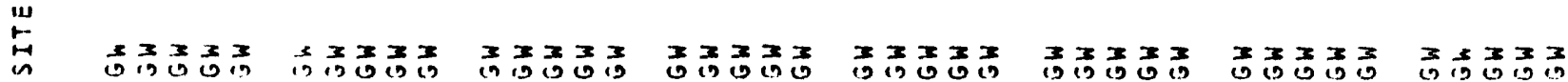

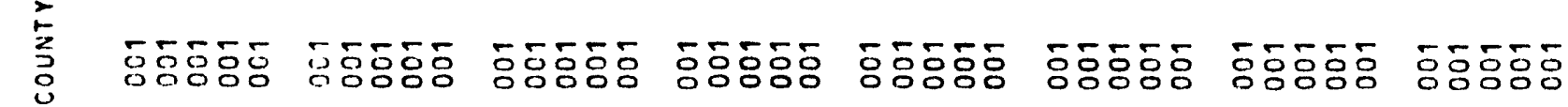

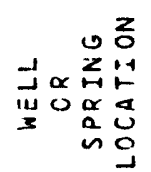

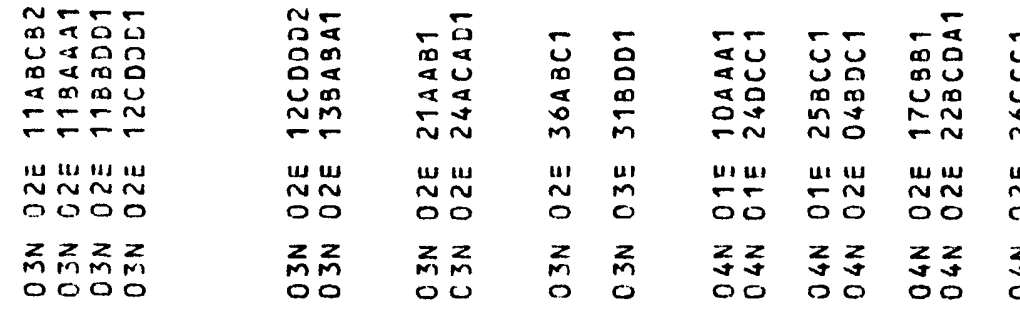

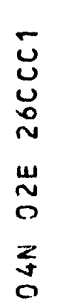

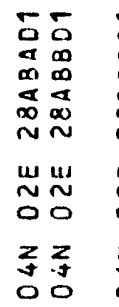

可可

문 a क क กับ

III) แI חת 중죠 000 


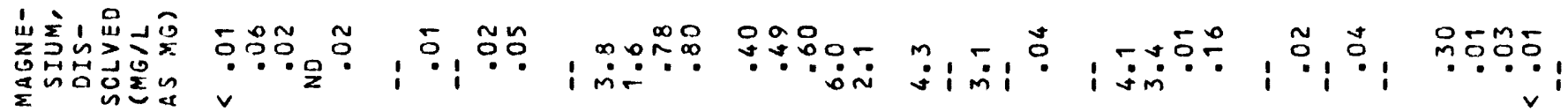

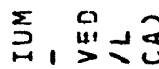

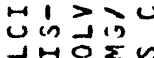

onson

$\because, \because \infty$,

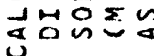

$\therefore \dot{0}$

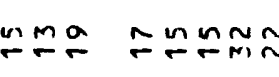

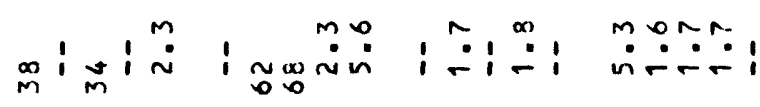

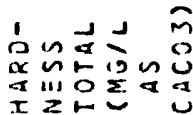

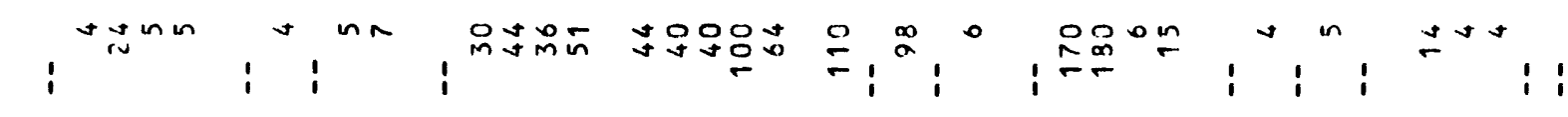

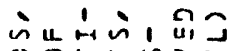

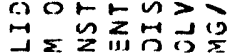

त绍

minn

\section{$m a$}

nom nntor

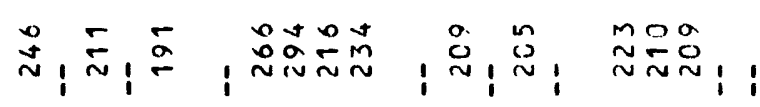

的苛。”品

只怘:

11

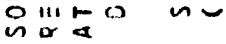

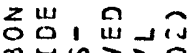

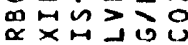

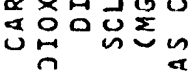

í: i i i i i i

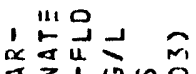

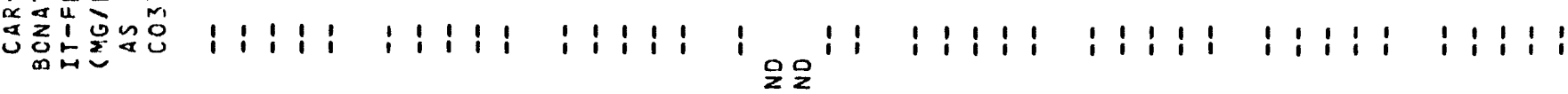

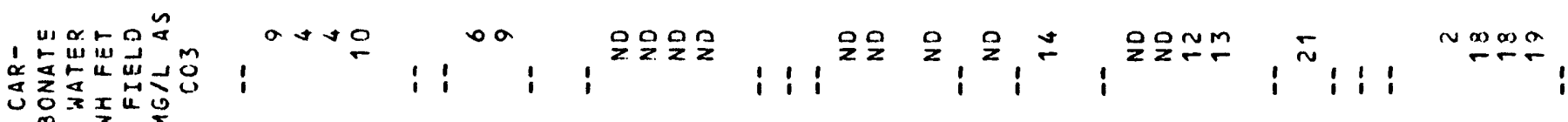

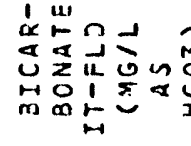

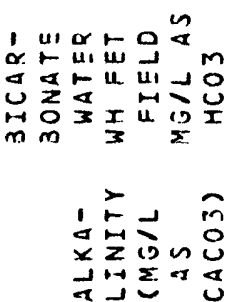

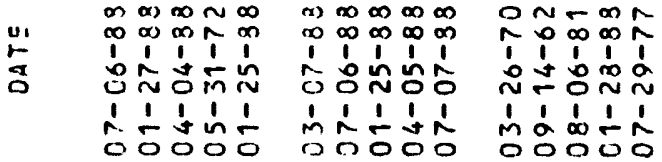

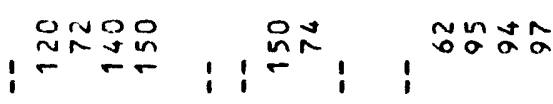

$1: 1: 1: 1: 1: 1: 1: 1: 1$

'용ㅇㅁ

1 1 : 요유

으, 은 a

1은음음

$\stackrel{\infty}{\infty}$

$\stackrel{\infty}{i}:$ i

ㅇํㅇำ

$1: 11:$

$1: 11: 11: 11$

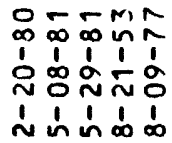

무숭

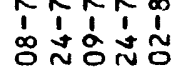

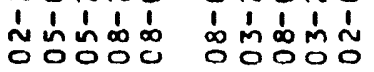

$0 \backsim-\infty \infty$

î́n

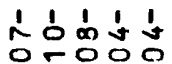

$0 m \infty \infty$

î:

崩余合

mํํำ

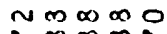
i $\infty$ on mñ丶 ํำ

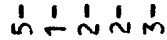
DOOOO 


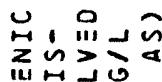

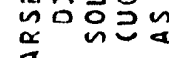

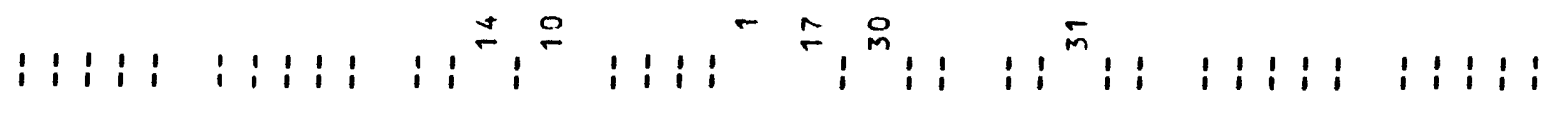

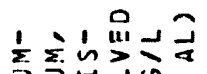

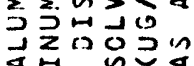

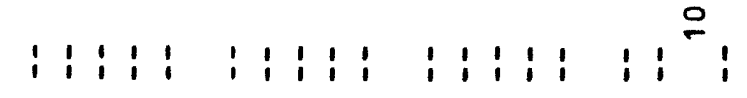

这, 品」

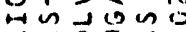

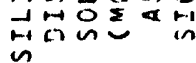

$m m m \infty \alpha$

$i \sim \lim _{\infty}$

i i n a

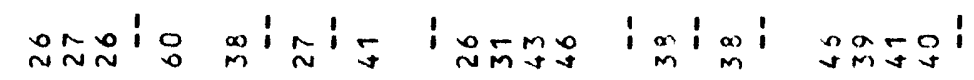

的苯さล

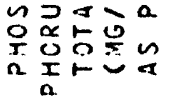

$\frac{0}{3} \frac{0}{0}, \frac{5}{0}, \frac{5}{5}, 1, \frac{5}{0}$

옹유

(1)

$\stackrel{0}{0} \frac{0}{0} \stackrel{0}{\frac{0}{\div}}$

$\frac{0}{5}, \frac{0}{0}$,

우음

둥 웅음

$\checkmark$

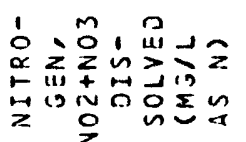

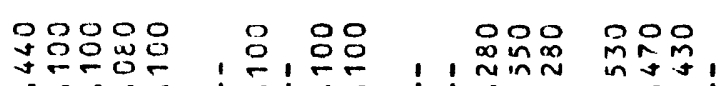

$\checkmark v$

․․

$\checkmark v$

?.? 1 !

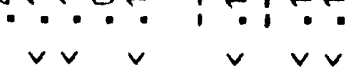

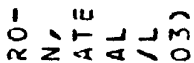

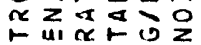

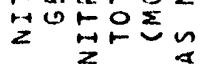

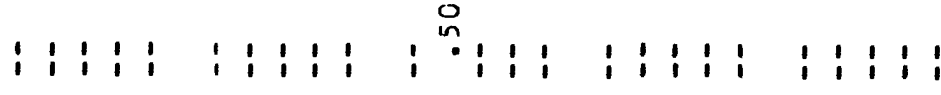

응 웅 웅응

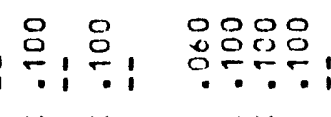

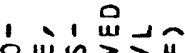

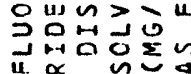

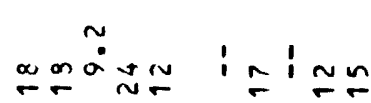

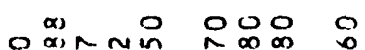

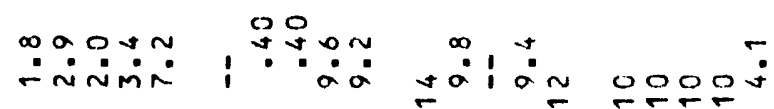

崖, 岃守守

4 出它的

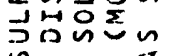

$m m r m \sim$

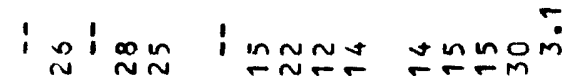

$\rightarrow i \infty i a$

$1+\infty \infty \infty$

$i \stackrel{\sim}{\sim} \dot{\sim} \underset{\sim}{\sim} \sim$

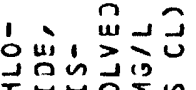

$m--m$

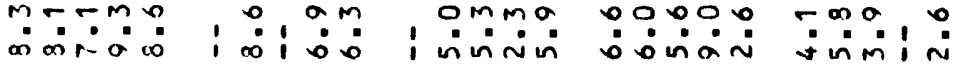

$\infty \sim-\infty$

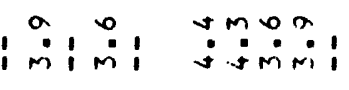

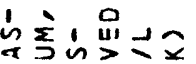

vormo $\quad$ o

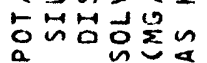

$\because \because \because \div \quad \div 1 \div$

I

$\because \cong \div$

$m-5 m$

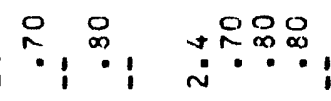

高品势

네

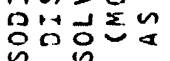

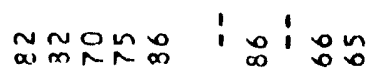

$1 \stackrel{\infty}{\sim} \sim \sim \infty \infty \infty \infty$

$\stackrel{\infty}{\sim}$ i 욤

iะกับ

'ำ

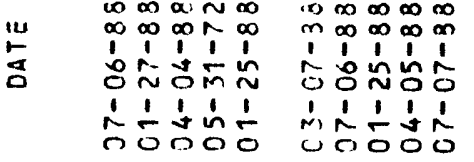

ONoñ Dorñ

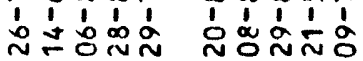

mán

ลก்̃̃

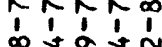

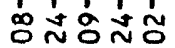

1́n்向向

omioñ

oin $-\infty \infty$

Tर́⿴囗十

$0 \infty \infty \infty 0 \pi \infty a \infty$

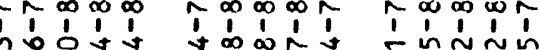

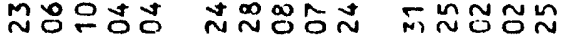

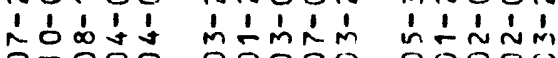
Somoo

กOON 


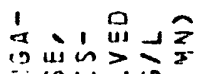

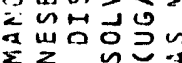

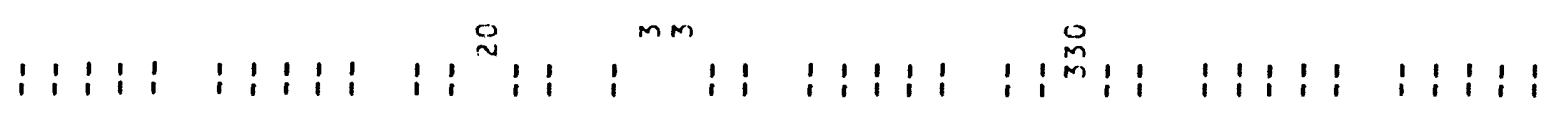

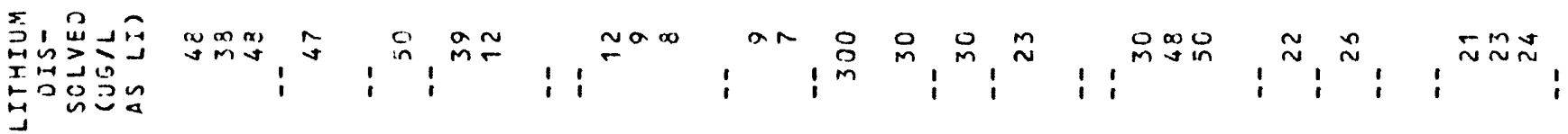

ini

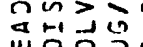

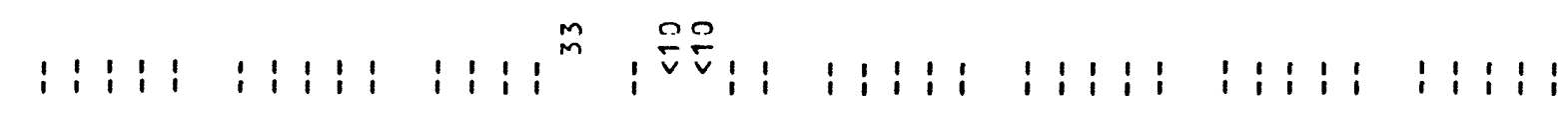

$\sum$ in

injo

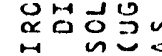

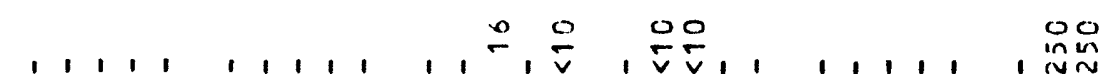

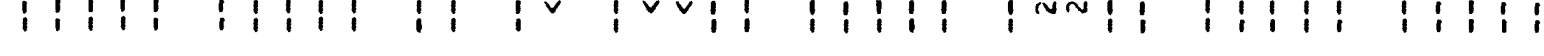

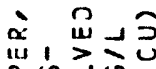

a田嵒的

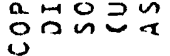

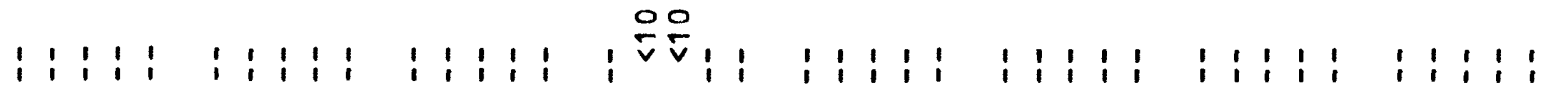

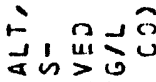

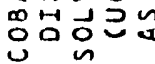

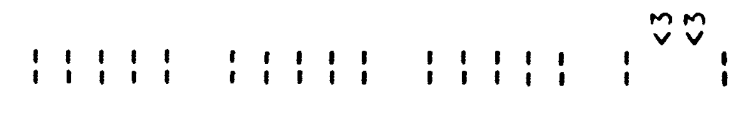

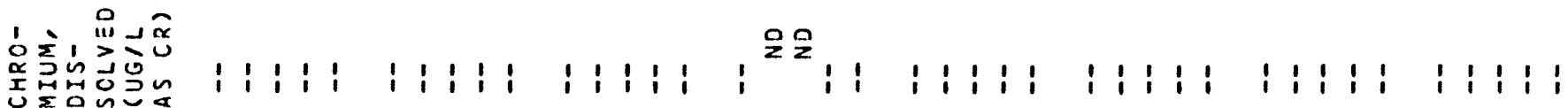

$\sum_{i=1}^{\sum}$ in

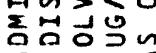

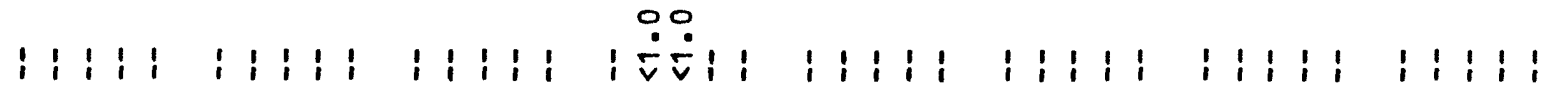

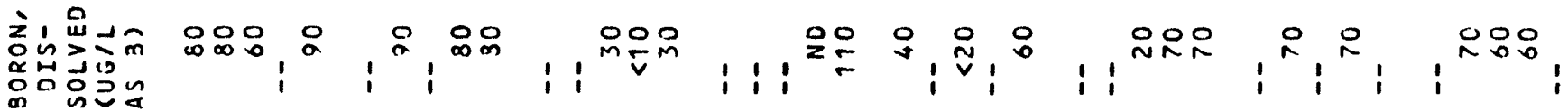

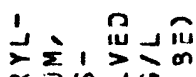

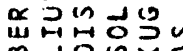

产, 品全

的>0

品兘氙

$1: 1: i 1: 11:$

i i i i i 9 i

$1: 11:$

! : ! !

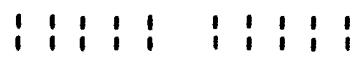

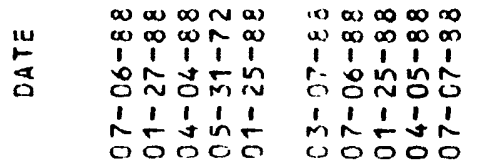

oñon

1! 10 1

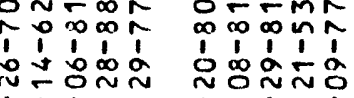

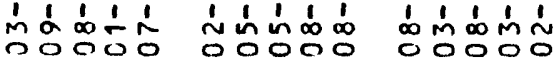

수에 $1\} 11\}$ 응응

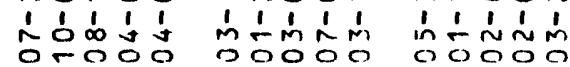




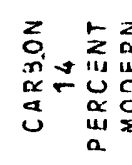

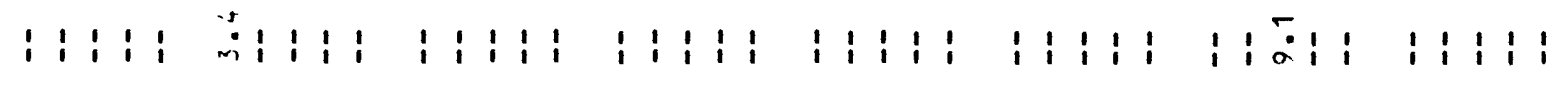

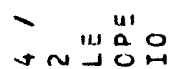

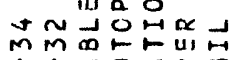

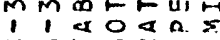

in is

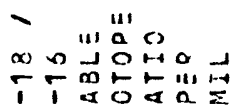

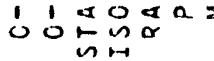

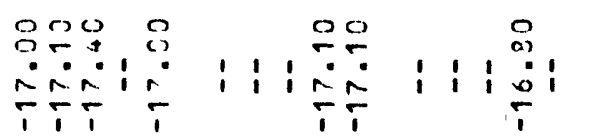

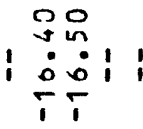

i 1 i :

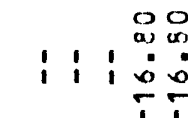

is

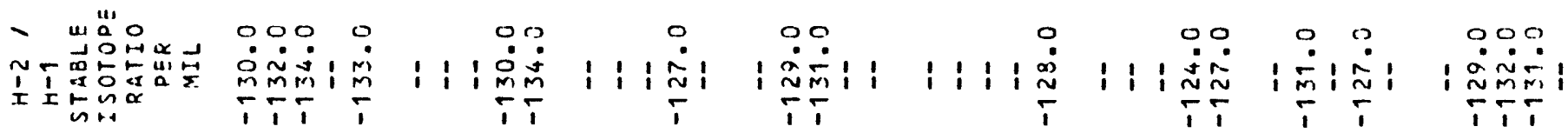

min

نำ

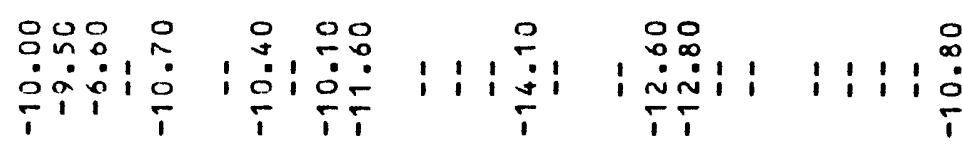

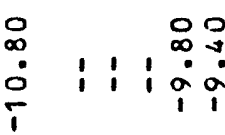

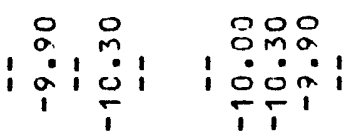

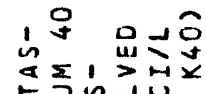

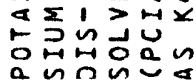

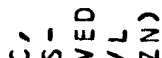

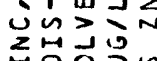

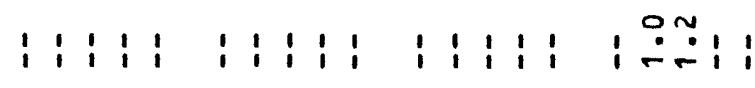

事跑

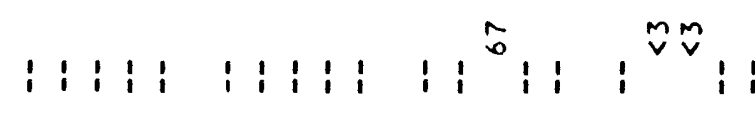

i: $: 1:$ :

i: $1: 1 \quad 1: 1: 1$ : $1: 1:$

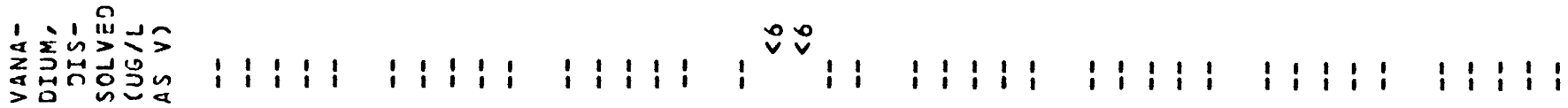

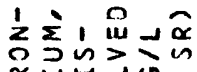

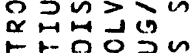

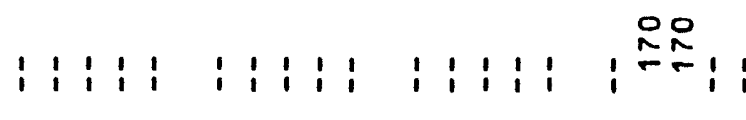

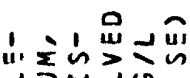

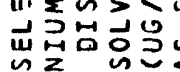

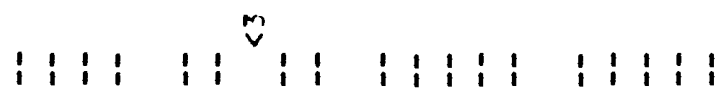

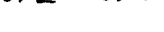

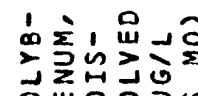

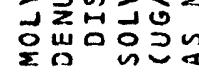

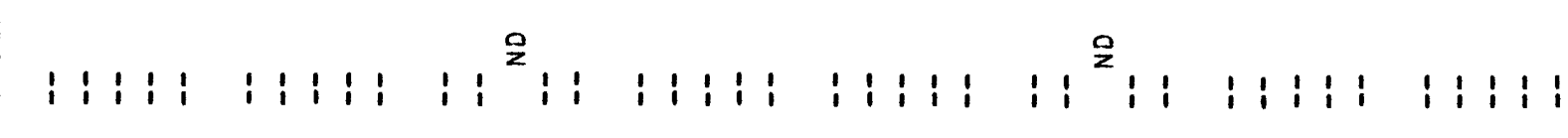




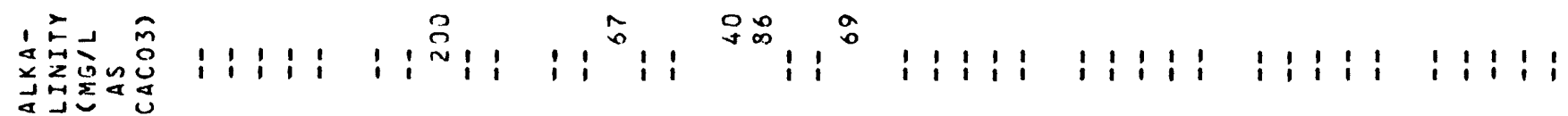

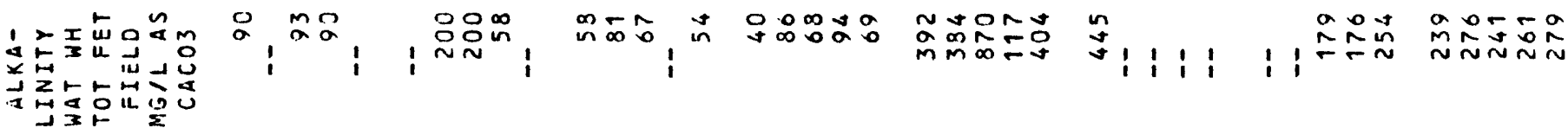

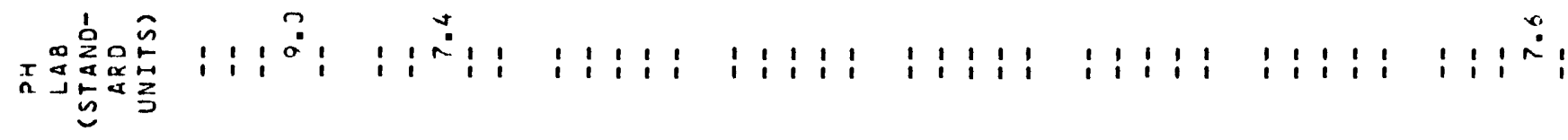

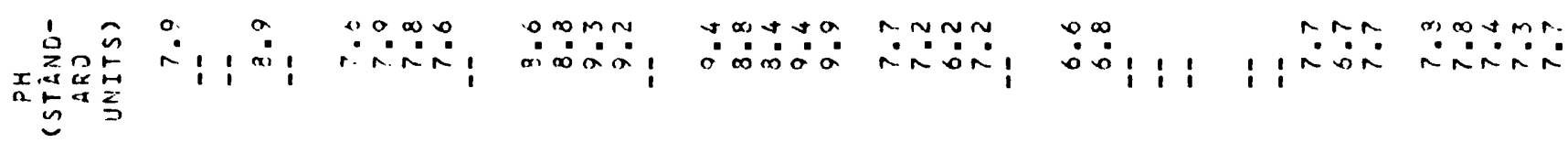

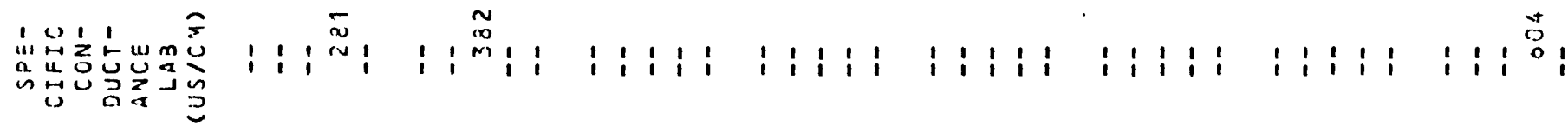

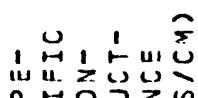

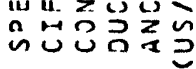

'́w $\propto \hat{U}$

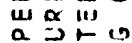
a.

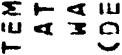

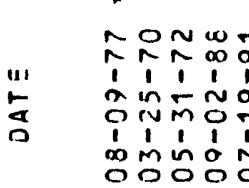
Om nuinim in 0 mol NNNm, NMMM

naña nooga 两

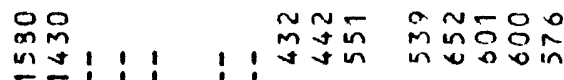
$\operatorname{rin} \pi \approx$ incis ก i

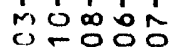

$\operatorname{rin}$ $\sim \pi \infty n$ ramín ñm 11111

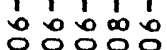

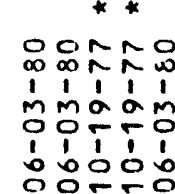

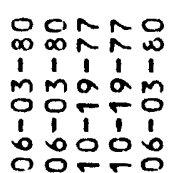

NoñN 1'míl NTNON 0
cono

in o. goinino

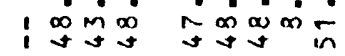

눙두 1'1 - nmm Doonon

- $\sigma^{2} \sim \infty$

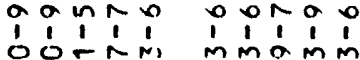

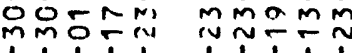
1'1 1 1111

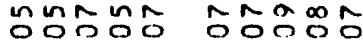




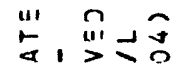

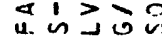

合祭品的

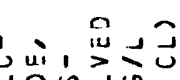

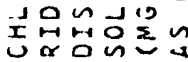

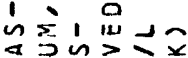

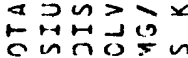

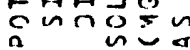

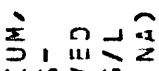

的出要的

영며요

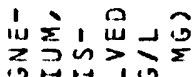

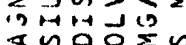
我的品少

品品 讨的主

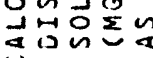

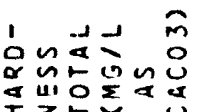

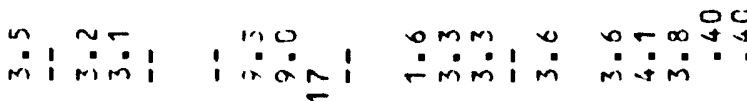

" ar, mas

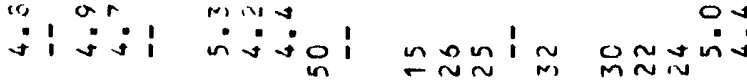

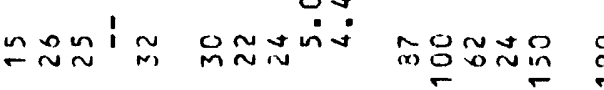

n! in

$i \underset{\sim}{\sim} \underset{\sim}{\sim}$

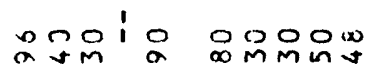

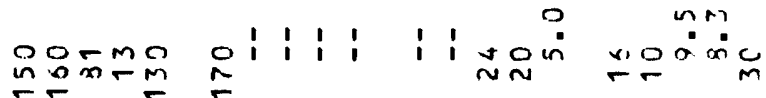

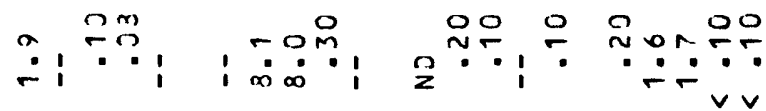

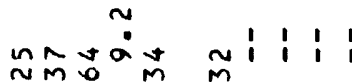

imn

ペNN요

$\approx i j \dot{m}$

'sina!

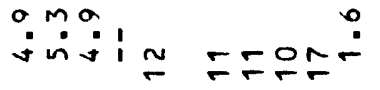

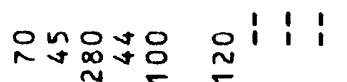

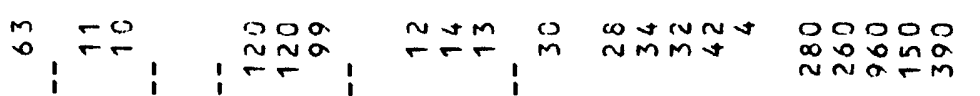

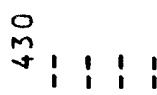

000 00000

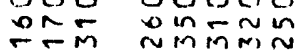

施它施品今

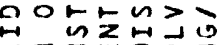

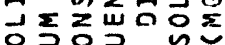

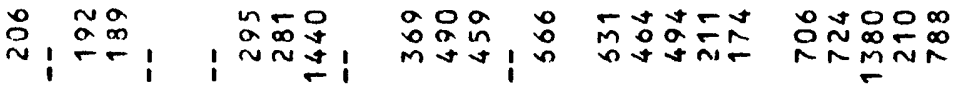
กั

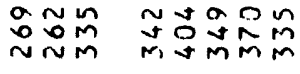

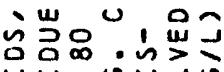
How

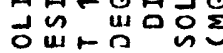

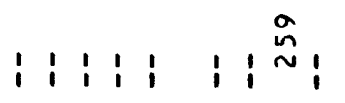

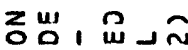

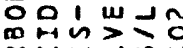

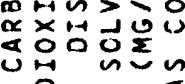

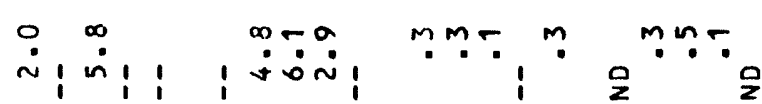

$\operatorname{mañl~}$

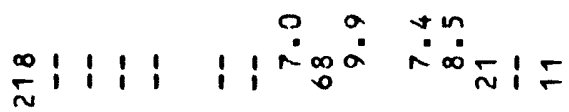

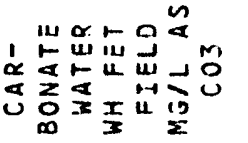

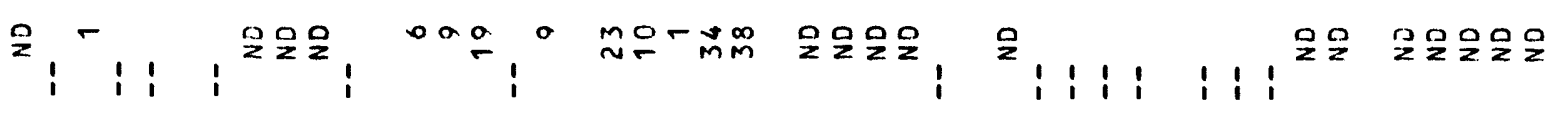

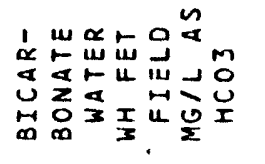

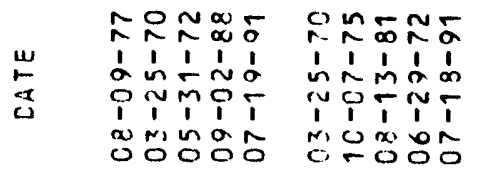

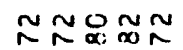
1́d向向a incuna

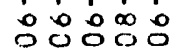

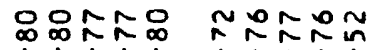

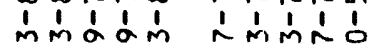
PPTTi ññN o응응

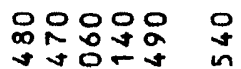

足 1 1 1

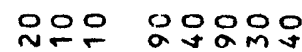

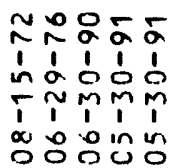

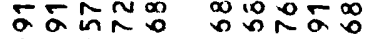

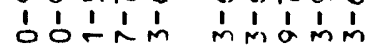
míp í unuta ríán 


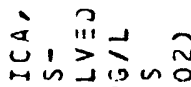

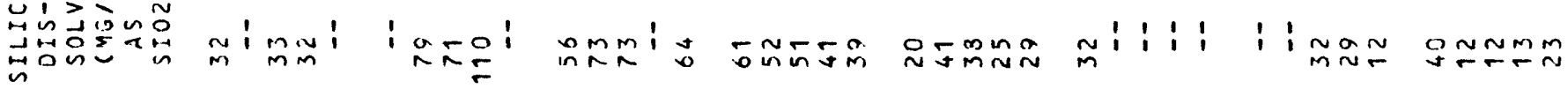

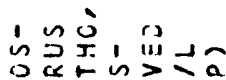

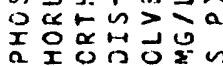

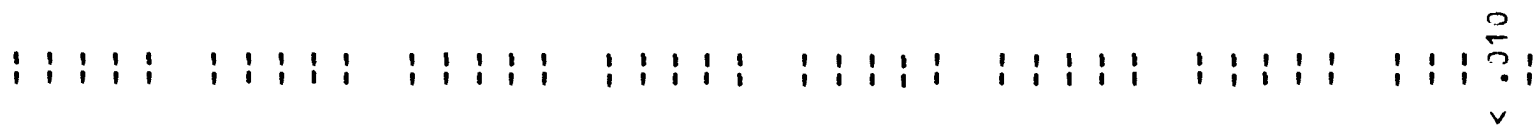

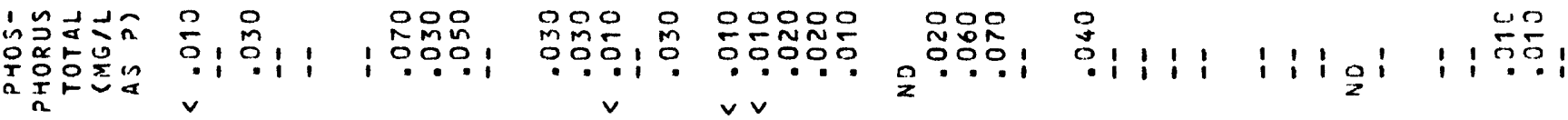

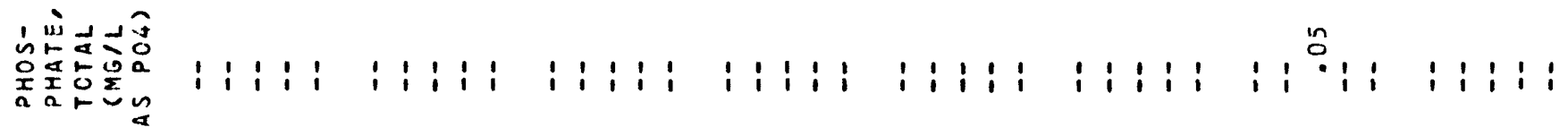

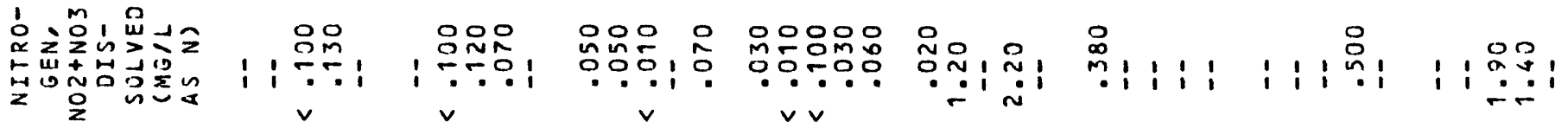

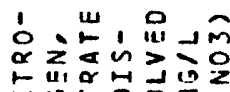

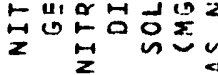

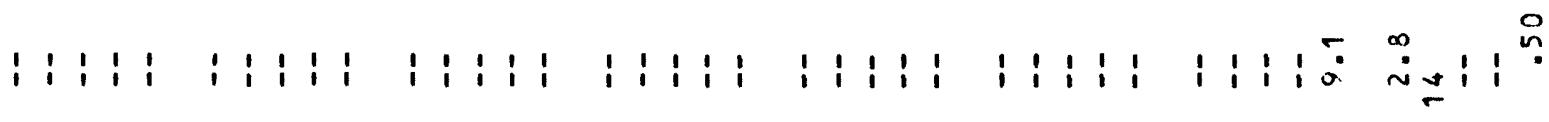

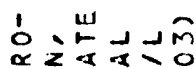

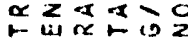

$\rightarrow 0,0 \leq$

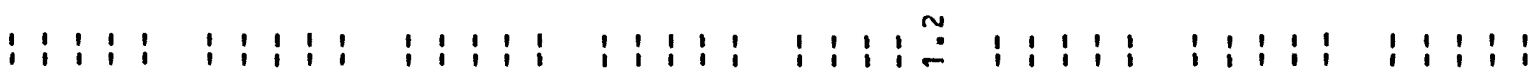

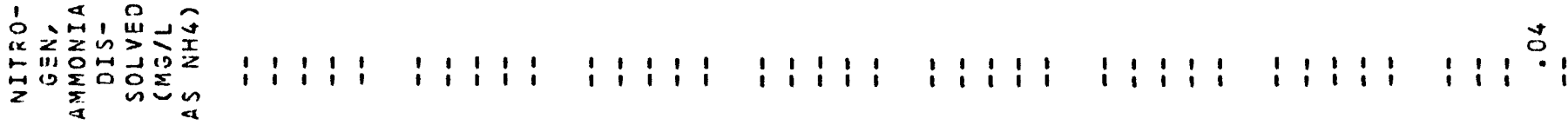

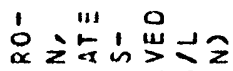

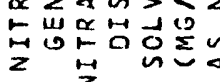

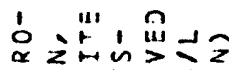

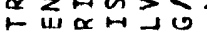

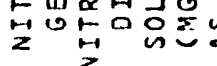

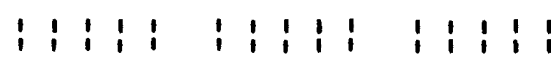

1:19:19:1: 1:11:

임

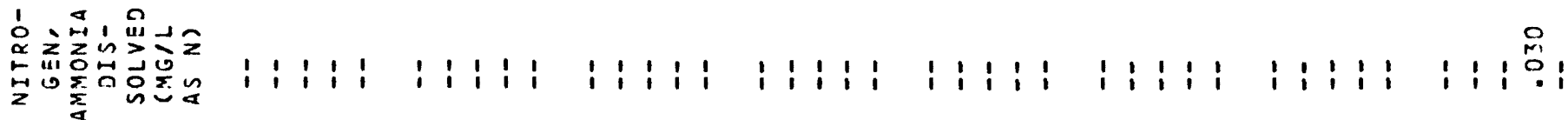

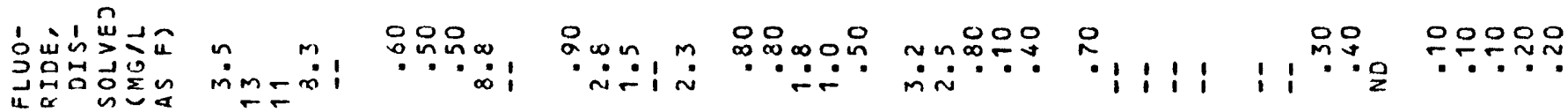

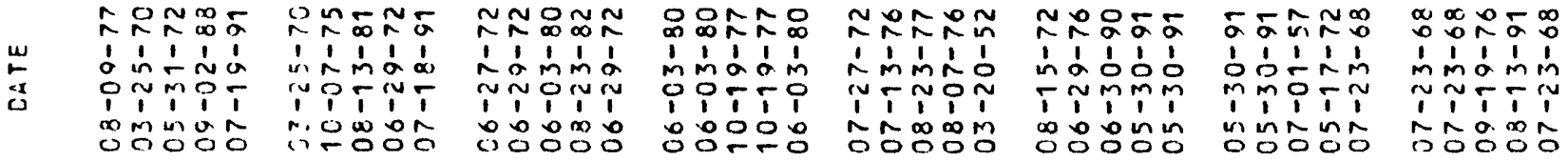




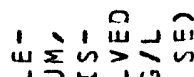

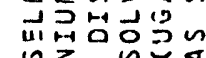

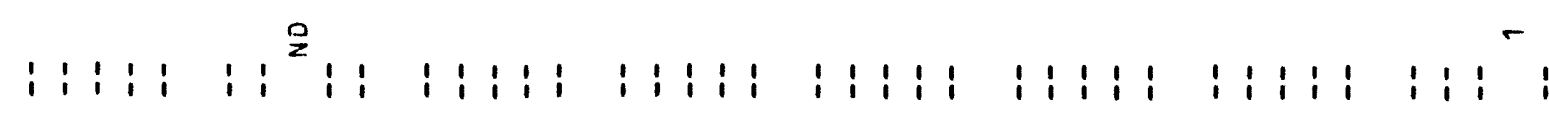

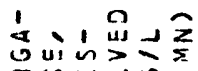

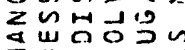

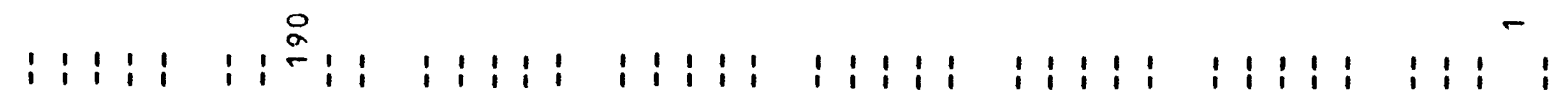

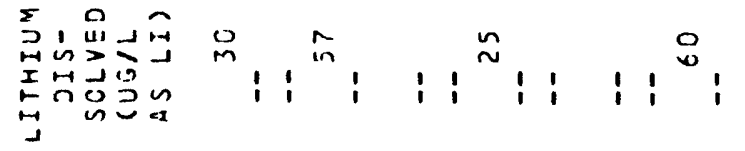

in 0 gogn

$1: \stackrel{0}{\sim} 1:$

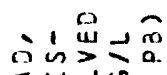

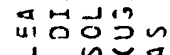

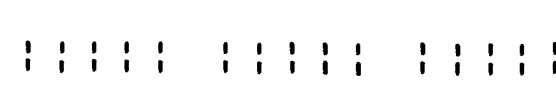

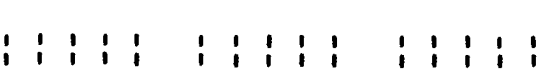

i is i i is

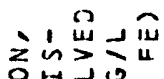

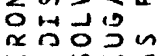

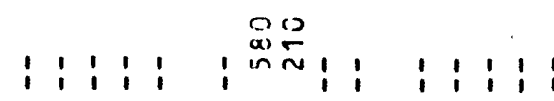

i:1i: i 운웃

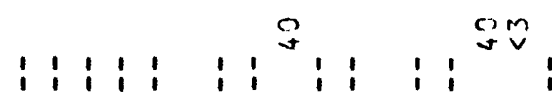

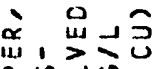

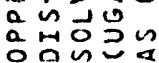

$1: 19: 21: 11911: 1$

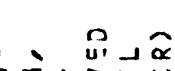

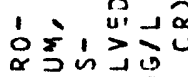

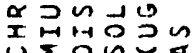

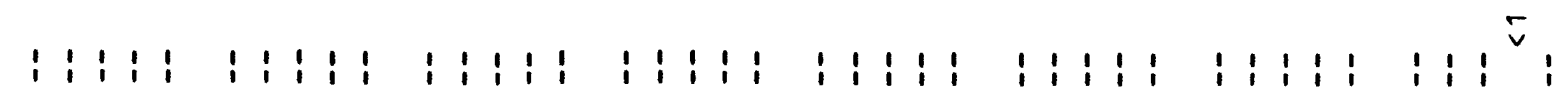

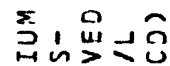

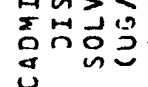

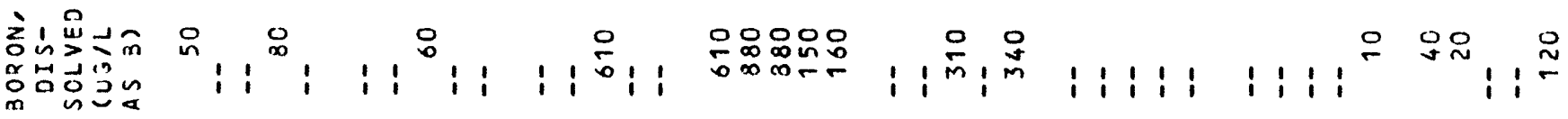

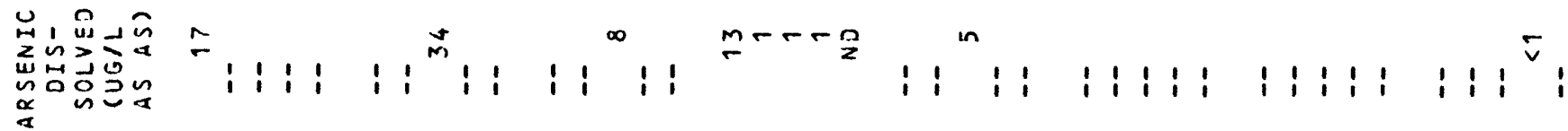

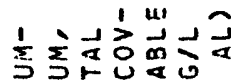

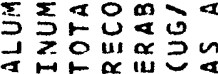

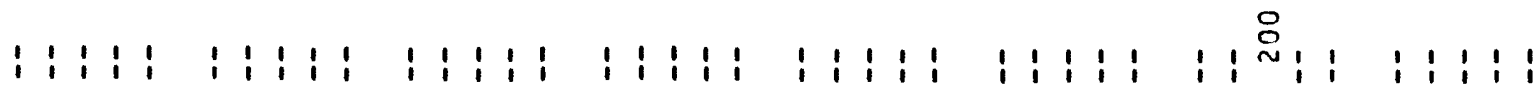

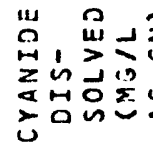

\begin{tabular}{|c|c|c|c|c|c|c|c|}
\hline 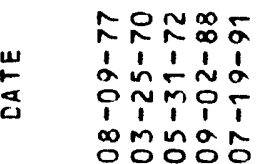 & 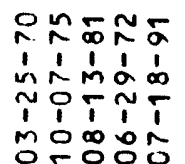 & 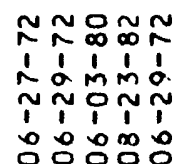 & 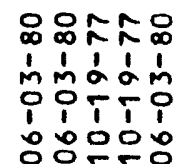 & 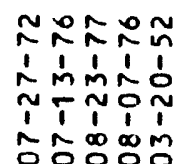 & 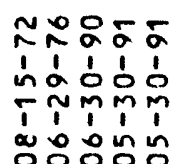 & 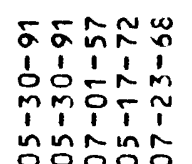 & 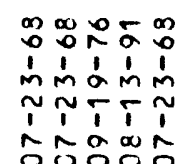 \\
\hline
\end{tabular}




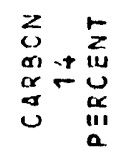

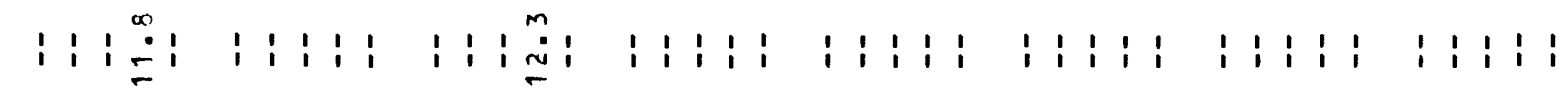

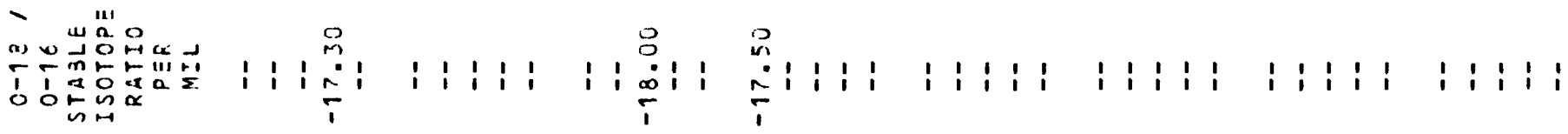

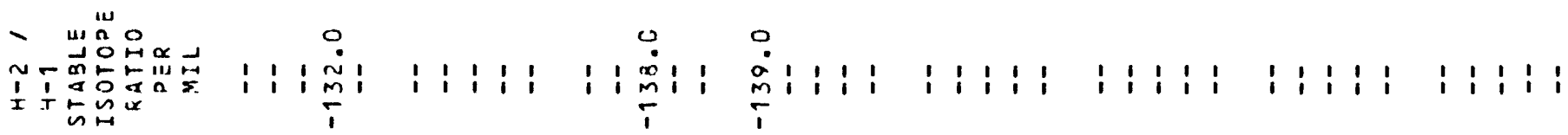

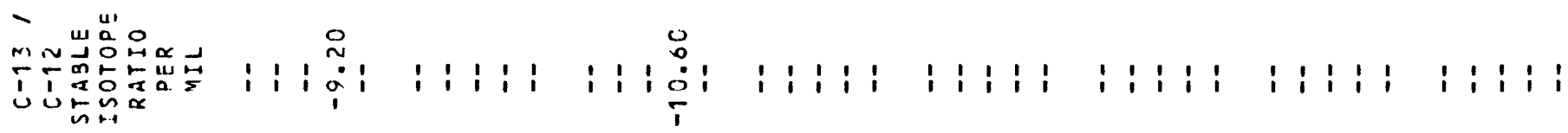

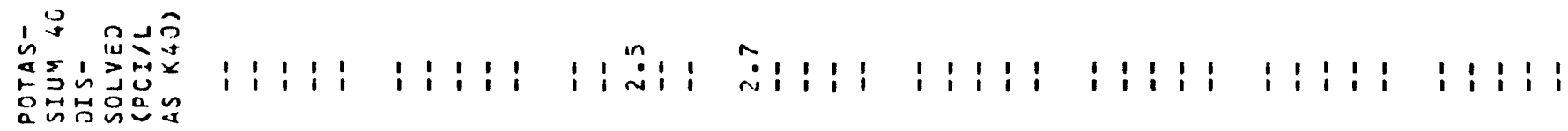

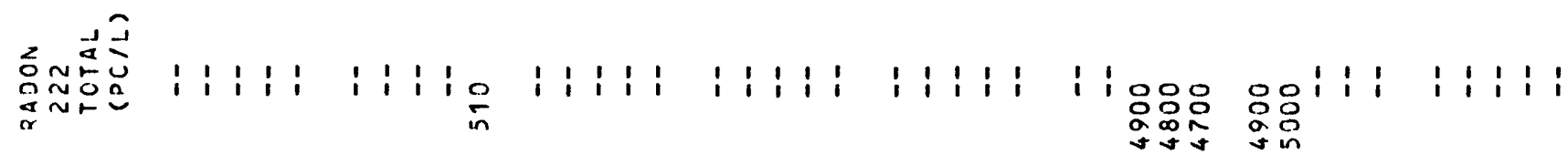

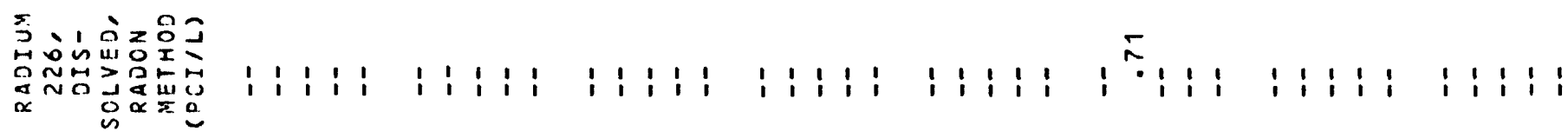

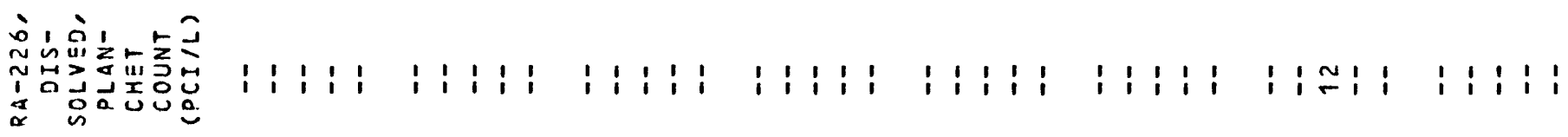

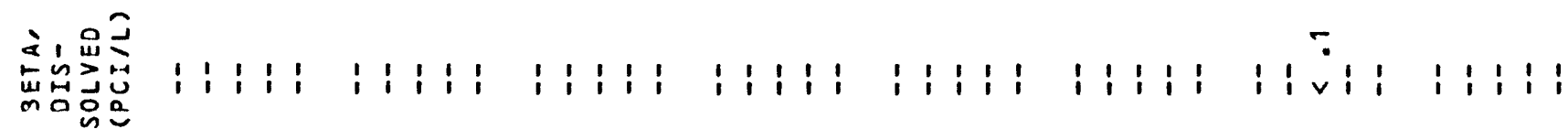

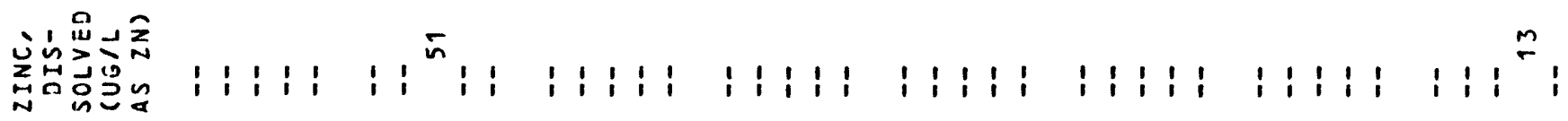

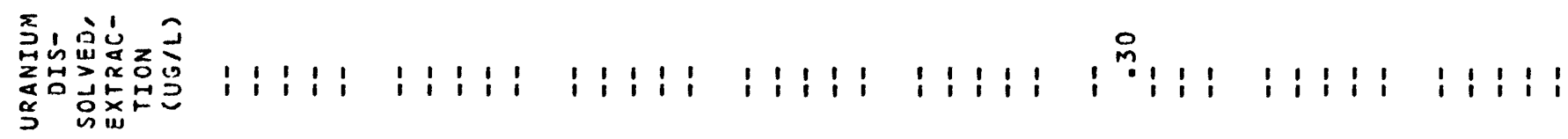
承产品品

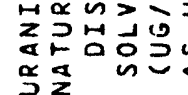

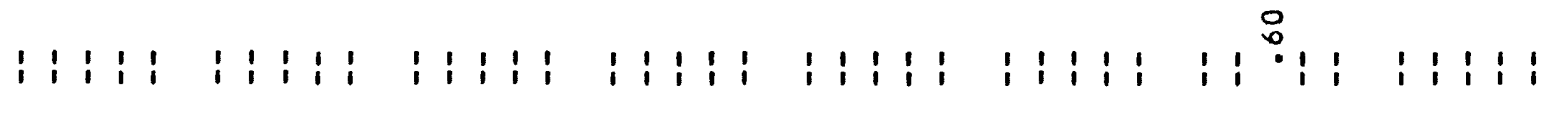

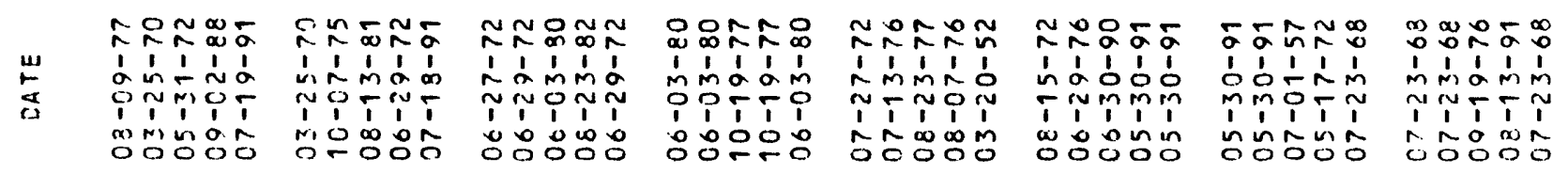




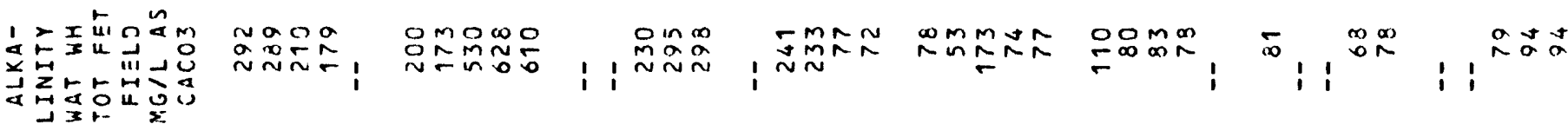

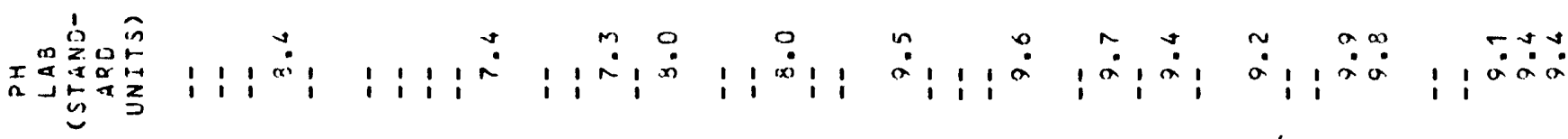

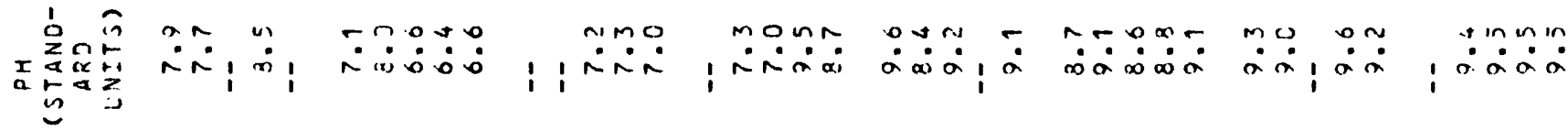

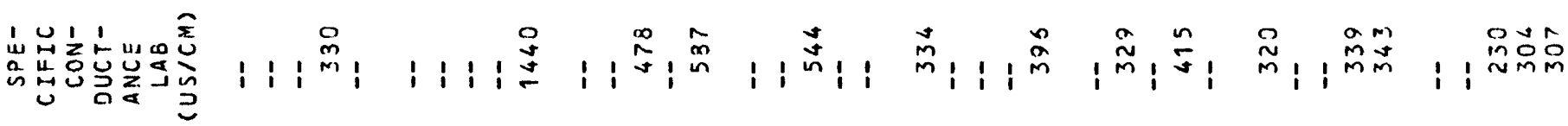

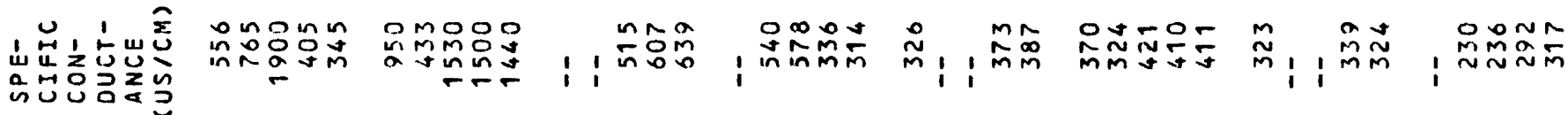
安山我

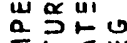

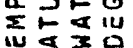

oomino noonin un noo

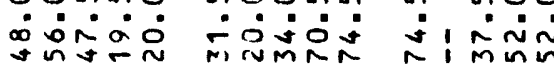

0000

- 90 onnm on no

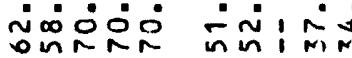

nomo

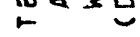
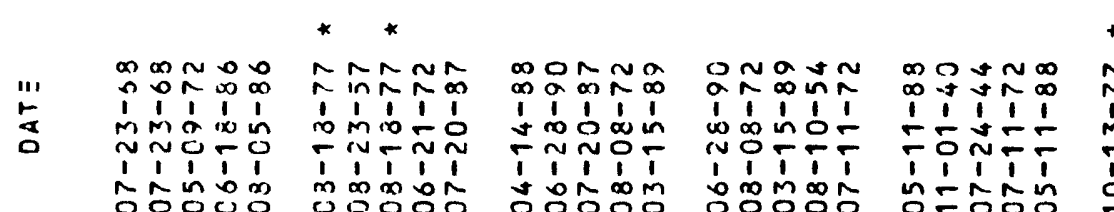

*

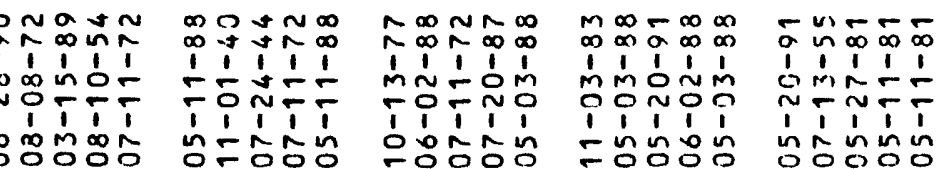

$\stackrel{u \prime}{n}$

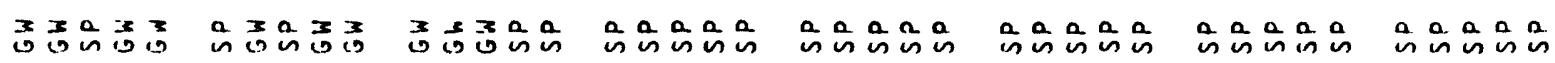

ז

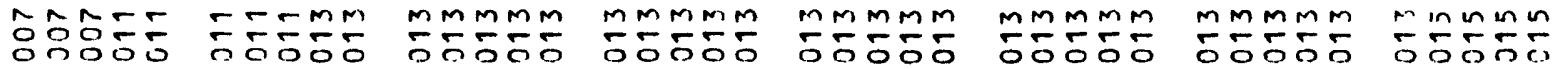

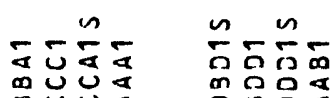

क

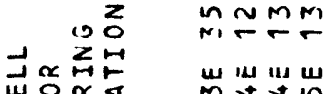

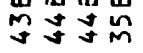

nnun

$\stackrel{\leftarrow \sim m}{\sim}$
ต

$\bar{m} \simeq \stackrel{\infty}{\sim}$

แI $u$ u

ติmm

nnn

m气士

\begin{tabular}{|c|c|c|}
\hline 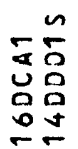 & $\begin{array}{l}n \\
0 \\
0 \\
0 \\
0 \\
0\end{array}$ & $\begin{array}{l}n \\
\infty \\
0 \\
0 \\
0 \\
\omega \\
-1\end{array}$ \\
\hline $\begin{array}{l}U_{1}^{\prime} \\
\stackrel{u}{\sim}\end{array}$ & $\underset{\sim}{\sim}$ & $\underset{\infty}{w}$ \\
\hline$\frac{n}{6} \frac{\pi}{0}$ & $\frac{n}{0}$ & $\underset{\sim}{z}$ \\
\hline
\end{tabular}

\begin{tabular}{|c|c|}
\hline 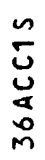 & $\begin{array}{l}n \\
\vdots \\
a \\
\tilde{a} \\
\sigma\end{array}$ \\
\hline & $\underset{\sim}{\sim}$ \\
\hline $\begin{array}{l}z \\
0\end{array}$ & $\begin{array}{l}z \\
0\end{array}$ \\
\hline
\end{tabular}

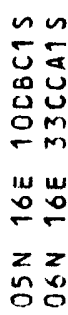

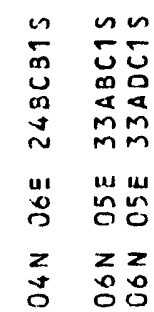




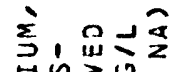

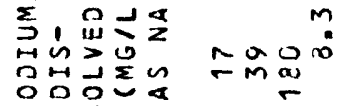

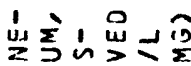
品我我

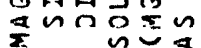

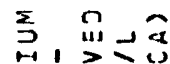

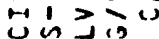

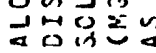

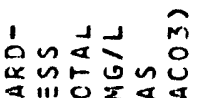

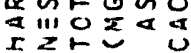

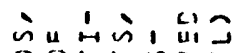

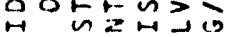

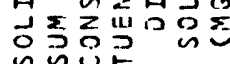

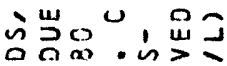

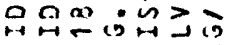

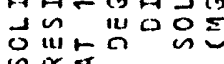

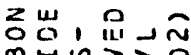

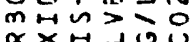

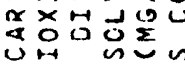

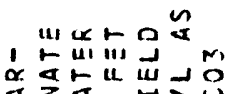

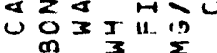

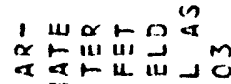

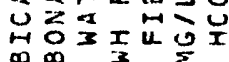

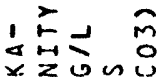

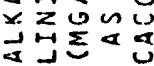

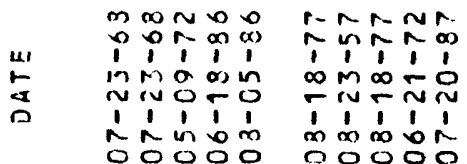

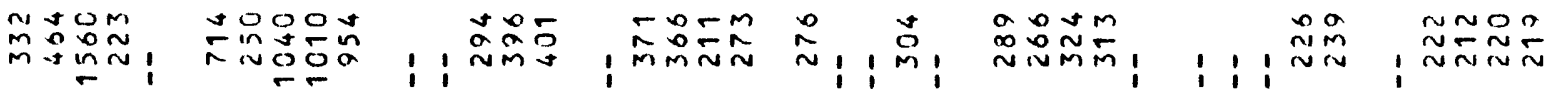

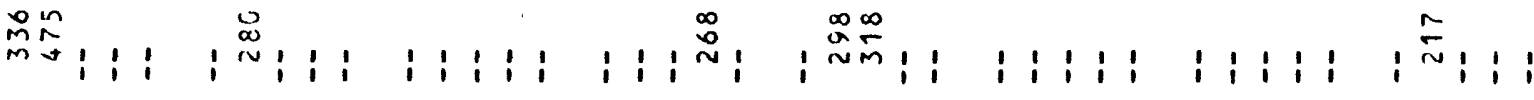

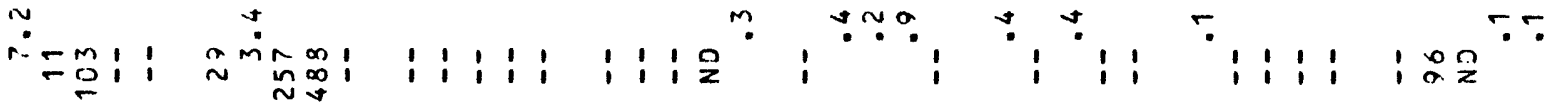

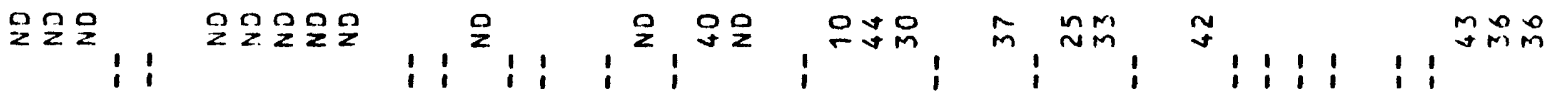

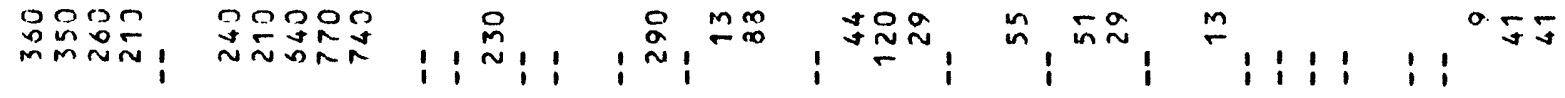

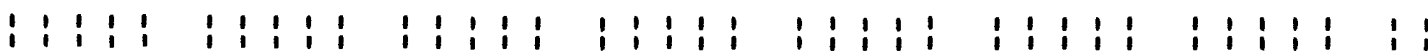
영

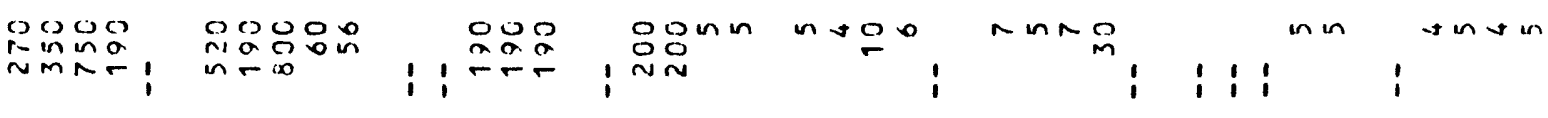

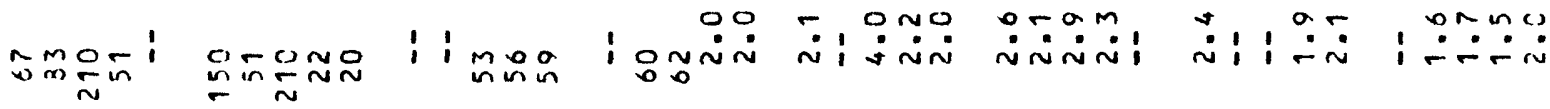




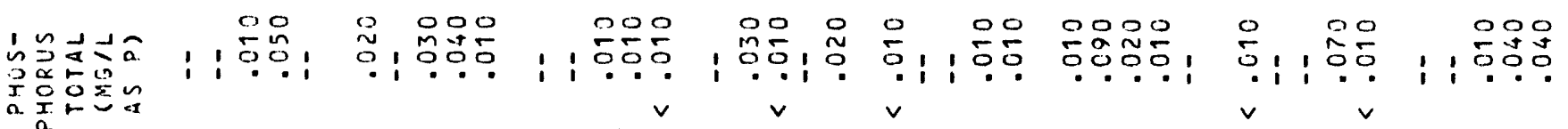

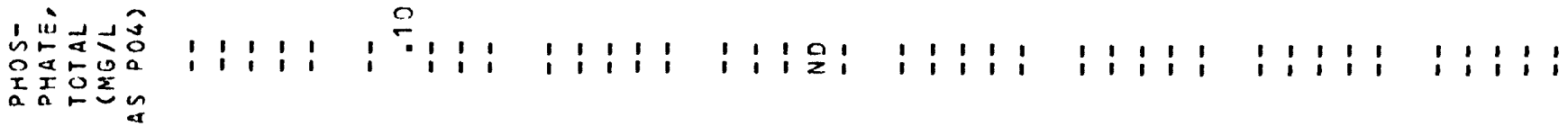

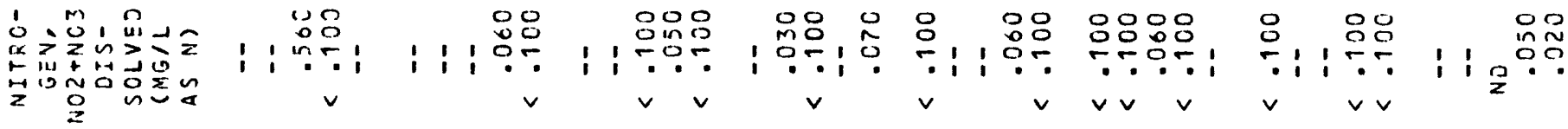

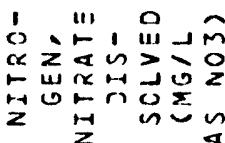

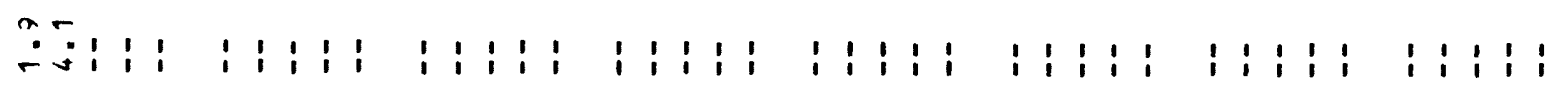

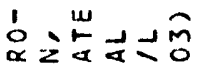
a

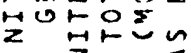

O $\sum_{\alpha}^{\prime \prime}$ in

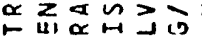

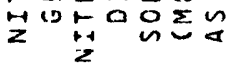

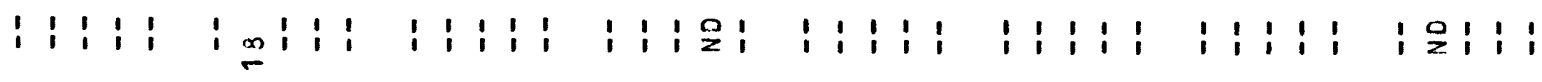

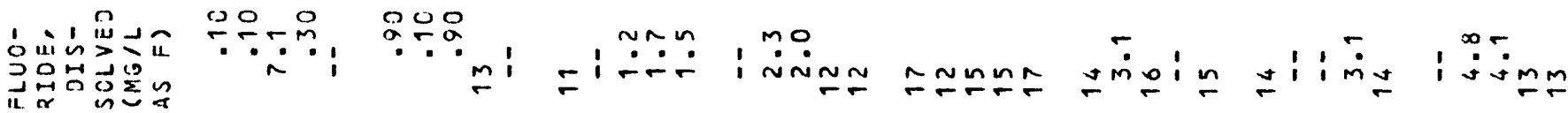

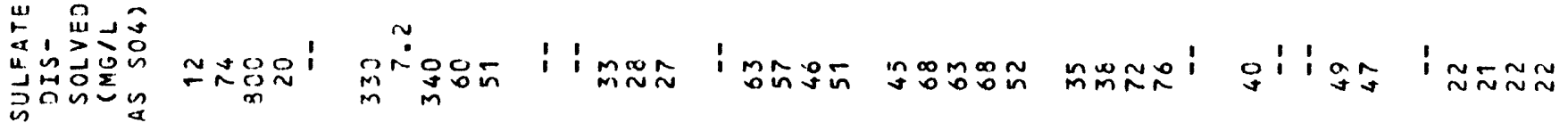

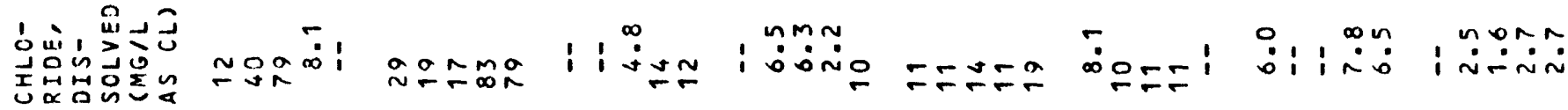

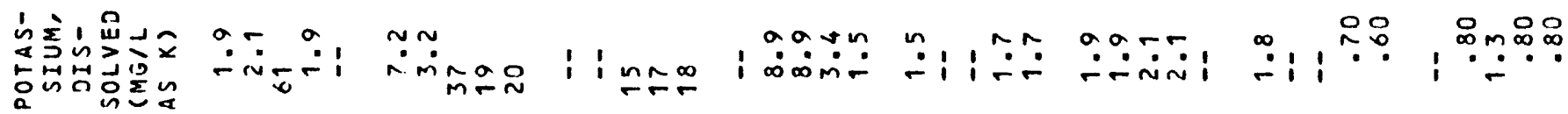

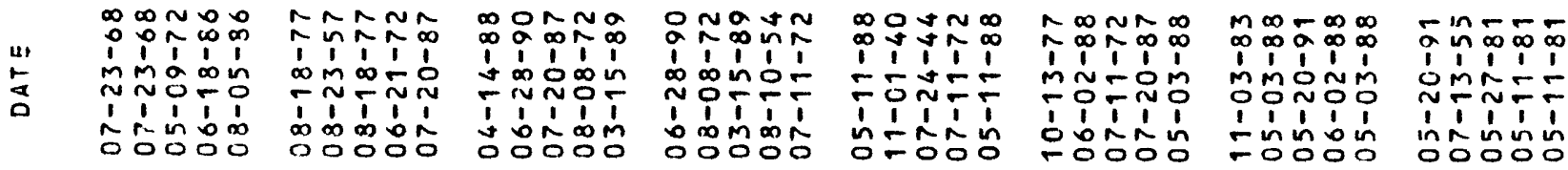




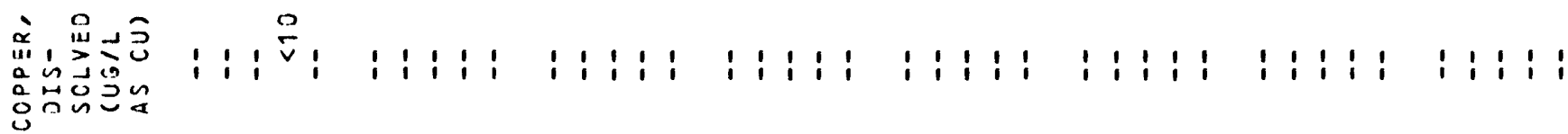

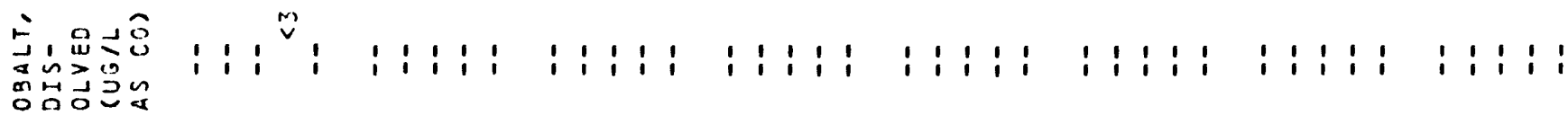

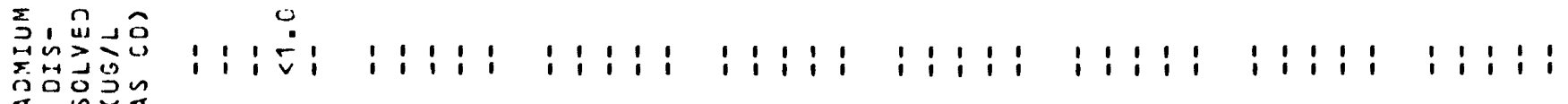

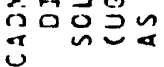

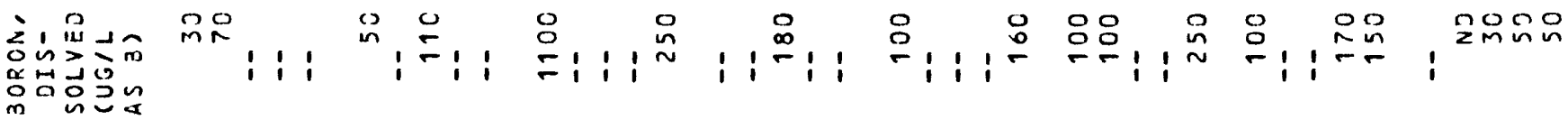

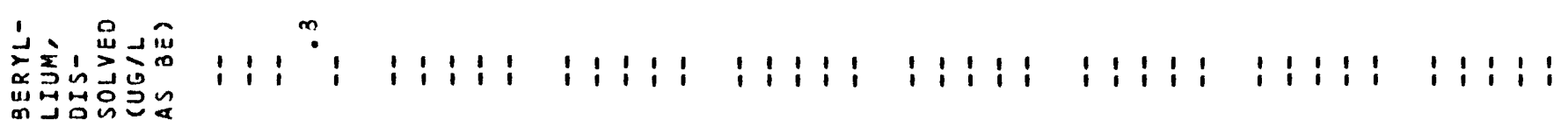

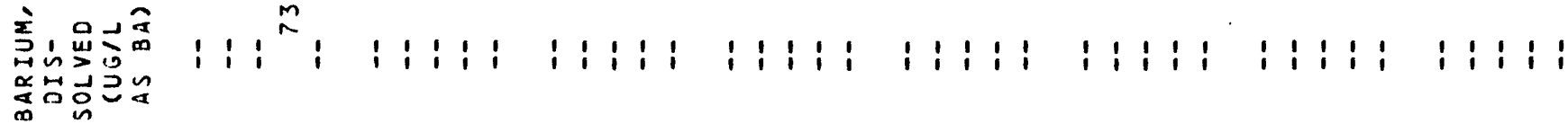

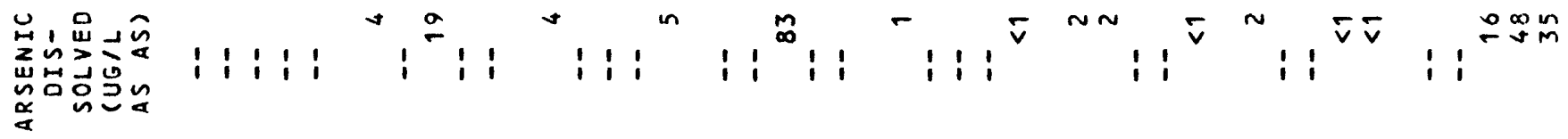

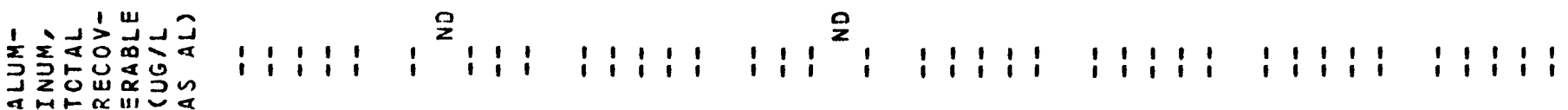

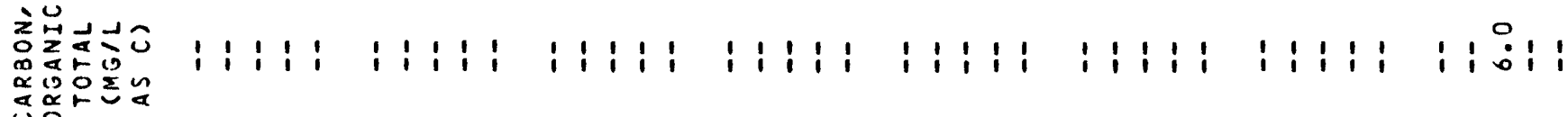

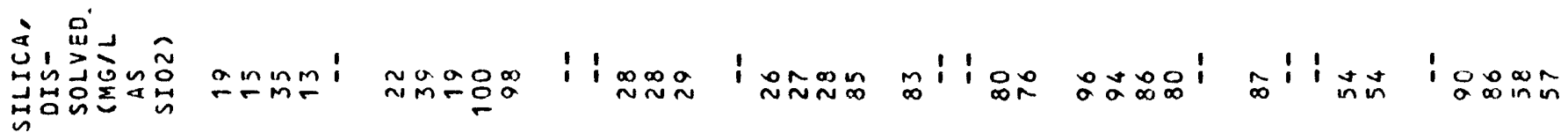

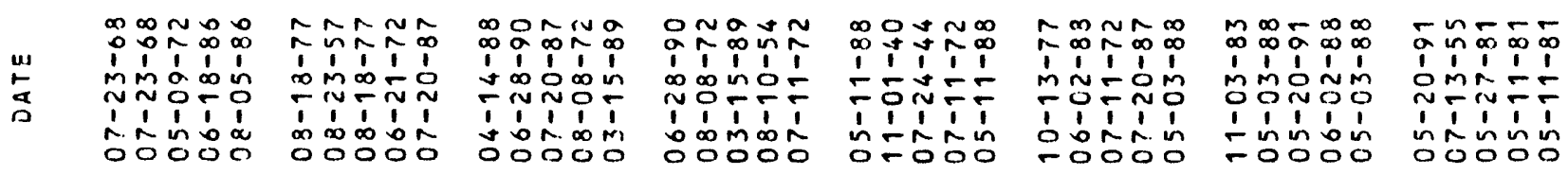




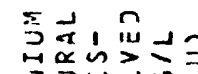

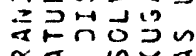

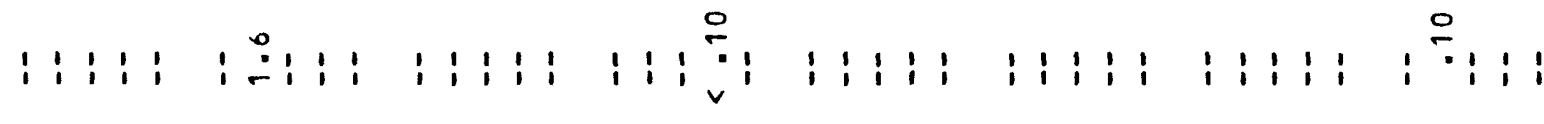
文主出出金

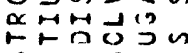

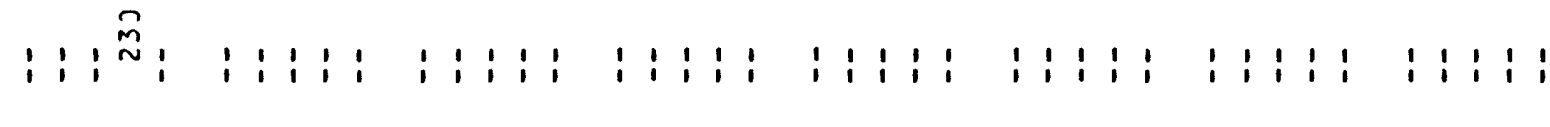

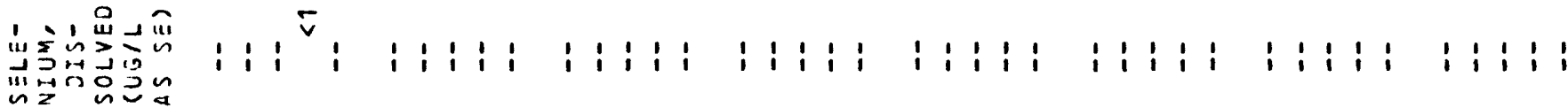

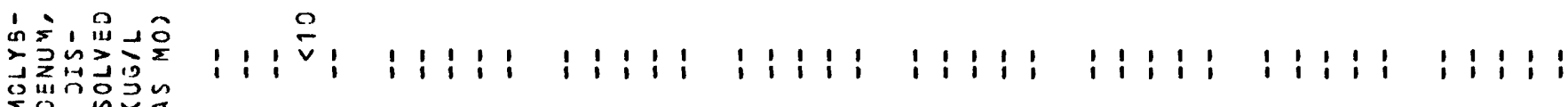

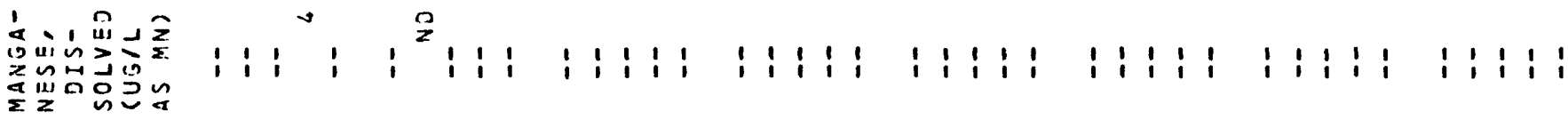

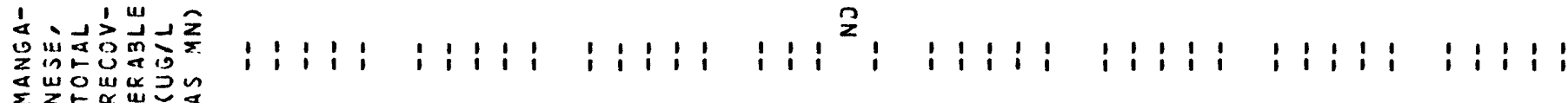

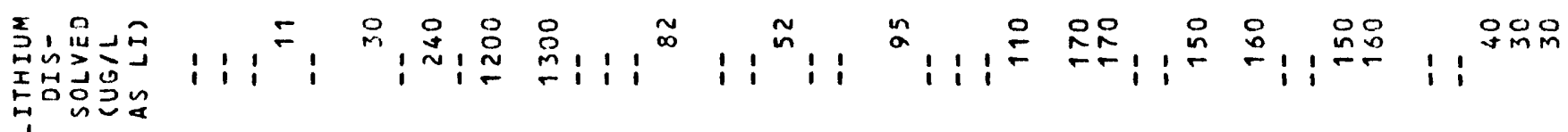

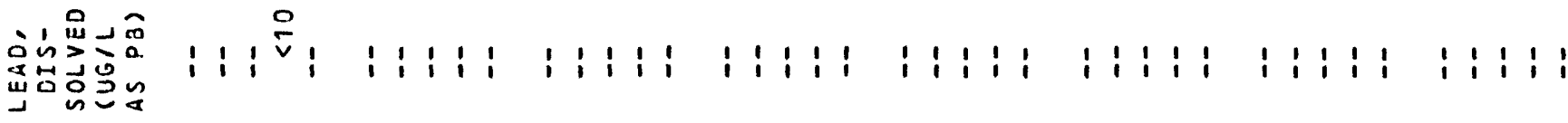

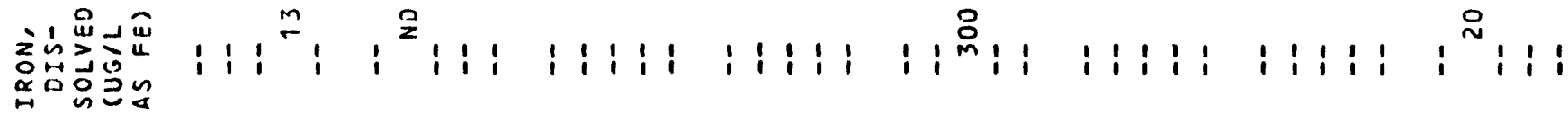

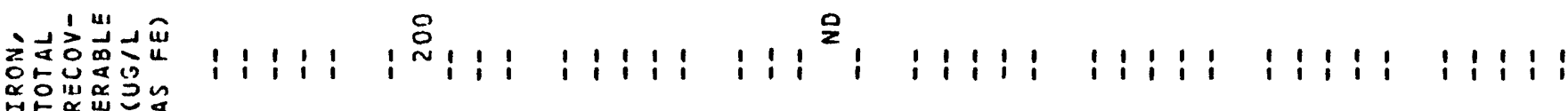

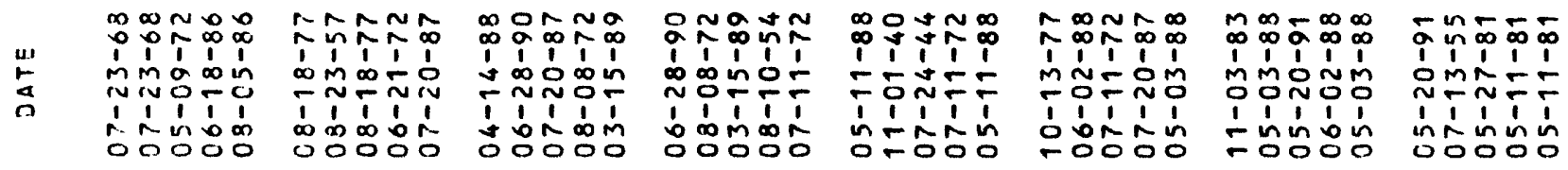




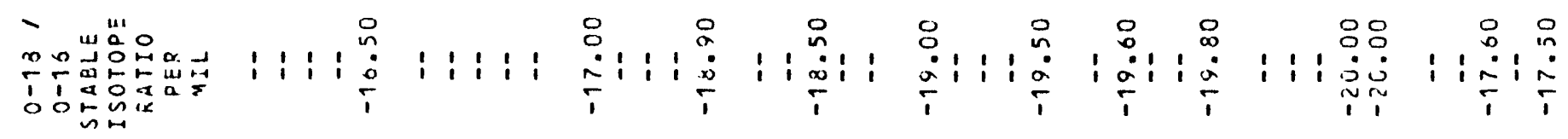

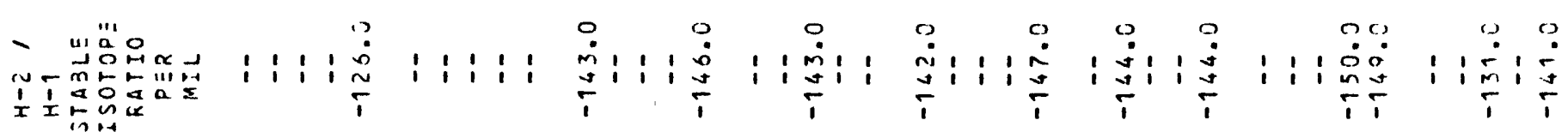

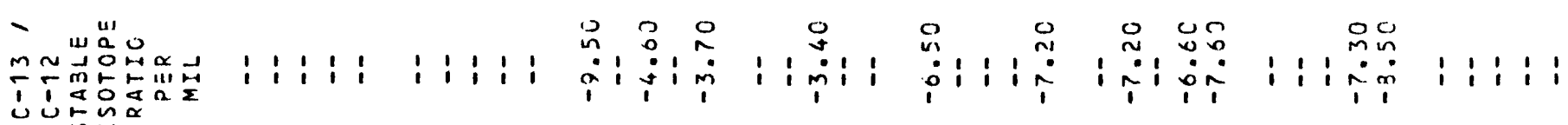

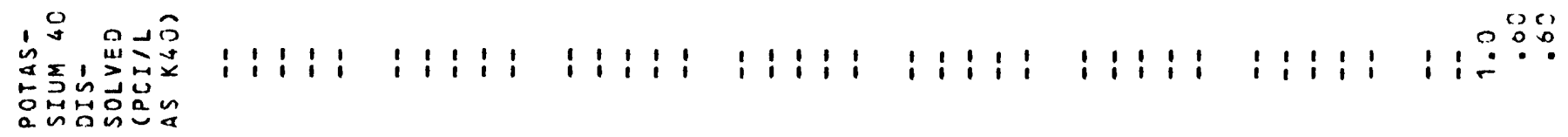

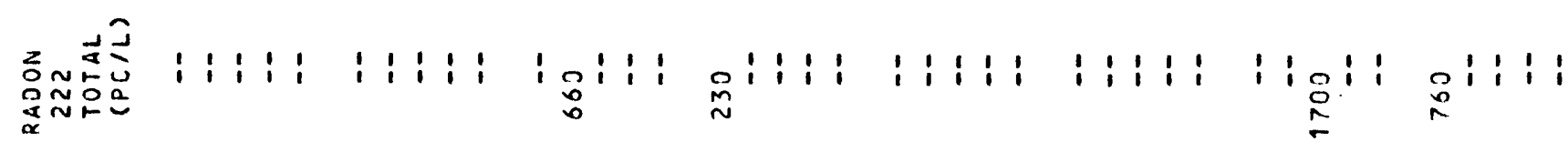

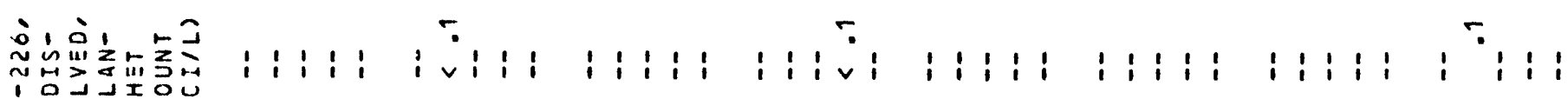

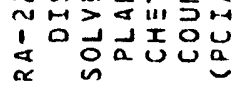

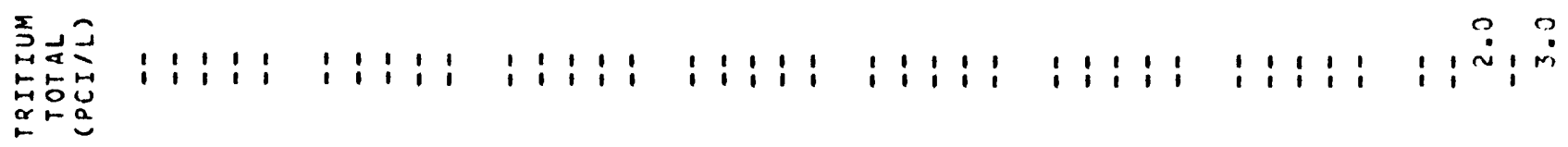

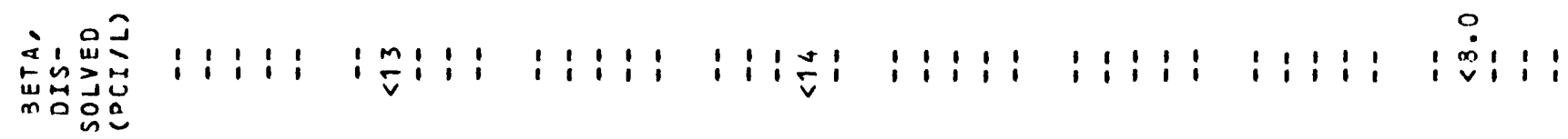

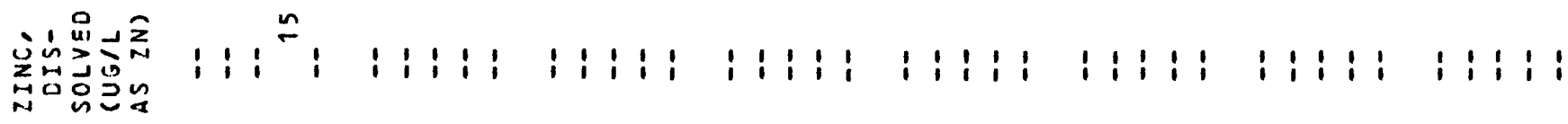

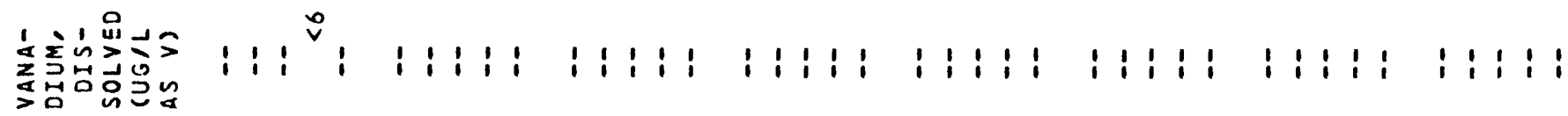

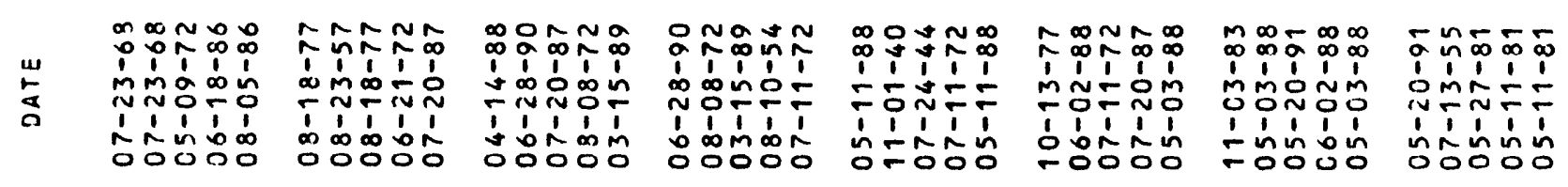


$\sum_{\text {i⿱ }}^{1}$

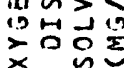
希

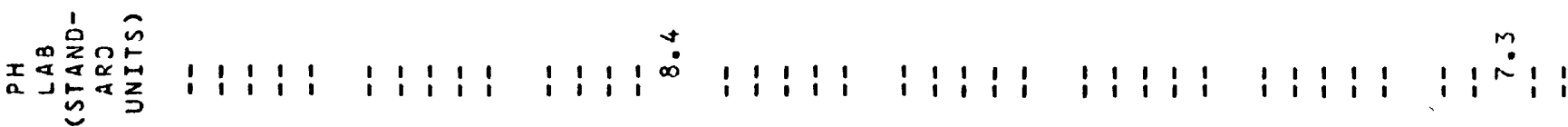

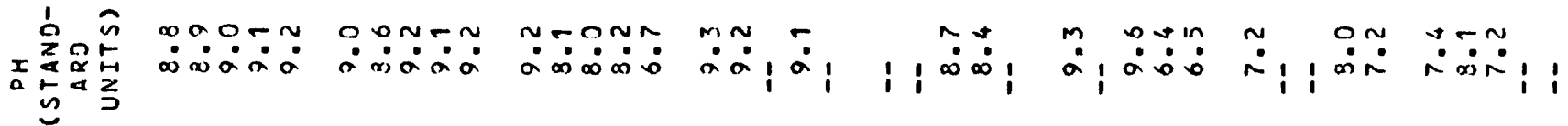

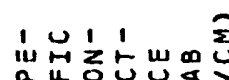

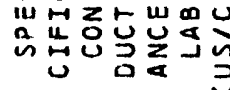

\section{i 1 i i i i i i i i \\ 1 $11^{\infty}$ \\ i i i i i i i i i}

i 1 1 i

$11111 ; 1: \overline{0}: 1$

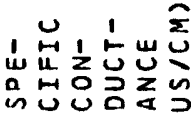

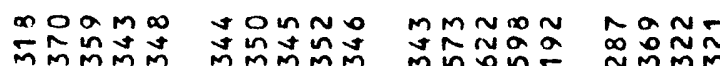

N

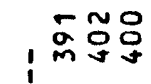

的, 事员员

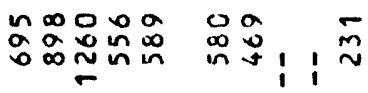

这

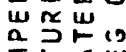

$\sum_{i=1}^{2}=10$

00000

Conom

nomoin

on 0

monoo

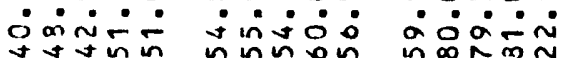

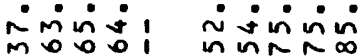

\& On?

oogno oomoo $+$

\section{‘}

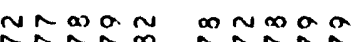

$\hat{1} \hat{1} \hat{1} \hat{1}$

oñ

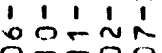

Tras and

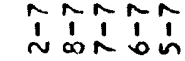

T̃ก⿻上丨

$\sim-N \sim N$

ñกㄴำ

$\begin{array}{llll}1 & 1 & 1 & 1\end{array}$

1
$=0 \frac{1}{0}=0 \frac{1}{0}$

กำ

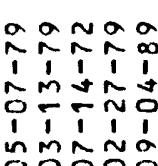

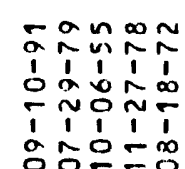

$\infty$, ํํำ

$\stackrel{i n}{5}$

a ana a

a. a. a a

a a a $x$

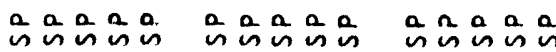

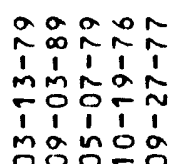

$a \approx a \sim n$

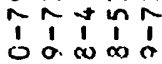

$\operatorname{cic} \frac{1}{1}$

$\begin{array}{llllll}1 & 1 & 1 & 1 & 1 & 1 \\ 0 & 0 & 0 & 0\end{array}$

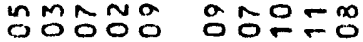

mano

mº엉요

aิ

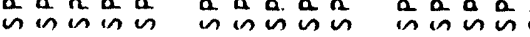

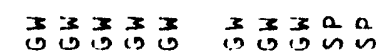

2

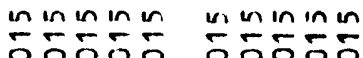

nn⿻上丨n

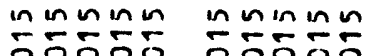

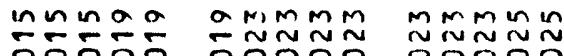

\begin{tabular}{|c|c|c|c|c|c|c|c|c|c|c|c|c|}
\hline ט & $\begin{array}{ll}n & n \\
n & n \\
0 & 0 \\
0 & 0 \\
0 & 0 \\
0 & 0\end{array}$ & $\begin{array}{l}n \\
\vdots \\
0 \\
0 \\
0\end{array}$ & 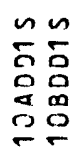 & 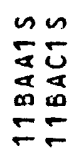 & 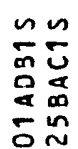 & 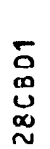 & 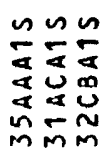 & 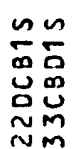 & $\underset{ن}{\dot{u}}$ & 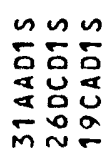 & 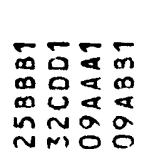 & 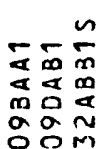 \\
\hline 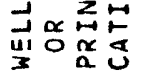 & 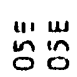 & in & 崩出 & 岕出 & 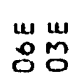 & 㔜 & 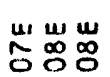 & $\begin{array}{l}w \text { w } \\
\text { a }\end{array}$ & 눙 & $\underset{\sigma}{\omega} \underset{\sim}{w} \underset{g}{w}$ & 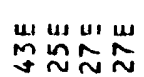 & 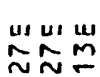 \\
\hline & $\begin{array}{ll}z & z \\
\infty & \infty \\
0 & 0\end{array}$ & $\begin{array}{l}z \\
\infty \\
0\end{array}$ & $\begin{array}{ll}z & z \\
0 & w \\
0 & 0\end{array}$ & $\begin{array}{ll}2 & 2 \\
\infty & \infty \\
0 & 0\end{array}$ & $\begin{array}{ll}2 & z \\
0 & 0 \\
0 & 0\end{array}$ & $\begin{array}{l}z \\
0 \\
0\end{array}$ & $\begin{array}{l}z z z \\
900 \\
0\end{array}$ & $\begin{array}{l}2 z \\
0 \% \\
0\end{array}$ & $\underset{2}{2}$ & $\begin{array}{l}z \sim \tilde{O} \\
0 \tilde{O}\end{array}$ & $\begin{array}{l}n z z z \\
\text { ज出负 }\end{array}$ & $\begin{array}{l}z z z \\
\text { Mó }\end{array}$ \\
\hline
\end{tabular}


$\sum_{3}, 0+\frac{a}{2}$

我我点

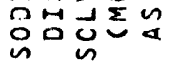

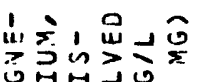

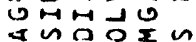

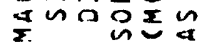

omñ

om

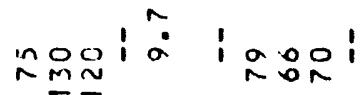

1 I 응 ก'

20

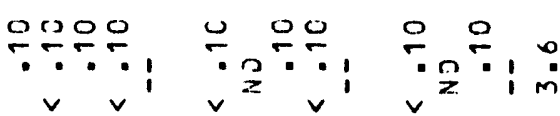

i.

i $12 \div \div \frac{0}{2}: 1:$

$\stackrel{0}{\div 0}$

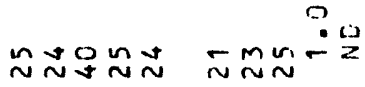

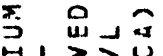

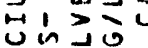

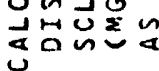

जom?

on

ن்

$\div ?$

i

กับ

?

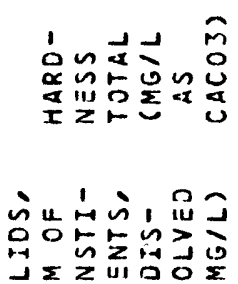

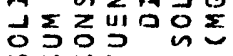

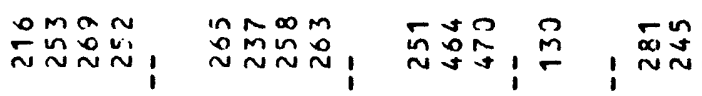

$00 m n$

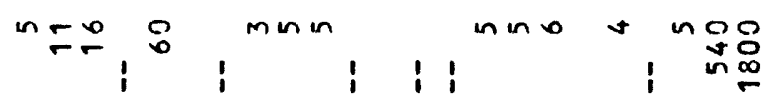

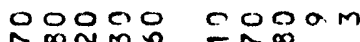
N

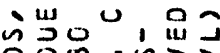

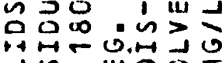

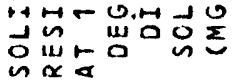

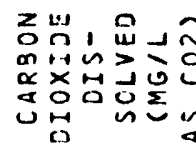

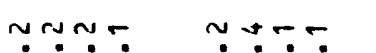

$\because \cdots$

$\because \div \div$

$\because \ddot{\sim}:$

in:

$\infty_{\infty}^{m !} \div$

ก̊!

$\stackrel{\infty}{\infty}, \quad$ in $i^{n}, \bar{n}$

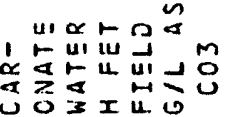

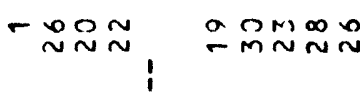

$\stackrel{a}{n 20}, \frac{0}{z} i^{\text {mañ }}$ i I $^{n} \stackrel{\infty}{m}, \stackrel{m o}{2}$

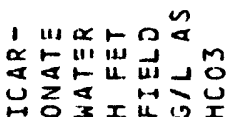

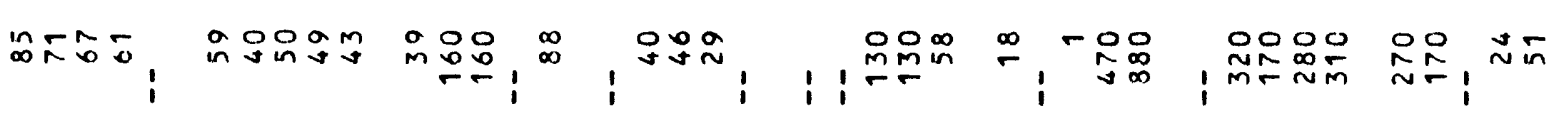

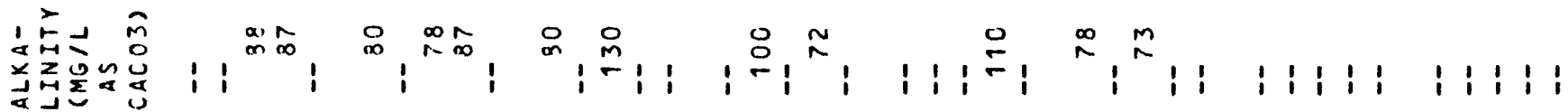

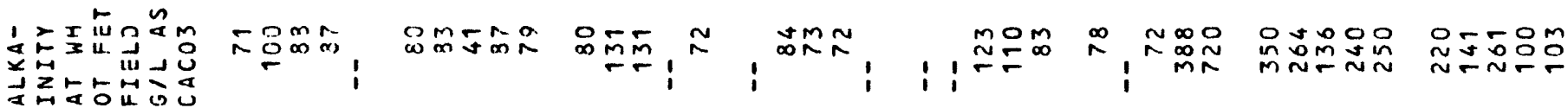

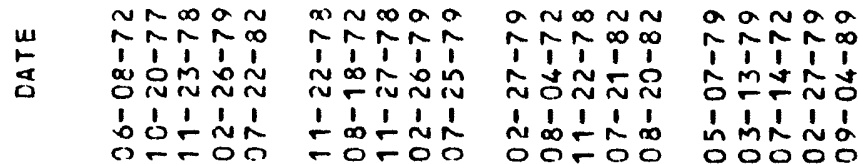

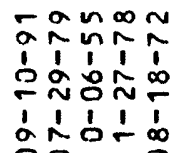

agaron $\uparrow_{1}^{\infty} \pi \hat{1}$ $m m \tilde{O}$

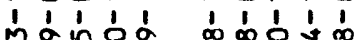

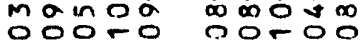

moran îi

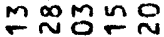

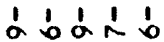
0000 


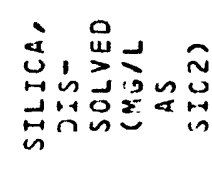

논응

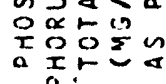

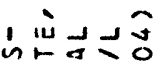

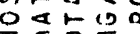

Tr의

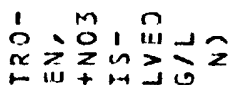

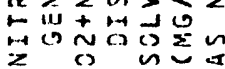

家之ồ

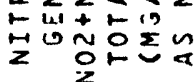

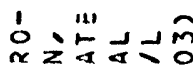

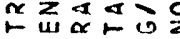
स단인

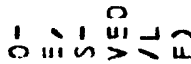

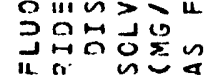

ㄴ,

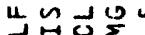

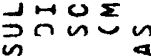

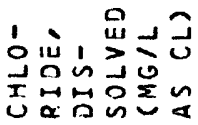

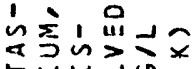

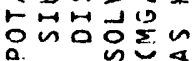

$\stackrel{11}{5}$

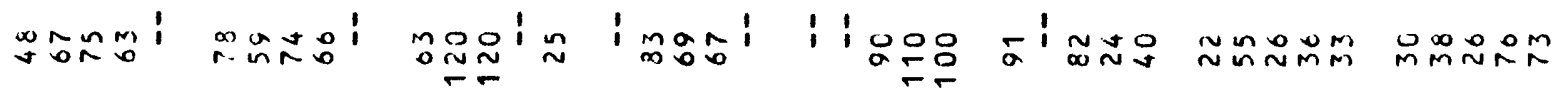

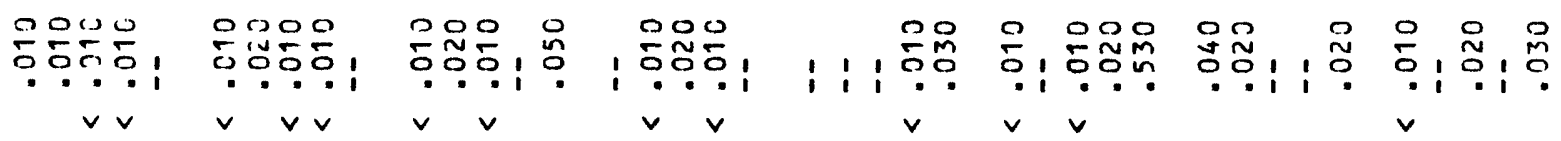

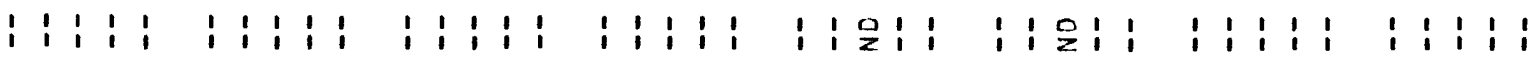
กํㅇㅇㅇ

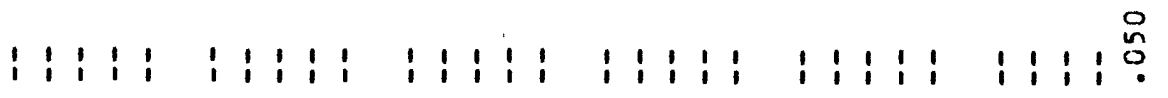

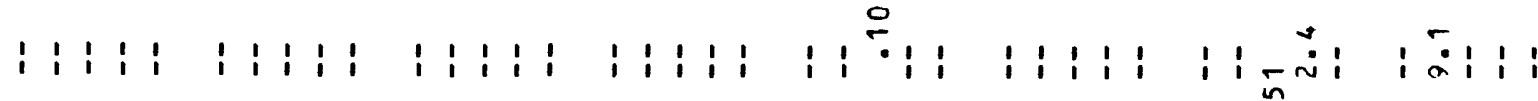

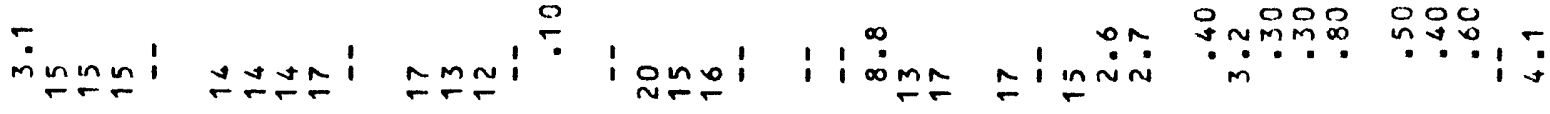

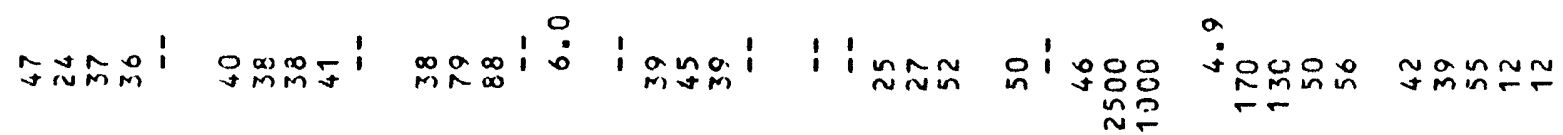

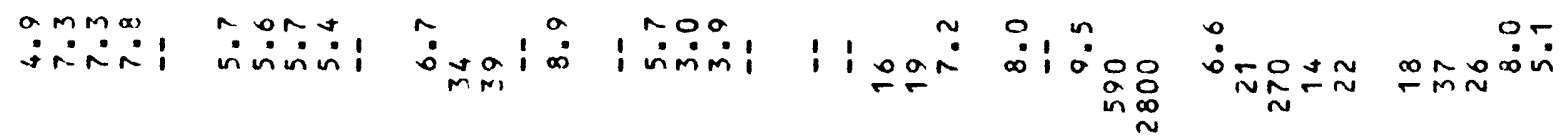
ํำ,

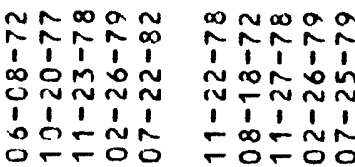

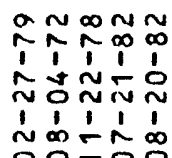
$\operatorname{sinas}$

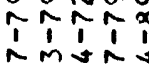

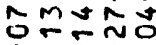
$1 ;$ i omana

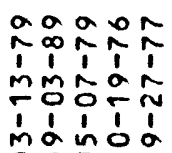

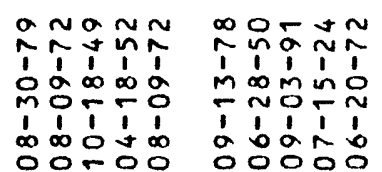




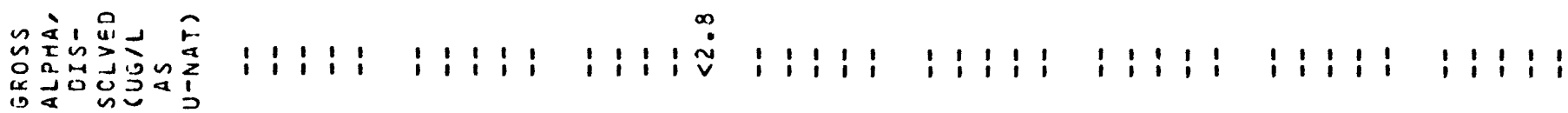

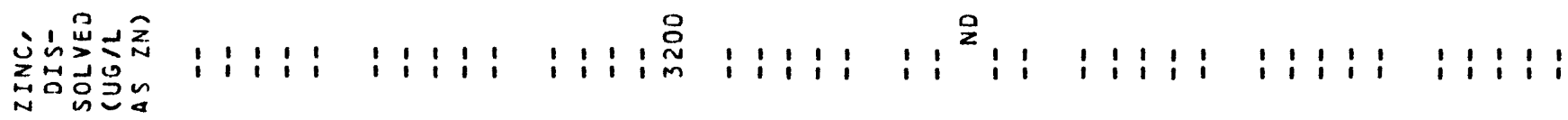

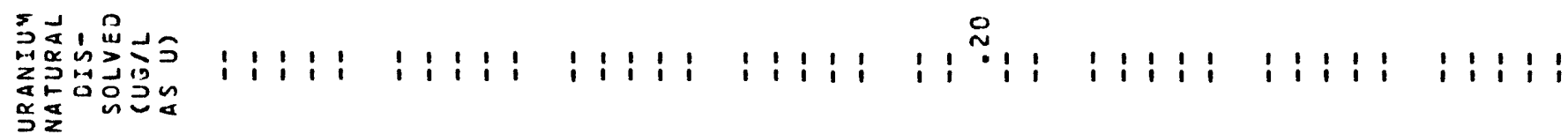

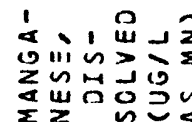

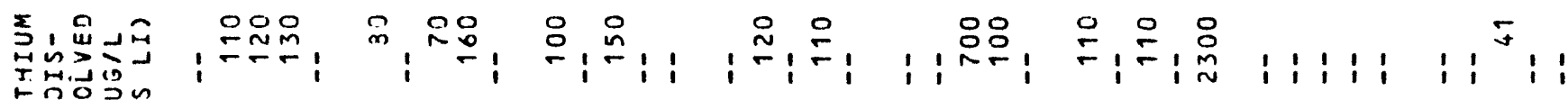

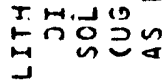

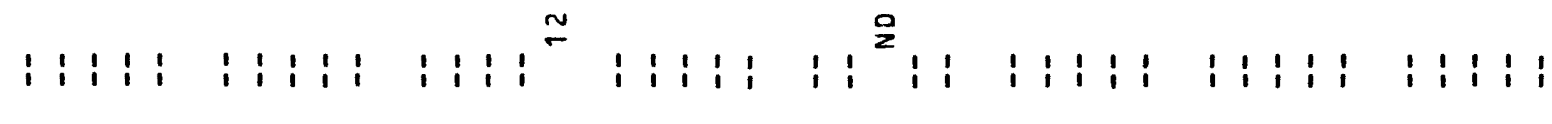

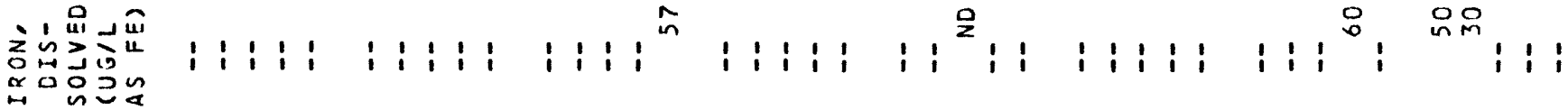

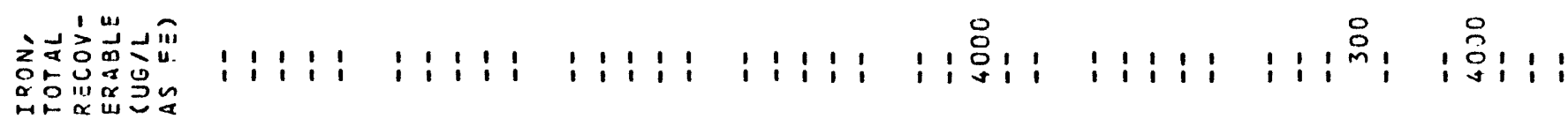

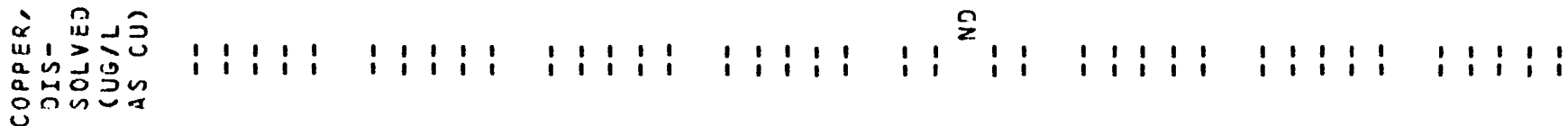

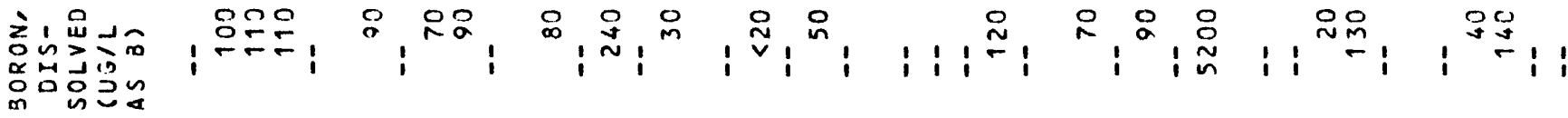

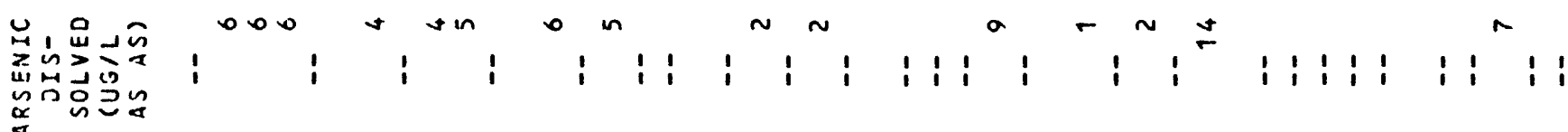

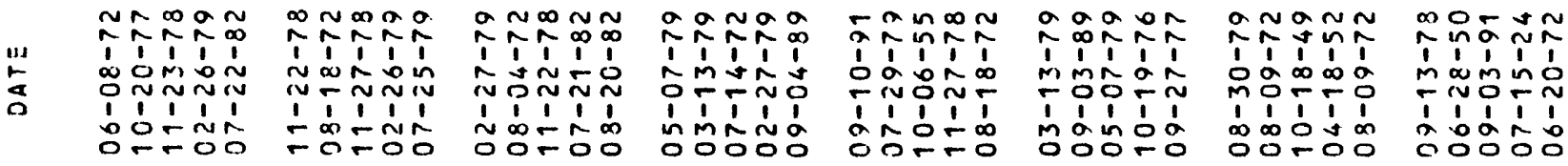




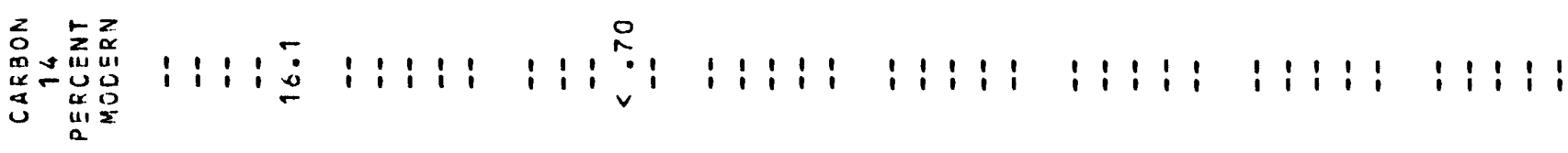

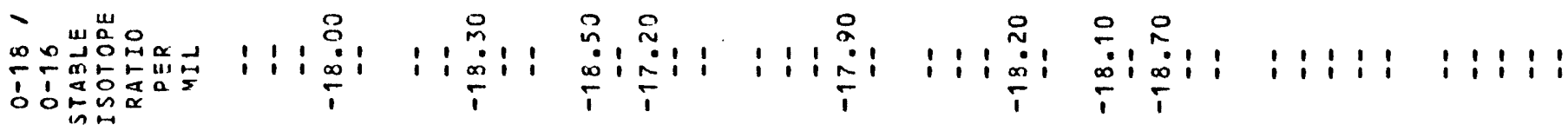

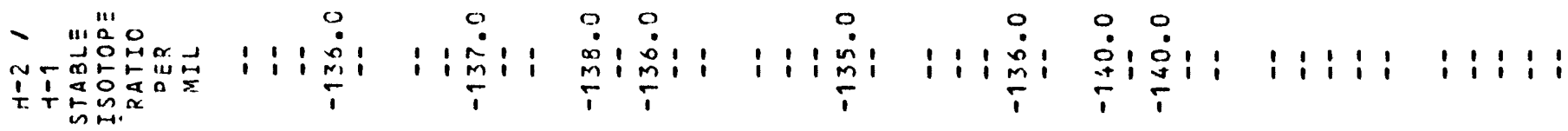

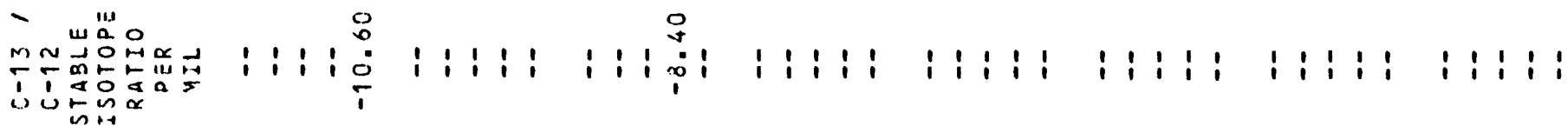

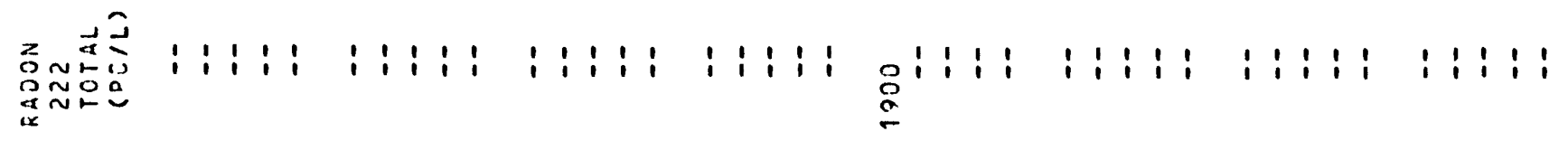

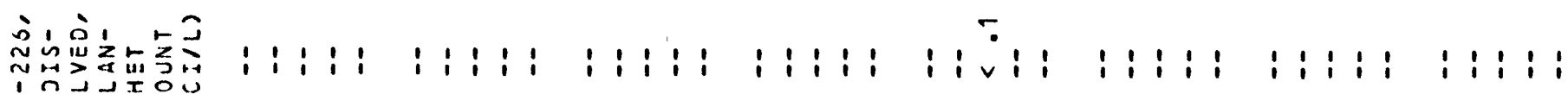

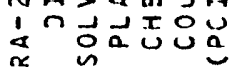

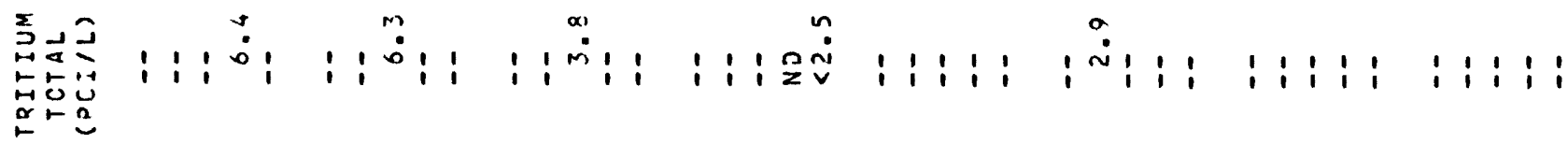

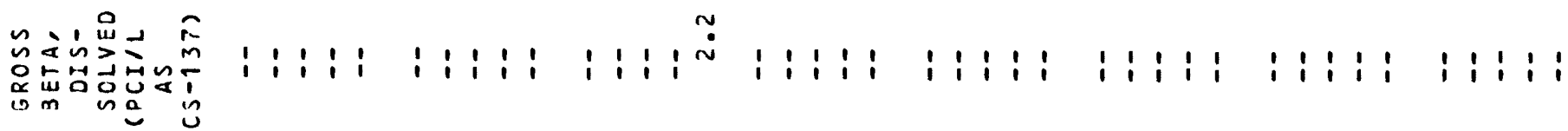

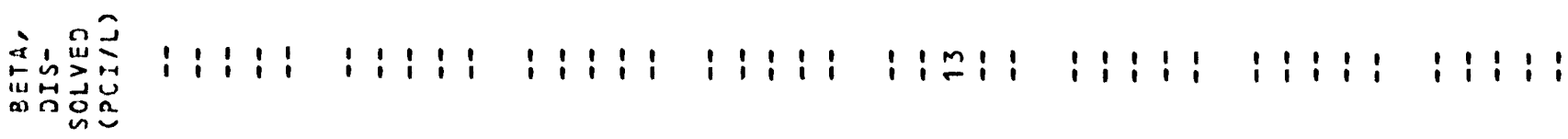

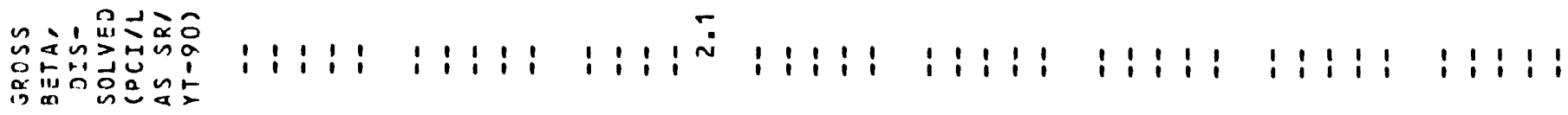

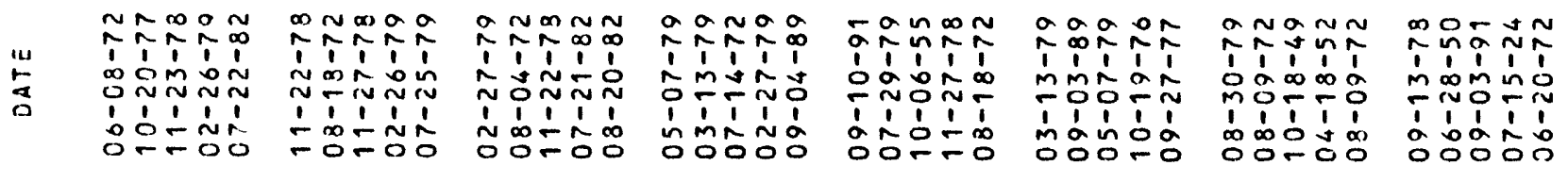




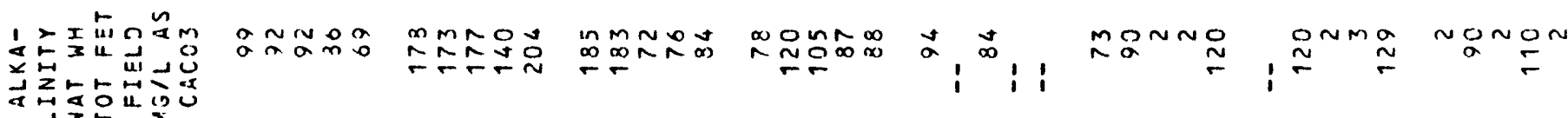

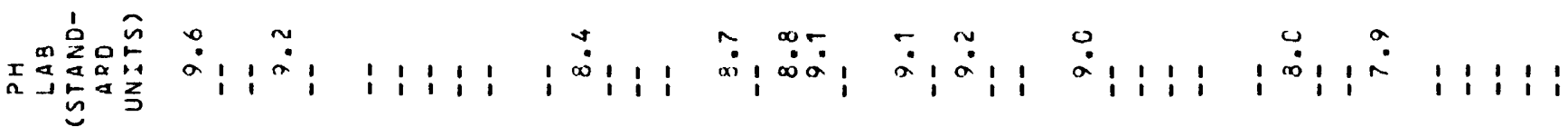

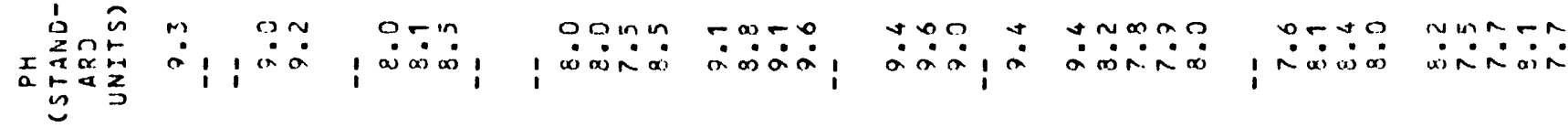

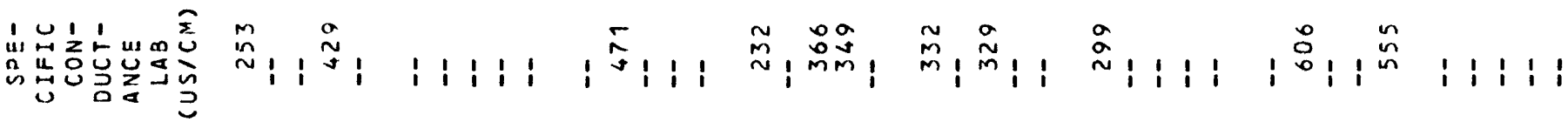

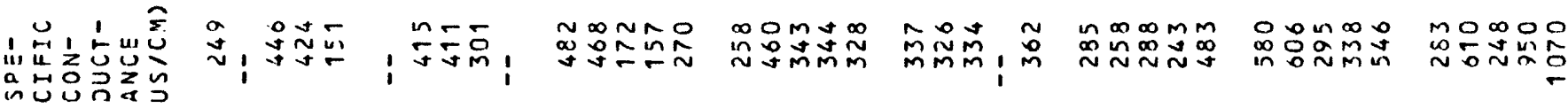
I us $\propto \tilde{\omega}$

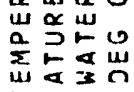

ominoo 00000000 ono . in in mis

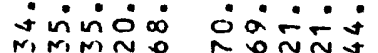
in: $00 \%$ un m 00

nnomin - inim

On m o in

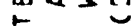
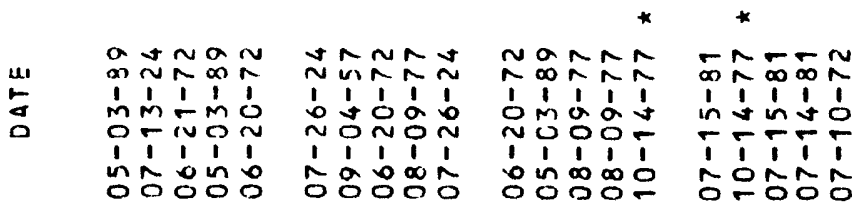
$\infty \infty \infty$ ! *゚oññ mín் $\stackrel{\omega}{n}$

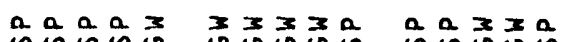

a a a a

a $a \propto a$

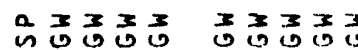

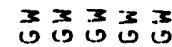

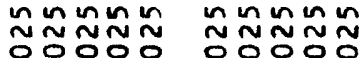

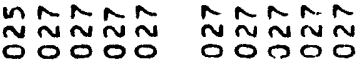

$\hat{N} \hat{\sim} \tilde{N} \tilde{N} \tilde{N}$

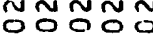

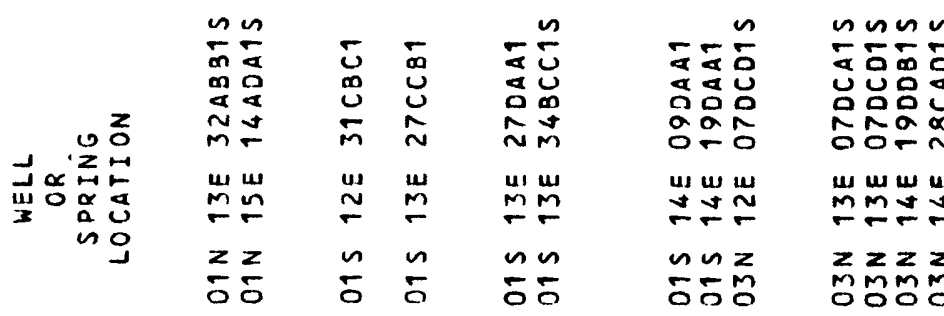

\begin{tabular}{|c|c|c|c|c|}
\hline $\begin{array}{l}n \\
\frac{n}{\alpha} \\
a \\
0 \\
0 \\
m\end{array}$ & 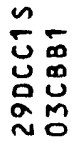 & 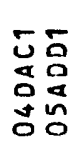 & 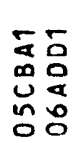 & 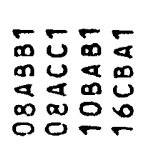 \\
\hline$\underset{\Psi}{w}$ & $\begin{array}{l}m \geq \\
\pm \tilde{O}\end{array}$ & 吾笠 & $\begin{array}{l}x \\
\tilde{0} \tilde{0}\end{array}$ & 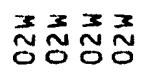 \\
\hline$z_{m}$ & $\begin{array}{ll}z & z \\
0 & 0\end{array}$ & $\frac{2}{0} \frac{2}{0}$ & $\frac{2}{0} \frac{2}{0}$ & $\begin{array}{l}2 z 2 z \\
5050\end{array}$ \\
\hline
\end{tabular}




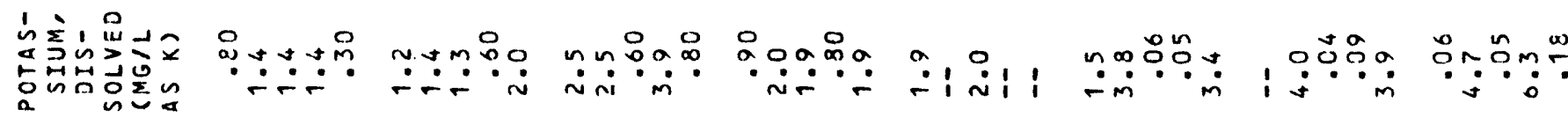

$\sum_{i=1}$

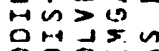

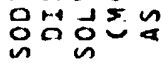

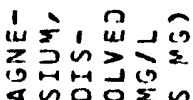

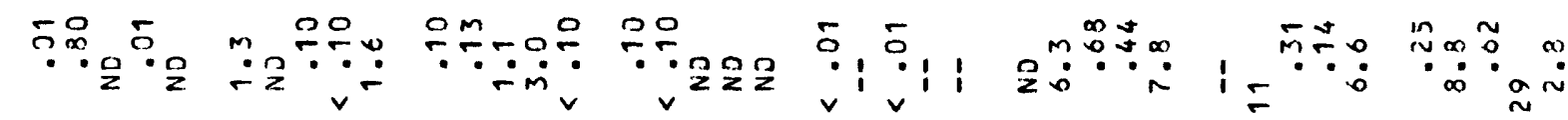

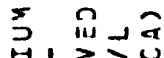

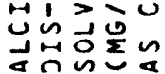

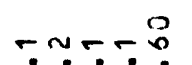

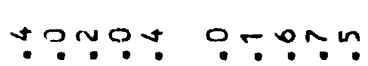

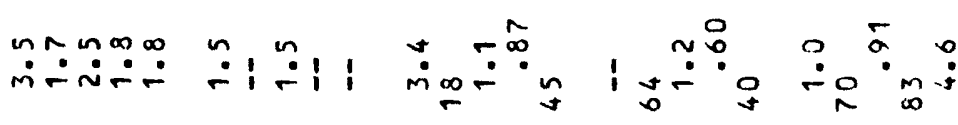

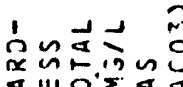

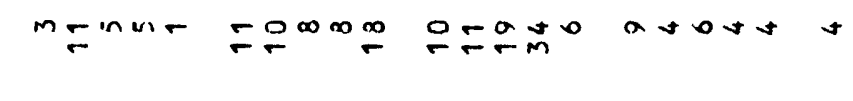

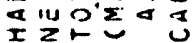

斻的品

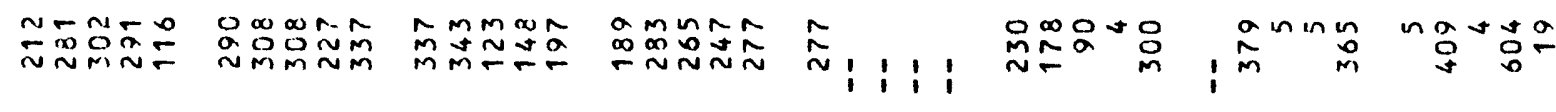

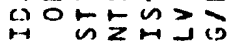

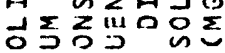

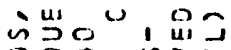

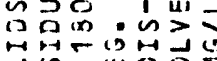

垈

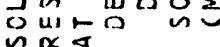

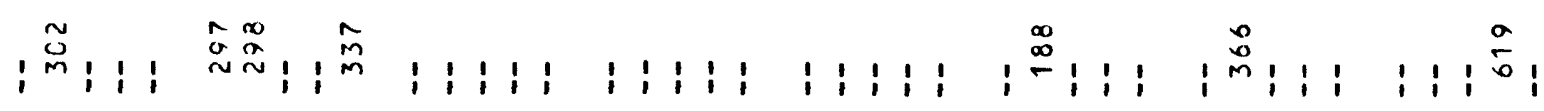

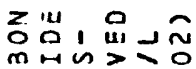

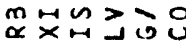

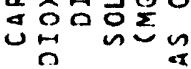

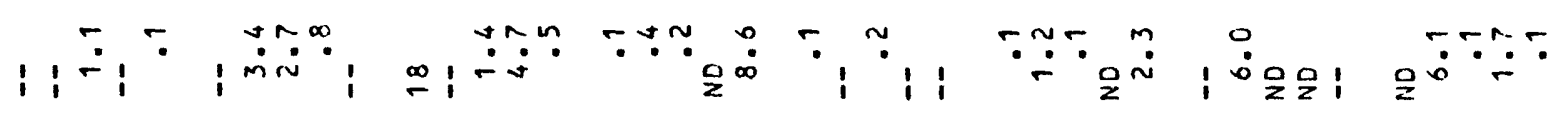

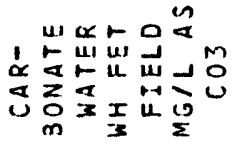

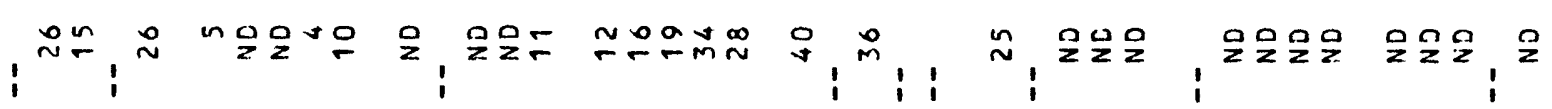

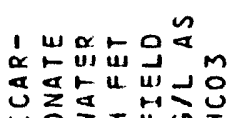

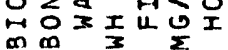

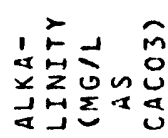

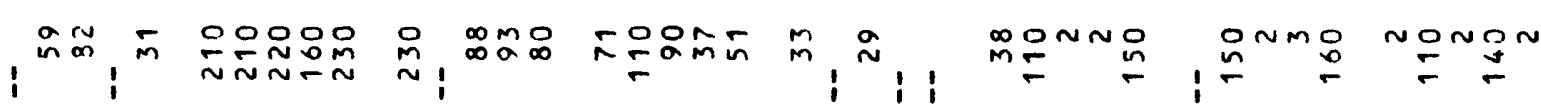

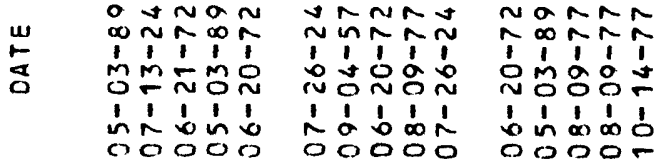

1:1: 1:1:1:1:

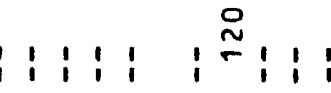

: i : i

00000

on

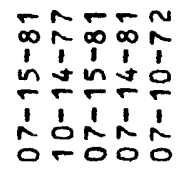

$-\infty m a-$

i

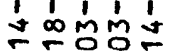

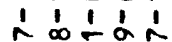

tin in in

in in

ํํㅇํํ요

네요 1

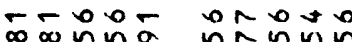

1

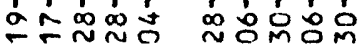

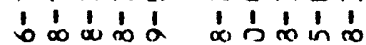

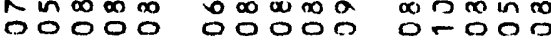




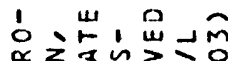

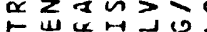

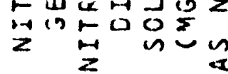

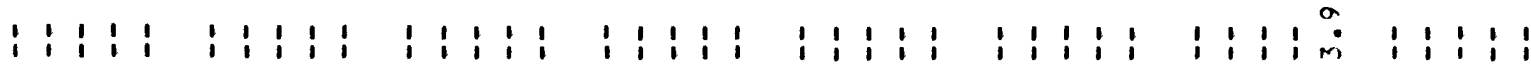

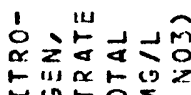

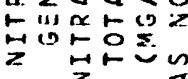

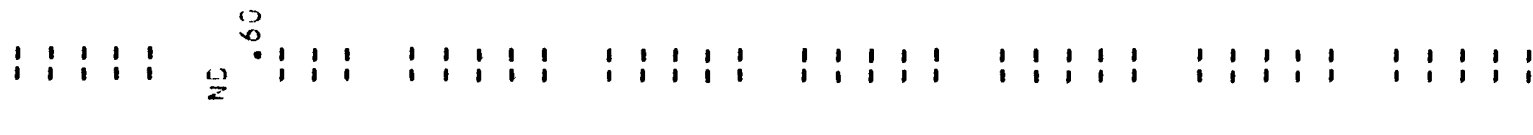

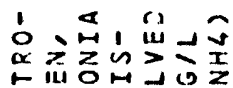

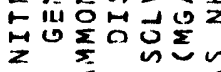

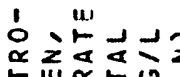

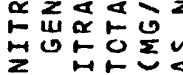

$1: 19: 11: 1: 1: 1: 1$

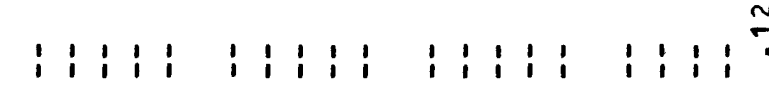

i i i i i

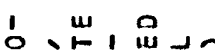

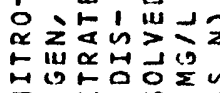

我"

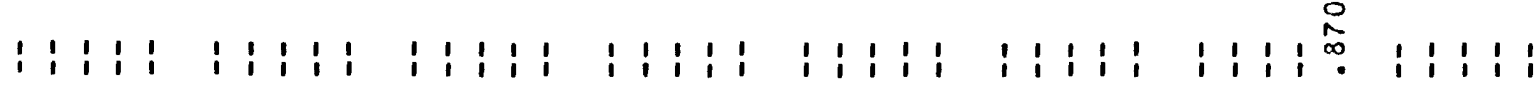

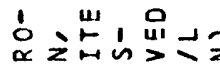

네

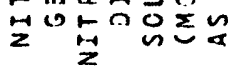

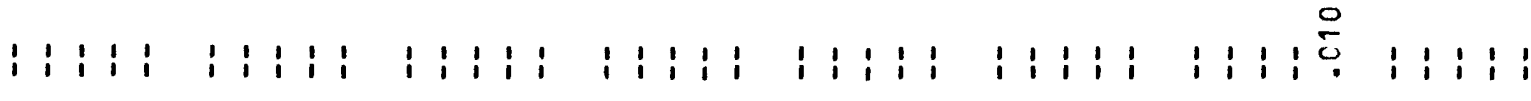

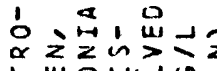

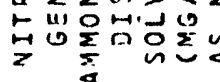

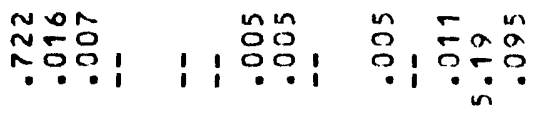

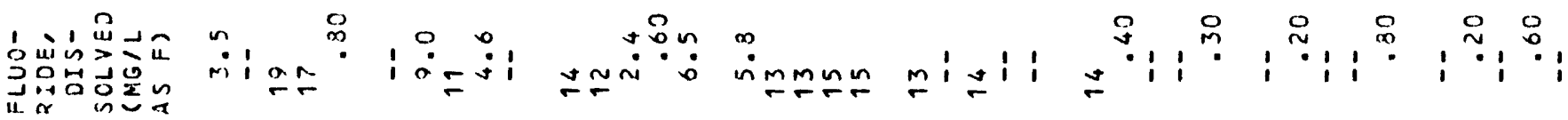

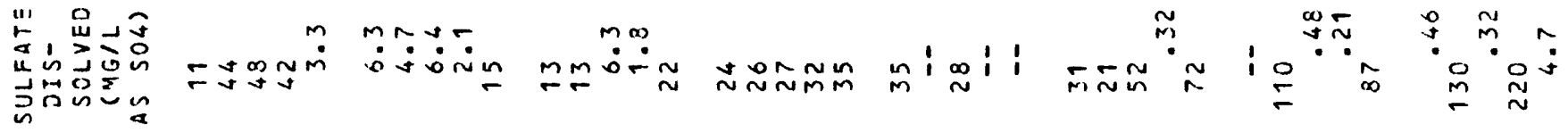

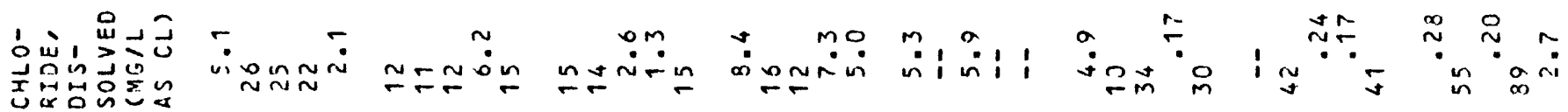

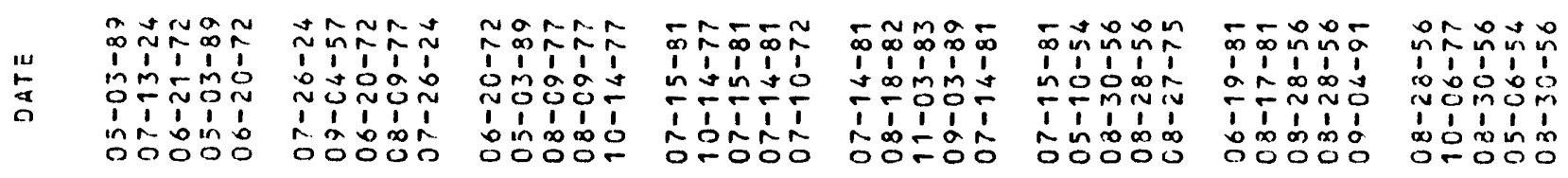




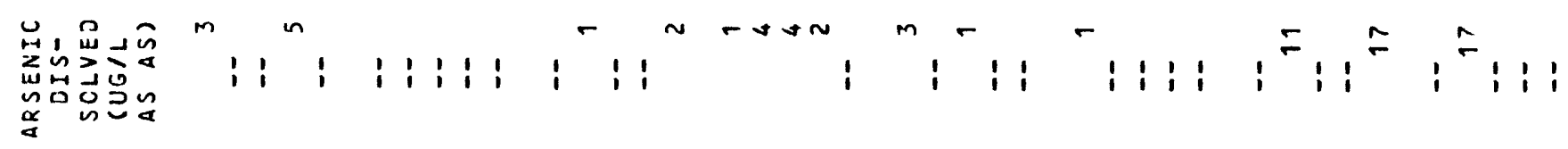

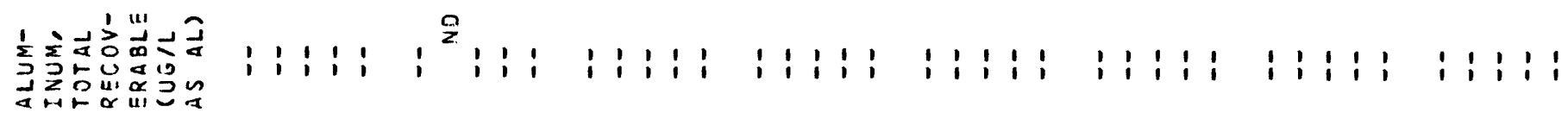

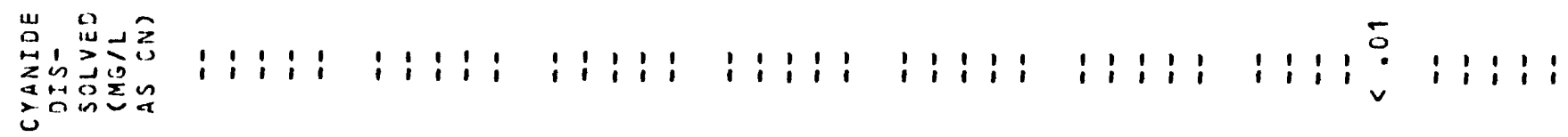

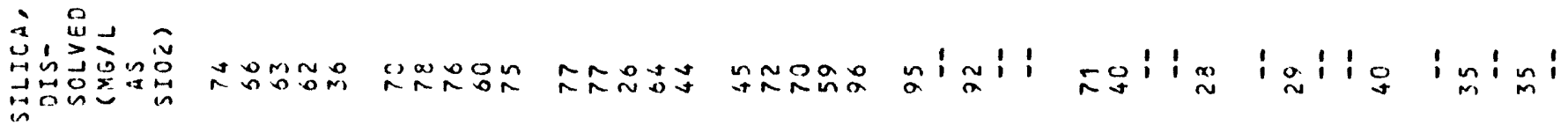

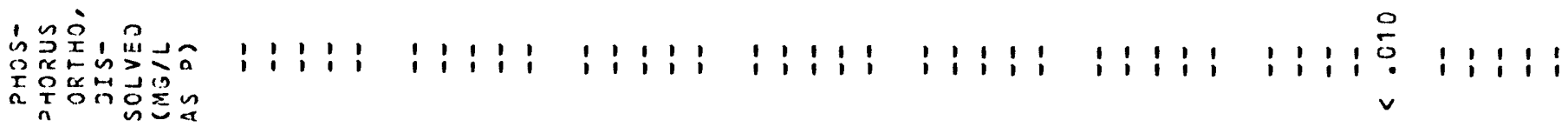

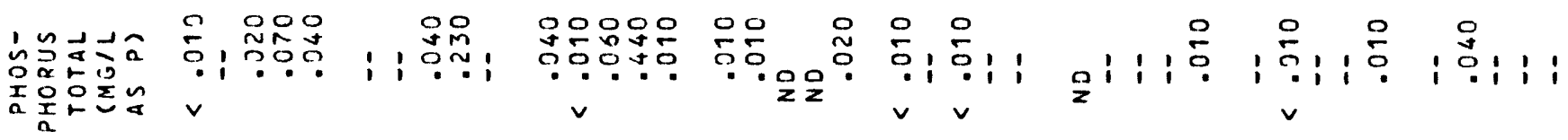

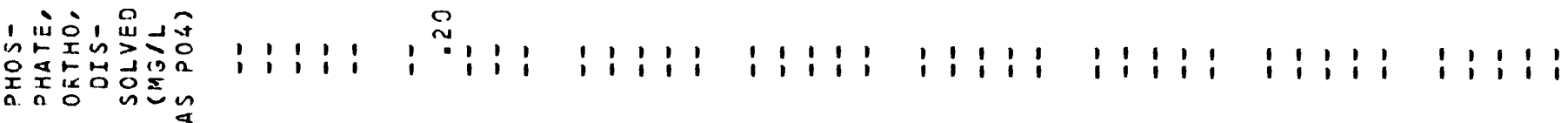

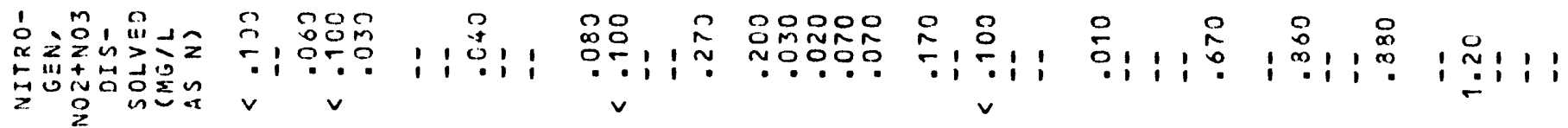

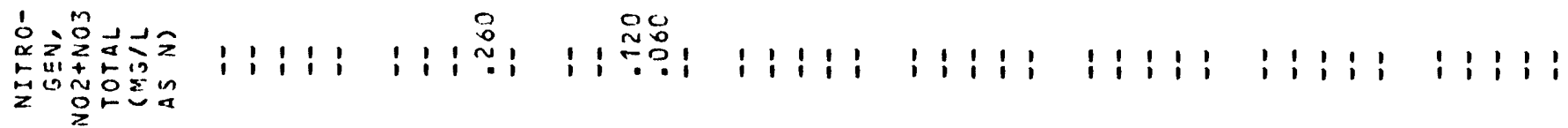

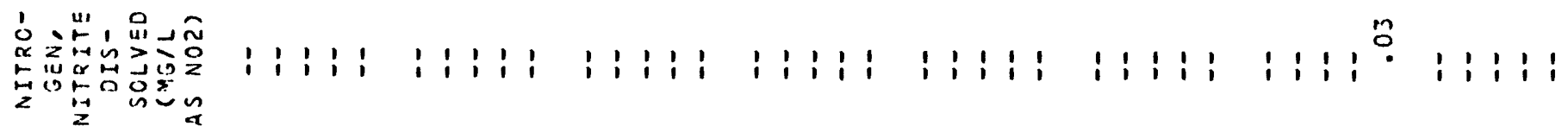

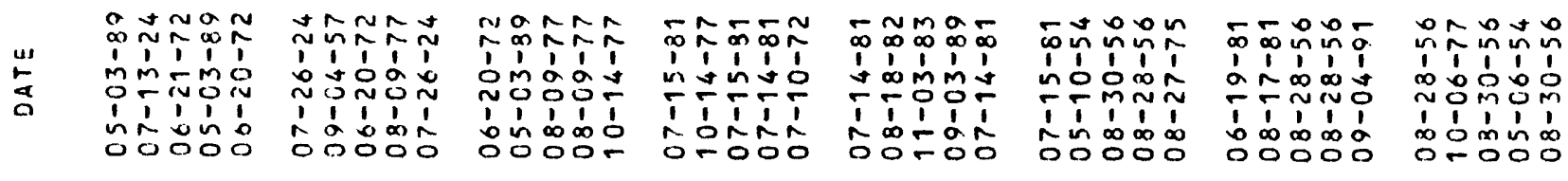




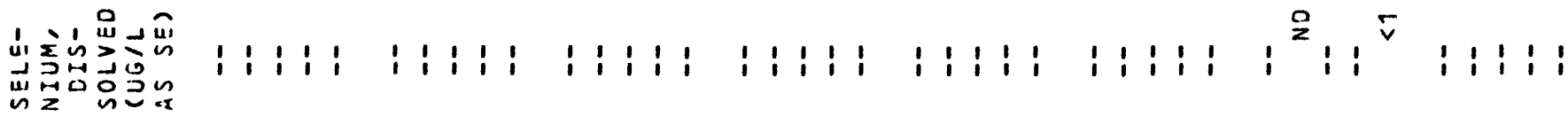

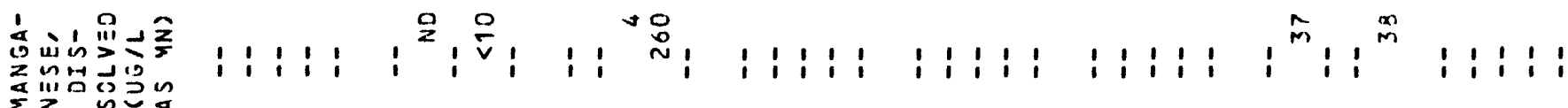

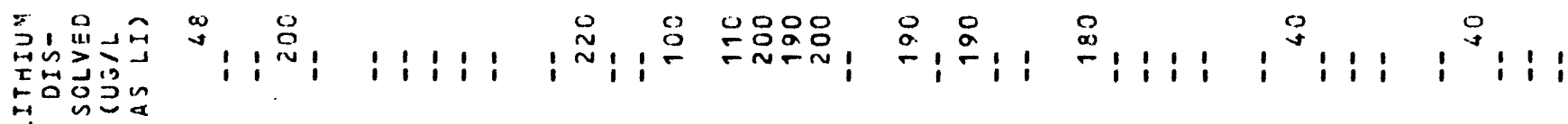

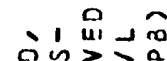
an $n>150$ यับำ

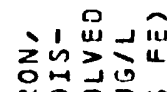
어요의

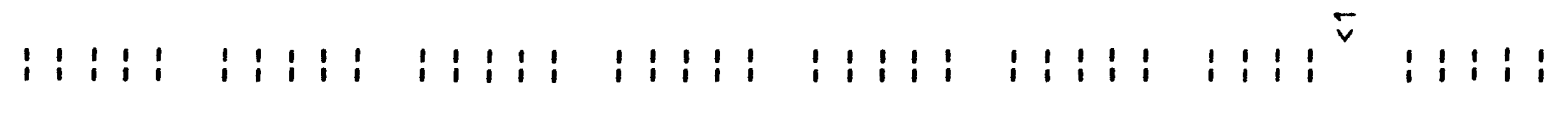

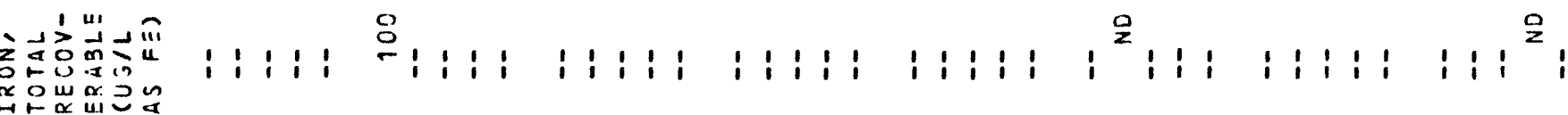

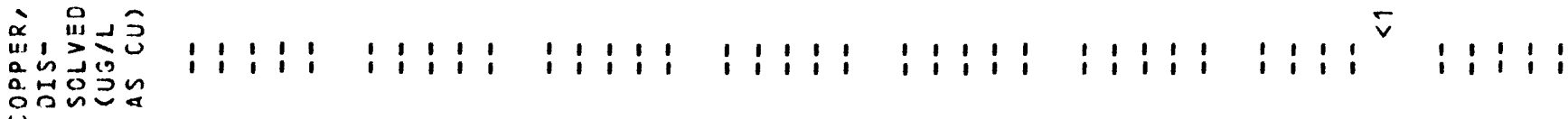

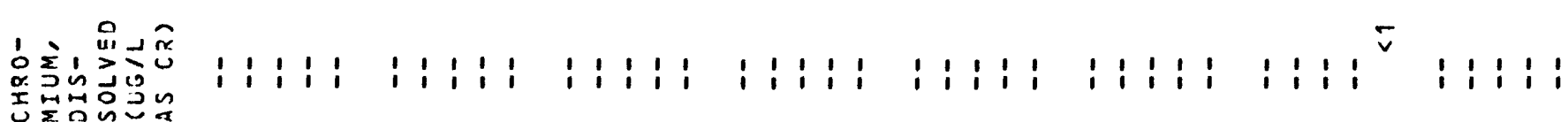

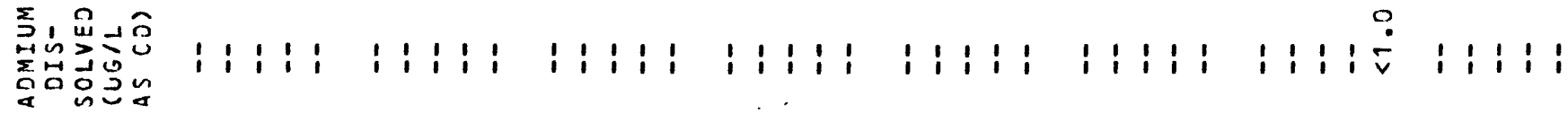

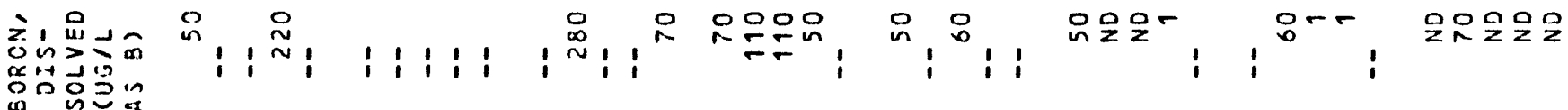

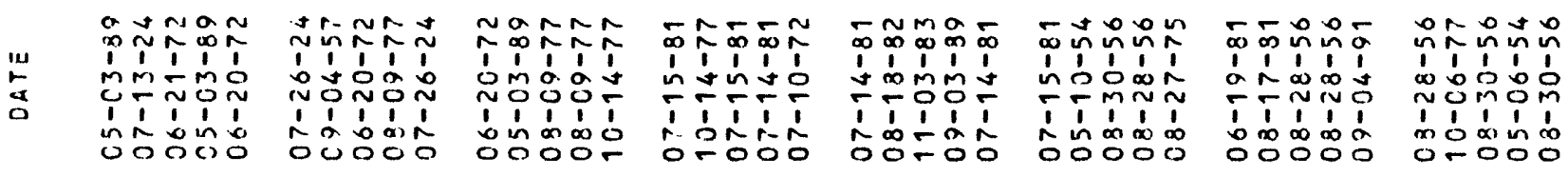




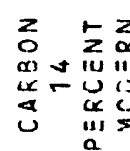

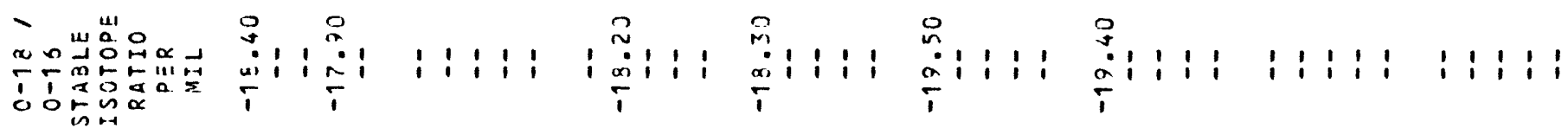

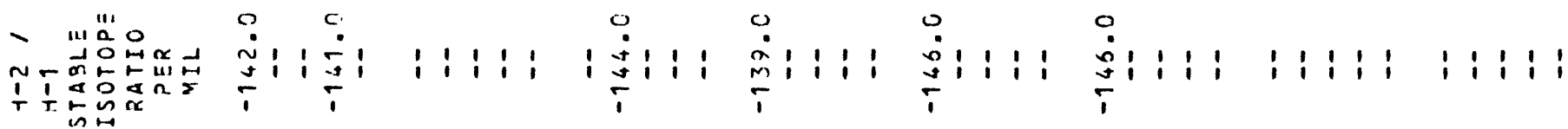

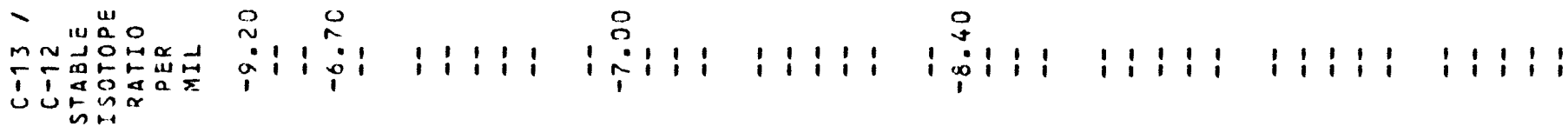

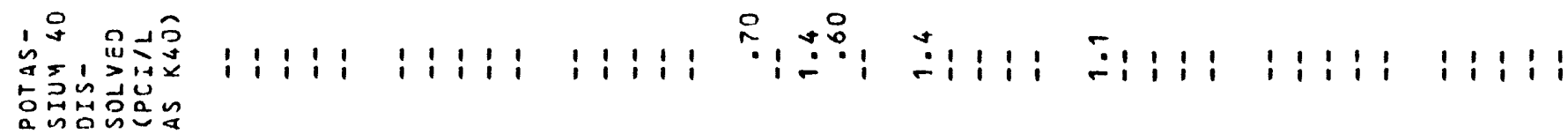

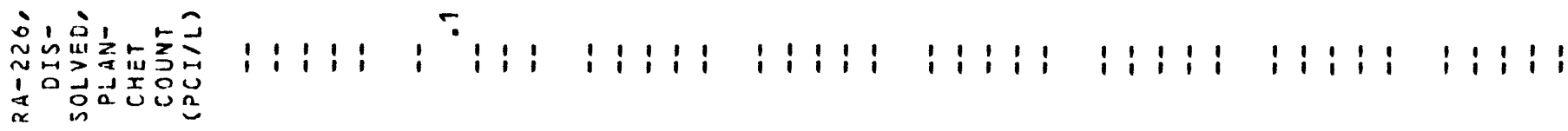

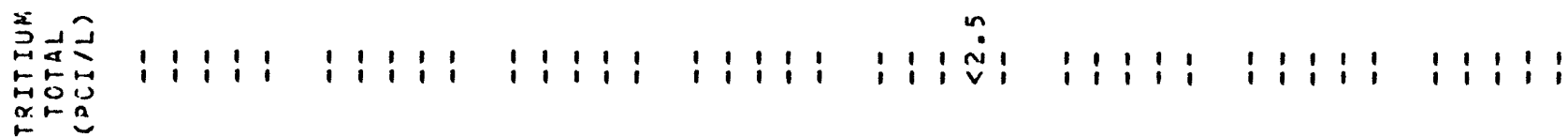

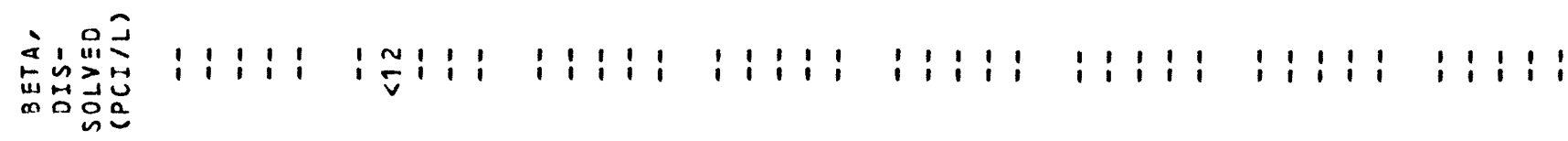

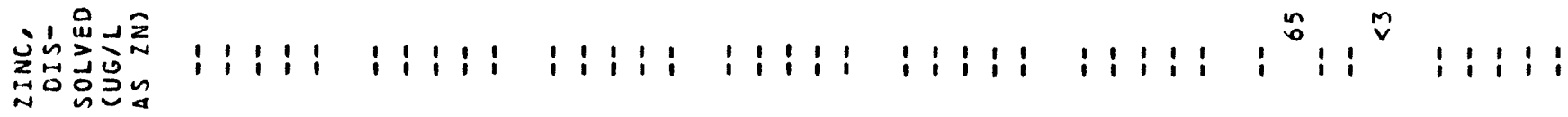

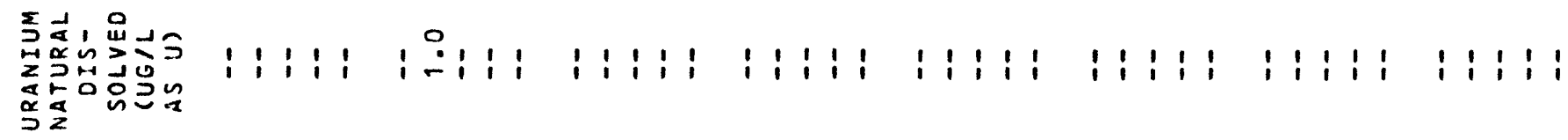

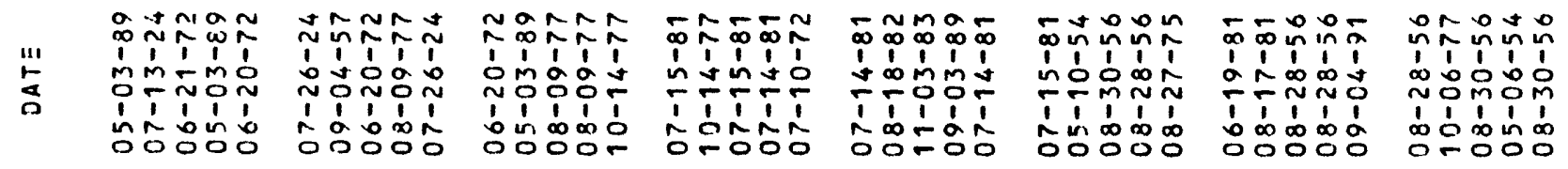




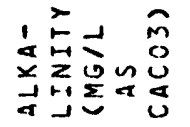

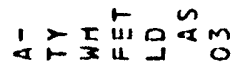

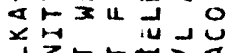

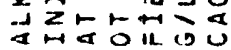

nmmon onOo

$\frac{1}{6}$

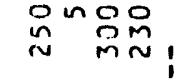

2000 raㄴ?

món

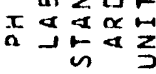

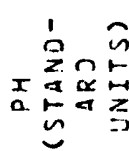

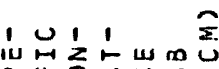

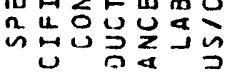

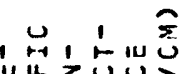

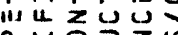
in

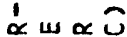
u) a $\sum_{11}^{\infty}=0$

00000

nogon

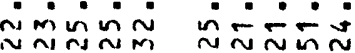

oิn bon

응용ㅇㅇㅇㅛ in N

oogmo mán in

o 00 in y $\lim _{\infty}^{\infty} \mathbb{N}$

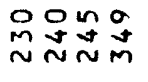

옹요 in mi⿻i弋in

O 0 nn Niming

- oNo iñ

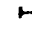

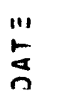 \\ $\stackrel{11 !}{5}$}

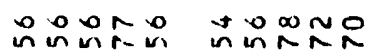
inut:

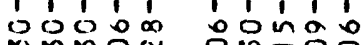
mị! y

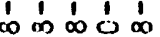

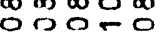
in

$\stackrel{a 1}{\leftarrow}$

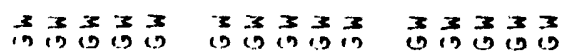

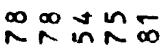
î, in mmon $-50 \pi$ 向向 in åำ

0.0 in 0 m

0000 n

000 in

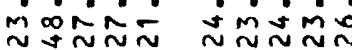

O in $\ln$ in mím

00000 iñ்in

ogoino

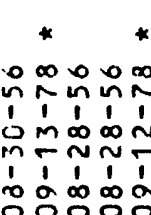
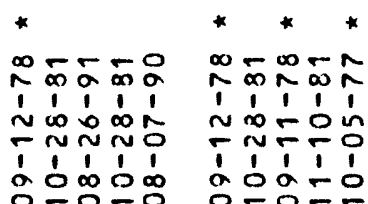
***

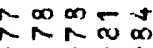
1 T nomm $0 \div-5 \frac{1}{1}$ oa 0 -

$+\log ^{2} 0$ औरำ nn un 2 in - $1 \frac{1}{1} \div$ $=R=\infty \infty$

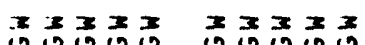

$3 x 33 x$

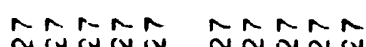

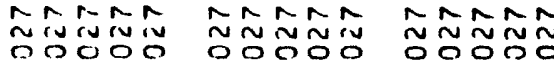

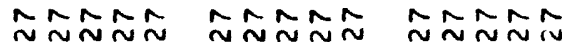
OOOOO OONOO O000ำ

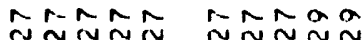

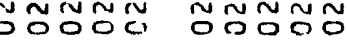

\begin{tabular}{|c|c|c|c|c|c|c|c|c|c|c|c|c|}
\hline & 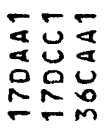 & $\begin{array}{l}\bar{\infty} \\
\substack{\infty \\
0 \\
\sim}\end{array}$ & $\begin{array}{l}5 \\
\infty \\
\infty \\
a \\
n \\
\simeq\end{array}$ & 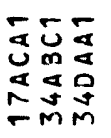 & 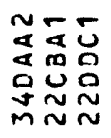 & 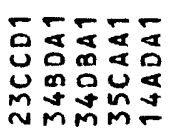 & $\begin{array}{l}\tilde{\infty} \\
\cup \\
0 \\
⿱ \\
\sim\end{array}$ & 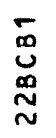 & 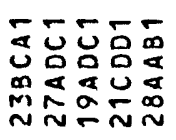 & 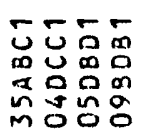 & 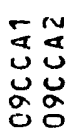 & 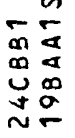 \\
\hline J结芯 & 焉管 & $\stackrel{m}{m}$ & $\underset{N}{n}$ & 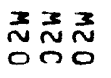 & 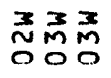 & $\begin{array}{l}x=3 \\
m \bar{m} \\
000 \\
000\end{array}$ & $\underset{n}{n}$ & $\begin{array}{l}x \\
0 \\
0\end{array}$ & 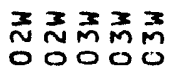 & 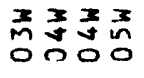 & $x_{n}^{3} x_{0}^{x}$ & \\
\hline & $\begin{array}{l}z \geq z \\
000\end{array}$ & $\frac{2}{0}$ & $\frac{1}{c}$ & $\begin{array}{l}n z Z \\
\sim \pi N \\
000\end{array}$ & $\begin{array}{lll}z & z \\
N & z \\
0 & 0 & 0\end{array}$ & 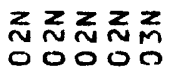 & $\begin{array}{l}z \\
m \\
0\end{array}$ & m & 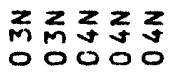 & 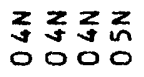 & $\begin{array}{l}z \underset{n}{z} \\
0 \text { in } \\
0\end{array}$ & \\
\hline
\end{tabular}




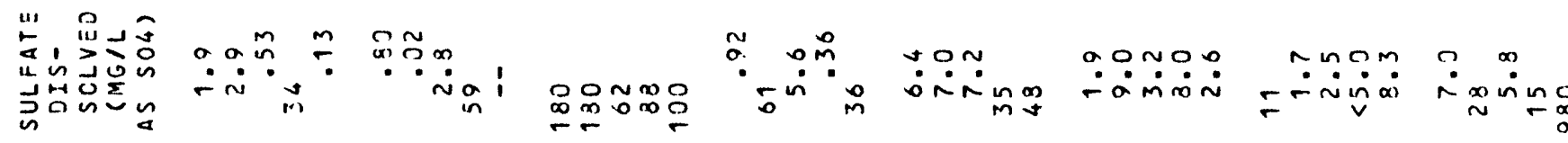

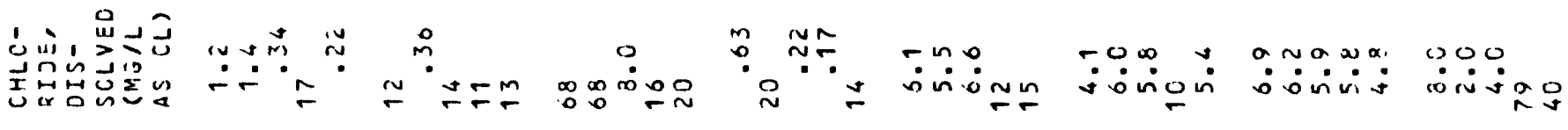

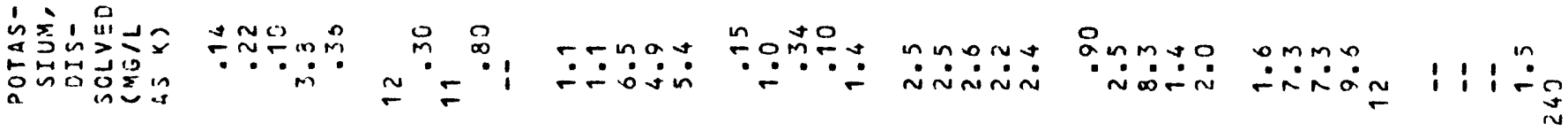

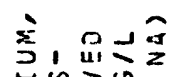

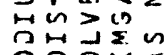
무워 ignimo m的的品点 o용ํำ

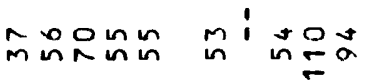

出交的出出

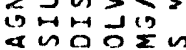

$\sim+\stackrel{\sim}{m m}$ $\therefore \dot{\sim} \cdot \dot{m}$

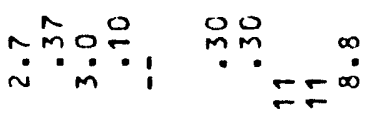

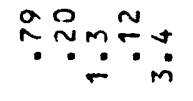
?omas: $800-\frac{0}{0}$ mando in in $\ddot{-} \dot{0}$ m

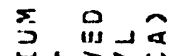

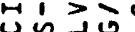

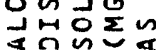

$\operatorname{oin} i n-\infty$ inis

?. $m$ $m a m i$

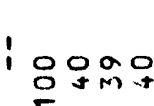
$\because 0 \div \stackrel{\infty}{?}$

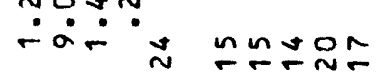
$\therefore 0$ ヘேำ ลำล $\div$ ำำ

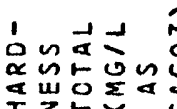

OOMnM trmon 00000 - nings

adara mand nod

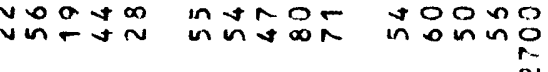

的的品今 战的衣

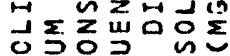

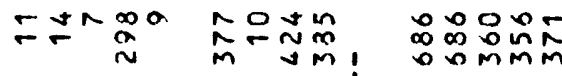
mon?

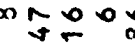

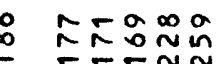
a $\rightarrow \frac{\pi n n}{2}$ orin 은은요

$\hat{\sim}+m \infty m$

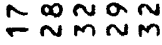

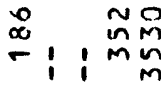

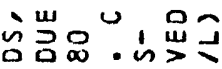

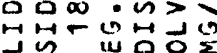
出出上出口品

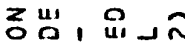
m $\propto \times \mapsto-100$ 떵유요의

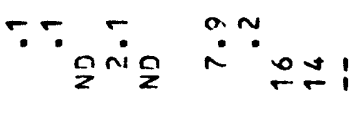
ơnum róñ à : i : i i in i in

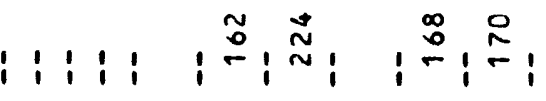
i $\stackrel{\infty}{\infty} !, \stackrel{0}{n} ! ! i$

웅aㅇ

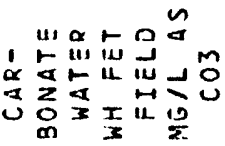
울를

1 1 x

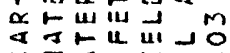

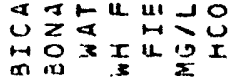

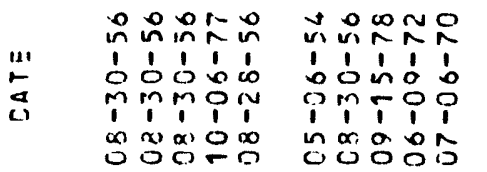

xisins mínd TFTT

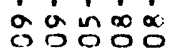

ํำ

向的占自

MTTTT

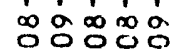

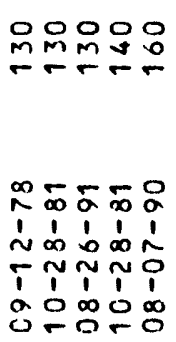

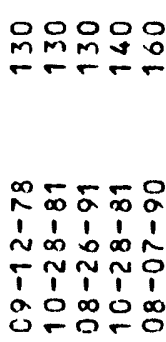

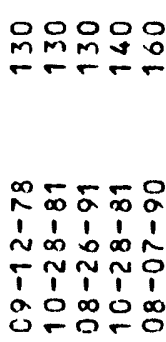

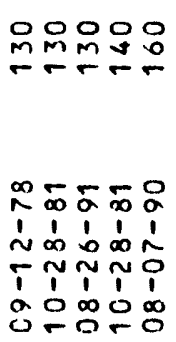

o 0000

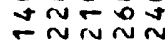

ONOOO ㄴํㅇํำ

$\stackrel{\infty}{\sim} \stackrel{\infty}{\infty}-\infty_{\infty}^{\infty}$

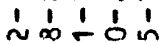
TT:Ti 응영두응

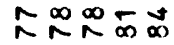

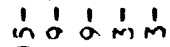
iTTT 응응영

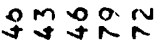
indín TTTit $=8=8$ 
的山我吉

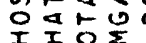

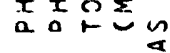

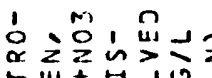

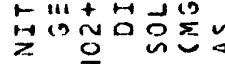

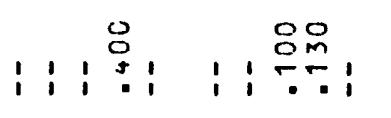

$\checkmark$

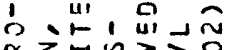

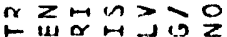

는은

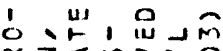

$x \geq x \sim \geq \geqslant$

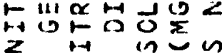

$z$ in

Oे

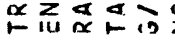

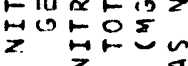

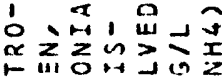

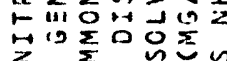

$1: 1: 13: 1$

1:11 1:19

1:1:1:11:

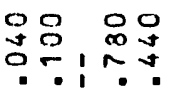

0
011
0

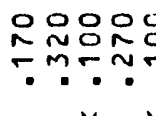

응응ㅇㅇ

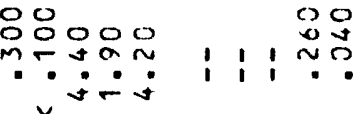

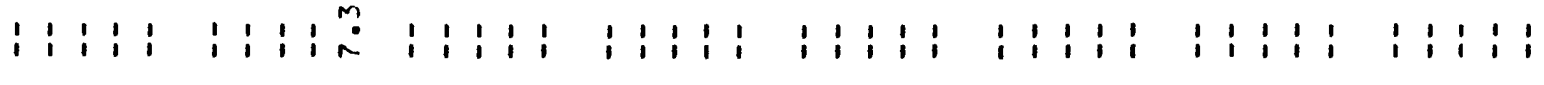

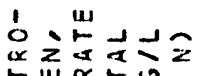

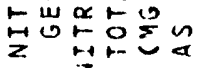

mon

11:19:11: 1: 1

i i i i

$\tilde{\circ}$

$\frac{\infty}{\infty}: \frac{\infty}{1}:$

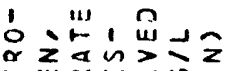

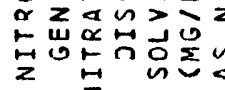

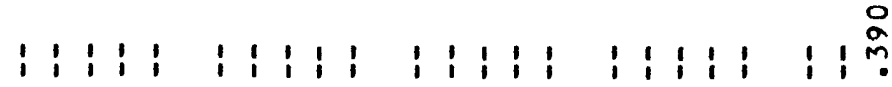

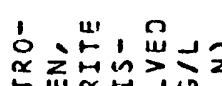

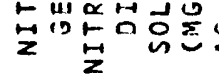

: 1:: i:1:

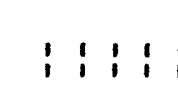

1:1:1:1:1:

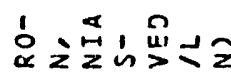

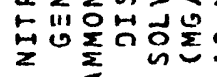

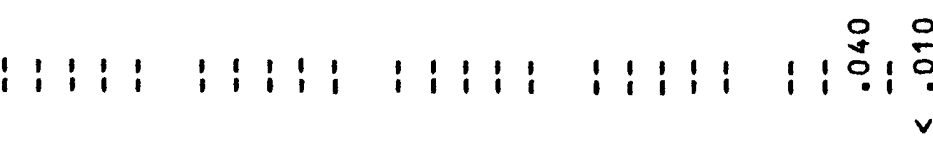

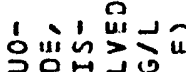

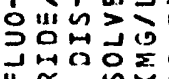

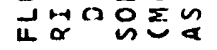

í:

1ำ ! ?

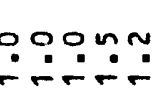

ㅇํํํำ

$\stackrel{0}{\infty} 0.00$

$\because$

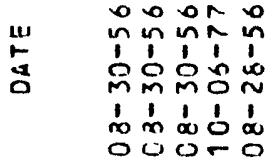

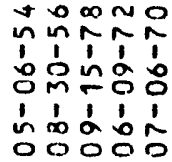

$\infty \sin ^{\infty}$

$0 \infty \infty \infty \infty \infty-\infty$

íticis

MMOÑ

d'áńs

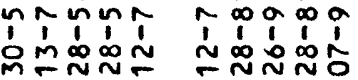

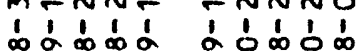

$\prod_{\infty}^{\infty} \prod_{\infty}^{\infty}-$

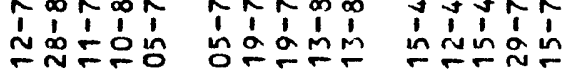

d lit

100ar

ํํㅇํํㅇํㅇ

a요의 


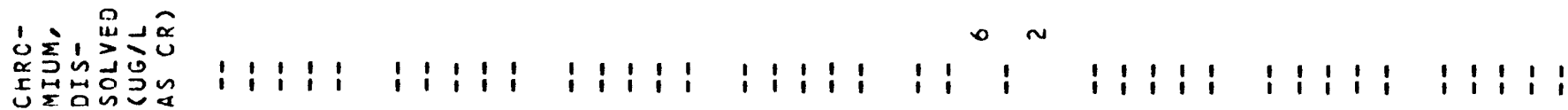

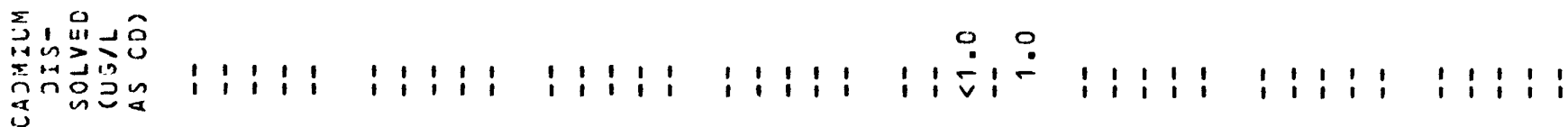

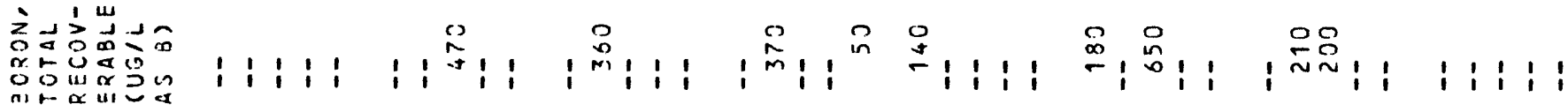

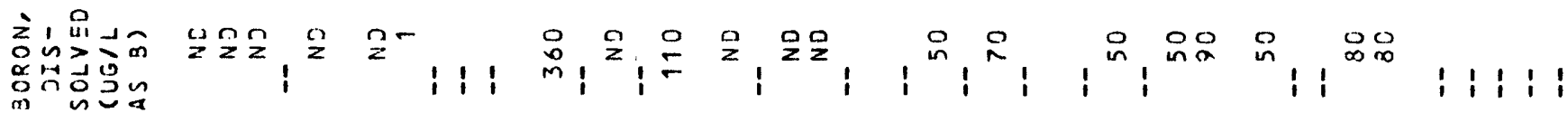

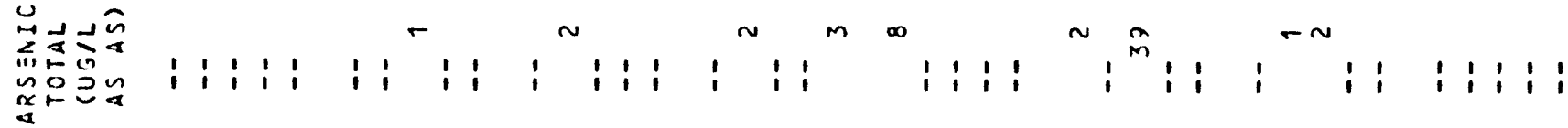

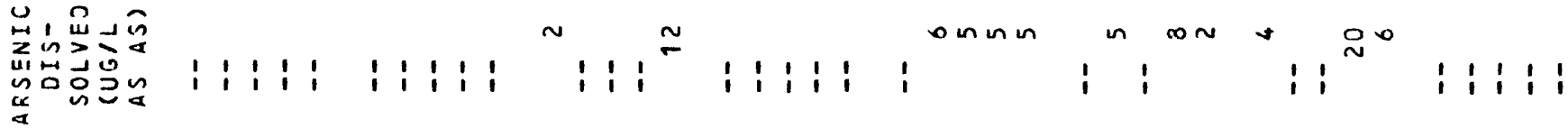

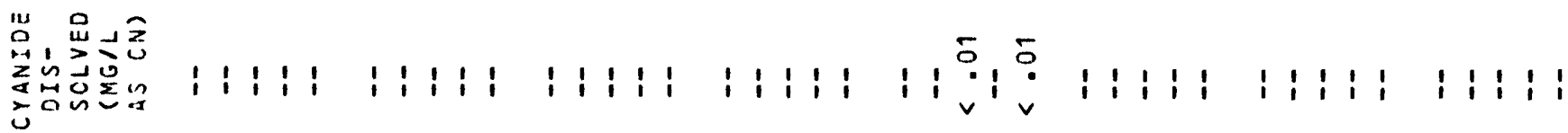

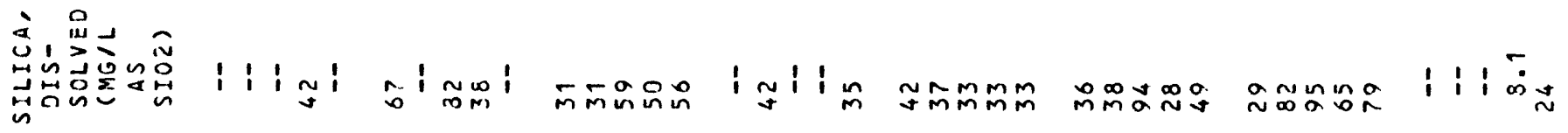

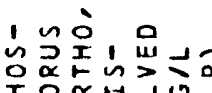

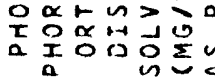

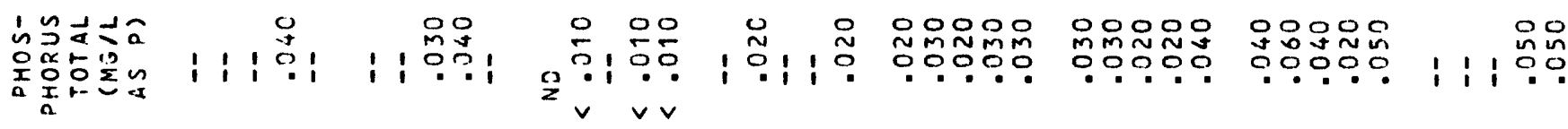

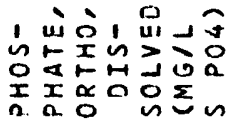

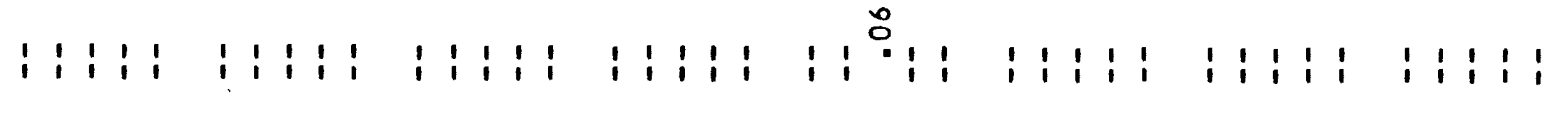

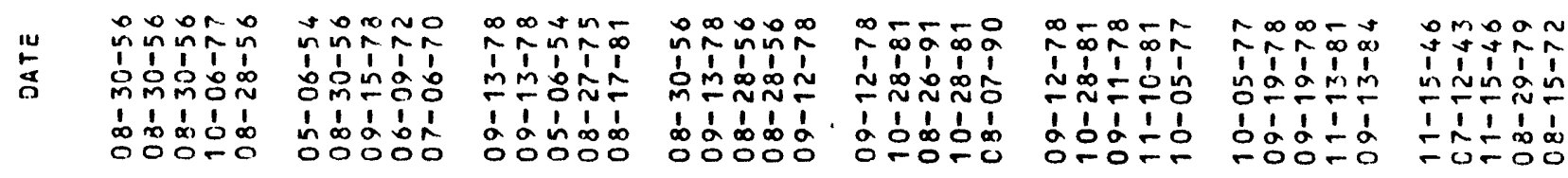




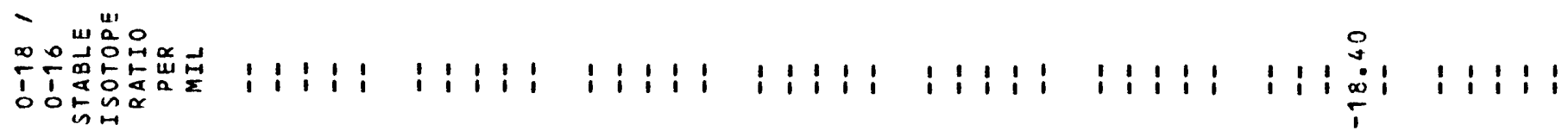

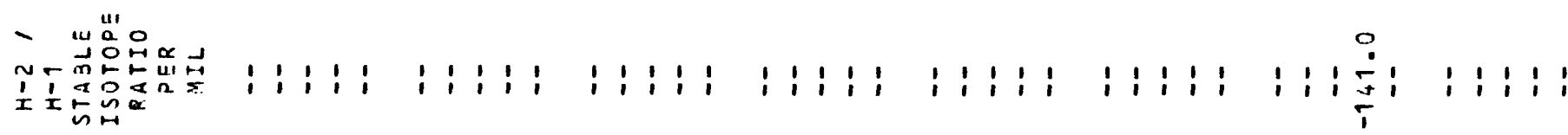

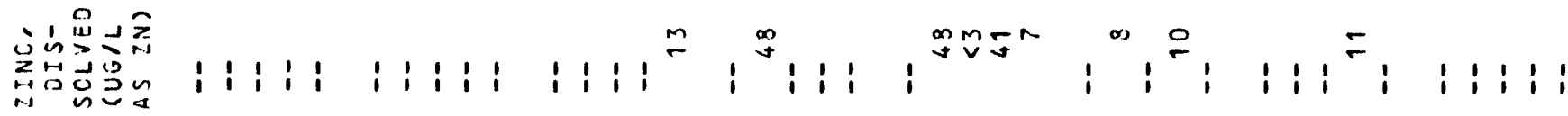

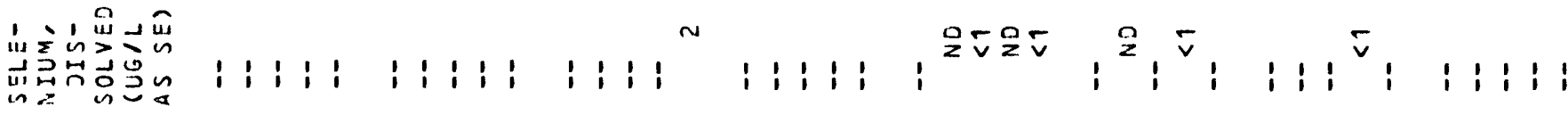

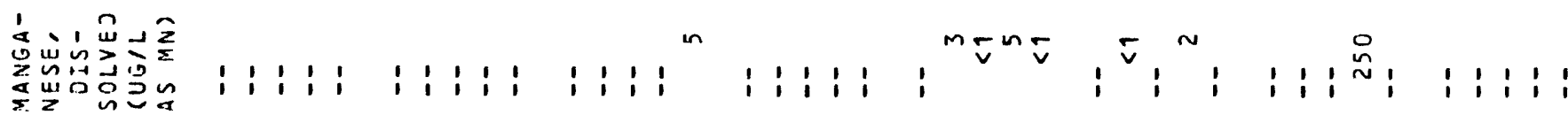

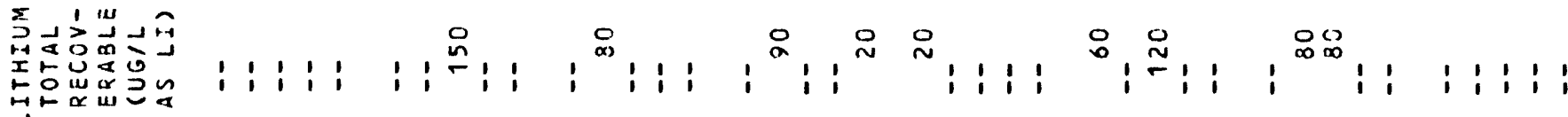

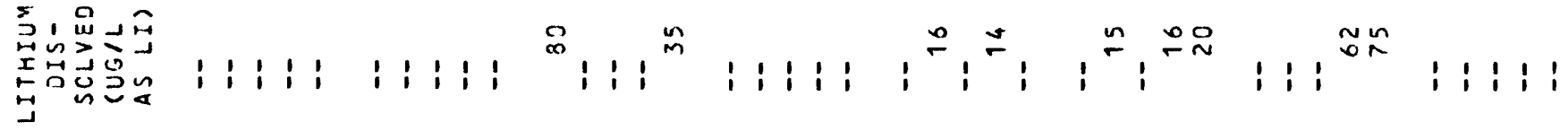

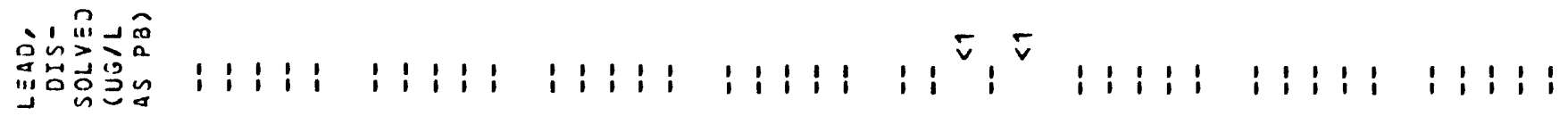

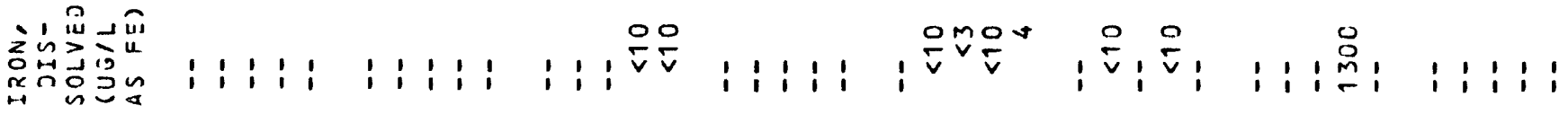

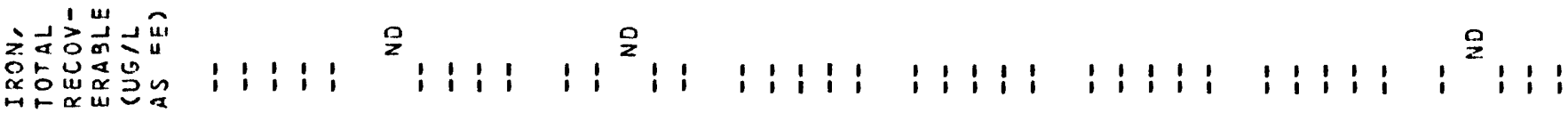

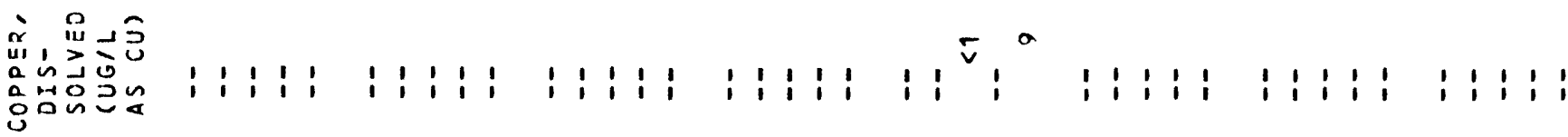

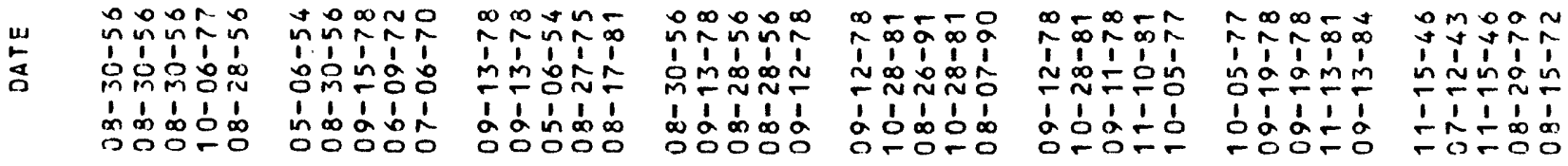




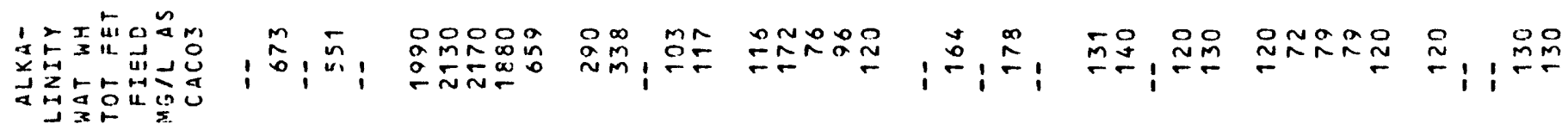

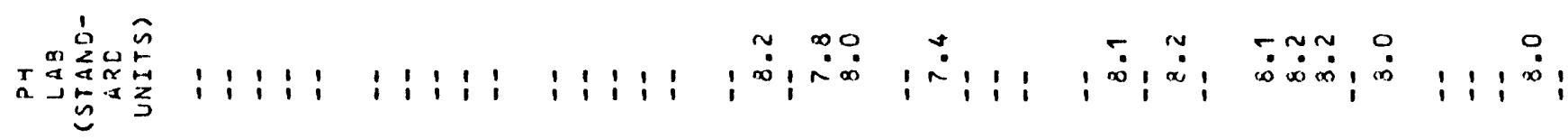

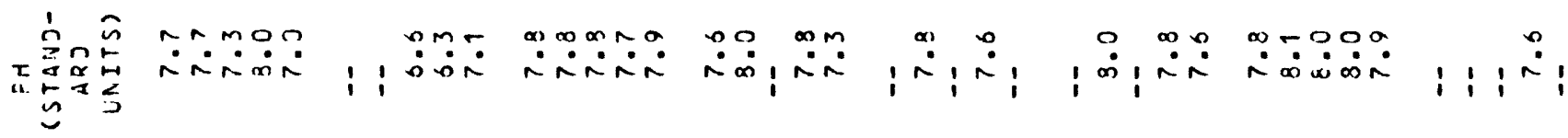
is $\begin{aligned} & 1 \\ & \text { a } 14\end{aligned}$

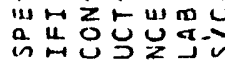
U 2 a

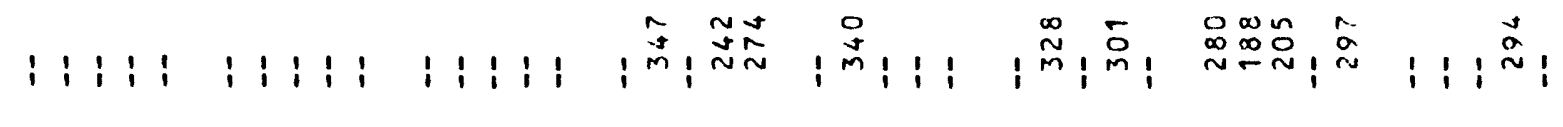

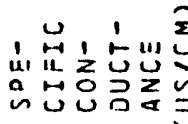

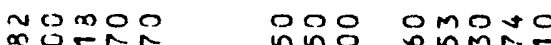

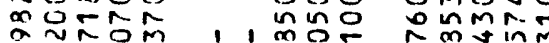

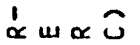
i1) $\propto$ แ $x_{i=1}=0$

oogou onooo o0000

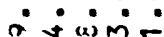

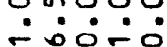
in i i i

o $000 \mathrm{~m}$ iñ

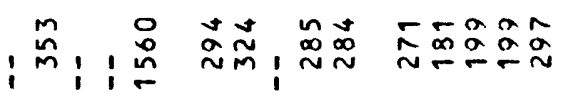
$i: \stackrel{\infty}{n} !$ $w a \geq 0$

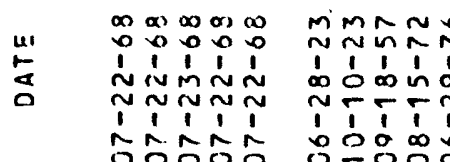

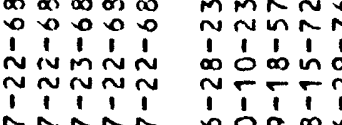
50.0ำ

$\infty \infty$ an $N$ in î ก N

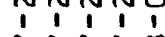

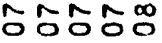

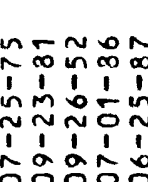

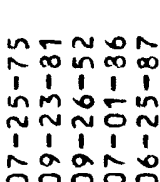

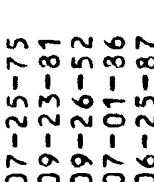

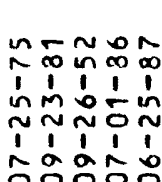
i i i

no $0 \mathrm{~m}$

in 000 in I

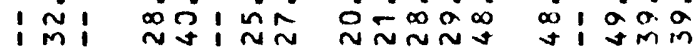

in $\min m$

$i$

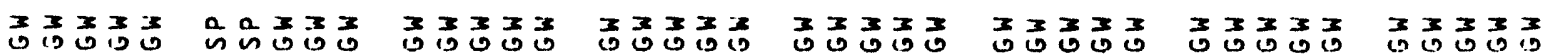

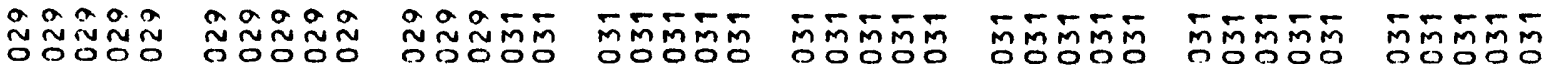

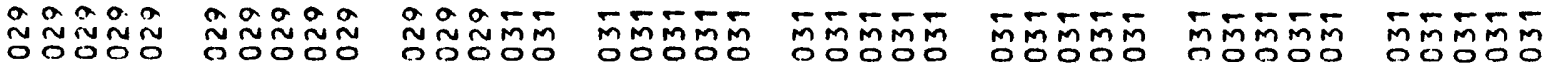

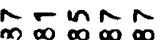

$\sim \sim \sim \sim \sim$ 1. Nㅜㄴ N i⿻日土

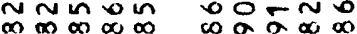
1111 1 111

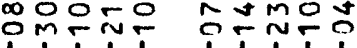

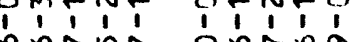

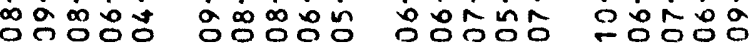

\begin{tabular}{|c|c|c|c|c|c|c|c|c|c|c|c|c|}
\hline \multirow{3}{*}{ 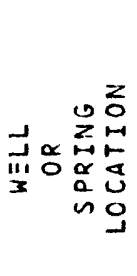 } & 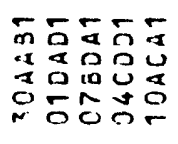 & $\begin{array}{l}\frac{a}{a} \\
a \\
\stackrel{a}{a}\end{array}$ & $\begin{array}{l} \\
\vdots \\
0 \\
\text { a } \\
\sim\end{array}$ & 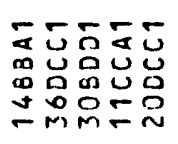 & 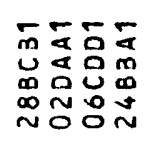 & 预 & $\begin{array}{l}-5 \\
0 \\
0 \\
0 \\
0\end{array}$ & 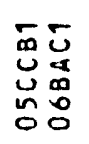 & $\begin{array}{l}\bar{\Xi} \\
\dot{a} \\
\end{array}$ & 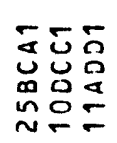 & 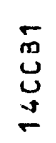 & $\begin{array}{l}\tilde{U} \\
\underline{O} \\
g\end{array}$ \\
\hline & 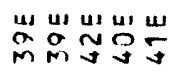 & $\frac{u}{\sigma}$ & $\frac{m}{\sigma}$ & 岁岩岩出出 & 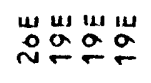 & $\stackrel{u}{\sim}$ & $\stackrel{u}{\sim}$ & 岩岕 & 屵 & 岩秥出 & $\frac{m}{N}$ & \\
\hline & 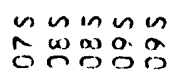 & $\tilde{a}$ & $\tilde{a}$ & 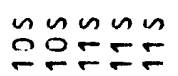 & 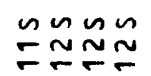 & $\cong$ & $\cong$ & $\cong \tilde{\simeq}$ & $\stackrel{\sim}{\sim}$ & $\cong \cong \cong$ & $\approx$ & \\
\hline
\end{tabular}




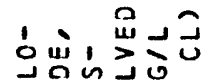

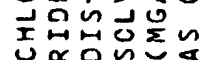

的空的坚立 过会分文

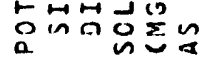

masor

wamin I' amm

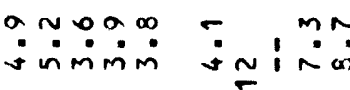

产, 品華

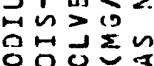

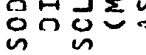

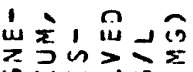

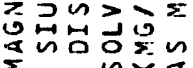

iñ 2 in

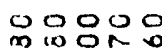

$\because$

ascina

$i: \simeq \simeq$

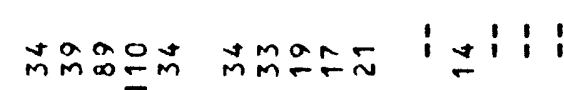

imimi ơ⿱宀⿻⺀大土

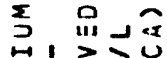

出的文向

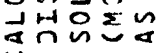

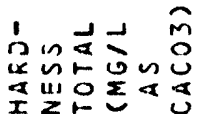

Donoo

$\simeq \approx n-m$

움음웅응

$\operatorname{anom}$

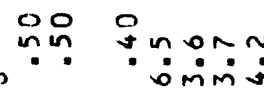

$\sin x$

omis $1=11$

ås,

$\dot{\infty} \dot{\sim} \hat{0}=$

$\because 11191$

Oonoo

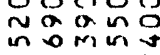

음응응요

mMNma

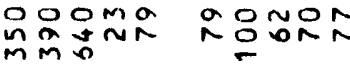

mूñ

i $\stackrel{\circ}{\circ}$ ! i

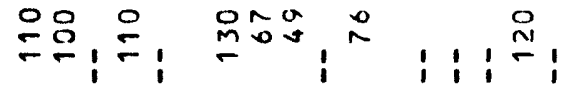

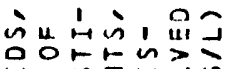

un in oo

iñ

요응

$m \infty a \sim N$

Doำ

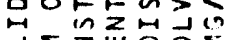

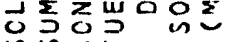

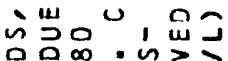

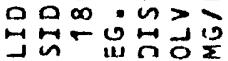

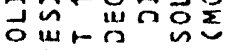

$\hat{a}-\sim \infty 0$

ar $\approx n m$

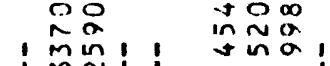

: $\bar{m} \bar{m} \stackrel{m}{\sim}$

$\checkmark$ ingm N N N

$\tilde{\sim}$

i $\sim$ ! !

ํㅗㄴ, $\stackrel{n}{\sim}$, mona,

$1: \stackrel{2}{2}$

zํㅣㅇㅛ man

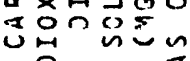

I

\&

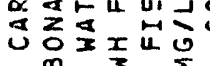

1 uxron

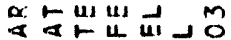

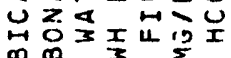

a) $200 \%$ in $\alpha$ ) 00

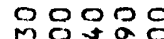
MO Nrin

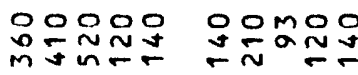

i 욤 :

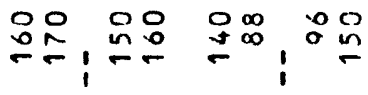

i i i :

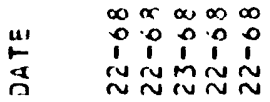

$12 \cos$

cos 000

$m M n N D$

1 1 1

은ำㅇำ

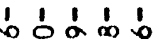

0ㅇํㅇ moñ

îj人

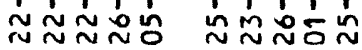

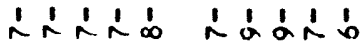

ố。ํㅇ

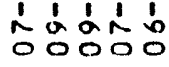

ก⿻上丨

$1: \infty \quad \infty$

은은

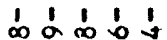

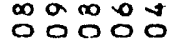

$\sim \operatorname{mn} N 0 \quad \sim N$ in 0 in

గn丨

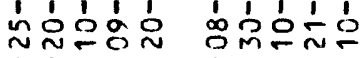

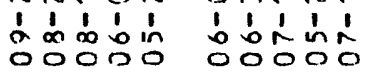

$\infty$ 은

19101 돈ㄷำ

$\begin{array}{llll}1 & 1 & 1 & 1 \\ 0 & 0 & 0 \\ 0 & 0 & 0 & 0\end{array}$ 


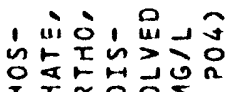

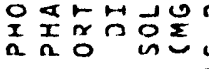

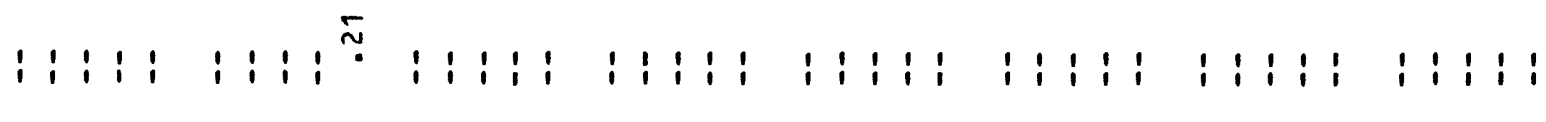

的藏古式

和起。

좀은?

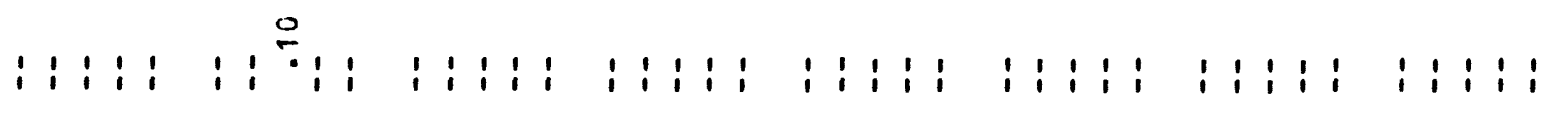

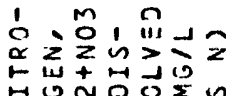

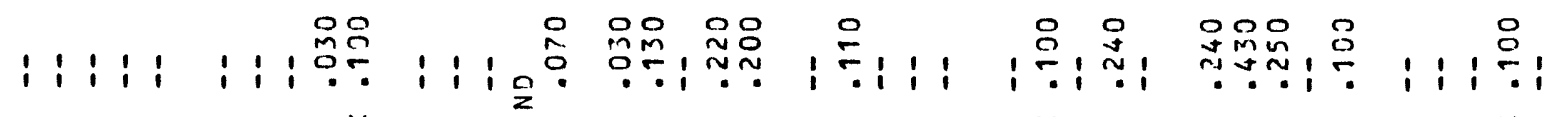

$z$

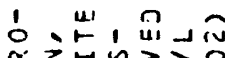

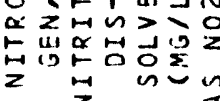

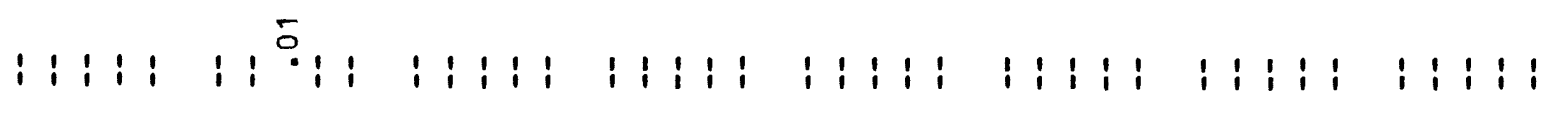

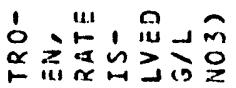

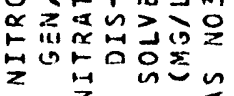

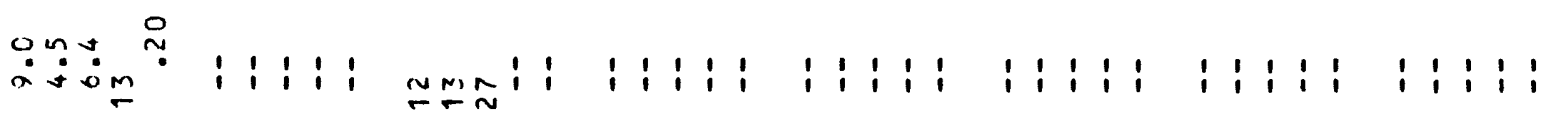

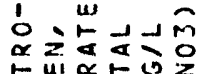

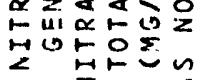

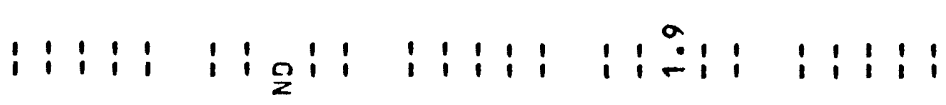

$:$

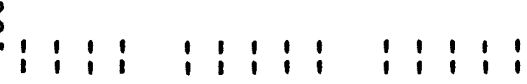

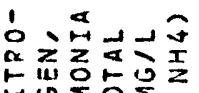

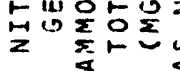

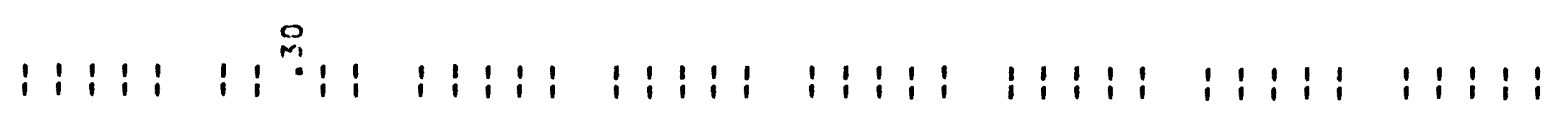

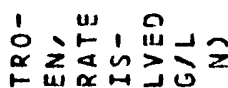

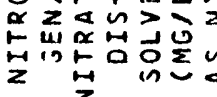

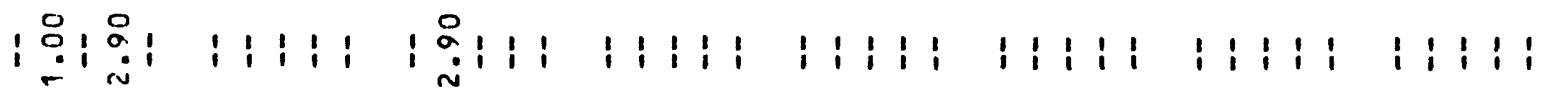

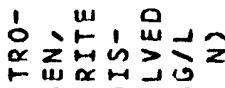

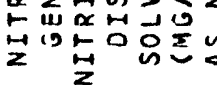

$\stackrel{m}{0}: 1 \quad i: 1 ; i$

i: $1: 1: \stackrel{m}{0}:$

i : i : i

1: $1: 1: 1: 1: 1$

1:1: i:1:i 1:1:

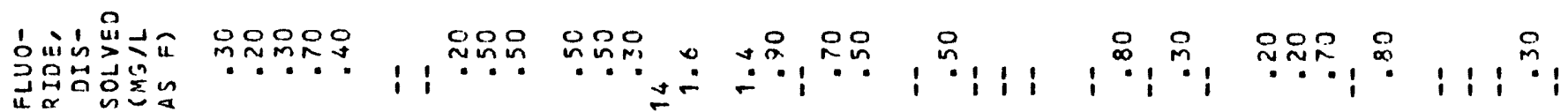

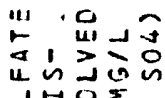

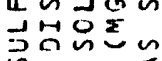

onnita gobog

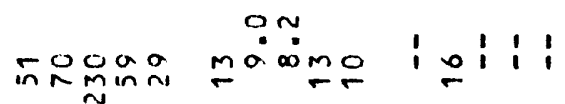

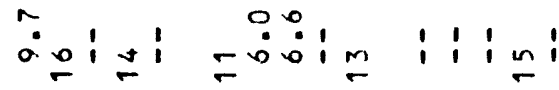

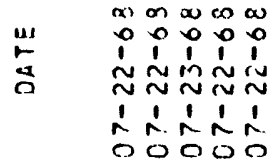

mmño momñ n-Nor

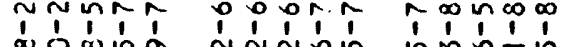

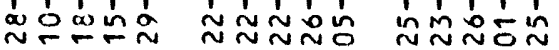

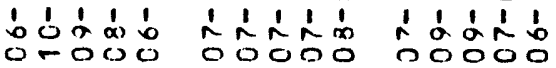

$\tilde{\infty}=\operatorname{nn}_{\infty}^{-\infty} \tilde{\infty}$

1

은ำ는

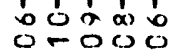

向向向占:

ทn

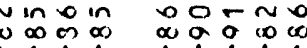

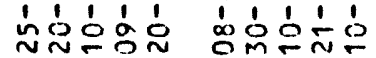

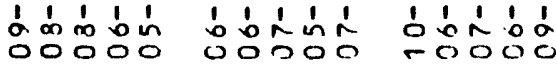




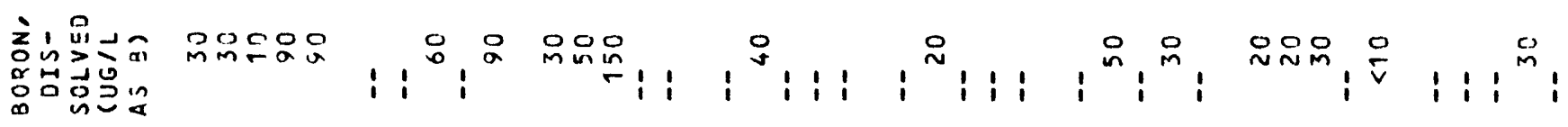

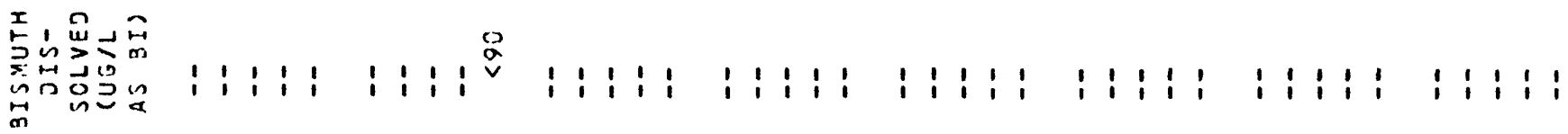

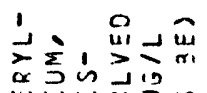

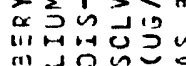

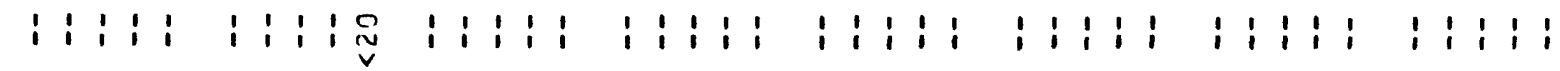

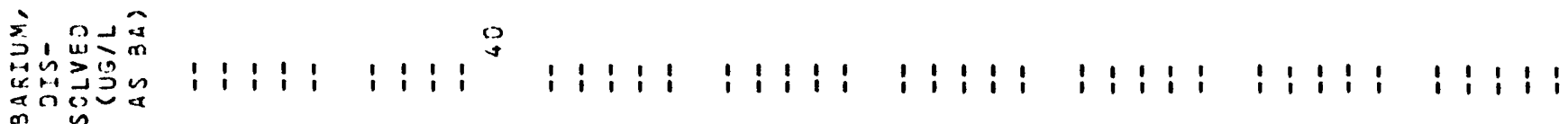

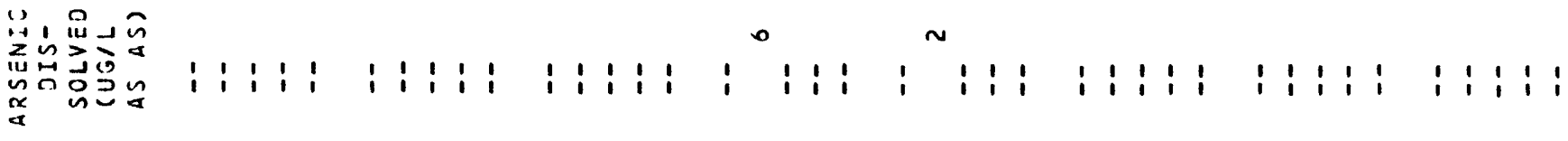

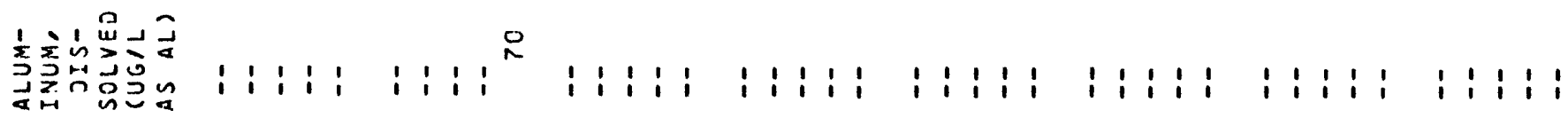

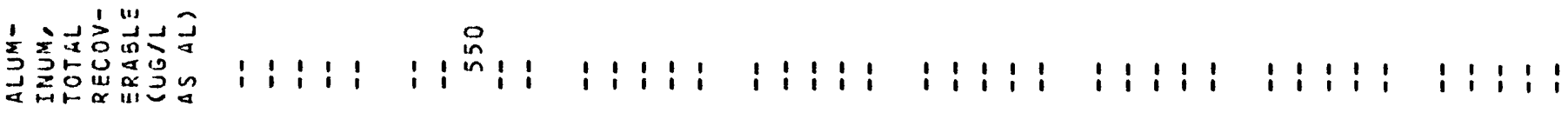

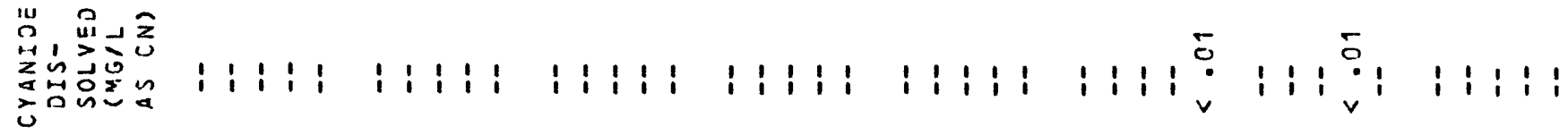

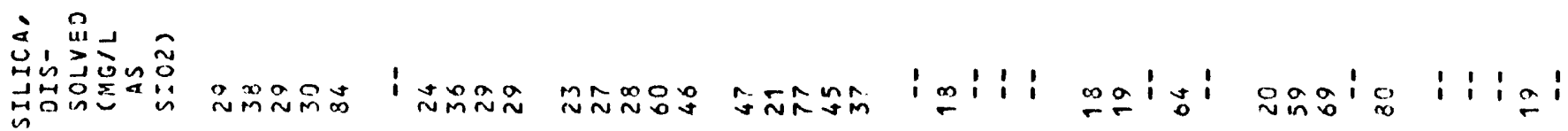

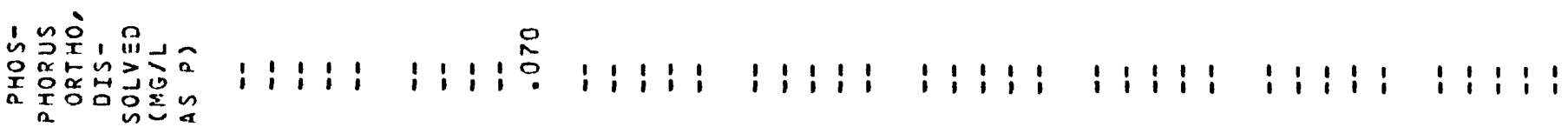

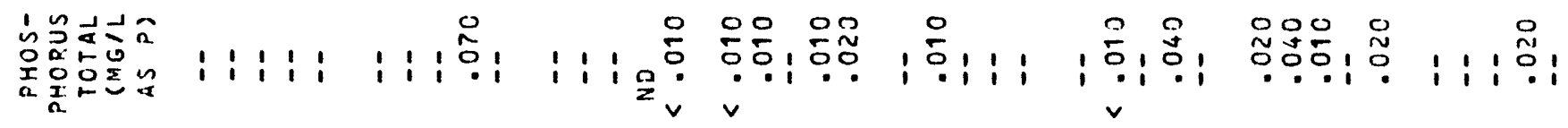

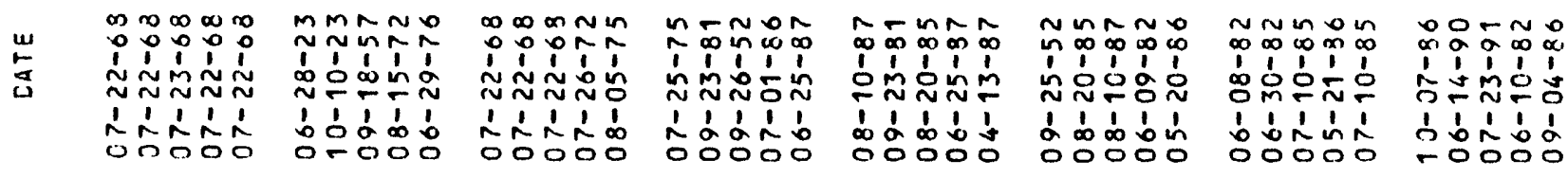




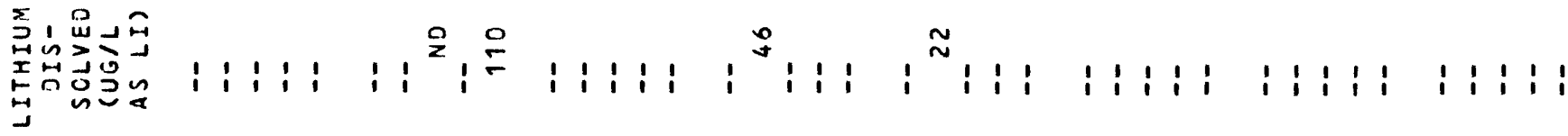

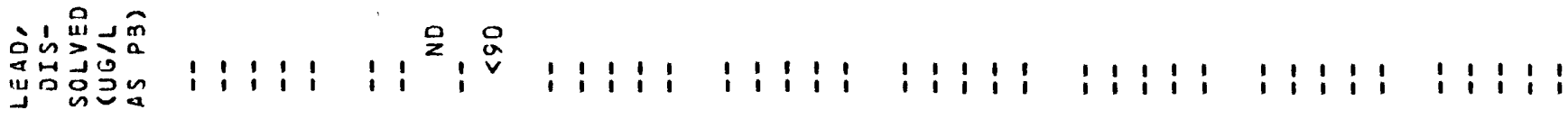

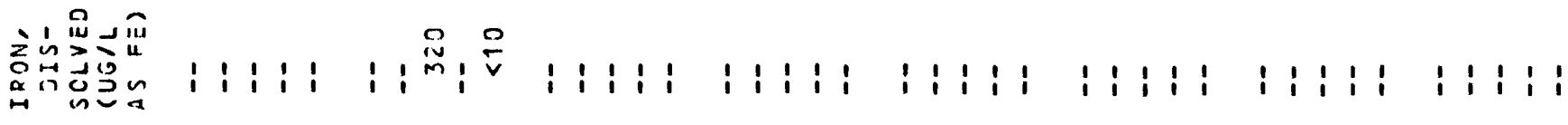

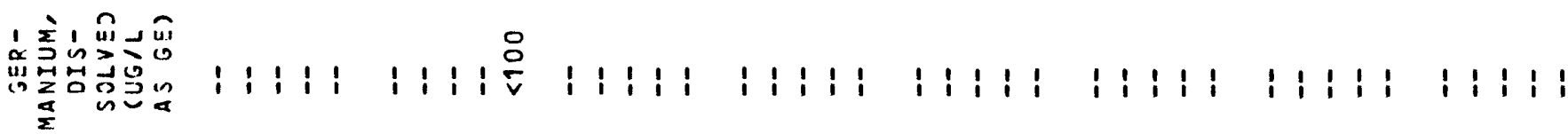

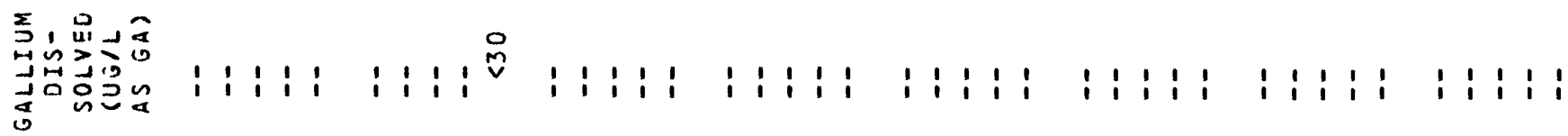

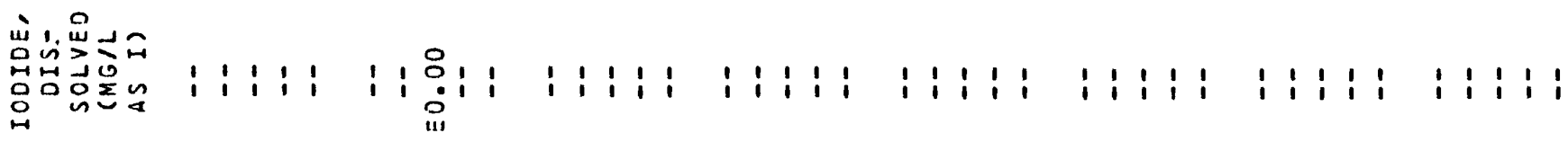

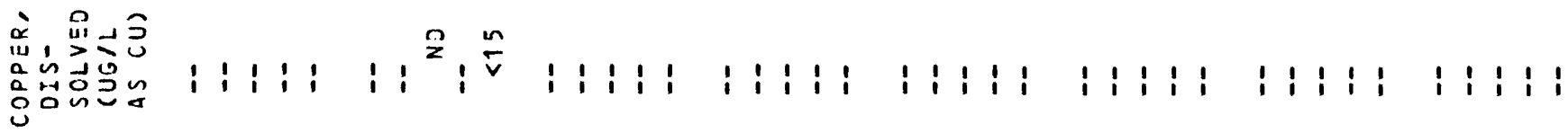

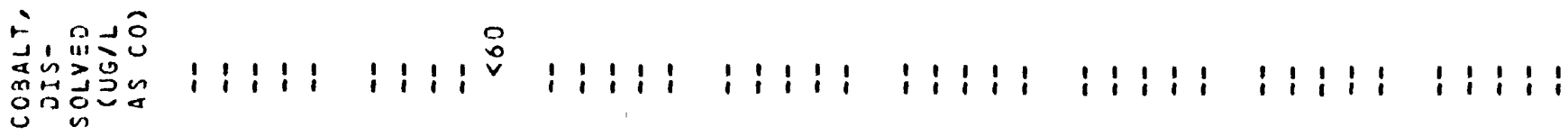

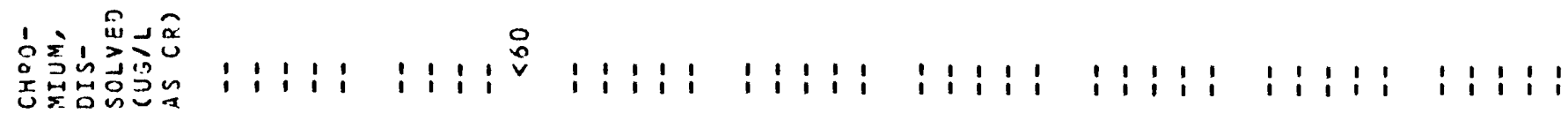

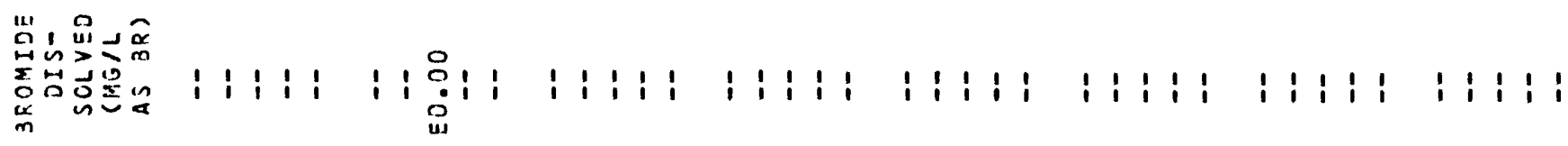

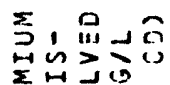

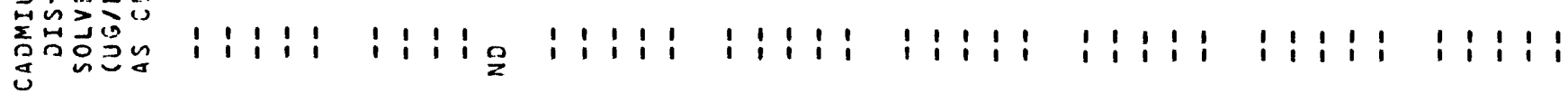

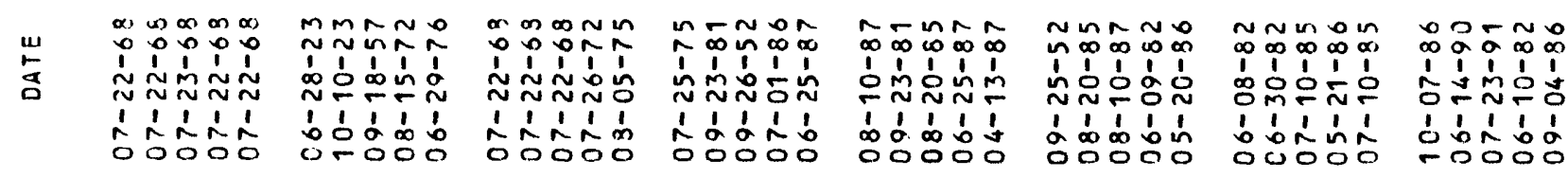




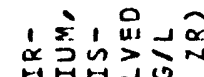

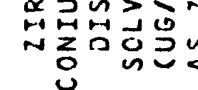

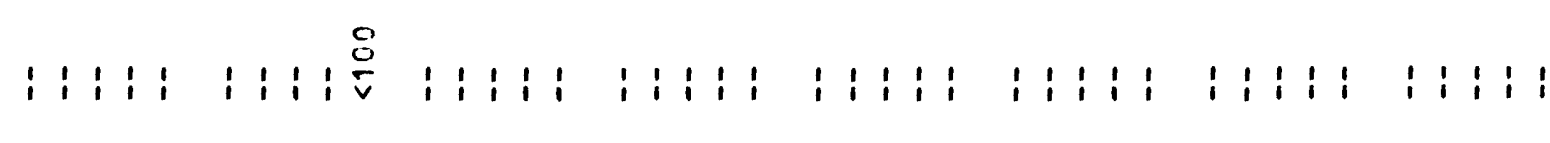

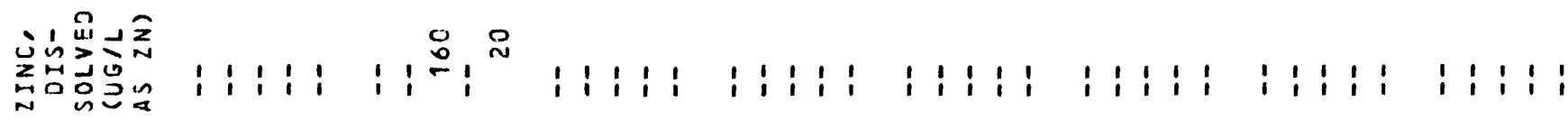

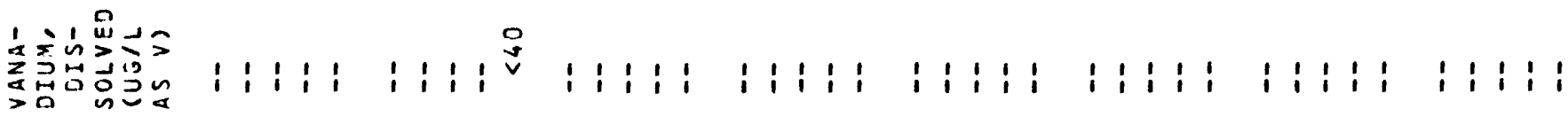

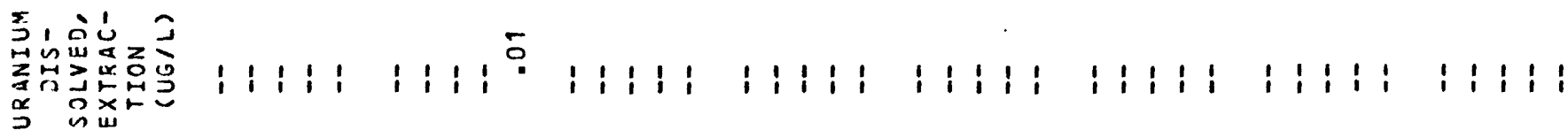

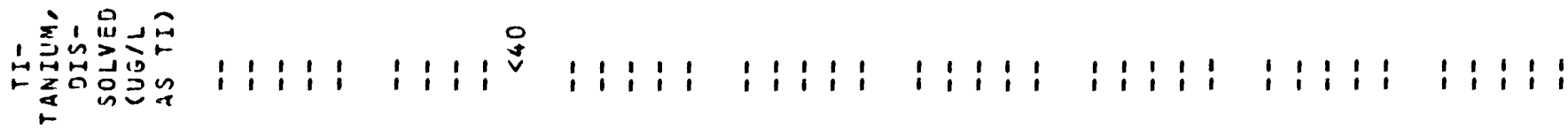

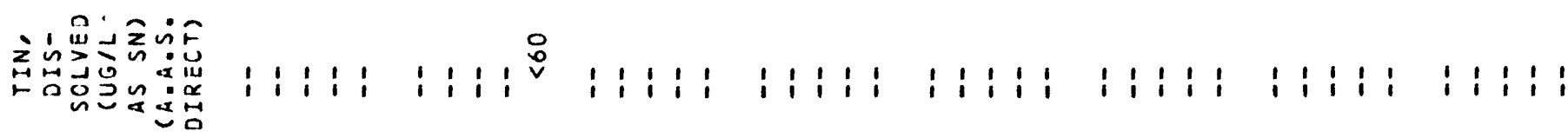

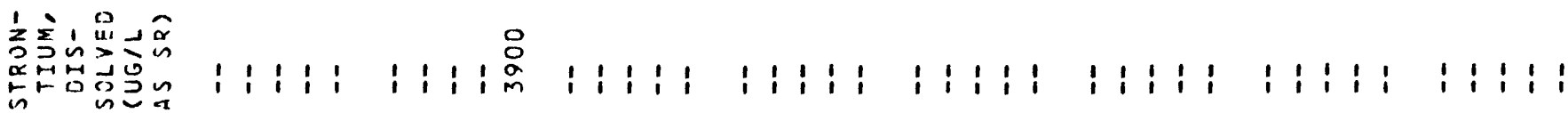

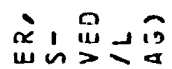

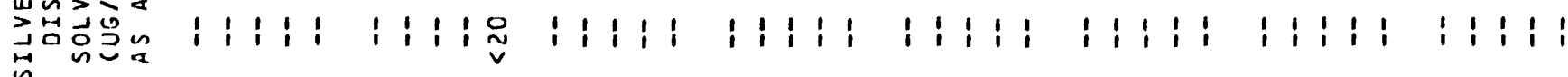

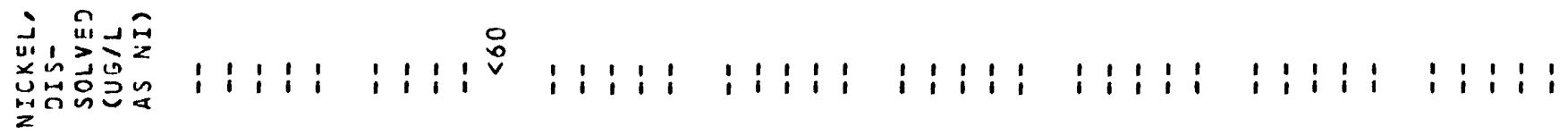

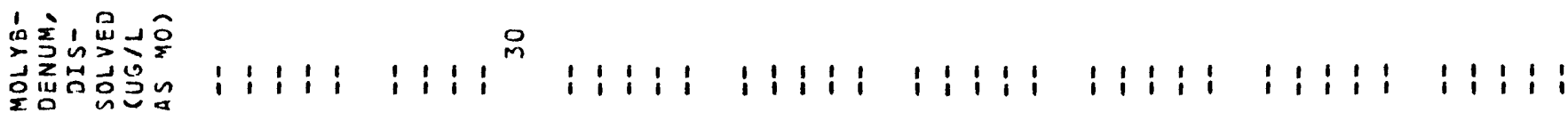

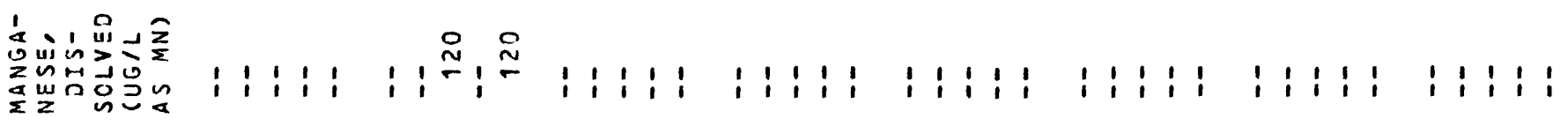

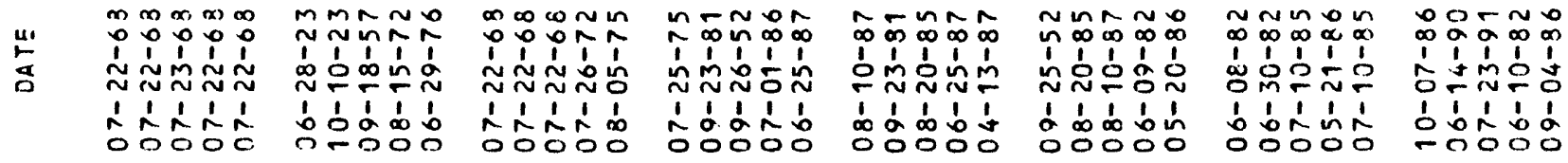




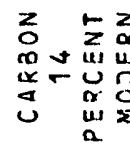

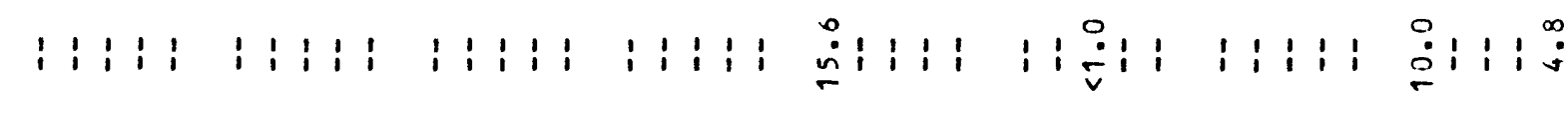

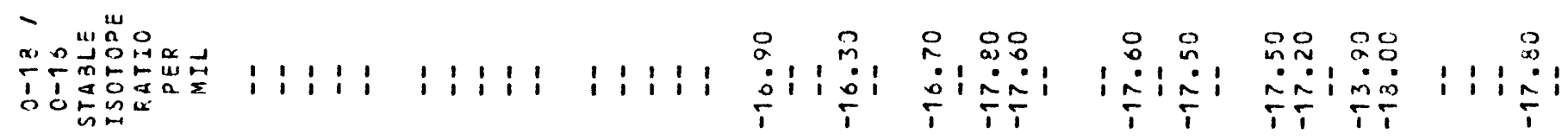

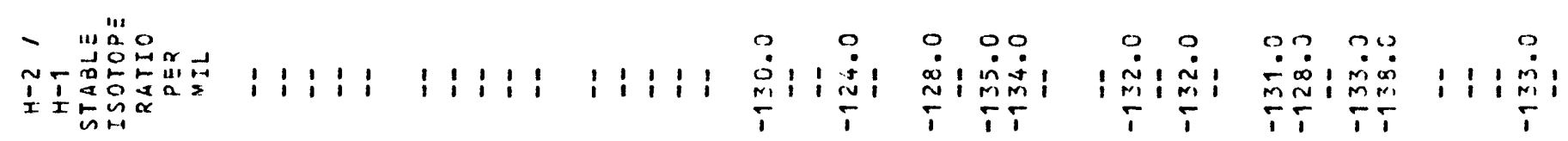

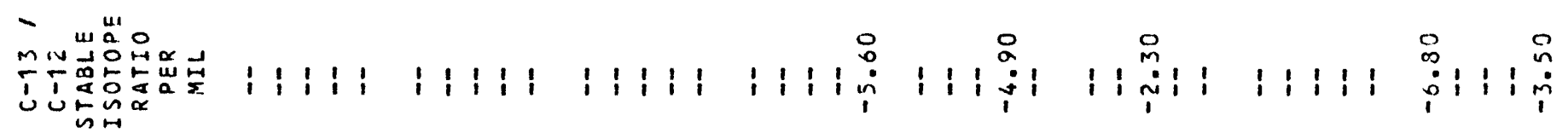

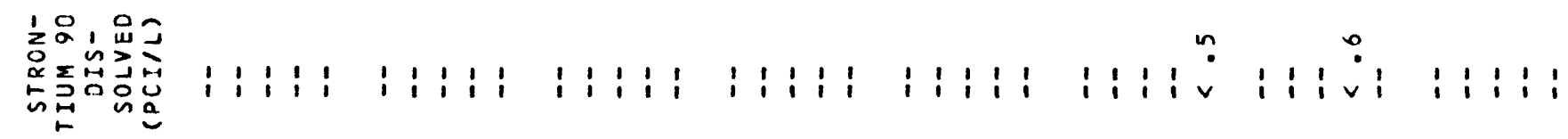

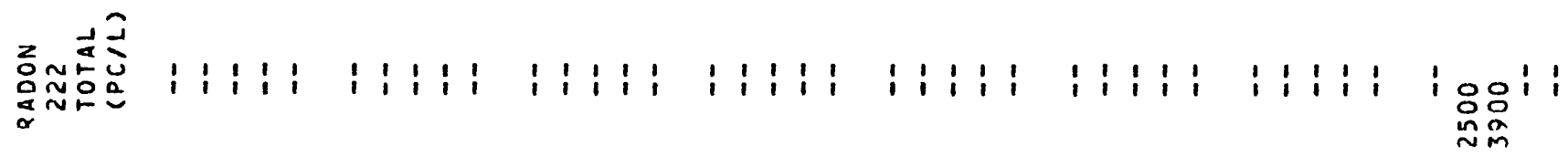

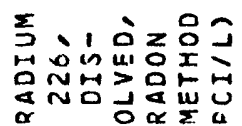

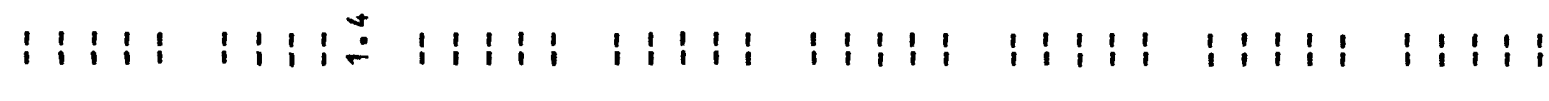

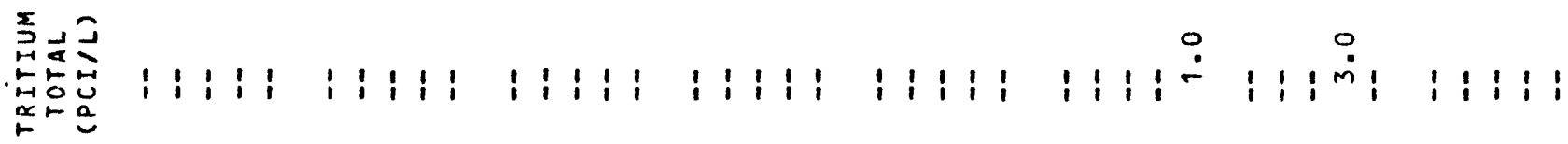

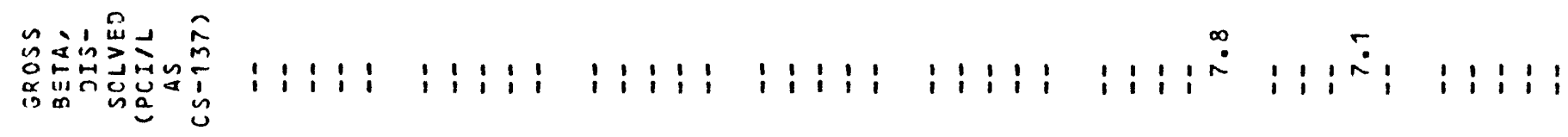

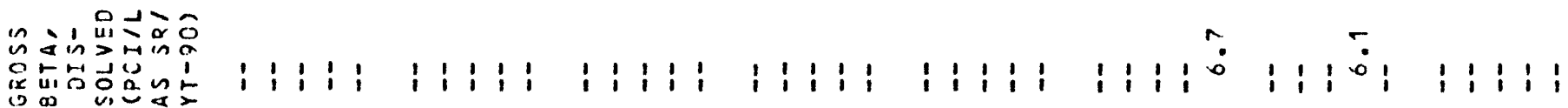

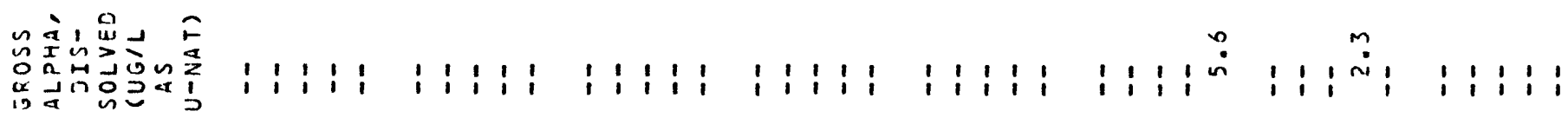

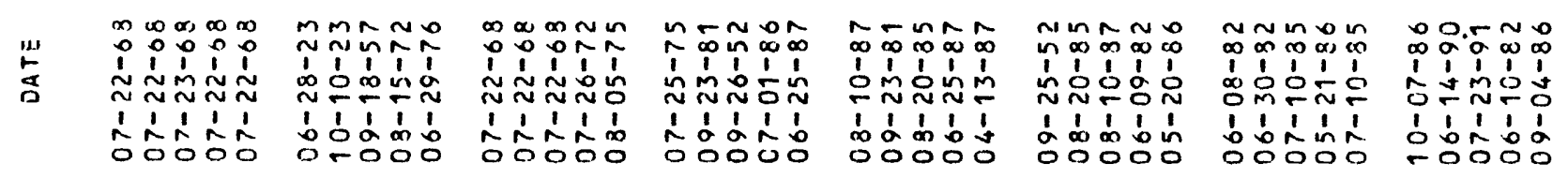




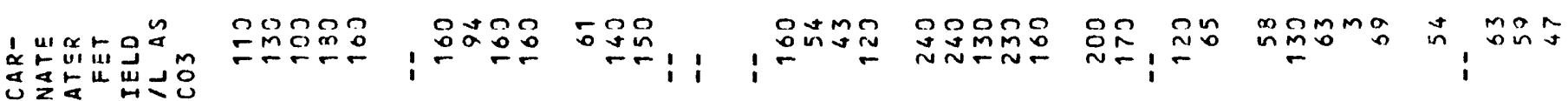

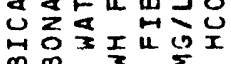

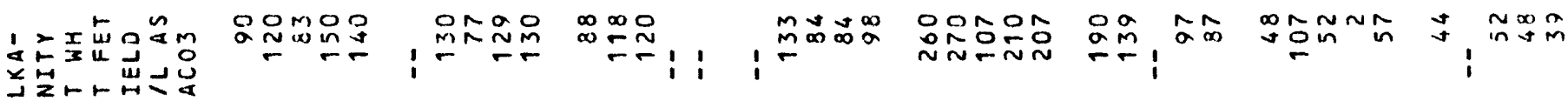

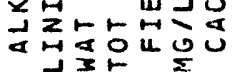

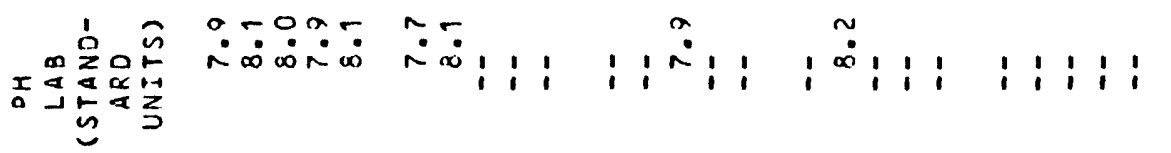

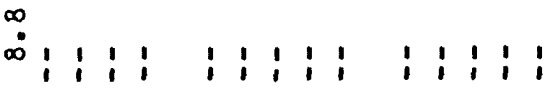

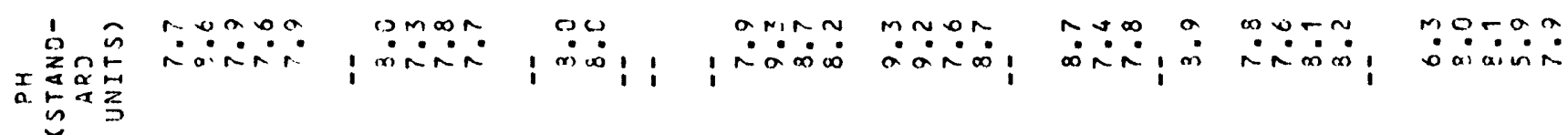

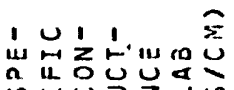

a o a) a ?

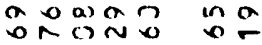
年,

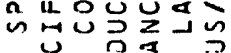

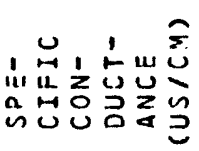

$0-a n$

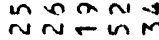

에 $\infty$ i i

$\stackrel{\infty}{\sim}$

m

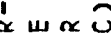

"1' 只出。

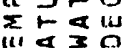

on 000

niñ

000 no

$i i^{n} i$

imi: $1: i 1 ; i$

NNㅁำ

ㄴํำ용

?o?

ono.?

09000

?0000

ing. ำ.

0.099

I11 39

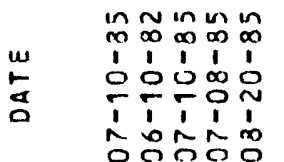

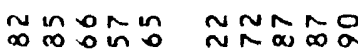

i

멍슨

i

1111

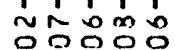

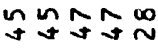

mã

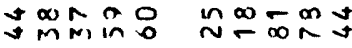

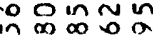

III

in

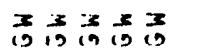

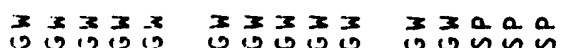

중 $\frac{x}{0} \times \frac{3}{6}$

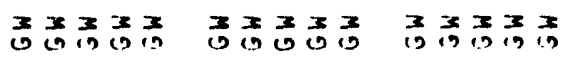

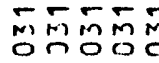

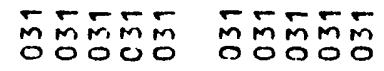

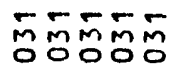

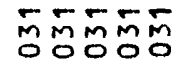

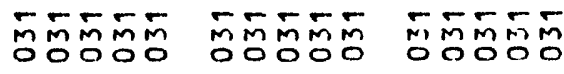

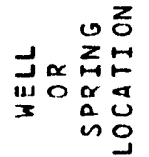

$5-5=$

ن

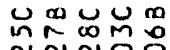

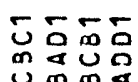

in

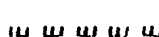

$\frac{n}{n} \pi \sim n$

$\approx \sim \sim \sim ⿻ 上 丨$

uा या

든

$\tilde{m} \tilde{m} \tilde{m}$
5
0
$\infty$
0
0
4
$m$
$m$

$w u$

iั

$\mathfrak{v} \mathcal{v}$

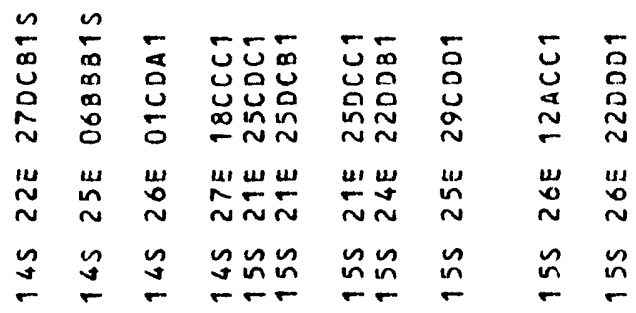

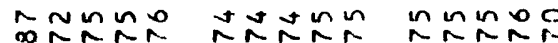

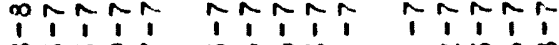
oning ñó NU⿺𠃊十

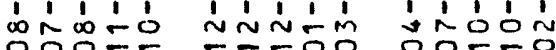

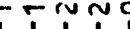
boñ $0+20$ 


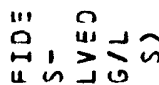

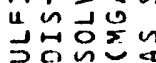

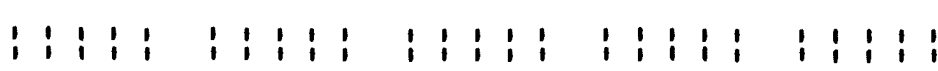

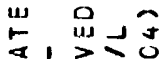

“4 的可品

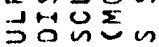

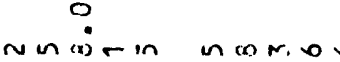

bii, 品出

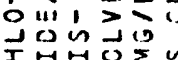

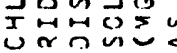

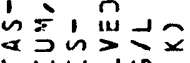

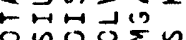
aำ经造

- onsen an $\infty$ $\left.\because \frac{1}{2}\right)$

$n m$ móno mín in?

i $=\infty \mathrm{mo}$ nhofa mo! m

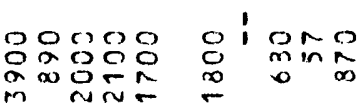
$\sum_{i=1}^{\prime}, \frac{a}{2}$ in in 19

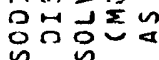
å?

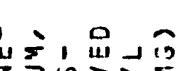

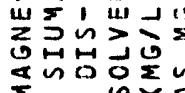

moo

mod m ans

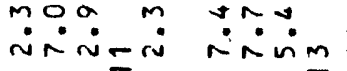
$\sin$ $\simeq 2 \pm i$

: om No

nกm

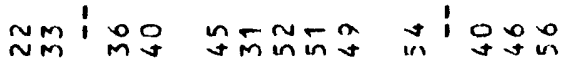

NOVME

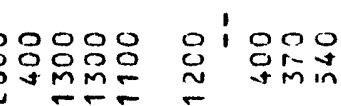
ㄷำ

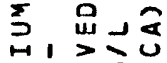

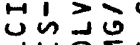
过台记立

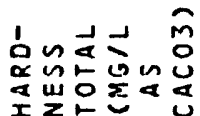

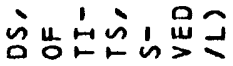

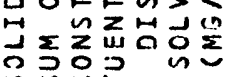

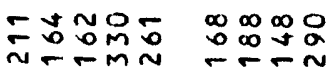

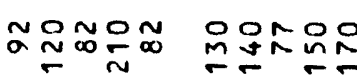

ram

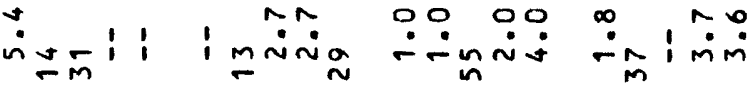
O옹ํำ in in min

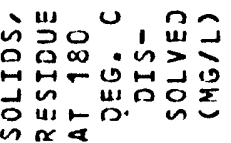

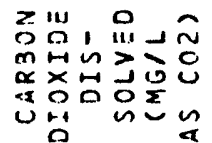
, \& U $2 \leq x$ I

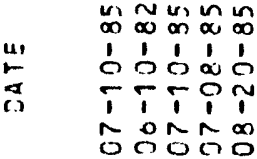

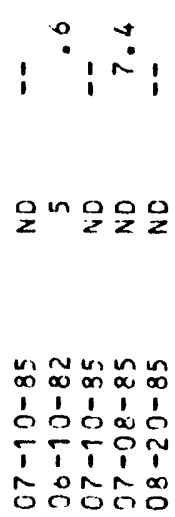




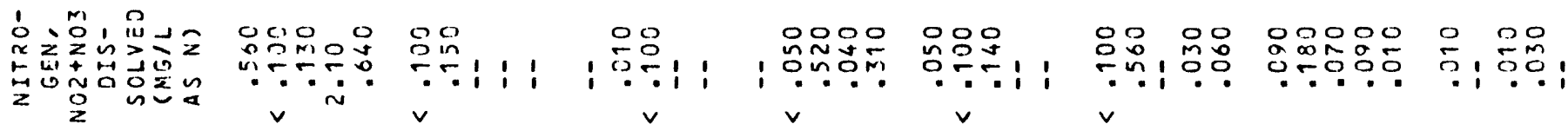

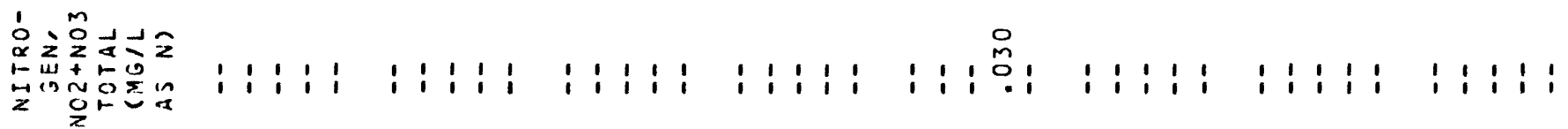

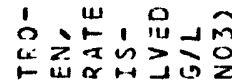

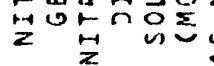

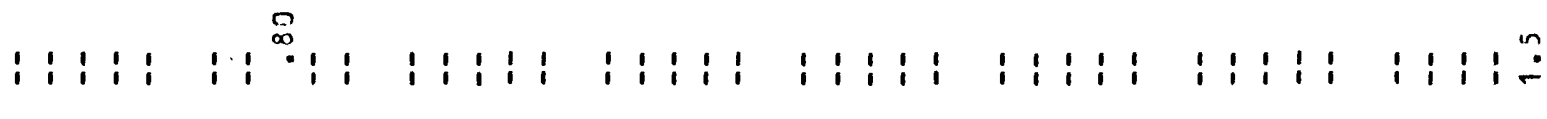

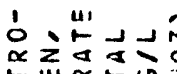

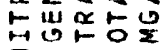
至은

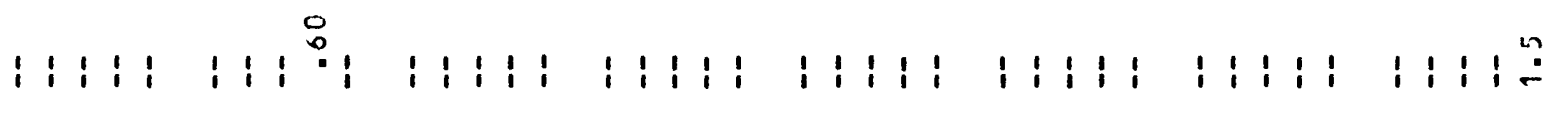

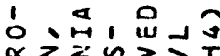

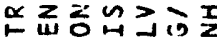

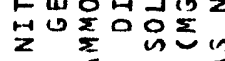

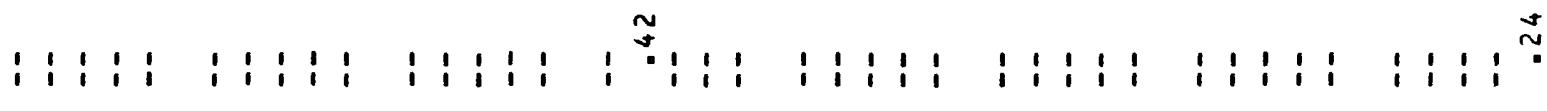

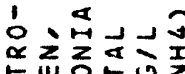
번응 2 列 $\begin{array}{lllll:ll:l:ll:}1 & 1 & 1 & 1 & 1 & 1 & 1 & 1 & 1\end{array}$ i i :

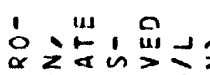

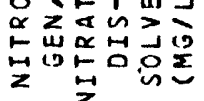

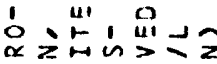

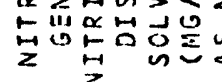

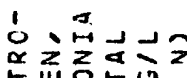

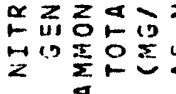
1:1:1 :1:1: $1: 19$

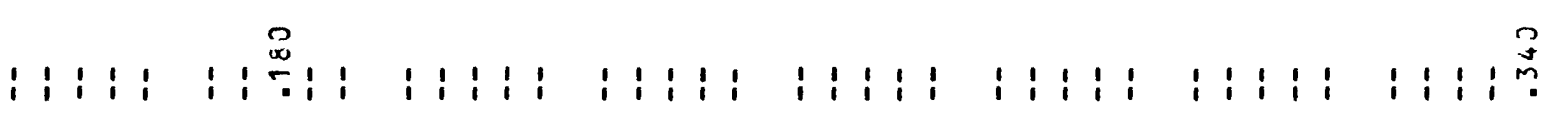

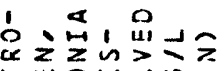

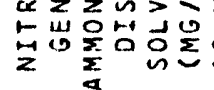

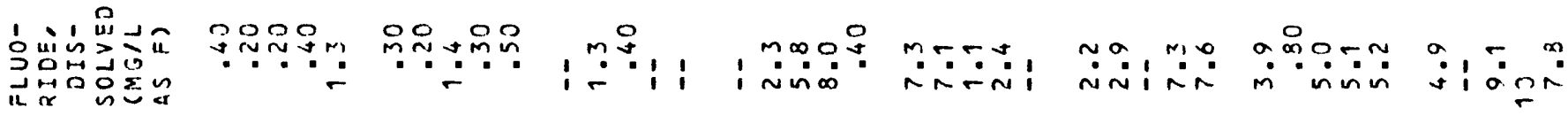

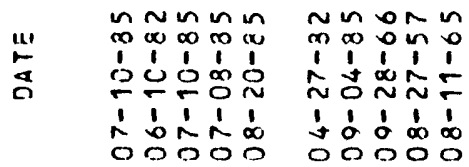

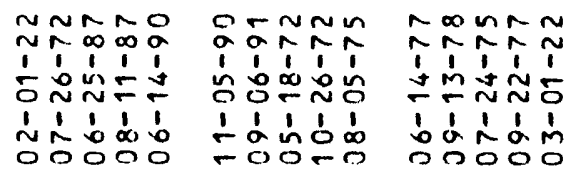

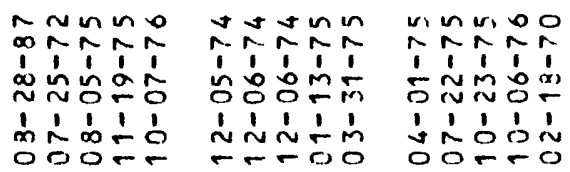




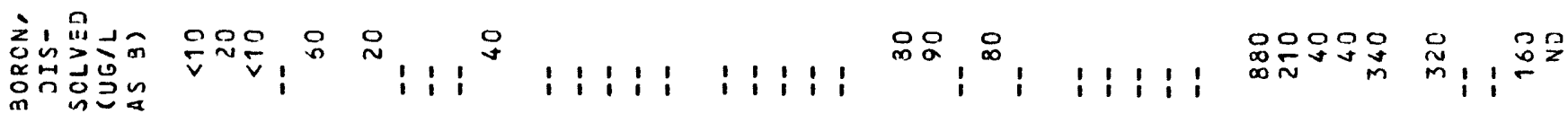

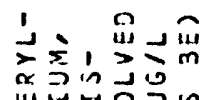
iis 1 in 0 , 0 \&

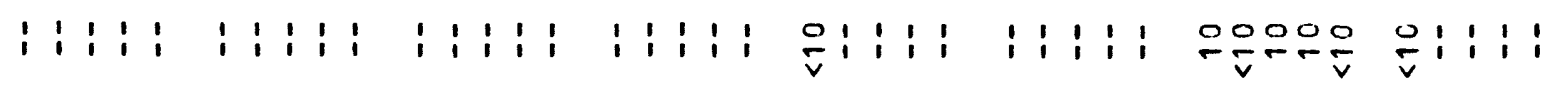

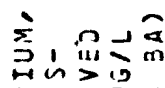

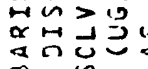

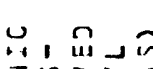
总品岂吉

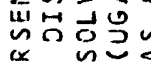

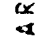

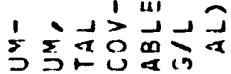

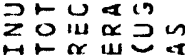

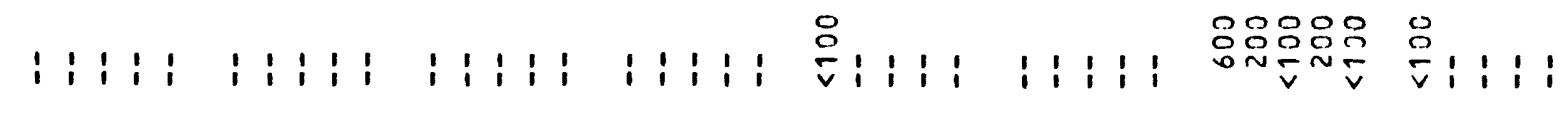

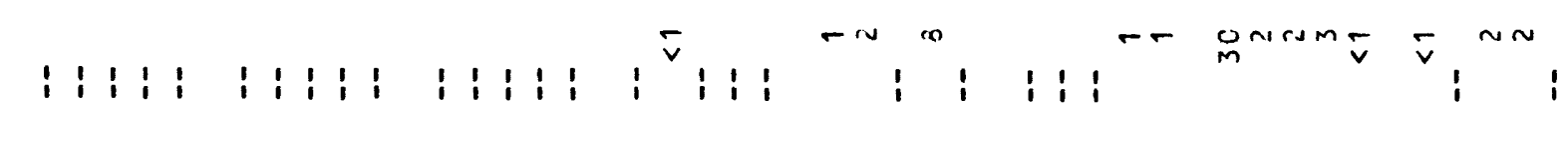

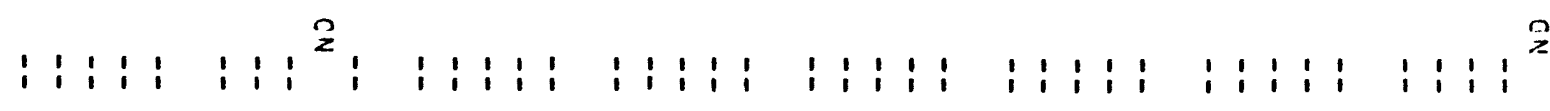

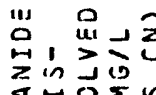

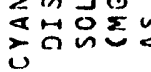

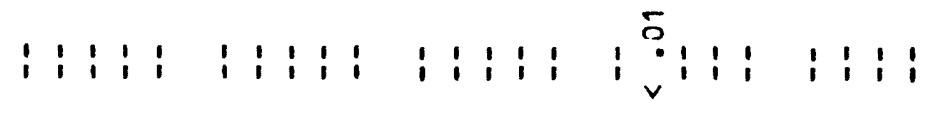

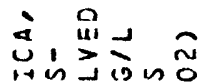

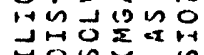
究

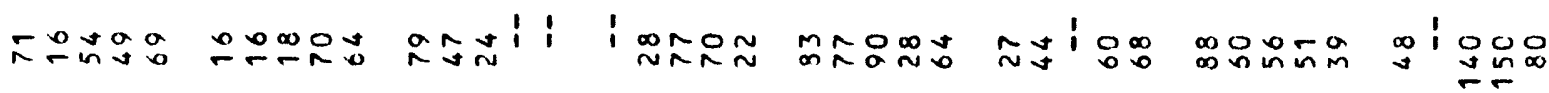

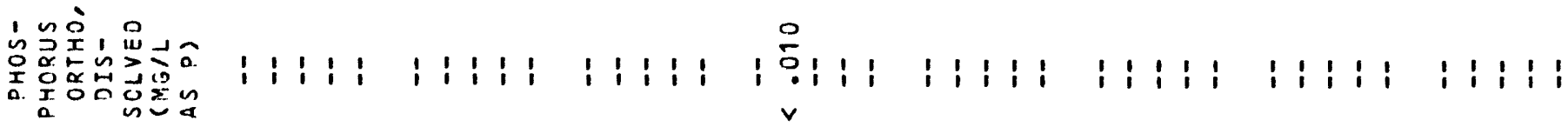

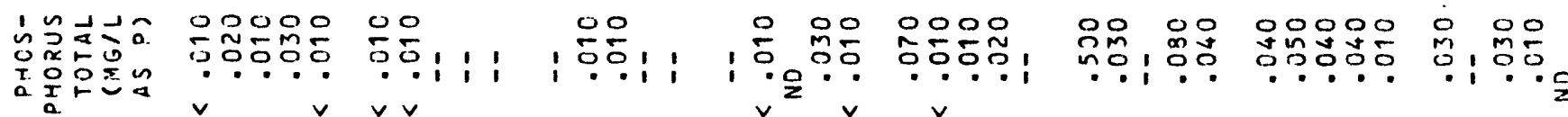

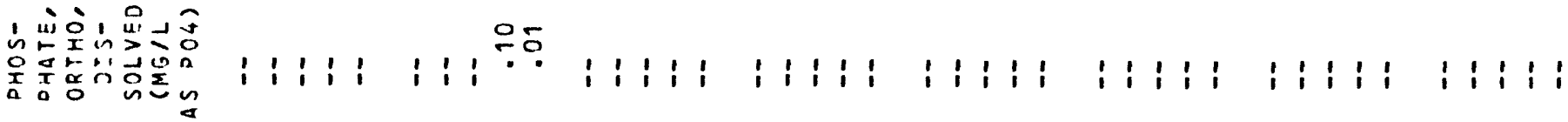

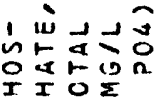

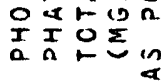

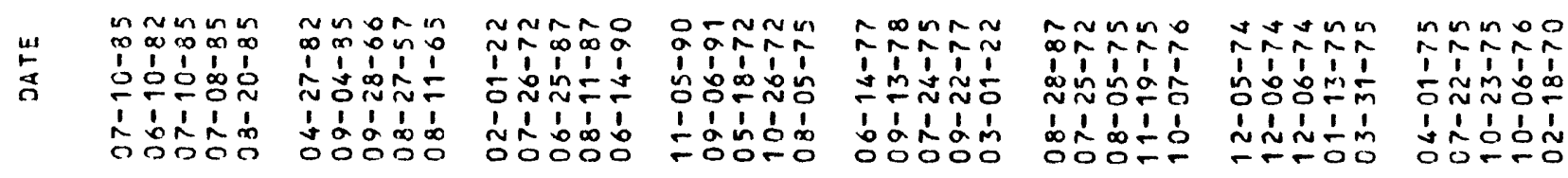




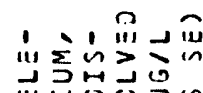

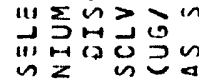

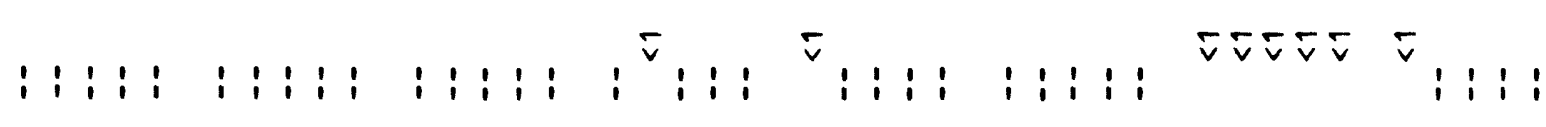

造的竖上文

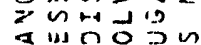

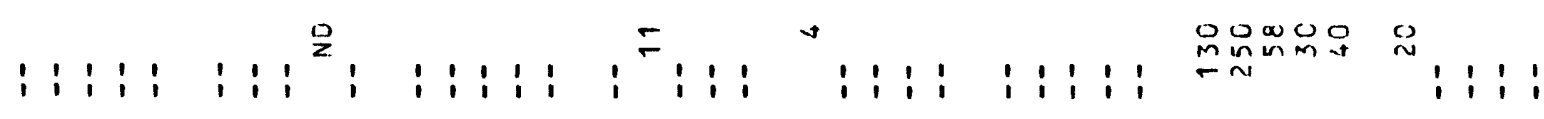

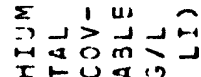

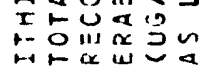

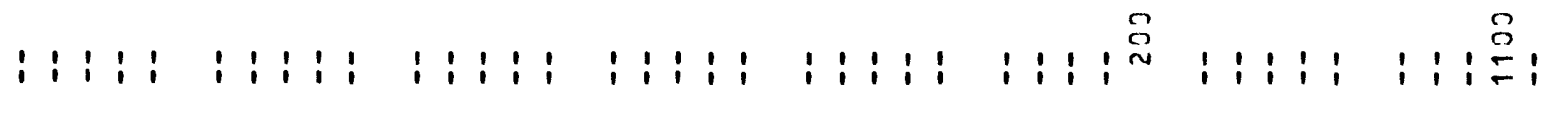

$\sum_{i=1}^{\infty}$ in $\begin{aligned} & 0 \\ & >\end{aligned}$

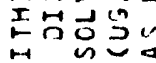

: : :

on 0

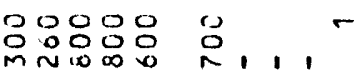

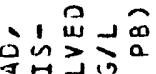

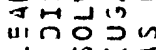

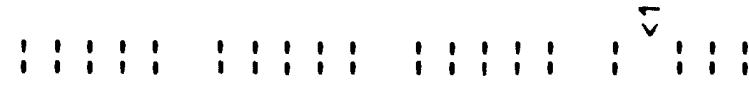

$: \div: 1: 1: 1: 1:$

$: 1: 1: \quad 1: i::$

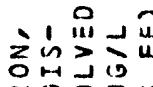

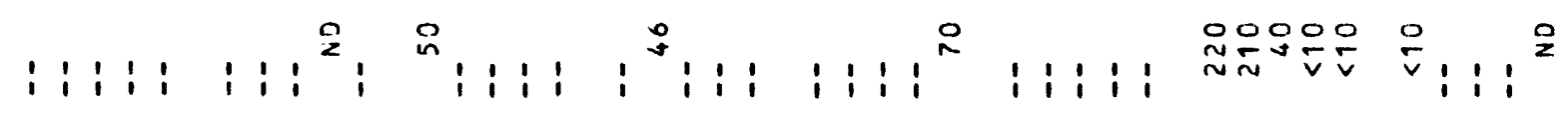

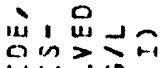

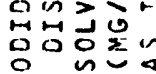

$1: 1: 1: 1: 11: 1: 1: 11: 1:$

$\frac{0}{6}$

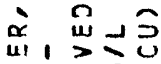

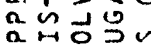

边出品采

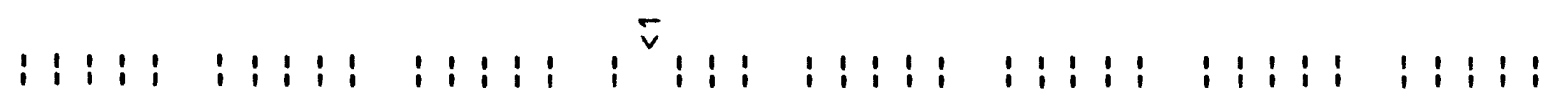

ìn

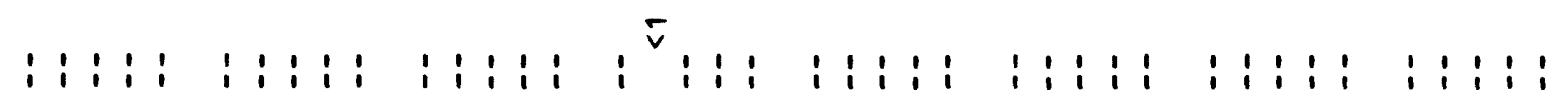

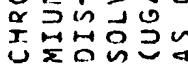

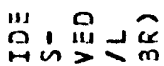

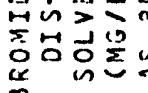

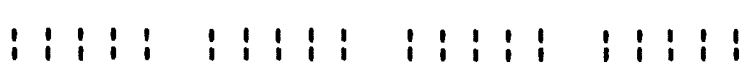

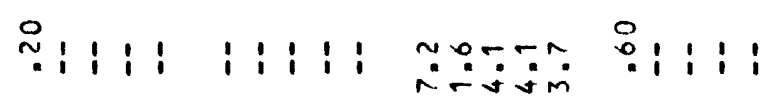

站出品

为牙

品品要

111119111111,111190 政

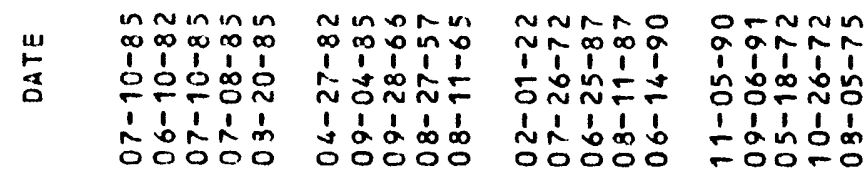

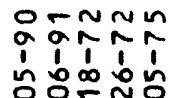

10ำ

กิำกำ

tîn

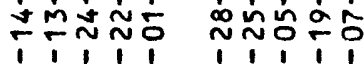

ถุㅇํำ

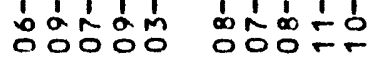

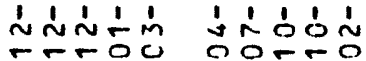




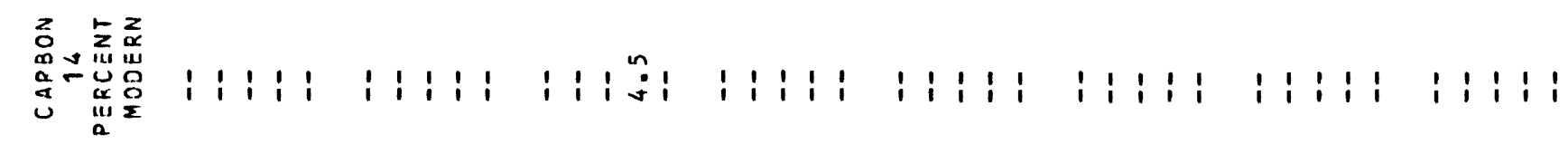

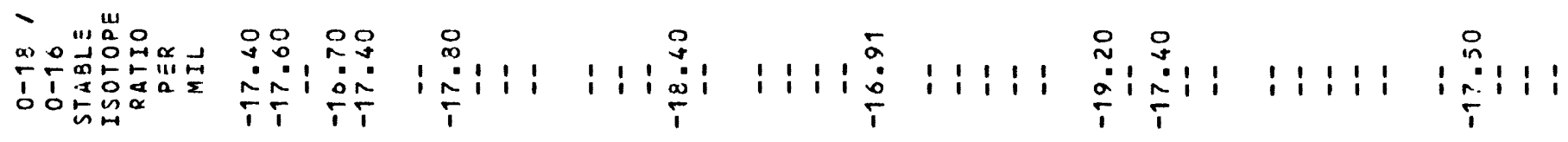

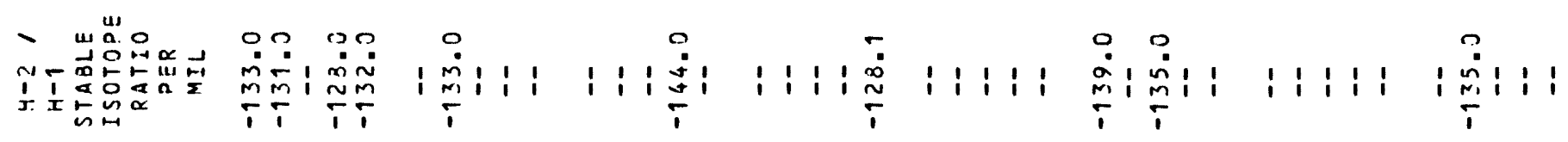

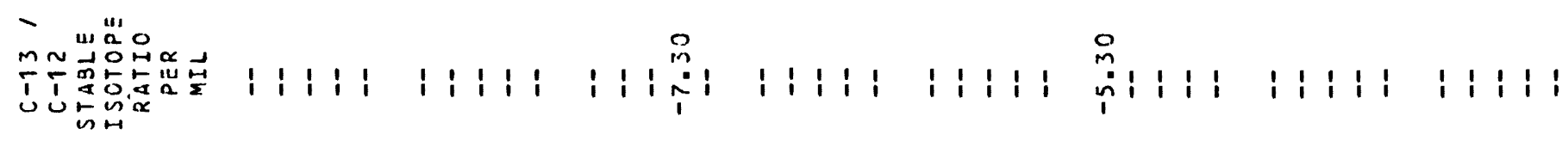

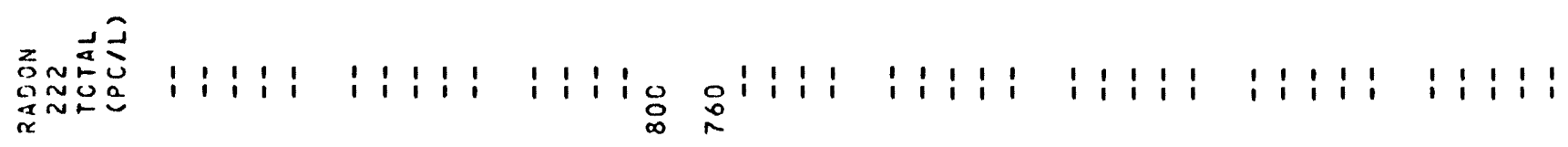

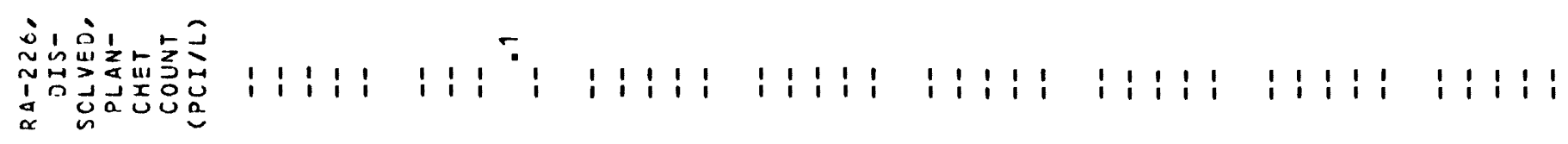

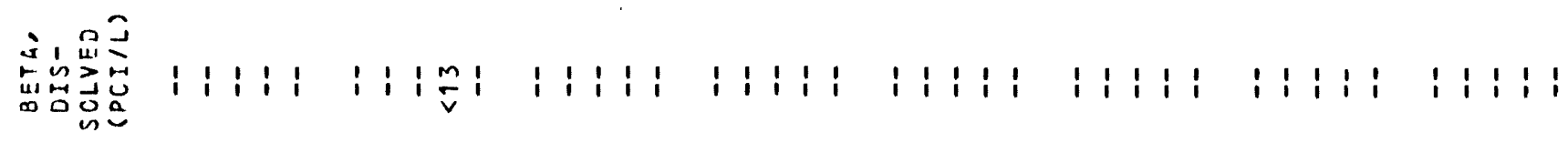

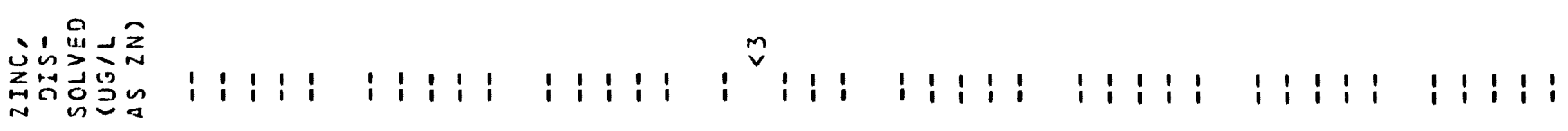

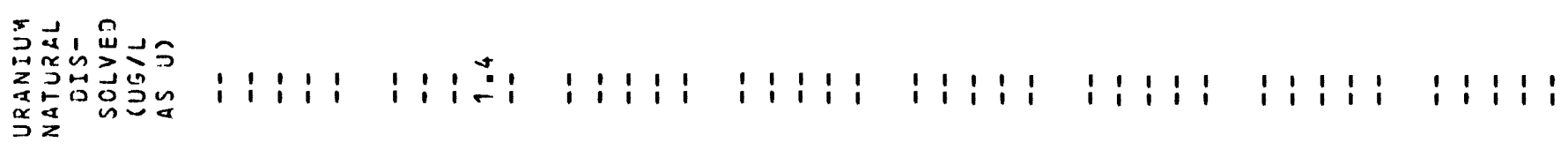

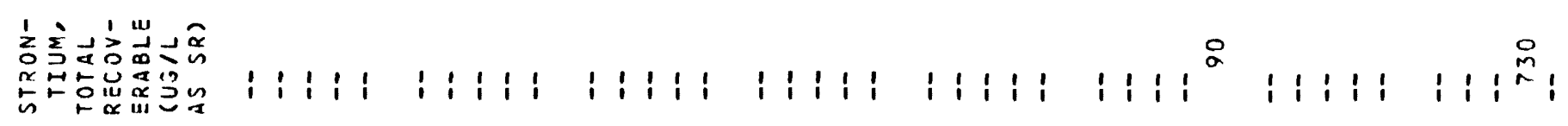

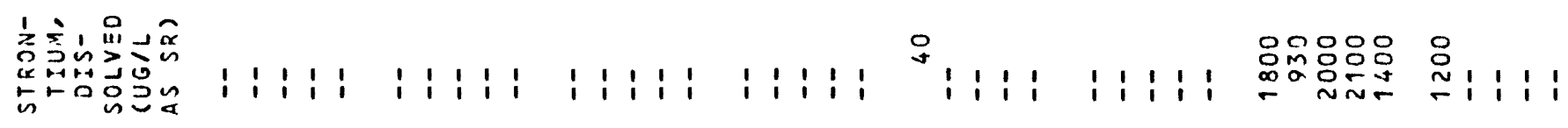

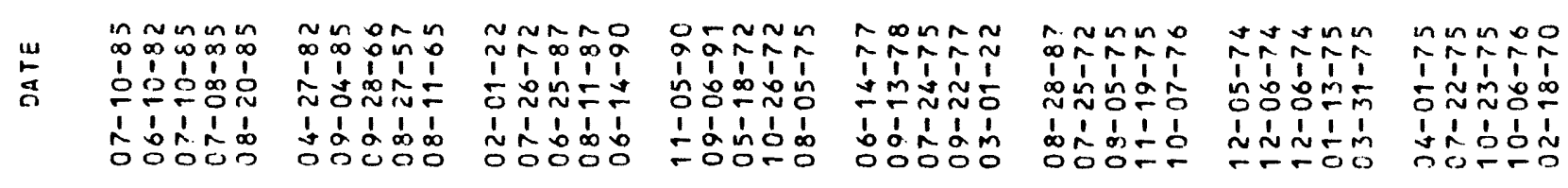




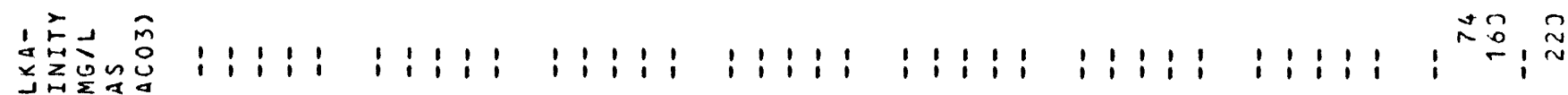

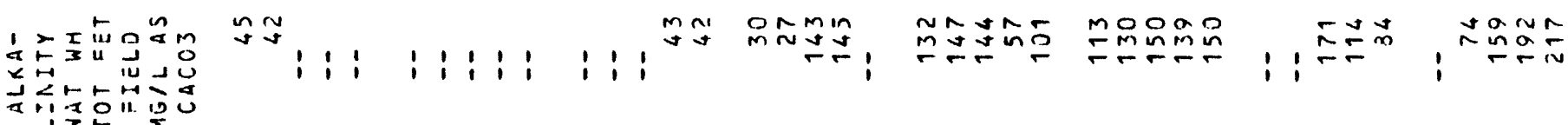

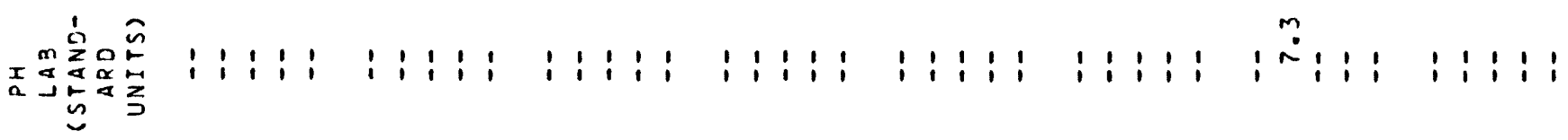

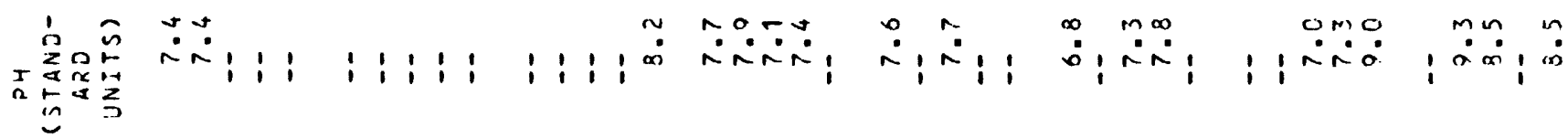

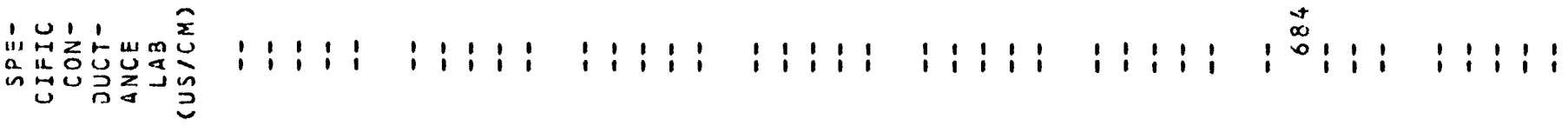

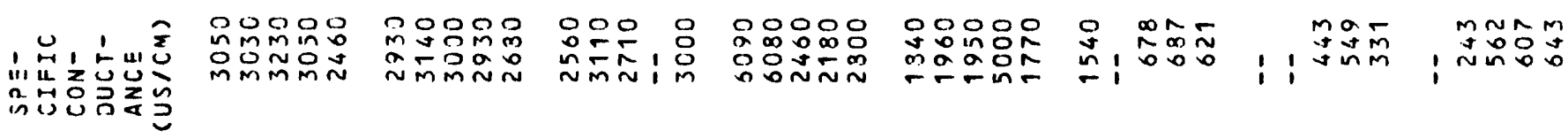

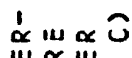
w出出0

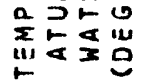

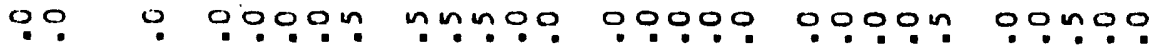

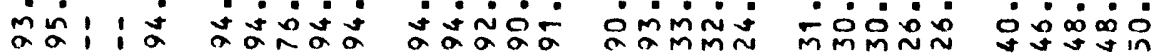

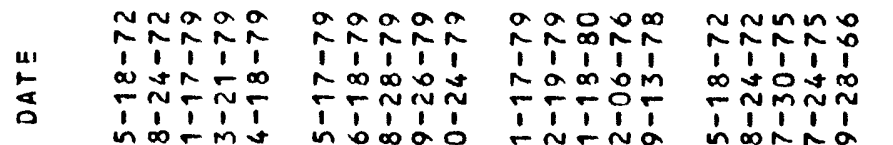

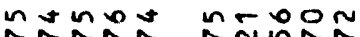

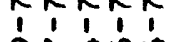
artog

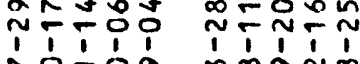

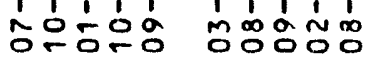

오กำ จำกำ

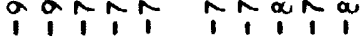

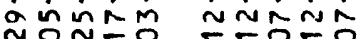
ñono

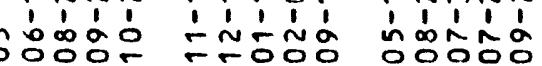

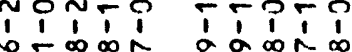

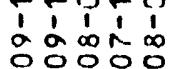

菂

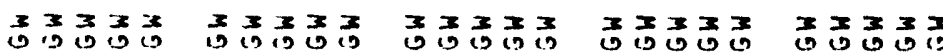

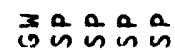

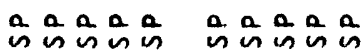

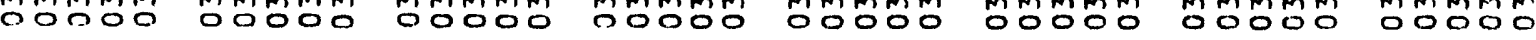




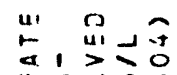

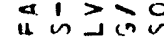

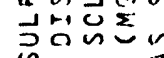

兠

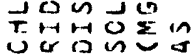

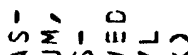

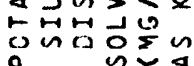

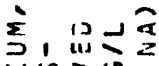

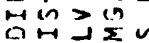

응요의

北之品要

$x_{n \rightarrow 1} \sim \sum_{10}$

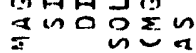

ño!

1191

$\begin{array}{ll}2 & 0 \\ 2 & 1 ! 1 \\ 2 & \infty\end{array}$

! ! :

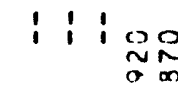

$\begin{array}{lll}0 & 0 & 0 \\ 0 & 0 & 0 \\ 0 & 0 & 0\end{array}$

잉요 a 0

w in O N

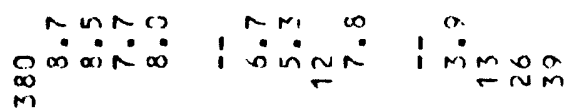
$m$

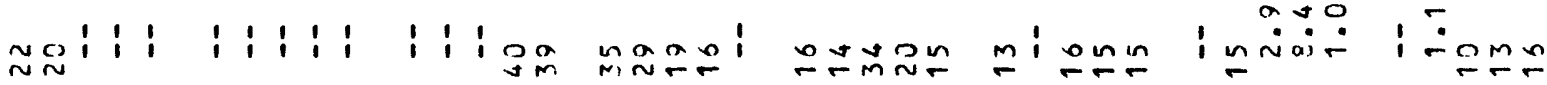

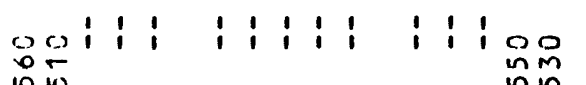

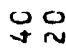
: 1 i i i i i i i i i :

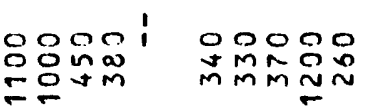

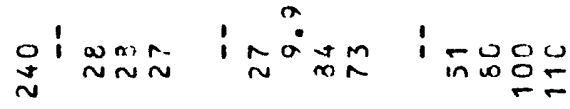

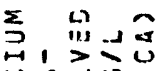

in

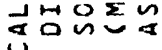

1

mm

的出的, 出

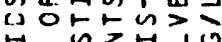

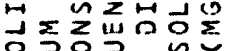

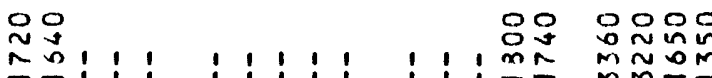

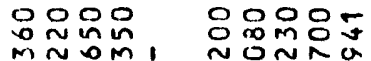

Mñm! $\cong 0 \cong N$

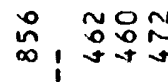

$\cos m \mathbb{m}$ $\tan \underset{\infty}{\infty}$

ํำำก 은은

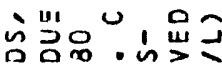

出 in

owヶ

zow,

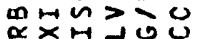

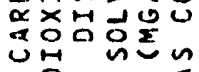

in

$\ddot{m} \dot{m}$

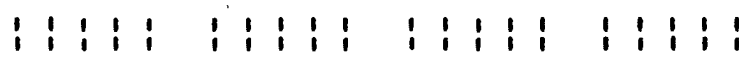

i i i i i :

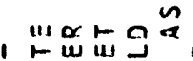

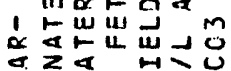

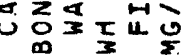

1

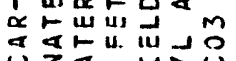

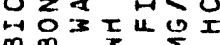

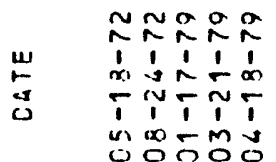

in $\sin$ i i : $1: 1: 1: 1$

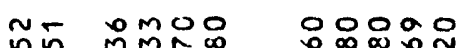
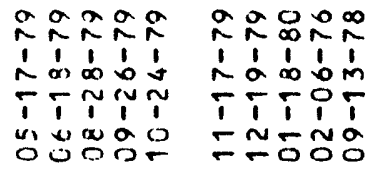

กัก์

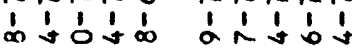

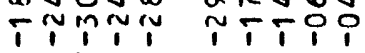
옹승영 동응ㅇㅇㅇ

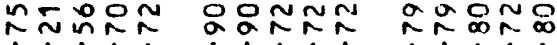

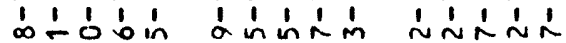
NTTTN NOMTO TTOT

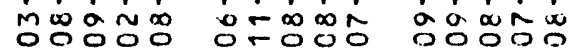




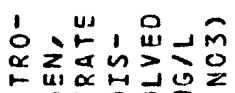

zot

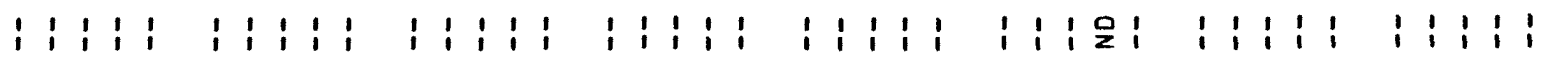

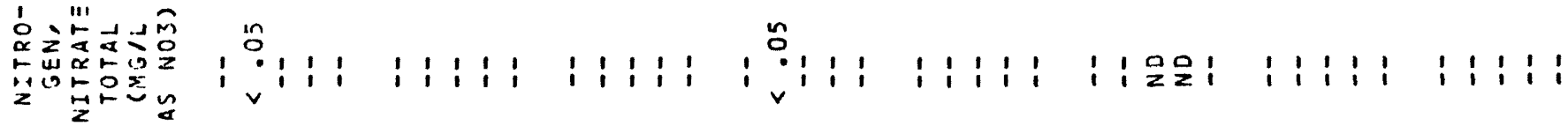

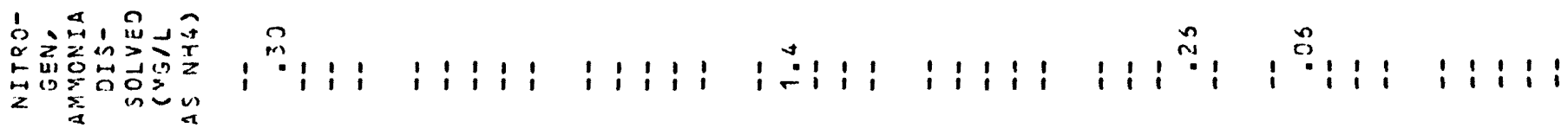

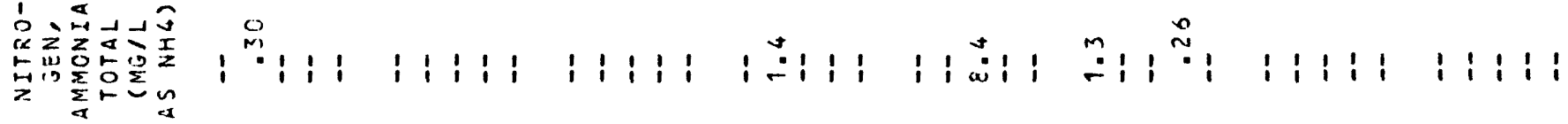

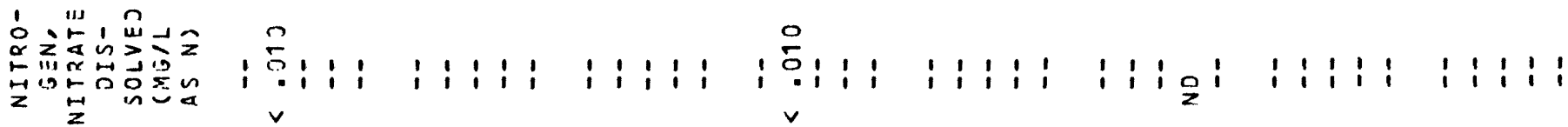

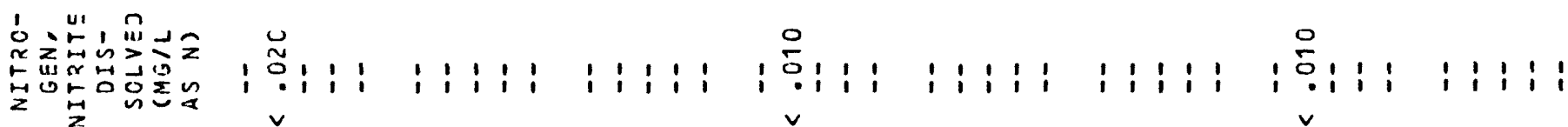

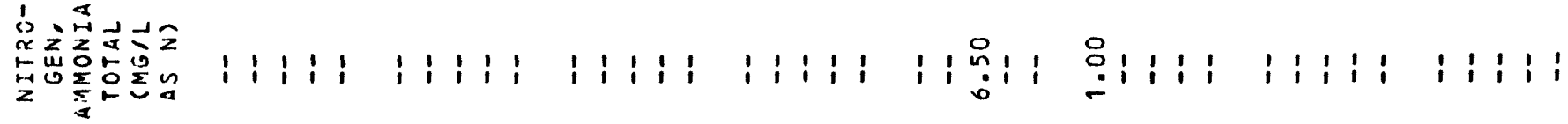

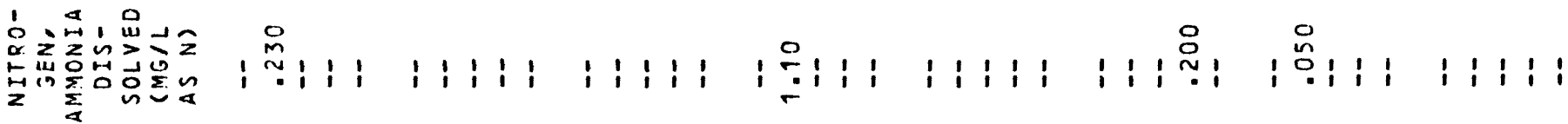

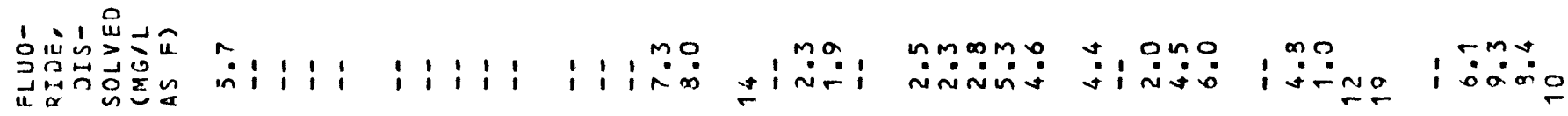

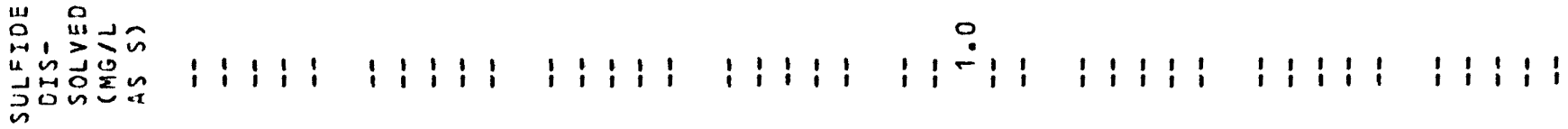

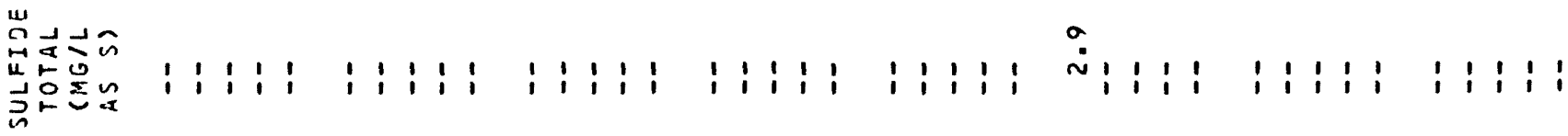

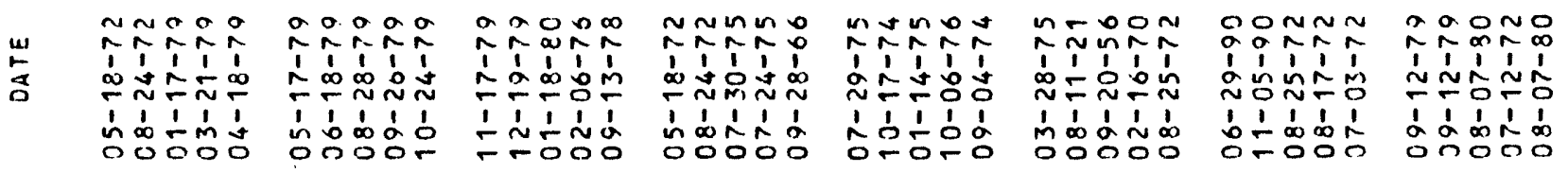




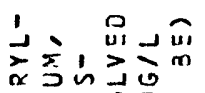

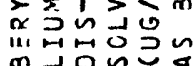

12:1: 1:1:1

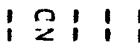

: $: \frac{0}{2}: 1$

위:1:1:1:1:1: $1: 1: 1:$

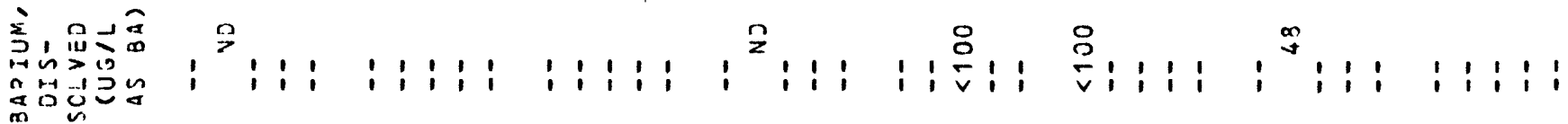

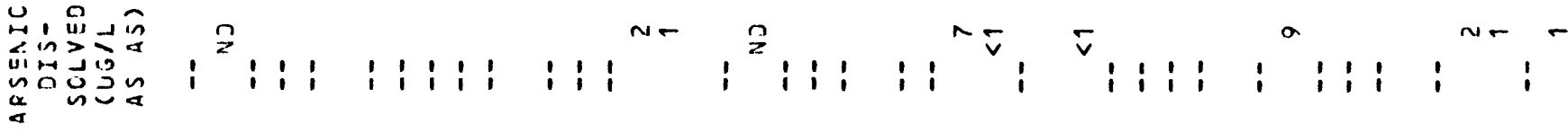

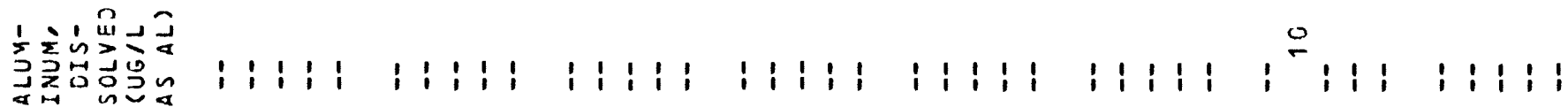

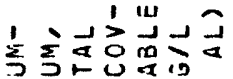

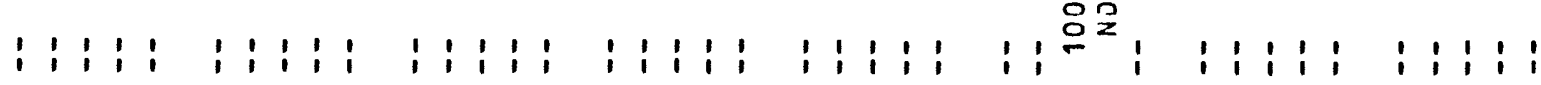

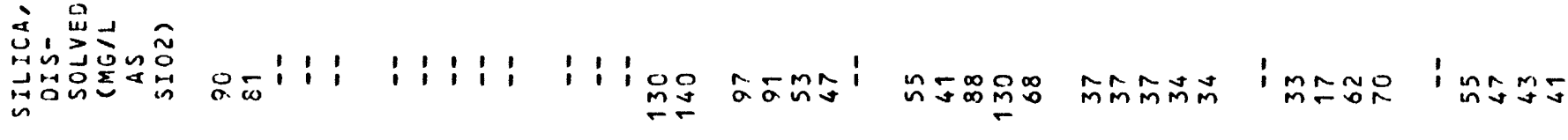

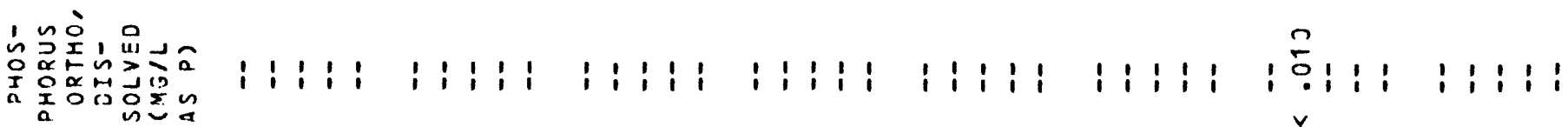
率

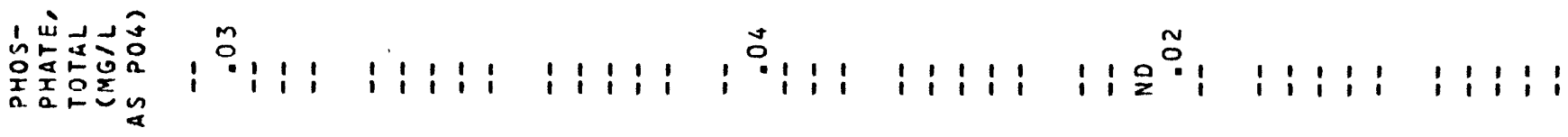

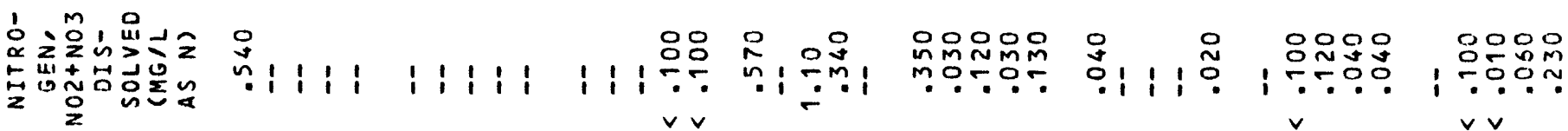

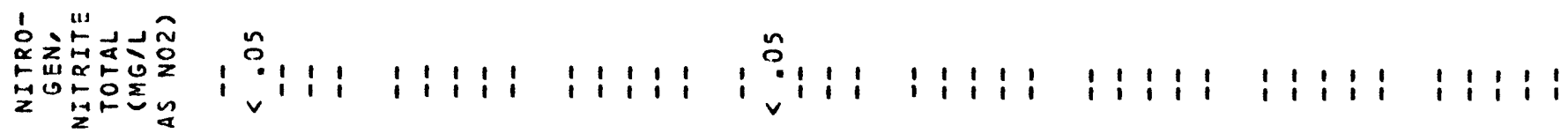

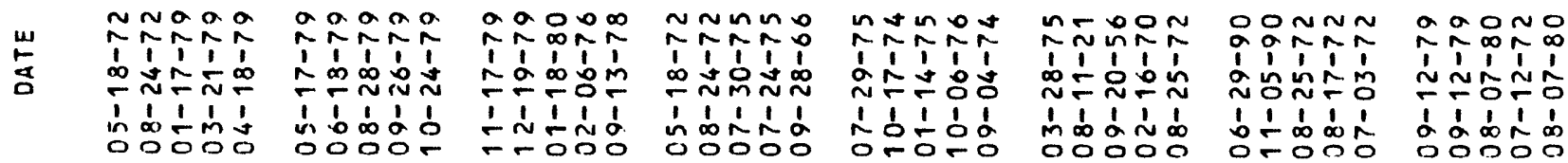




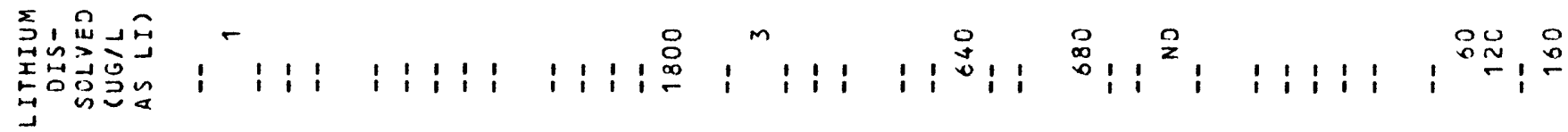

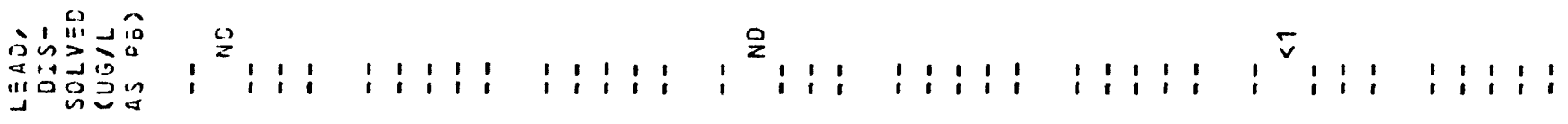

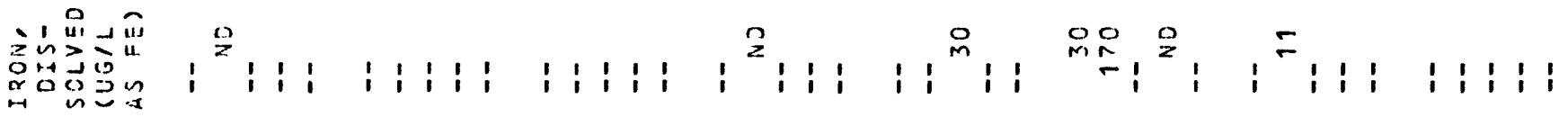

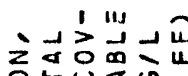

×

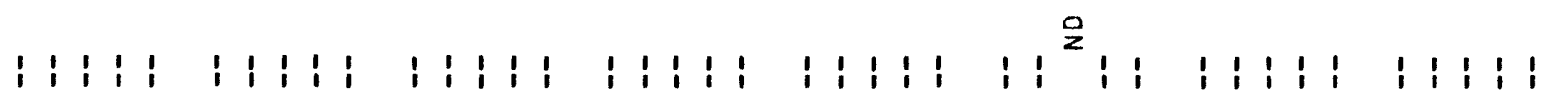

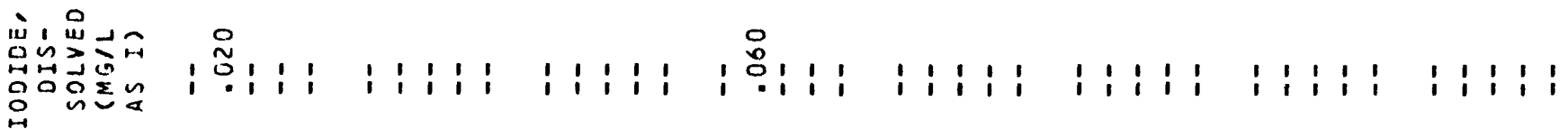

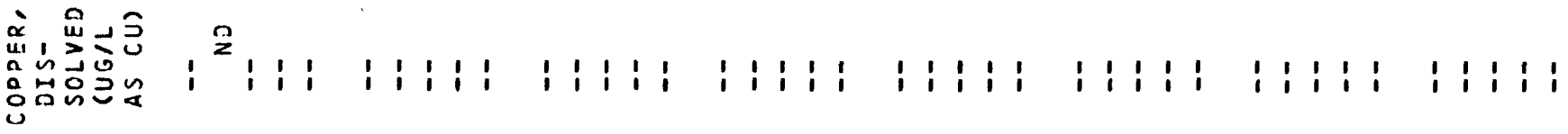

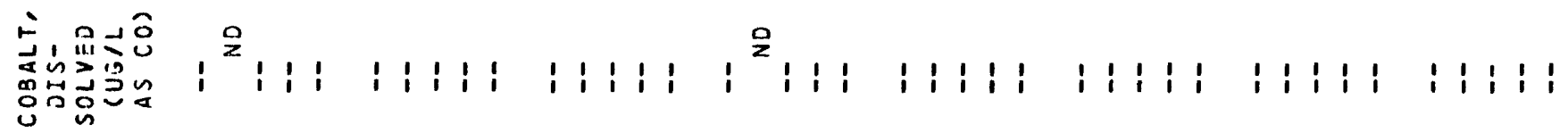

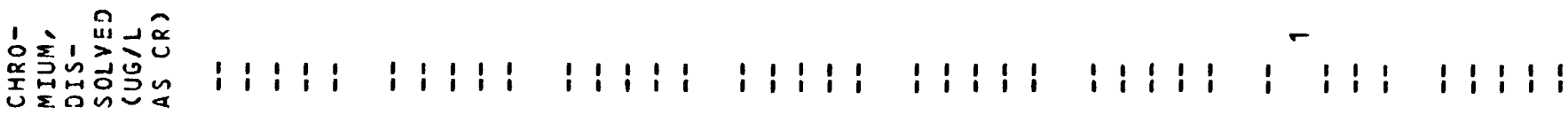

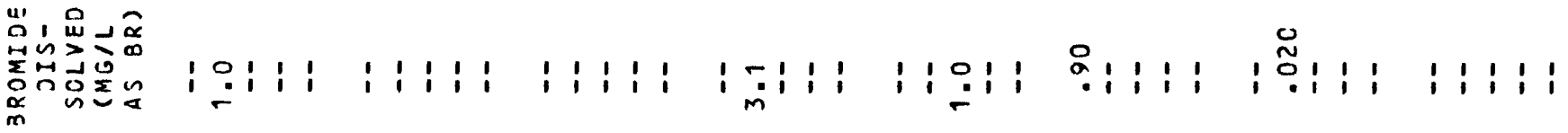

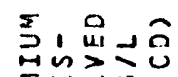

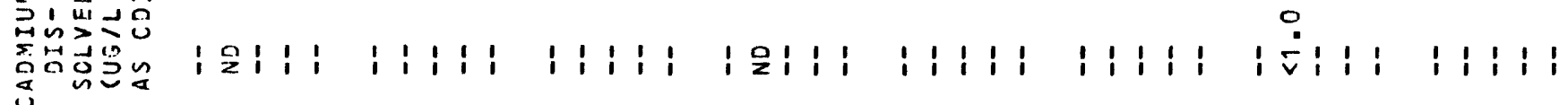

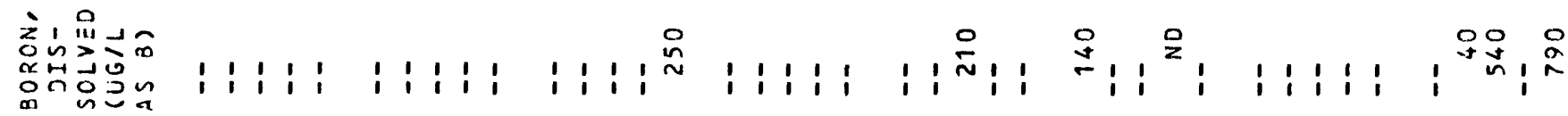

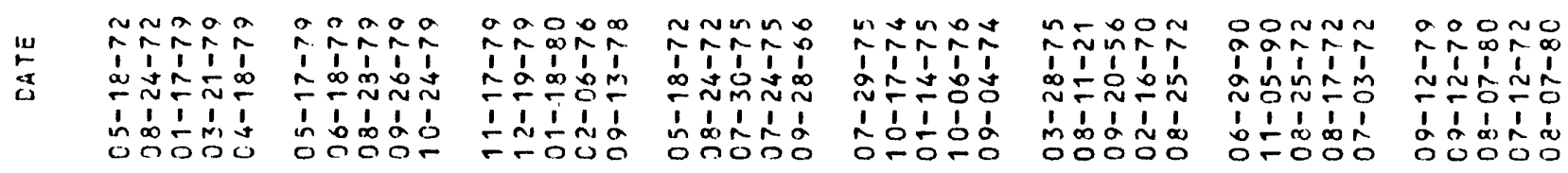




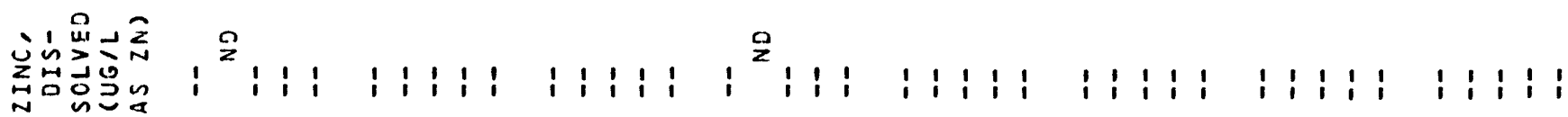

$\sum_{i=1}^{x} \underset{a}{a}$ is

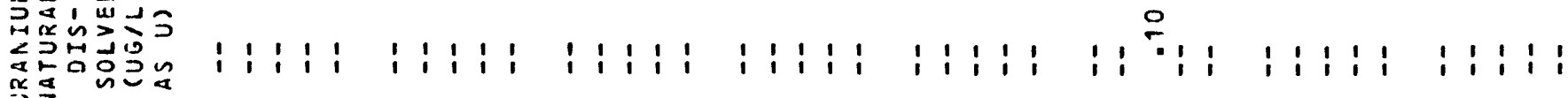

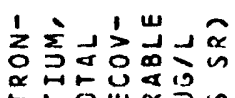

幺

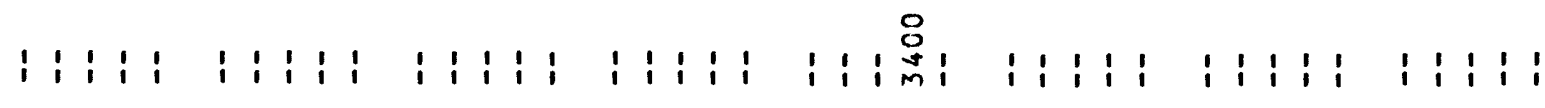

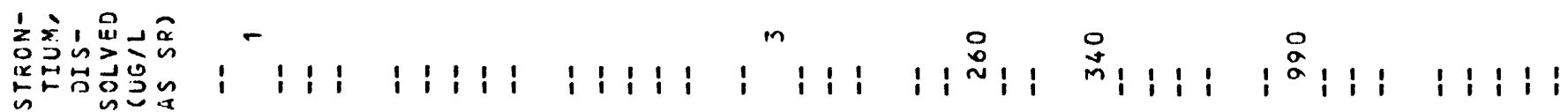

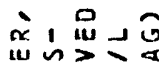

$>\sim 1.9$

in $n$ in

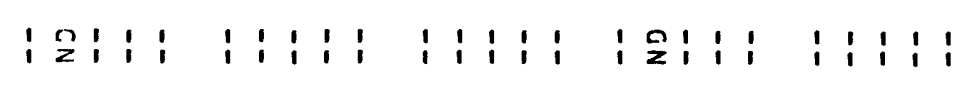

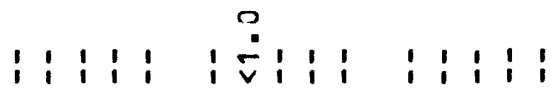

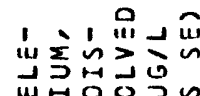

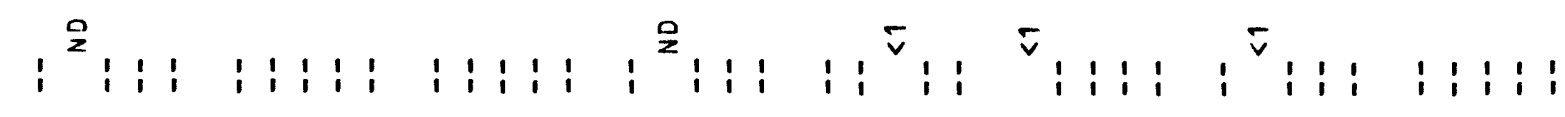

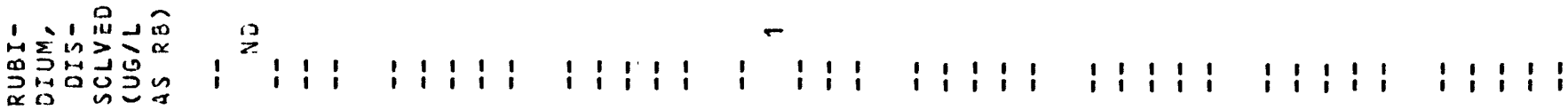

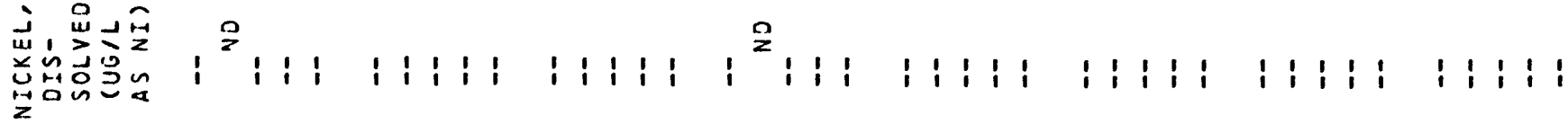

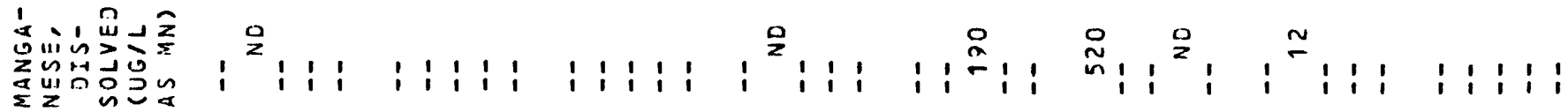

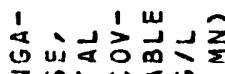

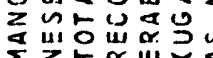

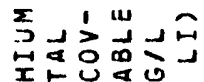

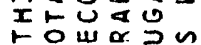

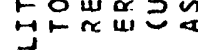

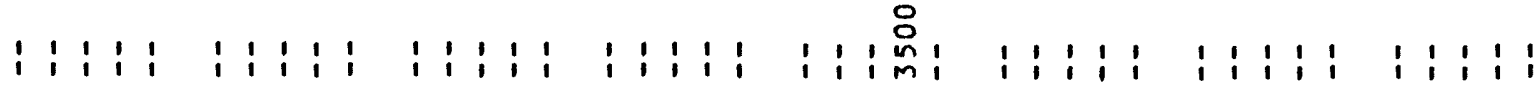

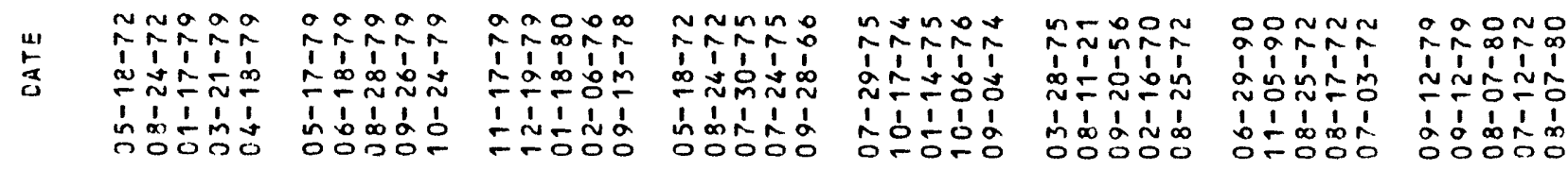




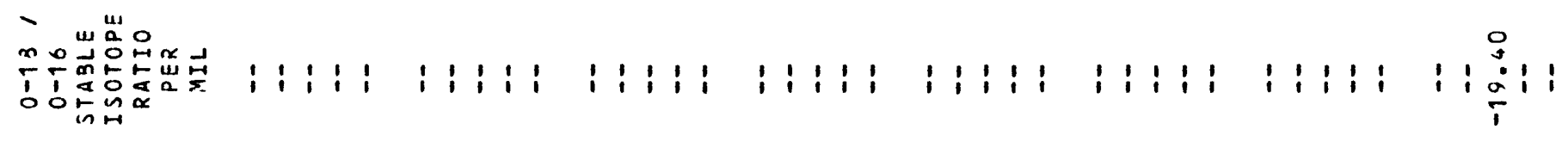

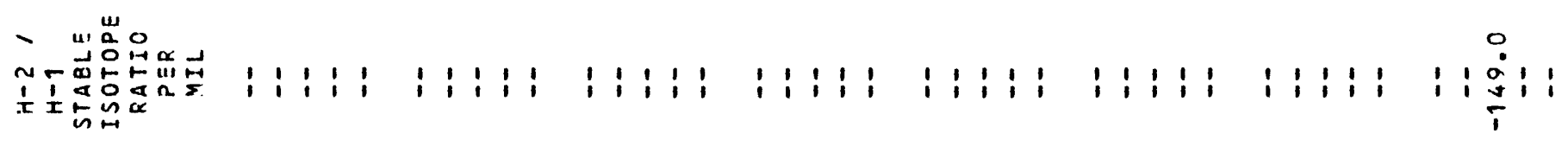

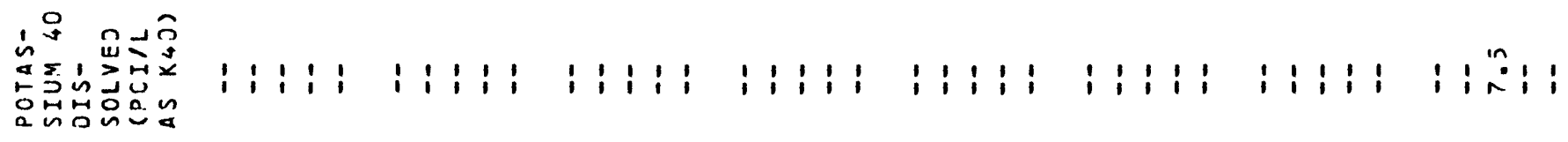

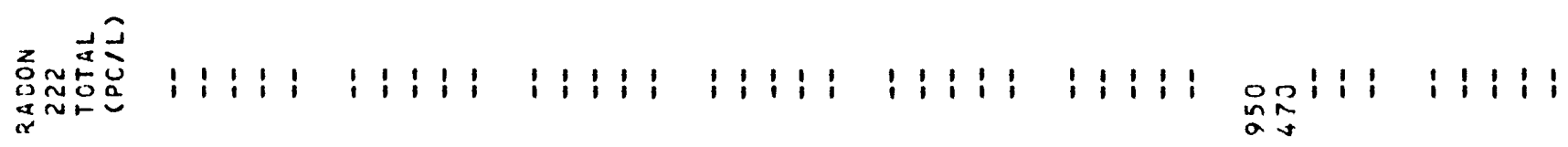

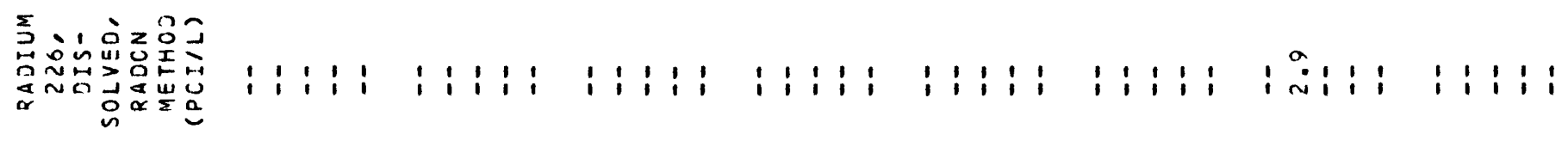

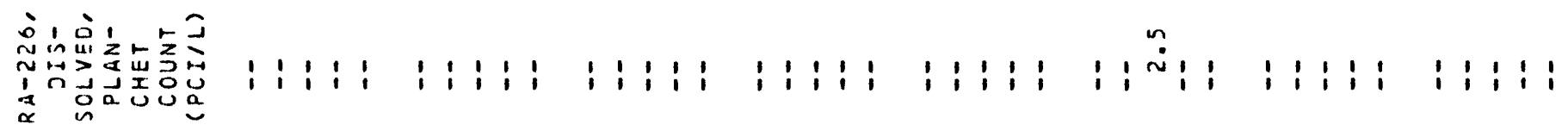

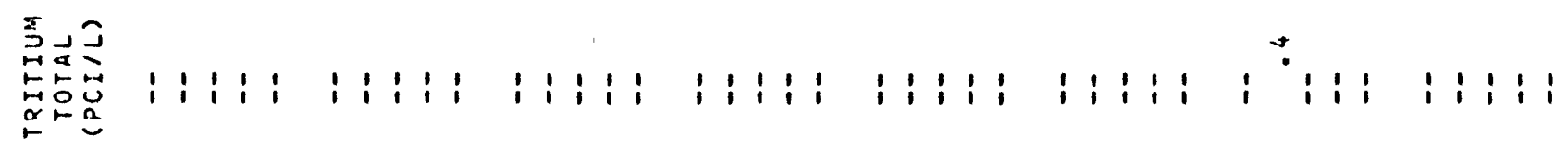

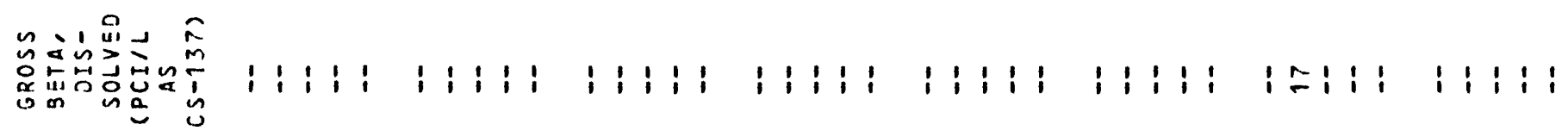

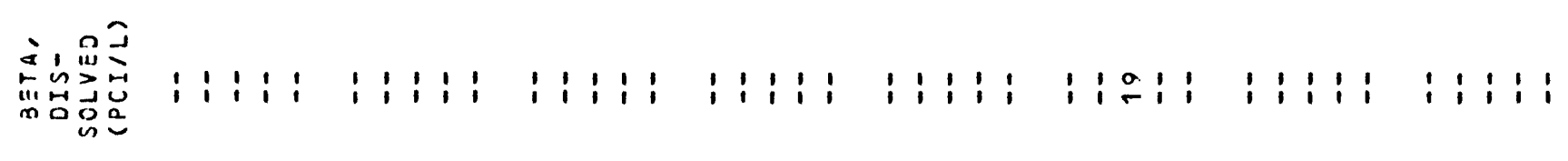
ndi

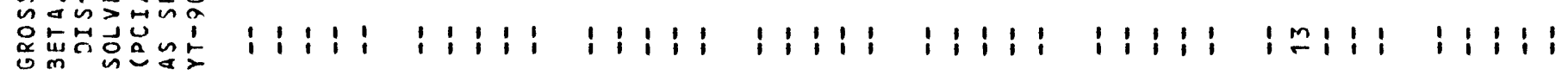

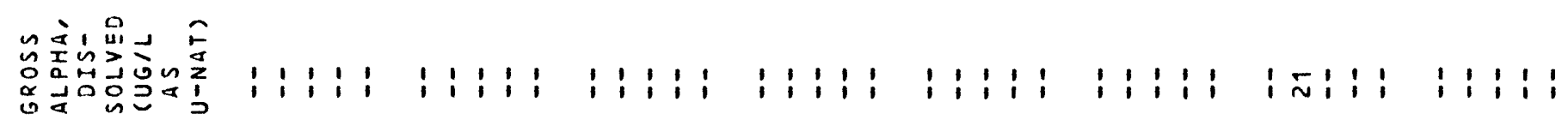

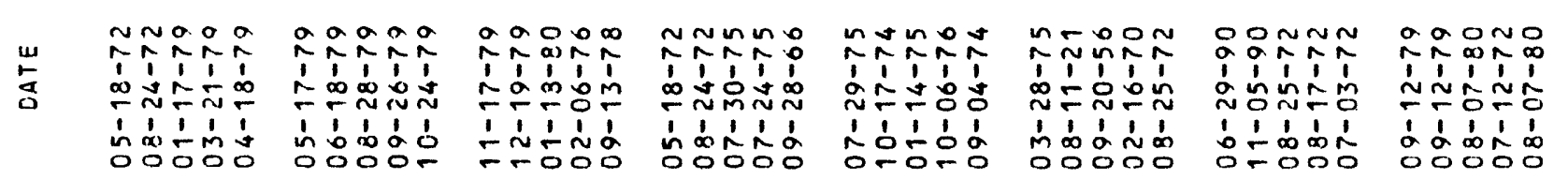




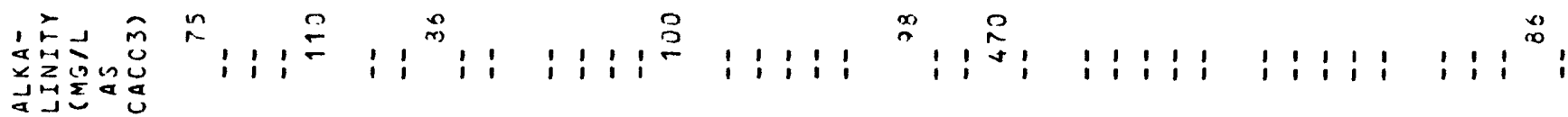

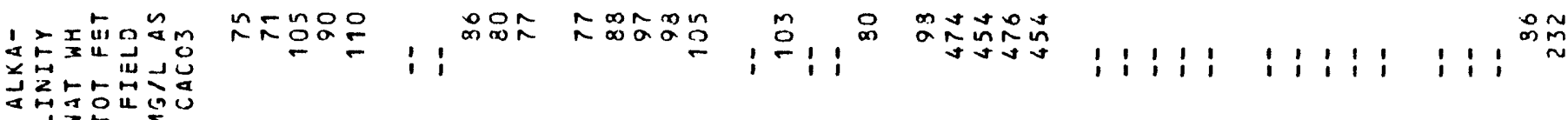

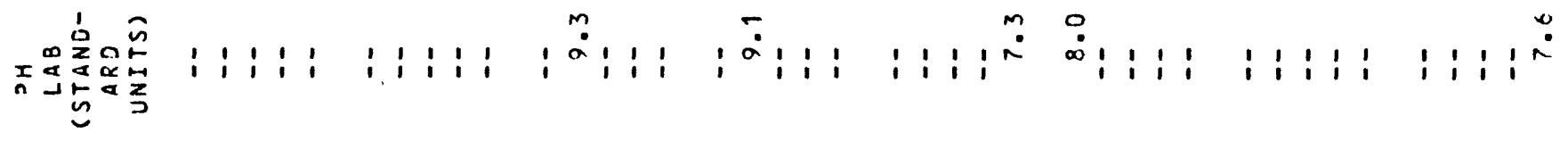

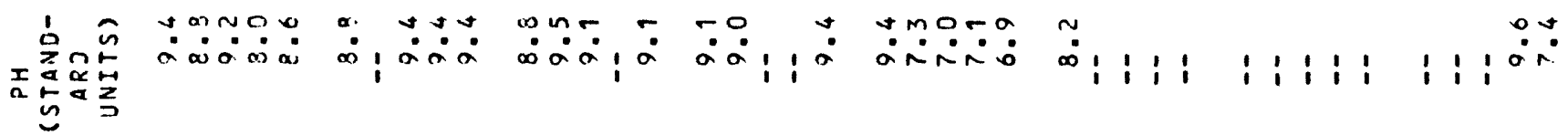

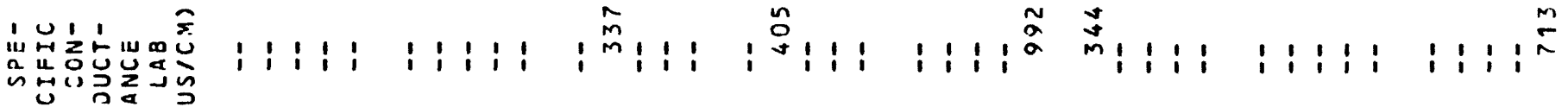

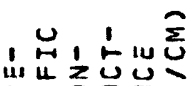

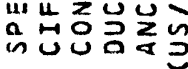

宓 $u \propto \hat{U}$

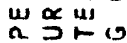
$\sum_{i=1}^{n} \begin{array}{lll}u \\ n\end{array}$

a

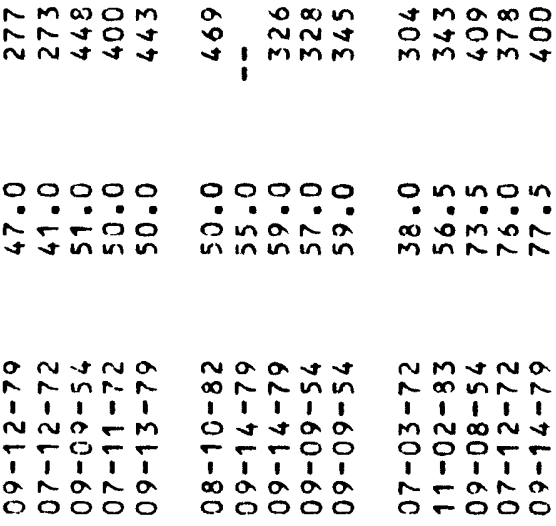

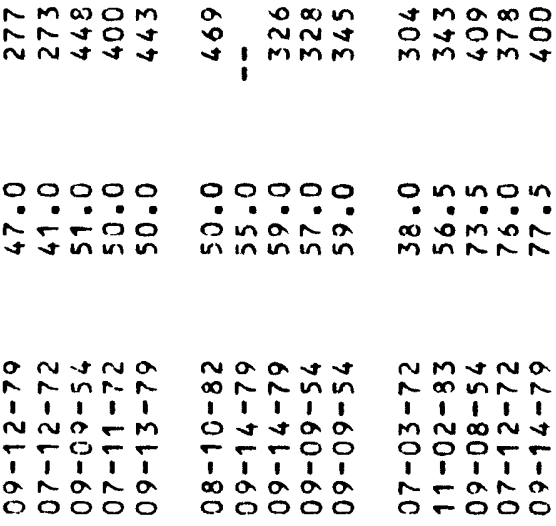

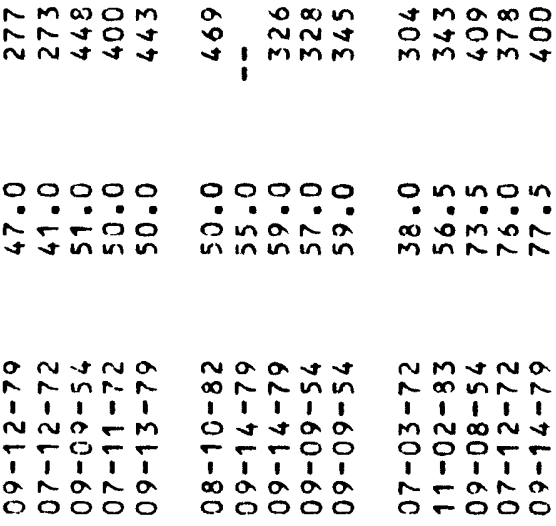

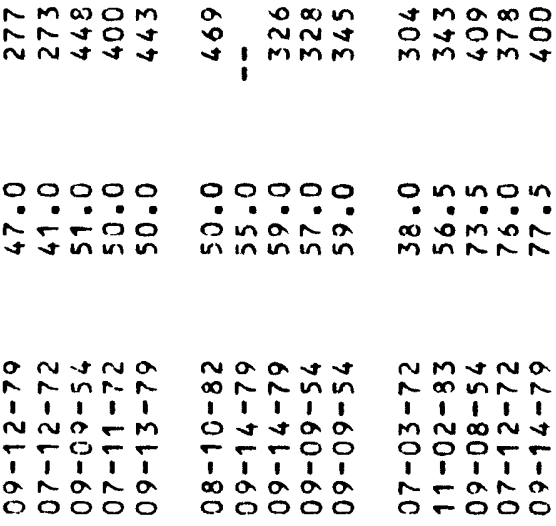

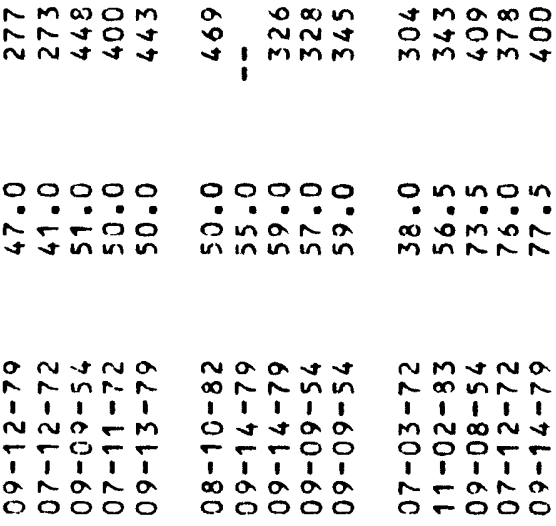

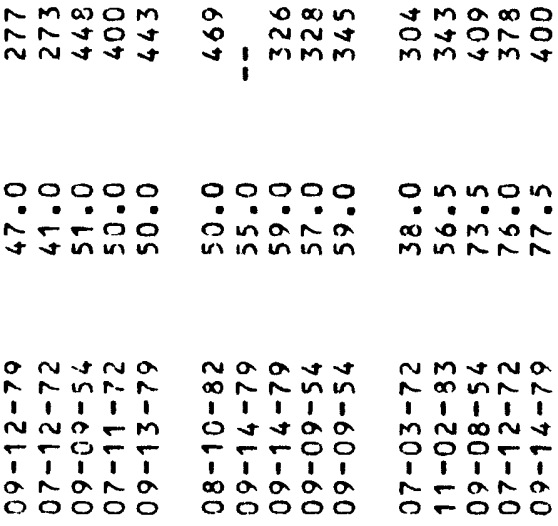

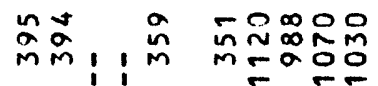

mm $m$ M $m$ N

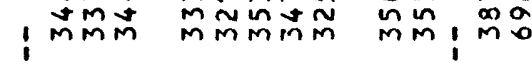

a $a$ a in $\sin \frac{a}{\cos }$

a a a a a a a a

a a a a

a a a a

a a a a

a a a a

a a a a. a

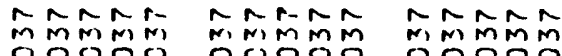

$\hat{m} \tilde{m} \tilde{m} \hat{n}$

minñññ

ñññ

M̂Mñññ

nNNM moñomón

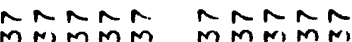

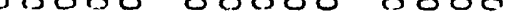

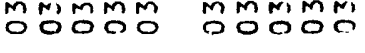

\begin{tabular}{|c|c|c|c|c|c|}
\hline \multirow{3}{*}{ 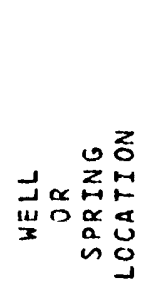 } & 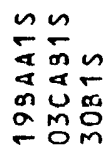 & 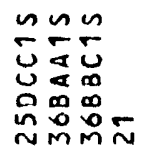 & 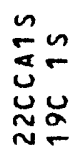 & $\stackrel{n}{m}$ & $\begin{array}{l}n \\
0 \\
0 \\
0 \\
0 \\
\sim v\end{array}$ \\
\hline & 㟧出㟧 & 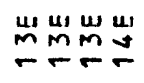 & Iu & $\stackrel{u}{\sim}$ & $\stackrel{m}{\sim}$ \\
\hline & $\begin{array}{l}z \vdots z \\
0 \\
0\end{array}$ & $\begin{array}{l}z z 2 \\
5 \pi\end{array}$ & $\begin{array}{l}z z \\
\square\end{array}$ & $\frac{2}{2}$ & 2 \\
\hline
\end{tabular}


III $\quad$ iII

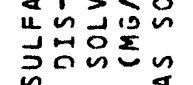

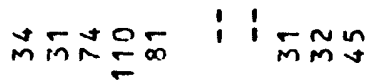

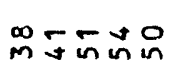

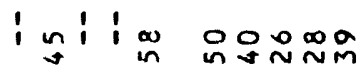

miti:iti:i ition

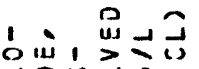

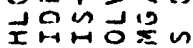

novo mino joo

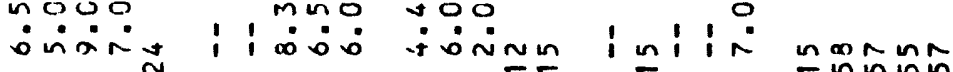

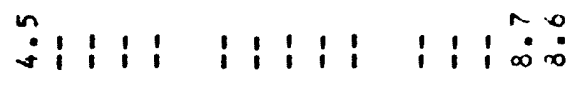

的文, 品

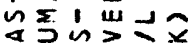

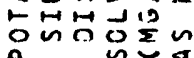

只只oinco

$\sin 0$

$\sin$

$m \sim \infty+\infty$

i cis is

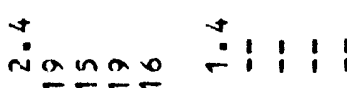

$1: 1: 191: 9$

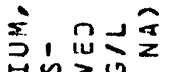

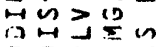

애요의

in cosang

I I

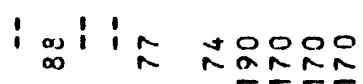

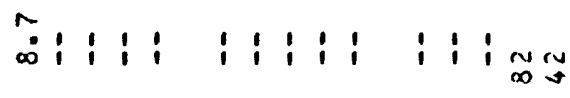

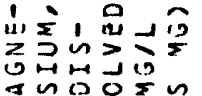

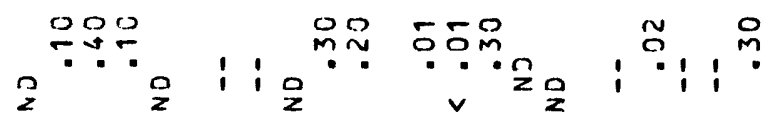

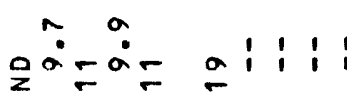

1:i:i i:io

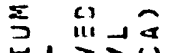

ज्ञñ

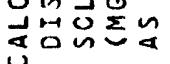

$\because \because \because \div, 1,9 \%$

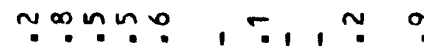

$n t r a$

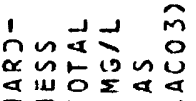

r.oñ

$1 i_{2+\infty}$

$i^{\ln v}$

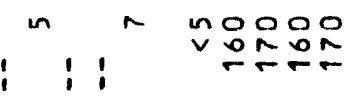

$\stackrel{2}{2}: 1$

mi! !

$1: 1911: \div 0$

的的品

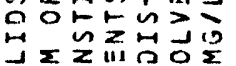

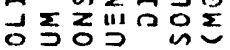

$\tilde{2}=\operatorname{nn} 50$

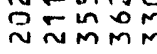
$: 1: \stackrel{m}{\sim} \stackrel{n}{N}$

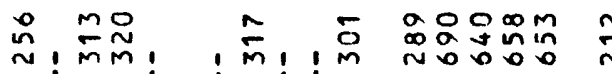

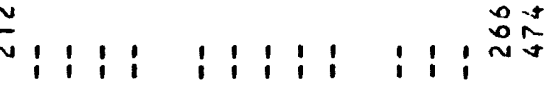

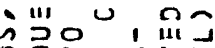

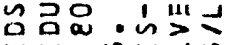

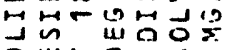
品䓃品

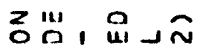
min>0

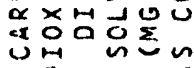

$\because \div$ : $\stackrel{\sim}{m}: !: i: \stackrel{\text { MNN }}{\sim}$ $i i^{-} i$ $1: \stackrel{2}{2}$ $\infty$
1
0
0
0

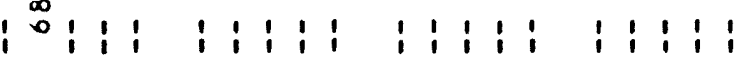

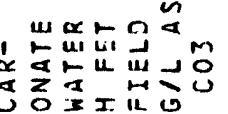

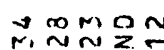

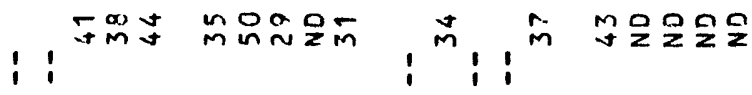
! $1: 1$ $1: 1 ; 1$ $1: i^{50}$

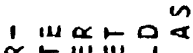

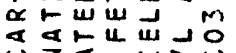

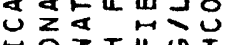

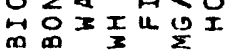

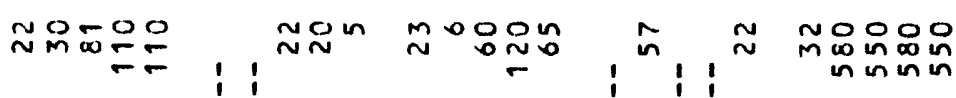

i i i i $\underset{\sim}{\sim}$ Nㅜำ

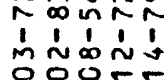
1 등ㅇㅇㅇ

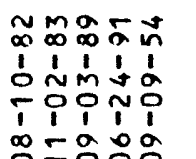

$2 \pm N a m$ mán - $1: T 0$ årí $\infty=0 \circ 0$

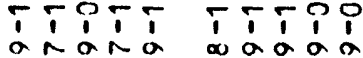
ㄷํㅇํㅇ영

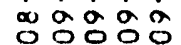

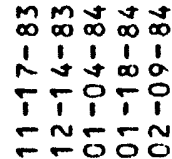

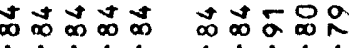

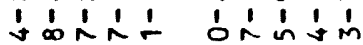
ipTí TiNT

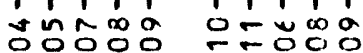




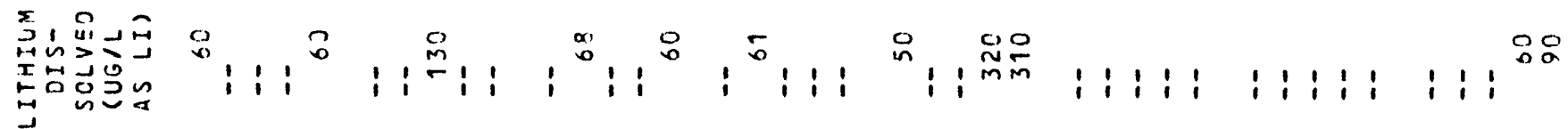

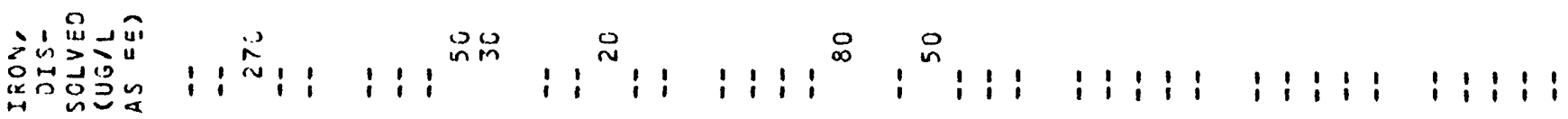

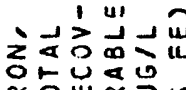

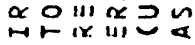

$11111,11111,11111,1,1, \infty$

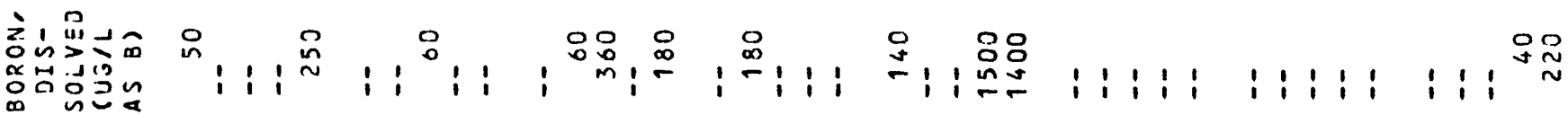

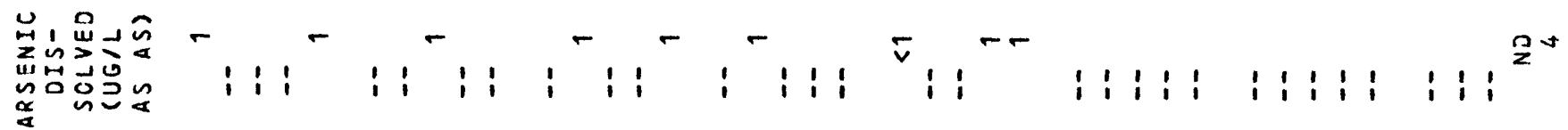

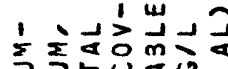

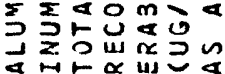

11:1: 1:1:

i: $1: 1: 1: i 1$

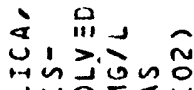

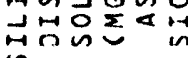

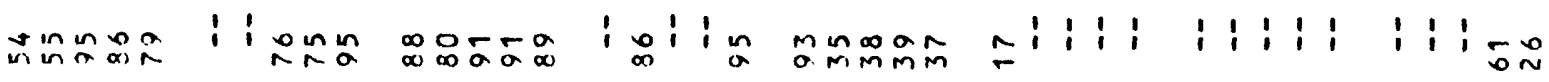

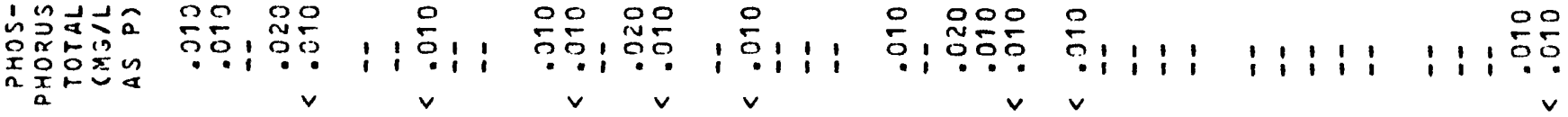

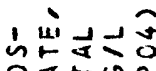

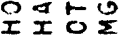

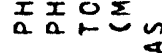

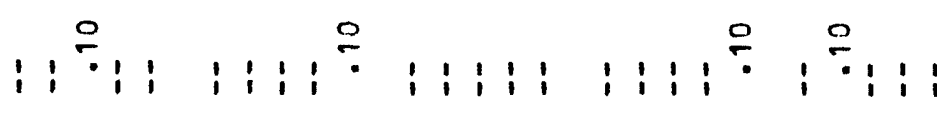

$1: 1: 1: 1: 1:$ : $1: 1:$

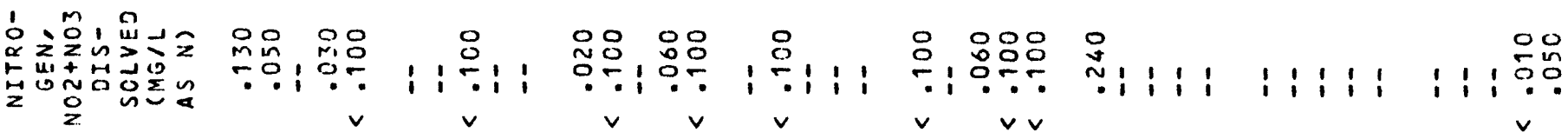

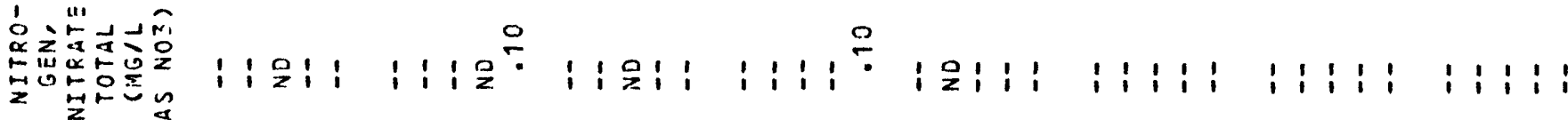

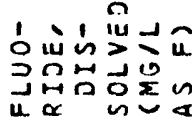

aㅡ영 ํomm ำ $\stackrel{0}{\because} 1: 1: 1: 1: 1: 1: 1: 1:$ 준ำ

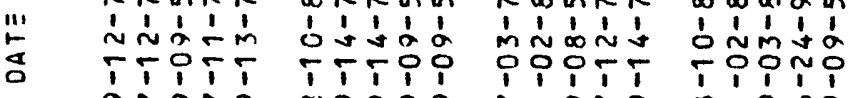

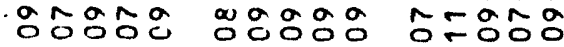

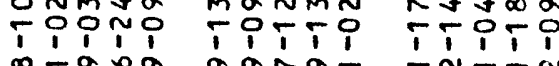

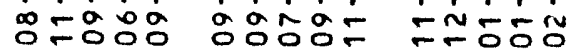

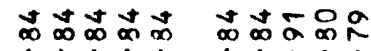

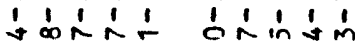
iptit ticit

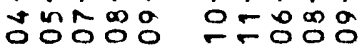




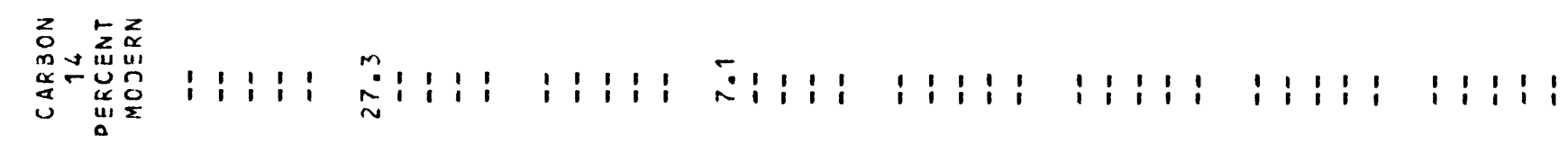

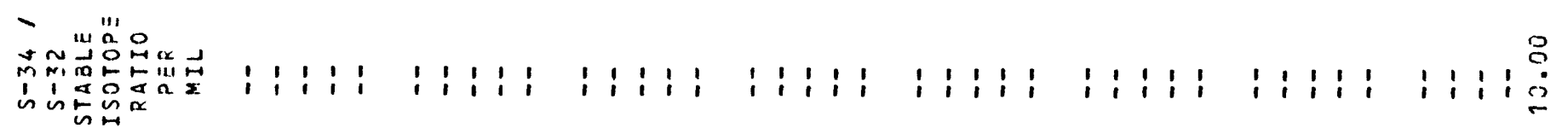

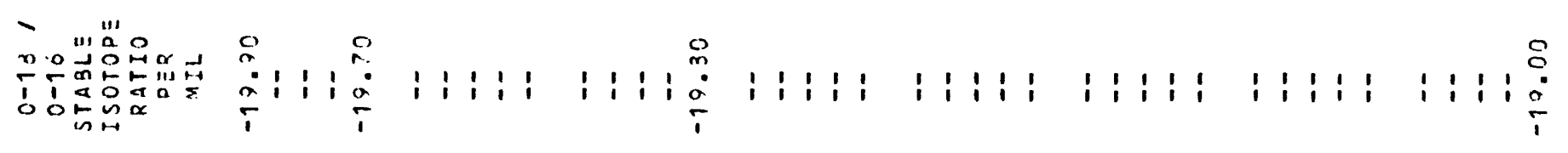

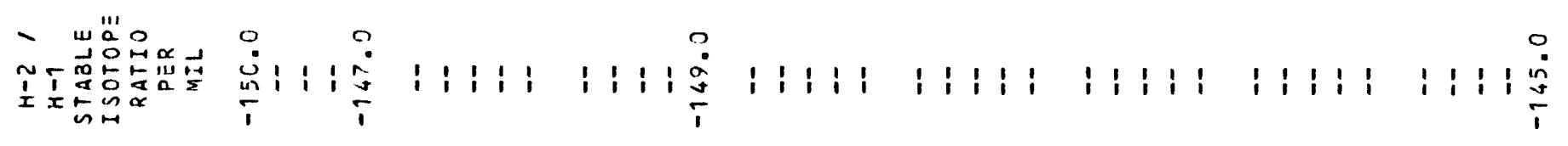

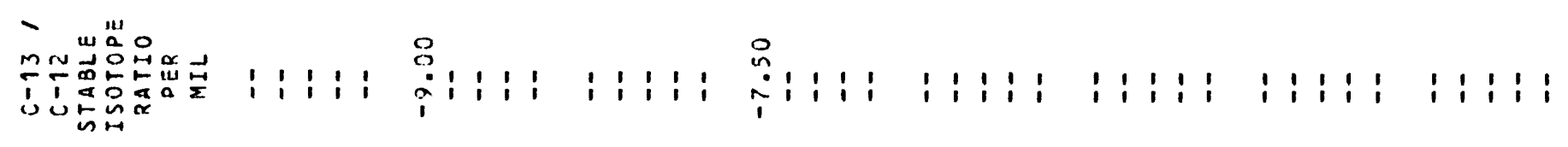

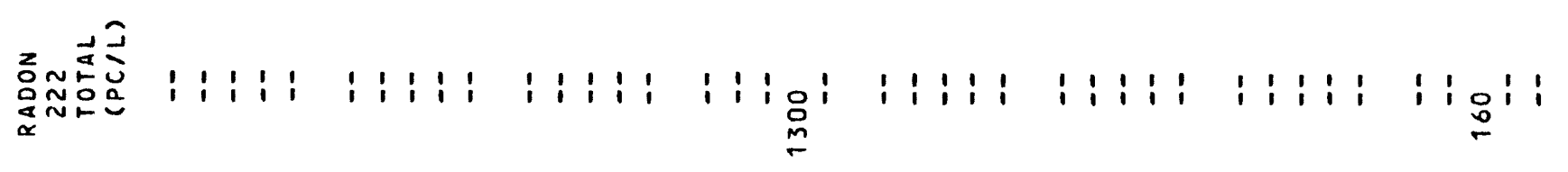

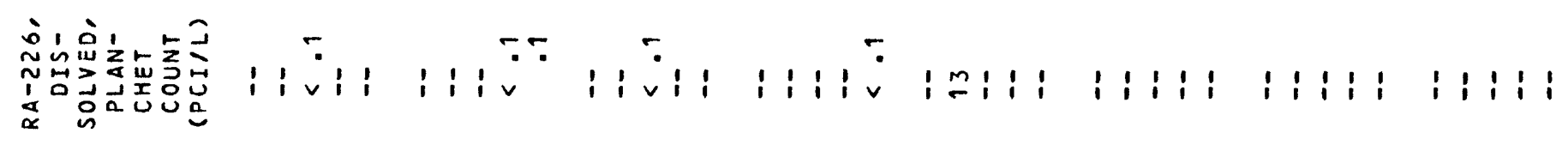

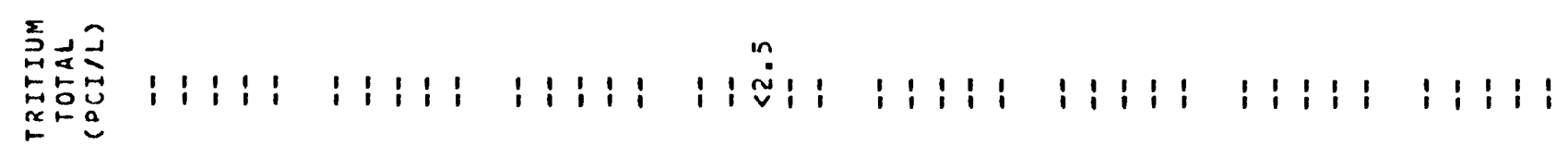

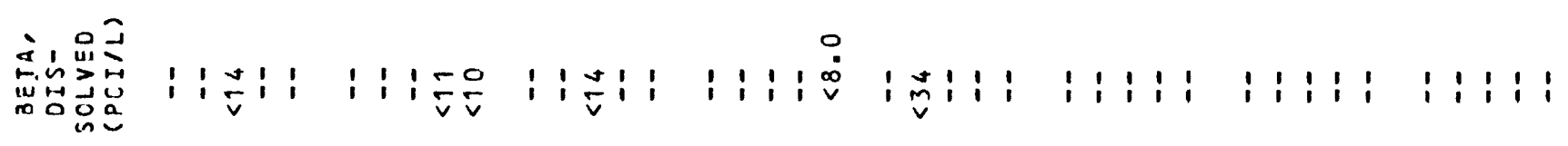

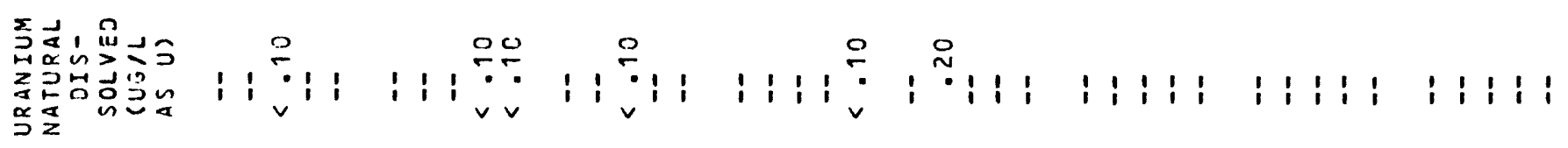

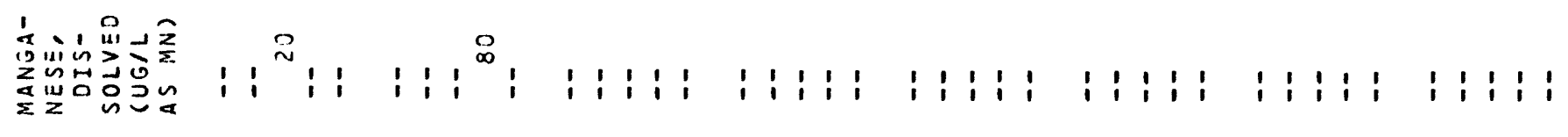

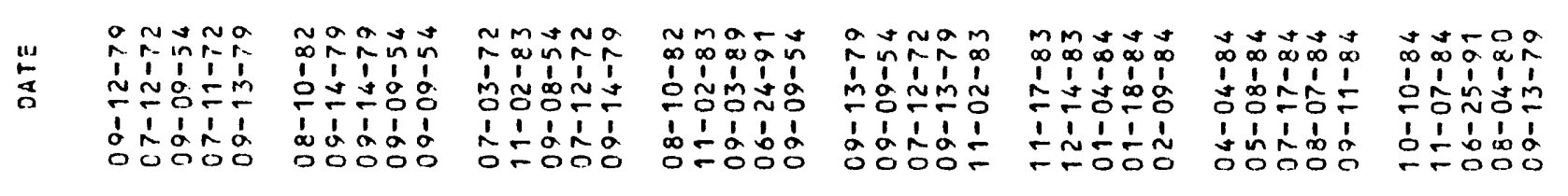




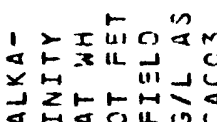

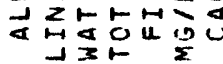

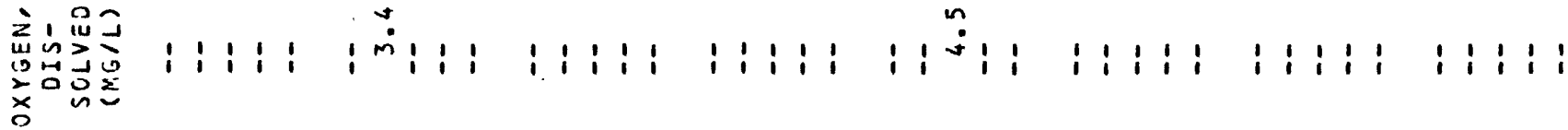

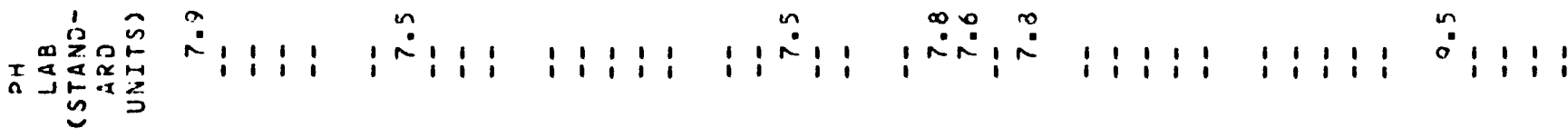

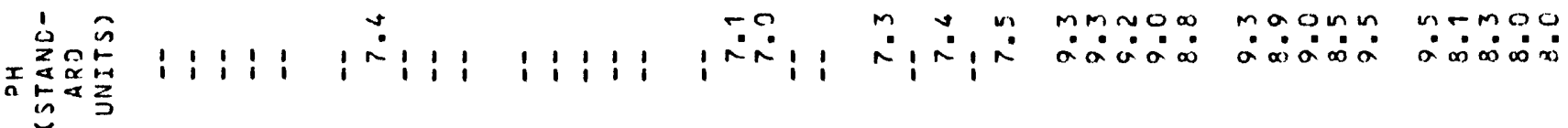

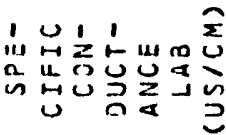
$\stackrel{0}{:}: 1: 1:$ :

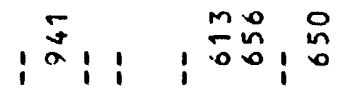
i: : : : i : i: : : :

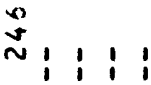

$\because 15$ u

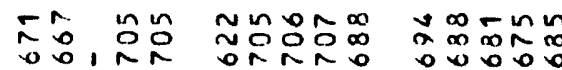

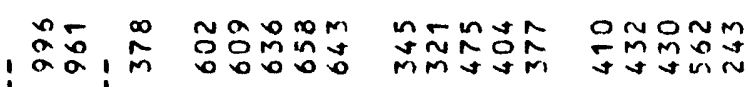

$\operatorname{man}+N$

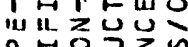

언곤

Ix $4 \propto \mathcal{U}$

i2, $\stackrel{\alpha}{\rightarrow}$ (1) is

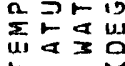

0.0.0. 0.0mm

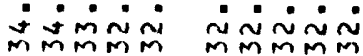

n? 0 in mo

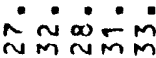

0. o ommoo

00000

O 0 in mo

iñio

$n m n \curvearrowleft m$

mana

0 in 00 in कก⿻上丨丶

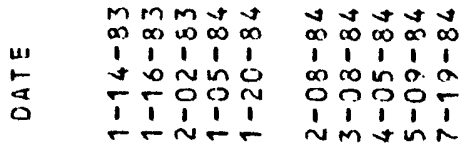

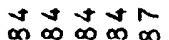

每

- $0 m-N$

$\infty m+5$

$0 \div-0 \stackrel{1}{0}$

I' i ' i

vioñ

列

11111

$\sim \infty_{1}^{\infty} \infty 0_{1}^{\infty}$

in $-\infty N m$

$=-2 \frac{1}{6}$

ỡờำ

बin

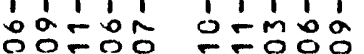

and

ii $1 ;$

in $0 N 50$

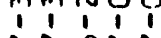

ónoำ

$\begin{array}{llll}0 & 0 & 0 & 0 \\ \infty & \infty & 0 & 0\end{array}$

11111

Nom

0
0

wrom on

1 i i i

Join 0

1 i 111

$\begin{array}{llll}\infty & \infty & \infty & \infty \\ 0 & 0 & 0 & 0\end{array}$

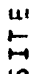

a a a a a a a

a a a a

a a a $=373=7$

a a a a a

$a$ a a a

a. $x \geq \frac{3}{10} \frac{3}{10}$

NANNR NNNNN NNRNN

ññ

ññ

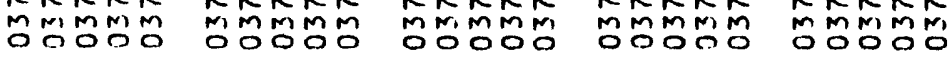

ñNnN nNNNo ómómó Mómómón

araan momom

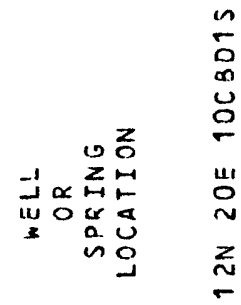

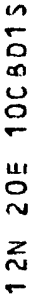

$\begin{array}{ll}n & \\ 0 & a \\ 0 & a \\ 0 & 0 \\ n & m \\ w & u \\ a & a \\ - & a \\ z & z \\ a & a\end{array}$

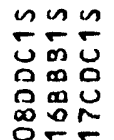

离足

แ山山

느는

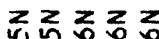

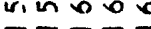

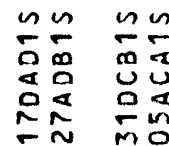

แI แ山 य

$\sim m \forall N$

$z_{0} \geq \geq z_{\infty} \geq$ $\frac{n}{2}-5 \div 5$

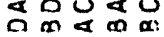

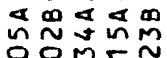

แม 世 엉ำ

胥等管 00000 


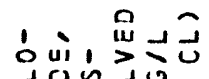

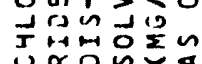
$\sim$ กั

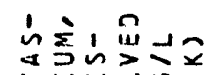

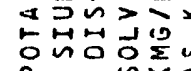

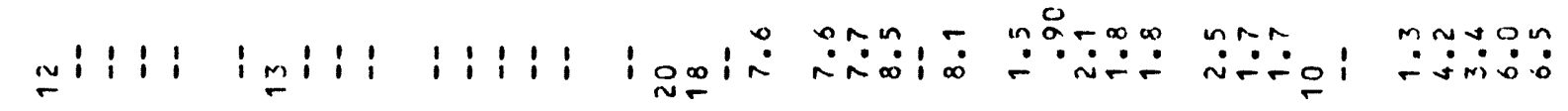

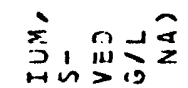

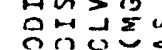

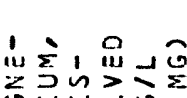

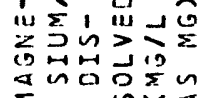
mili: iaiti i i i ingin nomit orana $\pm$

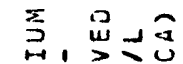
und

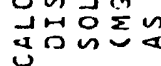

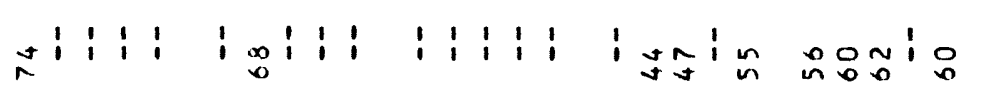
initim ñman

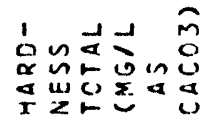
0

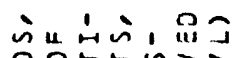

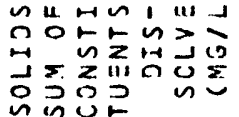

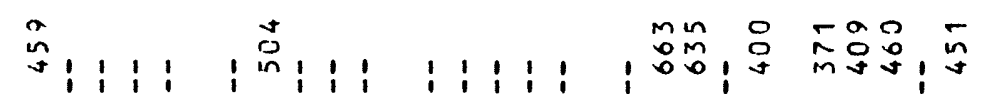
num
mon
Mommin
Nomin

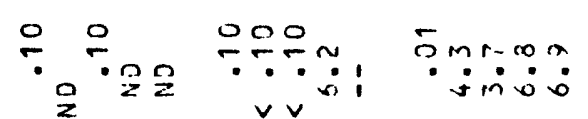

inou, on

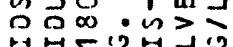

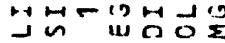

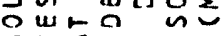
i i i i 2

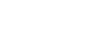
i i i i

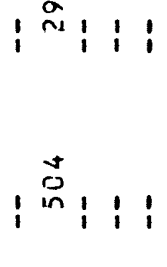
i i i i i

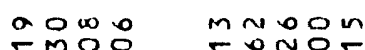
mMMO; N

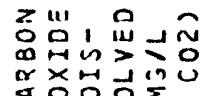

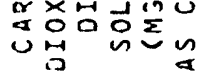

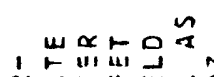

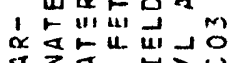

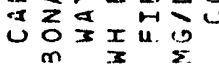

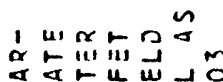

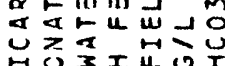
की

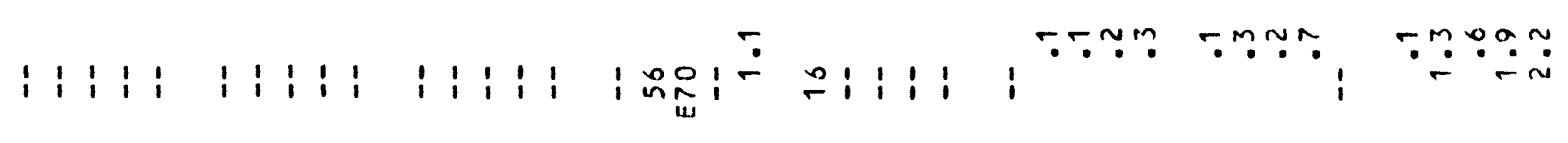

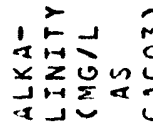
tu

$m m m s$

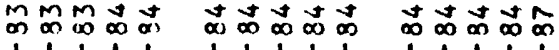

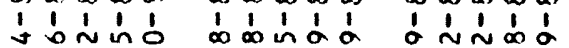
- 1 is 둔둥 axman is Titit 잉응 $\prod_{\infty}^{\infty} \underset{\infty}{\infty}-\bar{a}$ 1 in $\begin{array}{lll}1 & 1 & 1 \\ 1 & 1 & 0\end{array}$ MT 잉ㅇㅇ a요순

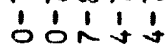
mî́, 등으응
잉요

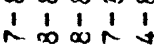
UNi 00000

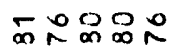
' 1 b in 1 '́ ?

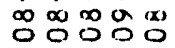




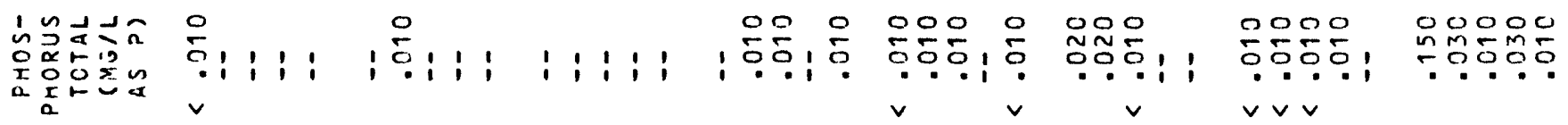
的“i它的出

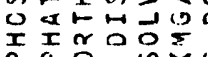

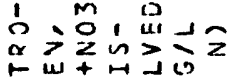

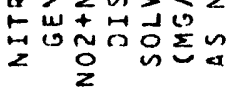

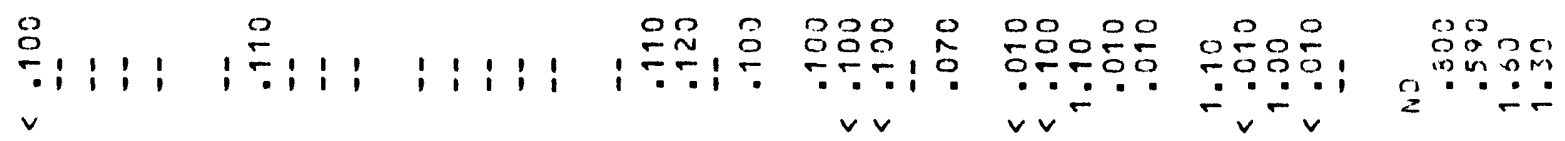

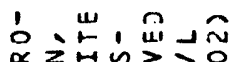
$\alpha \sum_{n \rightarrow 1}$

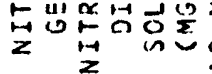

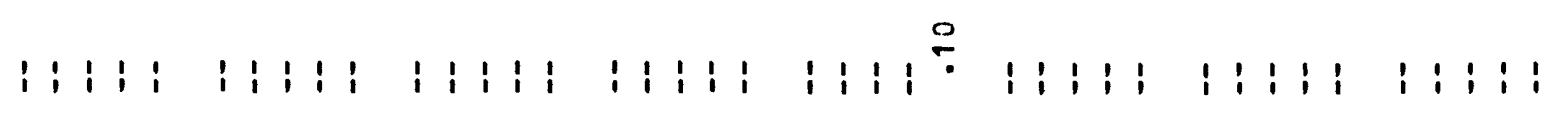

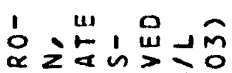

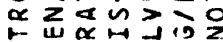

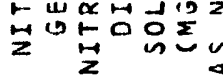

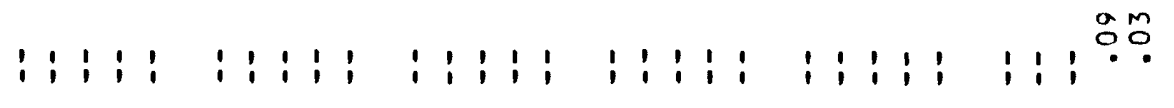

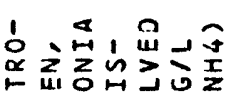

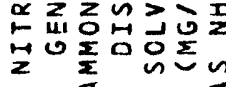

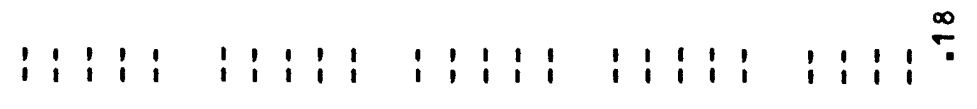
$\div: 11: 11: 1: 1$

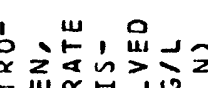

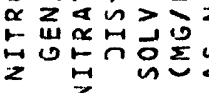

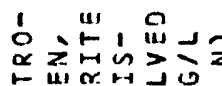

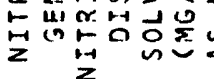

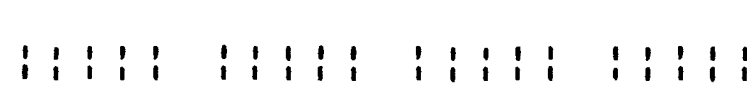
$1 ; 1, \stackrel{\infty}{0}$ 11:11:1:1:1:1:11

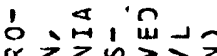

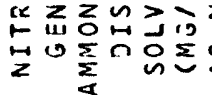

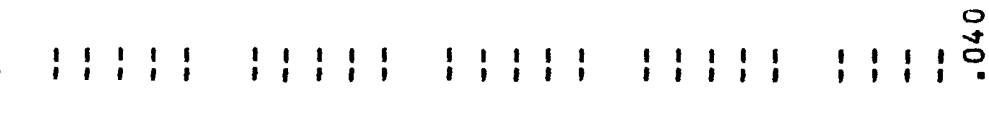
?

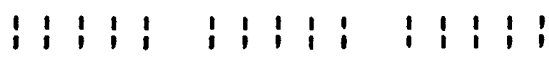

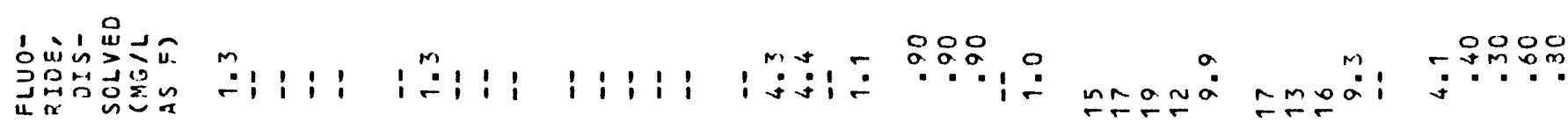

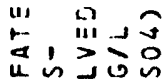

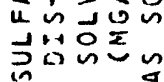

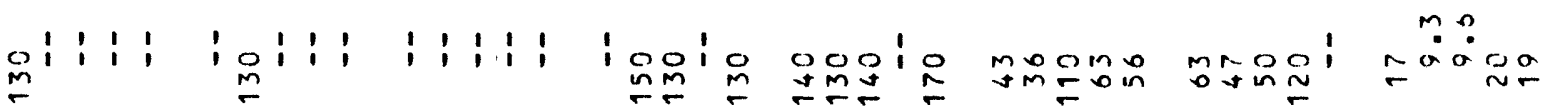

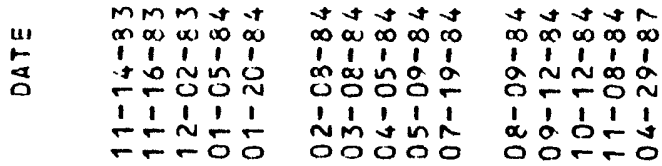
$-a m-n$ î́pín แับธกั bitis

minsoi pooj

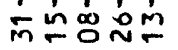

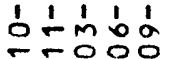

osoor

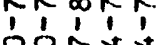

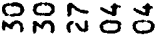

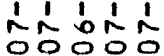

당- - 0000 1.

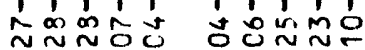
1
1
0 


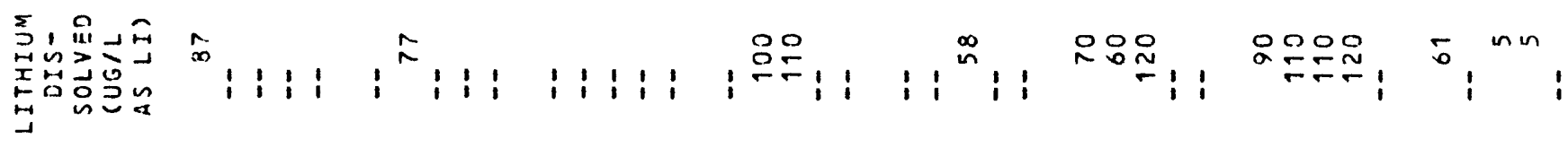

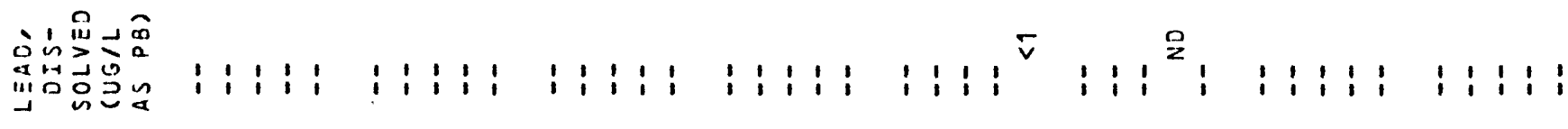

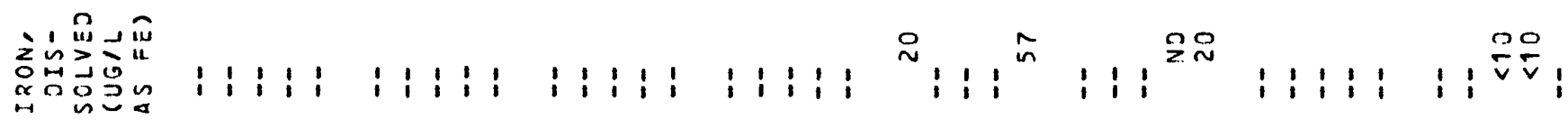

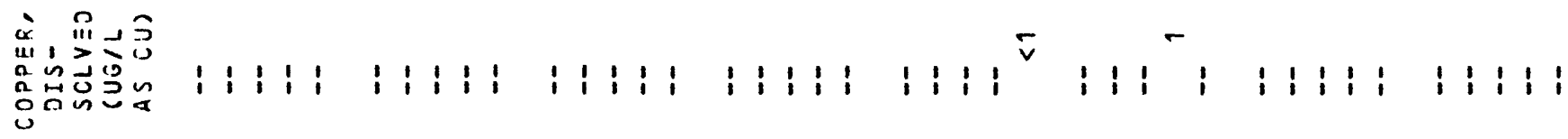

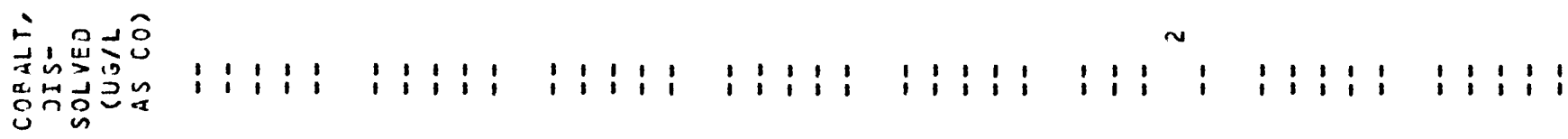

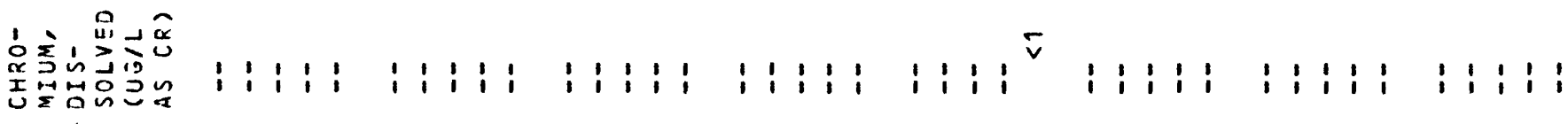

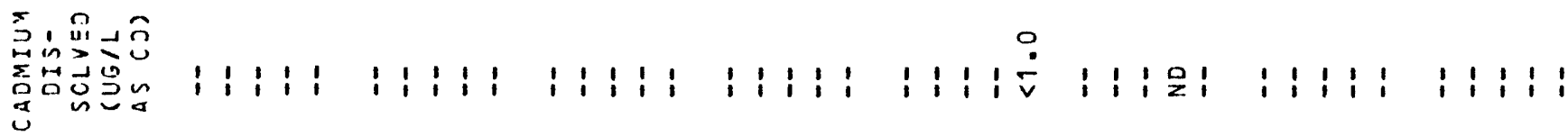

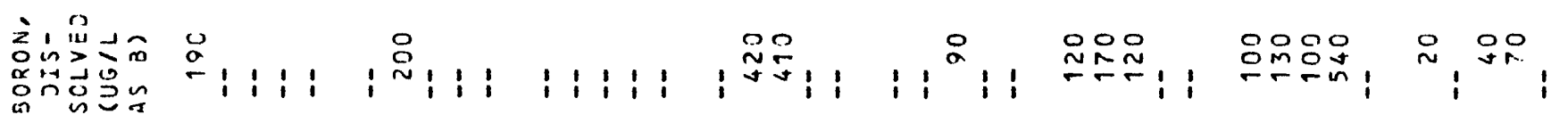

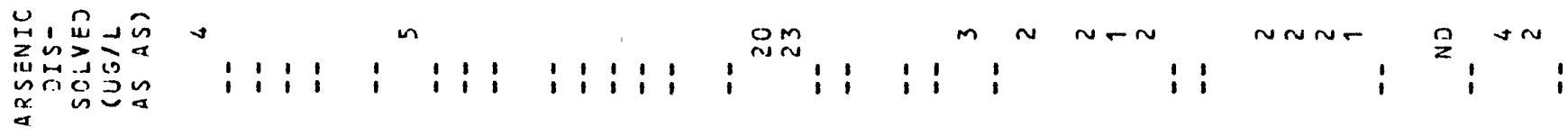

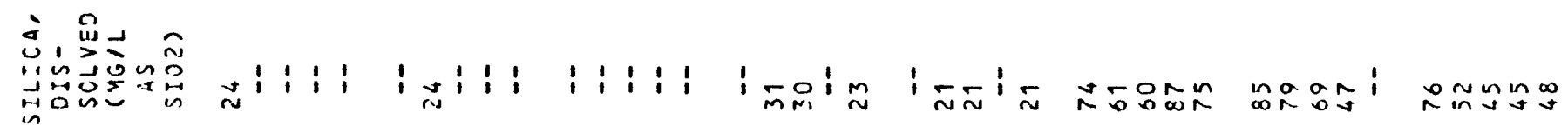

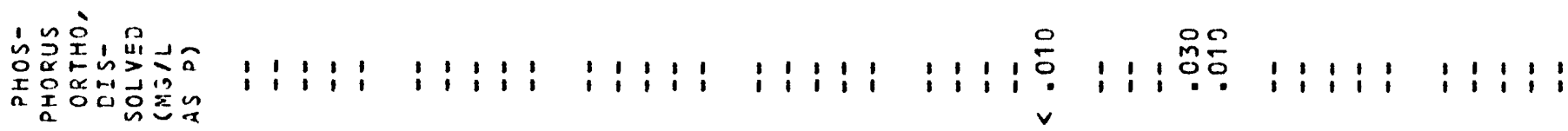

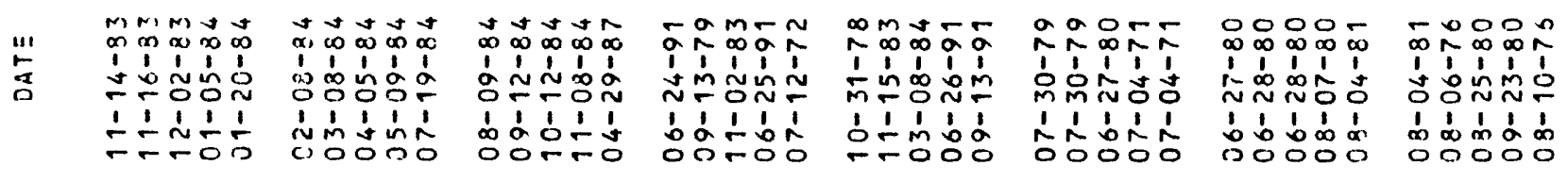




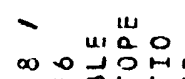

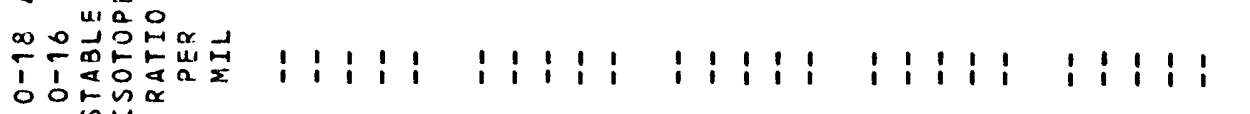

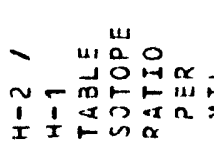

: : : : : : : : : :

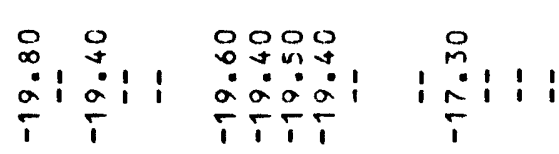

0
1

: : : : : : : : : :

: : : : : : : : : :

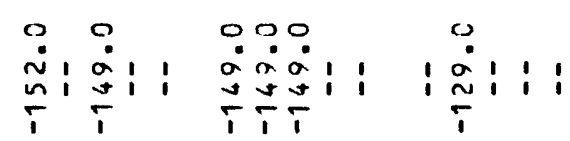

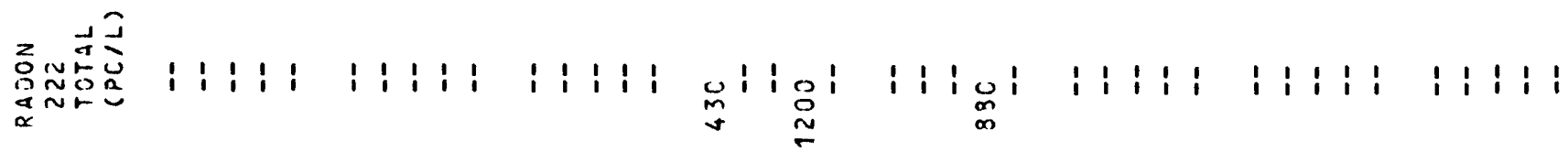

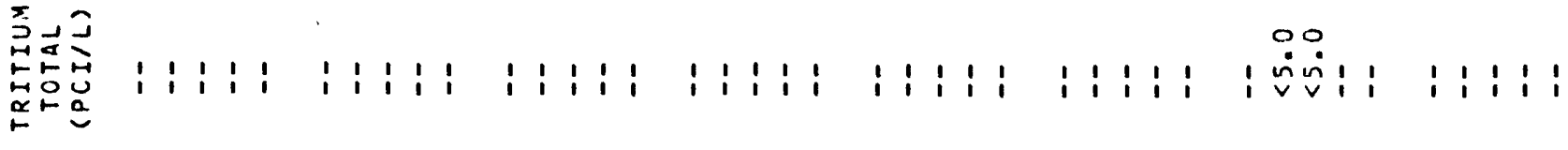

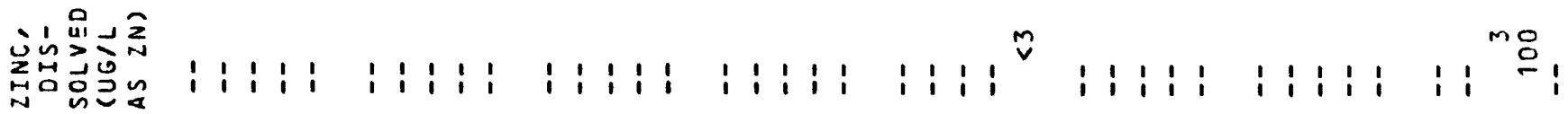

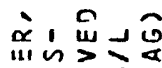

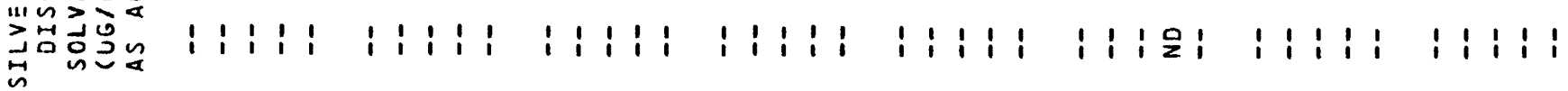

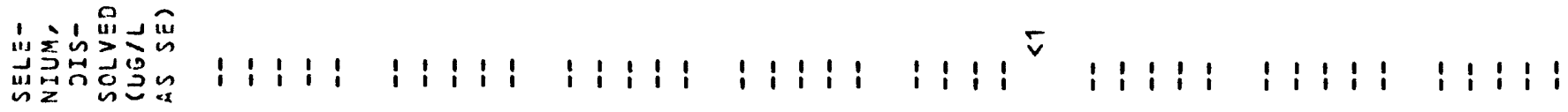

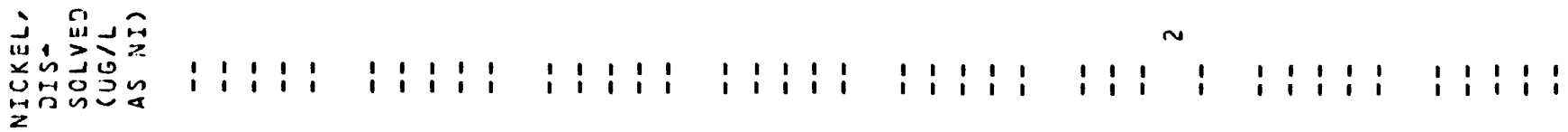

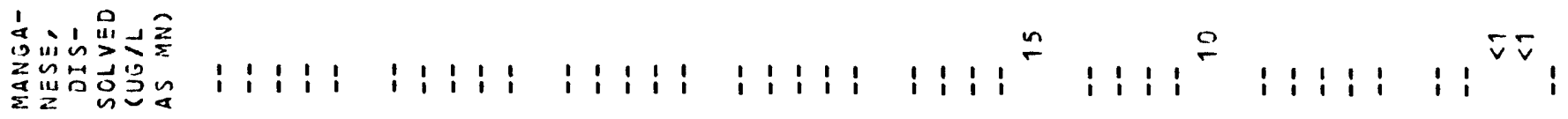

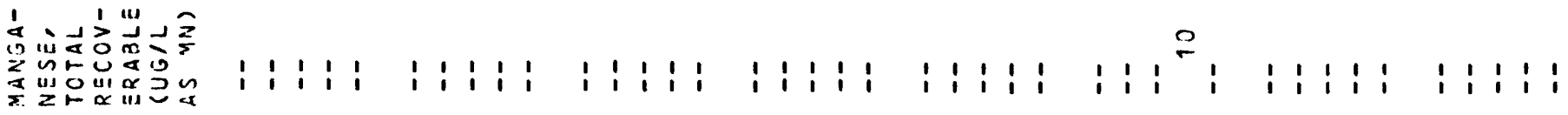

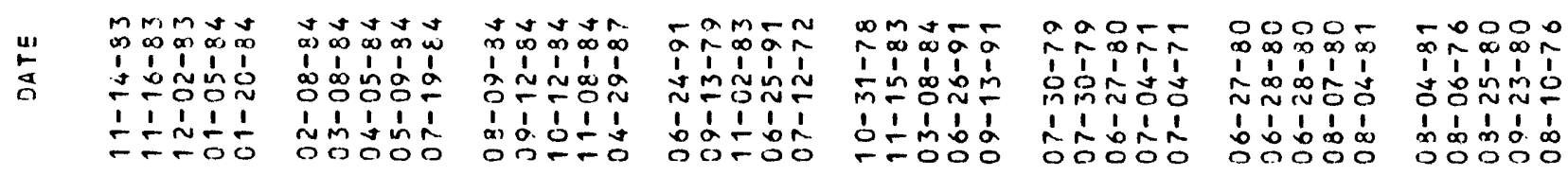




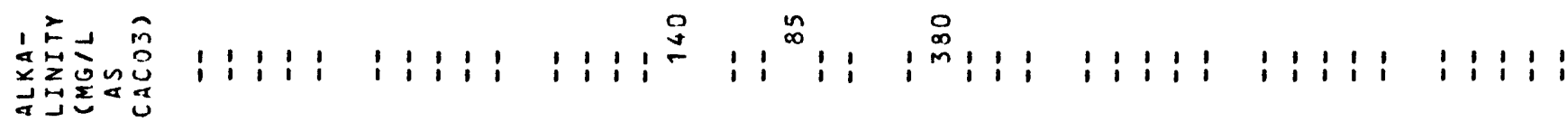

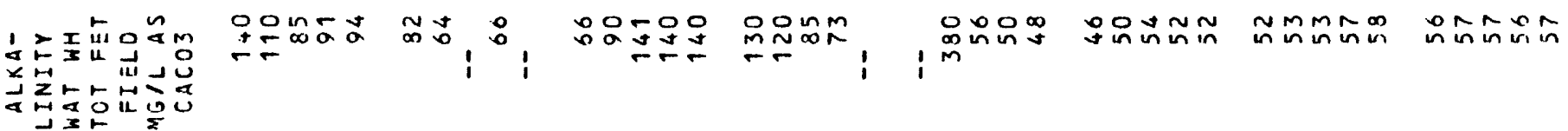

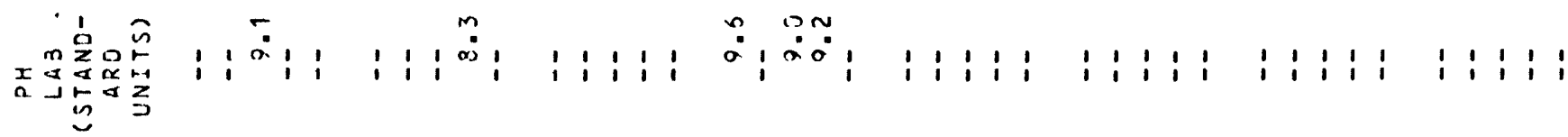

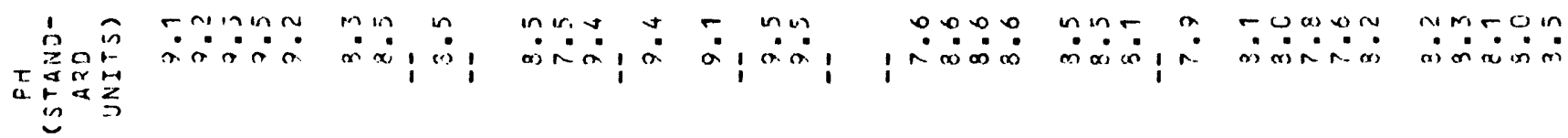

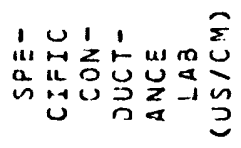
$i ! \stackrel{2}{2} !$
in
$\stackrel{n}{m} \underset{m}{\sim} \stackrel{\infty}{N}$
$1919111-1$ 1:11!

i i i i

i i i i

$\begin{array}{llllllll}1 & 1 & 1 & 1 & 1 & 1\end{array}$

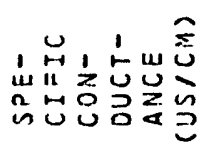

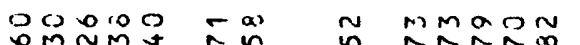

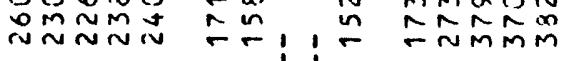

ㅁmin

iñN

$\operatorname{nat}=0$

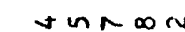

$\min \pi$ oinow

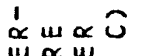

a'd

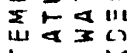

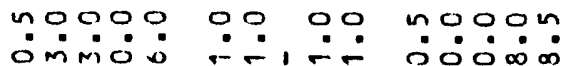

0000

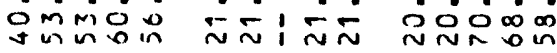

부요

0.00

nom

unin

non in

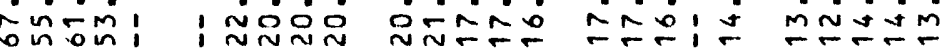

$* *$

NN-UN HaOOE OBNNO TNoma moogo or iño

1'́1'́n

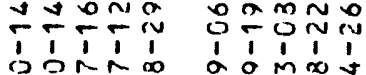

$1 \hat{1} 11$

movo

1.

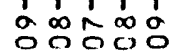

$\infty \simeq-\infty$

$\infty, 1,1$

- intoo

mon-5

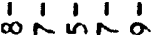

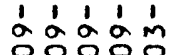

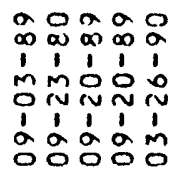

이요의

is 10

on $\sim$ 政

Tín

은응ํㅇ

tin on? Trmat unining mónon in otró TiNi NiTi

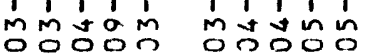
$\stackrel{a 1}{\leftarrow}$

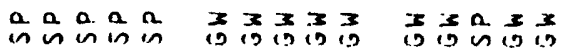

raaa

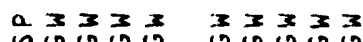

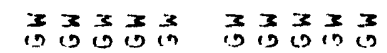

2
2
0
0

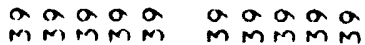

a a a o

Minm

a.oog mómón âa
mómón

åmag momón

$\cos 00$

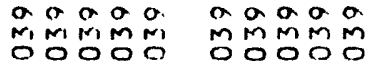

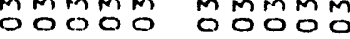
MOCOO DOCOO

\begin{tabular}{|c|c|c|c|c|}
\hline $\begin{array}{lll}n & n & n \\
m & n & a \\
n & u & 0 \\
a & a & m \\
0 & m & m \\
-m & m\end{array}$ & 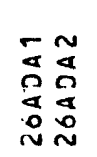 & 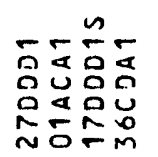 & 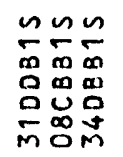 & 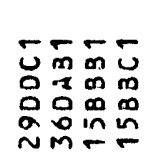 \\
\hline ü & 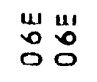 & 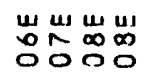 & 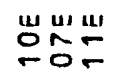 & 虫出出出 \\
\hline $\begin{array}{ll}z & 2 \\
m & m \\
0 & 0\end{array}$ & 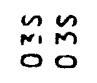 & 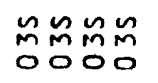 & 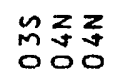 & 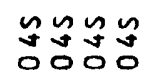 \\
\hline
\end{tabular}


$\because 1112 \pm 0$

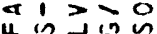

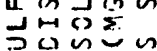

iii

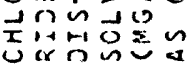

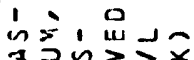

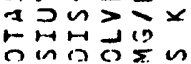
กำ

$\sum_{j}, 0 \neq \hat{a}$

$\rightarrow n>19$

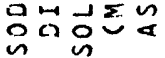

出文的品留 象出台过是品

I 214 出>

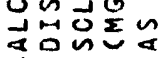

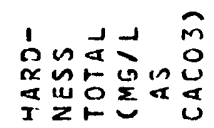

$m m+m m$

0000

$\div \div \div$

$\vee v \vee$

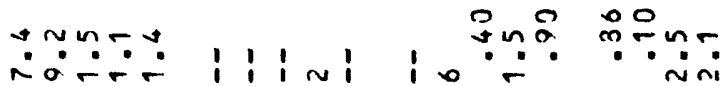

\section{OuN $A N \infty N$}

Nman

(3)

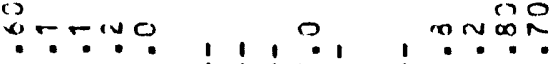

$r+r$

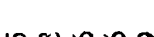

i i i

I

ion

i i

$\dddot{\sim} \sim \mp \sim$

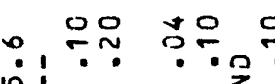

$\stackrel{0}{\square} 1,1,11$

कoo $-\infty 000 \mathrm{mana}$ I vinim miñin miñmi

in

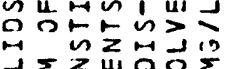

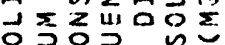
옹ํㅇ

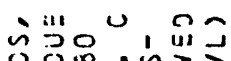

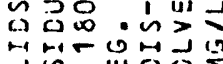

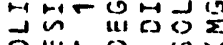

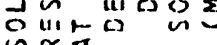

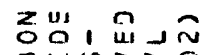
$m+n>\geq 0$

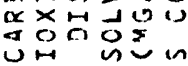

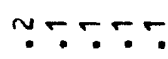

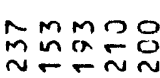

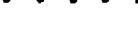

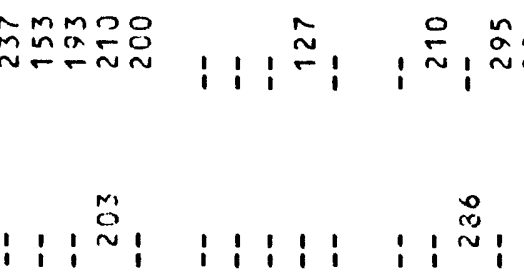
$1: 12$ ! $i i^{9}$

$i^{\infty}$

n ro in

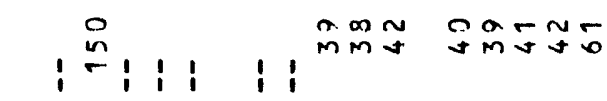

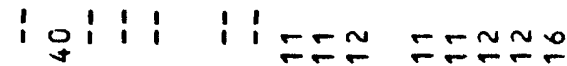

$\because \approx m \sim m$

n a

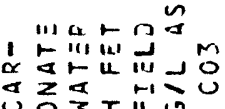

inmm $M$

$0 \underset{0}{0} x^{2}$ il

I $110 r 0 \%$ व

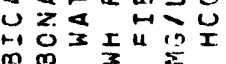

$\operatorname{mon}_{0} \sim \pi$

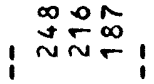

$\frac{n}{n}$ i i 동 m m

Ar- $\ln n$

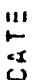
l 111 a noga

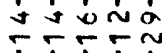
OON $\begin{array}{lllll}1 & 1 & 1 & 1 & 1 \\ 0 & 0 & n & 0 & 1\end{array}$ c. a $a m \infty$

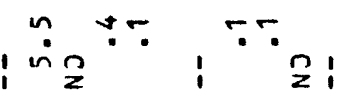

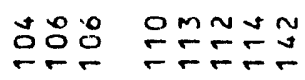

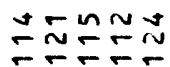

$-2000$ on $N \sim N 0$ $1 \hat{1}$ - mota ôn $\infty_{\infty}^{\infty} \approx-\infty \infty \infty$ 1 1 in 1 b ํํำ $\infty$ 出na
웅에

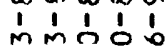
i

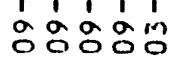

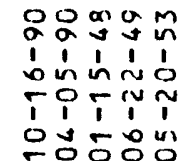

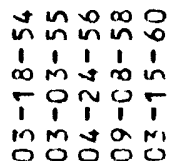

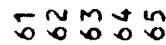
$1,1,1$ w⿻上丨-口 i. ' i i i in moton 


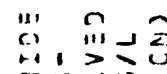

$z \sin$

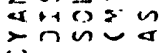

$: 1: 1: 1: 1 \frac{\overline{0}}{1}$

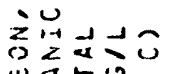

i1 4 古

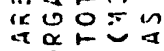

: $: 1 ;: 1: 1 ;:$

घं ,

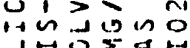

药䓃距星

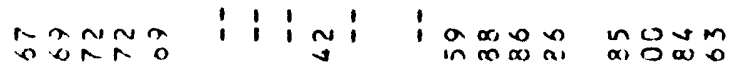

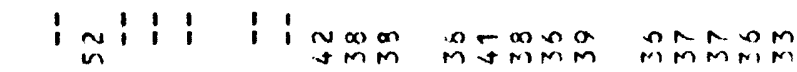

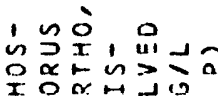

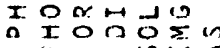

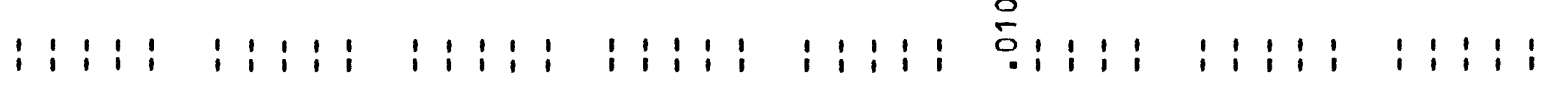

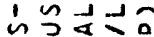

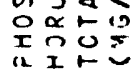

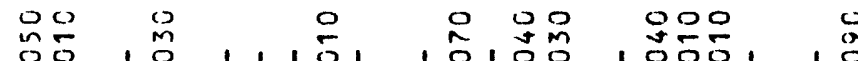

at

$2, ?: 1: ?$

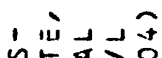

भिएव

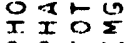

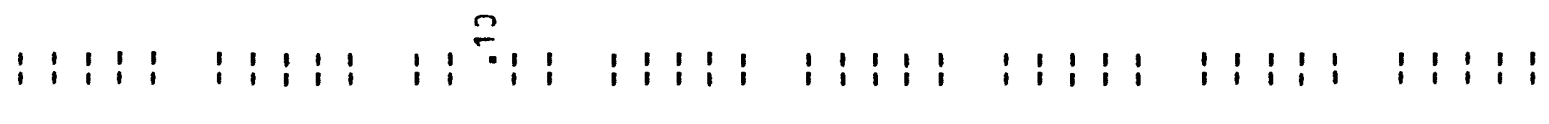

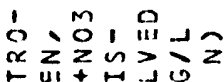

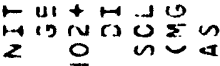

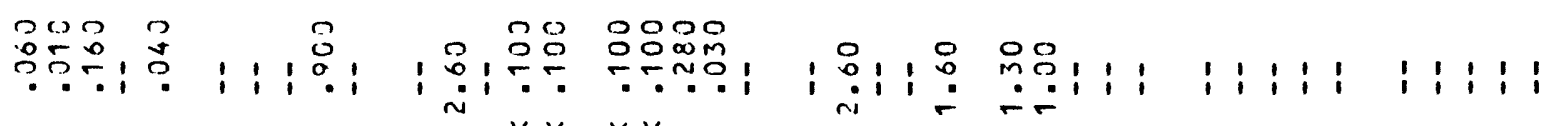

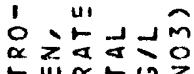

5410

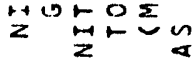

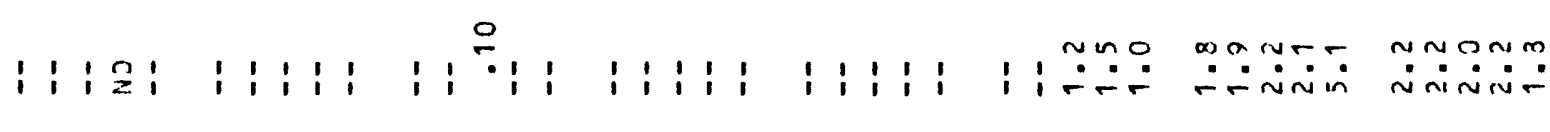

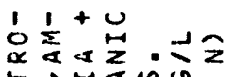

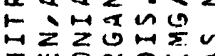

닝응

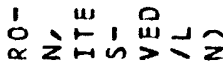

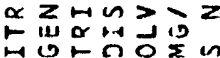

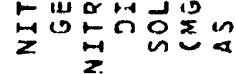

$191911: 19$

1:1:1 1911

i $1: 11$ i

1:11

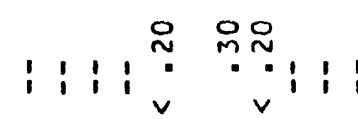

:11:19:11:

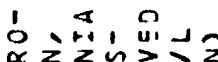

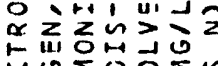

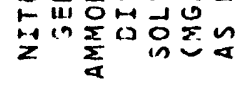

i: $1: i \quad i: i \frac{5}{3}:$

!

i i i

111

11111

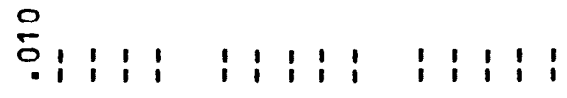

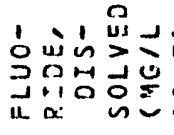

जimisim

$1: 1$
1
1

$i$

?man mosivi! ! ! ! ! !

$\div$ 운유문

윤ㅇN언 음윰유

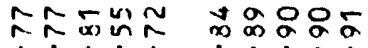

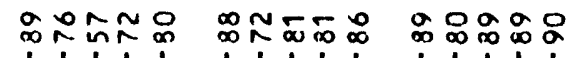

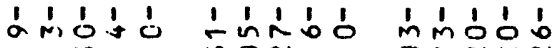

TTMTT MPNTT PNNTN

Tㄴำ

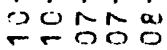

ơỡo

ระ8ะo

영워

少的少。

Titis

00500

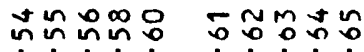
向向毛向 iprit pitip

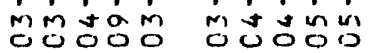




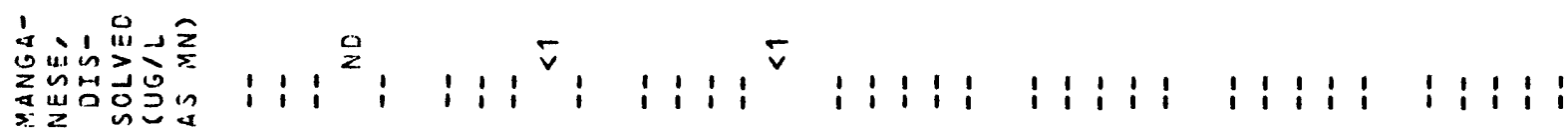

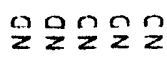

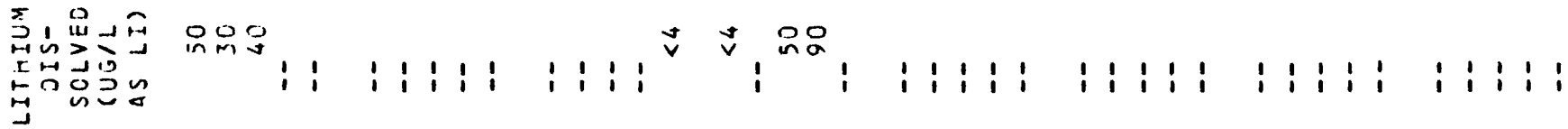

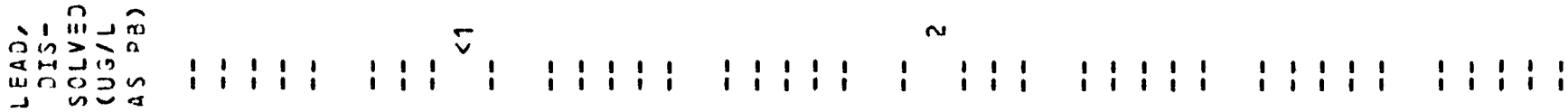

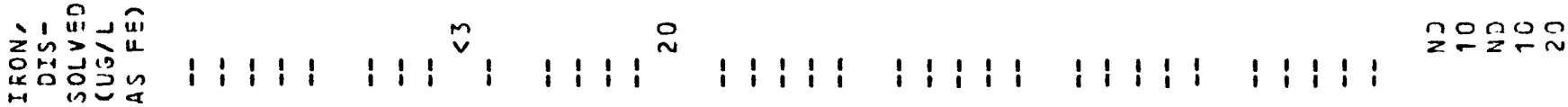

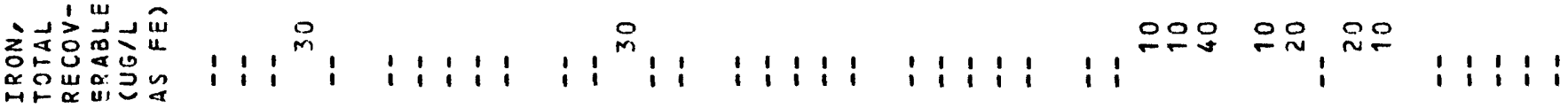

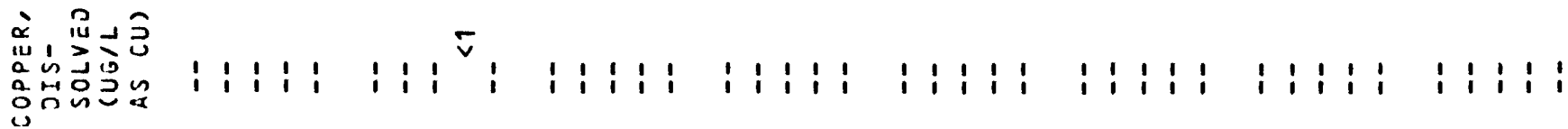
àn

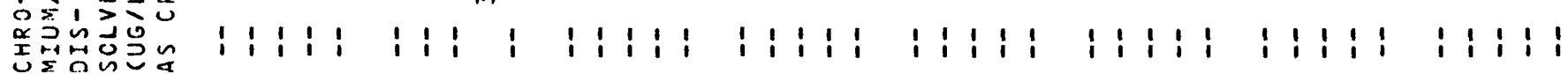

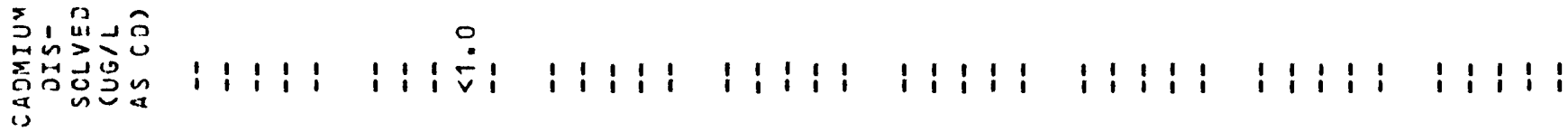

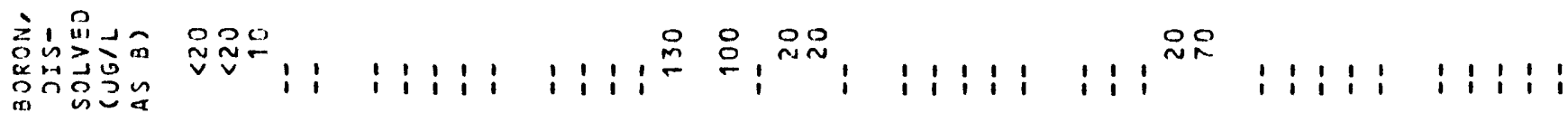

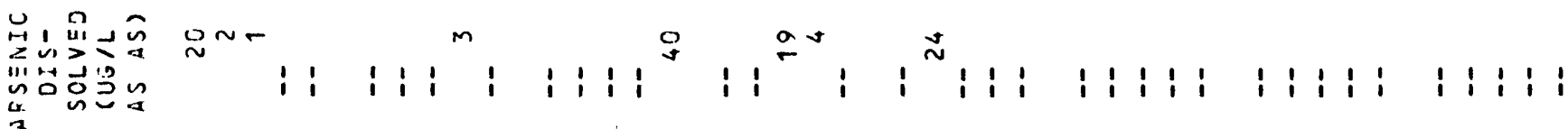

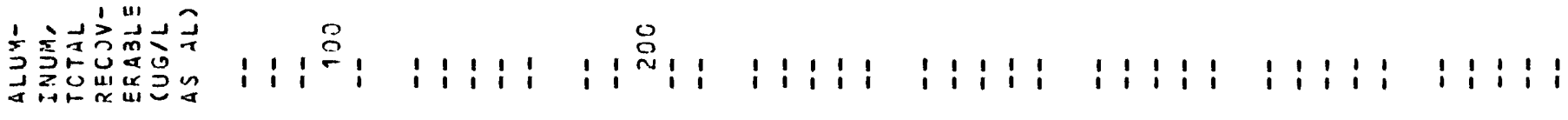

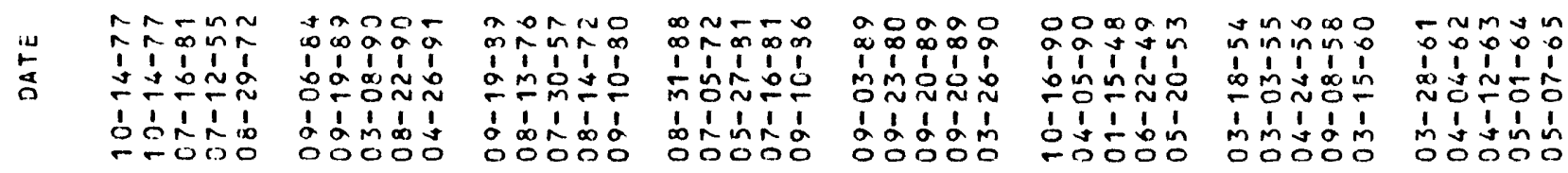




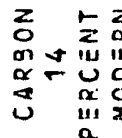

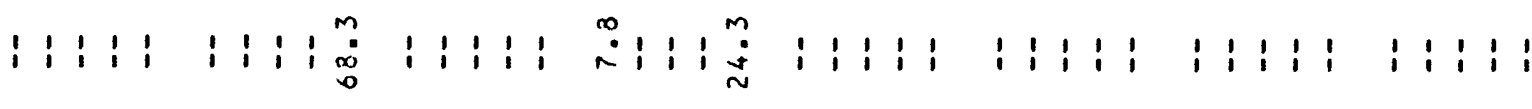

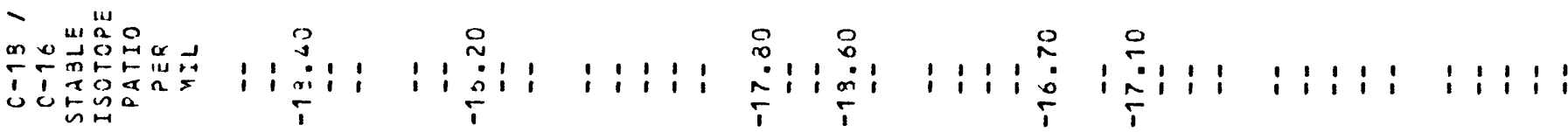

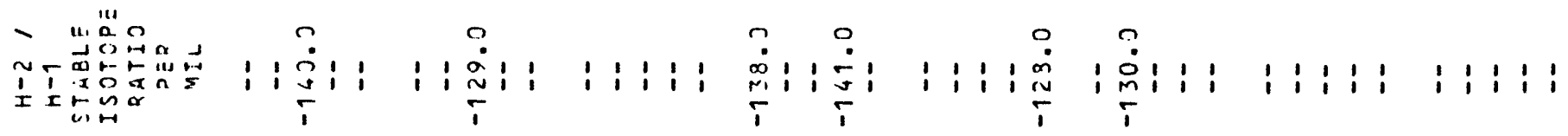

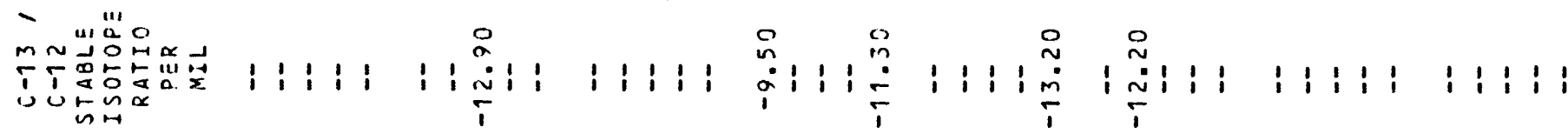

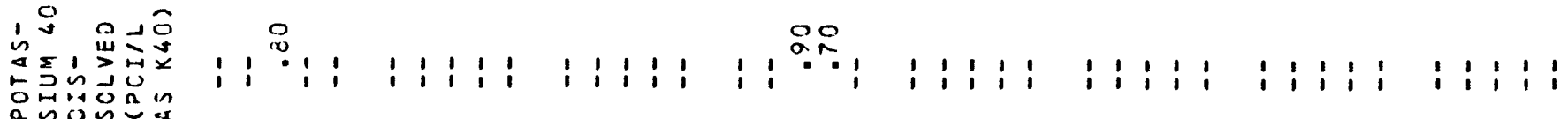

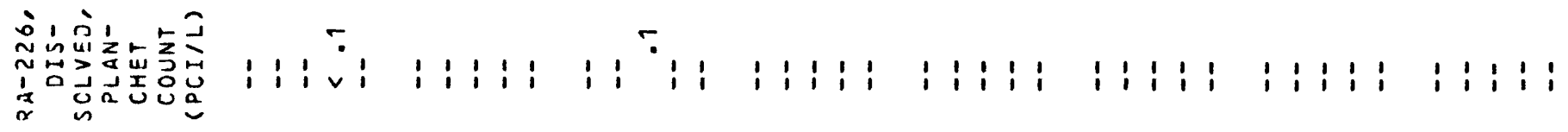

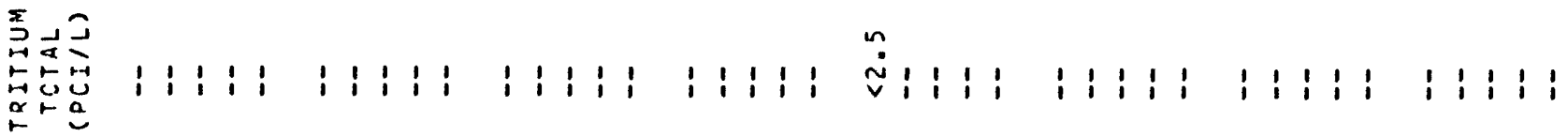

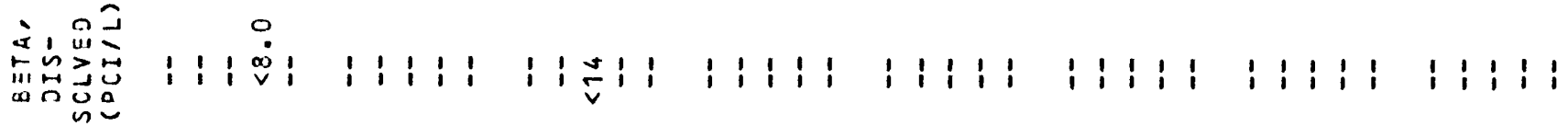

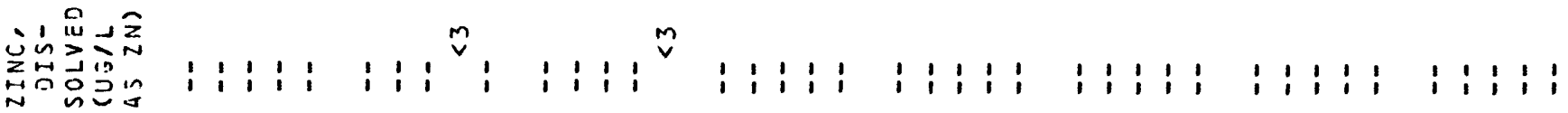

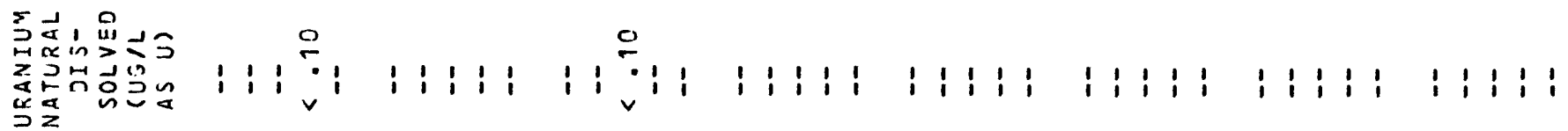

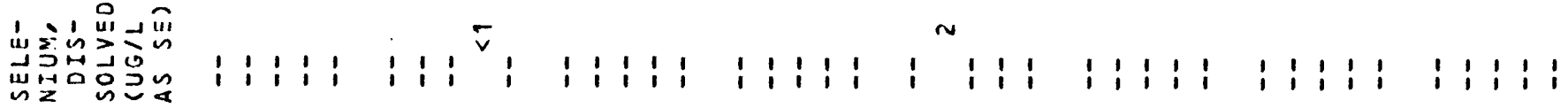

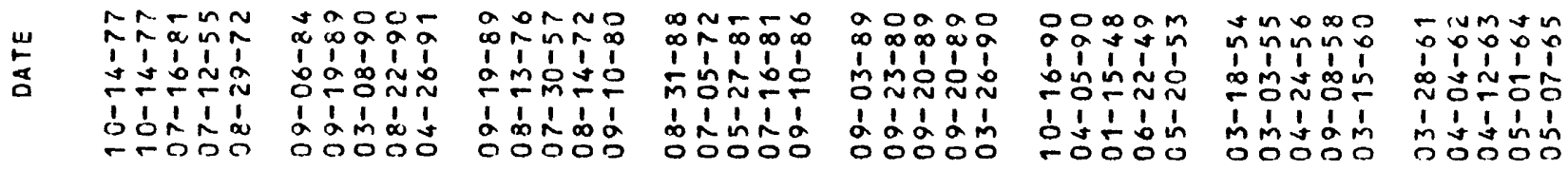




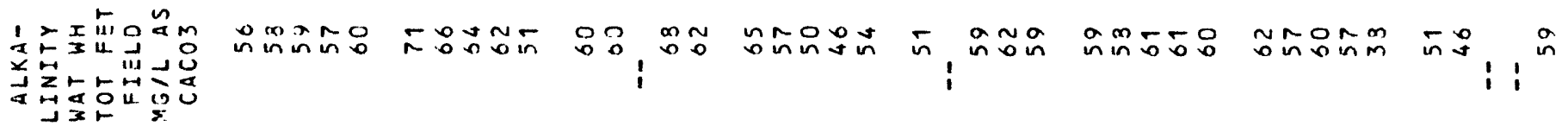

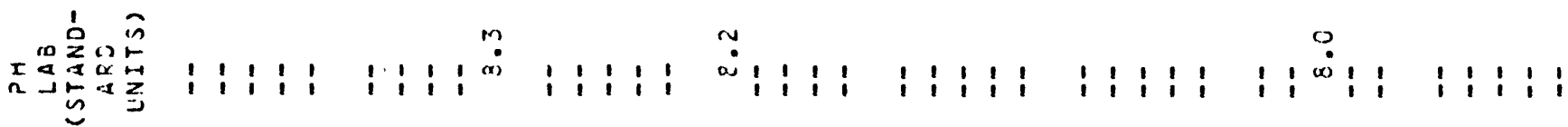

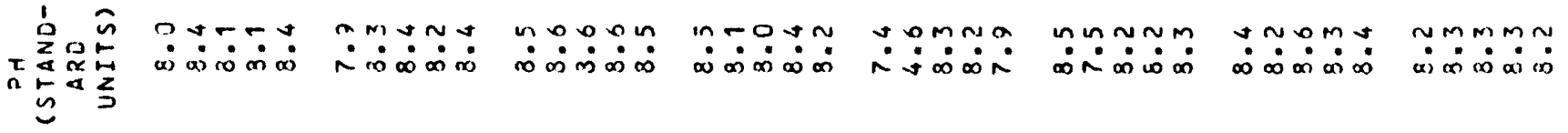

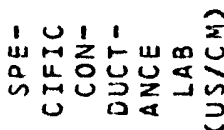

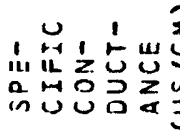

\& $u \propto \hat{1}$

u䊉

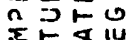

iu $x \leq 10$

i: $1: 1$

$\because 44^{\mathscr{a}}$

i i :

$\infty$

S:1:1: : in:

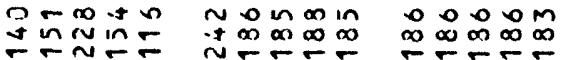
aning nor oarinm ntinna

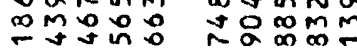

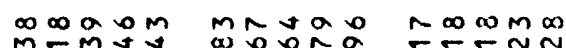
mEm $u 1$
$a$
0

ำ

$1,1: 0$

1

$1 \% 00 \%$

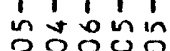

$\operatorname{lin} 2000$

is

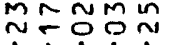

1 1 1

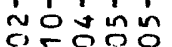

2000

i 1910 i

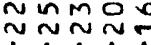

ㄲำ

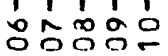

w

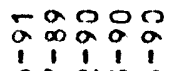

1ำ1 í

-

언ㅎํำ

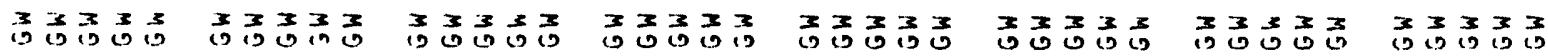

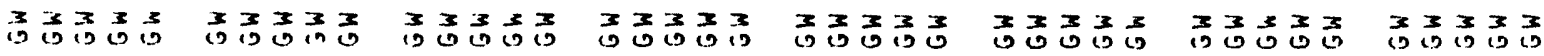

$\cos 200$

ias

ivo

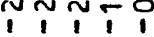

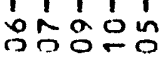

o

$1: 10: 10$

- 두워

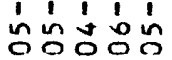

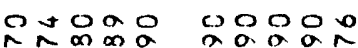

$\hat{1} 1 \hat{1} i \quad \hat{i} i \hat{i} \hat{i}$

N N-

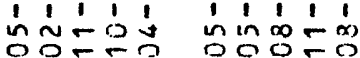

mâm

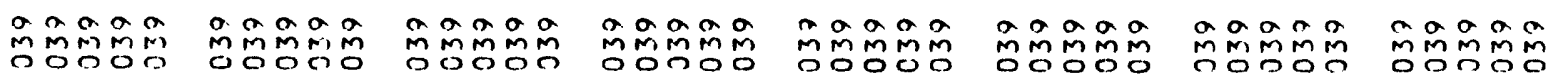

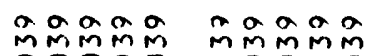

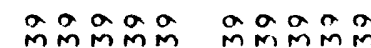

maoo

$\bar{y}$
0
$n$
$n$
$m$
$n$
$n$
$n$
0

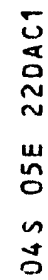




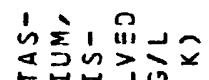
战出司要告

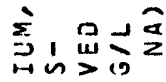

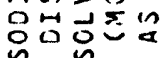

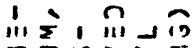
$\sum_{i \rightarrow 10} \sum_{10}$

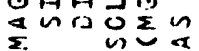

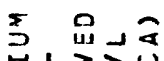

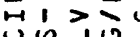

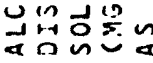

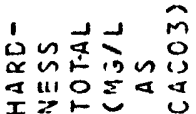

礼古的出

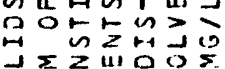

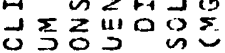

放。”

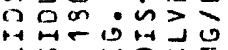

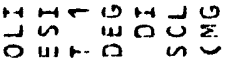
贾高

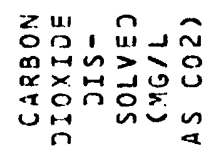

밤ำ

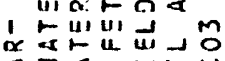

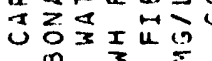

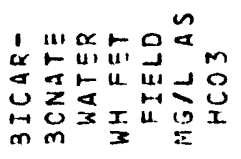

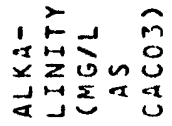

miñú

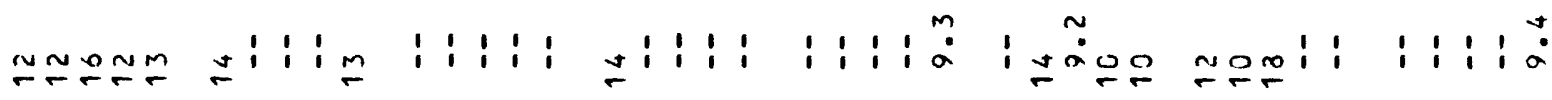

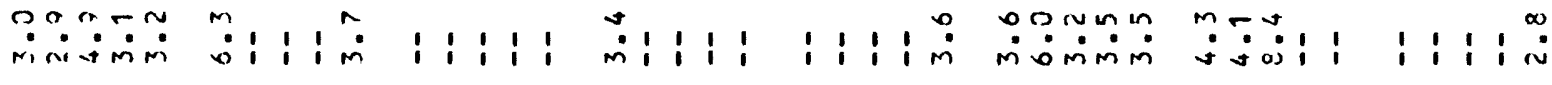

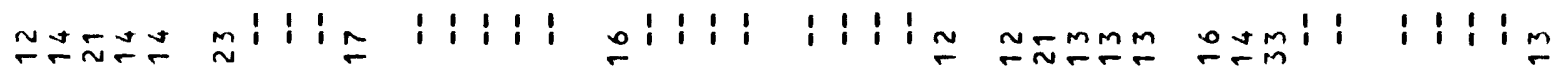

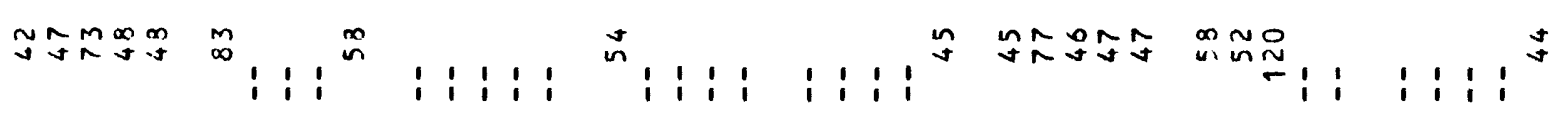

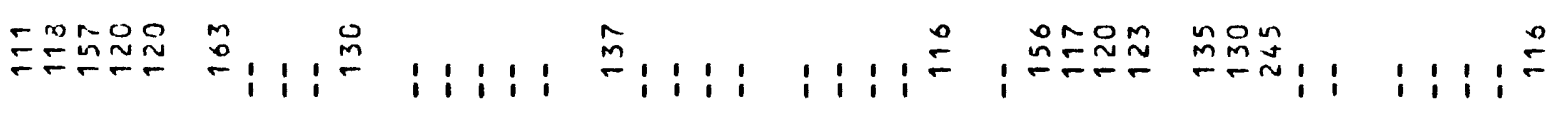

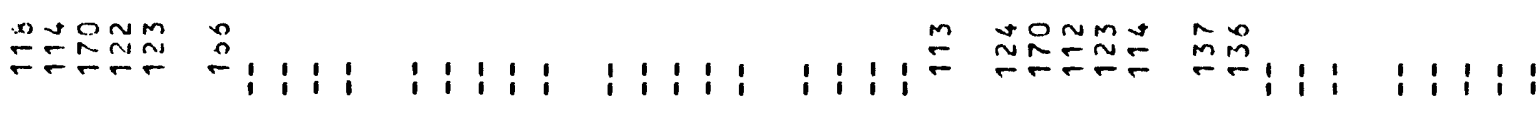

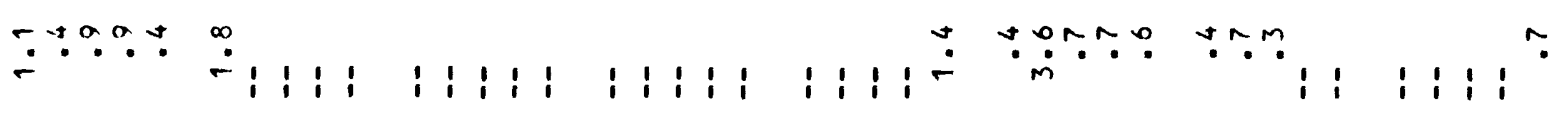

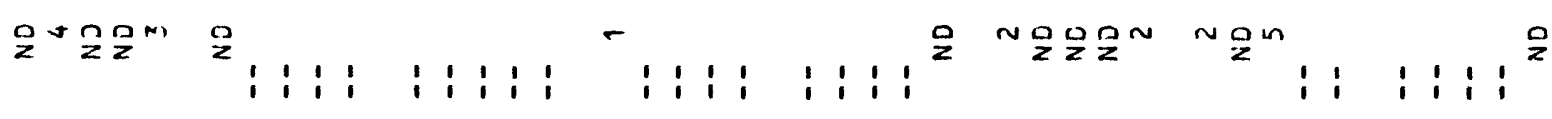
miñô

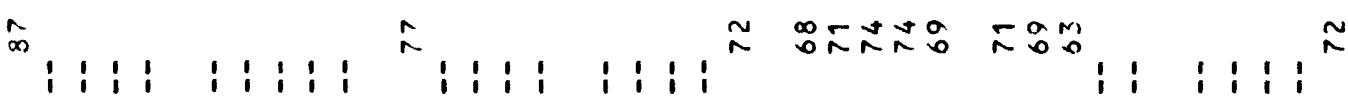

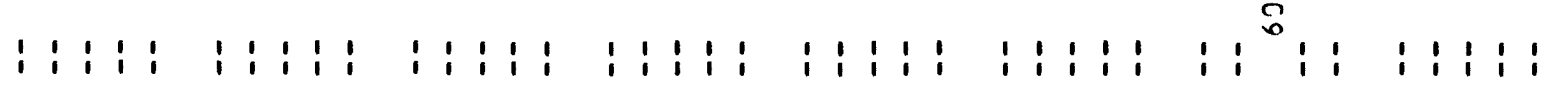

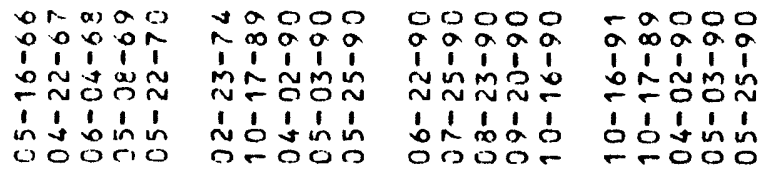

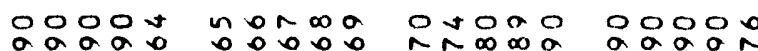

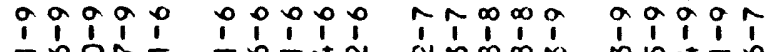

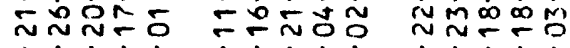

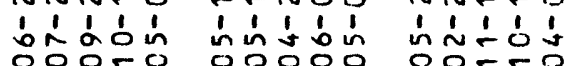
กำ in 


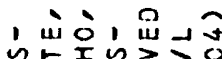

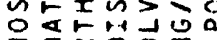
a $\begin{aligned} & x \\ & 0\end{aligned}$

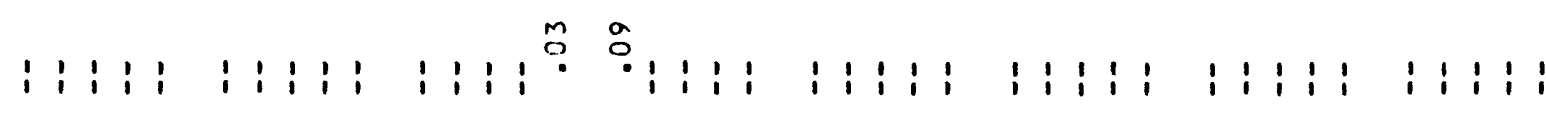

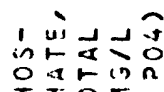
T.Tำ

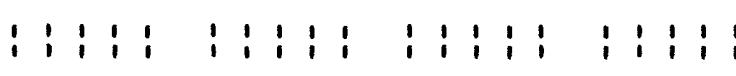

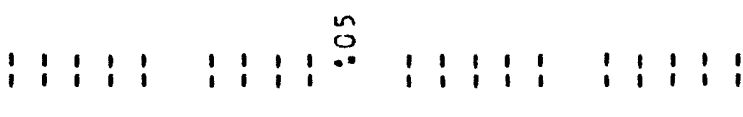

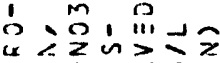

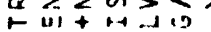
$z$ "

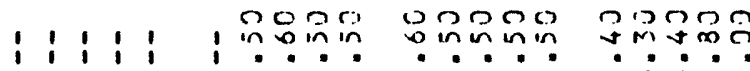

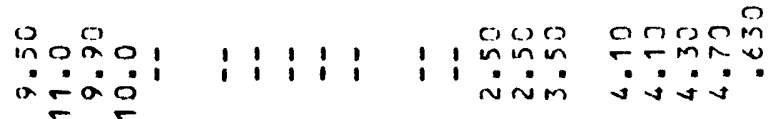
O)

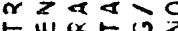

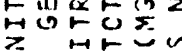
ñom:

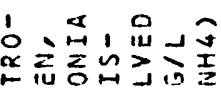

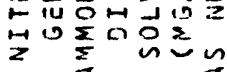

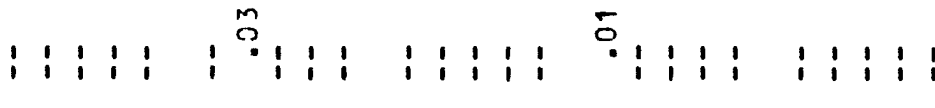

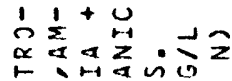

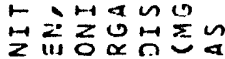

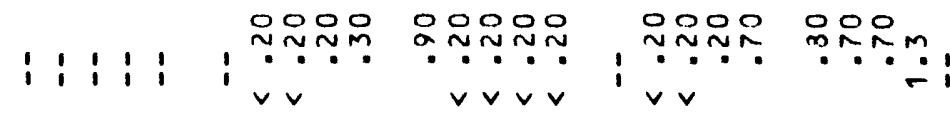

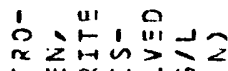

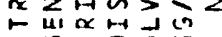

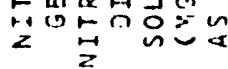

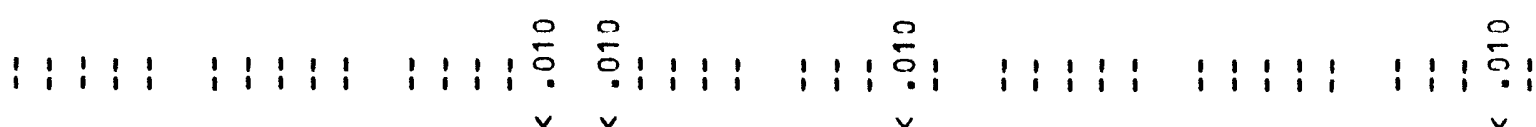

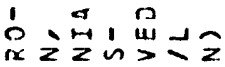
ix $z z \sim>2 z$

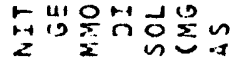

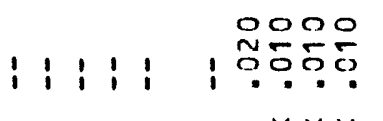
00000

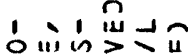

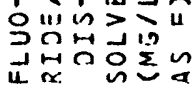

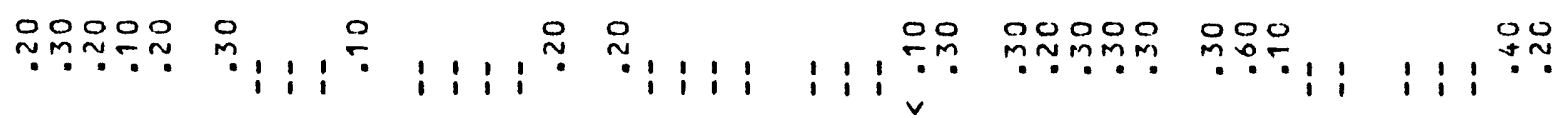
$\vee \vee v \vee v \vee v v$

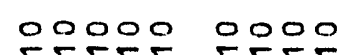

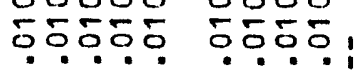
i i i i i i 190

0000 5555 !

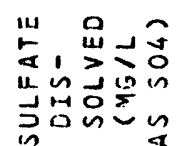

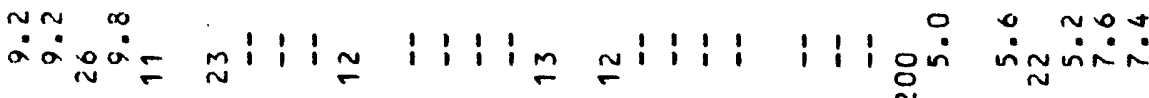
$\because \sim \operatorname{li}^{0} i$ i 1 i

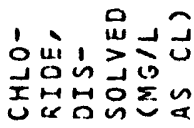

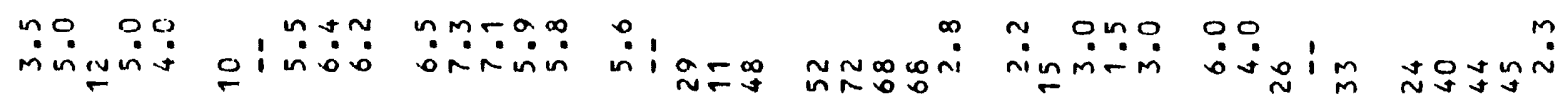

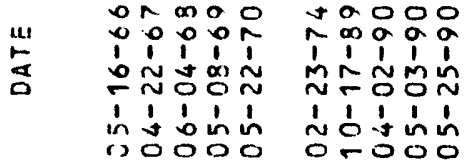

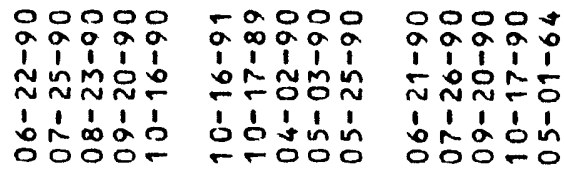

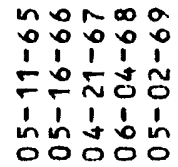

안ㅇㅇㅇㅇㅇ 20여유

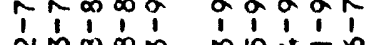
N

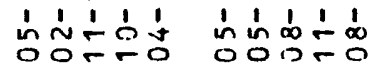




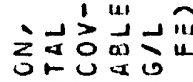

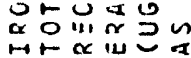

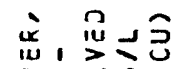

的

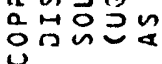

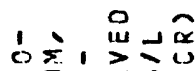

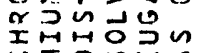

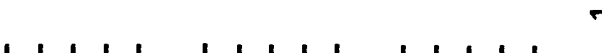

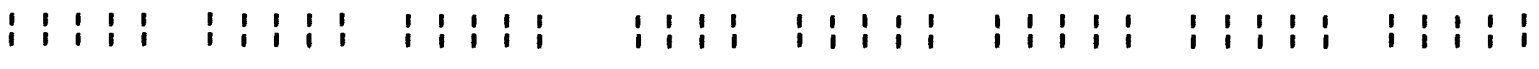

$\sum_{\rightarrow \rightarrow \infty}^{5}$

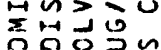

造踏

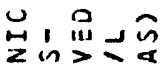

unj

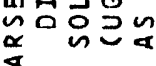

$\begin{array}{llllllllllllll}1 & 1 & 1 & 1 & 1 & 1 & 1 & 1 & 1 & 1\end{array}$

$r$

i i i

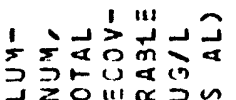

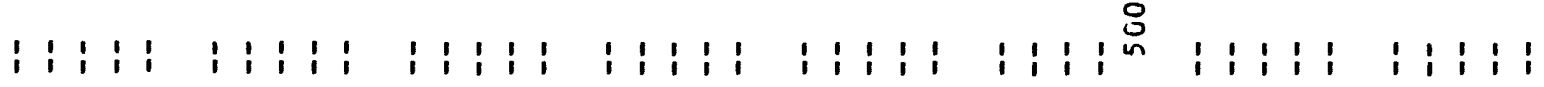

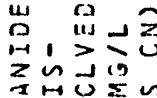

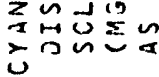

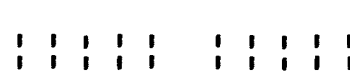

5

这,

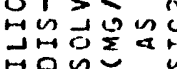

出

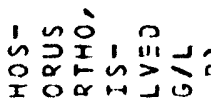

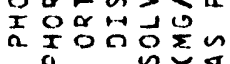

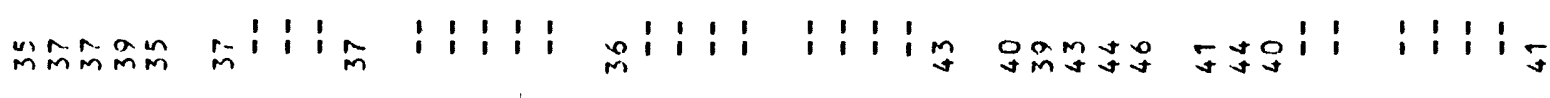

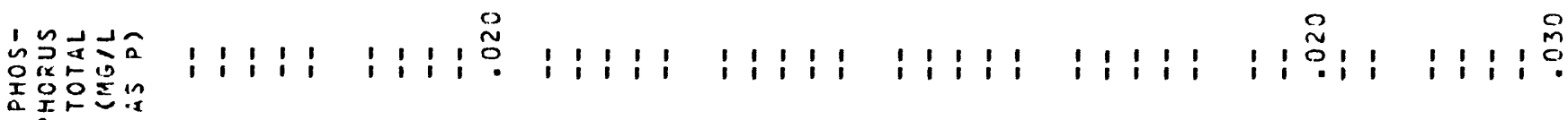

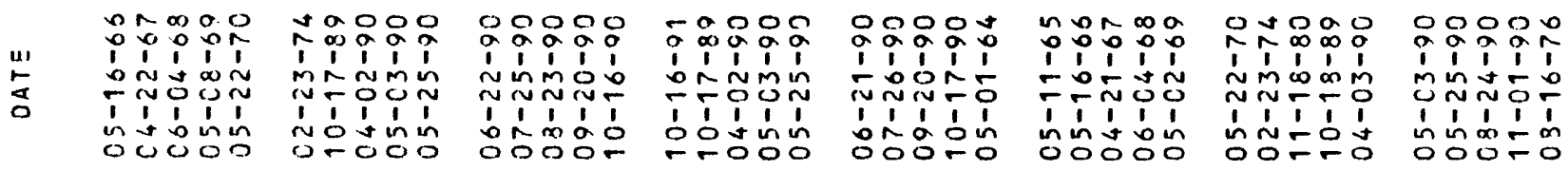




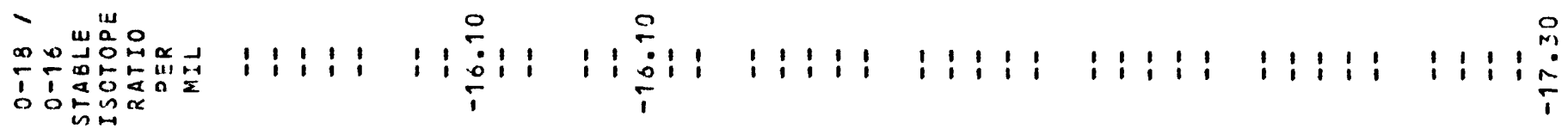

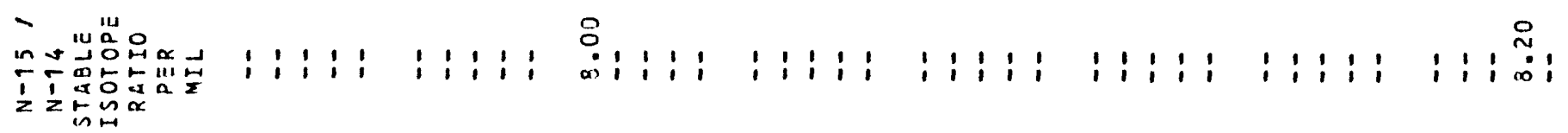

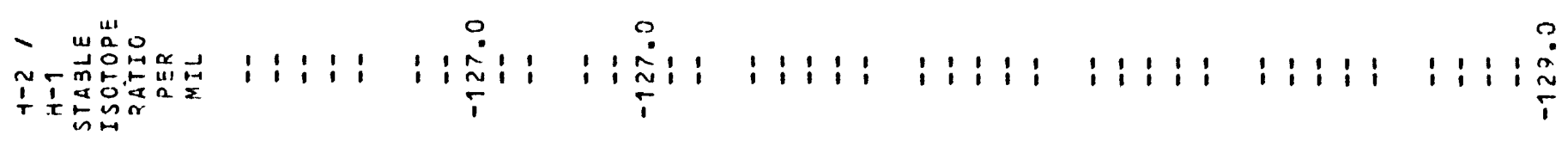

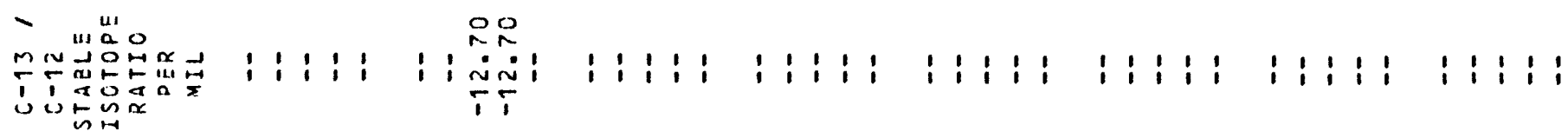

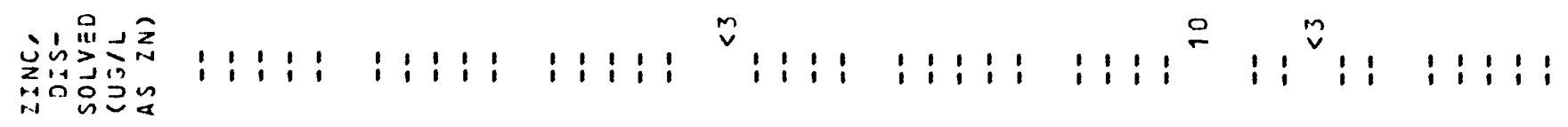

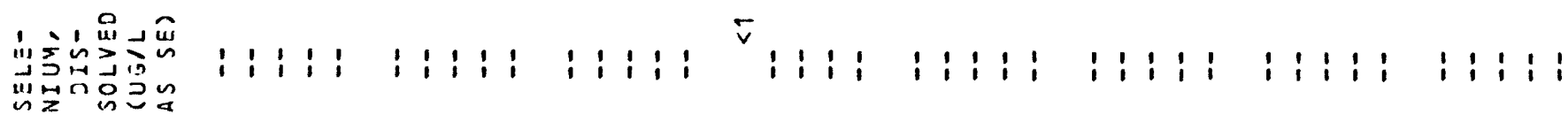

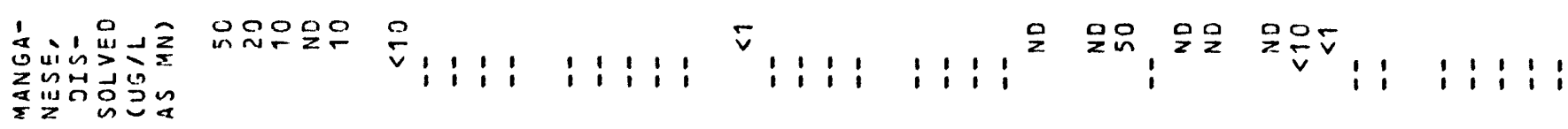

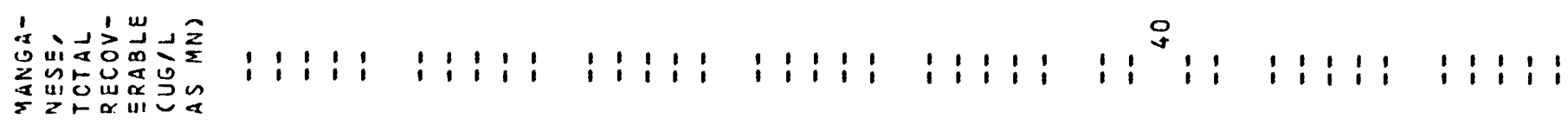

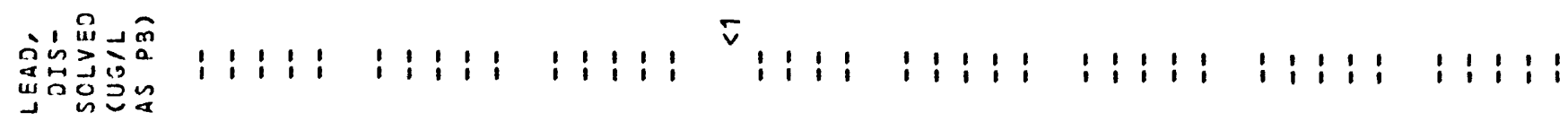

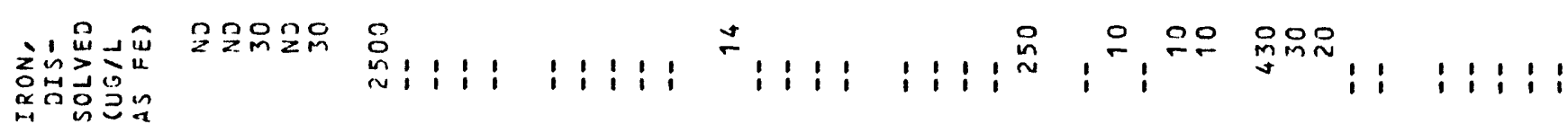

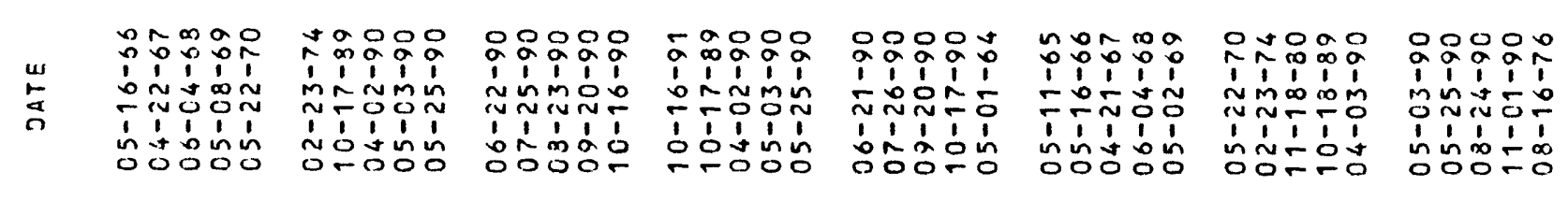




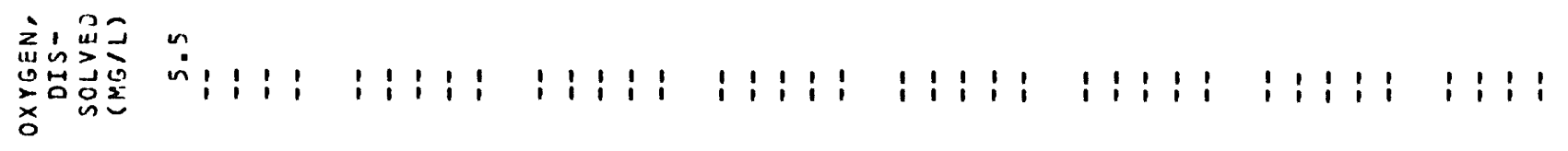

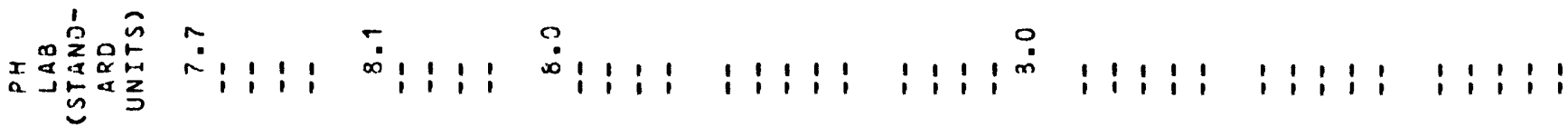

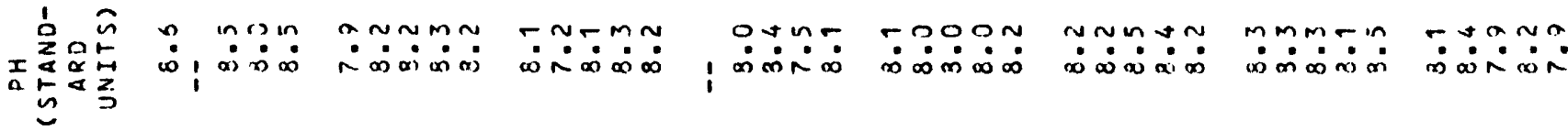

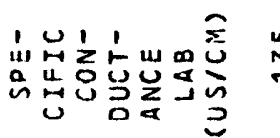

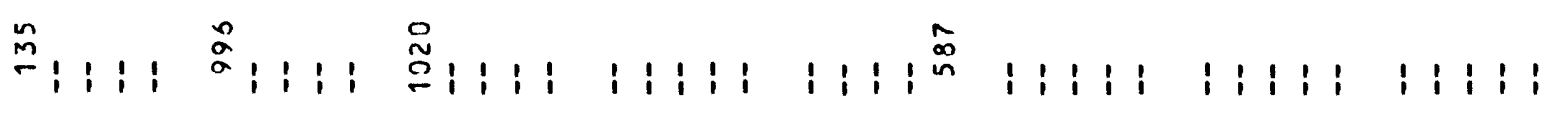

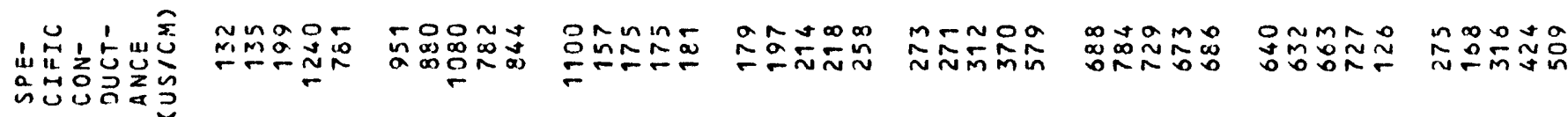
d w 㟧号些。

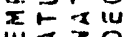

ounoo inini: wññ

0000 in ¿ெ0்:

- coo

minumin 0000

oninum

00000

minooo 世政0 nonnon azmad ajón ¿0ंáa

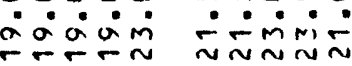

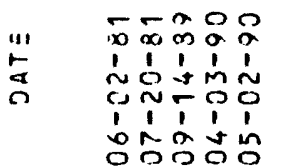

50502 5 motn

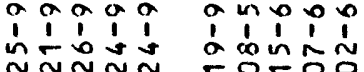

กNNN

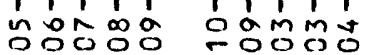

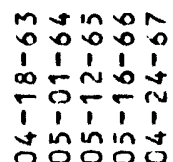

$\infty 0000$

DOAno mogon

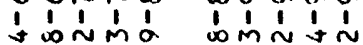

0Nㅜำ

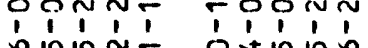
toning

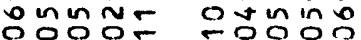

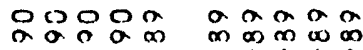
i. 1,1 को 1,11 bin. 1,11 - 1 1

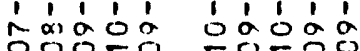

$\stackrel{\mu s}{\leftarrow}$

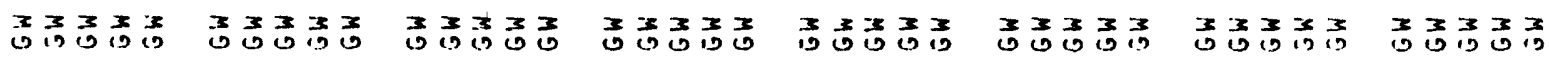

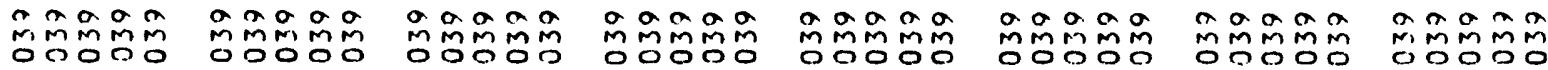

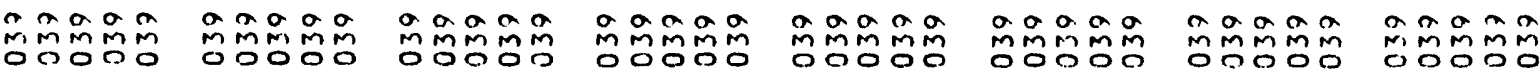

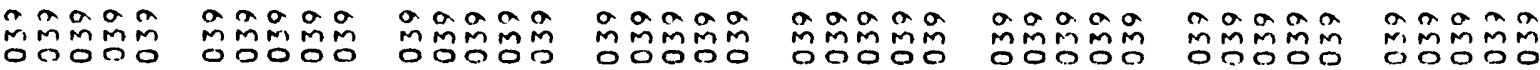

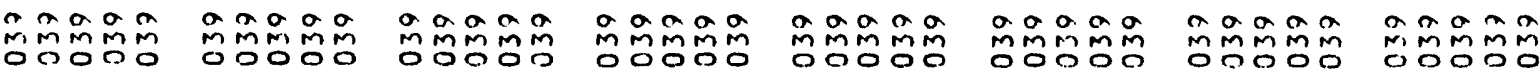

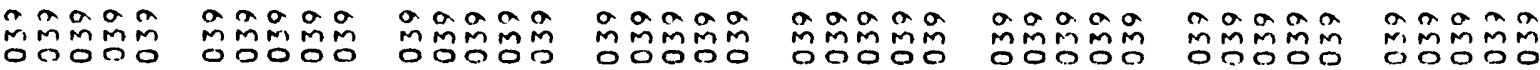

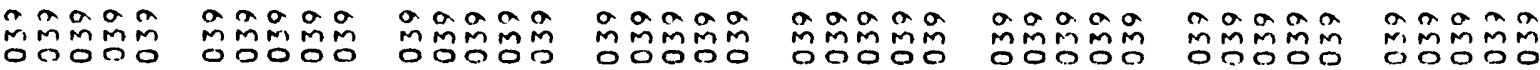

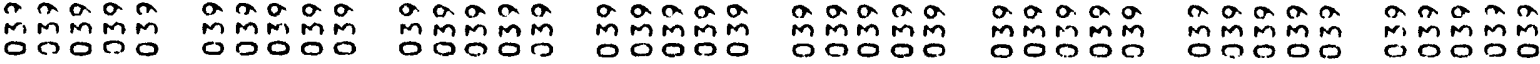

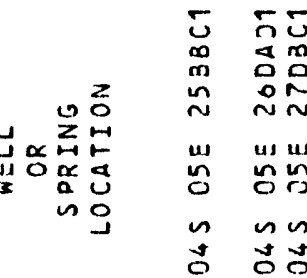

5
0
$u$
$m$
$m$
$w$
0
$n$
0

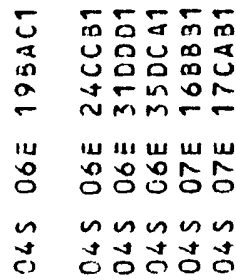




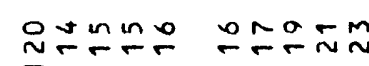

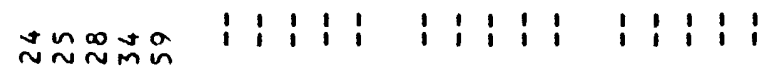

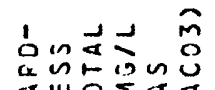

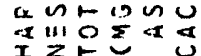
in $11: 1$ 品 $1: 1:$

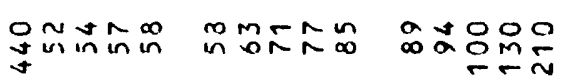
1:1:1: $1: 1: 1: 1: 1: 1$

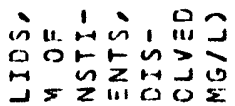

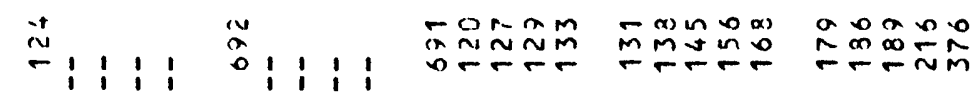

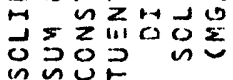

zu! , 足小芩

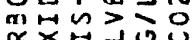

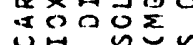

m

i: i i

$n m m$

o-mon

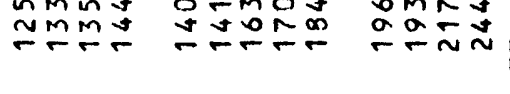

$1: 1: 1: 11: 1$

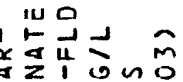

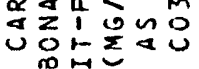

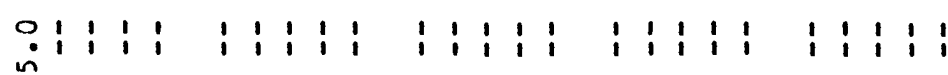

iั?o?

1:1:1 $1: 1: 1$ 1:1:1

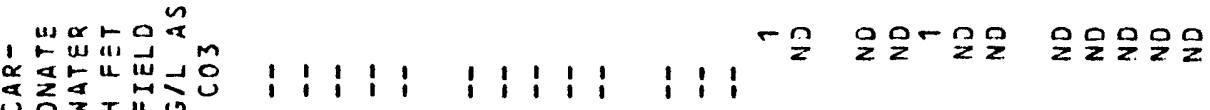

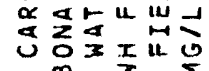

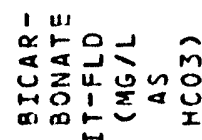

$n+1: i \quad i: i: 1$

i $1: 1:$

: $1: 1:$

i i i i

1

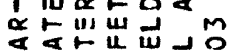

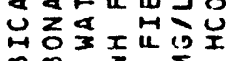

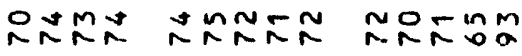

$: 1: 1:$ : $: 1: 1: 2$

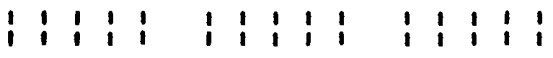

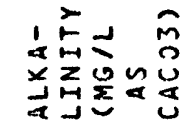

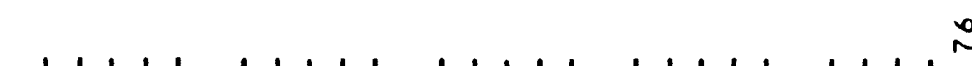

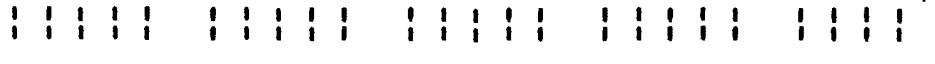

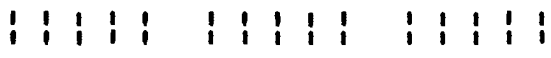

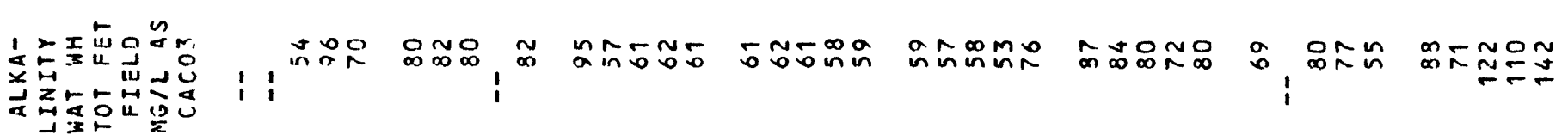
$\begin{array}{ll} & \\ 0 & \end{array}$

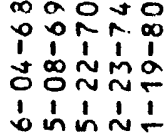

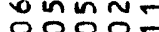

11:11 1:11: 11:11 1:1:1 1:1:1 1:11: 
1
0
0
2

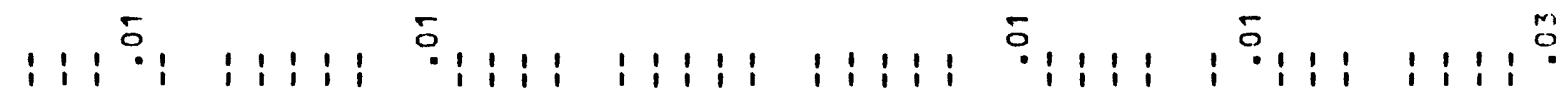

1 $\sum_{\alpha}^{1}+10$

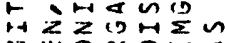

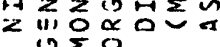

i i iุ?

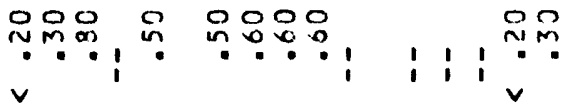

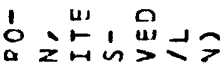

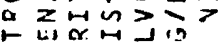

$z$ is on

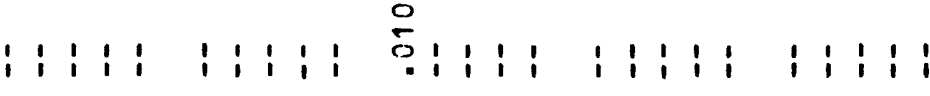

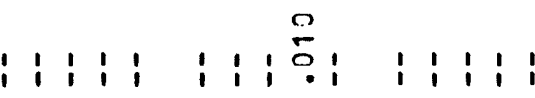

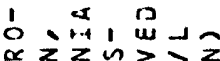

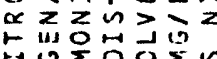

i 1 1 50.

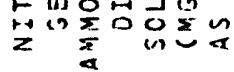

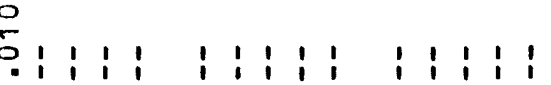

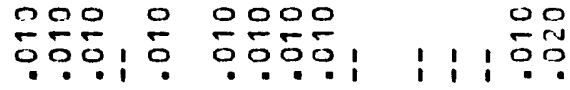

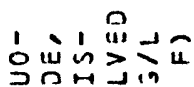

$\stackrel{0}{\because}$ i i :

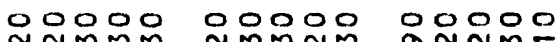

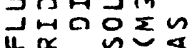

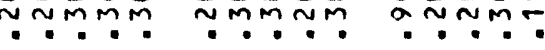

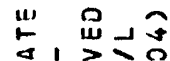

in 190

引ัต

miti: o: $1: 1: 1$

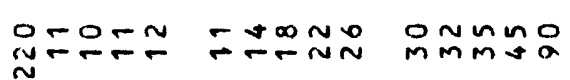

i:i:i i:ioi $1: 11:$

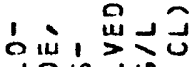

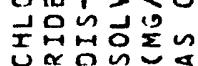

n! I

imomia nn

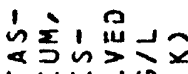

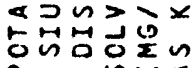

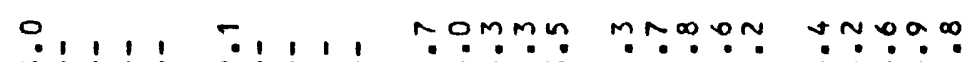

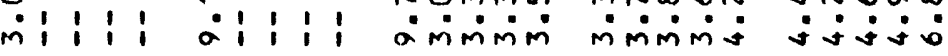

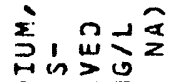
iㅔ

:i i i i mi i i i

$m$ mon

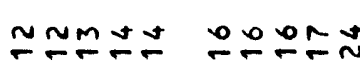

$1: 1: 1: 1: 1: \quad 1: 1: 1$

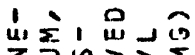
$\sum_{0 \rightarrow+1} \backsim \sum^{5}$

-i: i :

$\because 0$.

monom -00

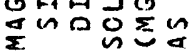

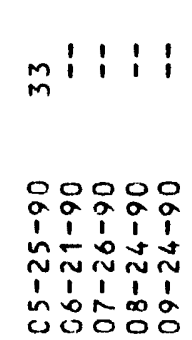

$\begin{array}{lllll}5 & 0 & 0 & 0 \\ 0 & 0 & \alpha & 0 \\ 1 & 1 & 1 & 1 & 1 \\ \sim & 0 & 1 & m & 0 \\ 0 & 1 & 0 & 0 \\ 1 & 1 & 1 & 1 & 1 \\ 0 & 1 & 1 & 1 & 1 \\ 0 & 0 & 0 & 0 & 0\end{array}$

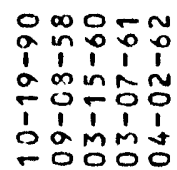

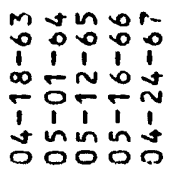

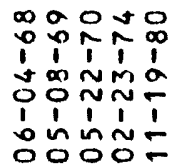

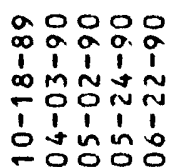

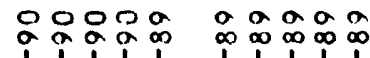
om-or n

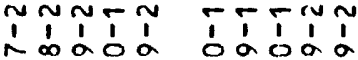
잉영 영영 


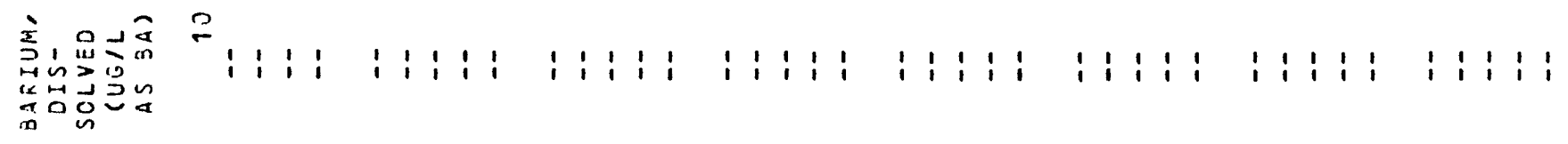

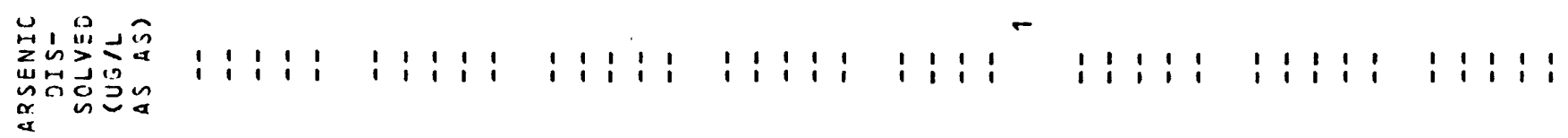

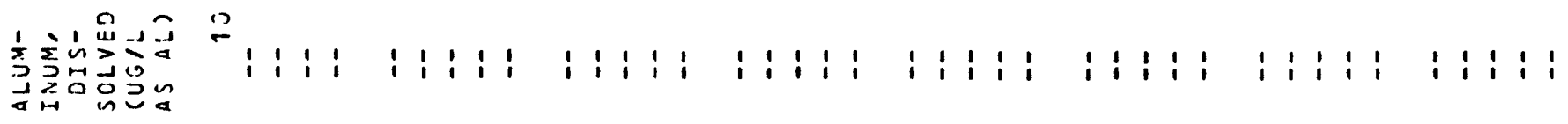

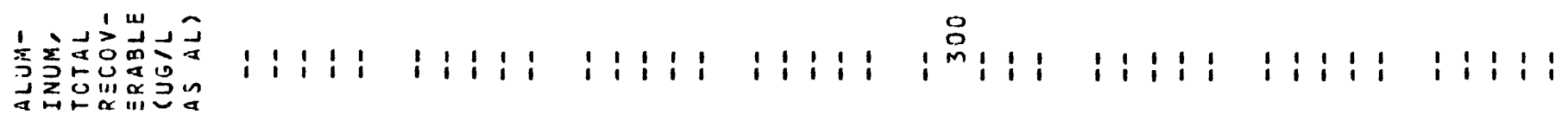

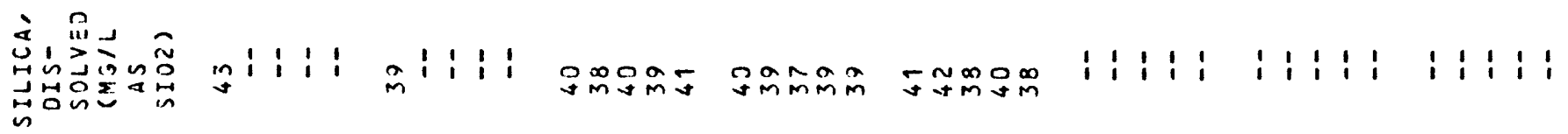

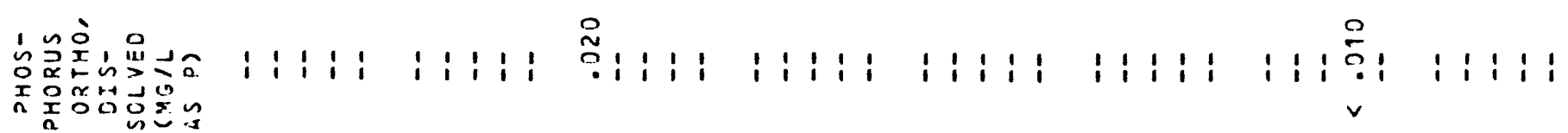

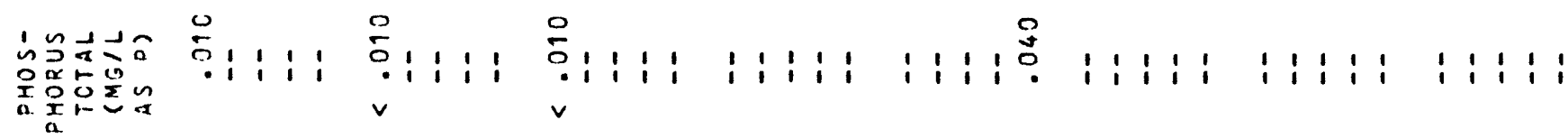

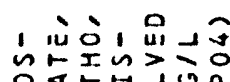

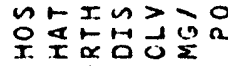
T.

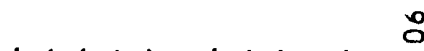

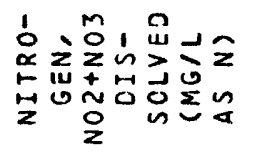

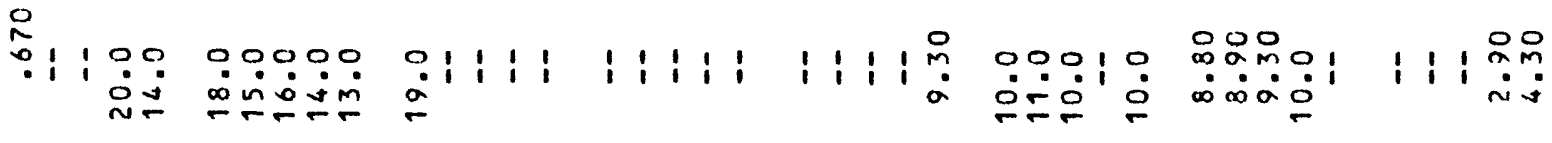

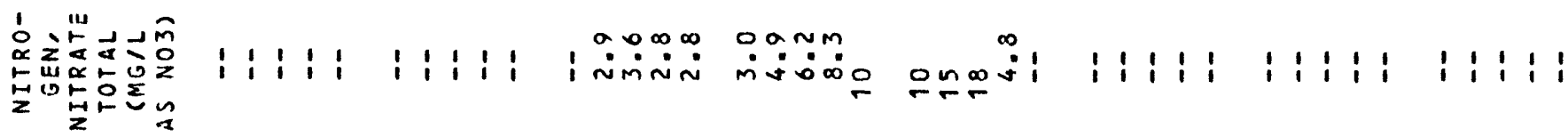

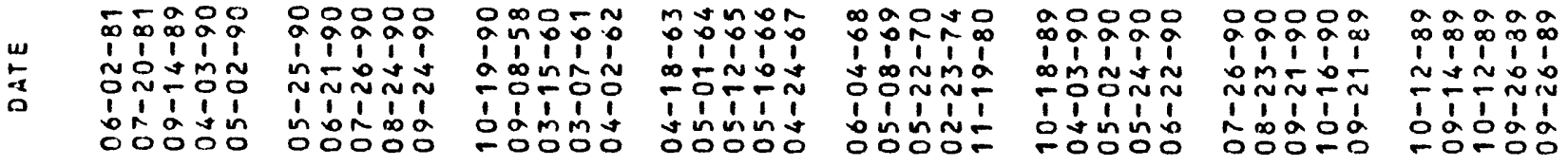




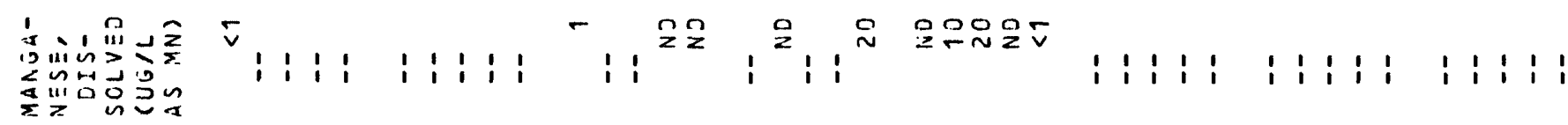

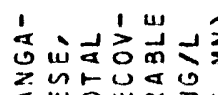

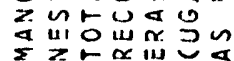
${ }^{2}:{ }^{\circ}:$ : : : : : : : : : : : : : : : : : : :

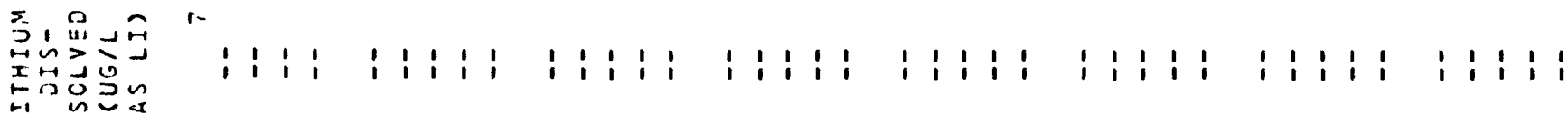

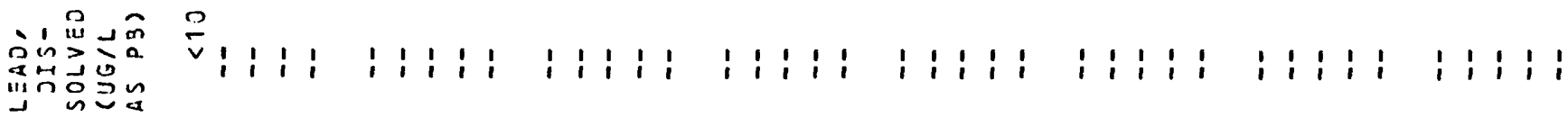

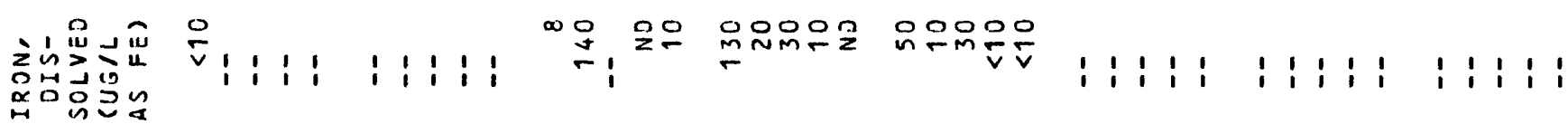

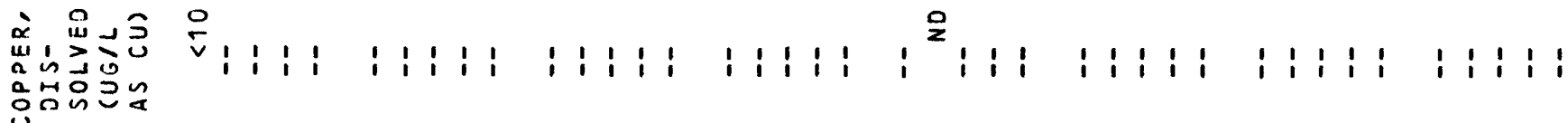

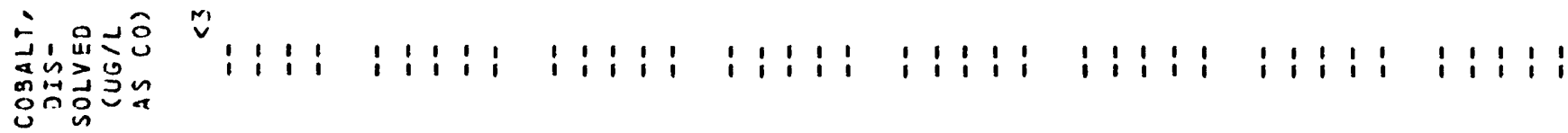

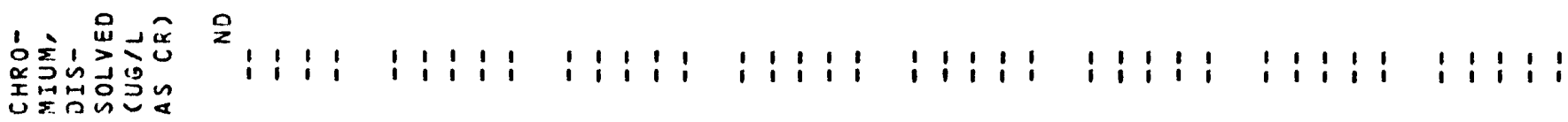

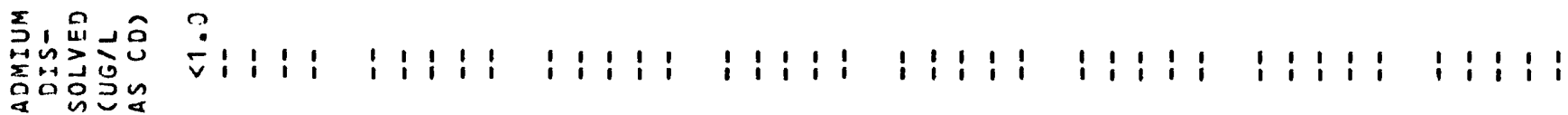

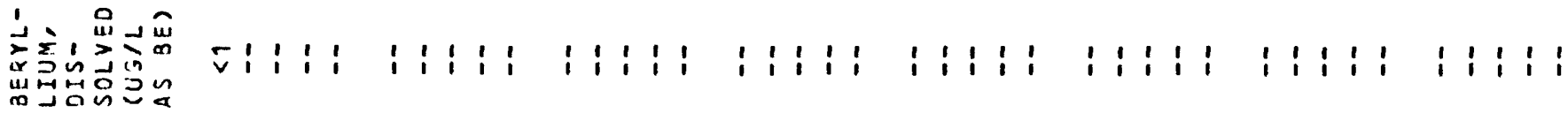

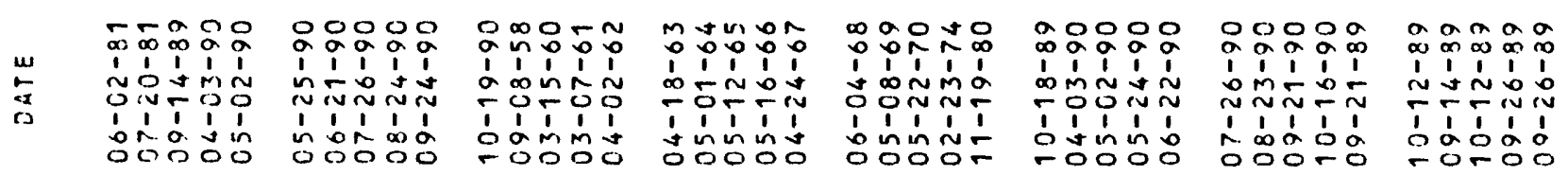




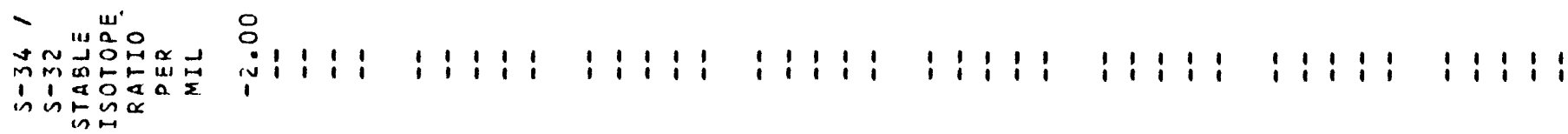

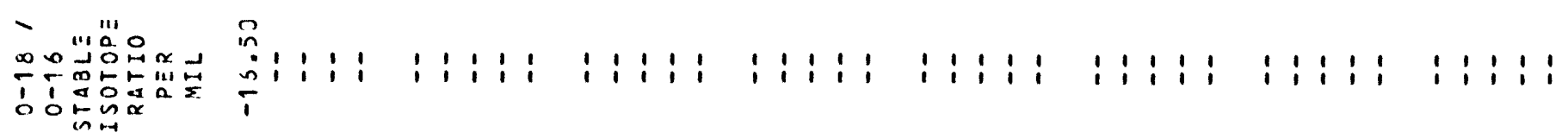

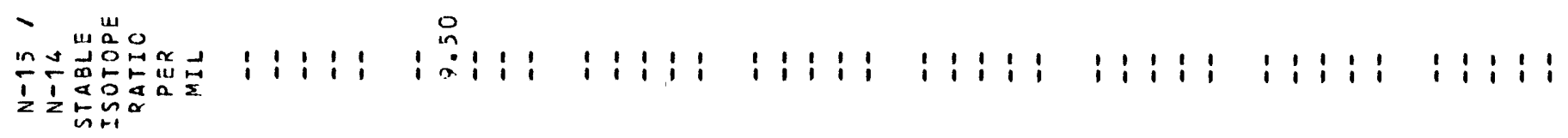

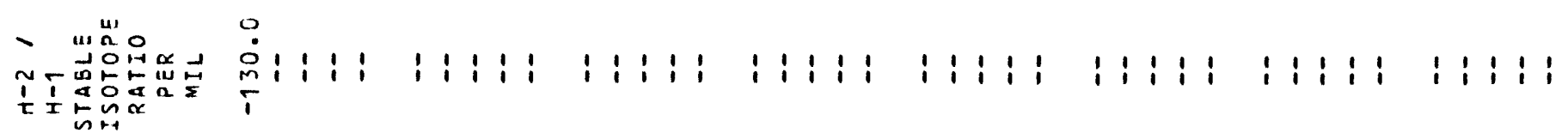

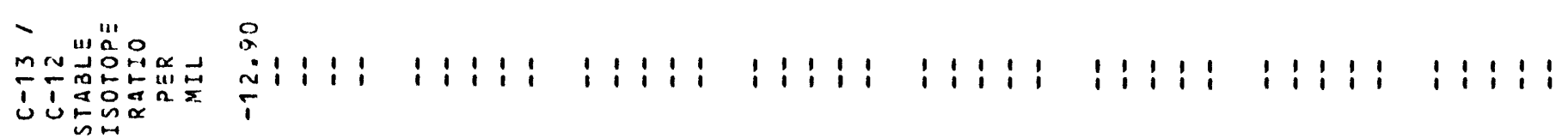

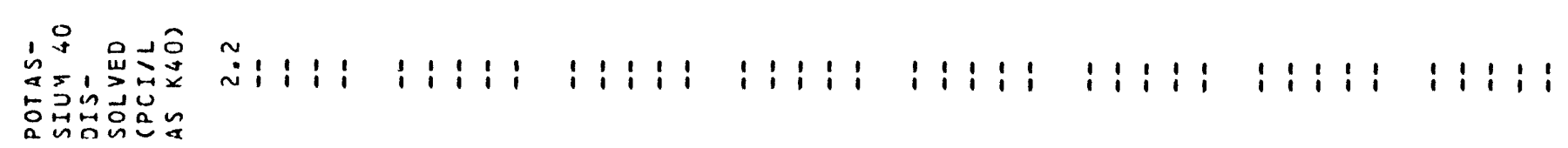

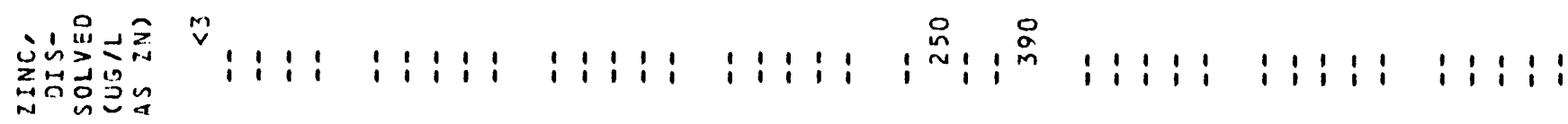

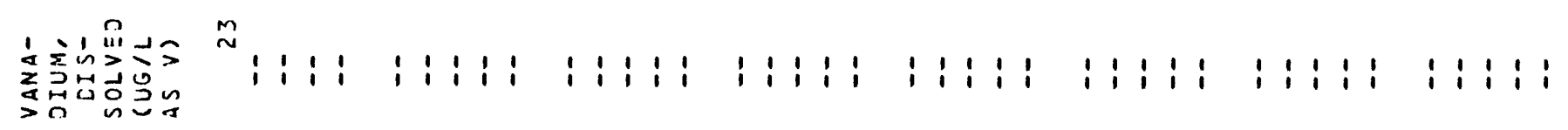

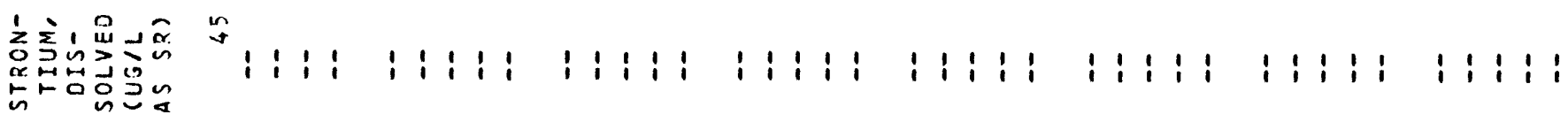

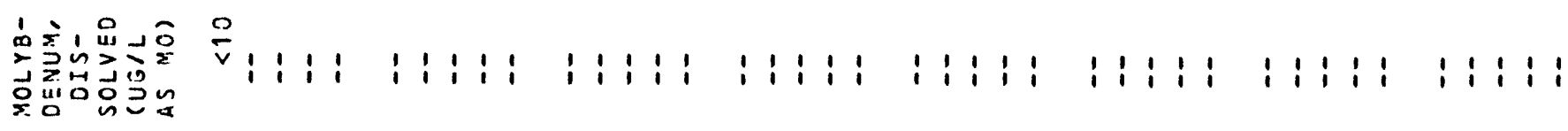

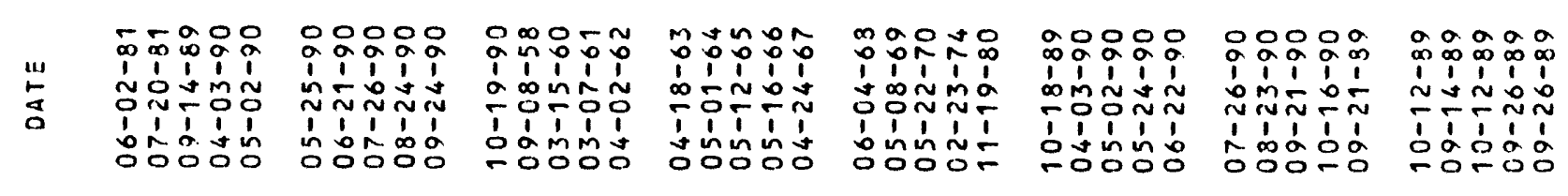




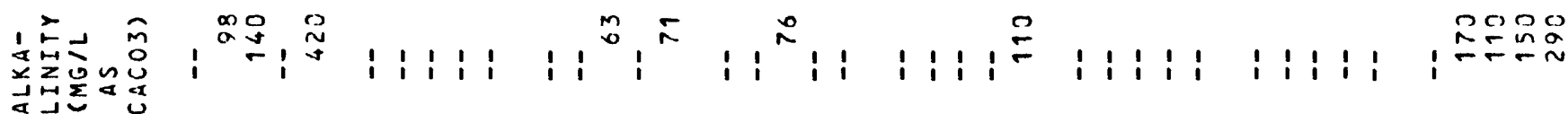

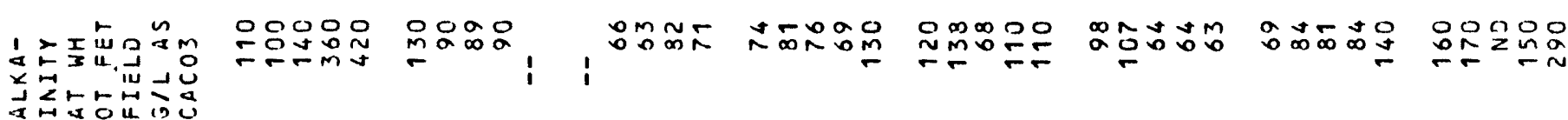
$\rightarrow=54$ is

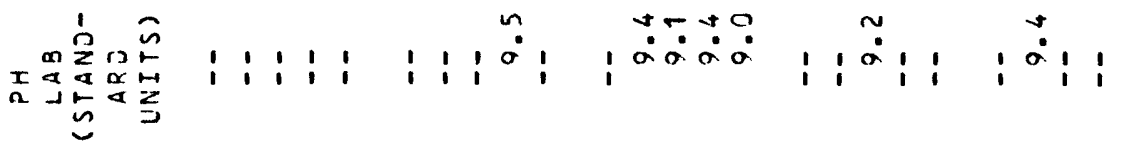

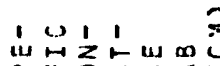
ล แ. $050 x$ in

1. 1,2 us

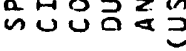

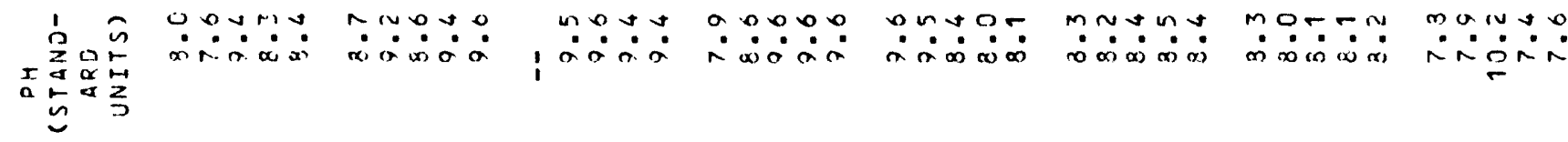

1" w

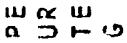
$\sum_{i=a} \rightarrow 0$ III $<\geq 0$

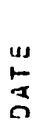

- 000

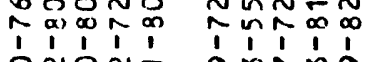

onON= 2 Namo

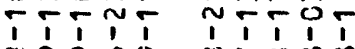

$\begin{array}{lll}10 & 0 & 0 \\ 0 & 0 & 0\end{array}$

$\begin{array}{lll}\infty & \infty & \infty \\ 0 & \infty & 0\end{array}$ $\infty \pi-\infty \pi$

$\begin{array}{lllll}1 & 1 & 1 & 1 & 1\end{array}$

กัก๊ก

11 i i i i

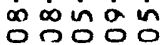

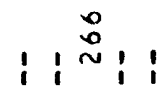

in
$1: 1: 1$

$1: 151: 1: 11:$

in

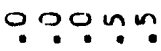

in iñ

nno nn

o. o. 0

o0ini imi-ini

nnm no

ำㅇำ

$n \pi \sin ^{\infty}$ îin $m \sim \infty \sim ⿻-1$ TTTT oxoñon \begin{tabular}{llll}
$M$ & 0 & 0 & 0 \\
\hdashline & $\infty$ & 0 & $\infty$ \\
1 & 1 & 1 & 1
\end{tabular} $\stackrel{m}{\sim}=-\pi$

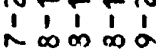

$0-a n \infty$ minnin $\frac{\pi}{2} \frac{1}{2}$

$\ln 250 m$ in $\operatorname{tin} m a$

$\underset{\sim}{u}$

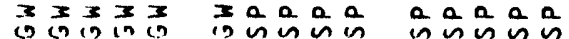

a a a a

$3 \geq 3 \geq 3$

0
0
0

1111

NN$\tilde{N} \tilde{N}$

11111

mogmo

क के०ू?

1111

춘ำ

ád

agmon

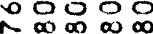

$1,1,1$ ONONR i $15 \div 5$

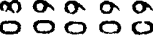

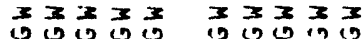

$\frac{3}{10} 373$

a a a

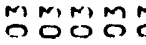

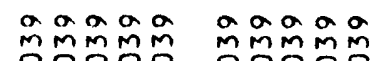

anoa

monmó

mago

araa

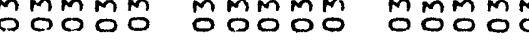

ํㅗำima

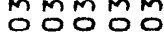

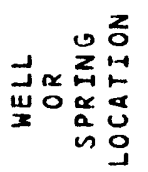

\begin{tabular}{|c|}
\hline 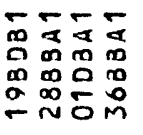 \\
\hline 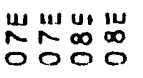 \\
\hline 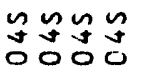 \\
\hline
\end{tabular}

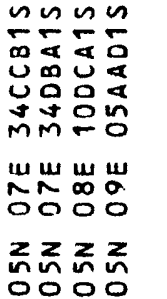

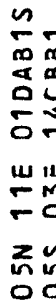

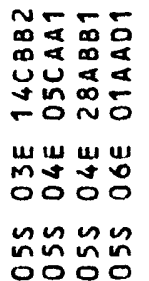

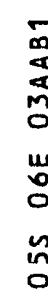

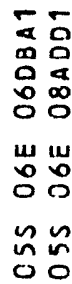

它

in

in

ณ

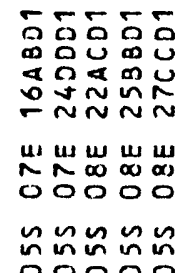


"11.

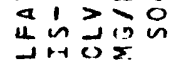

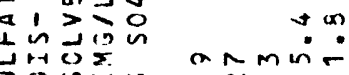

贫的果

bii, 娄

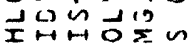

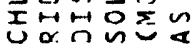

统, 品

น

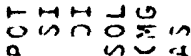

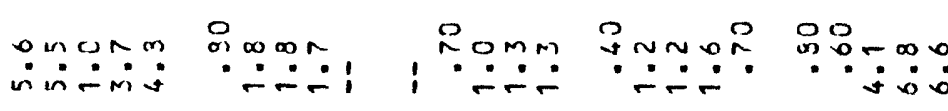

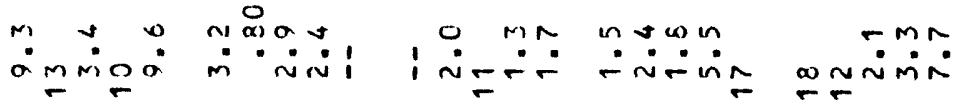

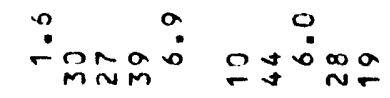

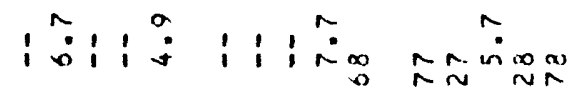
$\therefore \quad n=\frac{\pi}{2}$

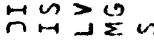
Oก 0 舟

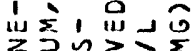
$\sum_{i \rightarrow n} \backsim>0$

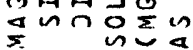

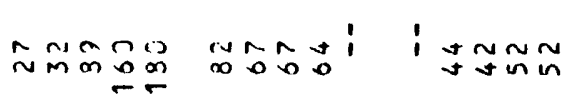

ำกำ

i i i i i i a munge

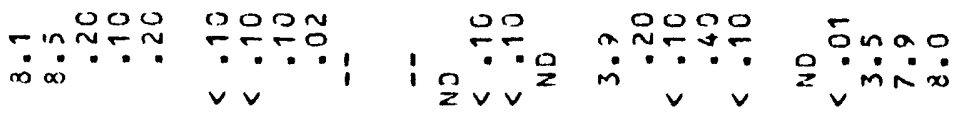

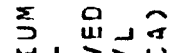
1'

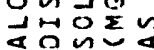

man 2 a.

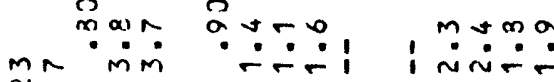

ñ

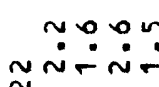

$\because ?$ nntnR nim=0

anmognams

Oñ

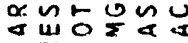

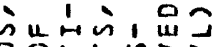

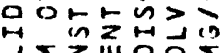

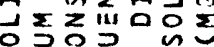

N-no- OON

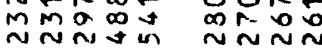

$\sim \sim ⿻ 上$

웅ㄷㄴ

o in in

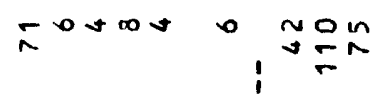

i i i

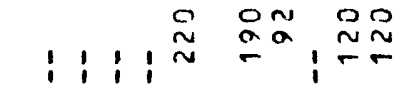

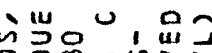

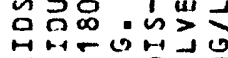

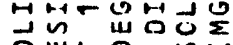

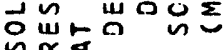

zัu, 品山

m

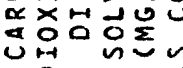

i i i i i $\stackrel{n}{n} i$ i i i i

i : i i

i i i :

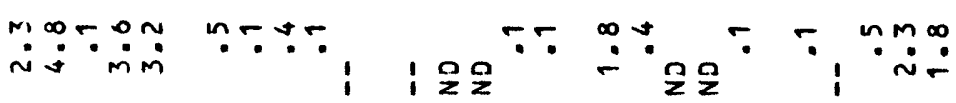

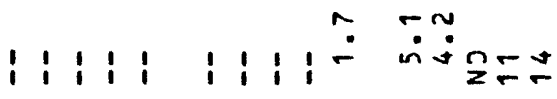

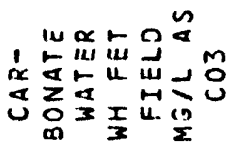

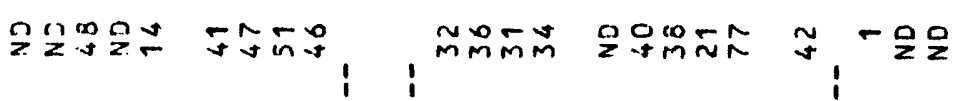

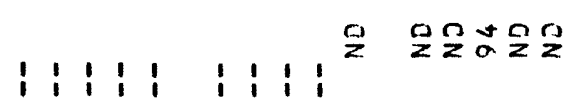

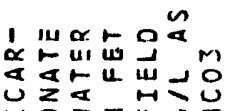

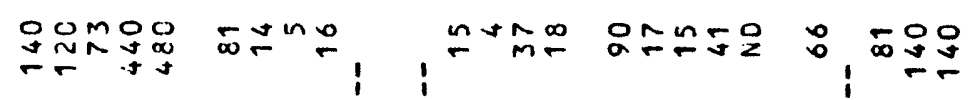

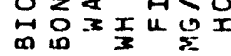

엉요 $\cong$ ก

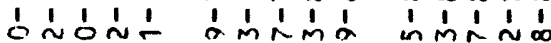
TT TT TTFOT NONON

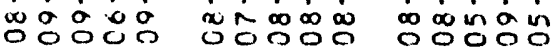

经更经 $\underset{\sim}{m \infty}$

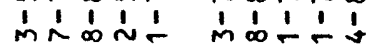
Titi กั0 i

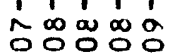

영영영영

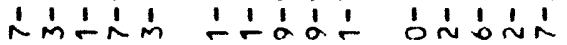

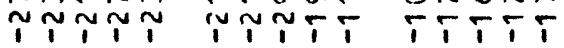

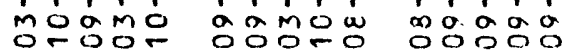




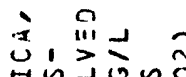

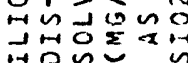
is

'纪主, 品上 or

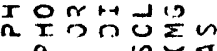

ถรุมีล

जिए?

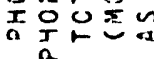

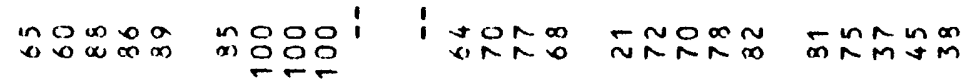

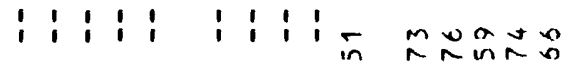

in

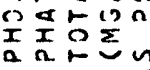

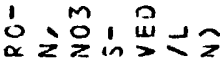

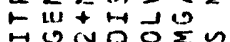

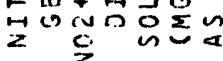

으음웅

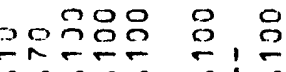

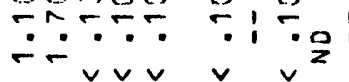

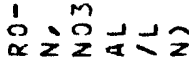

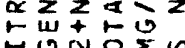

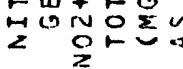

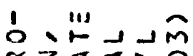

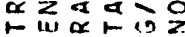

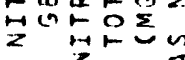

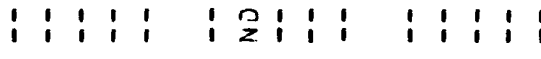

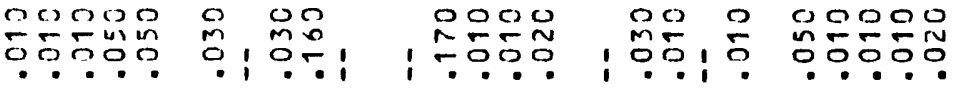

$\checkmark$

i: i i i :

1:10: $1: 11: 1$

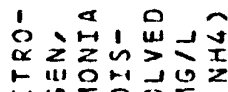

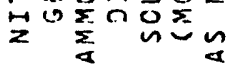

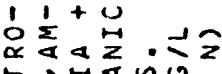

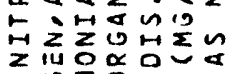

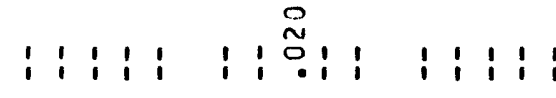

ํํำ

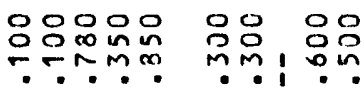

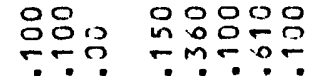

$\checkmark v$

i

$\checkmark$

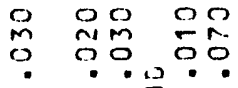
प्र

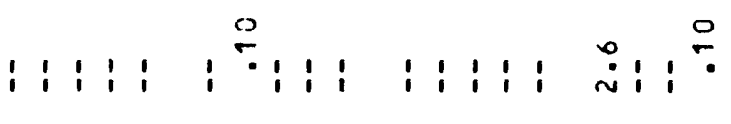

i i i i

: 1: $1:$

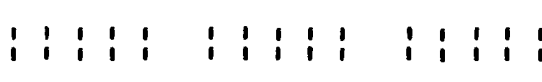

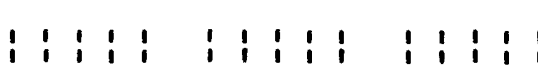

1919191919

융 융

iviv

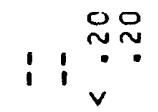

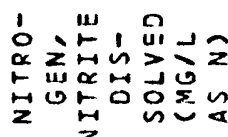

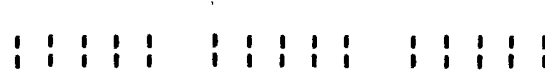

11111 11:11 1:

응윰, 웅웅

$\vee v \vee v$

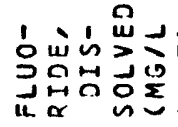

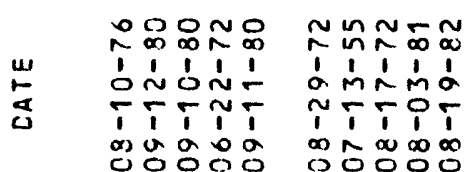

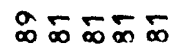

向向向向1

NONON

owing

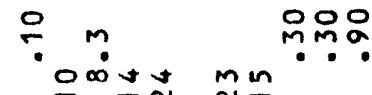
운ㄷㄴ

$\sim r$

ถกำ-

1

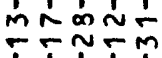

Donn

mind
YTF

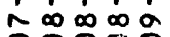

$1 \stackrel{\circ}{\div} 1^{\circ}: 111^{\circ} \stackrel{\circ}{?}$

영옹영음

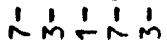
N NNN 뭉으의
옹영요 웅요 1́ád ćádín NNTTT TTTT

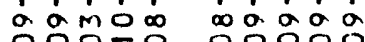

$\because 0 \div 80$ $\therefore \dot{\sim} \cdot$ 


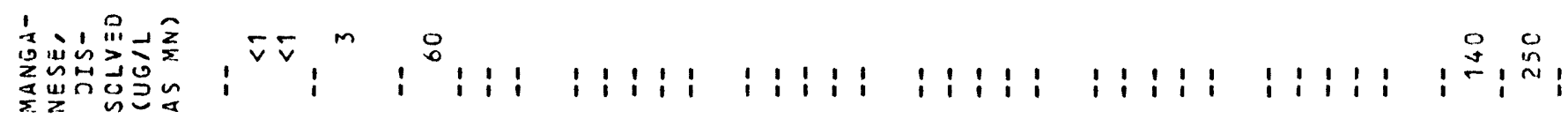
1
3 u

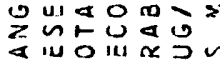

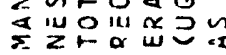

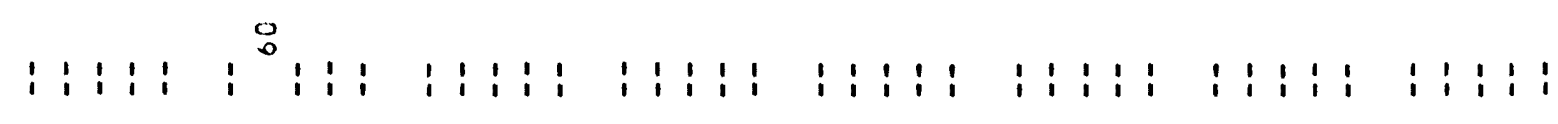

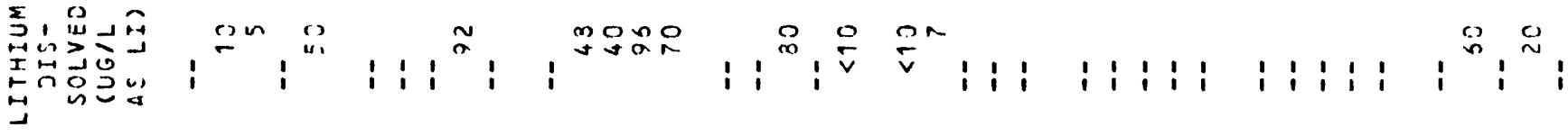

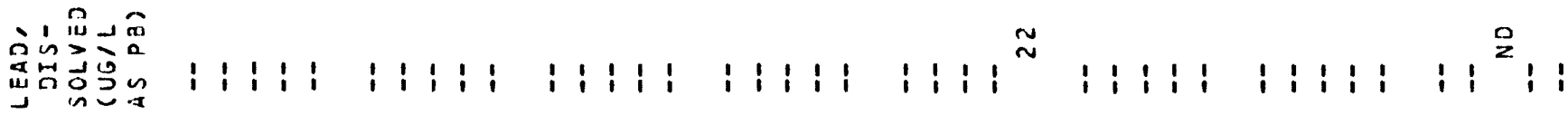

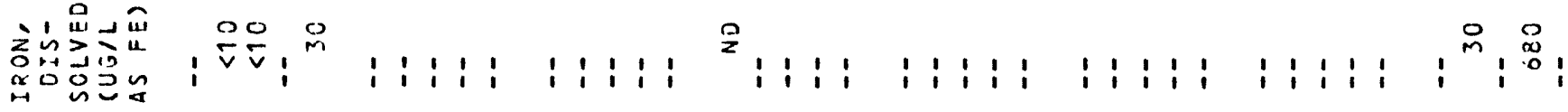

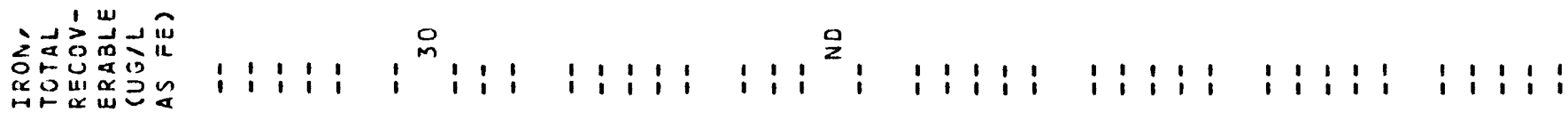

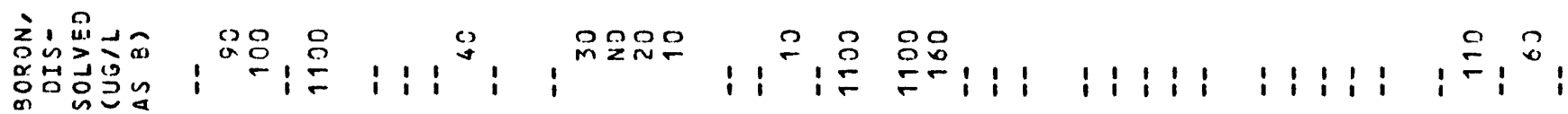

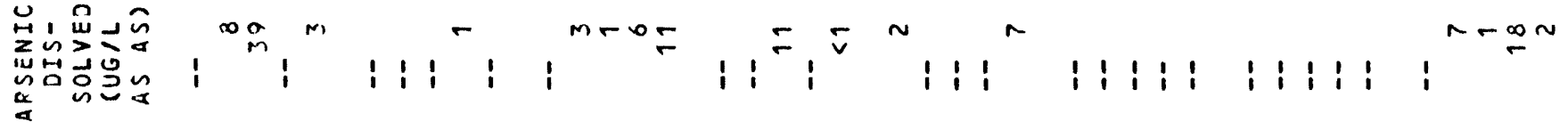

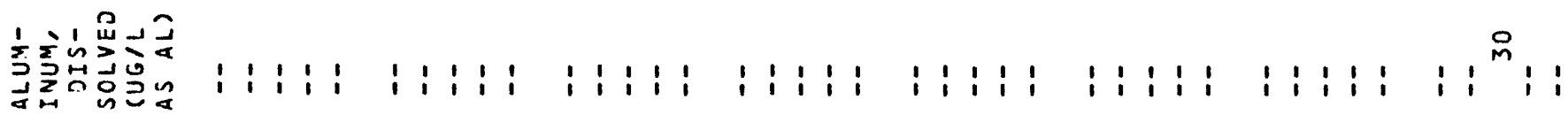

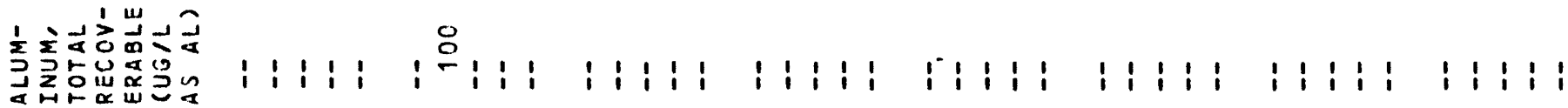
敦重起

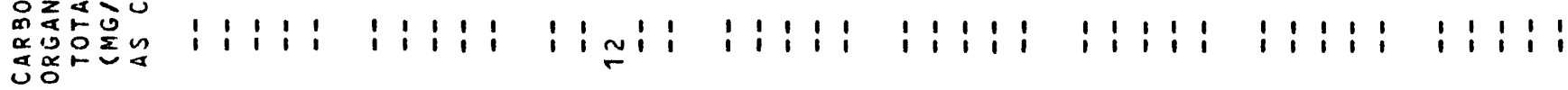

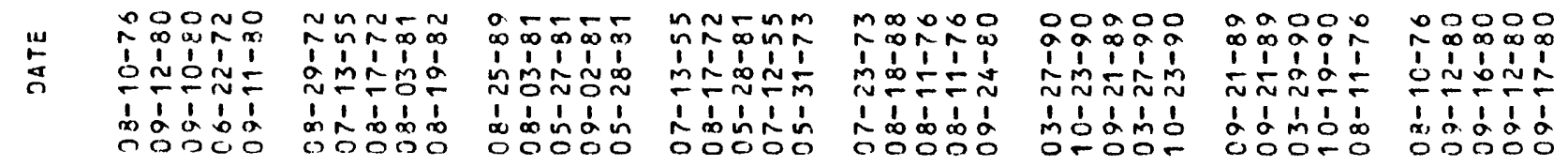




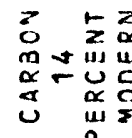

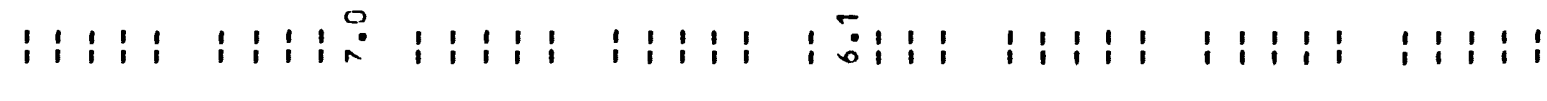

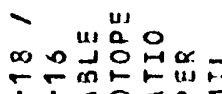
它墭象

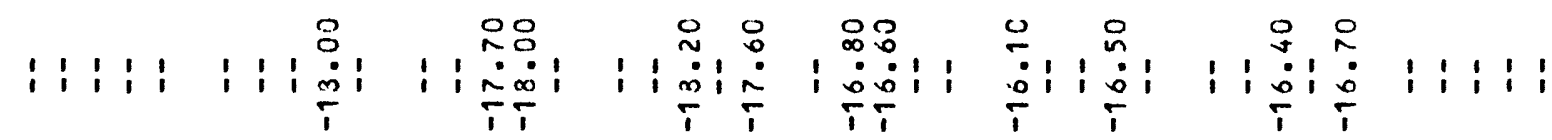

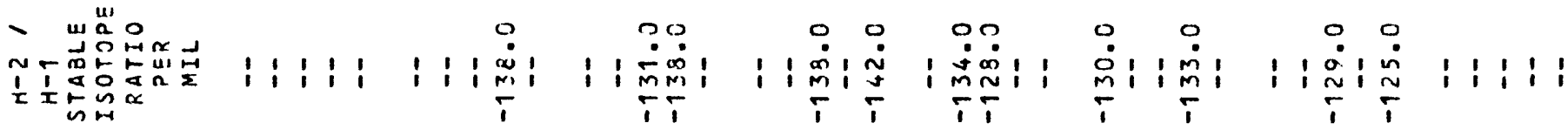

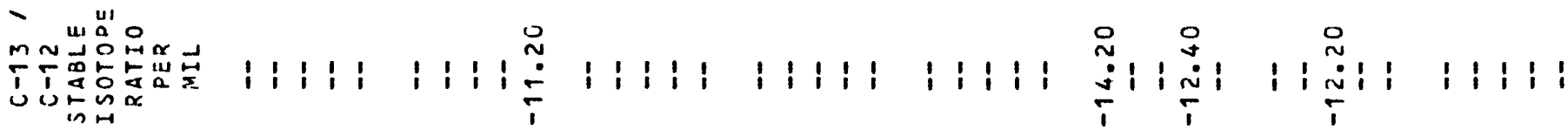

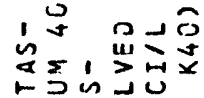

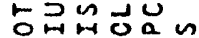

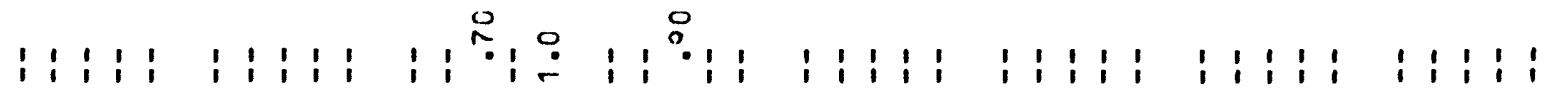

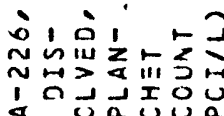

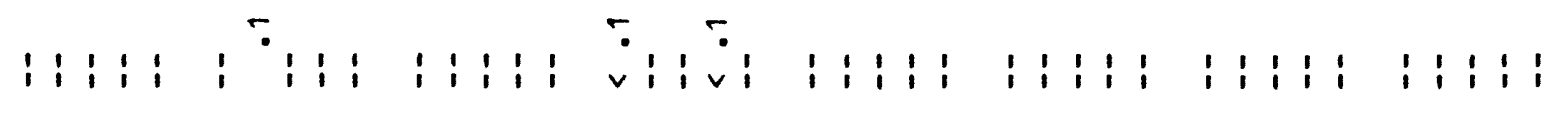
$\sum_{3}$ 난:-1 aro

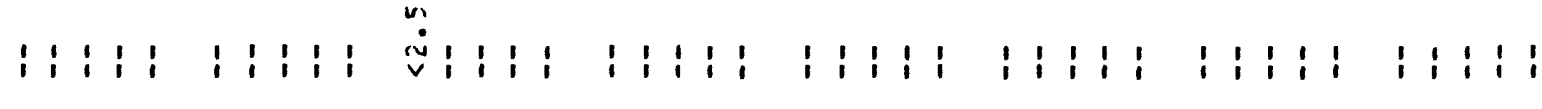

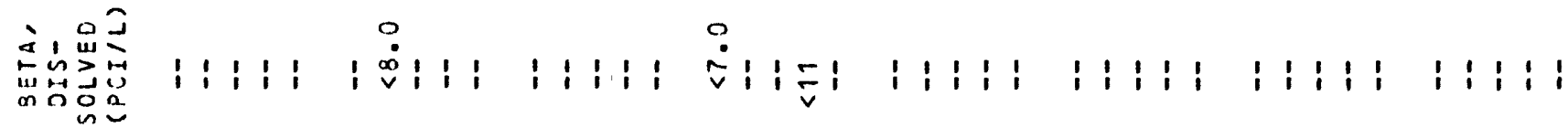

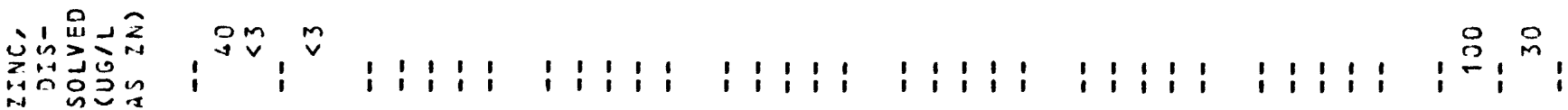

至者的品」三

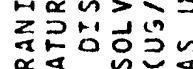

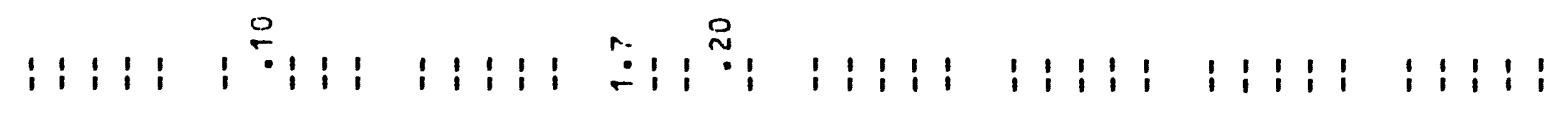

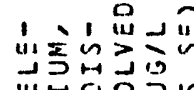

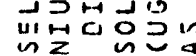

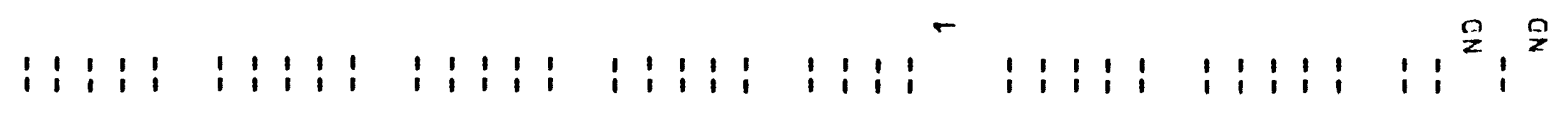
$\underset{\substack{m \\ \stackrel{a}{c}}}{m}$

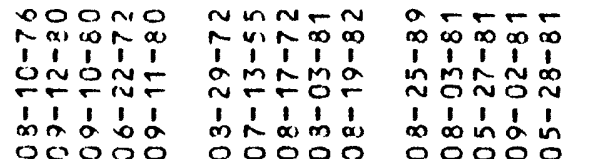

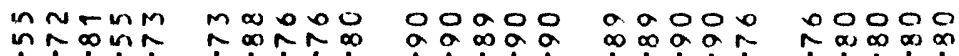

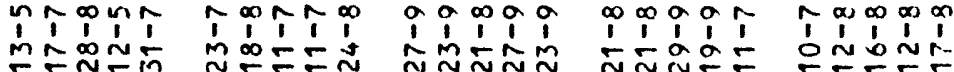

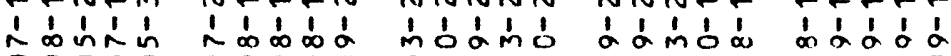

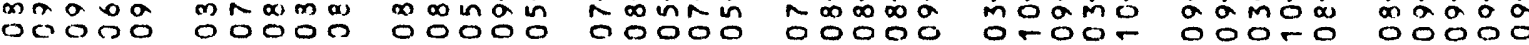




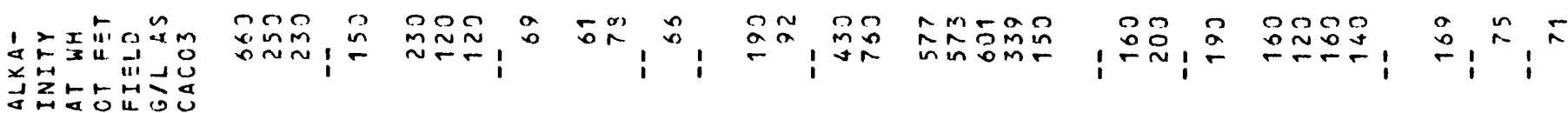

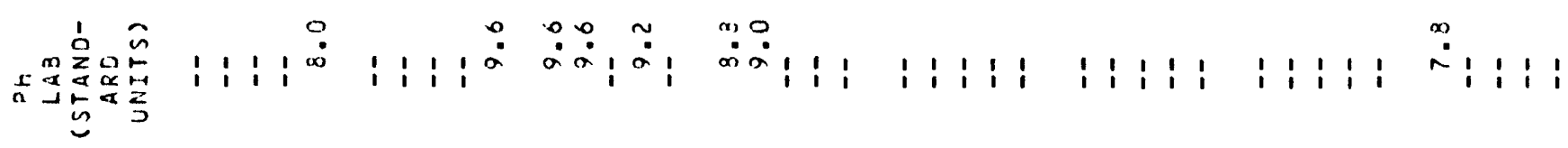

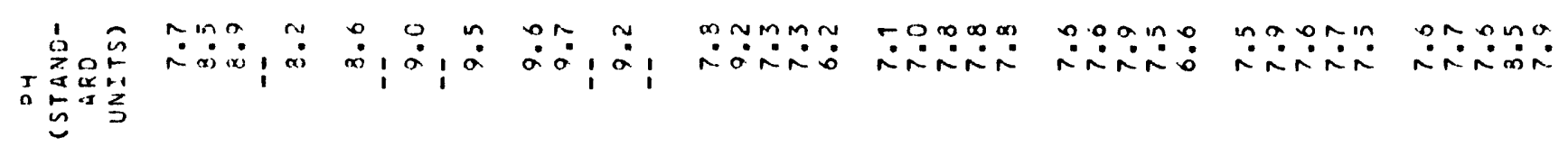

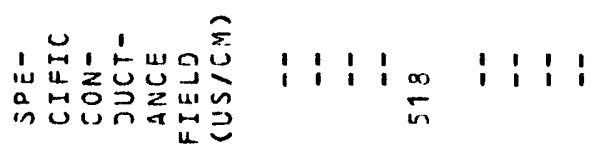

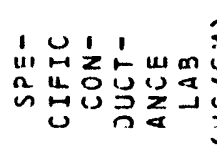

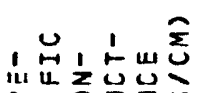

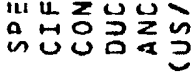

\& un $\propto \mathcal{U}$

w $\propto$ it

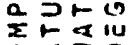

w $\alpha \Omega$

in

$\sim 0000$

1000

in $0 \div$ in

1 $15 \div 5 \frac{1}{1}$

$\begin{array}{lllll}1 & 1 & 1 & 1 \\ 0 & 0 & 0 & 0 \\ 0 & 0 & 0 & 0\end{array}$

$M \pi N-\infty$

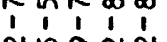

nNañ

1. 1151

列

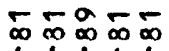

1111

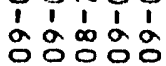

N5ก 55
NoNNR

둥우눈

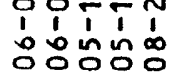

$\infty \infty_{1}^{\infty} \pi \hat{1}$ iก̃บ

ํㅕㅇ웅워

11

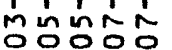

i: $1: 1: 1$

i i : i :

i i i i :

"I!

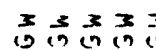

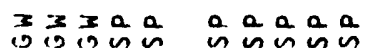

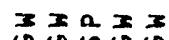

a a $3 x=$

Tลำก

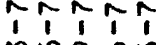

눈웅웅

1 11051 กํㅇํㅇㅇํㅇ $\bar{n}$ $n_{1} 1 ; 1$ onova oñá 1ิ1 1'1 1 1 1 1

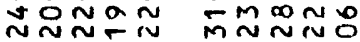
1111 ordra dín

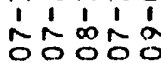

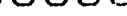

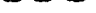

inaa moñom

araa oagar

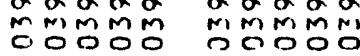

miñ

iziziz

mmmmm

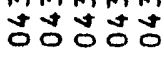


'ㅎ '

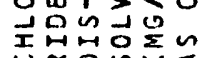

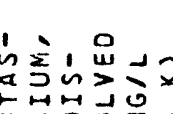

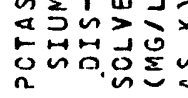

点, 品

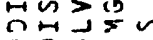

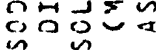

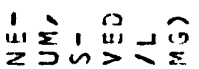

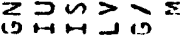

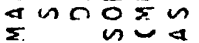

I $\stackrel{0}{\mathcal{H}}, \hat{x}$

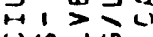

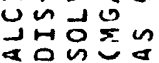

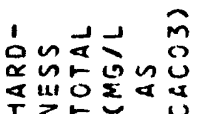

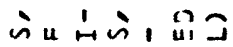

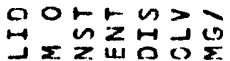

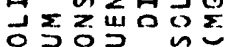

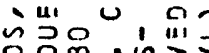
(in

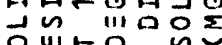

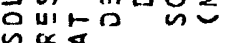

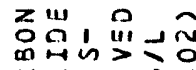

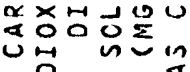

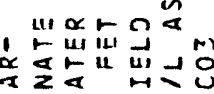

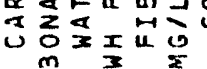

1 woron

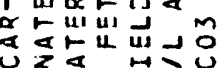

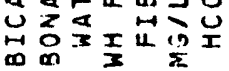
กั:

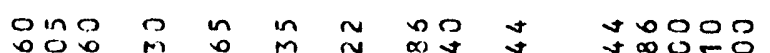

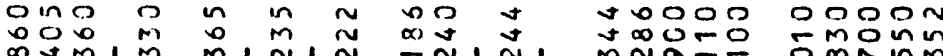

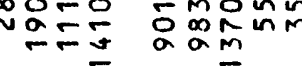

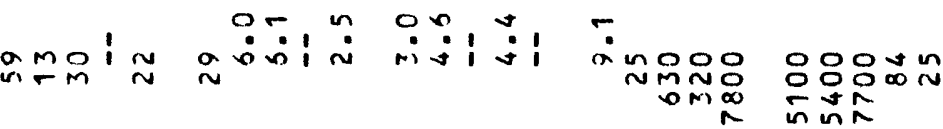
iñin

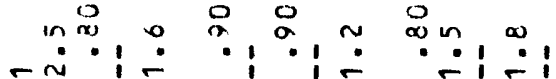

oralo ciala orini atoon

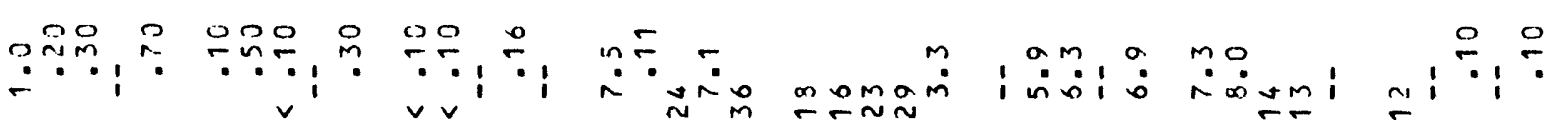

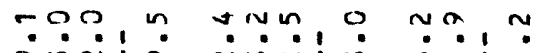
añía niminim mínil

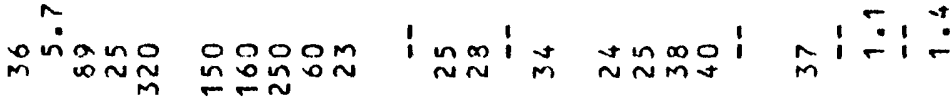

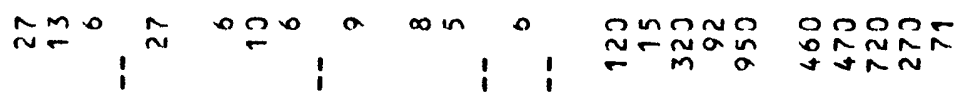

:

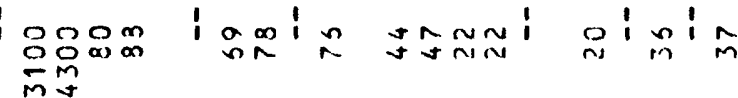

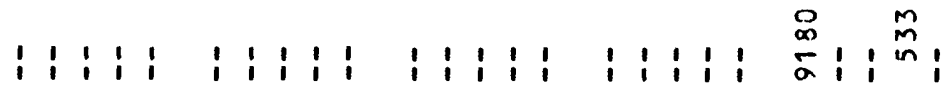

$1: 1$

i i i i i i i i i

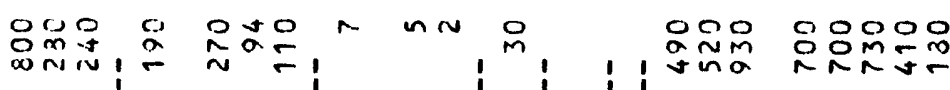

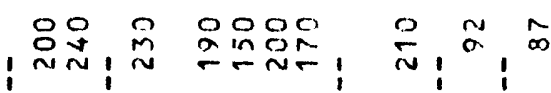

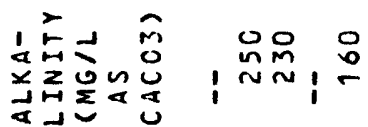

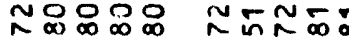

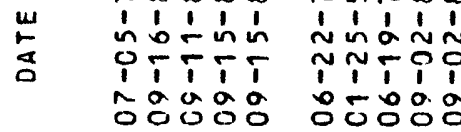

$\infty \infty_{\infty}^{\infty} \infty$

in $121 \frac{1}{1}$

윤?

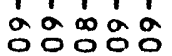

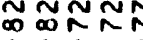

11015

웅듀

oํํำ

$\infty_{0 N \infty}^{\infty}$ mód Ti Ti minno

NANAN R№N

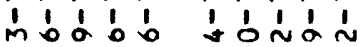
NTOTO NNNT

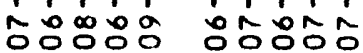

anNAN - $\begin{aligned} & 1 \\ & \text { m }\end{aligned}$

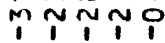
กิํํㅇํㅇ 


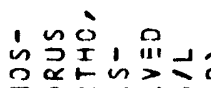

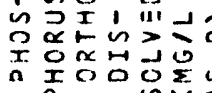

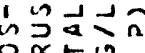

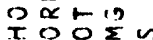

T.ำ

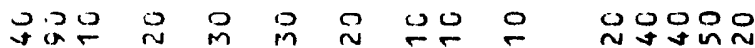

o.0.:

$1: 30$

華, 足

으음유음

곡요

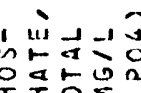

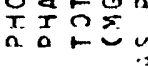

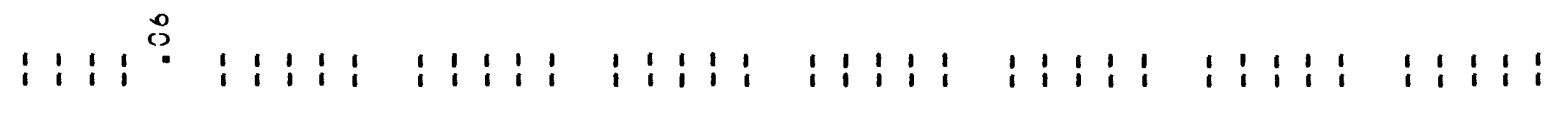

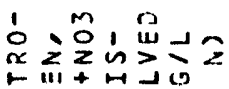

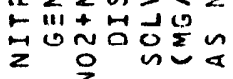

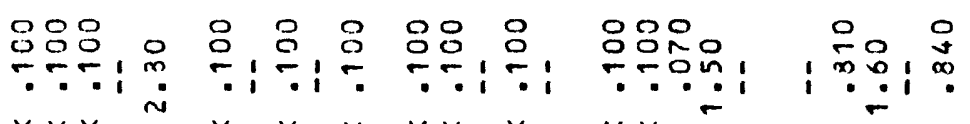

,

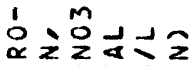

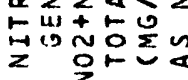

$$
\checkmark v
$$$$
\text { 洒 }
$$
$\vee v \vee v$
$\checkmark$$$
\text { - }
$$

$1: 11: 11: 1: 1: 1: \%$

is 1 is

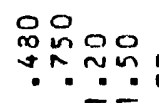

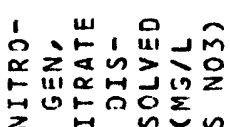

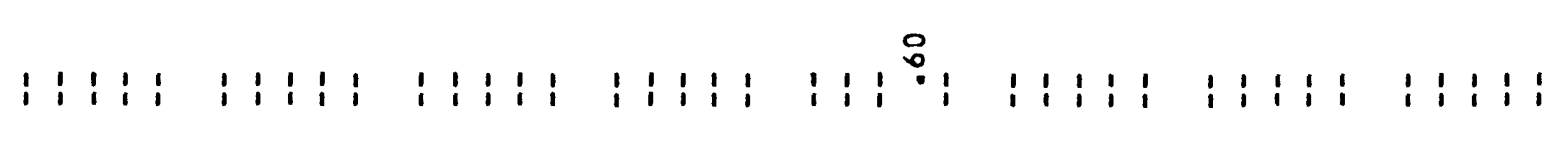

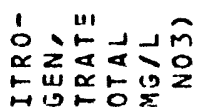

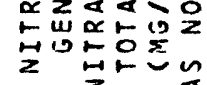

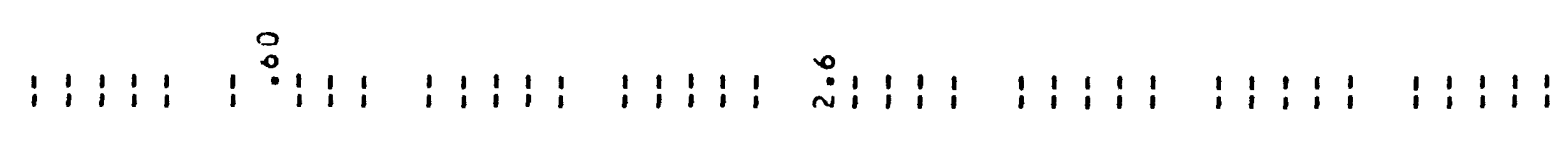

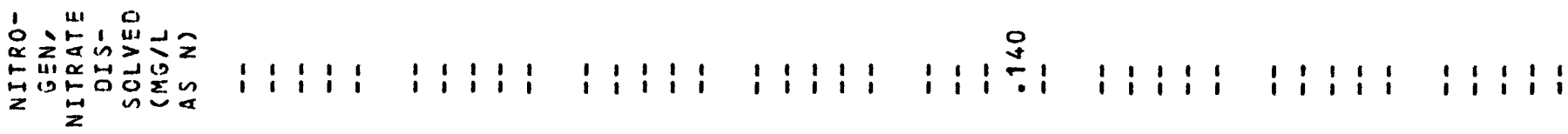

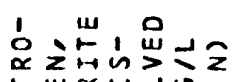

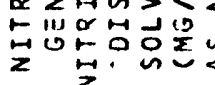

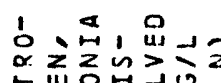

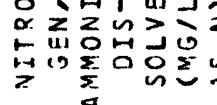

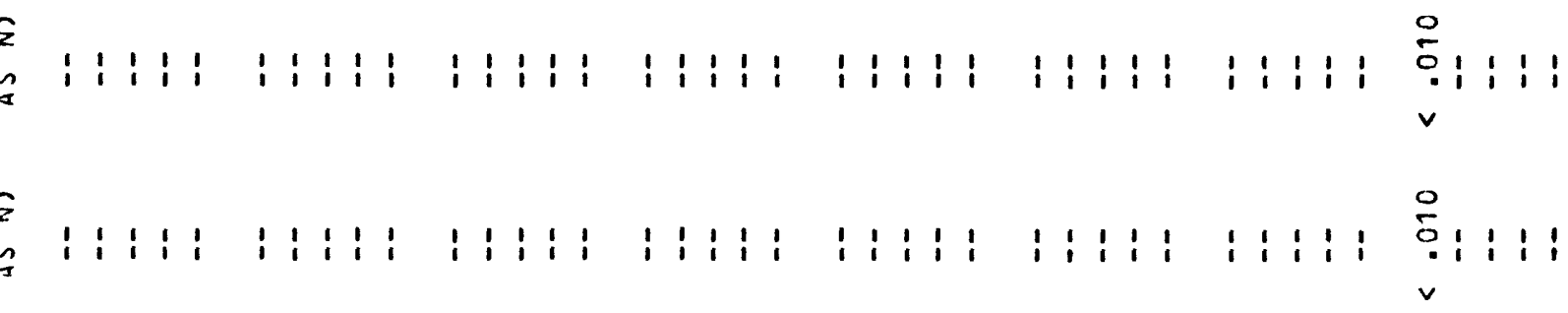

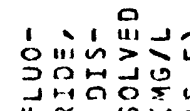

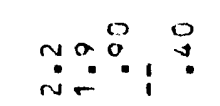
móni

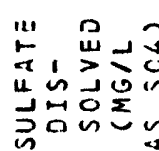

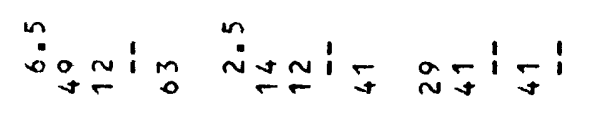

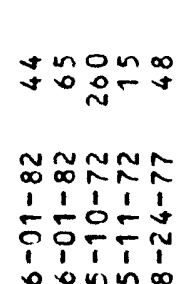

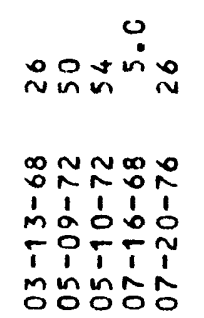
ㅇํㅇํํㅇ

i imín ํํ요

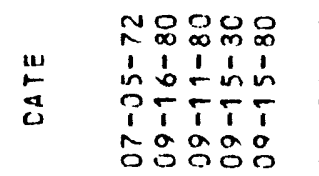

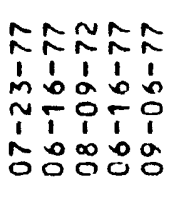

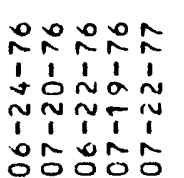

-กลกล์

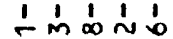
mĩ 동ํㅇㅇㅇㅇ 


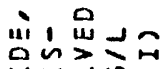

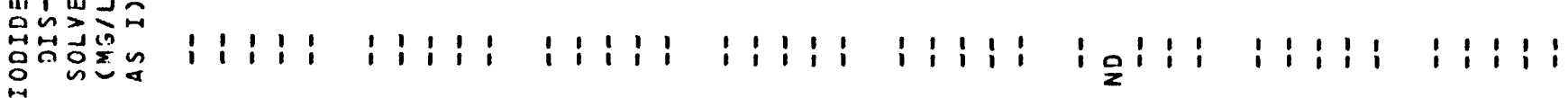
离, 엉요의

$1: 1: 1: 1: 1: 1$

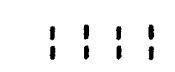

i : $:$ :

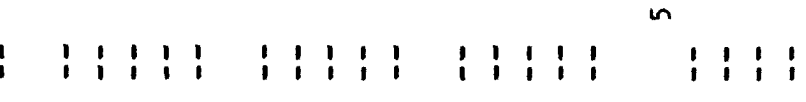

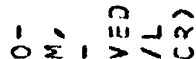

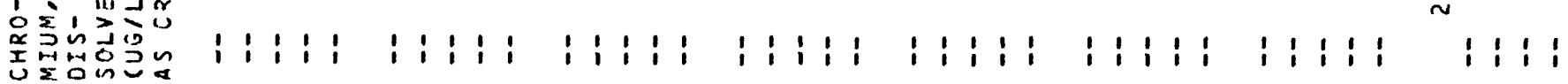

님, 에

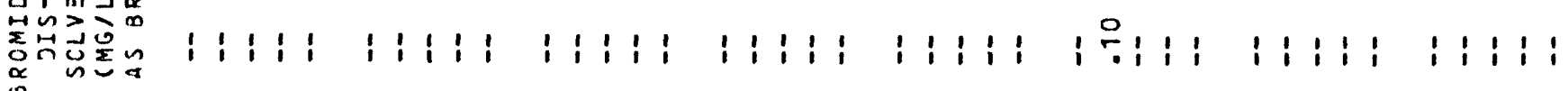

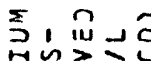

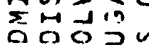

ădon

i: $1: i: 1: i: i$

i i : i i

i i i i i

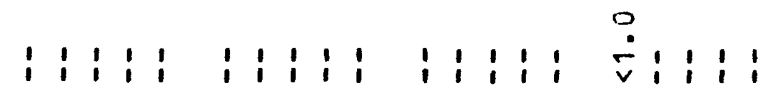

辛品是通

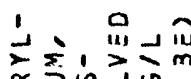

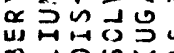

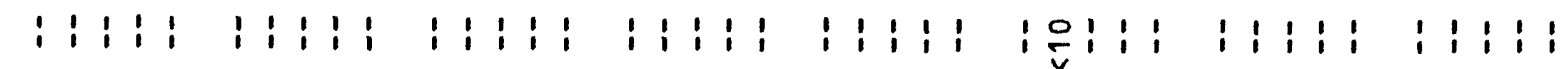

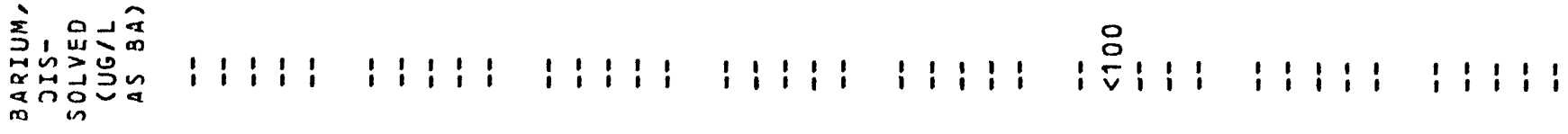

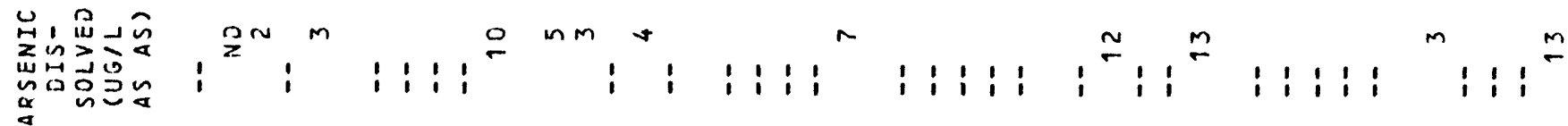

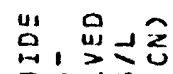

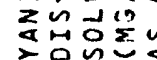

分的乎

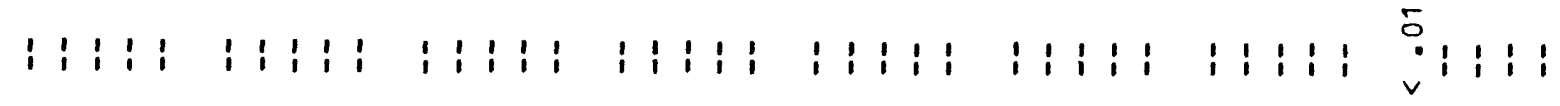

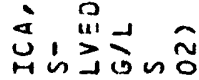

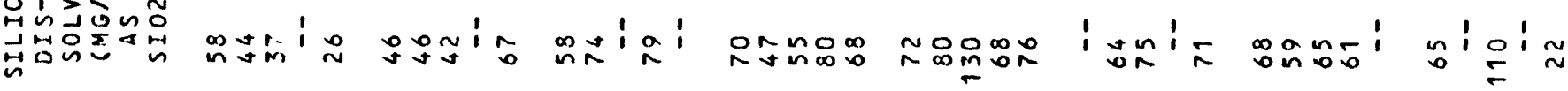

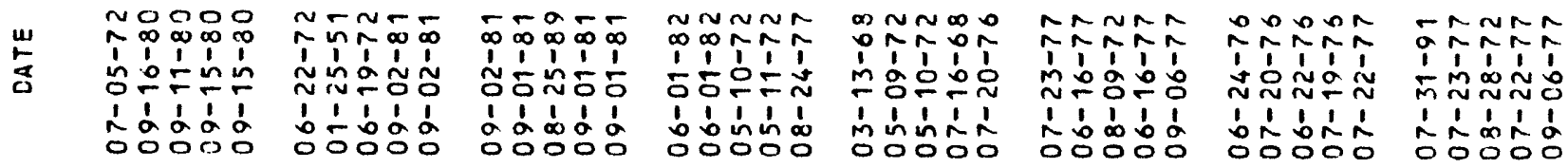




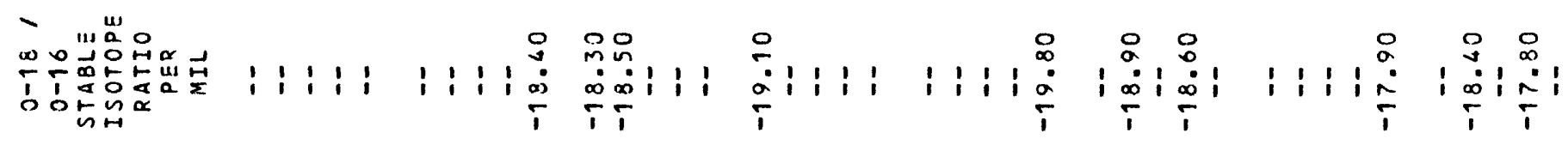

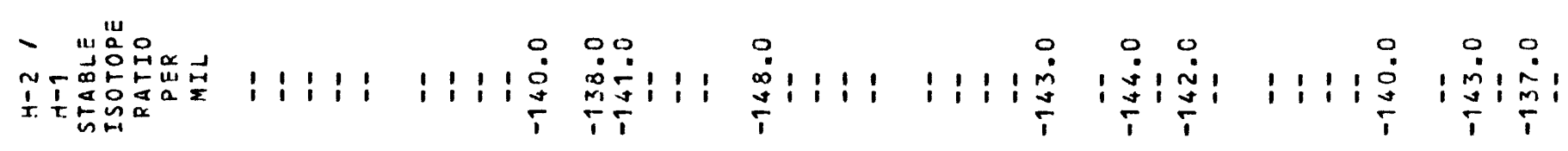

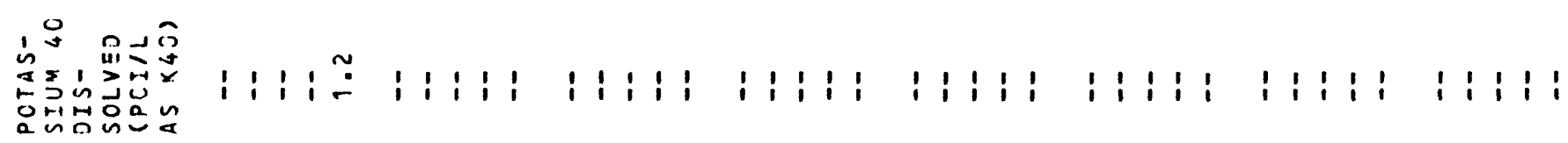

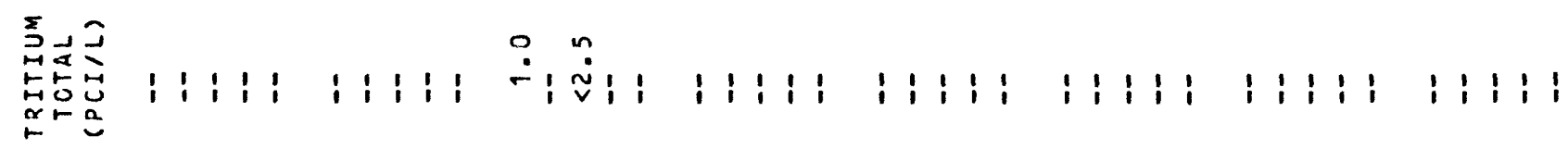

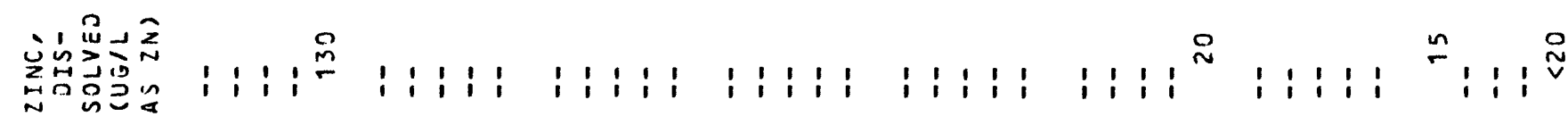

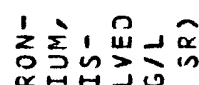

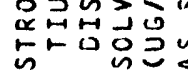

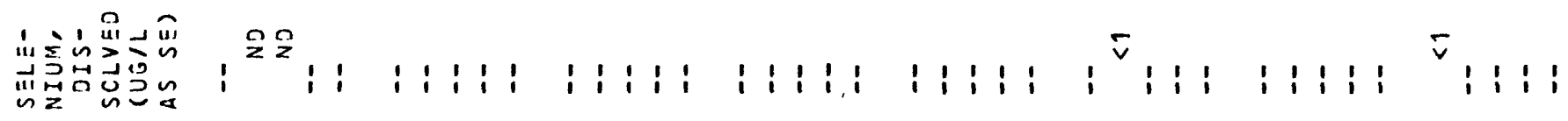

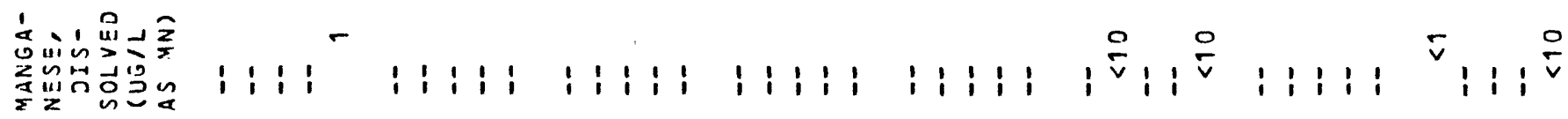

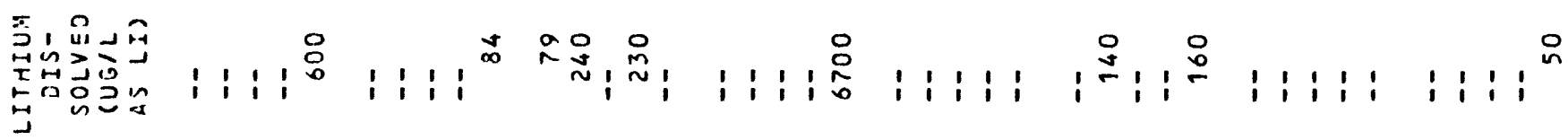

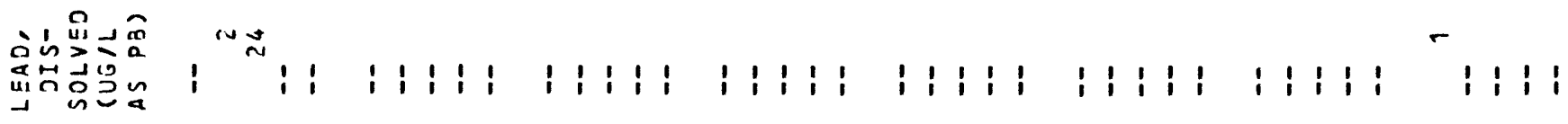

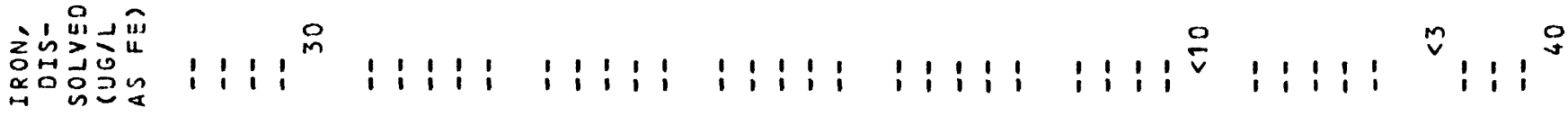

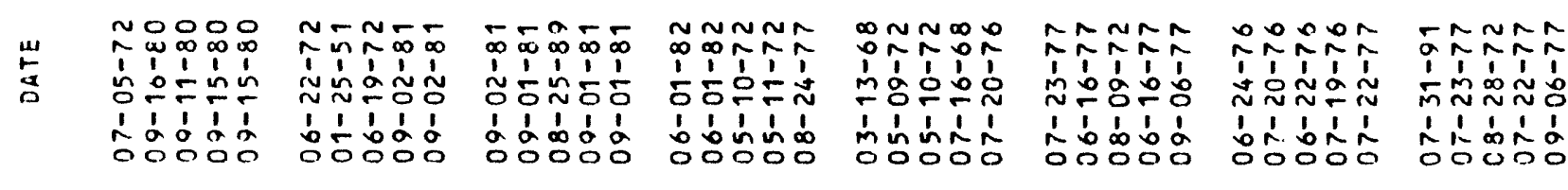




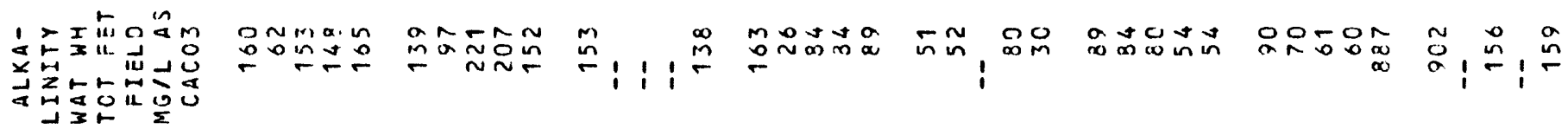

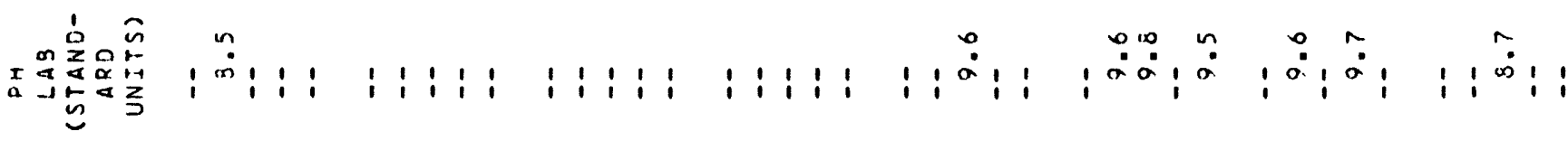

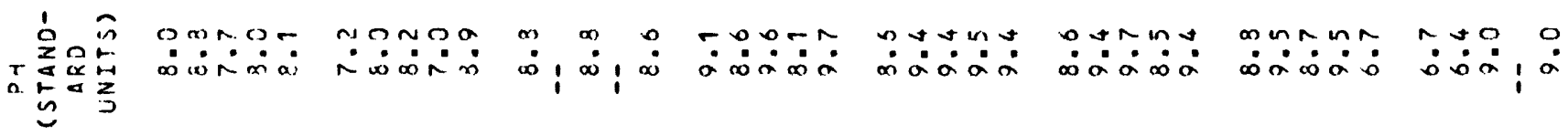

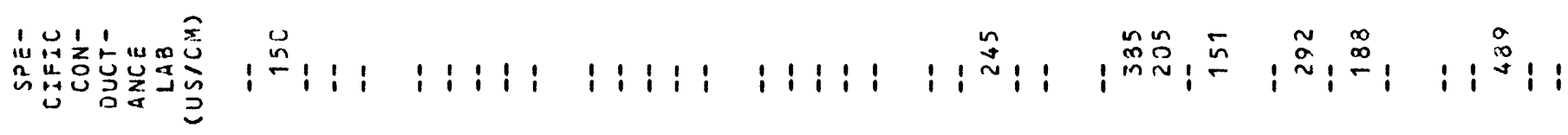

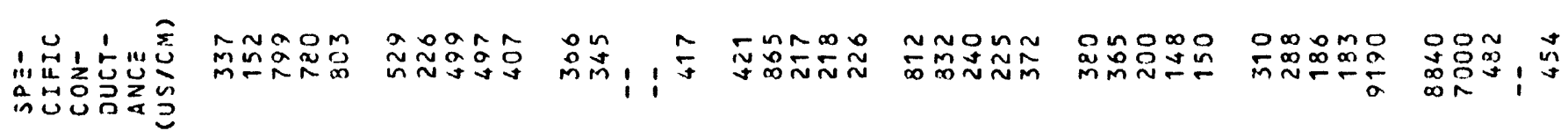

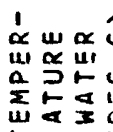

movom oogoo oom o gom o oommo

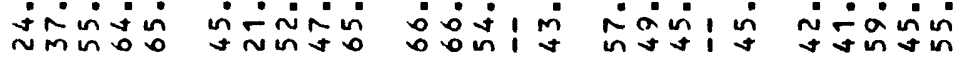

o. 0 . n nim $\min$

000 in 0 in $\operatorname{in} \infty \dot{\infty}$

o00 in

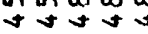
a $2 N$ in

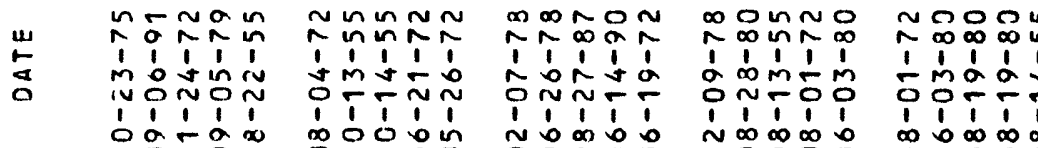

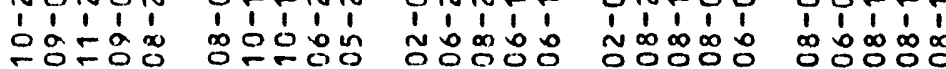

$\sim \sim N \sim N$ I

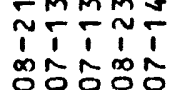

NNNNONNEmM $\hat{i} \dddot{i}$ $n \pm m \pm r \quad n \infty m o n$ i 1 i

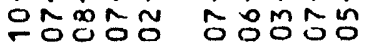

$\stackrel{\sim}{n}$

a maa a a a $3 a$ a a a

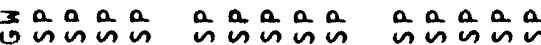

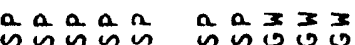

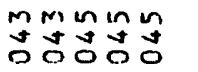

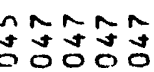

先告旅主

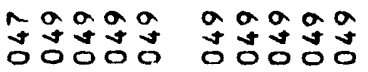

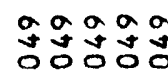

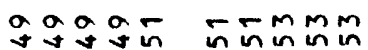

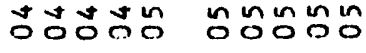




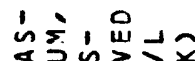

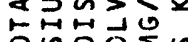

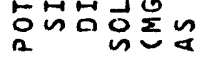

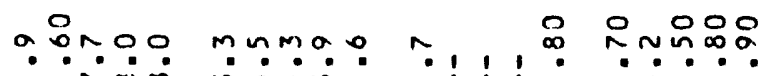

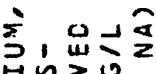

두에

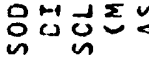

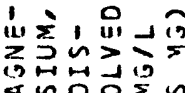

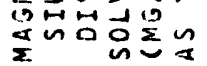

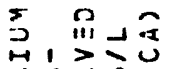

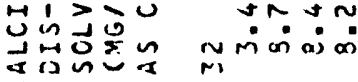
ํํํํํำ toma

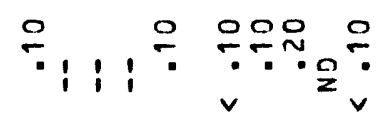
$00 m a n$

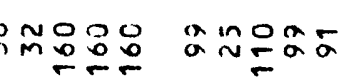

i. 1

$\sin 2$

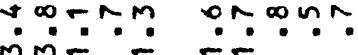

nน

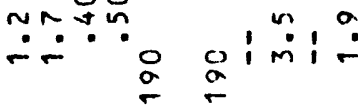

西 Oथี

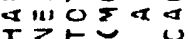

施斗施, 足 은

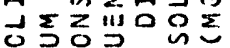

minma a n mmo Mำn MINmḾn

$\sim$ i ortar nnanm $m ! 1, \stackrel{\infty}{\sim}$ 은ำ

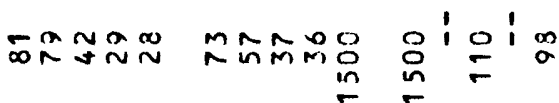

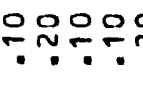

ำต กั ำำ

$\because \min _{\infty} \operatorname{mi}_{i}$

onsn- nanom

ni ti nnoton onta

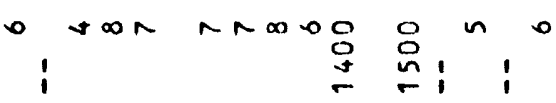

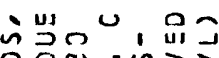

a

药药比

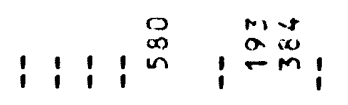

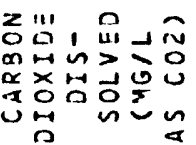

in:

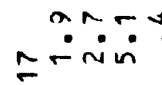

?

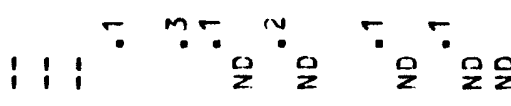

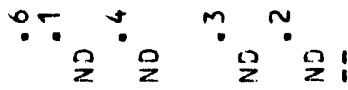

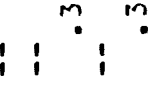

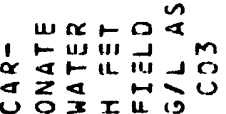

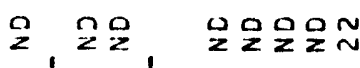

$\stackrel{\infty}{1}, \stackrel{\sim}{*}$ m

$\min \infty N$

$\tilde{N} \min _{n}-$

$i i^{n} i^{n}$

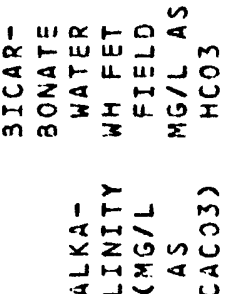

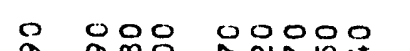

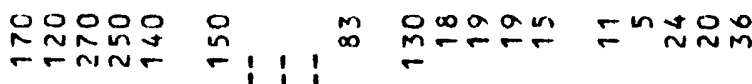
$r: r-n$ i 1 $\stackrel{0}{n}, 1,1, i^{n}, i^{\infty}, i^{n}$,

MNㅗ뮴

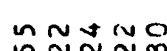

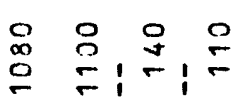
$1: 1 \stackrel{0}{1}:$

: : : : :

ñ noin $\hat{1}: \hat{1} \hat{i} \tilde{i}$ คิํํำ

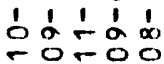

$N \operatorname{nnnN}$ กิ Oำ눈

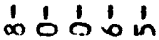

$\infty \infty n 0 N$

$\hat{1} \hat{1} \mathfrak{1} \hat{1} \hat{1}$

กํㅠㄴ

' $\begin{array}{lllll}1 & 1 & 1 & 1 & 1 \\ 0 & 0 & 0 & 0 & 0\end{array}$

Tonno NoOO

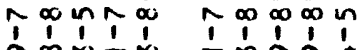

ด舟塄出

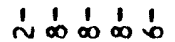

ธํํㅇํำ

16151

$\sim \mathscr{N} \sim \sim$

T⿻一⿻⿻口卄乀八

$\check{\sim} m \stackrel{m}{\sim}$

$-\pi n=$

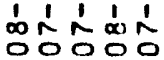

$\begin{array}{lll}0 & \sim & 0 \\ , & \mathbb{N}, & \frac{m}{m}\end{array}$

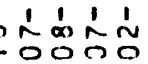

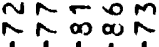

ヘำ

1' 


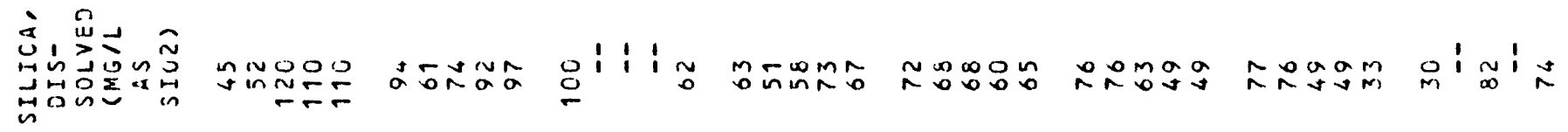

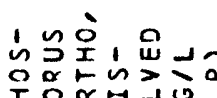

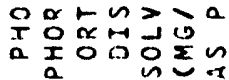

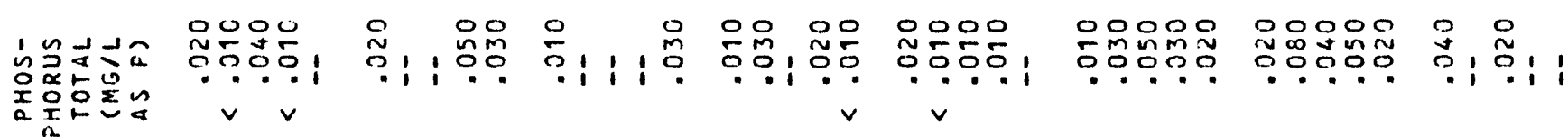

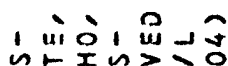

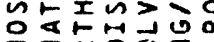

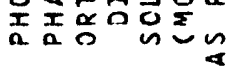

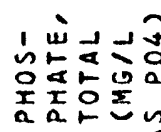

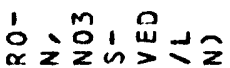

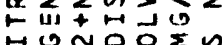

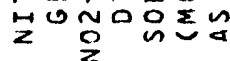

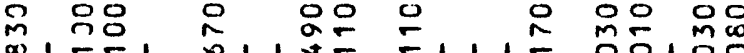

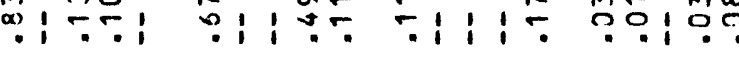

$\vee v$

$\checkmark$

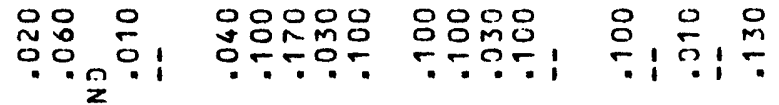

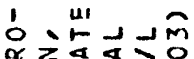

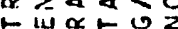

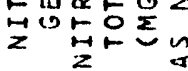

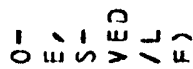

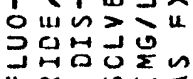

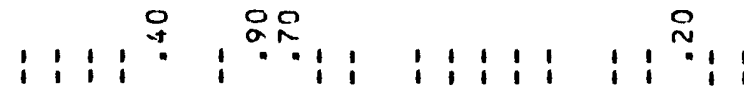

$1: 11 \%$

$\stackrel{0}{?}$

iII

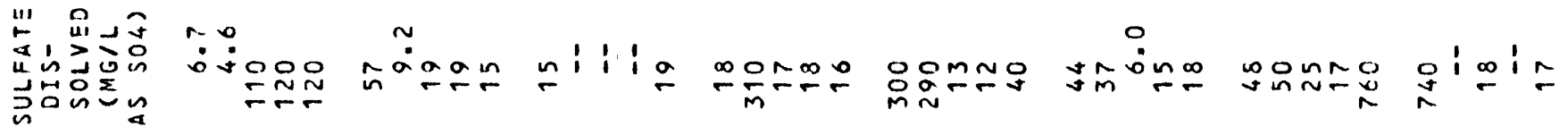

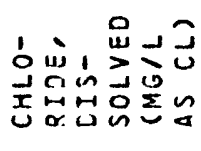

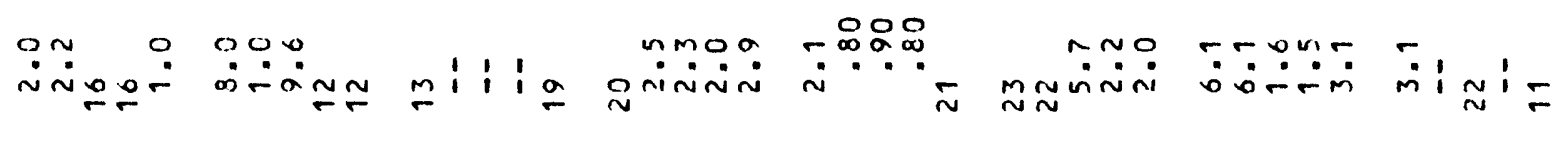

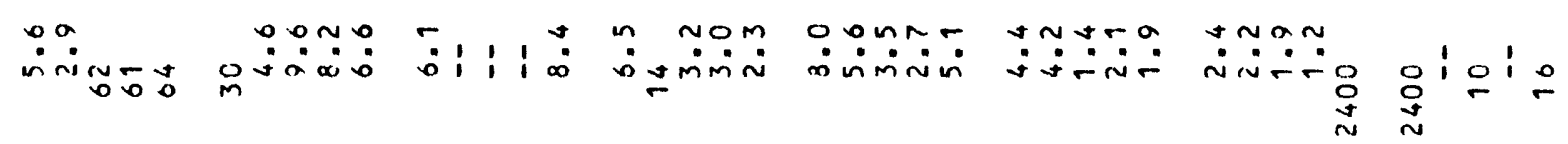

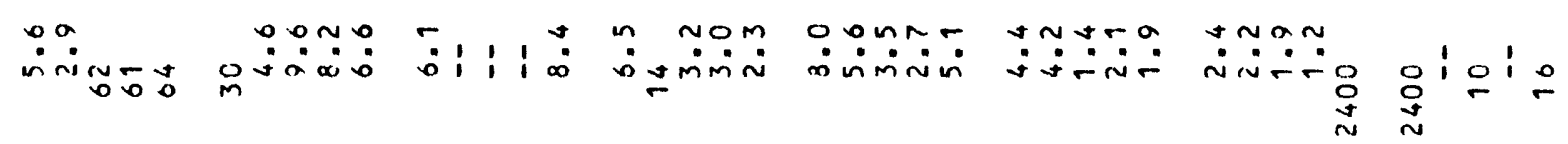

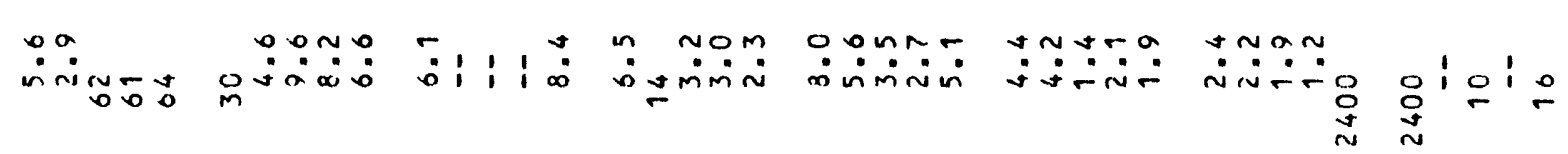

mingot o

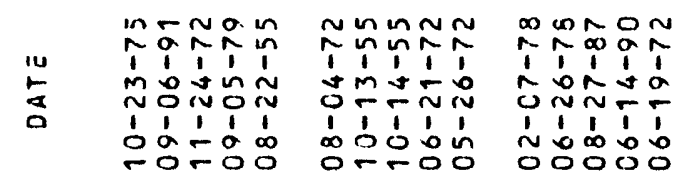

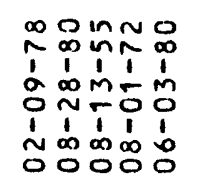

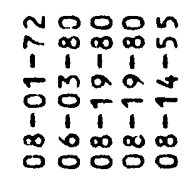

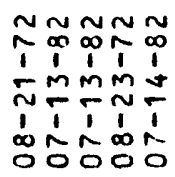

ก

$\sim n-\infty m$ 1ิ1\}

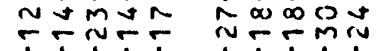

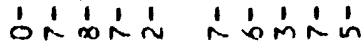
oñoñ åog̃on 


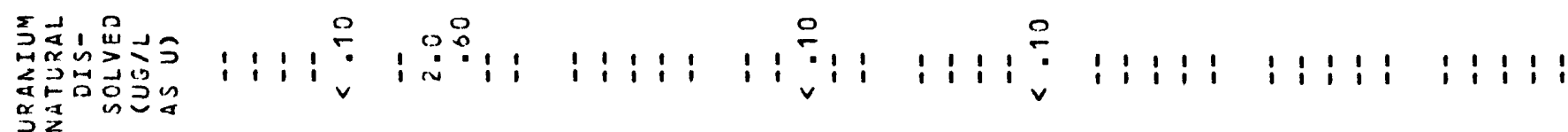

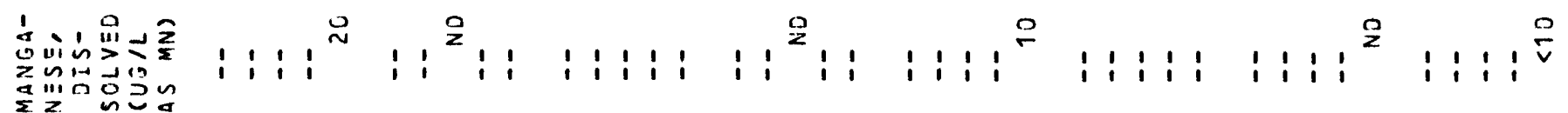

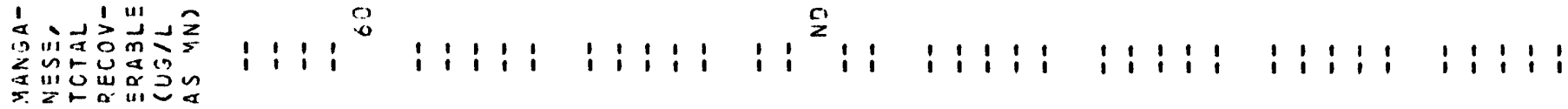

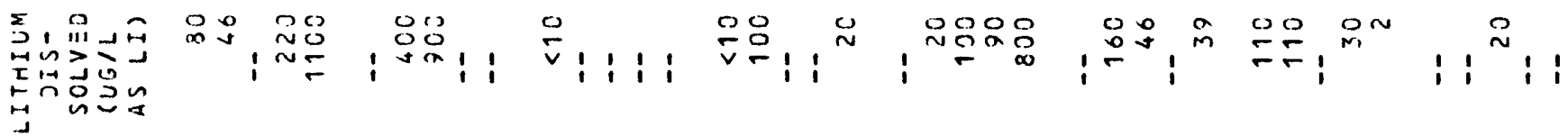

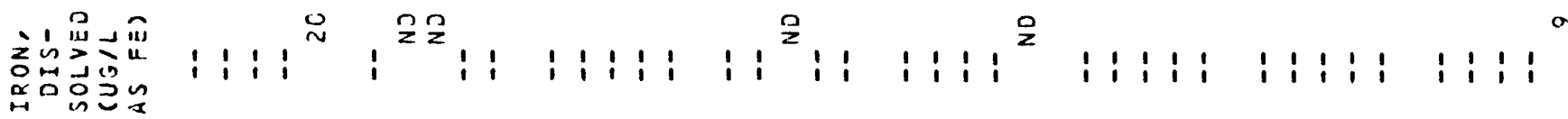

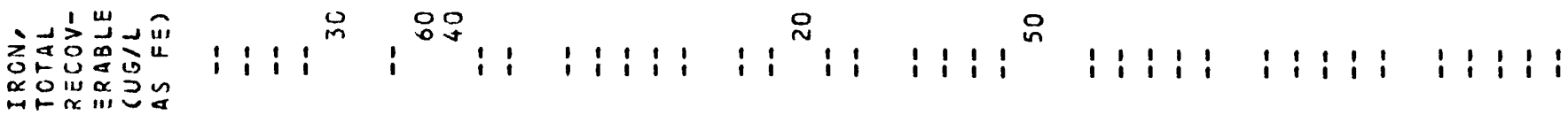

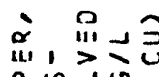
aำํำ

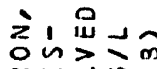

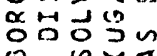

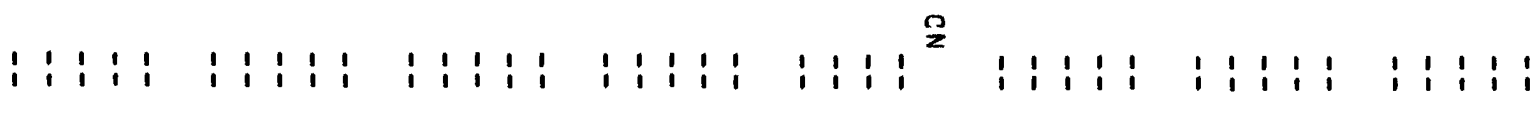

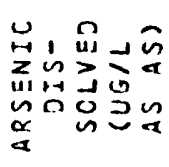

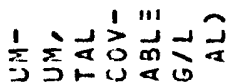

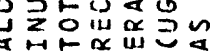

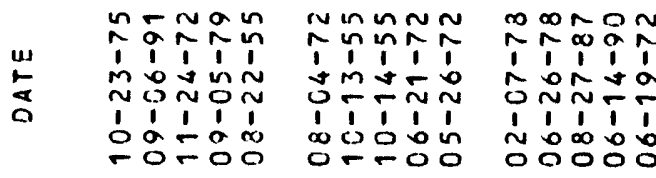

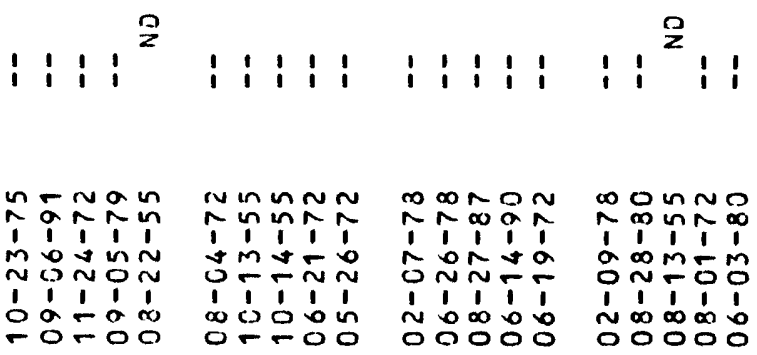

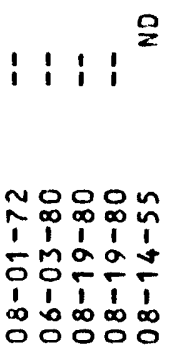
: : : : : : : : : : : : : : : : :

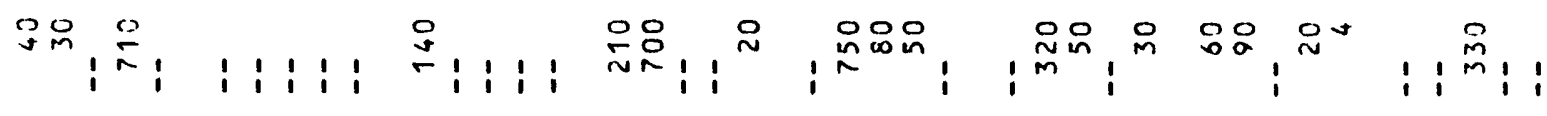

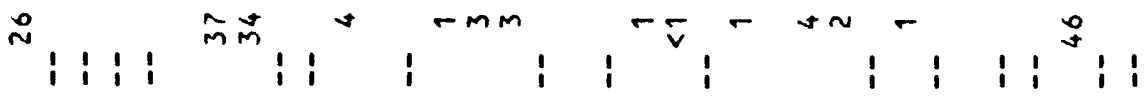
$r=\frac{0}{i}$ i i i i 象

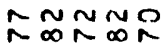

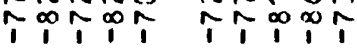

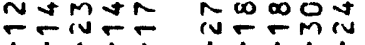
1'1 1 1 1 111

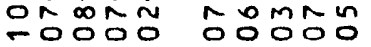




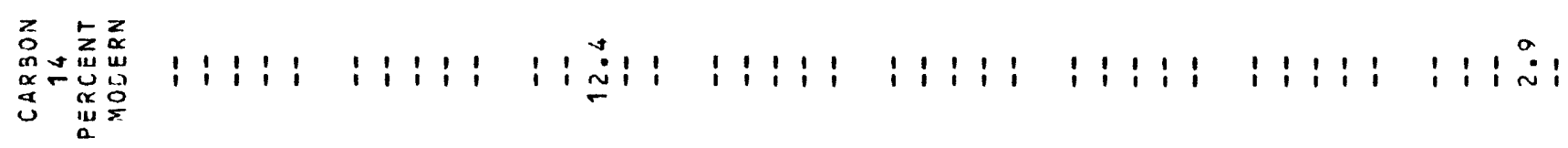

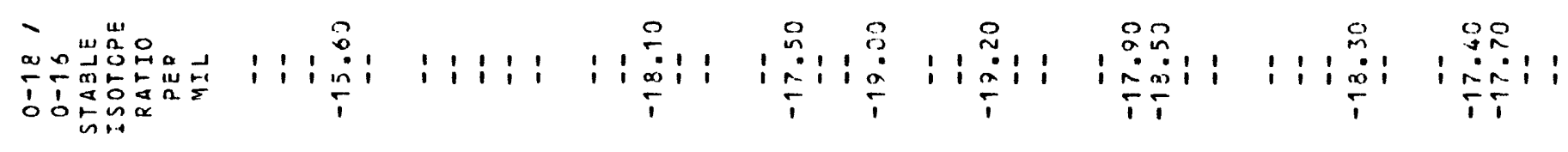

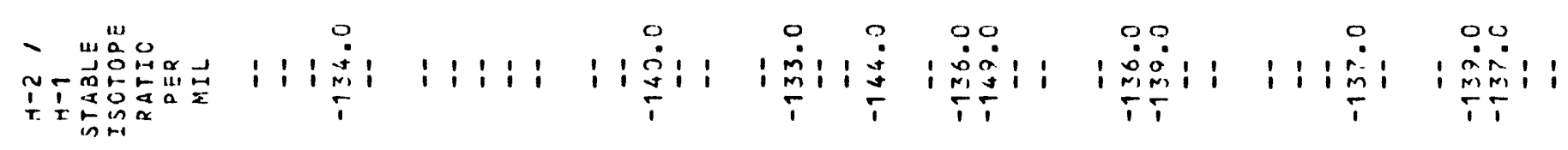

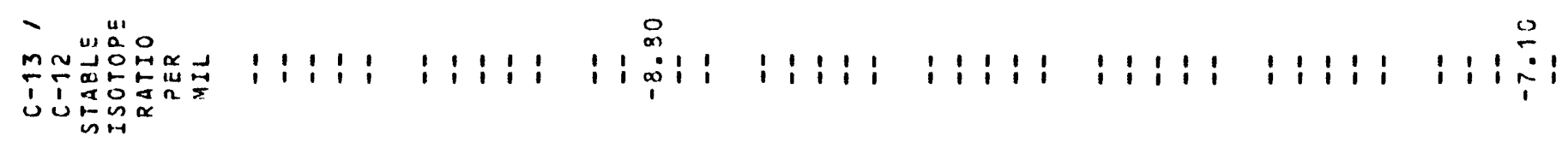

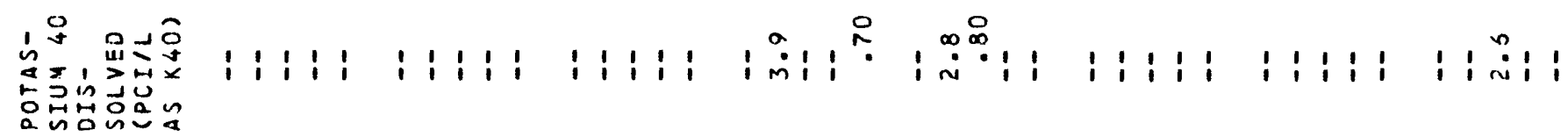

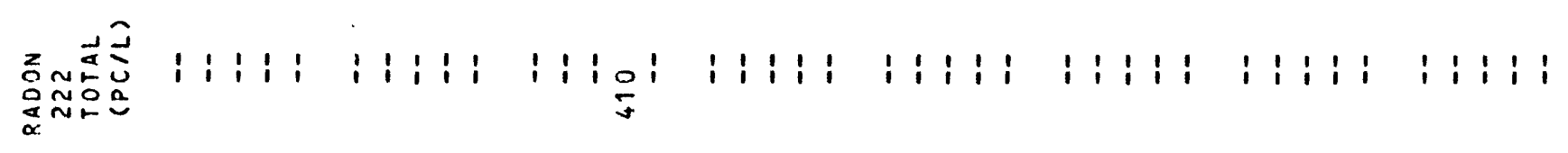

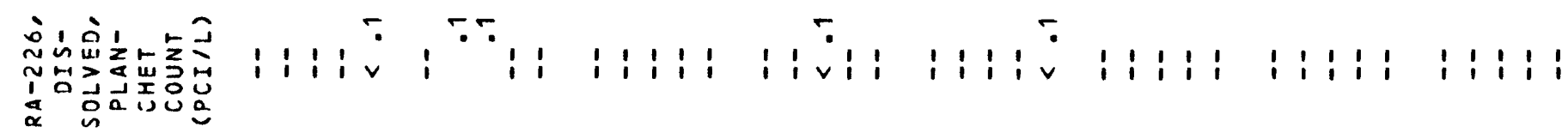

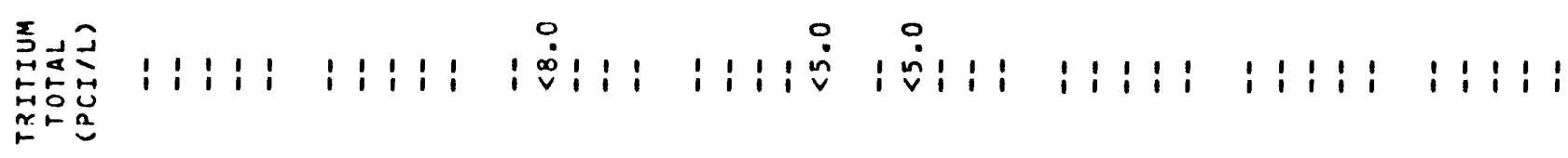

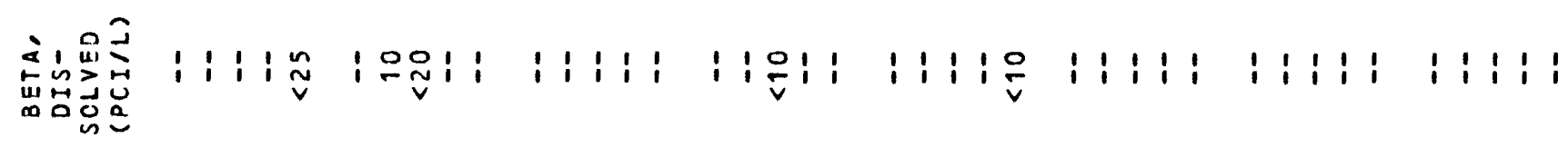

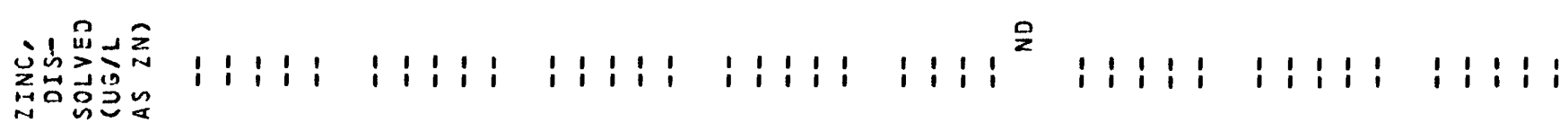

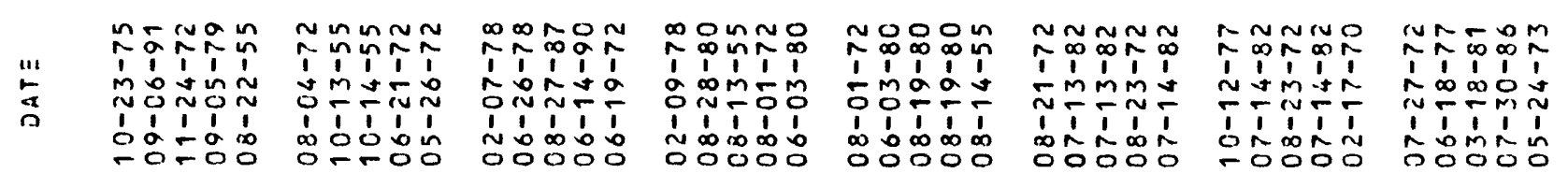




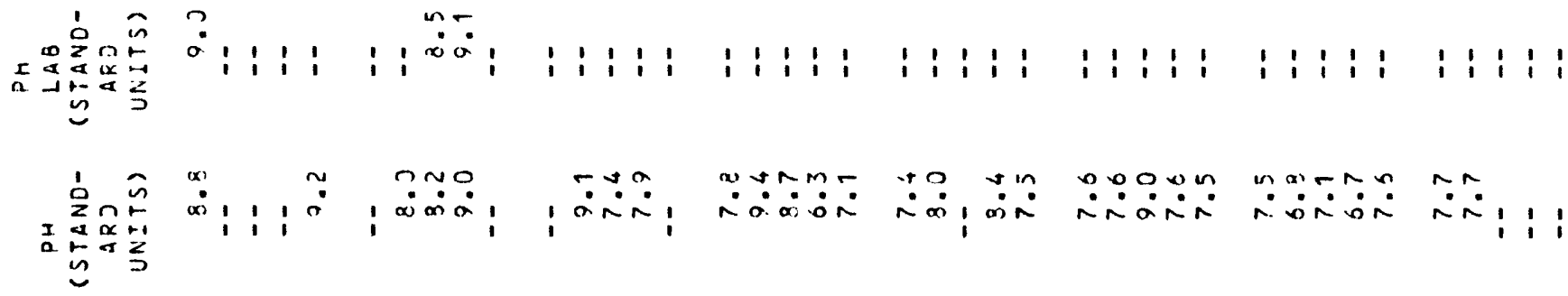

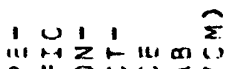
n. $u$ o in

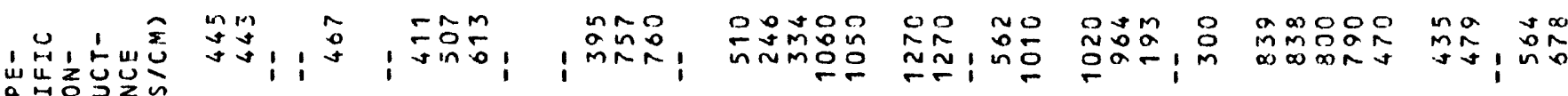

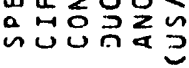

'uㅗ

i11

员范出

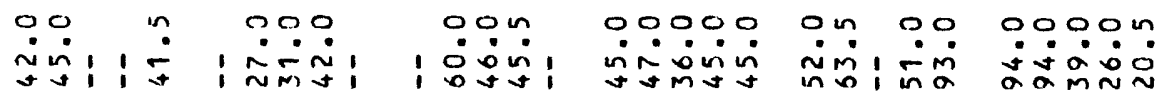

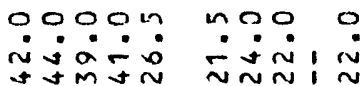

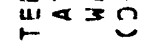

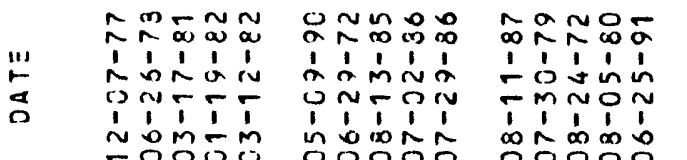

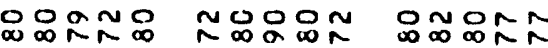

11111

กñoñ

in $\begin{array}{llll}1 & 1 & 1 & 1 \\ 0 & 0 & 0 & 0\end{array}$

i. 1111

bín

No윰ำ

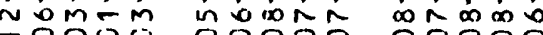

mºmo

$\infty \infty n$

1's 11

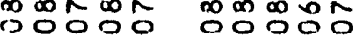

oñ̃a ñmâ

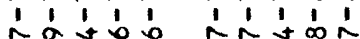

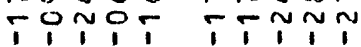

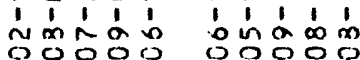

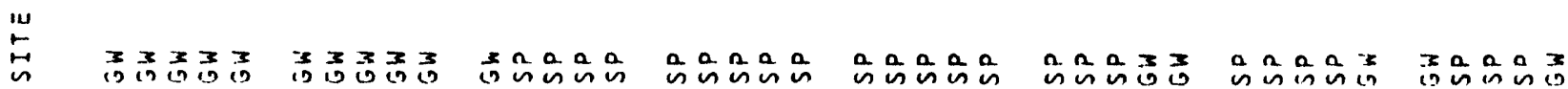

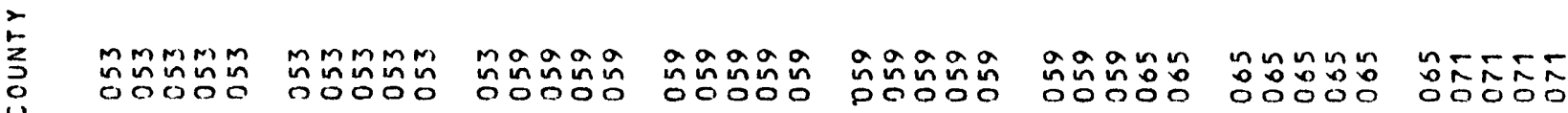

\begin{tabular}{|c|c|c|c|c|c|c|c|c|c|c|}
\hline ט & $\begin{array}{l}5 \\
0 \\
a \\
a \\
\sim\end{array}$ & $\begin{array}{l}\text { mo } \\
00 \\
00 \\
\text { mo } \\
00\end{array}$ & $\begin{array}{l}n \\
\vdots \\
0 \\
0 \\
\infty \\
-\end{array}$ & 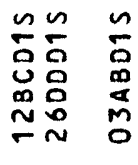 & $\begin{array}{l}n \\
\dot{U} \\
\dot{y} \\
m\end{array}$ & $\stackrel{n}{\sim}$ & 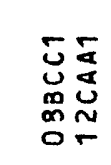 & $\begin{array}{l}n \\
\vdots \\
\vdots \\
0 \\
0 \\
0\end{array}$ & $\begin{array}{l}5 \\
0 \\
\text { n } \\
0 \\
0 \\
r\end{array}$ & 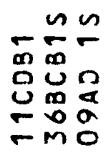 \\
\hline 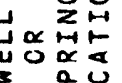 & $\stackrel{111}{\sim}$ & 虫 & 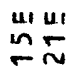 & $\begin{array}{l}w \\
\tilde{N} \\
\sim\end{array}$ & $\stackrel{u}{\sim}$ & $\stackrel{m}{\sim} \underset{\sim}{m}$ & $\begin{array}{l}w_{w}^{w} \\
\sim O\end{array}$ & $\underset{v}{m}$ & $\frac{11}{4}$ & 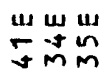 \\
\hline ธo & $\tilde{a}$ & 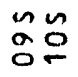 & $\begin{array}{l}z z \\
n \\
n\end{array}$ & $\begin{array}{l}z z z z \\
00 a \\
\sigma \\
\sigma\end{array}$ & $\underset{\sim}{z}$ & $\underset{m}{m} \underset{\sim}{m}$ & $\begin{array}{l}2 z z \\
\text { in }\end{array}$ & in & $\begin{array}{l}2 \\
0 \\
0\end{array}$ & $\begin{array}{l}z \sim \sim \\
0 \simeq \pm \\
0 \leftarrow \sim\end{array}$ \\
\hline
\end{tabular}




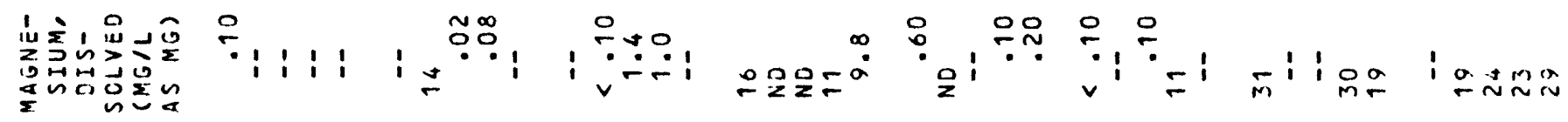

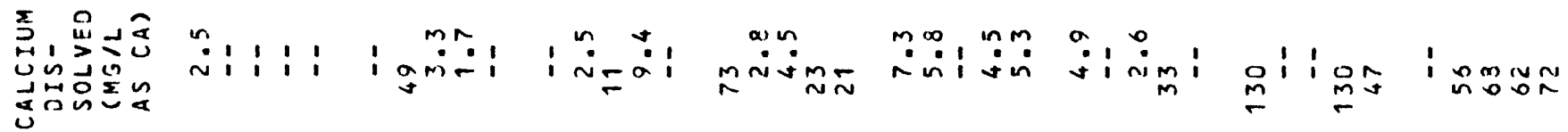

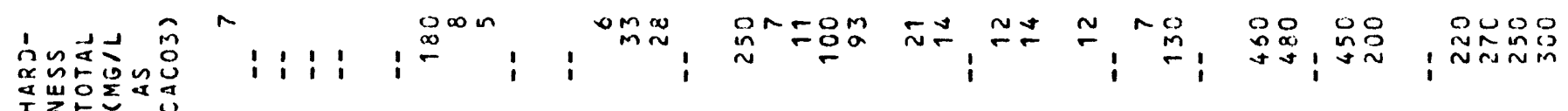

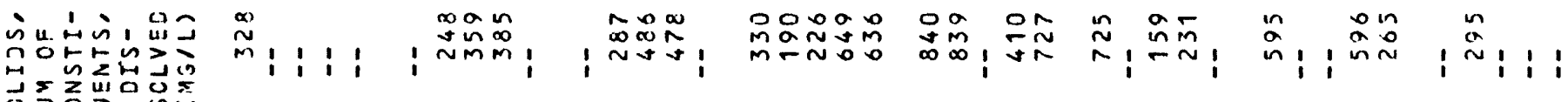

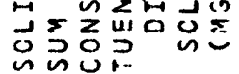

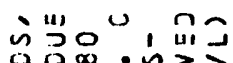

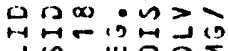
古出

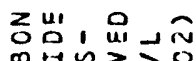

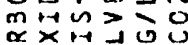

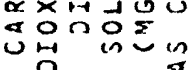

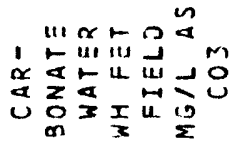

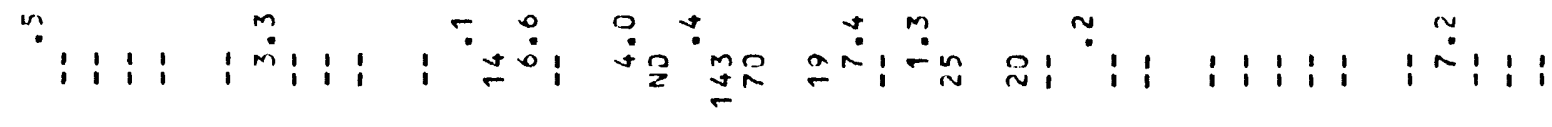

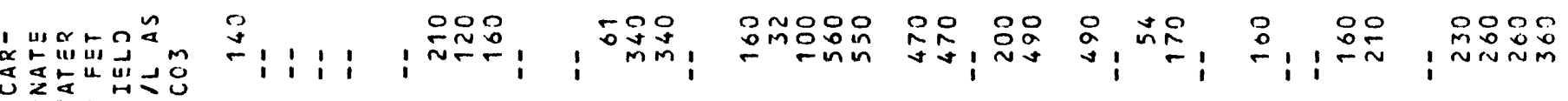

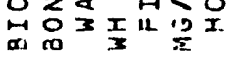

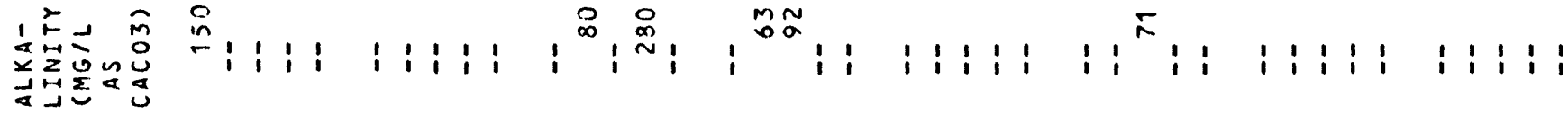

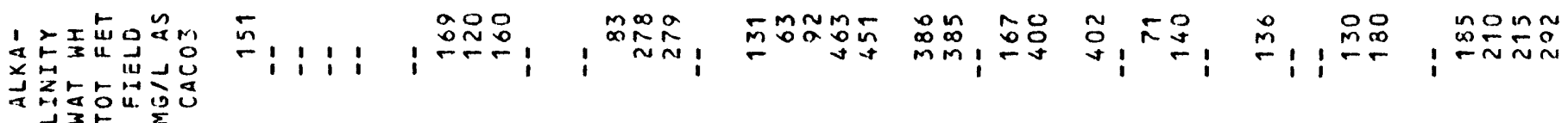

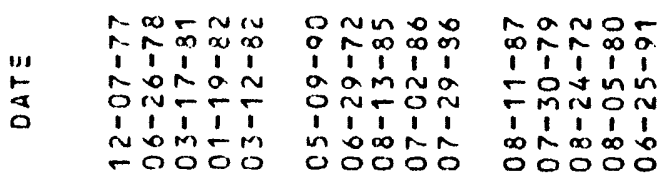

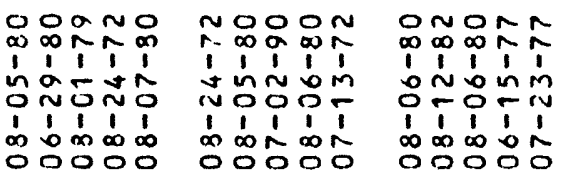
ONR̃ri $\hat{1} \hat{1} \hat{i} \hat{1} \hat{1} \hat{1}$

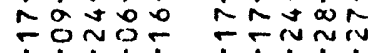
T11 1 1 1 1111

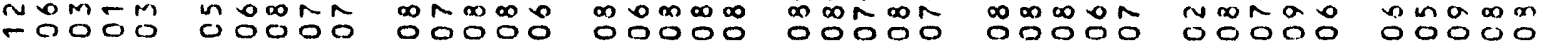




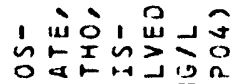

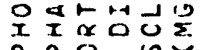

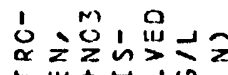

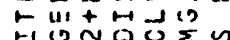

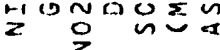

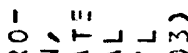

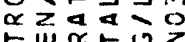

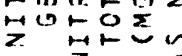

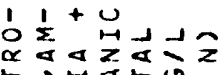

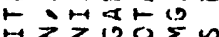

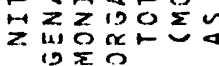

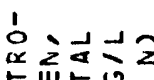

분돈

它的品士

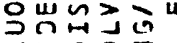

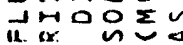

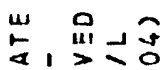

的分品

声皆品步

니 $1:$

$\div: 11$

i

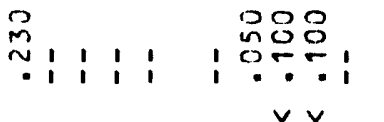

웅요

언웜윰음

웅음, 웅옹

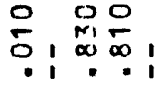

$v \quad v$

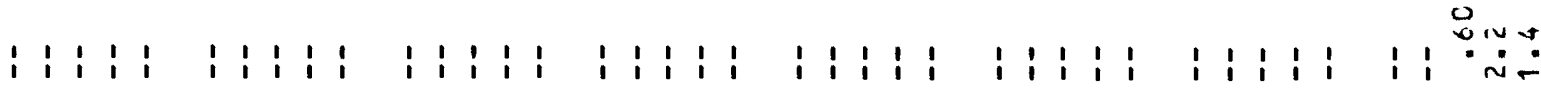

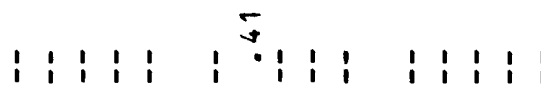

1911

i i i

i i i i

$\begin{array}{llllllllllll}1 & 1 & 1 & 1 & 1 & 1 & 1 & 1 & 1\end{array}$

i i i

i : $1: 1$

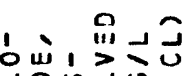

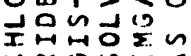

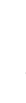

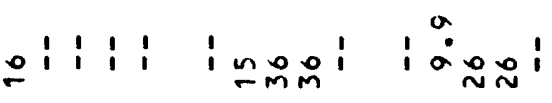

nmo

mion:

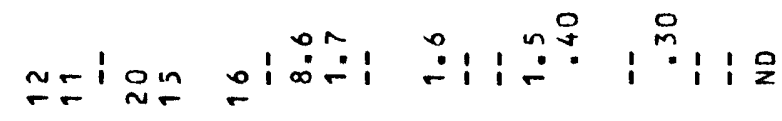

向部芸士

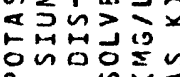

$\because 1111$ iñoi i̊n

in $\infty$

$n^{\infty}$

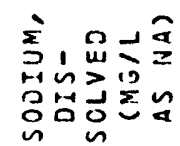

$\underset{2}{2} 1:$

'mogín

i

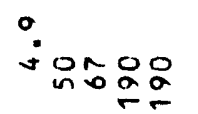

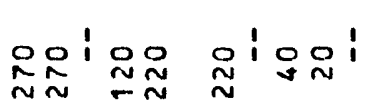

milóm ing i i

$\hat{\wedge} \hat{N}_{\infty}^{\infty} \tilde{\infty}_{\infty}^{-\infty} \tilde{\infty}_{\infty}^{\sim}$

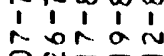

ivFF

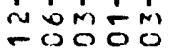

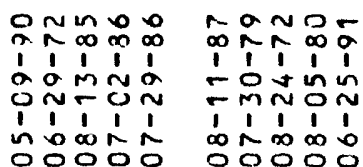

NOOOON O№̛

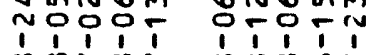

b́l

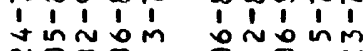

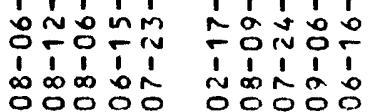

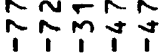

ratar

TTNN

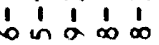




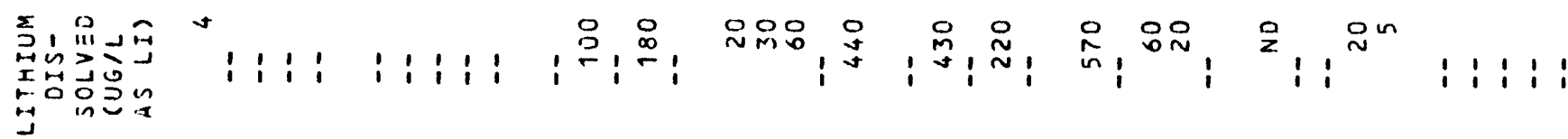

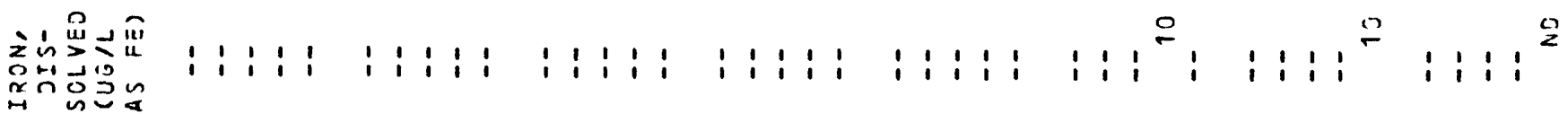

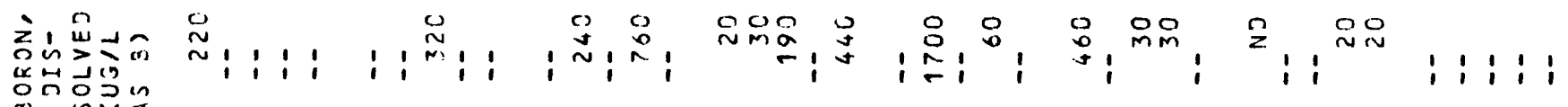

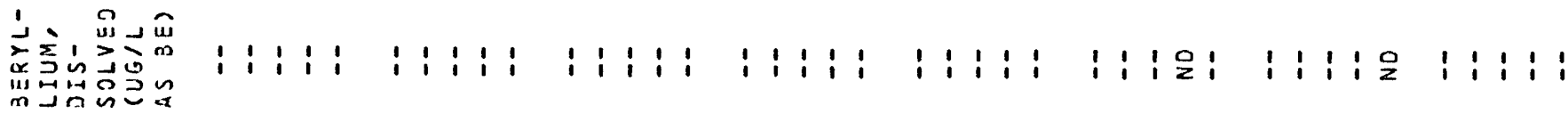

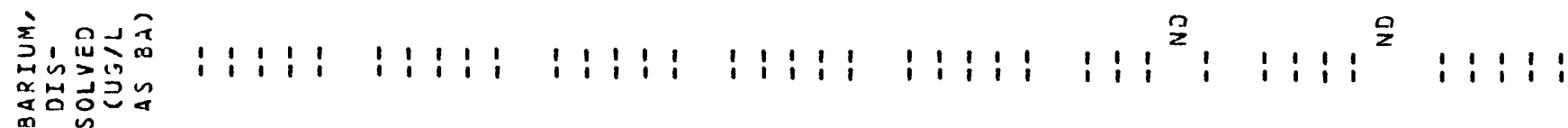

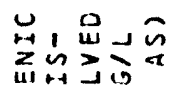
$\approx 0$ 잉

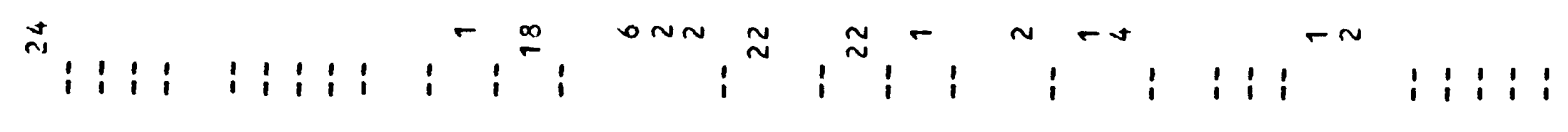

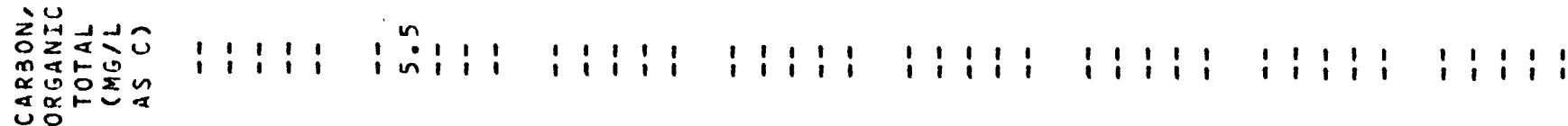

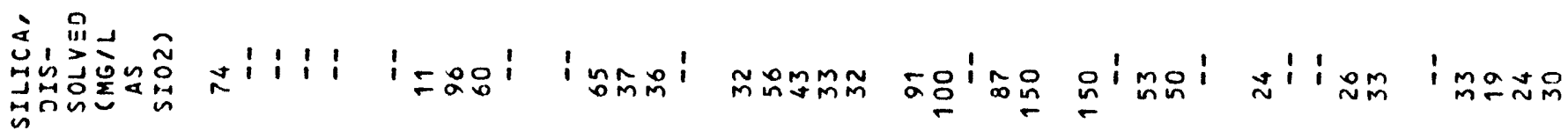

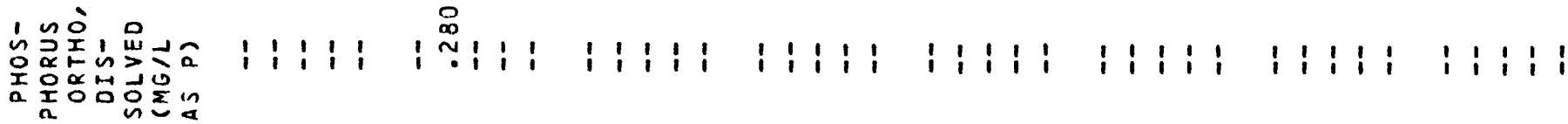

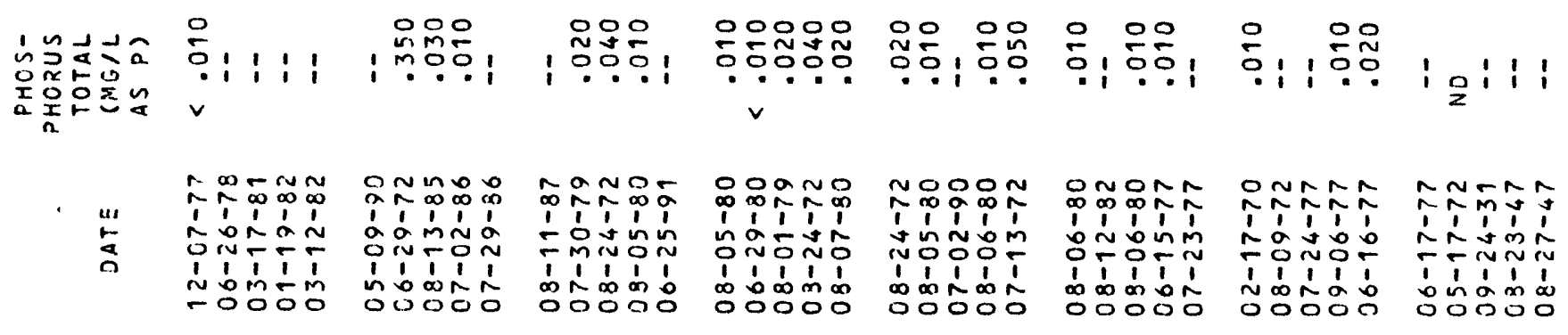




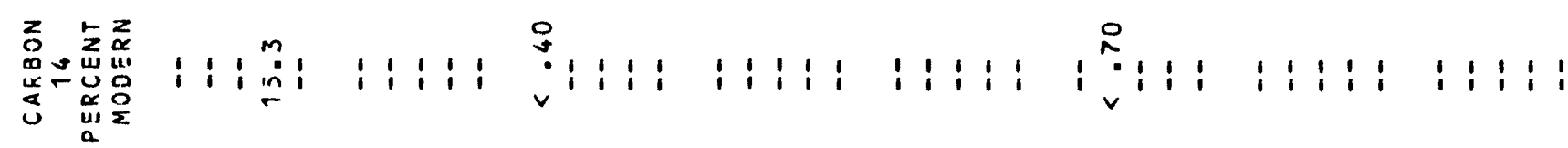

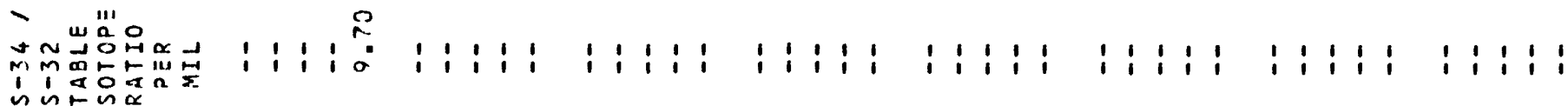

$\operatorname{tn} \infty$

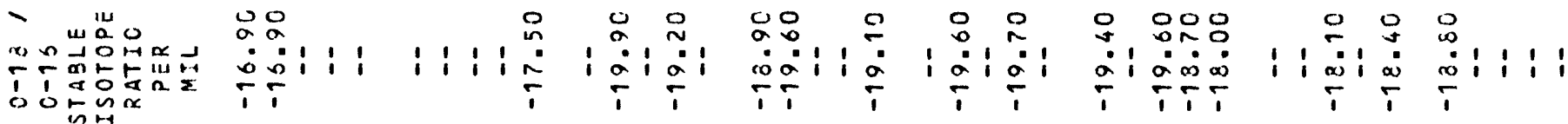

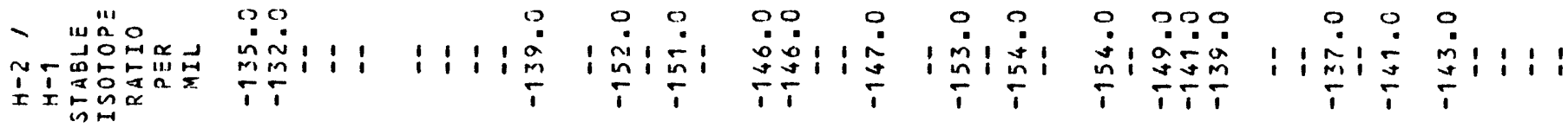

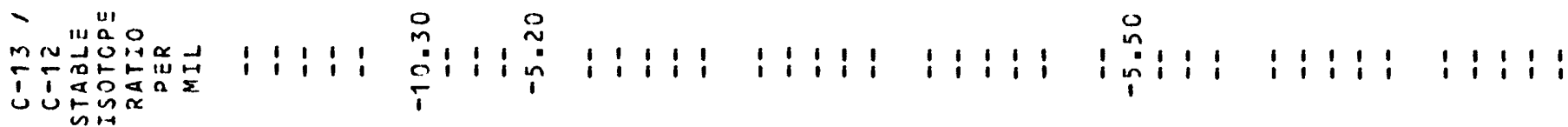

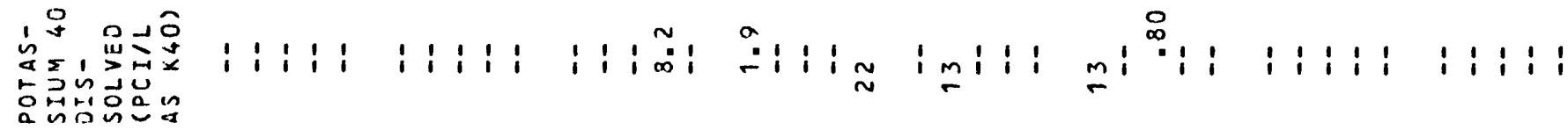

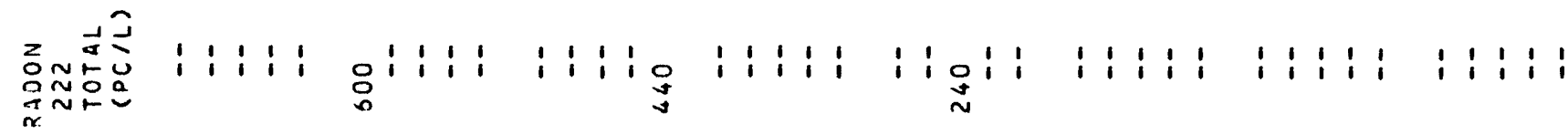
至憵

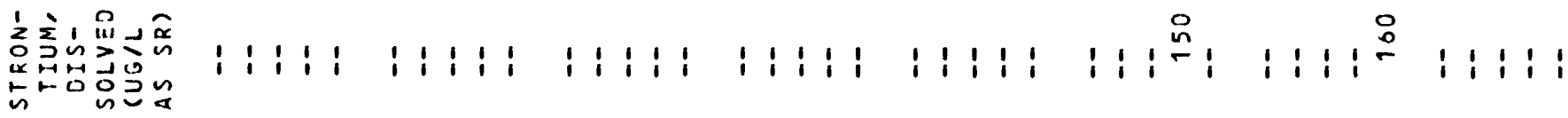

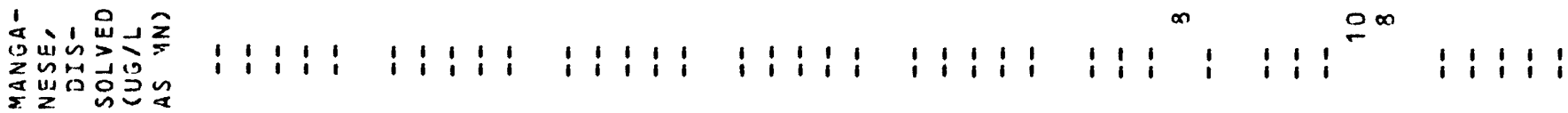

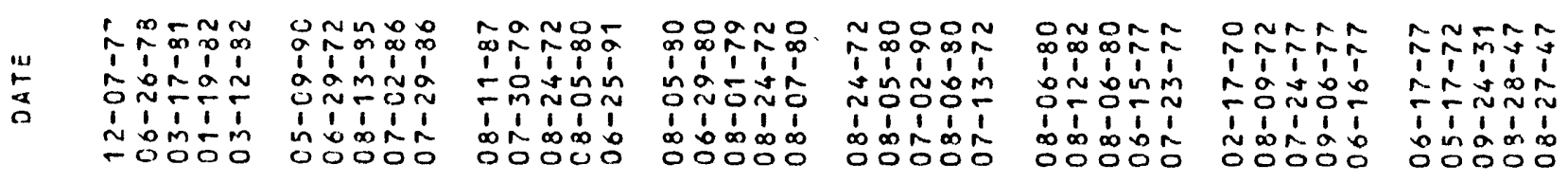




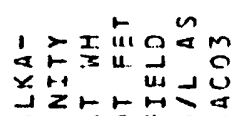

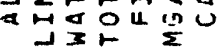

m n-r

ocoñ̃ñ

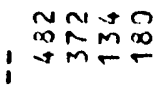

어요의

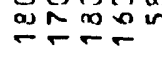

엄으요

ando요

ñ

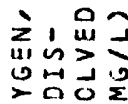

x

$1: 1:$

111111111

i: $1:$

$x$ 我公余点

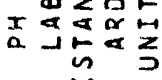

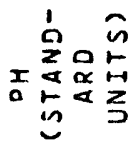

14

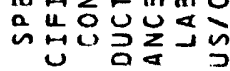

,

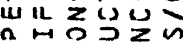

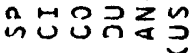

$\propto u \propto S$

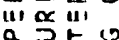

III

$\stackrel{n}{a 11}$

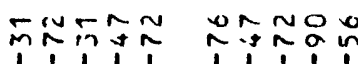

nos o on

TTNTF MTTT

gud

us o ou?0?

管 : in

on $n$ 응

in minis

in o ono

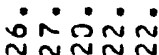

0.000 iñon

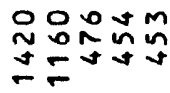

min

mand

$\bar{N} \tilde{N} \tilde{N} N$

o I Mm

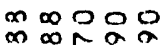
mMñ 둔

Tîñ

$\geq m$ nm

1505\%

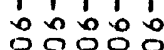

능

in $\infty 11$,

$\sim m+\infty$ in

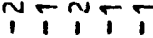

ผ

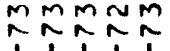

' 1 in '

voooo

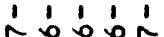

กิํํㅇำ

nomoo

00 in 0

in 0000 nin

Ti in $\min \alpha$

무요

$\underset{\substack{m \\ i-1}}{n}$

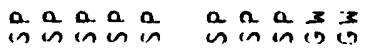

$3 \frac{5}{19}=10$

a $\begin{aligned} & \text { in } \\ & \text { in }\end{aligned}$

$\frac{\pi}{10} \times 30$

mañ

î 1,19

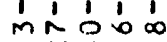

Tim5

ํํำ

$005 m-4$

कo 100

in mon

눈

m not

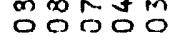

*

$m$ in $-x r$

-

in in 0 m

o o-0o
3333

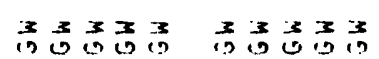

rĩ̃̃

MMMMm MmMmm

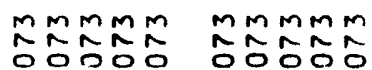

Mmmmm mmmmm 0000 00000 0.0000 00000

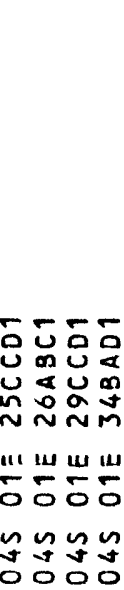

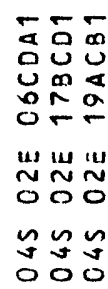

$\begin{array}{ll}\vdots & \vdots \\ 0 & m \\ u & 0 \\ 0 & 0 \\ v & \sim \\ 111 & 111 \\ \sim & 0 \\ 0 & 0 \\ n & n \\ 0 & 0 \\ 0 & 0\end{array}$ 


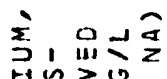

in

in 요

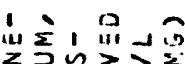

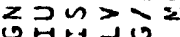

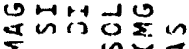

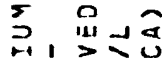

Un 10

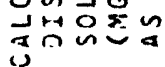

in $\vec{a} \neq \hat{m}$

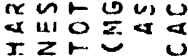

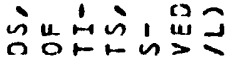

ค은

过现出口证

i

ณ 20. in

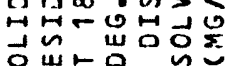

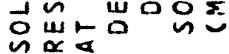

劭, 品山

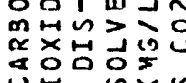

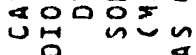

t16

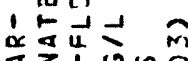

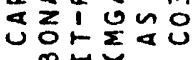

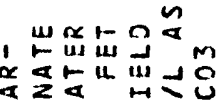

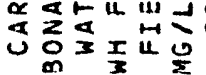

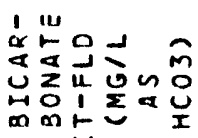

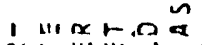

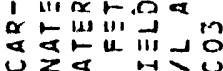

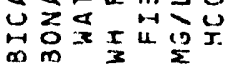

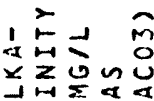

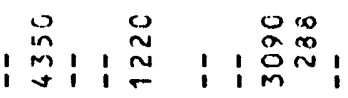

a.ton

$\operatorname{arman~ogran~}$

$1,1 \div 1 \mathrm{~m}$, v

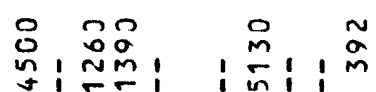

1

lo! i

ími i

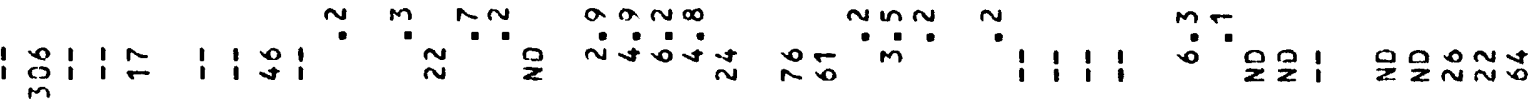

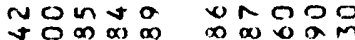
N

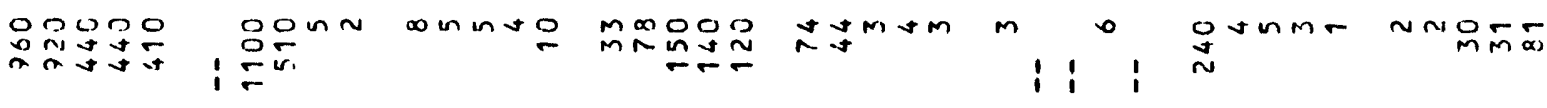

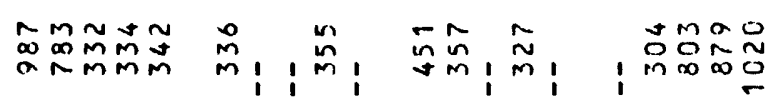
! minm ñm! :

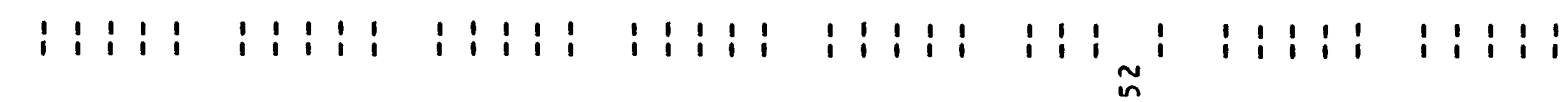

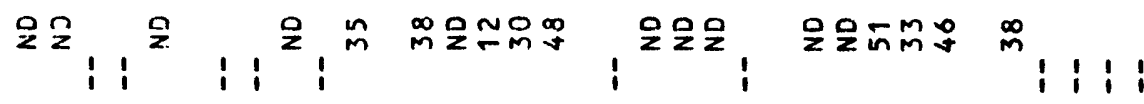

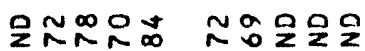
1:1:1 $1: 11$ i: $1: 1:$ i $1: 1:$ : $1:$ : 1 1 1 1 191

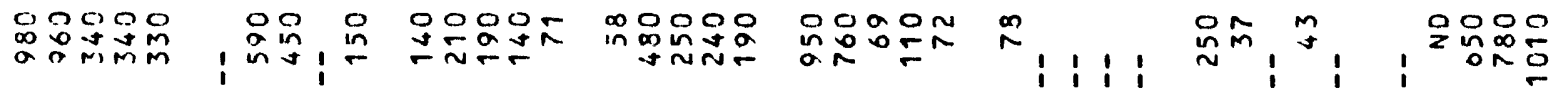

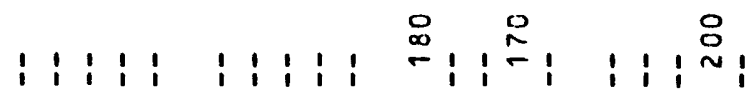
온 : i :

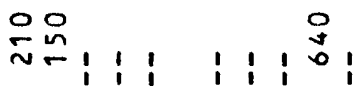
$-N-n N$

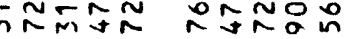

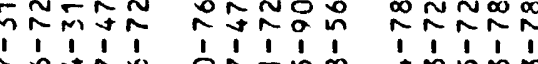
oÑ PTNTT MTTFT 에의 bong

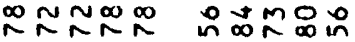
n'ms 1 's in T⿻ กTกT 잉ㅇㅇㅇㅇㅇ

年 + ^o: TกMT

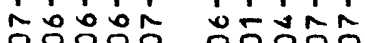

엉ํํㅇำ

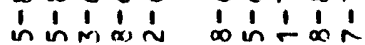
NTTYT TYTTY

1
$\infty$
0 


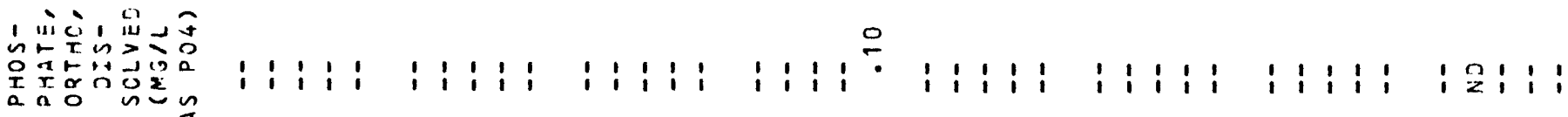

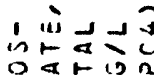

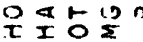

a. a.

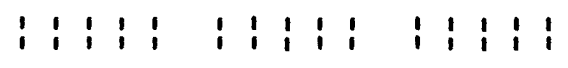

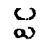

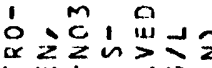

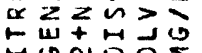

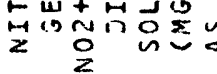

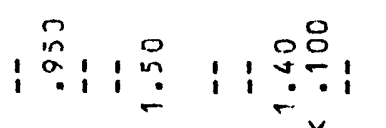

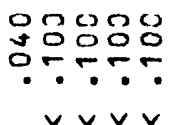

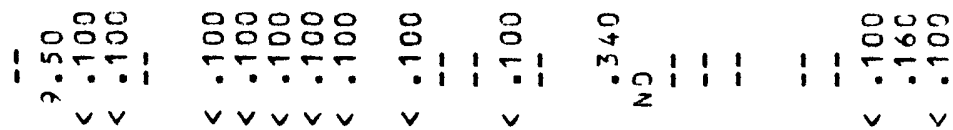

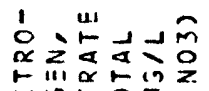

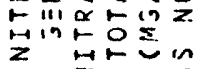

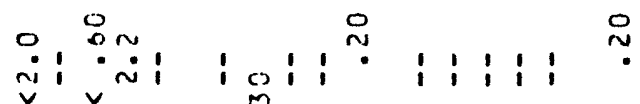

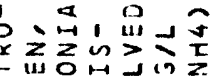

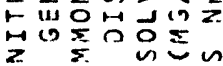

$i: 1: i: i: 1 \stackrel{9}{\div}$

自势+u

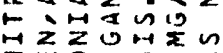

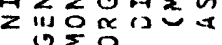

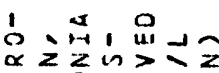

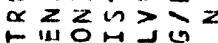

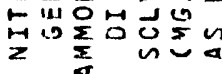

1:1 1:1:1:1:

$1: 191$

: 1 : 1 :

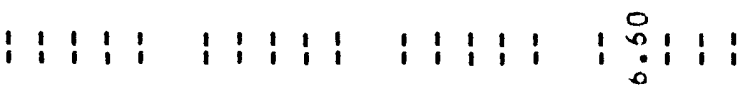

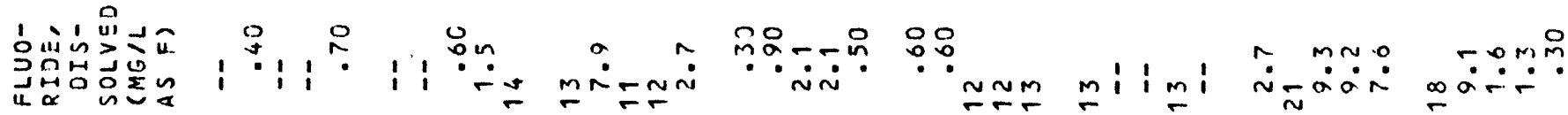

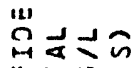

u. 둥ํำ

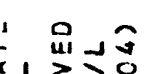

造的立它品

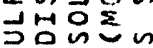

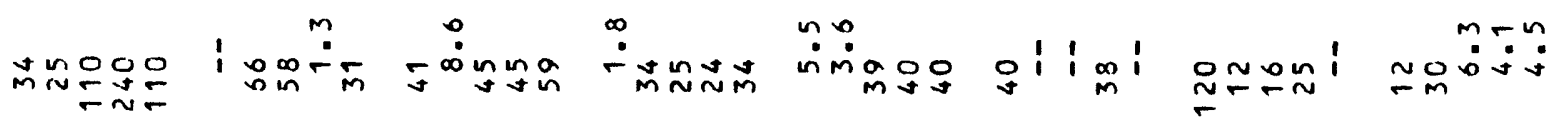

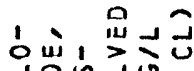

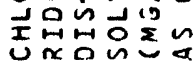

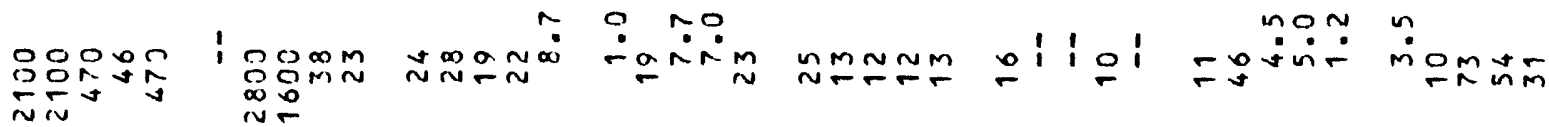

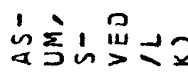

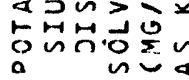

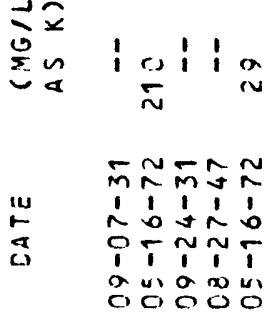

i í⿴囗十

mån

$\stackrel{0}{\infty}, \stackrel{0}{\infty}: 1$

๓ேஸ்

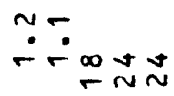

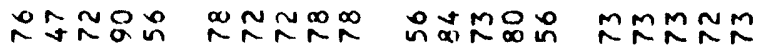

1,111

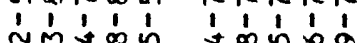

NTNT NTOTi

"TTFT TTYT

ㅇํㅇํㅇㅇํㅇ

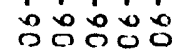

มุด

$\stackrel{\infty}{\sim} \underset{\infty}{\infty} \tilde{\infty}$

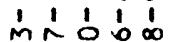

$\div \dddot{1} 1 \div \div$

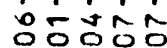

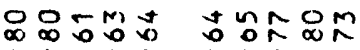

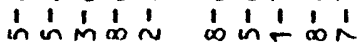
NTTPT TOTT

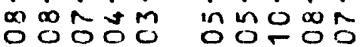




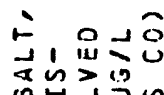

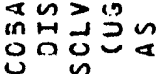

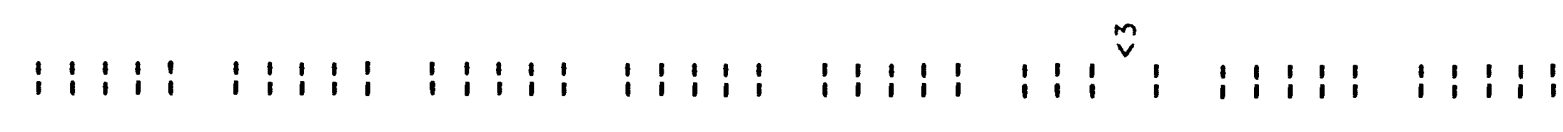

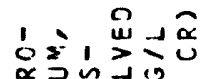

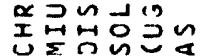

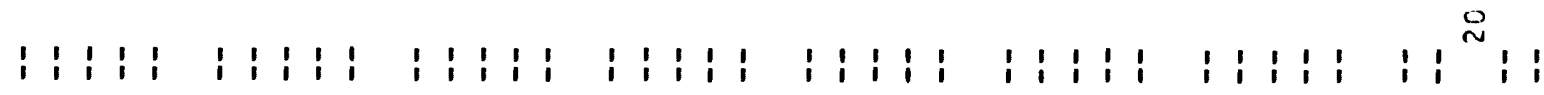

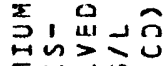

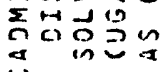

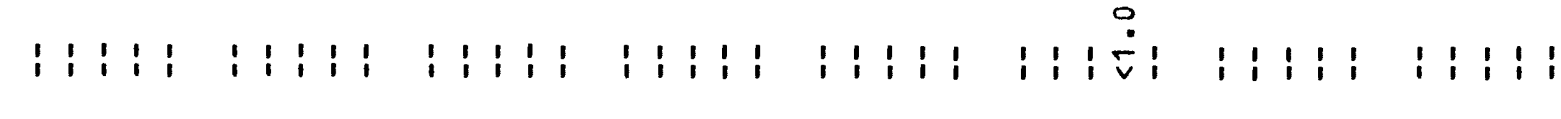

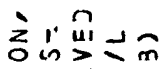

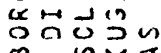

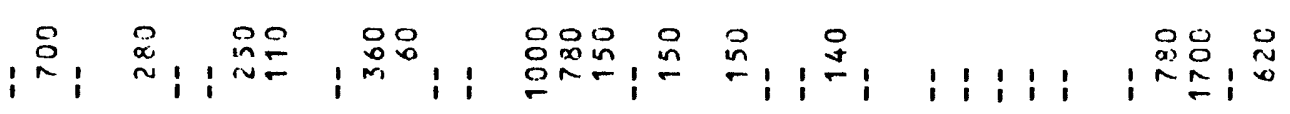

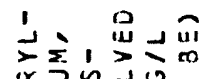

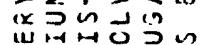

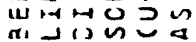

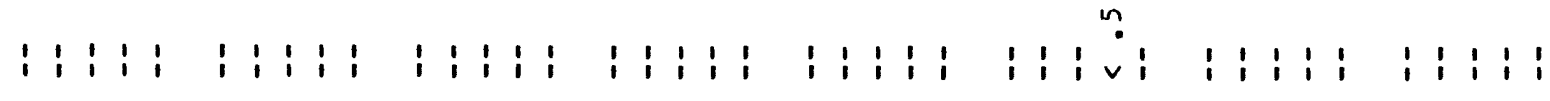

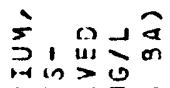

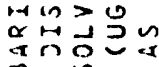

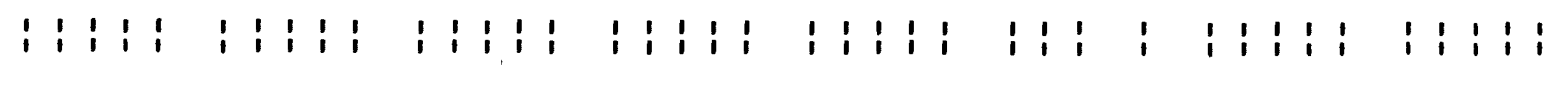

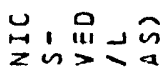

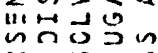

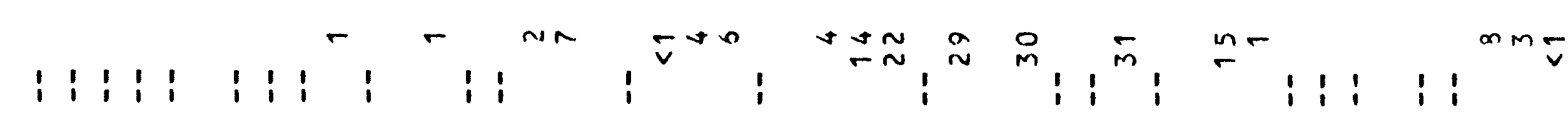

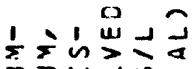

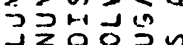

a

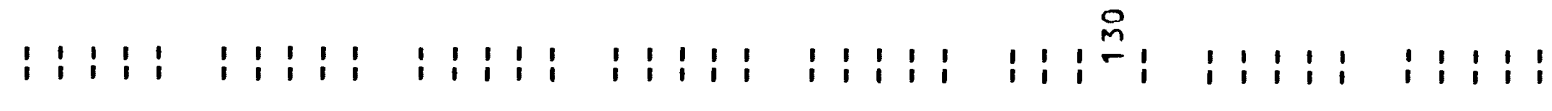

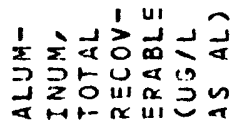

i i i i i i i i i

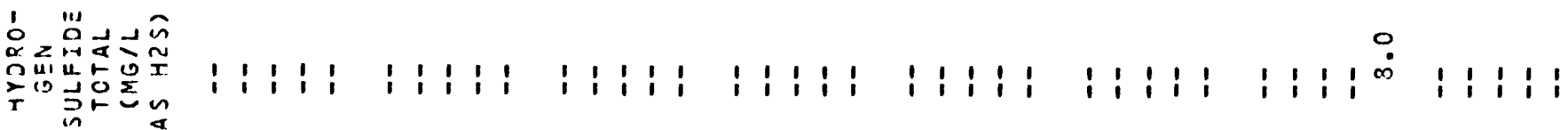

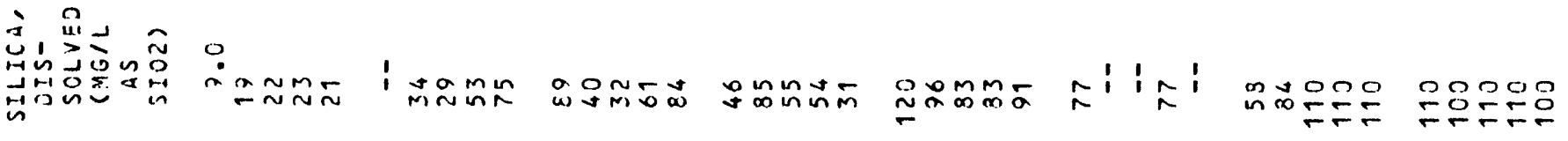

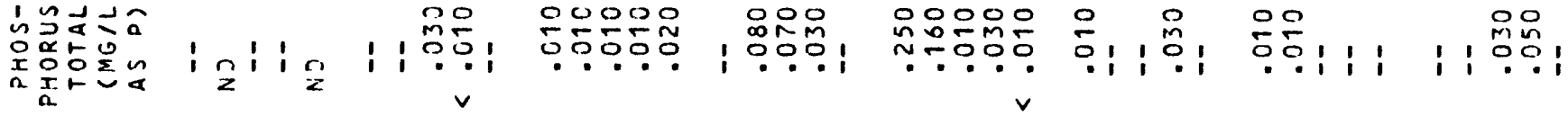

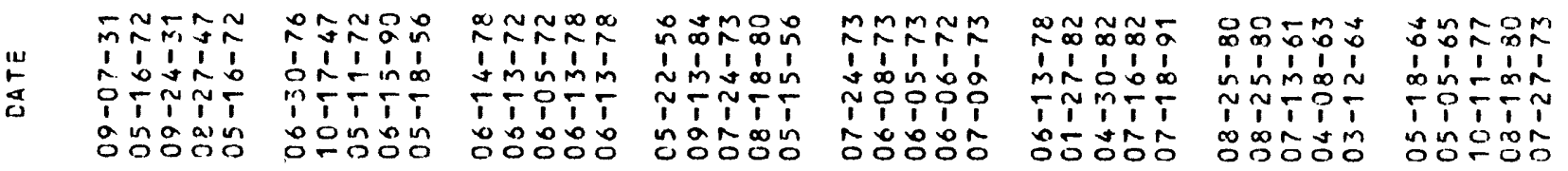




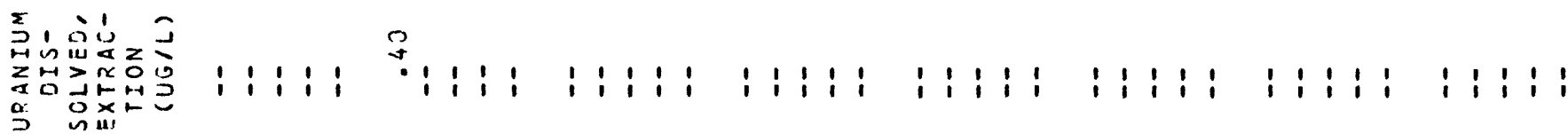

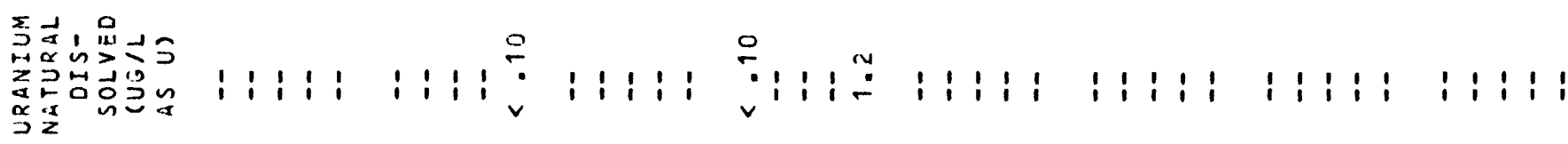

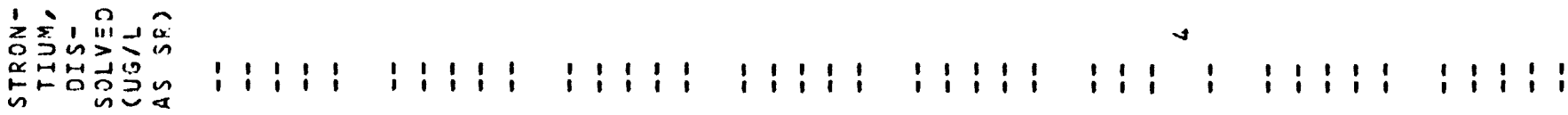

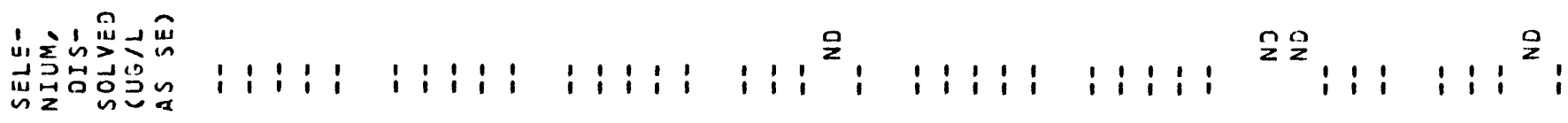

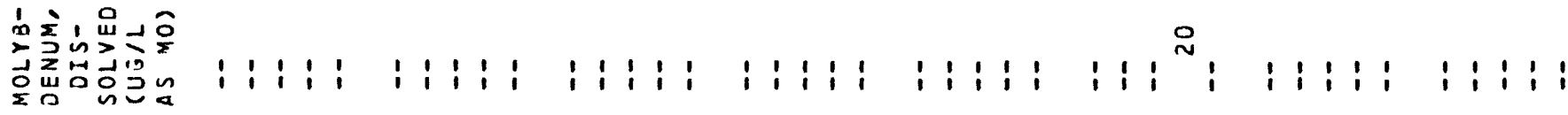

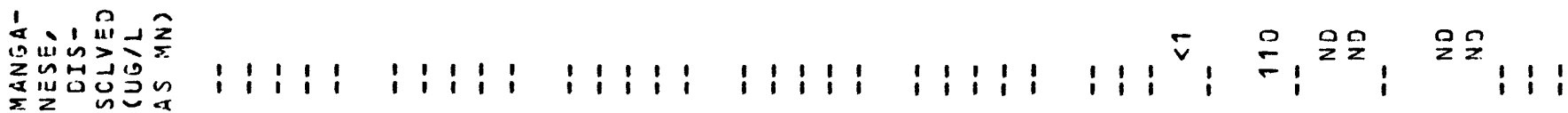

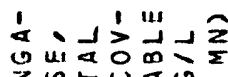

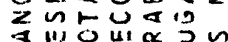

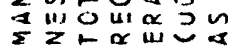

$\sum_{i \rightarrow 1}, \prod_{i=1}^{0}$

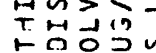
to 03

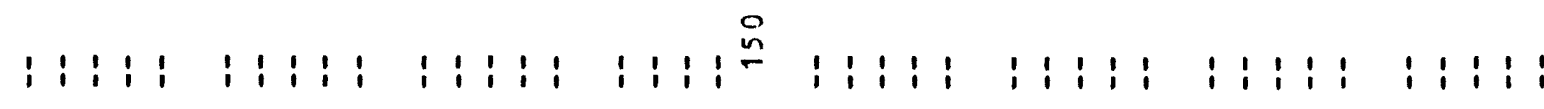

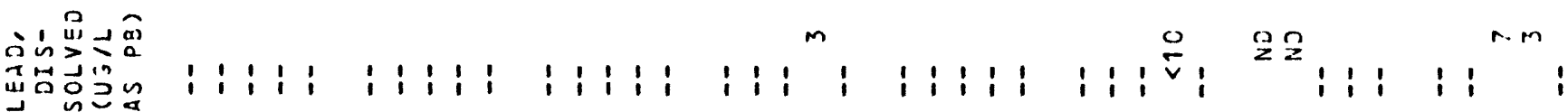

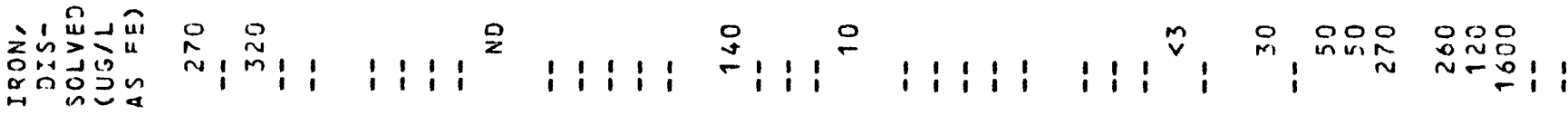

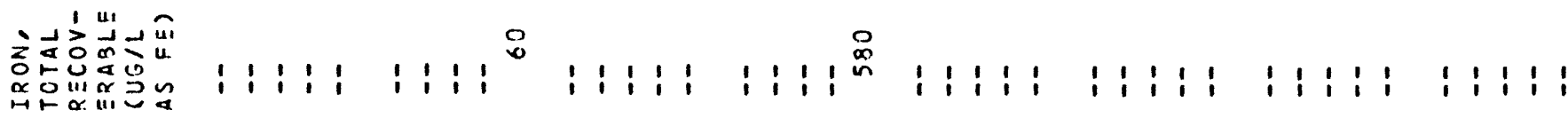

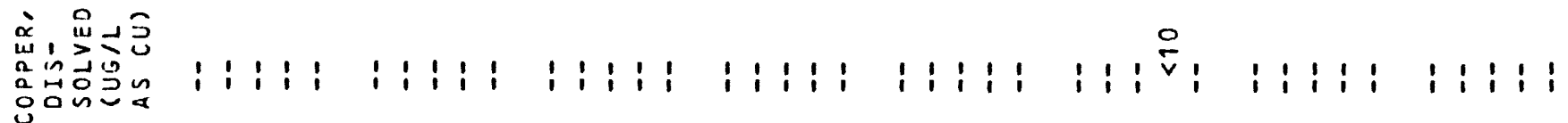

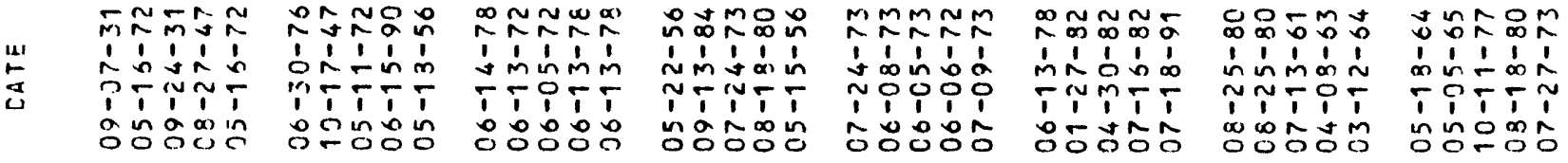




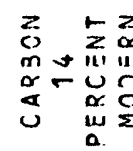

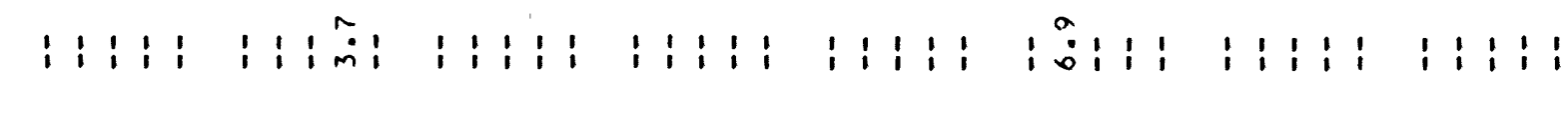

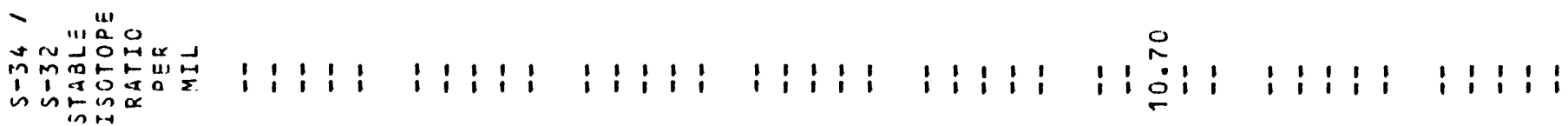

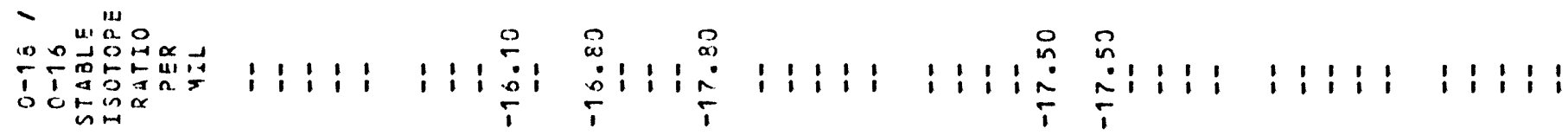

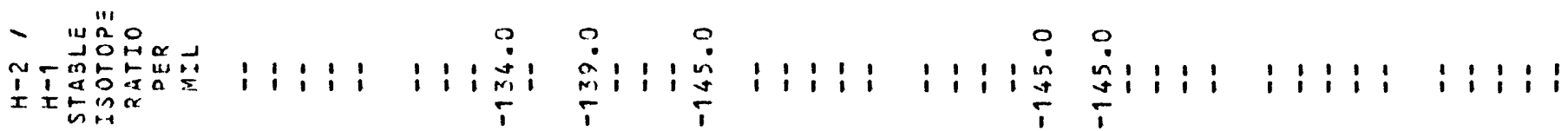

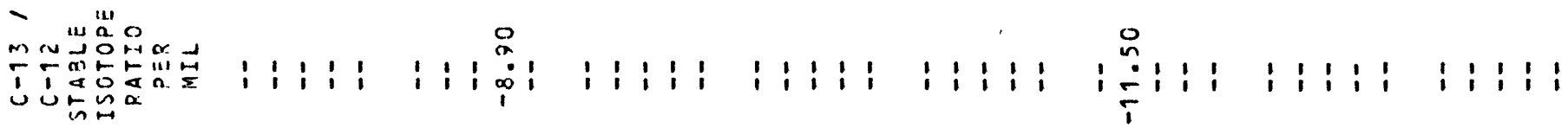

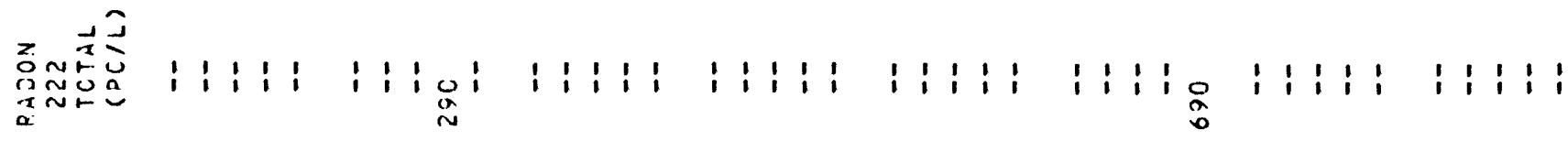

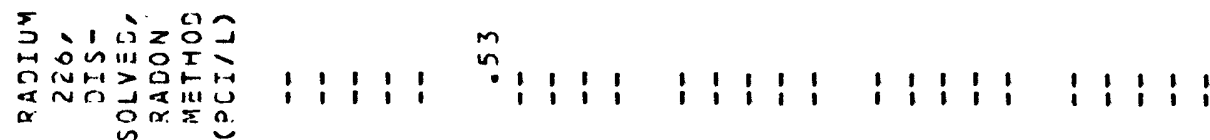

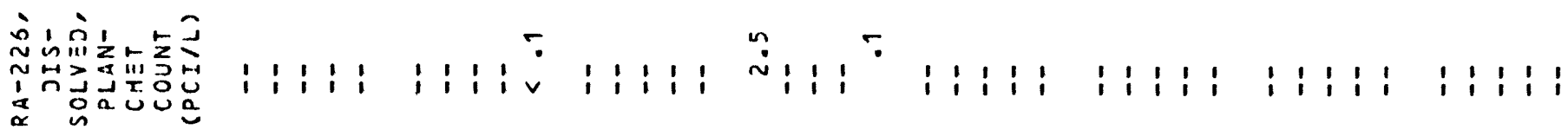

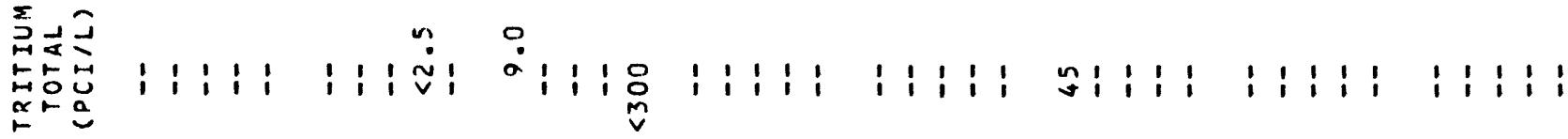

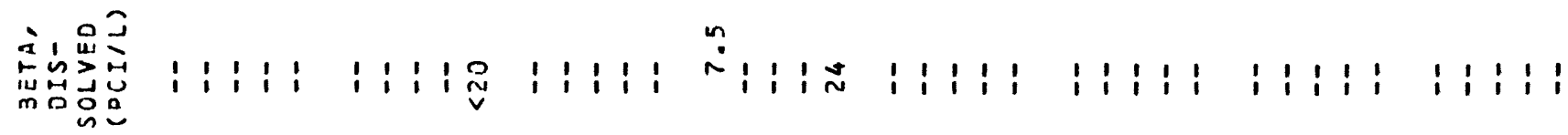

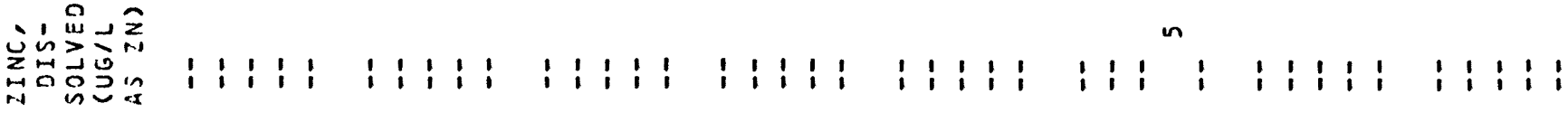

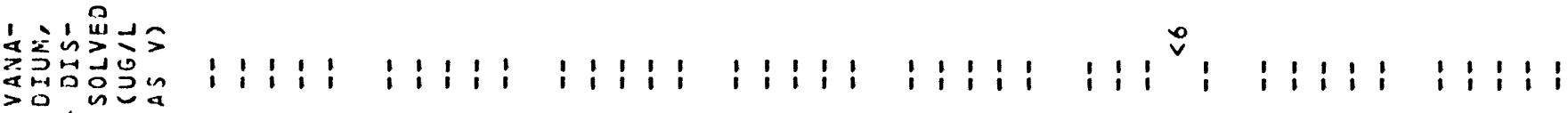

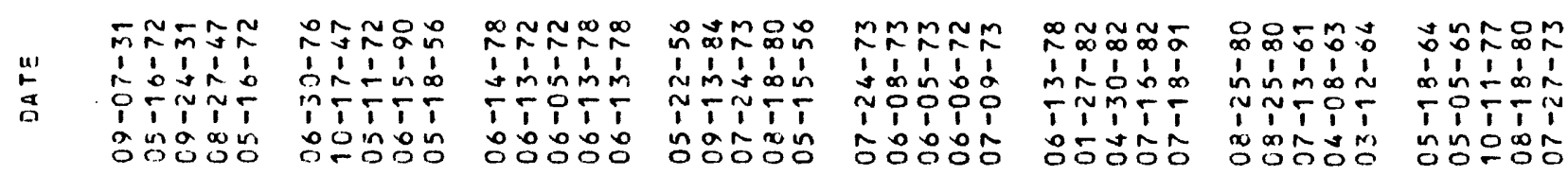




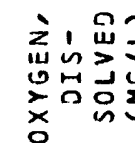

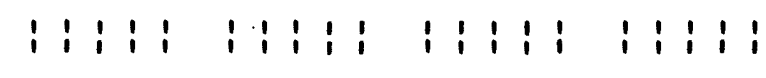

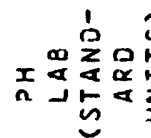

总余占

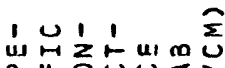

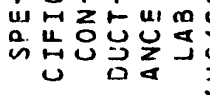

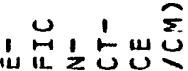

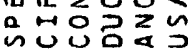

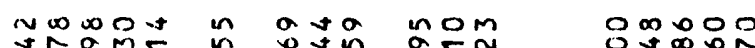

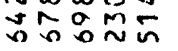

on

11111

$\because \dddot{i}$

i: $1: 19: 1:$

: $1: 191: 11:$

$\ddot{a}: 1 ;: \stackrel{\infty}{a} 1, \dot{a}$ !

1) $\propto$ U

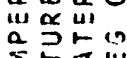

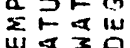

00000

iinis:

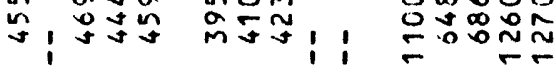

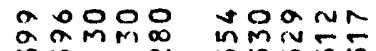

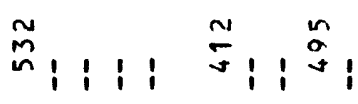

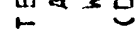

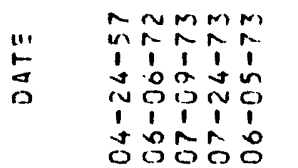

$\infty-m, n$

00000 Dinmon

nnono

oognu noo non

0.0000

o oinoin

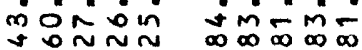
-

ition matán

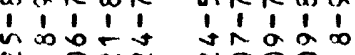

interis

NiPi:

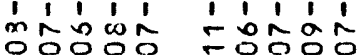

mmomm

Tิton tis

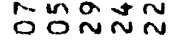

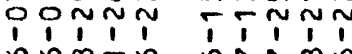

or̃́n

$\infty \infty \infty \infty \infty$

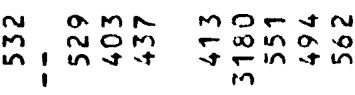

$\stackrel{n}{n+1}$

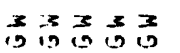

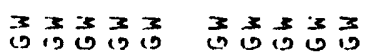

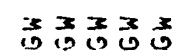

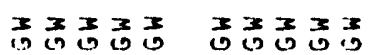

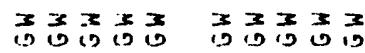

2
3
3
0

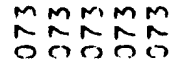

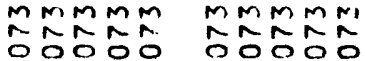

$m M m m$

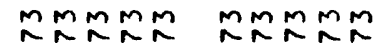

mMmmm mmmmm

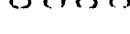

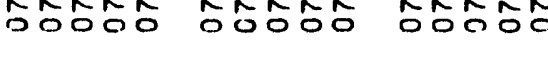

ooooo oogóo

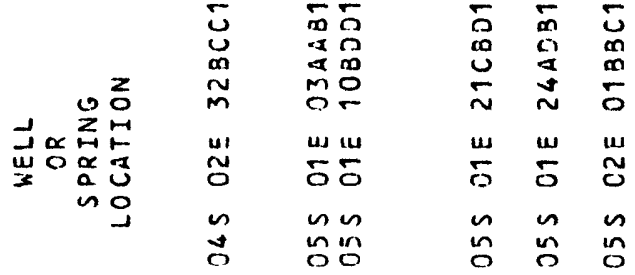

\begin{tabular}{|c|c|c|c|}
\hline 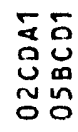 & $\begin{array}{l}\bar{a} \\
0 \\
0 \\
m \\
m\end{array}$ & 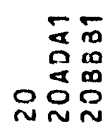 & $\begin{array}{l}5 \\
0 \\
a \\
\sim \\
\sim\end{array}$ \\
\hline$\underset{\sim}{\sim} \underset{\sim}{\sim}$ & $\stackrel{w}{\tilde{\sigma}}$ & m茨出出 & m \\
\hline $\begin{array}{l}n \sim \\
\sim \\
0\end{array}$ & n & 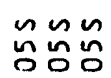 & n \\
\hline
\end{tabular}

๓

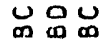

兄m

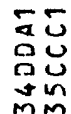

แ

Mm心

um

nูn 
$\sum_{i} \stackrel{0}{\rightarrow} ⿻$ ज的出

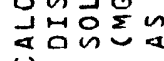

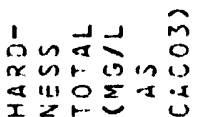

的北的, 合 not $n=1$

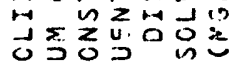
은라

ing "II

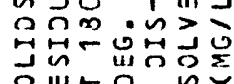
in $x=$

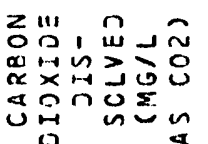

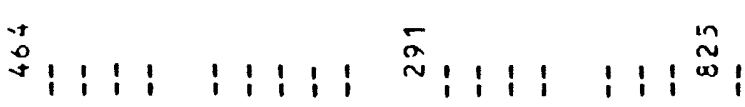

inm M

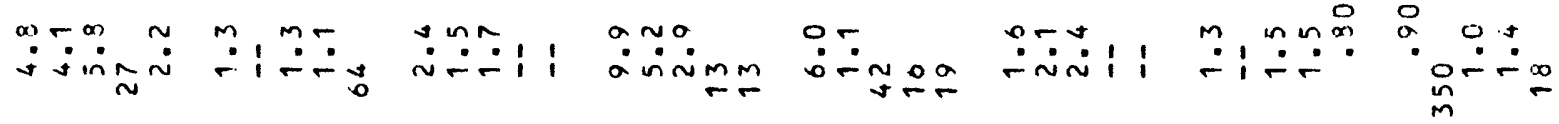

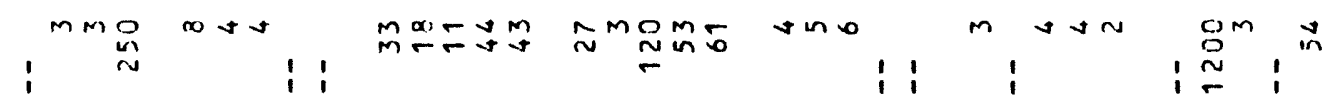

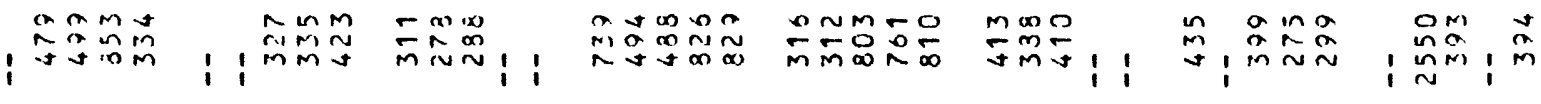

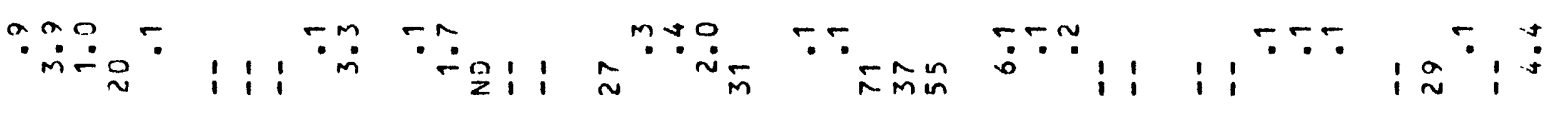

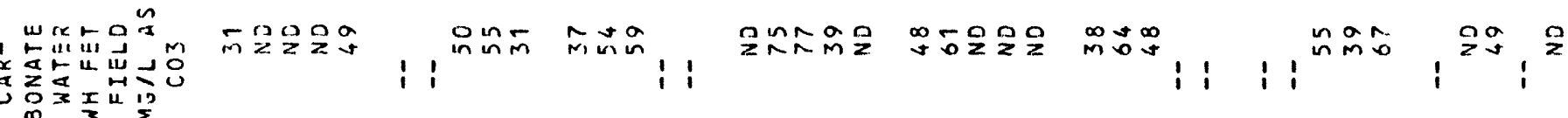

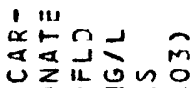

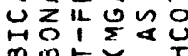
an

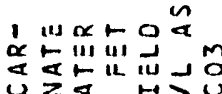

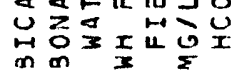

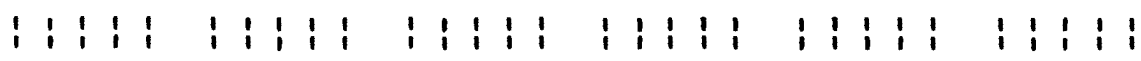
$\underline{0}^{11} 1: 11: 111$

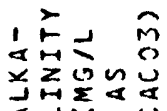

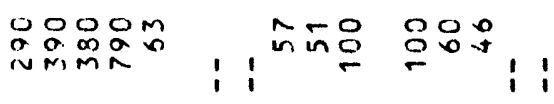

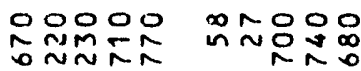
$\underset{n N o}{N}$ $1 i^{m m n}, i^{\circ}, \stackrel{0}{N}$ 可留区过

i:1:i i:

$: i: i:$ 1 总! $1: 11^{\circ}$ $1: \stackrel{\stackrel{D}{\sim}}{:}:$ 总品, 竞

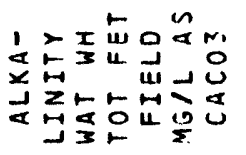

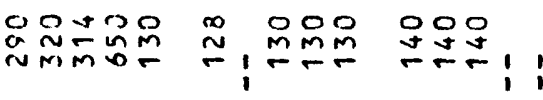

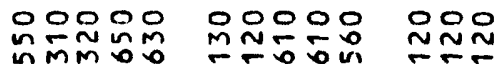

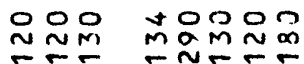

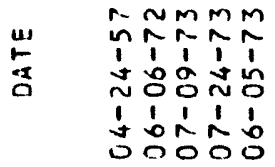
m-MON MNMN क

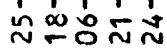
ciciticis

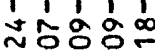

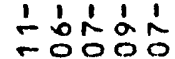

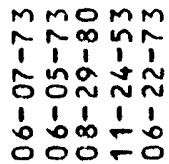

mmmom TิT人一 $\sim \underset{N}{2} \tilde{N}$

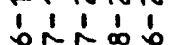

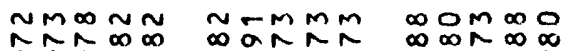

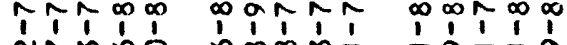

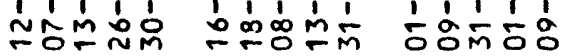

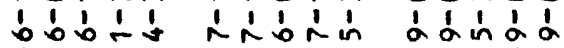

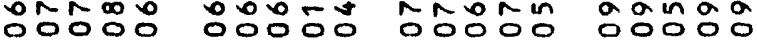




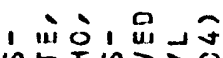

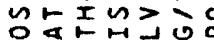

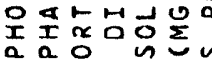

본ㄷㅇ

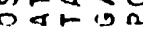

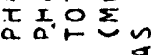

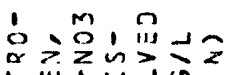

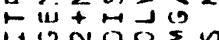

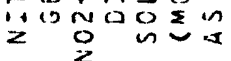

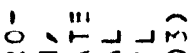

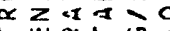

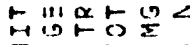

$z z^{2}$

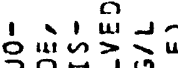

保证

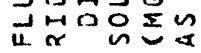

III

吕求さ会

녹은호

गे

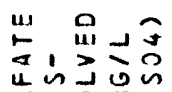

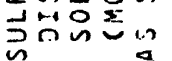

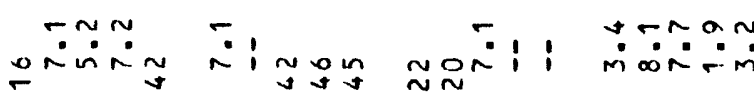

$\stackrel{0}{4} 11: 1,1: 19$

ỡ்"

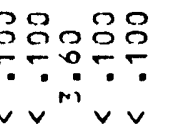

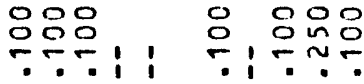

$\vee \vee v$

$v v$

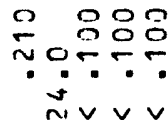

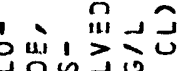

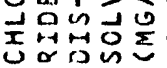

nunom olimum n-o! !

的文, 品十0

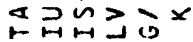

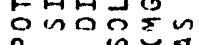

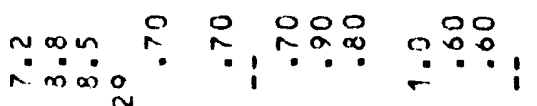

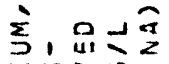

$\min >\cos$

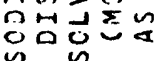

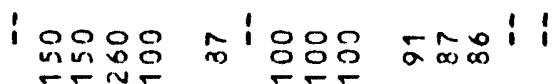

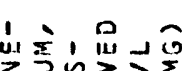

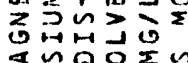

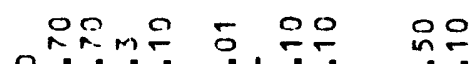

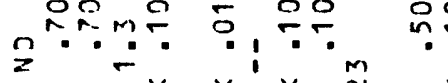

n NMMM

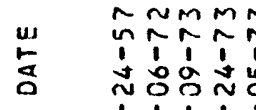

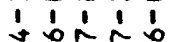

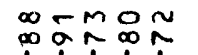

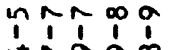

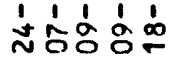

$\stackrel{\infty}{\sim} \div \frac{\infty}{\sim} \approx$

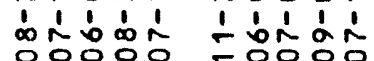

i i i

ํำㄴ. ํำํำ

$\div 2$

운ำ

tinoom

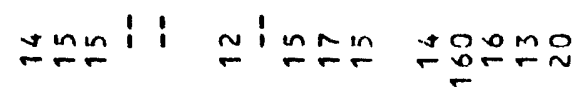

$\sim \dddot{0} \sim \stackrel{\infty}{\sim}$

$\because ?$

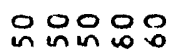

$\ln \operatorname{mog} 0$ $\stackrel{\sim}{\sim} \sim \sim \sim ⿻ 上 丨$ NMN

응ㅇㅇㅇ

: i !

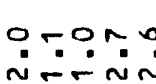

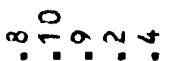

은운

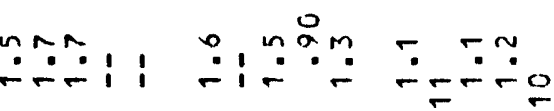

$m \sim M N-$

mmomm

1ิ1

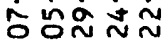

b́ำ Tî́,

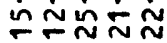

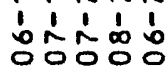

I:1 $1:$ i:1:1 $1: 1: 1$

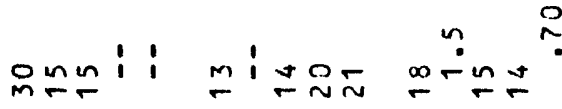
¿̇: $1: 11: 1: 1$ - O000 000-0

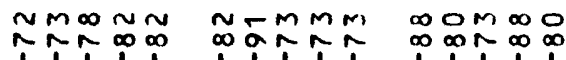

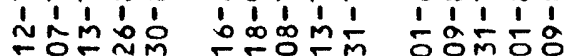

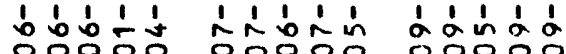


学, 品

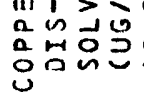

i: : :

: $: 1:$ :

I:

i: $1: 1$

i: $1:$ :

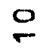

$\therefore$, 品

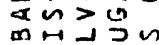

봄ํำ

$1: 1: 1: 1: 11$

:1:1: :1:1:

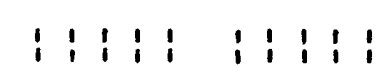

m

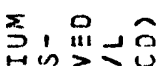

勿

远跑

$1: 1: 1: 1: 1: 11: 11: 1: 1$

1:1:11:11

之文荘十

on>

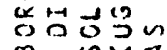

1303

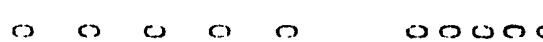

\section{5c 0}

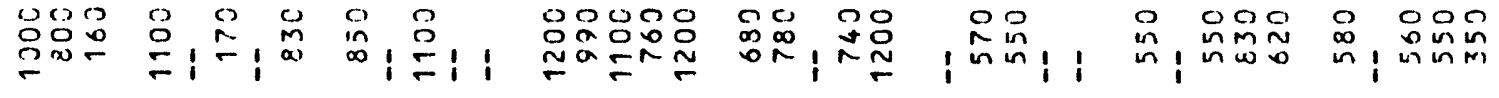

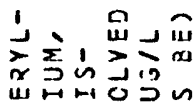

$1: 1: 1,1: 14$

: $: 4:$ :

: : : : :

: : : : :

i i i: :

?

m尸

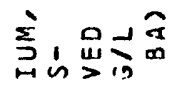

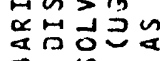

$1: 14: 4: 1: 4$ i: : :

i i i : :

i : : :

: i : : :

$\infty$ i: $1:$ : $:$ :

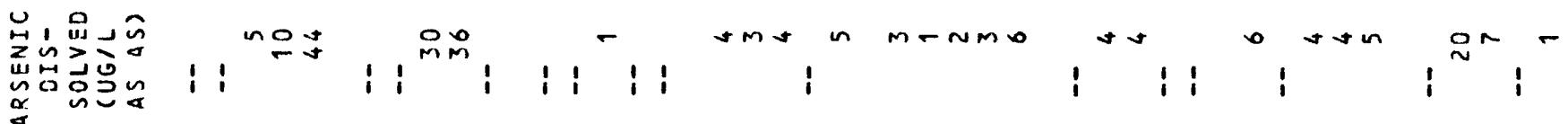

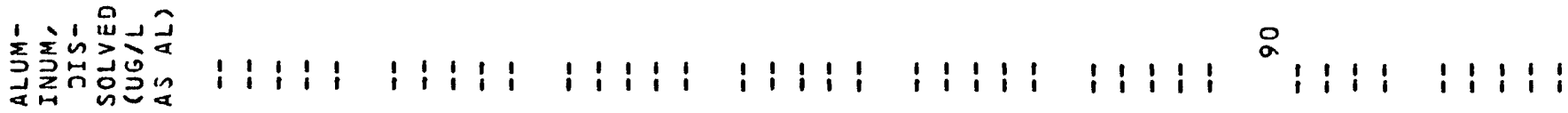

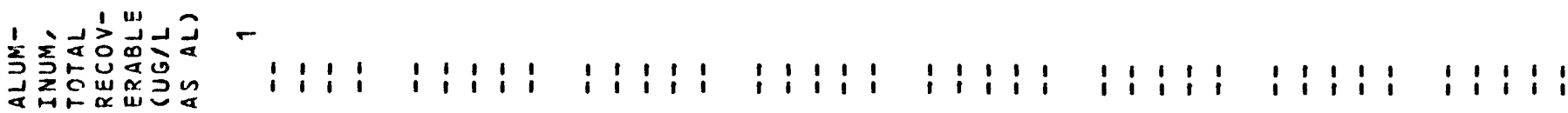

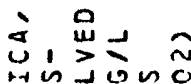

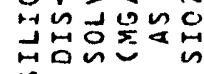

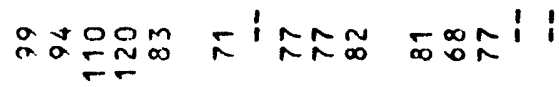

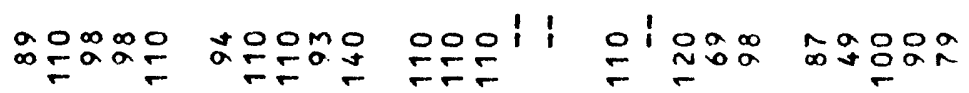

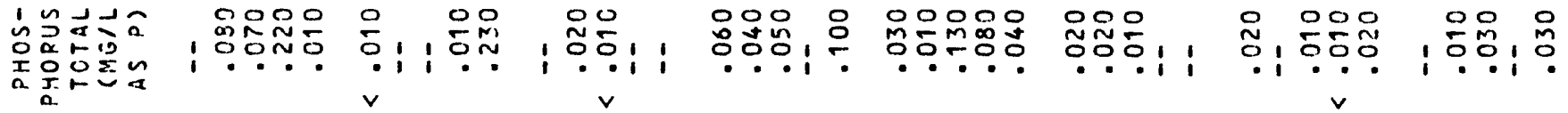

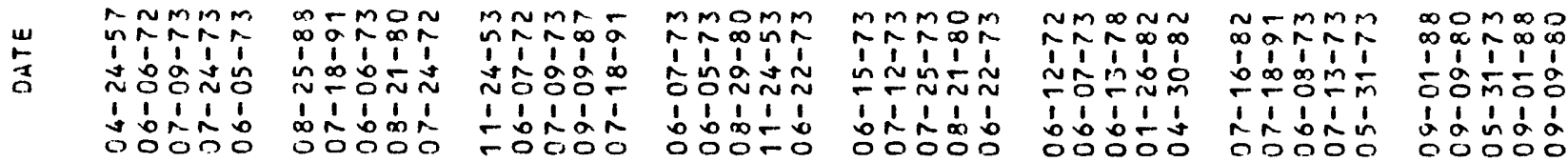




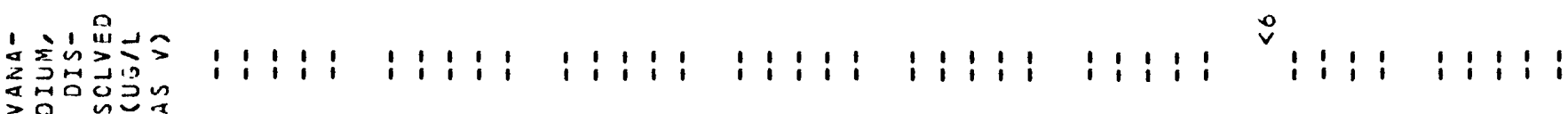

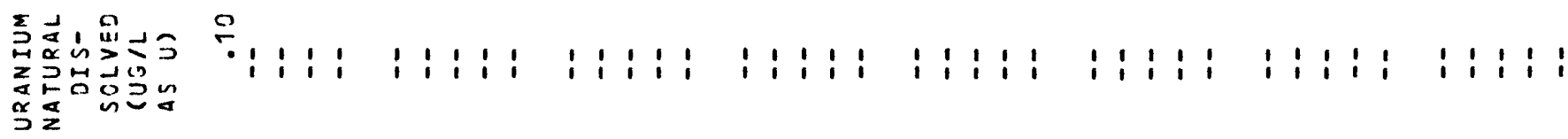

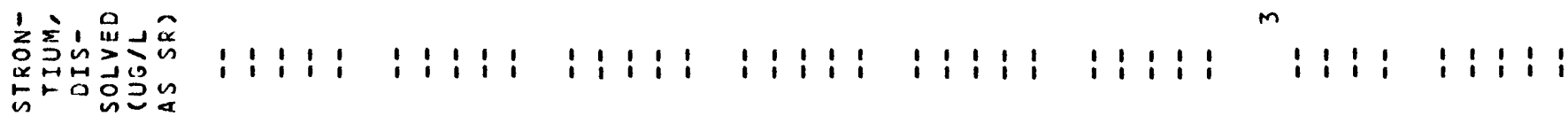

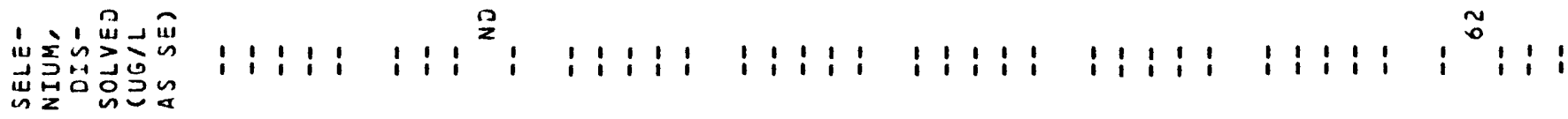

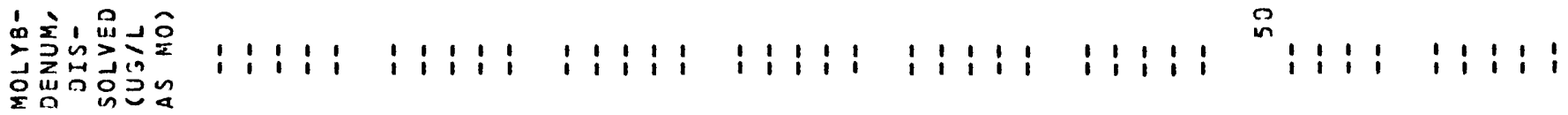

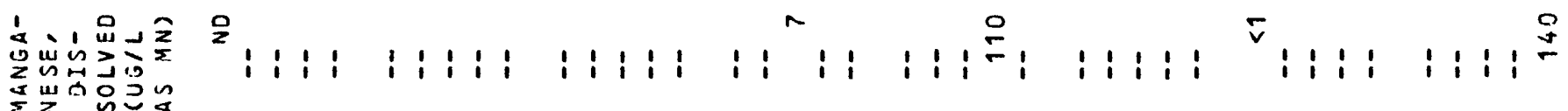

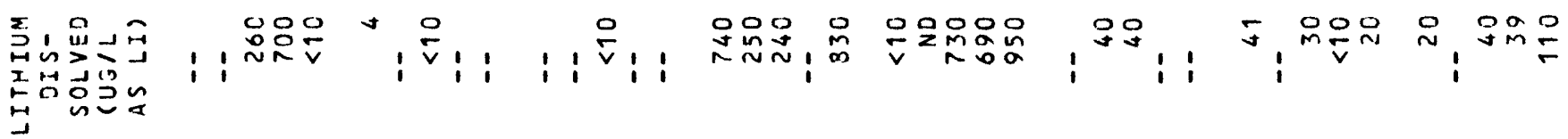

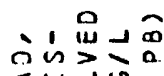

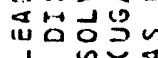

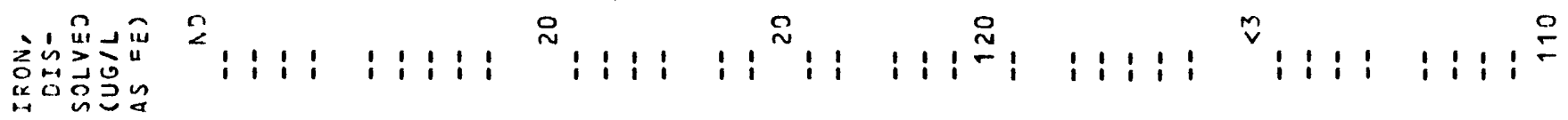

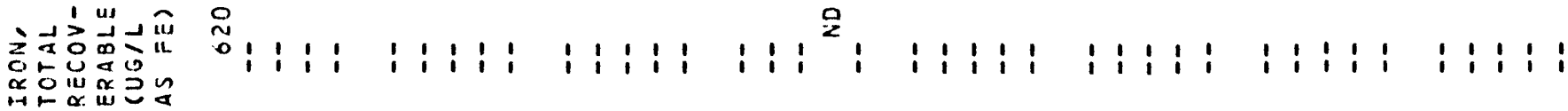

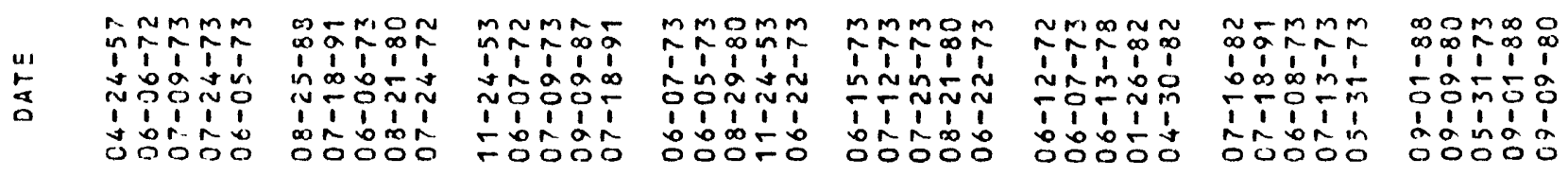




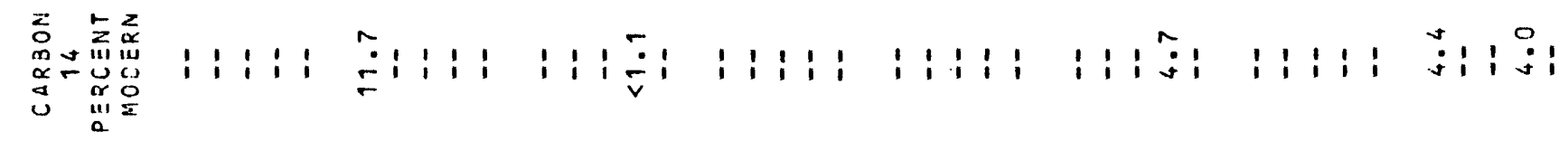

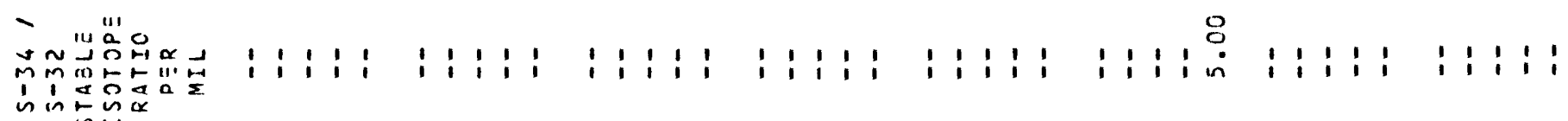

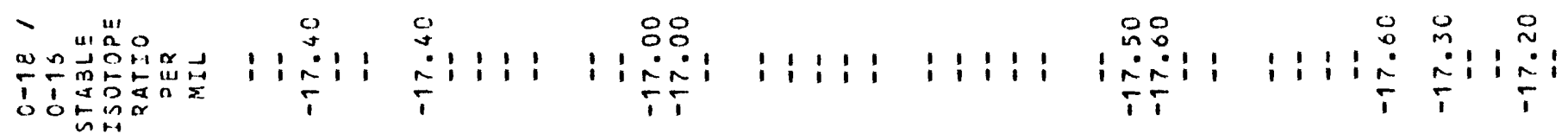

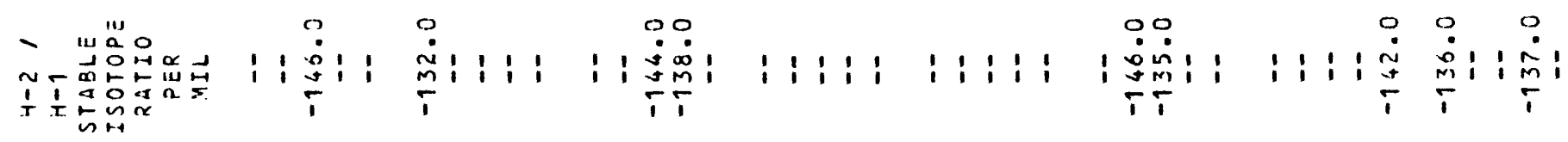

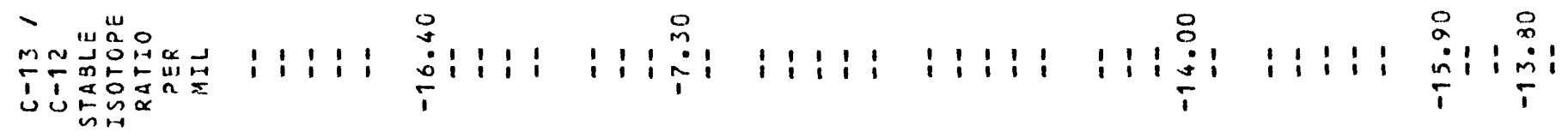

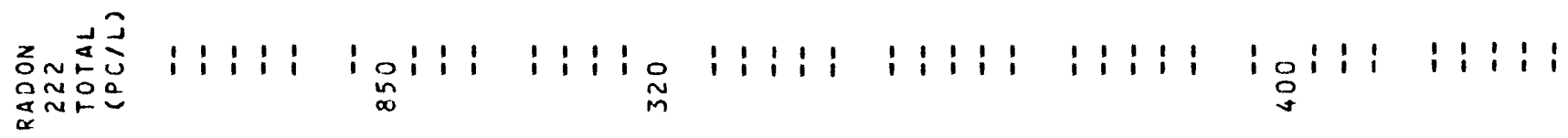

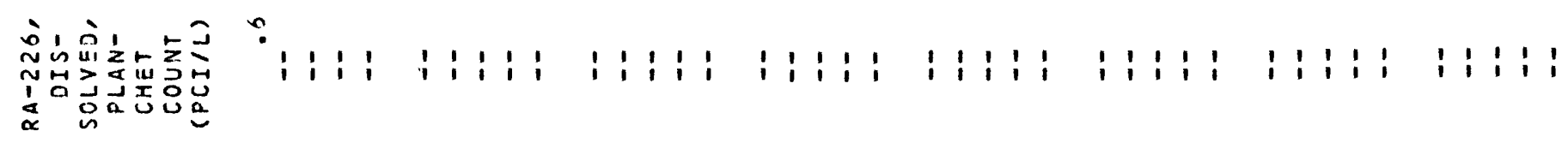

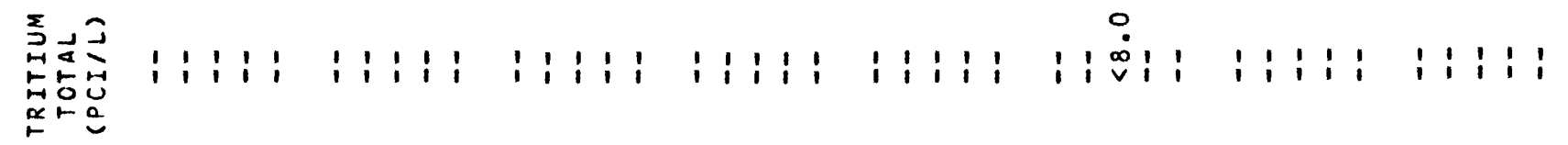

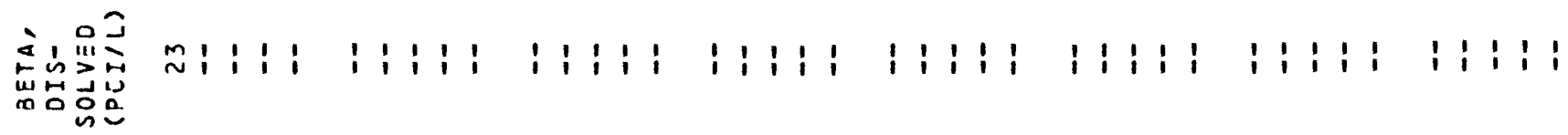

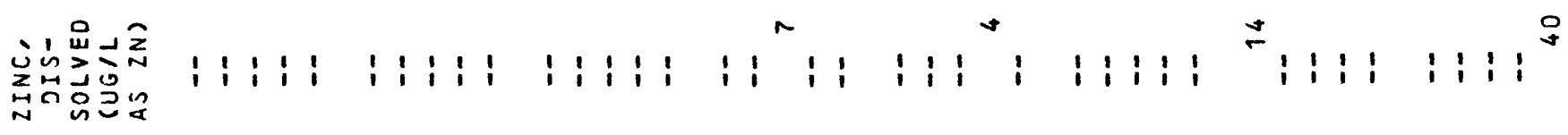

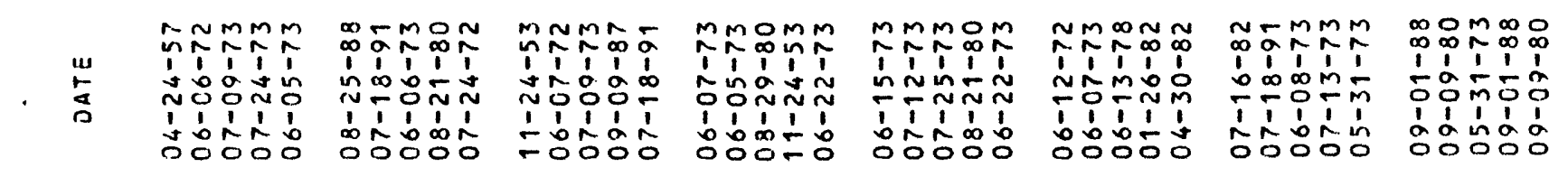




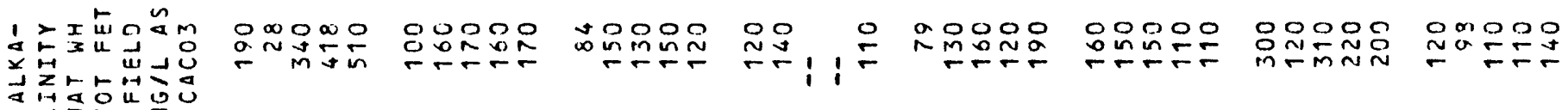

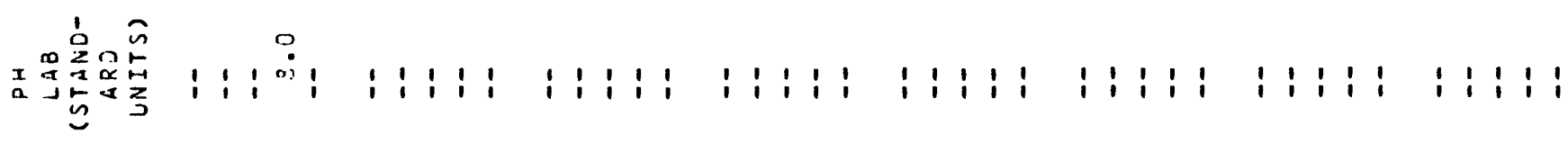

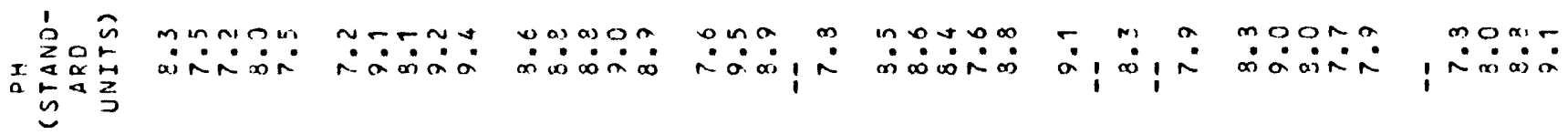

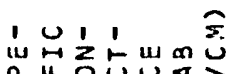

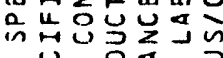

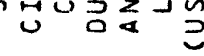

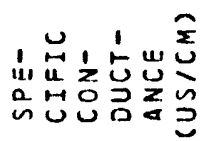

i $u \propto \hat{U}$

us $\cong$

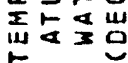

!"1

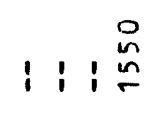

in o O O MO

o 0 w in rtr

*a

$i^{\infty}, i^{N}$

$m m \infty O n$

Nininiñ

品, 㔯, 古

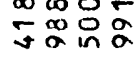

$\ln \alpha \operatorname{in} n$

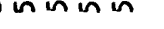

00000

$\dot{0} \dot{0} \dot{0}$

00000

onomo

inom o

N Nan N

車出! 定

$\operatorname{mingon}$

misiñ

nonom

mininis

o inino 0

ini-io

$\wedge$

$m \infty m-m M m N m m$

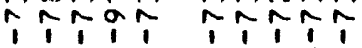

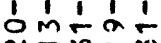

NFm-M Tm

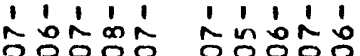

$m \sim m m m$

in

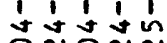

ำ

1.11 1

m $\sim \sim \sim m$

i

Nón

nmก

ํำ

MNMM

b' í

눙 的

1'1 11

웅ㅇㅇㅇㅇํㅇ

MMNMm

$M N M N M$

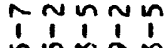

๗พำ

$1=01$
1
1
0
0
0

in moO

ก $\infty \infty \infty$

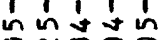

ono oc

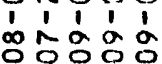

mNM

11111

원다

oㄷํㅇํㅇ

$\stackrel{m}{\stackrel{m}{H}}$

$33 x 37 \quad$ d. $3337 \quad 3 \times 33$

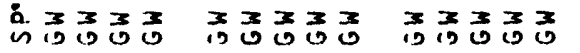

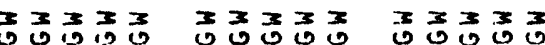

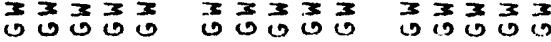

2
$z$
0
0

$M M m M m M M M m M M M M$

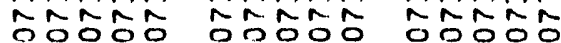

$\stackrel{m M M m}{M}$

$m m m m$

$\stackrel{M}{M} \mathfrak{\sim} \sim \mathcal{M}$

$M M M M M$

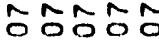

$M M m M m$ กิดกิ์

\begin{tabular}{|c|c|c|c|c|c|c|c|c|c|c|c|c|c|c|}
\hline o & 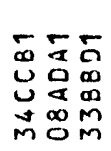 & $\begin{array}{l}5 \\
0 \\
0 \\
0 \\
\vdots \\
m\end{array}$ & 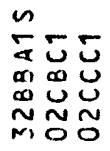 & $\begin{array}{l}5 \\
\dot{0} \\
0 \\
\dot{J}\end{array}$ & 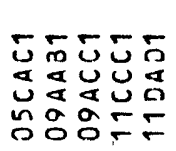 & 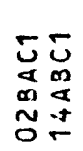 & $\begin{array}{l}\tilde{U} \\
\dot{0} \\
i n \\
\sim\end{array}$ & 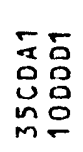 & 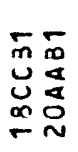 & 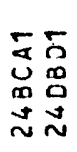 & $\begin{array}{l}\sigma \\
0 \\
0 \\
0 \\
\vdots \\
\sim\end{array}$ & 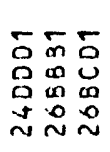 & $\begin{array}{l}\tilde{u} \\
u \\
0 \\
0 \\
\text { v }\end{array}$ & $\begin{array}{l}5 \\
0 \\
0 \\
u \\
\text { m }\end{array}$ \\
\hline 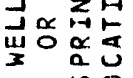 & 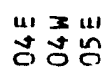 & in & 嵌虫出 & $\stackrel{m}{m}$ & 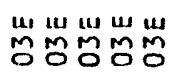 & 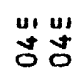 & $\stackrel{u}{0}$ & 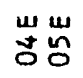 & w & 㟧出 & 㟧 & 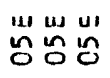 & in & \\
\hline & 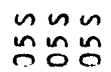 & in & $\begin{array}{l}\backsim \sim n \\
000 \\
000\end{array}$ & $\tilde{0}$ & 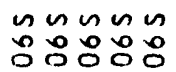 & $\begin{array}{l}n n \\
\circ \div 0\end{array}$ & ڤo & $\begin{array}{l}n n \\
0 \\
0\end{array}$ & $\begin{array}{l}n \tilde{0} \\
00 \\
0\end{array}$ & $\begin{array}{ll}n & n \\
0 & 0 \\
0 & 0\end{array}$ & $\stackrel{n}{\circ}$ & $\begin{array}{l}n \sim \sim \\
000 \\
0\end{array}$ & $\begin{array}{l}n \\
0 \\
0\end{array}$ & \\
\hline
\end{tabular}




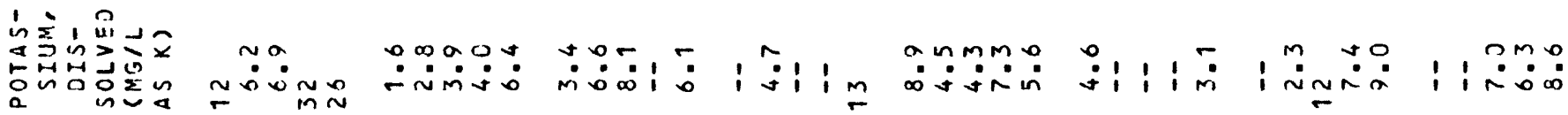

辛, 出这余

tis

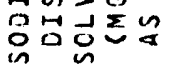

mogoo

$\sin 1000$

mํㅡㄴ응

뜬은은

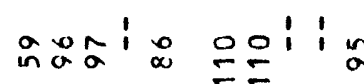

꿁옹으 윤

1 2000

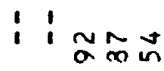

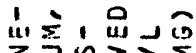

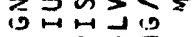

$\infty 2$

$\stackrel{\infty}{\sim}: 0 \div$

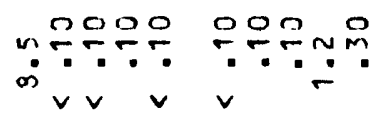

ํำ

음요

noto

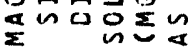

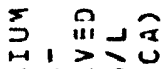

式出这的

amoso

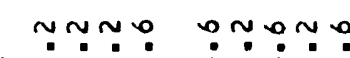

0

o omoan

mo:

I

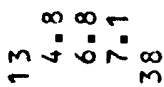

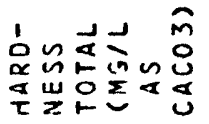

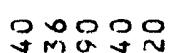

mmmms

$=\infty a m i n$

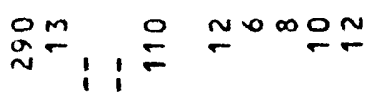

$a \approx \operatorname{nin}=$

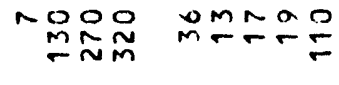

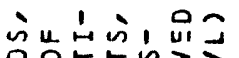

now 000

$\operatorname{mos}$

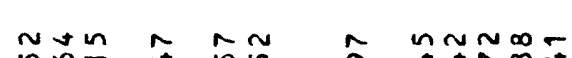

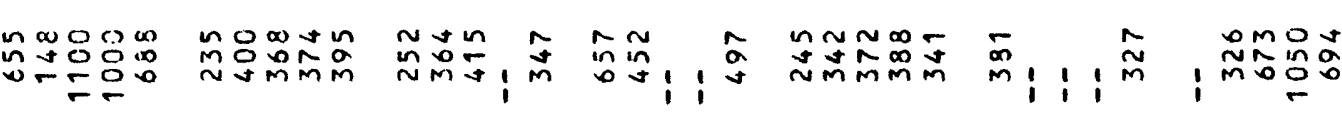

$\log$

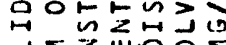

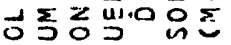

immin

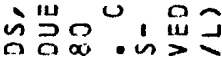

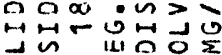

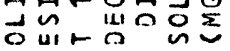

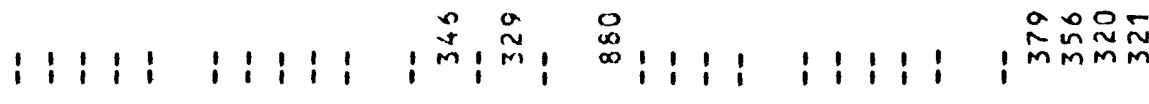

i: $1:$

ñ

nm $m:$ :

z뼝,

m $\rightarrow \geq \geq 0$

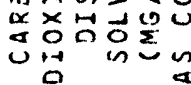

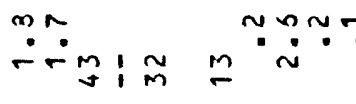

ษะmm

$\because ! i^{m}$

$\because \stackrel{\infty}{\sim \infty}:$

$\because \because \frac{\infty}{1} \because \because \div \infty$

onm?

$\min$

แบ xเoน

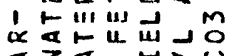

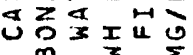

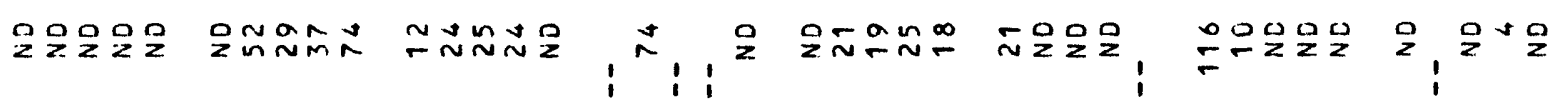

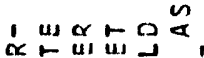

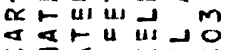

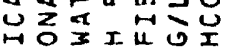

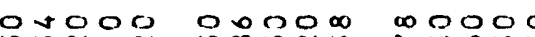

m

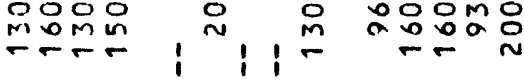

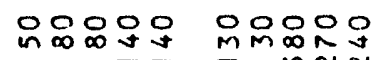

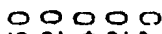
in

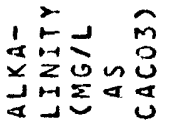

$i^{\stackrel{\alpha}{\sim}} i: i ; i ; i$

$::::$

$11: 11$

$1: 1 ; i$

i:i: i im

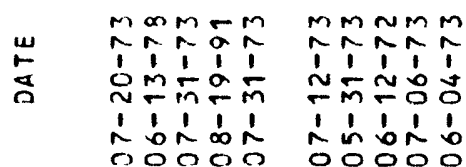

$m \sim m m m$

îฺ

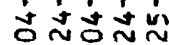

ân $\sim \mathfrak{\sim}$

$m \sim m m m$

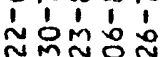

b!

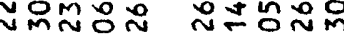

1 1 i

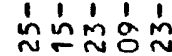

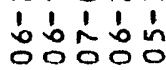

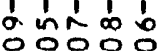

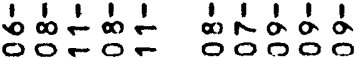

$\sim m \sim m m$

Nî̃̂́

ธㄴำำ

m1 111 


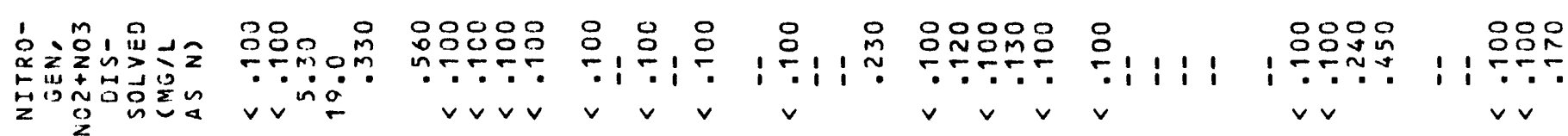

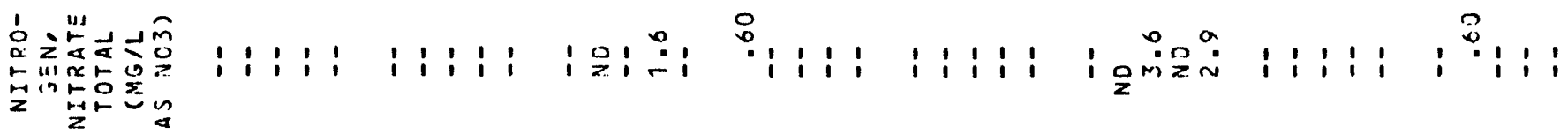

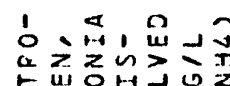

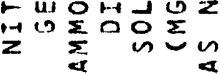

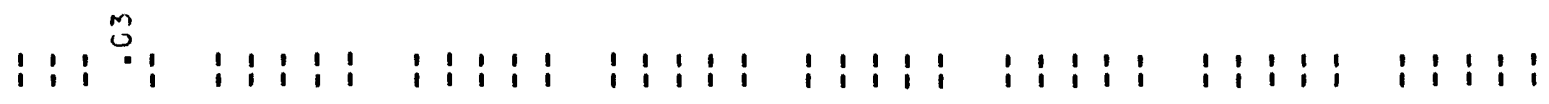

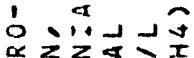

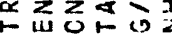

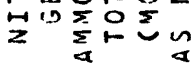

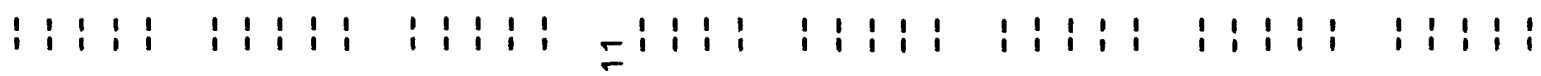

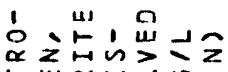

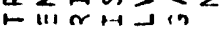

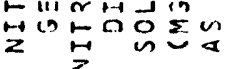

$1: \frac{0}{1}: 1, i: 1 ;:$

bे

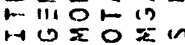

1:1: $1: 11:$ 1:1:

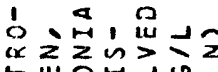

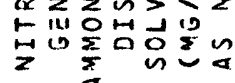

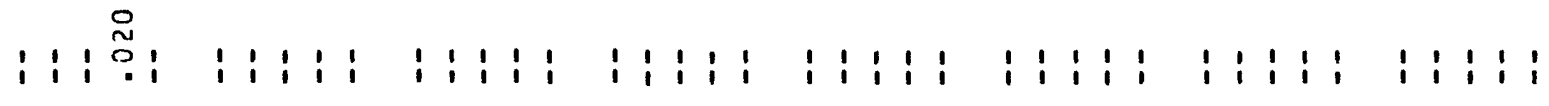

iㅔ

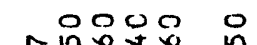

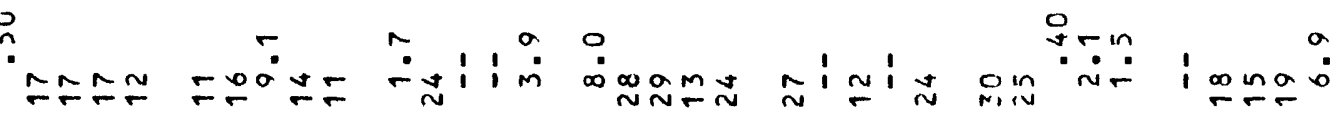

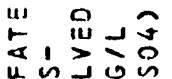

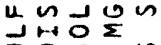

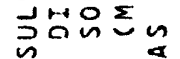

옹요요

$m \pi n \pi$

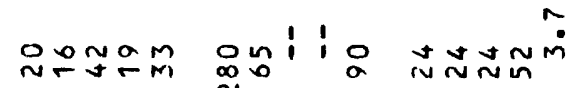

$\stackrel{\infty}{\sim} \underset{m}{\sim} \operatorname{inm} i m$

I

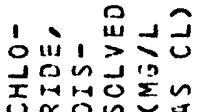

m-iona

$\tilde{a}_{n}=0=n a i l+a$

anmo

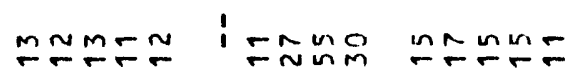

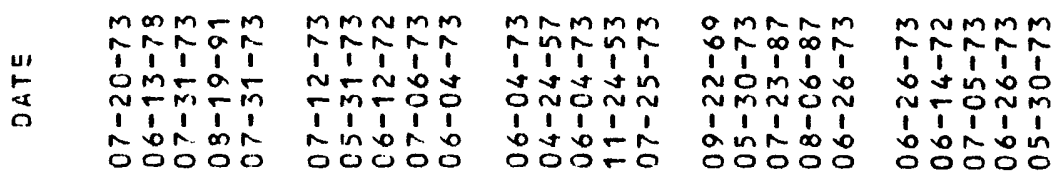

mNMNM

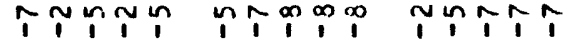

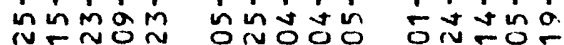
1Yi 1 1 1 1 1 1 1

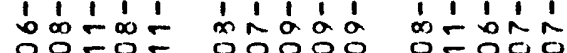


吾的紫全

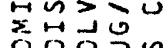

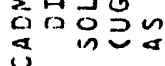

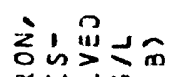
希台哥包

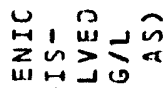

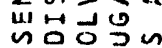

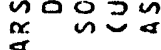

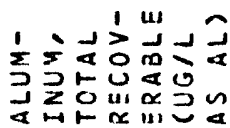

哭品足 z的要的 动然品告

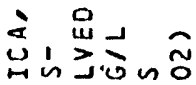

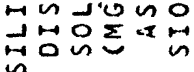

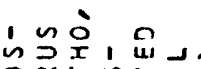
으의 ․ㅜㅇㅢ

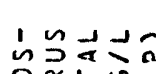

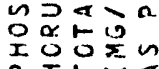

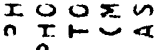

$111,1,111,2$ $\frac{2}{2} 1$

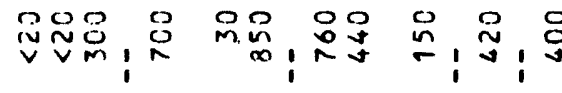

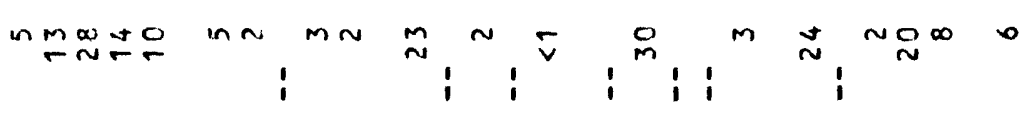$$
\text { i i }
$$$$
i^{2 \ln \infty}
$$$$
\text { i }
$$

\section{: : : : :}

:i:

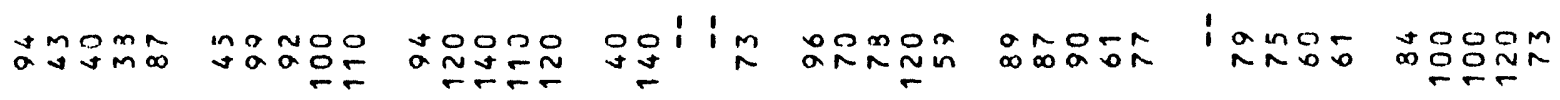

$1: 1 \frac{0}{9}: 1: 1 ;:$

i: $: i:$

$1: 1: 1:$

$1: 1: 1$

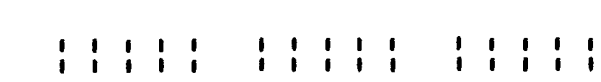

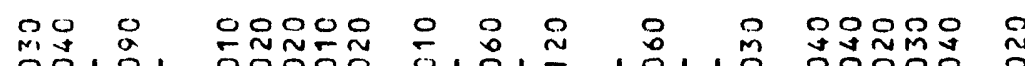
ก.:1, 0.,

: $1: 1 \%$

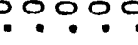

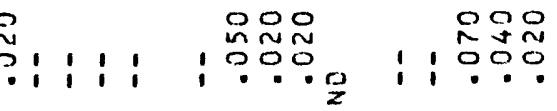

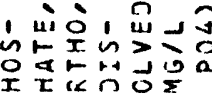
, i i i i i i:i: i:i:i i i i i i i i i: $1:$ : $1: 1$

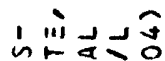
นู้

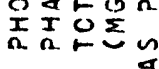

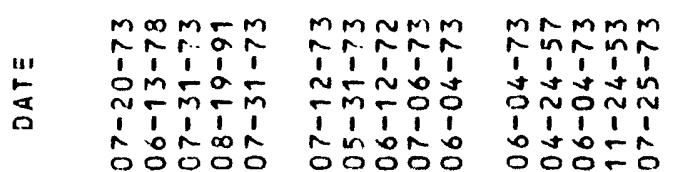
1:1:18:1:1: 10:11
¿ 211

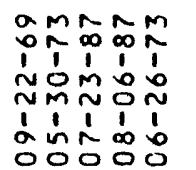

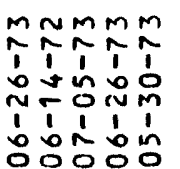

$\stackrel{m}{\sim} \tilde{m} \sim \mathcal{m}$

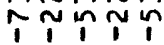
inmam TTY

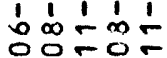

웅 $\because 11: 111 ; 1:$

Mㅜㅇㅇㅛ $\approx M N M m$ กิ๊

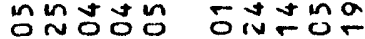
ón 


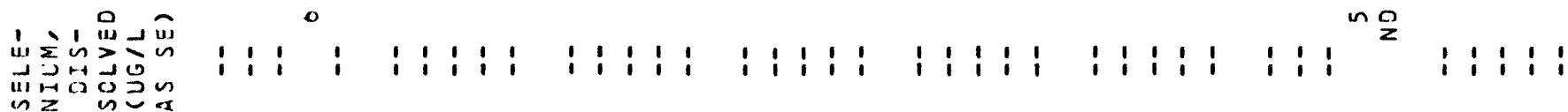

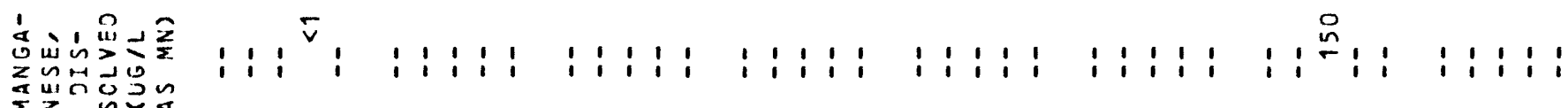

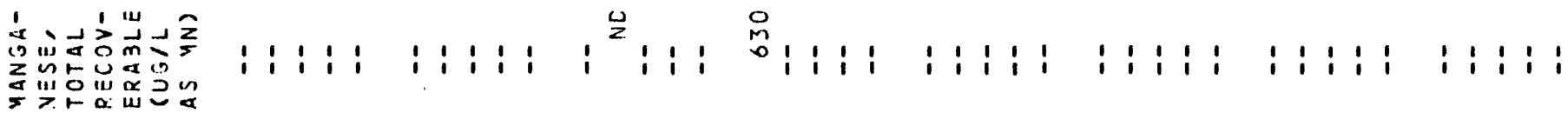

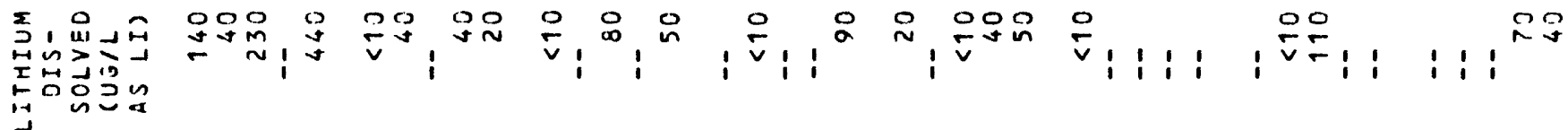

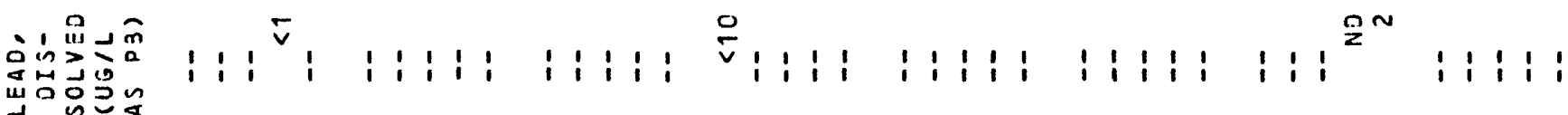

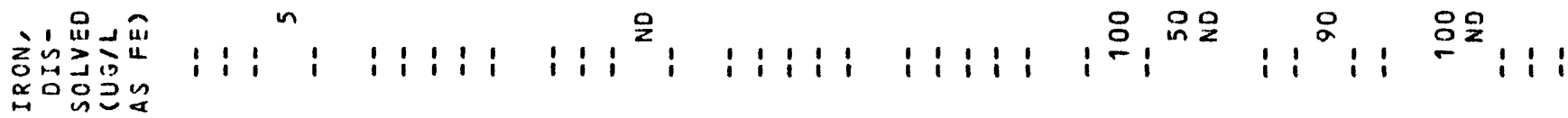

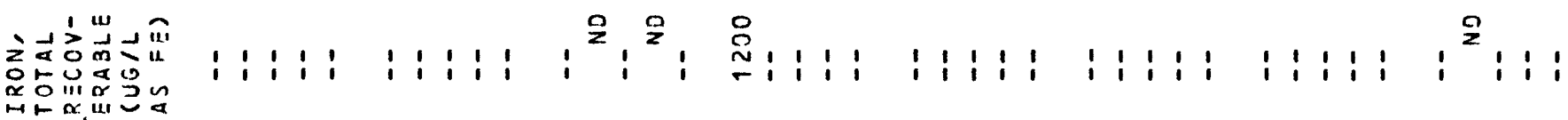

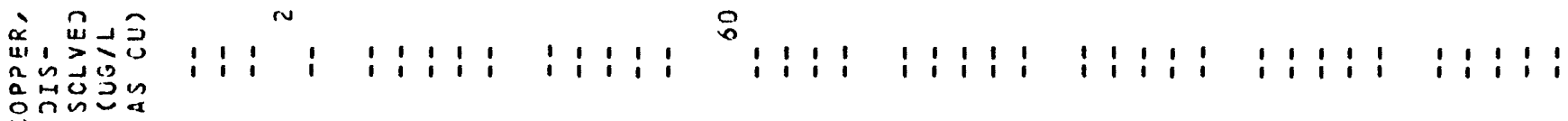

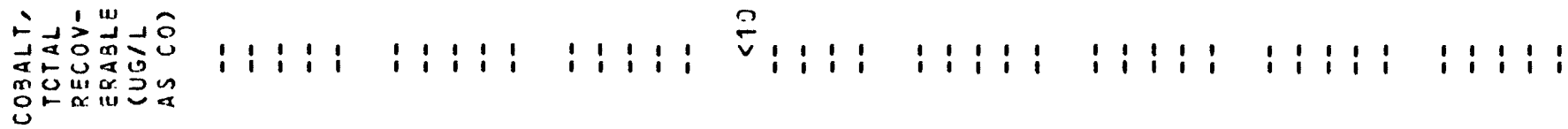

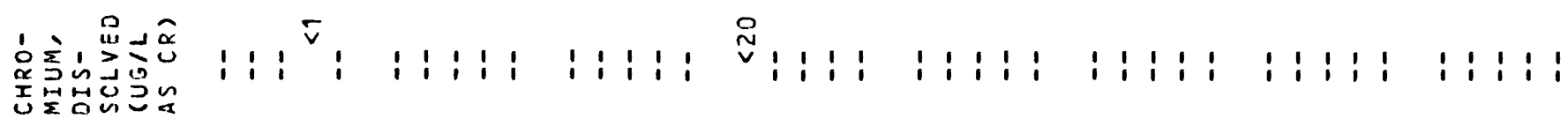

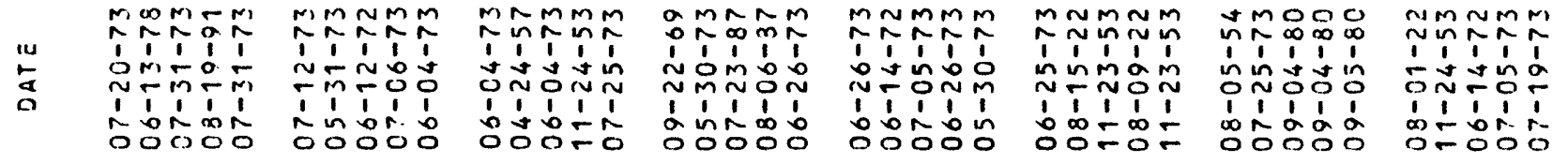




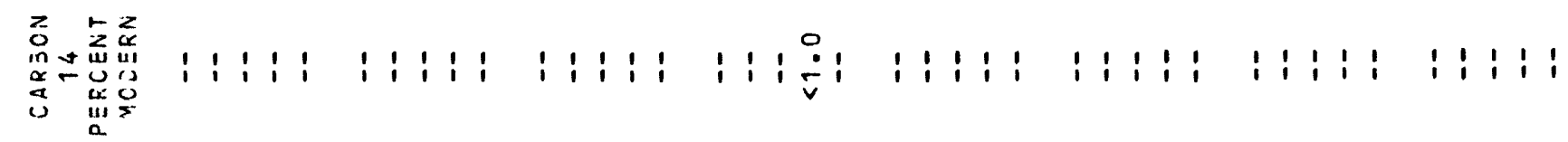

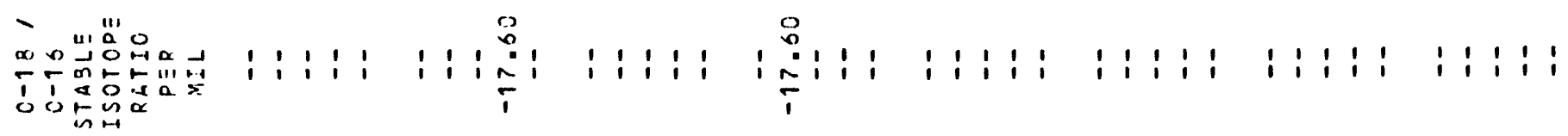

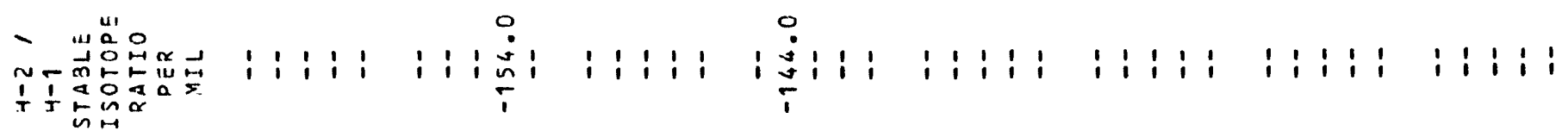

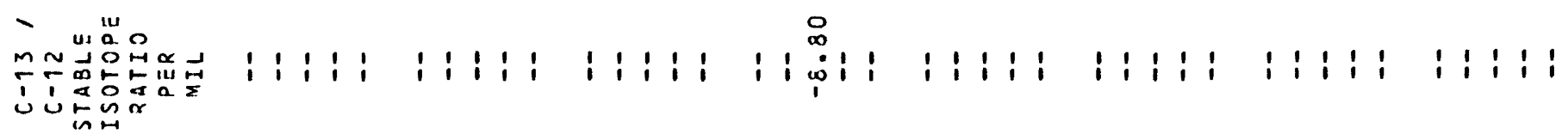

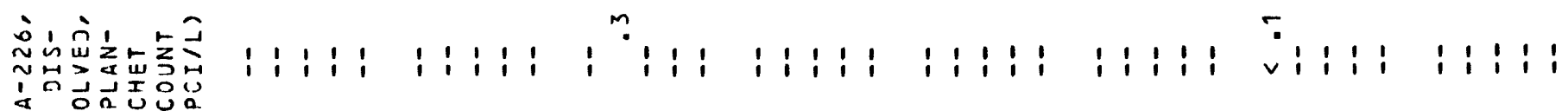
or os

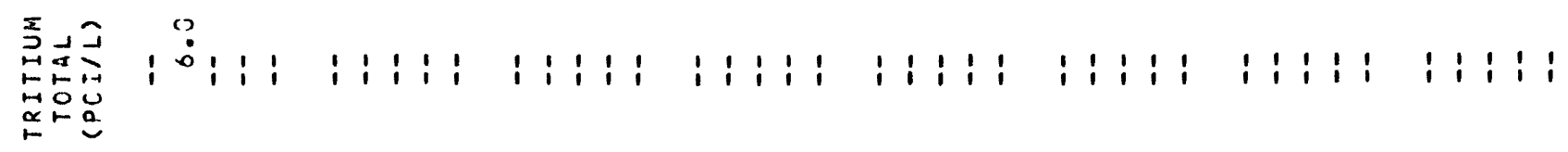

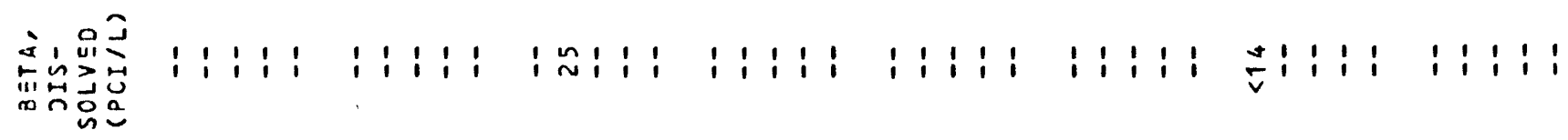

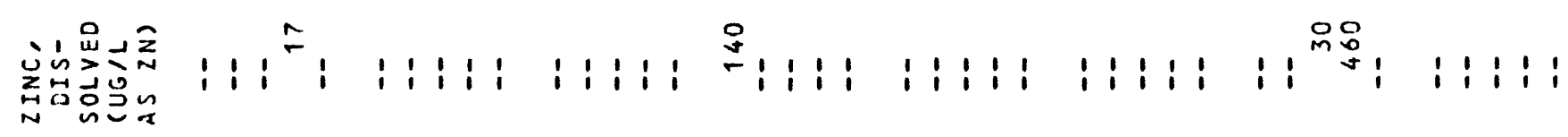

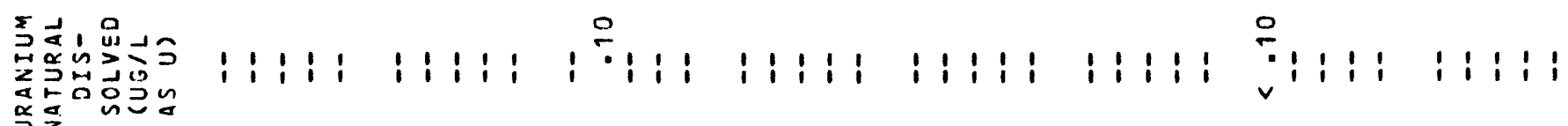

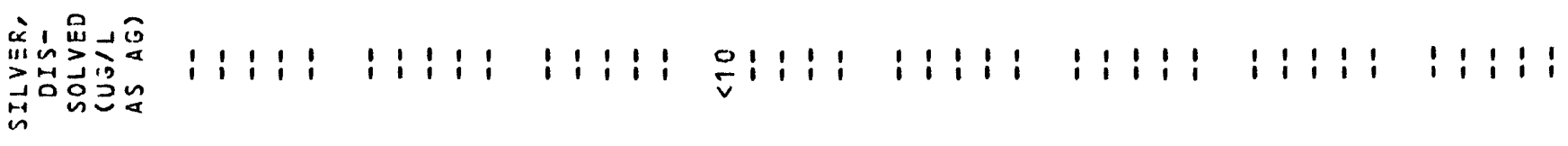

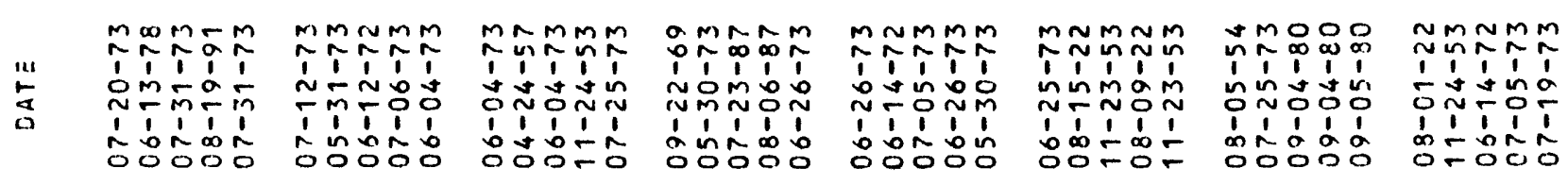




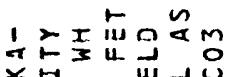

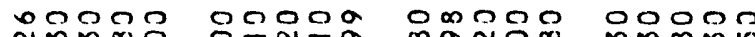

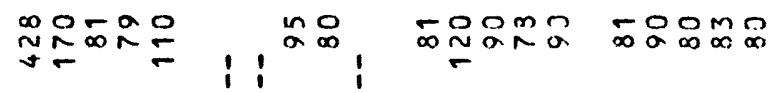

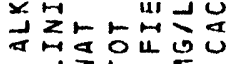

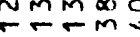

$B=\simeq \frac{\sim}{\sim}$

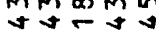

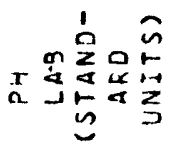

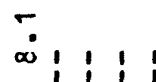

$1: 1: \hat{2}$

$\because$

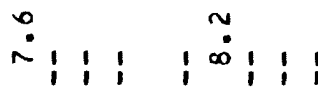

$\ddot{n}: \dot{\infty}:$

$\because \stackrel{0}{\infty}$

i!

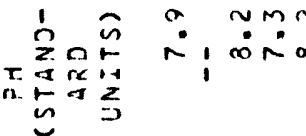

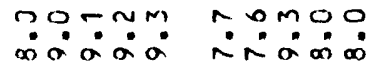

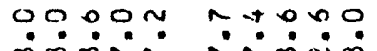

i

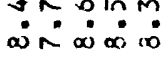

an?

1.t, t wo

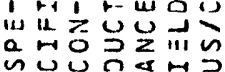

n⿺辶一兀

! : 1:

i i i i

ioi i i

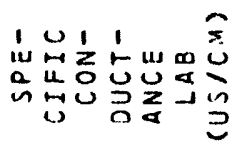

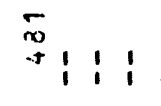

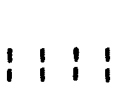

2

1 品, 1

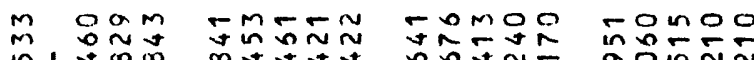

$m$ 일

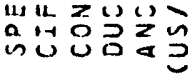

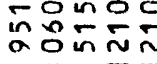

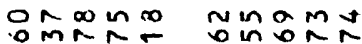

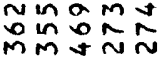

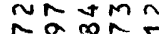

กลกัก

$\operatorname{sam} O N$

$\stackrel{1}{\alpha \cdots} \propto 0$

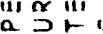

$>25$ iा

in wingo

$0000 \mathrm{~m}$

noinco

"Oino

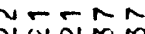

in $\dot{0} \dot{0}$

$\sim \sim m \dot{m}$

minimin

0000 m

ino:

oonoo

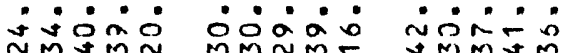

ongom

iI $4: 3$

㸟

a) $\approx N$

OMCMO OTMmM

ititi $150 \hat{1}$

MOOPMO

1111

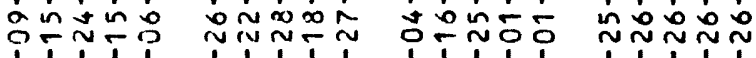

15!

1 1 1 1 1 1 1

1'1 1

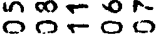

oño

å

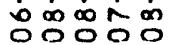

ammao

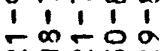

TOTNM

占占向的思

जMMUm

Smm

$\stackrel{\text { II }}{\text { in }}$

$333 \times 3$

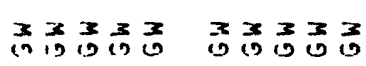

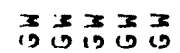

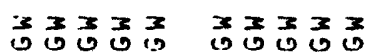

3
0
0

monoso mingom

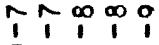

워ำ

$\begin{array}{lll}0 & 0 \\ 1 & 1 & 1 \\ 0 & 1 & 1 \\ 1 & 1 & 1\end{array}$

1,11 !

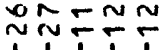

1.

ammog

1111

ำㄴำ

i 1 i

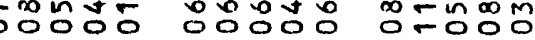

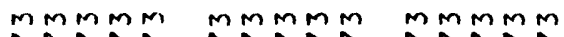

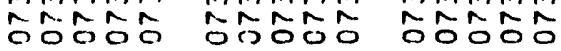

$M m m M m$

MMMmM MMMmm

mMNMM

Mmmmm

mmmmm 00000

OOOOO

ỗỗố

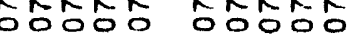

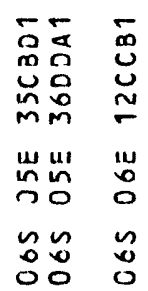
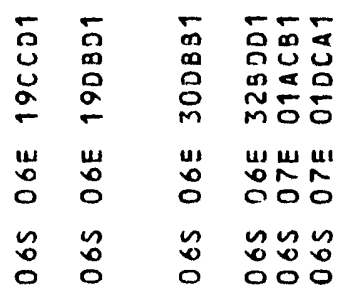

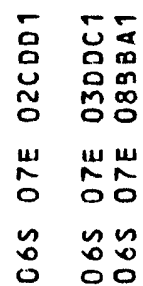

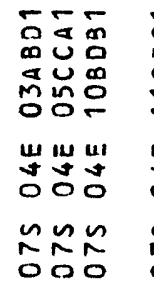

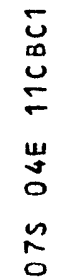

क⿺

品

¿ับ

嵓

ш ш ш

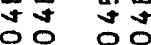

$\tilde{2} \approx \sim$ 


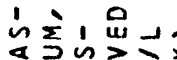

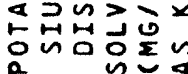

$\sum_{i=1}, 0 \geq \frac{a}{2}$

$\rightarrow \backsim>0$

مคำ

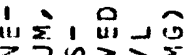

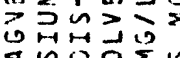

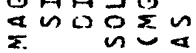

$\sum_{11}^{n}, 0$

in.>

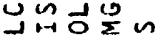

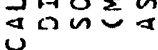

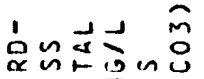

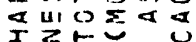

被的, 品与 not5

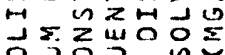

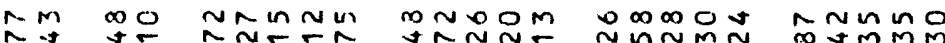

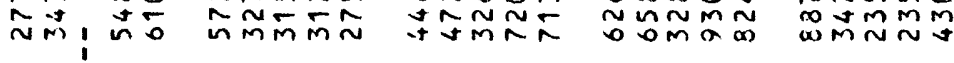

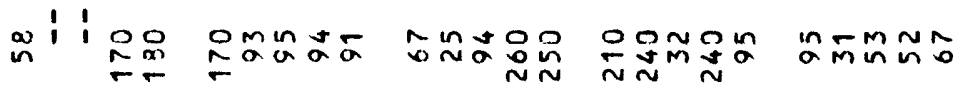

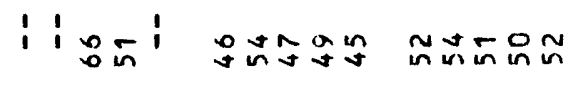

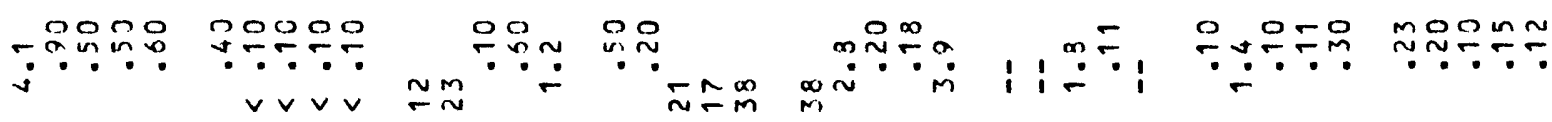

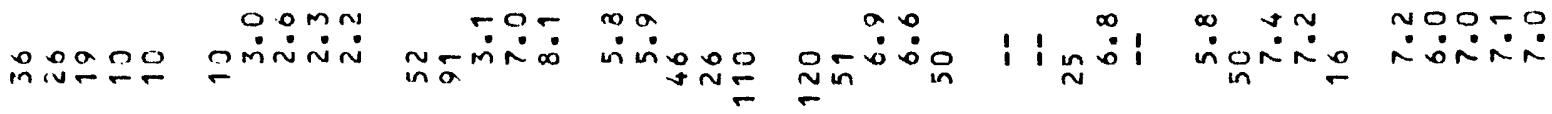

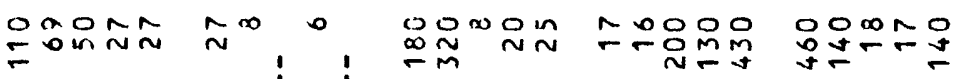

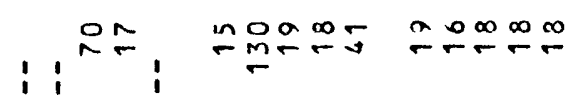
에인

i un $^{\prime}$, , 品

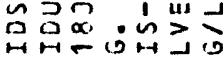

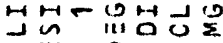

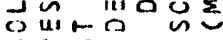

중몸ㅆ

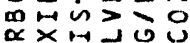

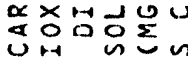

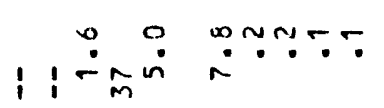

$=1: \stackrel{\infty}{a}$

$\because \because \infty$ : $1 \pm \frac{1}{2}$ i i i

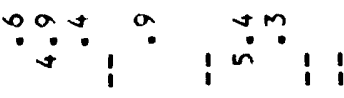

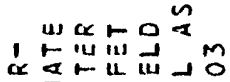

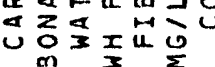

1 $\alpha \leftarrow$ u山 $m$ 可比出

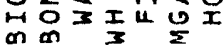

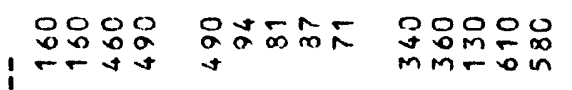

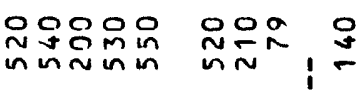

i i i

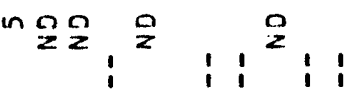

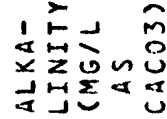
i i i i

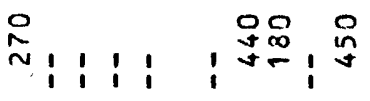
i i i:

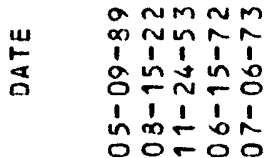
OMOMO $1: \infty i 1$ $\stackrel{\sim}{\sim} \stackrel{\infty}{\sim} \stackrel{\infty}{\sim}$ $\begin{array}{llll}1 & 1 & 1 & 1 \\ 0 & 1 & 1 \\ 0 & 0 & 0 & 0\end{array}$
CómMm MOOM

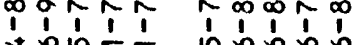
ปำำ

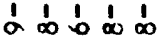
0000
N윰ำ

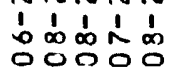

TMmaO

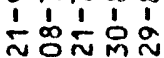
占

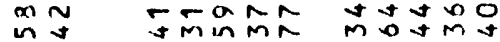

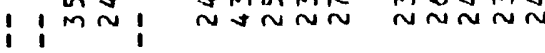




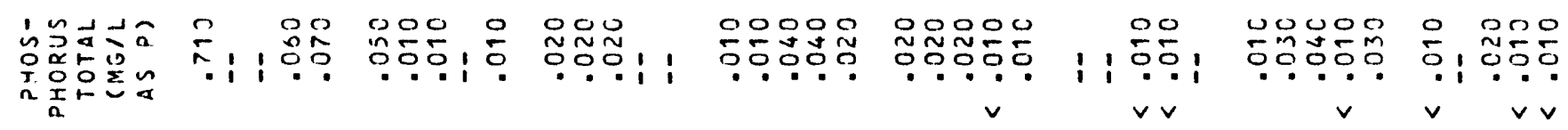

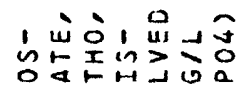
xac00

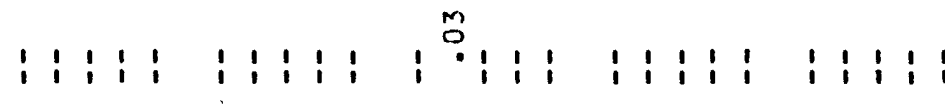

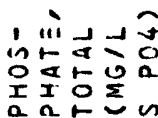

b.

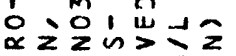

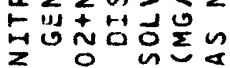
운 1 응

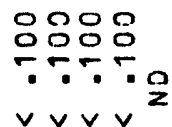

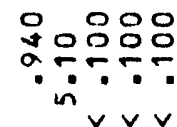

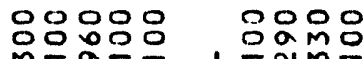
ํํำ?

no요 m 11 i $1: 1:$ i i i i 1:1:1:1:11

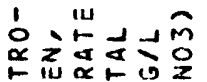

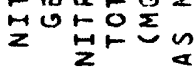

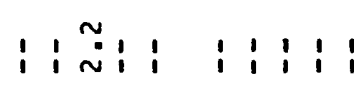

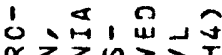

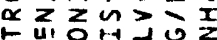

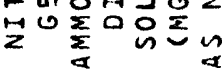

$1: 11: 191: 1$ m i i

向诘的落さ之

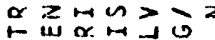

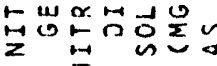

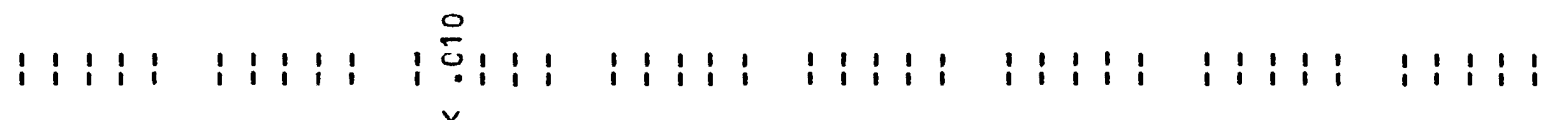

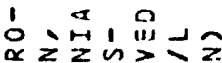

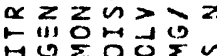

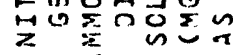

i 1 i 1 11111 ก Oั.

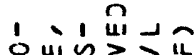

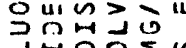

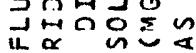

$\because$, on?

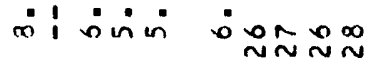

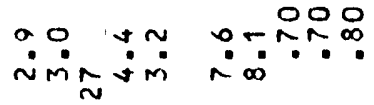

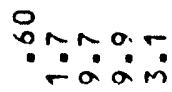
i ing ! ånision orimon

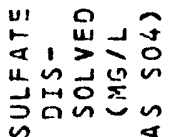

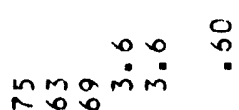
?

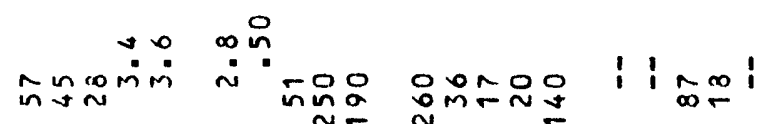

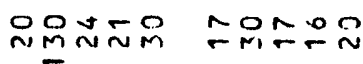

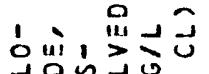
牙的可可

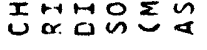

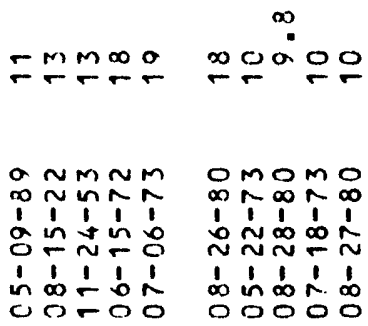

norna

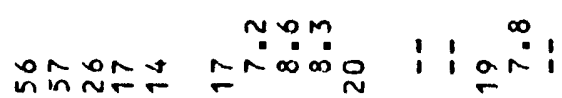

कि कि क्ष

ro.t. ratror

ómma moomo ît̂́ în ㅇำำ

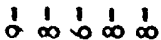
ఓ

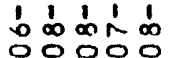

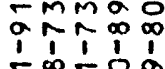
동무워 $\begin{array}{llll}1 & 1 & 1 & 1 \\ \infty & 1 & 1 \\ 0 & 0 & 0 & 0\end{array}$ Tीक

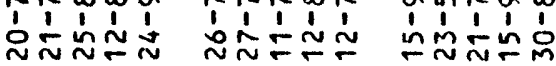

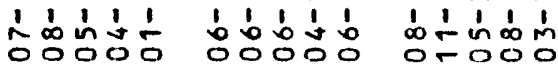




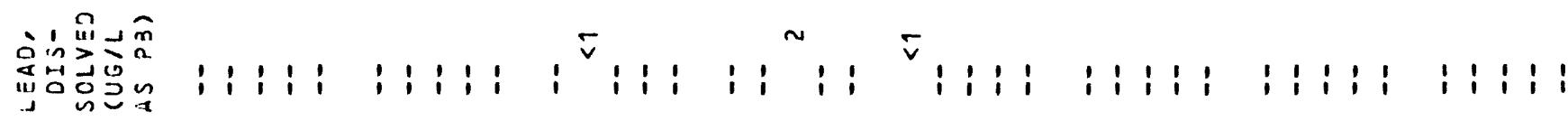

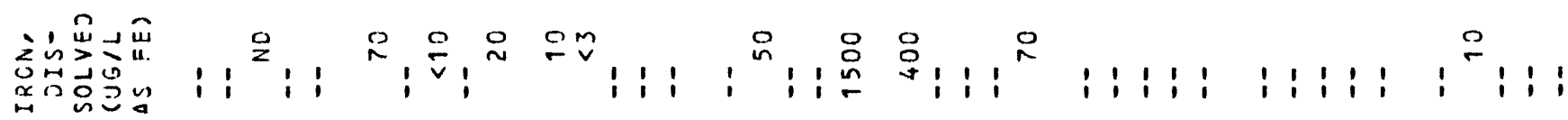

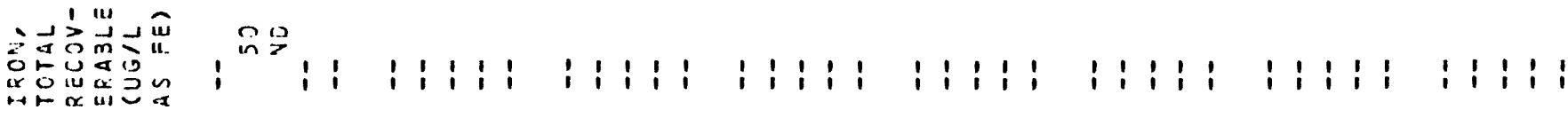

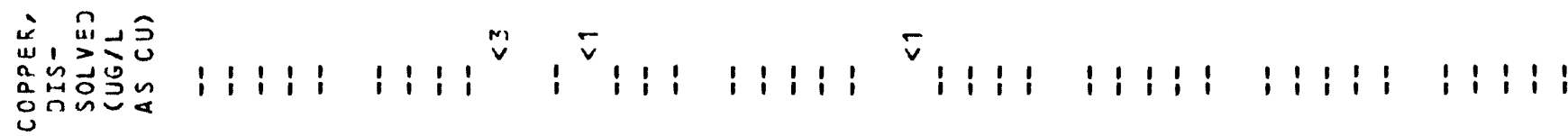

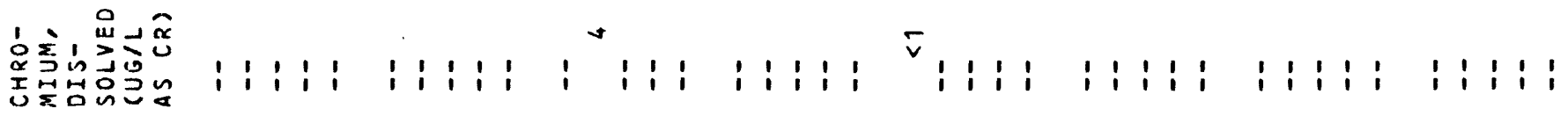

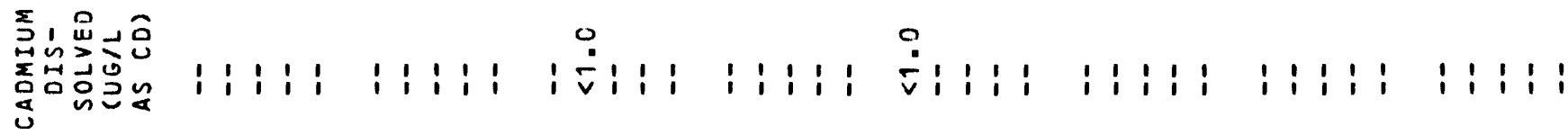

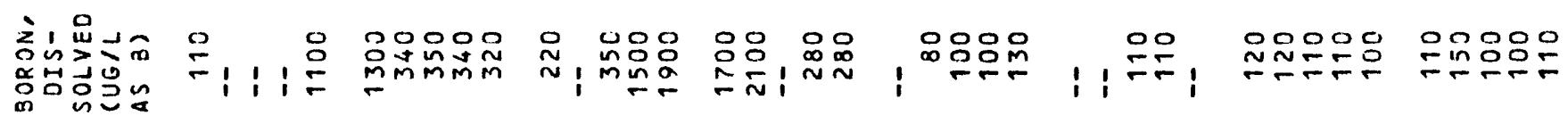

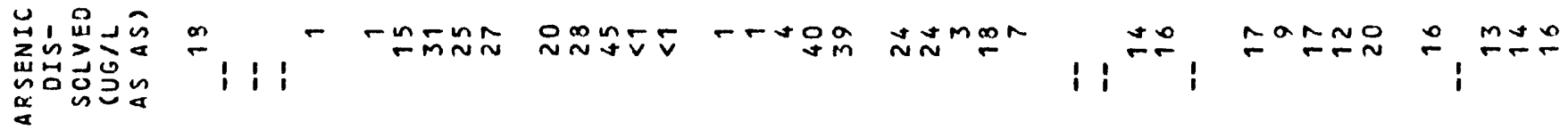

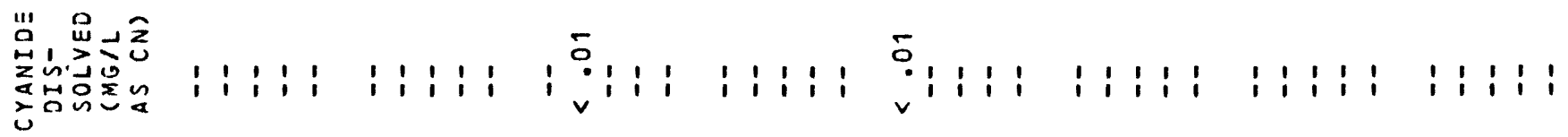

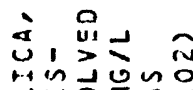

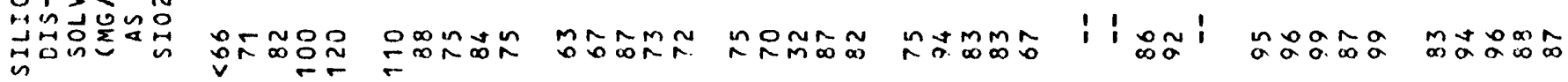

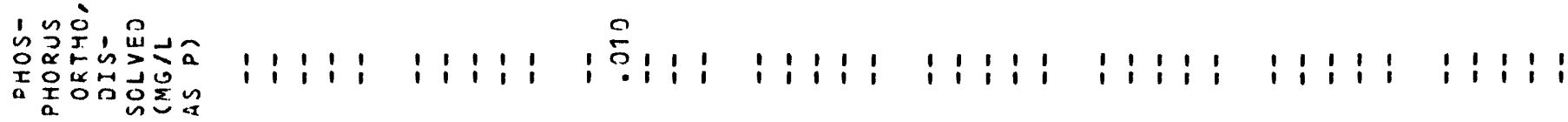

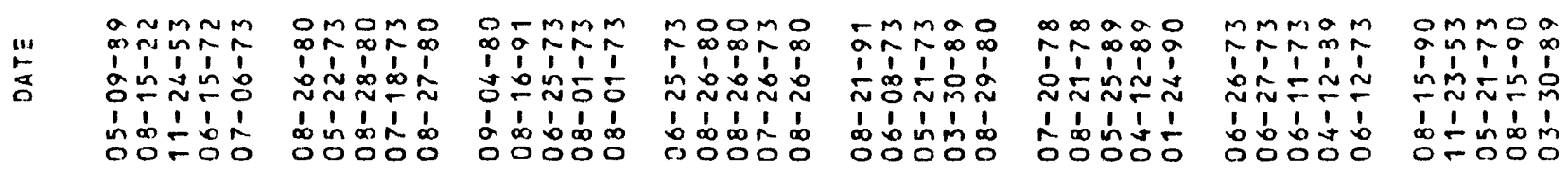




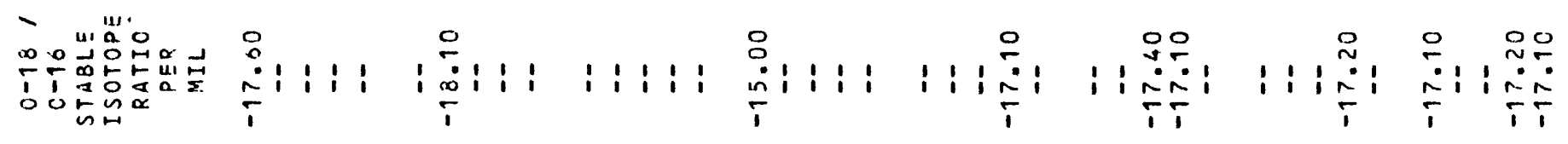

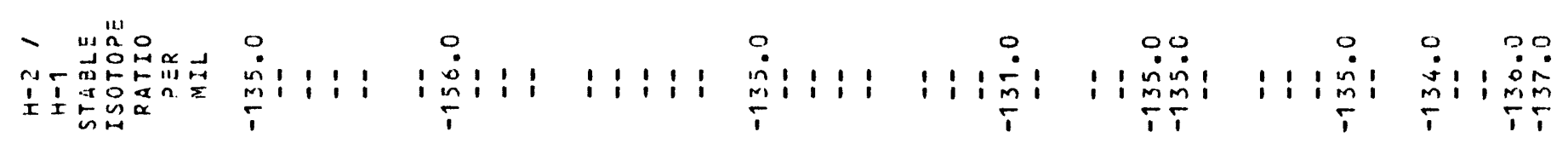

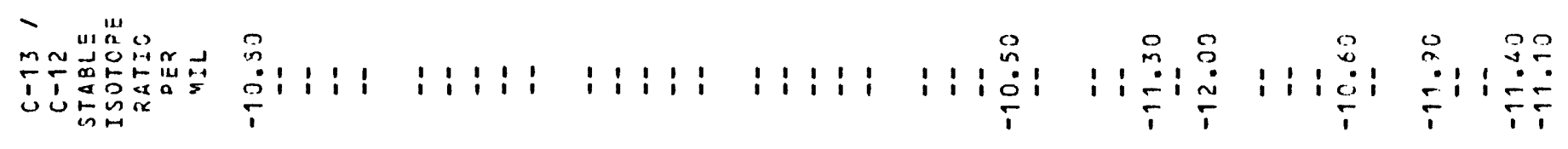

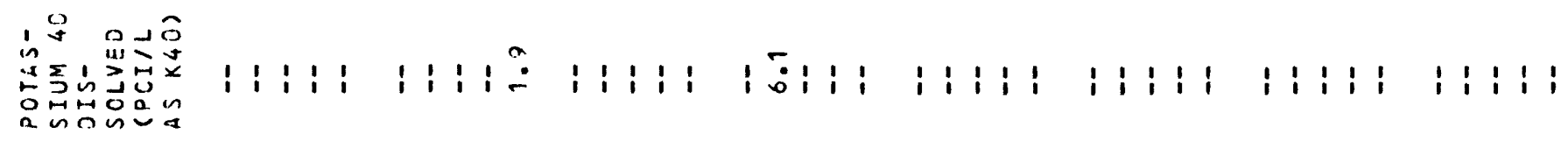

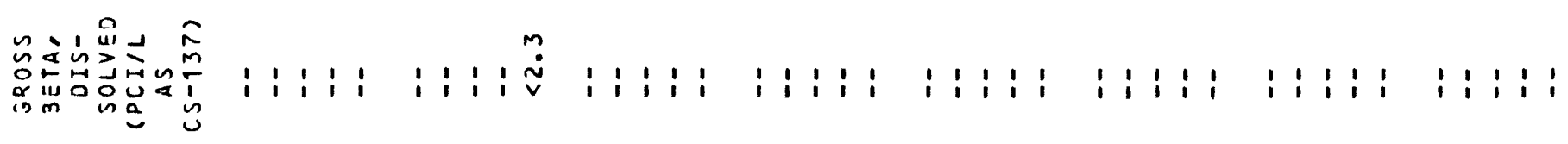

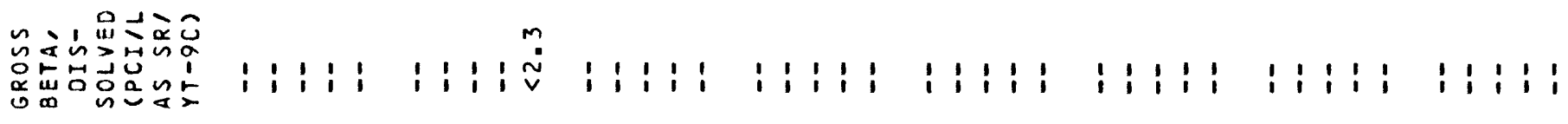

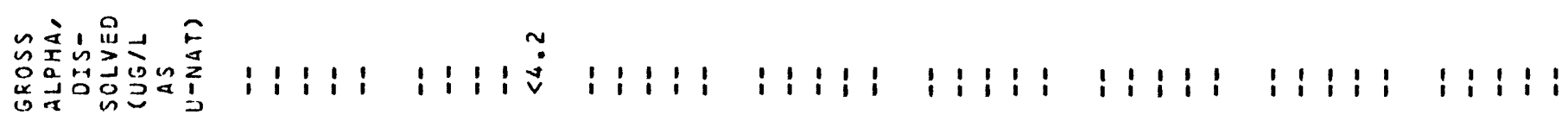

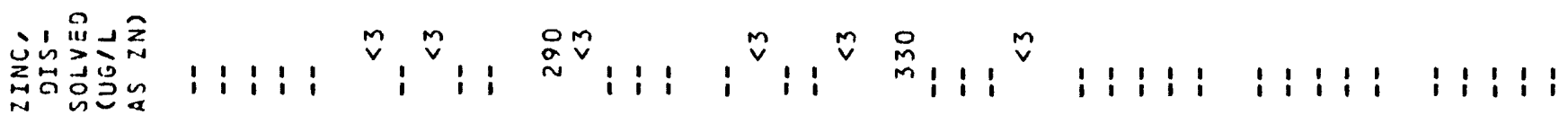

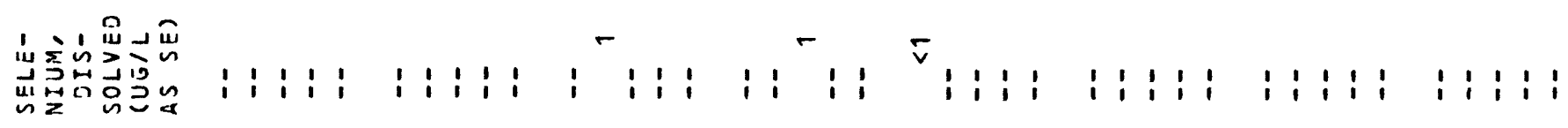

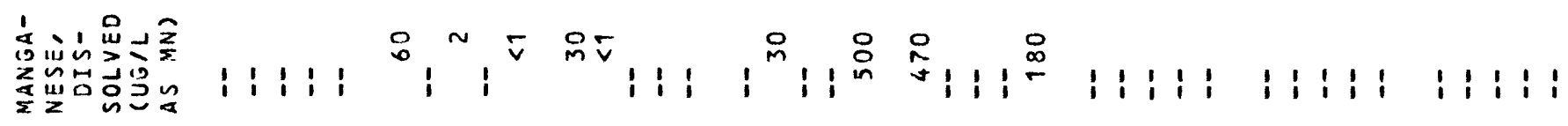

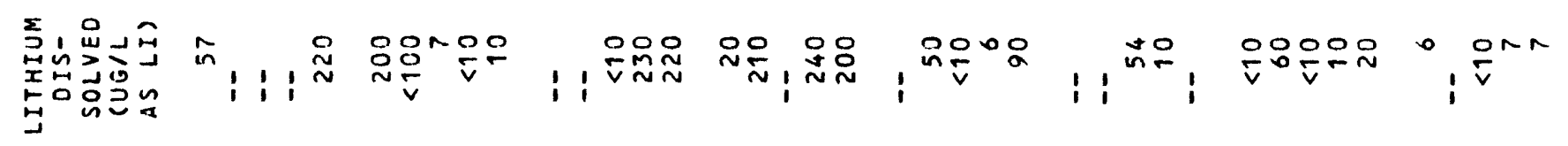

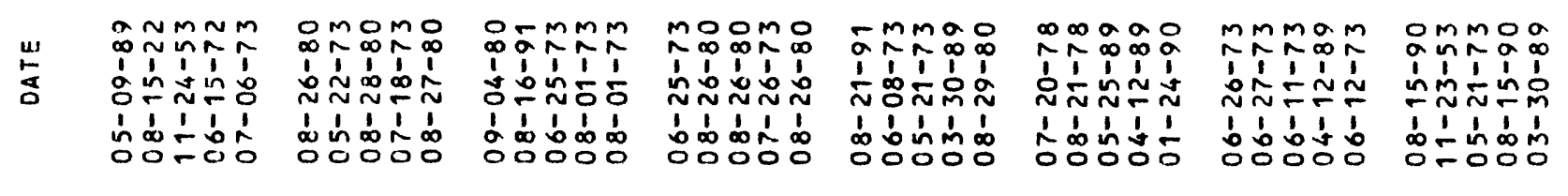




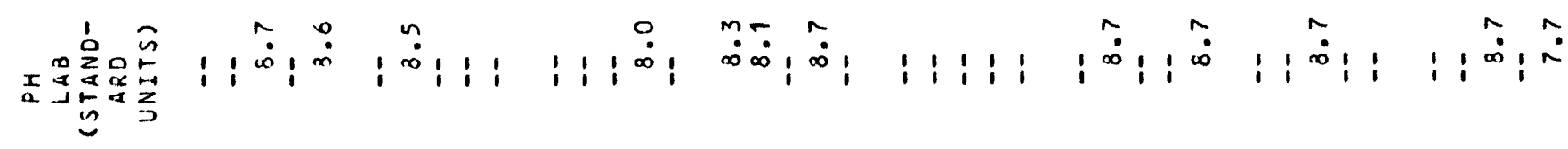

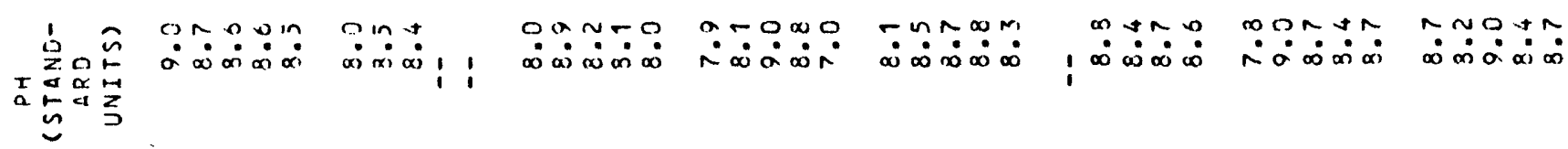

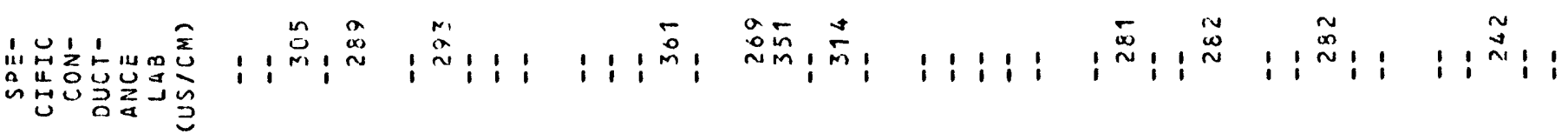

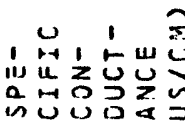

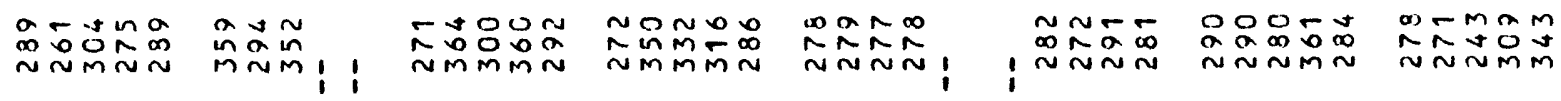

i. us $\propto \mathcal{U}$

gomoin oum uno

- $: 0$.

Mimin

omomo o.? : ? :

o o no

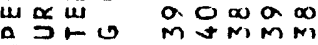

MMMNN

noró O-nina

araici

ono n

in 0000

in in in in 0

$\sum_{110}^{0}=0$

$m M m M N$

$\sim N M m M$ MmMm

mónio

àmion

$m \sim-m m$

MMNMN

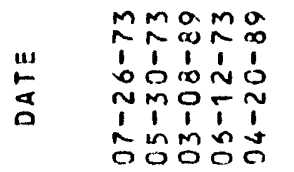

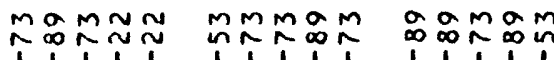

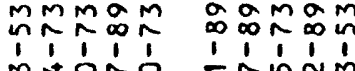

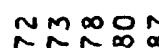

$\sim \sim \sim, \infty i$

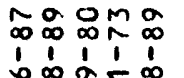

$\operatorname{Tn} \hat{0}$

mUTNO TNmNm

$-0 m \sim m$

0 $0 N \pi N$

i个ip

$11 ; 1 ;$

ticis

11111

$m_{M \infty}^{M} M$

11111

$N-m=\pi$

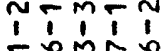

$M M O M Q$

i i i i

MNTnN

西

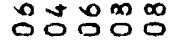

-

年

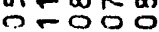

$\stackrel{m}{m}$

$33 \div 3=$

$x \geq \geq 0$

$\frac{3}{10}=\frac{3}{10}=\frac{x}{10}$

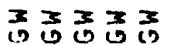

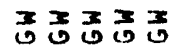

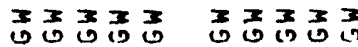

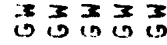

$m m m m$

NONÃ

mmmmm

mmmmm

mmmmm

$m m m m m$

$m m m m m m m m$

$M M M M M$

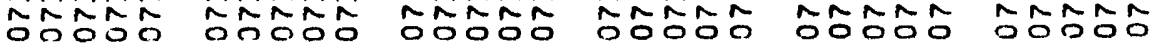

ำลำดิ

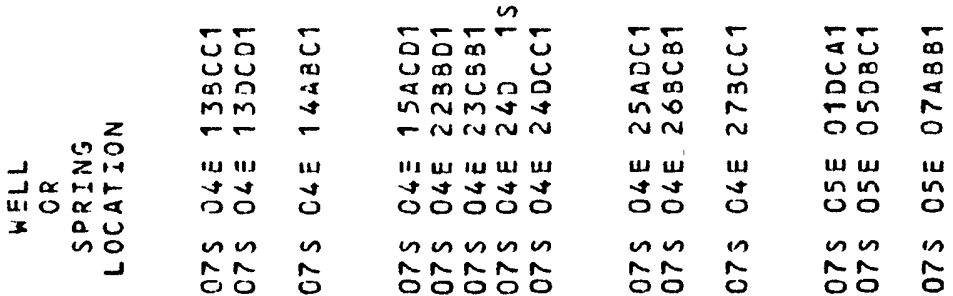

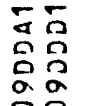

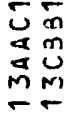

$\underset{\substack{0 \\ 0 \\ 0 \\ 0 \\ 0}}{0}$

०0

iII II

wn

nn $n$ n

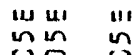

ณn

กิธก กิ์ 


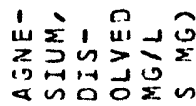

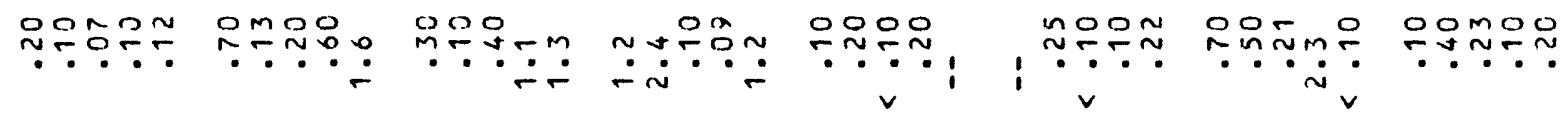

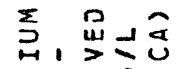

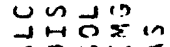

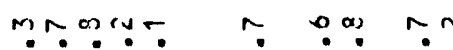

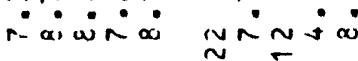

$\because N$

$\forall n$

$m \operatorname{mn} m$

?non

$\because m \because r+? r$ तु

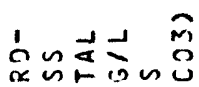

annmo dorta

$\infty$ a. $\sin$ $\infty \infty: 1$ $\underline{n} \sim \infty$

isirio

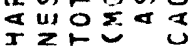

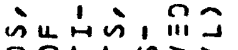
30t

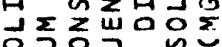

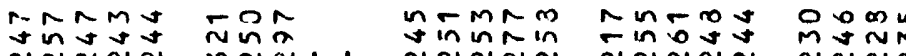
ññn m nan:

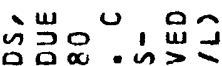

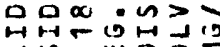

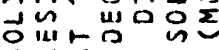
i i i i i i i is $\stackrel{\sim}{\sim}: i: i: i: i \stackrel{0}{n}$ i i i i i i i $\stackrel{a}{n} i, i ! i \stackrel{a}{n} i$ i

z虫, 品小主

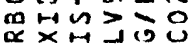

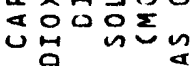

$\because \cdots$, : III

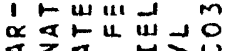

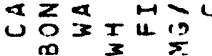

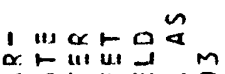

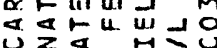

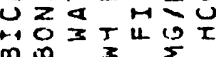

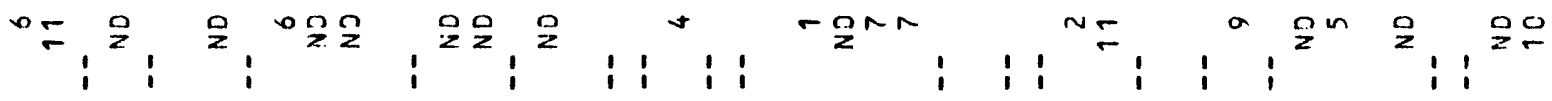
๙)

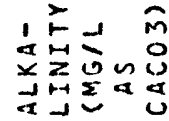
i i i i i i i i i i i i i i i i i $i^{\infty}, i$

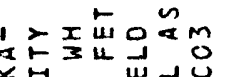
Mㄴㅇㅇㅇ

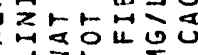

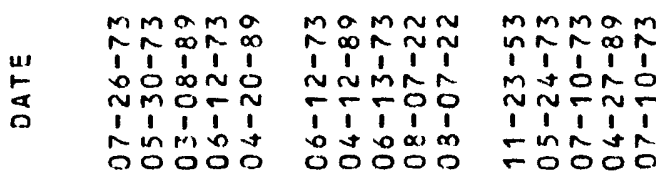
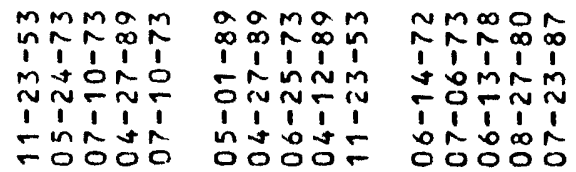
$\begin{array}{lllll}N & 0 & 0 & m & 0 \\ \infty & \infty & \infty & \sim & \infty \\ 1 & 1 & 1 & 1 & 1 \\ 0 & \infty & 0 & 1 & \infty \\ 0 & 0 & v & 0 & 0 \\ 1 & 1 & 1 & 1 & 1 \\ \infty & m & \infty & 1 & m \\ 0 & 0 & 0 & 0 & 0\end{array}$

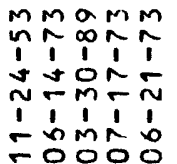

ming Tी

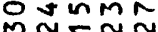
miñ in 1111

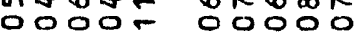

궁에 


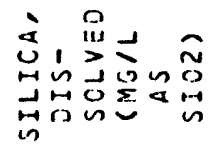

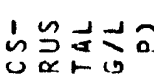

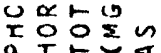

a욘두

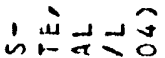

वास्व

IT인

을 ax $\sum_{i=1} \sum_{n=1} n$

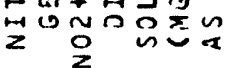

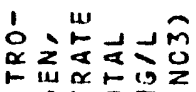

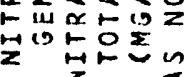

언논 马口it

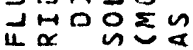

$\stackrel{w}{4}, \stackrel{0}{\rightarrow} \leqslant 5$ is is $>$ in

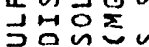

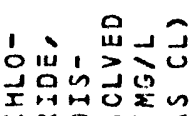

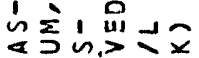
5

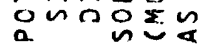

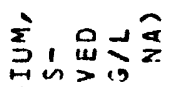

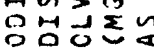

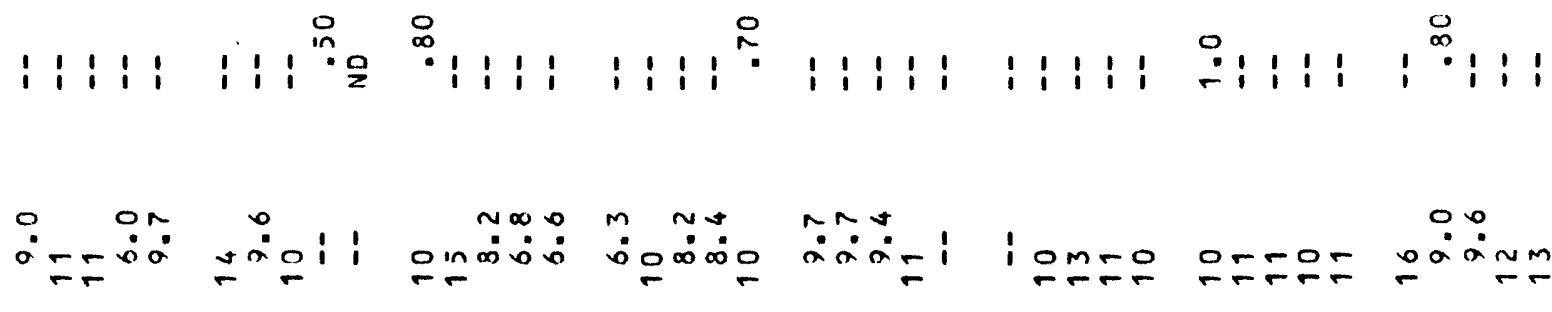

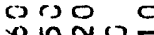

nutne 020

ำบำ

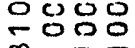

क⿺⿻一𠃋十

$\checkmark v \vee$

mings

m.?

응 잉요 응 ogo $m$ ñ , 염ำ INกN

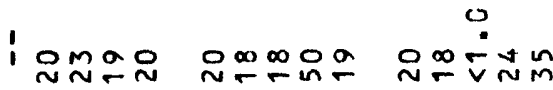

$004-0 \quad 0000$

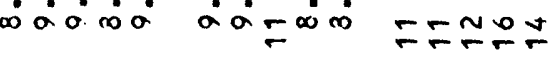
$\because r m \infty 0 \quad m \infty a r$ $\dddot{\infty} \infty \min ^{\infty}$

$\infty 0 \%$

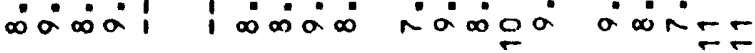

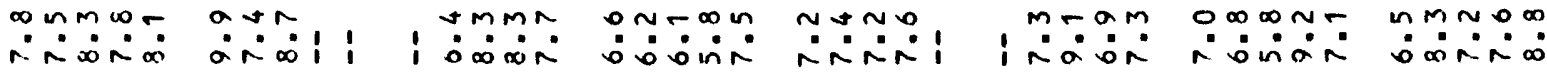

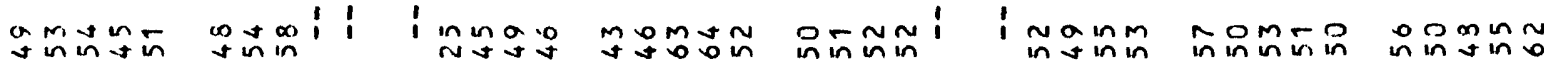

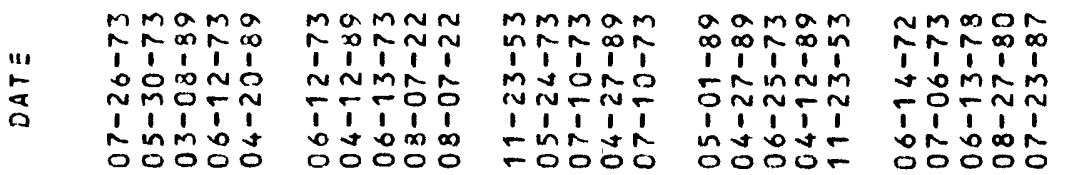

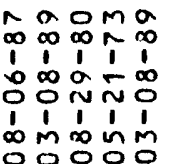

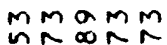
îi * $0 \Omega$ Timi Ib má

MmOMO î ovmm 1 111 $n-\infty \pi \infty$ of $=000$ 


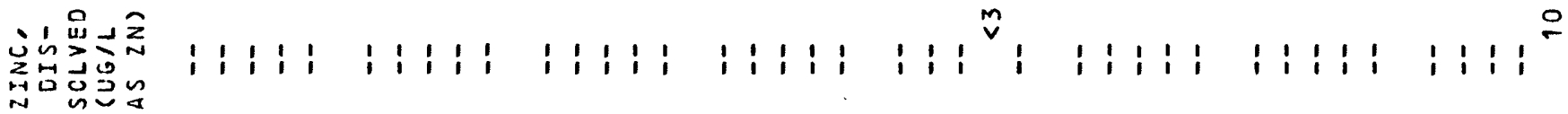

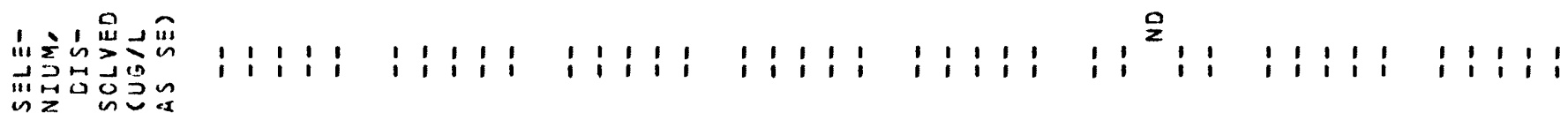

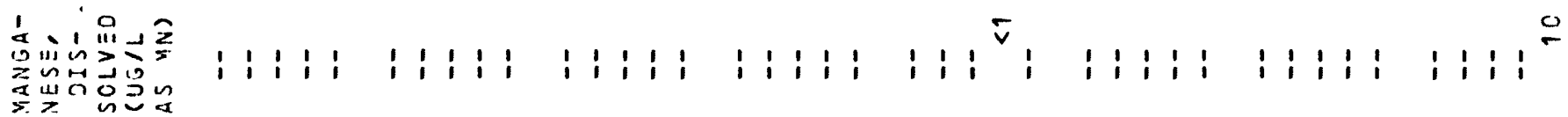

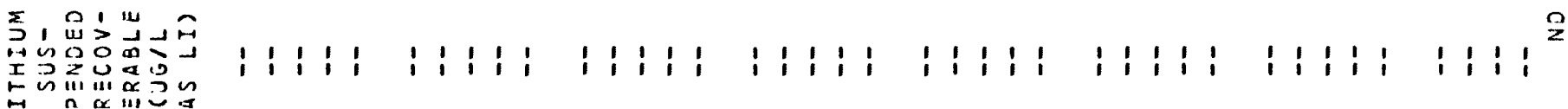
a 0 i11 0

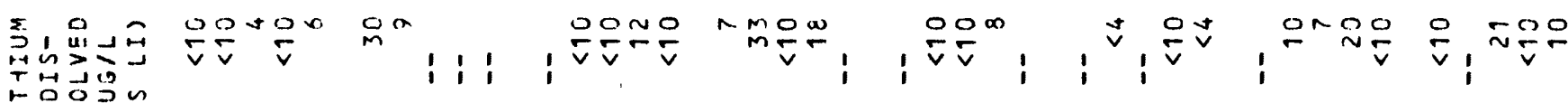

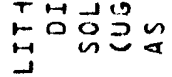

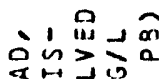
is $\begin{array}{ll}0 & 0 \\ 0 & 0\end{array}$

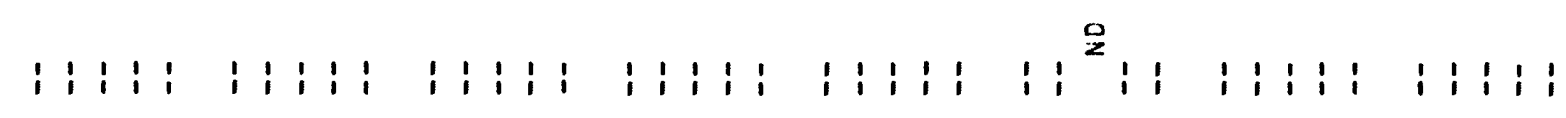

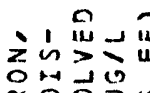
ํㅐㅁำ

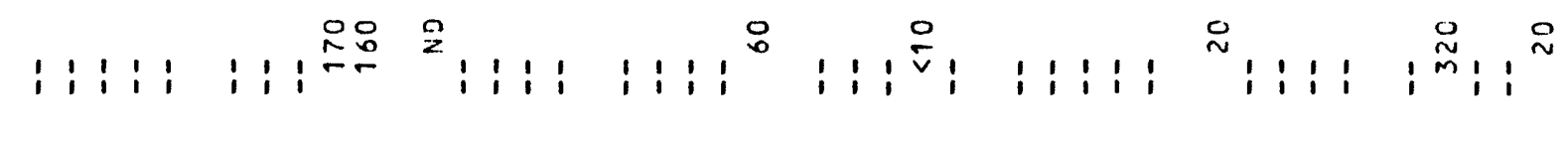

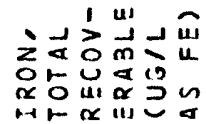

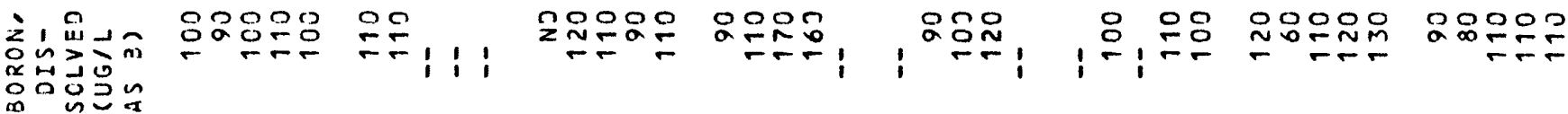

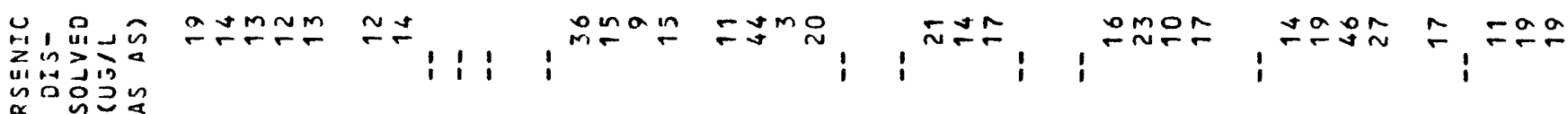

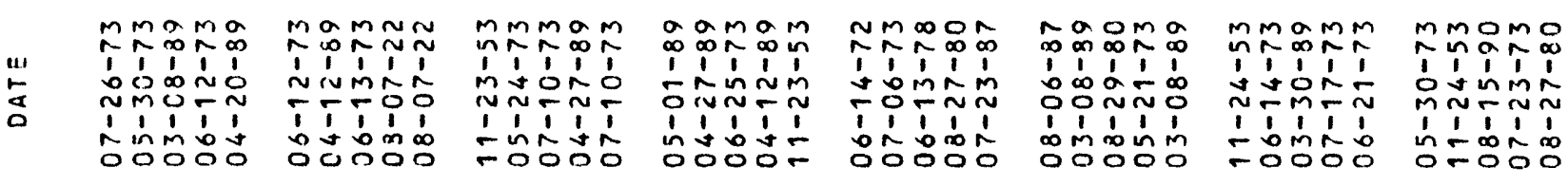




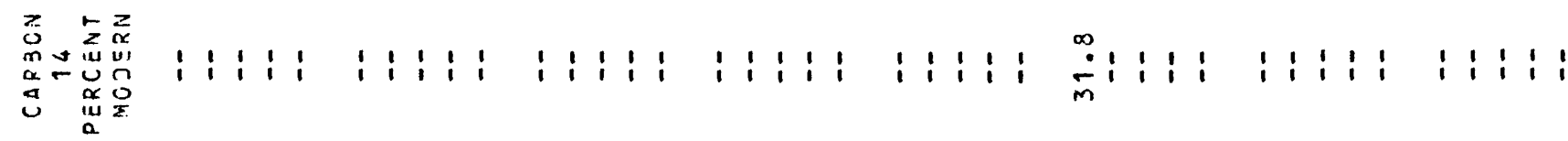

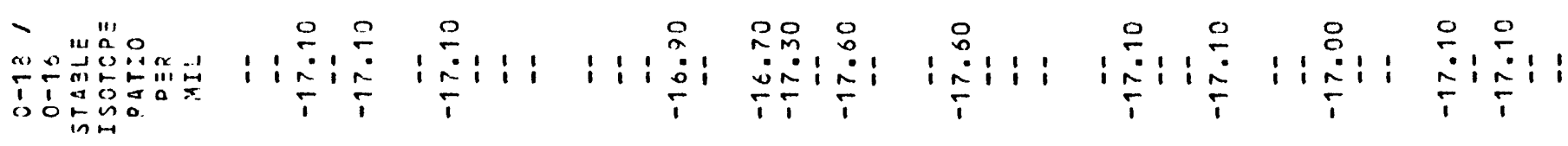

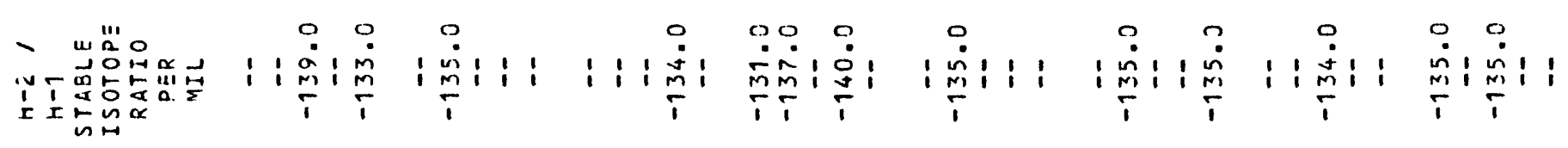

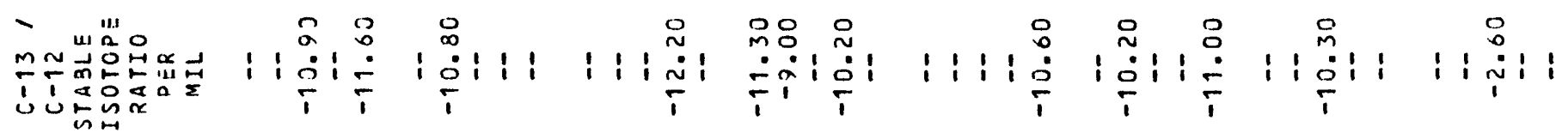

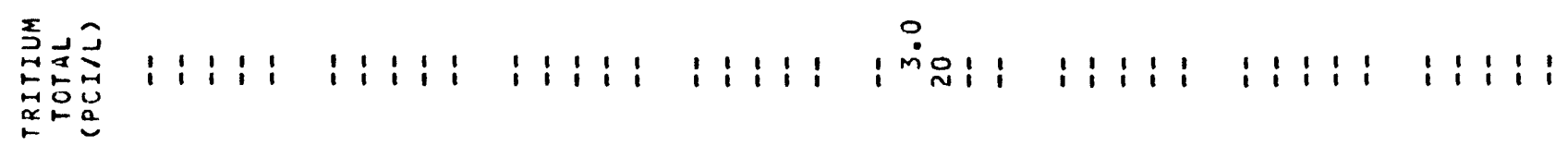

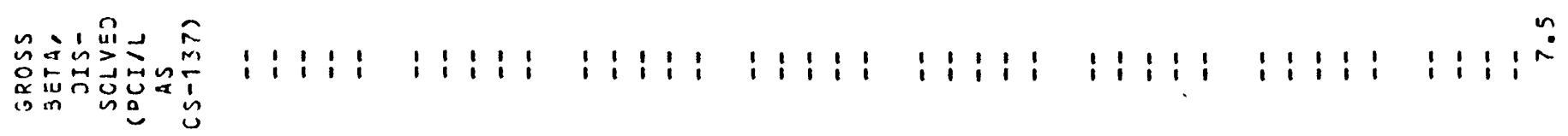

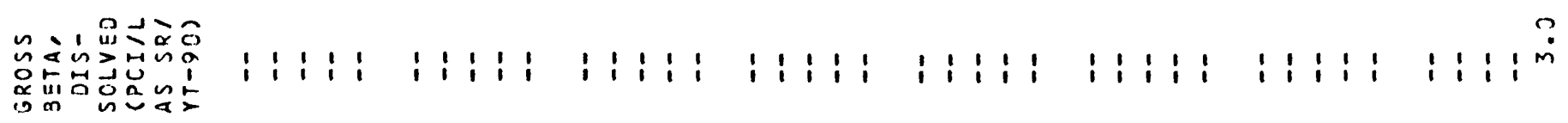

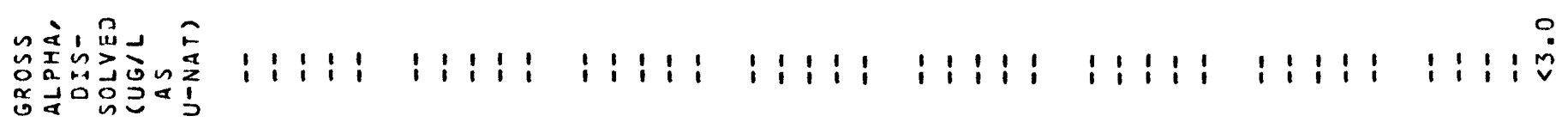

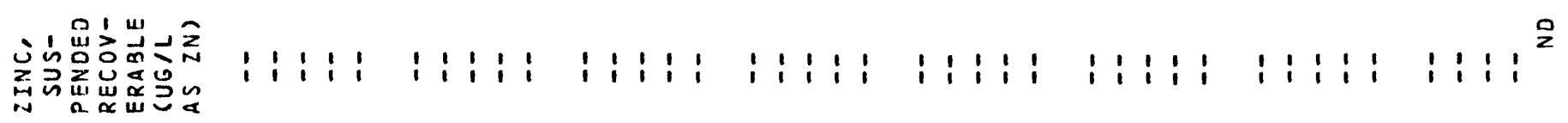

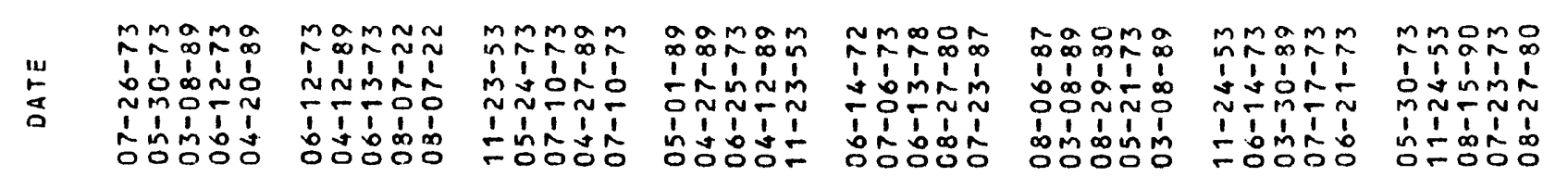




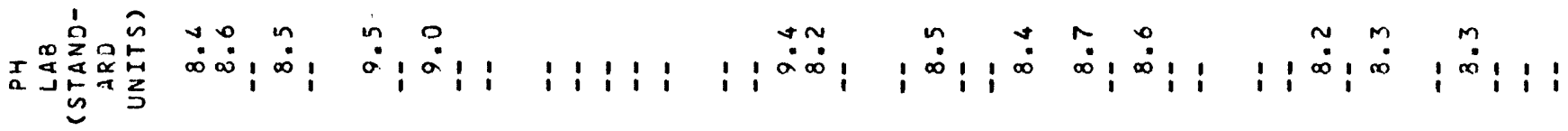

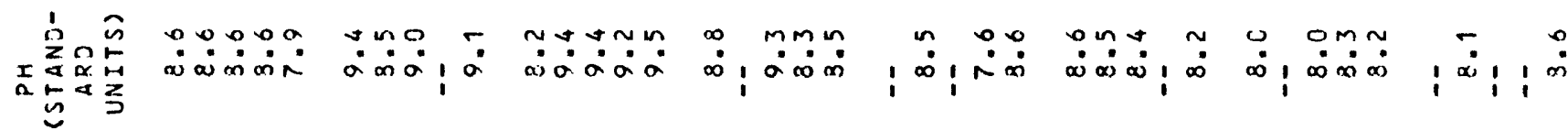

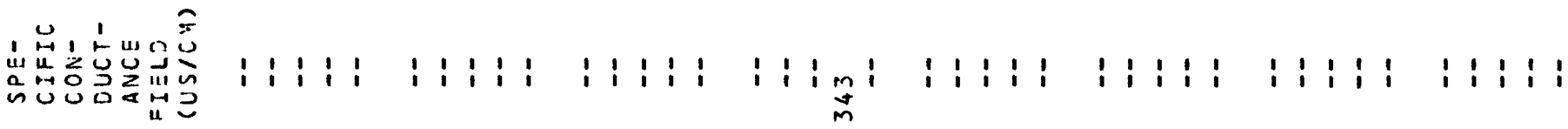

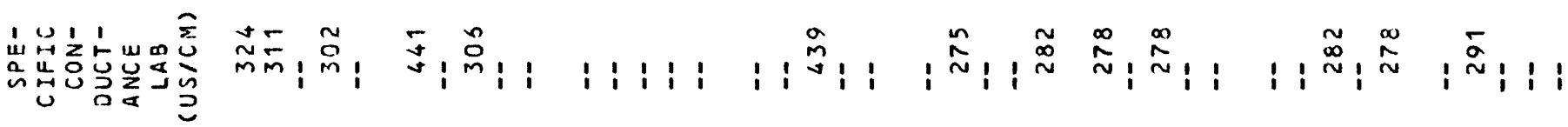

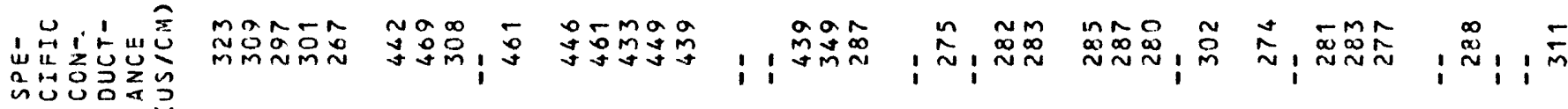
毛山我 u' $\sum_{i=1}^{m}$

noono ouinoo 0 ino00 o nnn no000 nicimin giñón

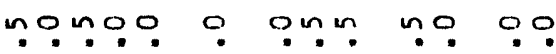

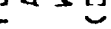

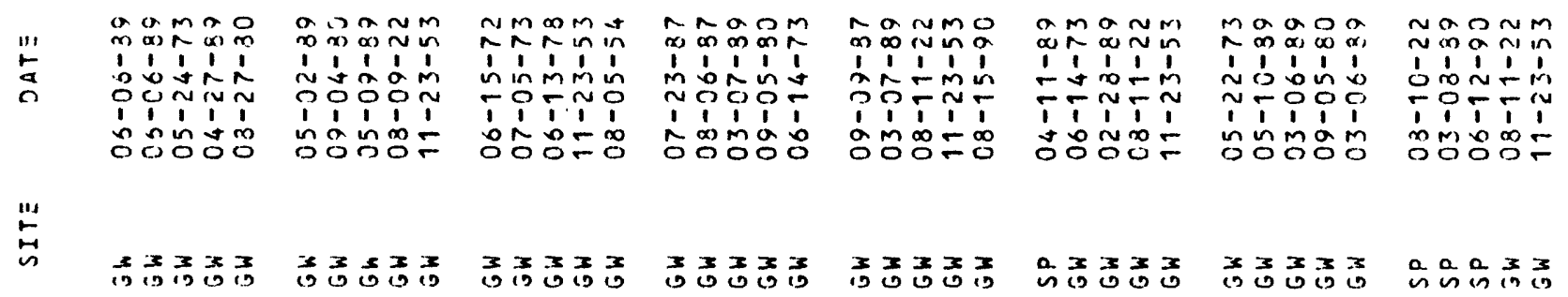


$\sum_{i=1}^{\prime}$ in

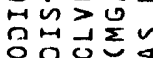
in in a

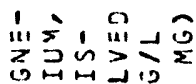

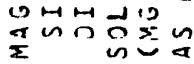

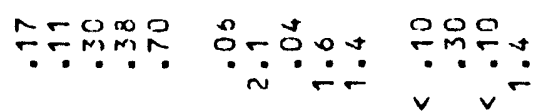

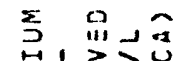

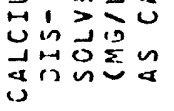

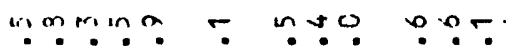

$\because \underset{\cos }{n}$

$\because \because \dot{n} \dot{n}$

$1: \stackrel{2}{2}$

ingó mar̃a

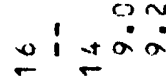

$\underline{2=1} \div$

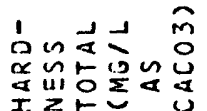

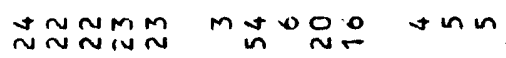

$\stackrel{1}{1} 1^{\sim} 1^{\sim}$

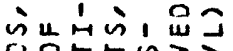

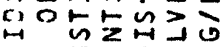

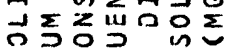

Dinñm ing

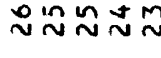

mM̃ó !

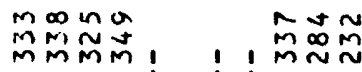

min

늑운

ก

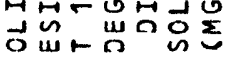

$1,1,1,1, N_{M}^{N O}$

$i: i^{\pi}$

$\pi$ i i i i i i i i i

$\stackrel{\sim}{N},: \stackrel{\infty}{N} \stackrel{0}{\sim}$

i $\stackrel{\sim}{\sim}, \stackrel{m}{n} \stackrel{m}{m} \stackrel{m}{n}$ i i

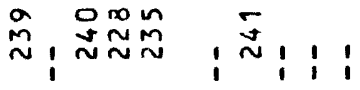

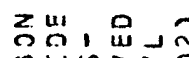

की $\rightarrow \sim \sim \geqslant 0$

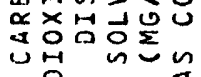

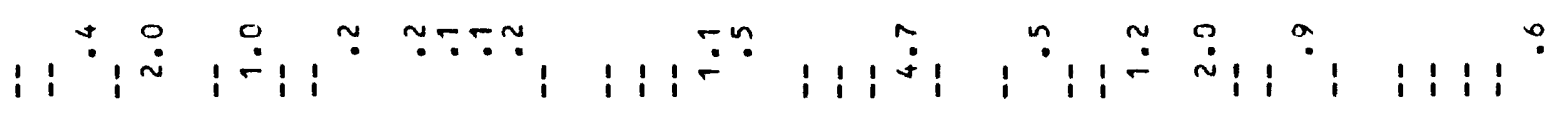

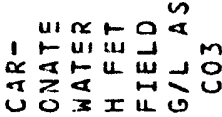

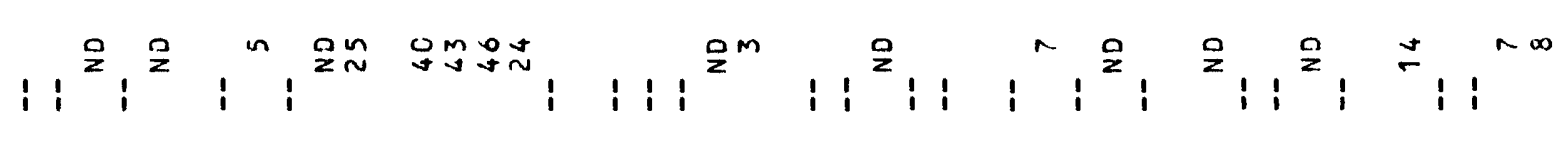

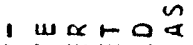

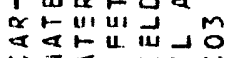

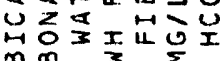

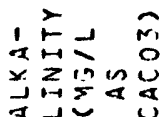

i i $i^{\infty} i^{\circ} i^{\circ}$ 웅

N in in

00
+20

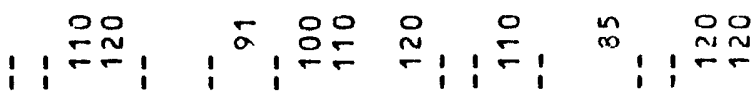

$1>x \div 10 \approx m$

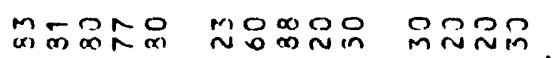

i i i i $i^{\circ} \stackrel{\circ}{1}$ i i i i i i i i i i i

i 1 i i

i i i i i i i

1 1 i : i

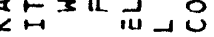

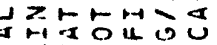

$\because \infty \stackrel{n}{n}$

mָ̃ñ

กัดूณ

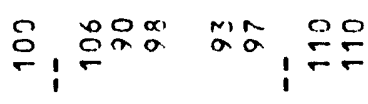

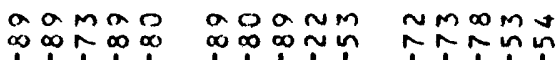

1. 1111

coñ

$\begin{array}{llll}1 & 1 & 1 & 1 \\ 0 & 0 & 1 & 1 \\ 0 & 0 & 0\end{array}$

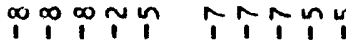

녕웡

ตnำ

$\infty \approx 00 m$

i 1011

ำㅇํㅇㄴ

in

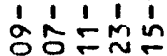

1'mmáb

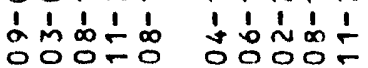

manm

î̃

maO 00

$\begin{array}{lllll} & \infty & \infty & 0 & 0 \\ 1 & 1 & 1 & 1 & 1\end{array}$

先응ํㅇㅇํㅇ

NoONm

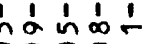

ํํำ

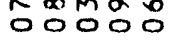

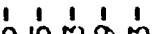

Nian

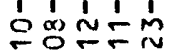

1'⿺⿻一𠃋丶 


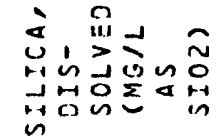

in 공ㅎㅁㅇa

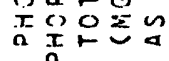

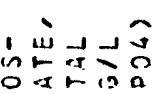

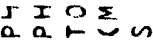

')

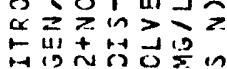

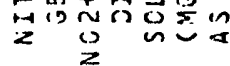

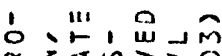

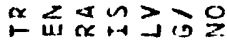

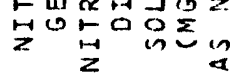

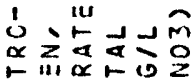

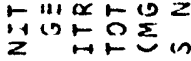

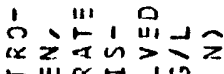
:

'́丶万

览,

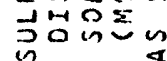

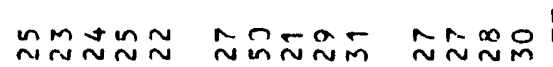

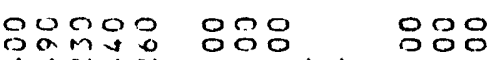

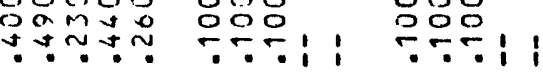

$\checkmark v v$

$v v v$ i i i i i i :

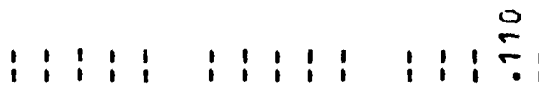

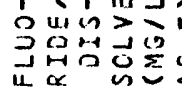

i!i! i i i i i i i !

İas I

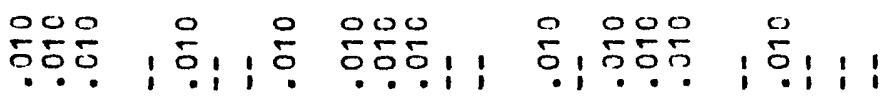

$v v i v v v$ is i i i i i: $1: 1$

$1: 1: 191: 11$ 1 , 紧

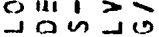

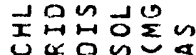

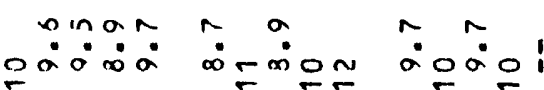
$0 \% 0$ i! i i i i ?

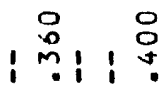

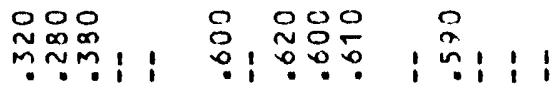
$1 ; 1 i^{\infty}$ :1:1: $1: 1: \div$ 2

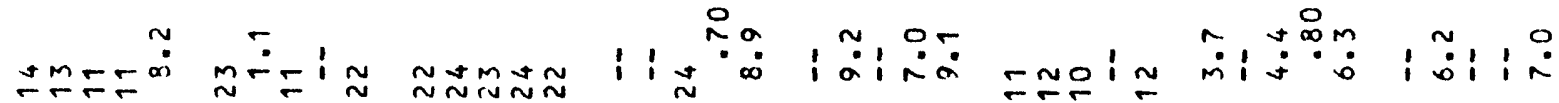

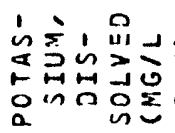
ஐบฺn $m ?, \quad \infty \infty \infty a$ $\sim \infty i$ iniñ i: ini: in $\because \because m$ is i

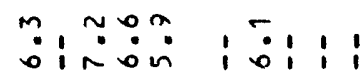

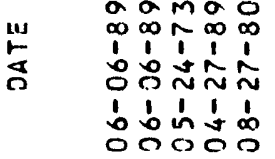

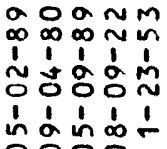
$\approx m \infty m *$ îîi in ํํำกำ ป่า

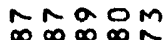

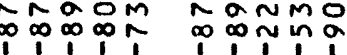
ํํㅇํำ

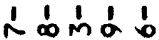
átín 은 ㄴ.

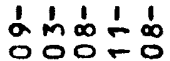
amom 11111

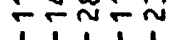
I o응ㅇㅇ
Nonm 11111 웅다 l'mb

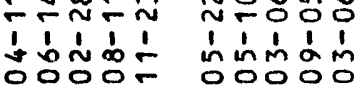




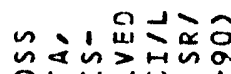

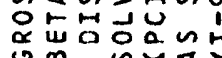

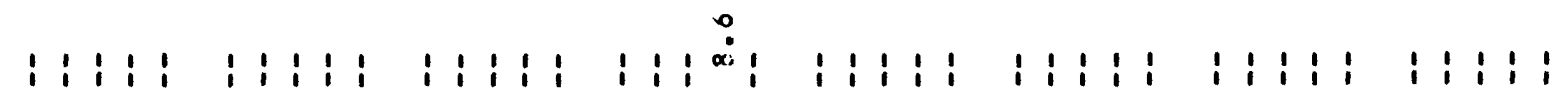

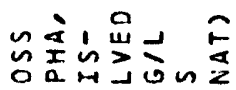

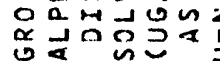

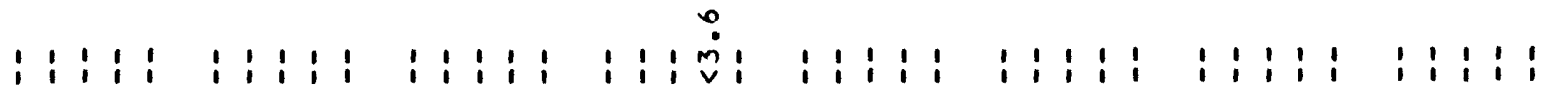

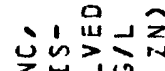

în

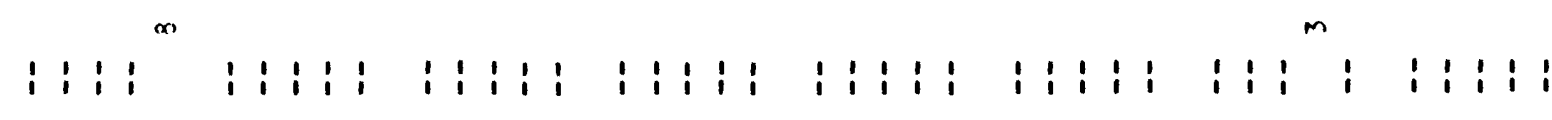

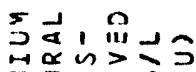

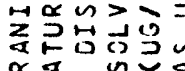

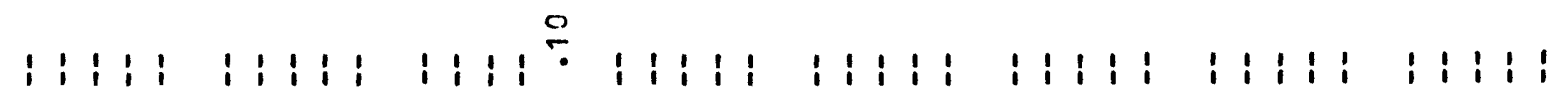

iu $\sum_{i}$ in

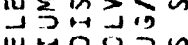

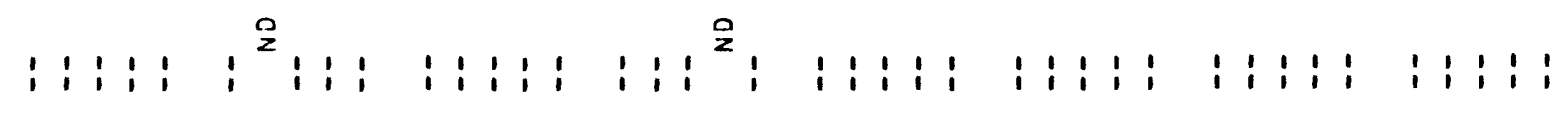

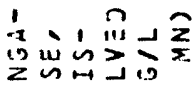

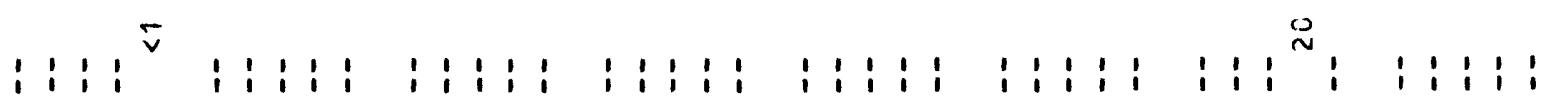

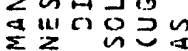

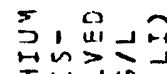

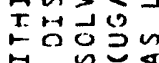

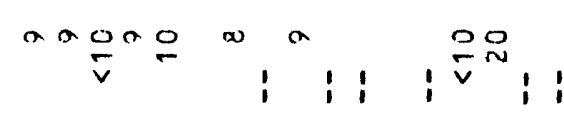

$\sqrt{0}$

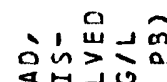

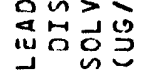

之in

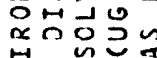

$1 i^{+} i^{\frac{2}{2}}$

$i^{*} i i^{\text {n }}$ no $^{2}$

$\stackrel{\circ}{\sim}$ mon, i : i

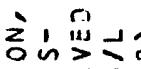

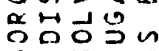

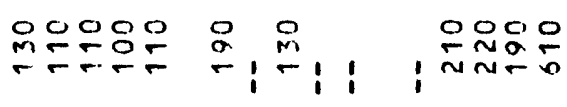

i 哭, 品

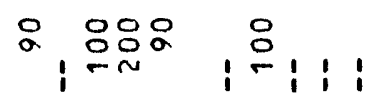

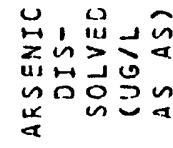

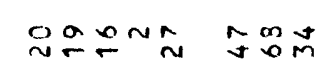

i i $i^{\infty} i$

iñ

$i_{i}^{m} i i^{n} \stackrel{n}{n} i$

$+\stackrel{n \infty}{\sim}, \stackrel{\circ}{\sim}$ i i i

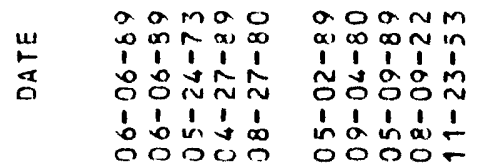

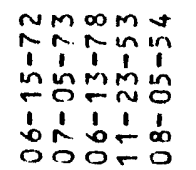

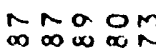

ow

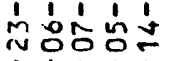

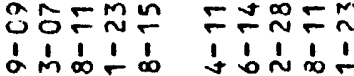

masoo

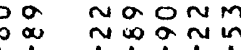

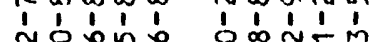

궁ㅇㅇㅇㅇ 웅푼

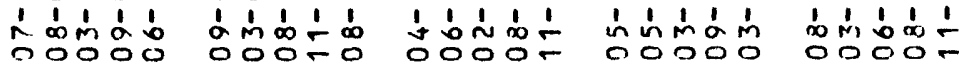




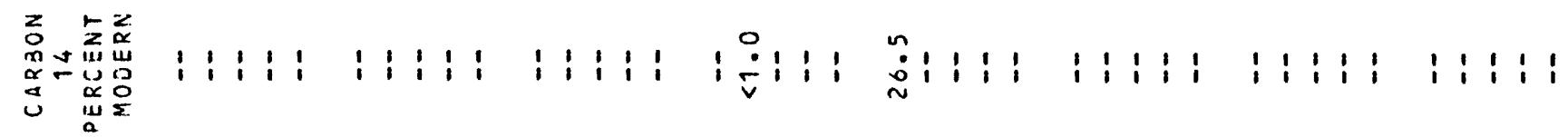

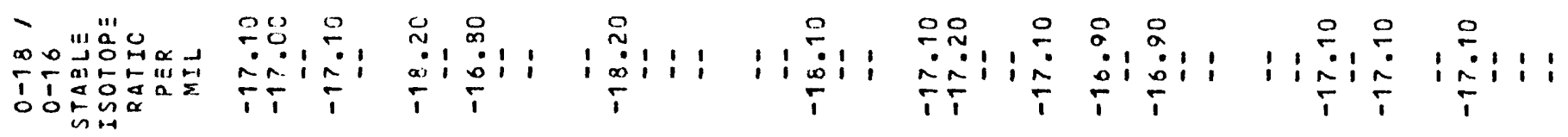

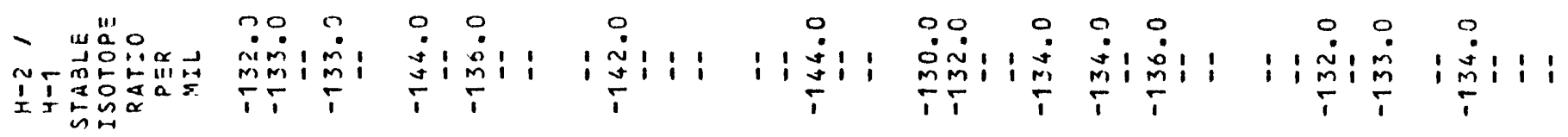

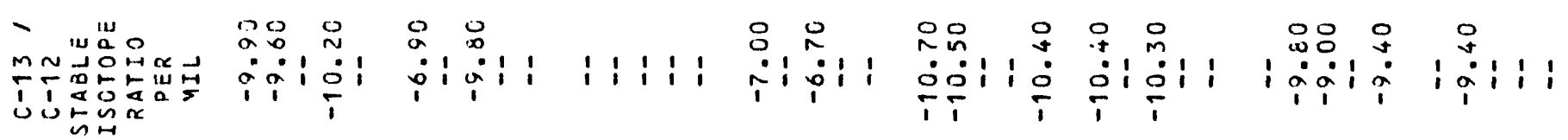

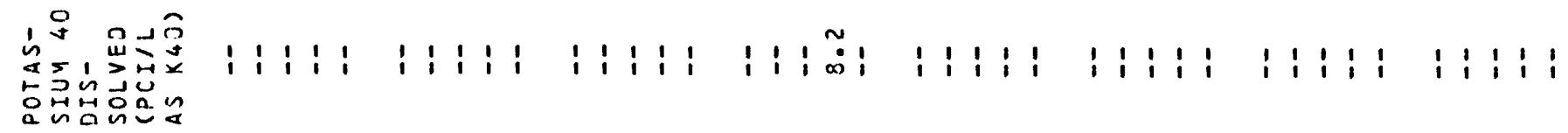

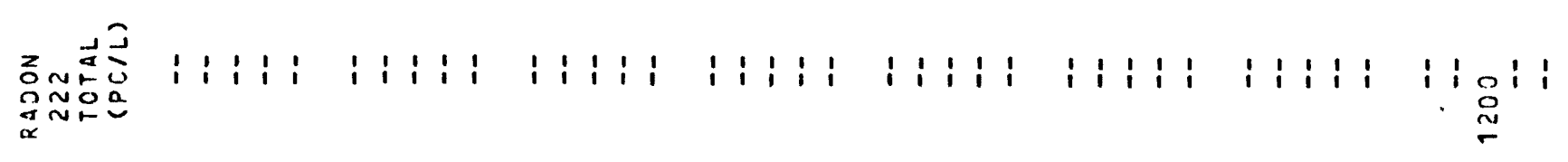

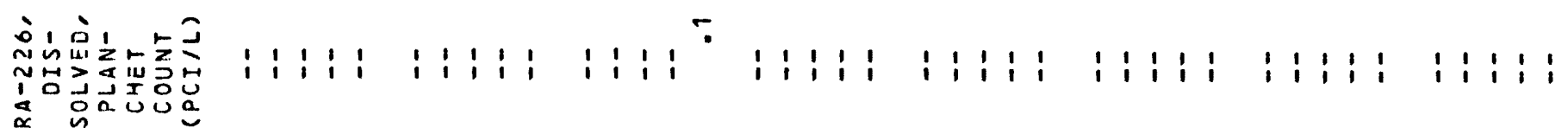

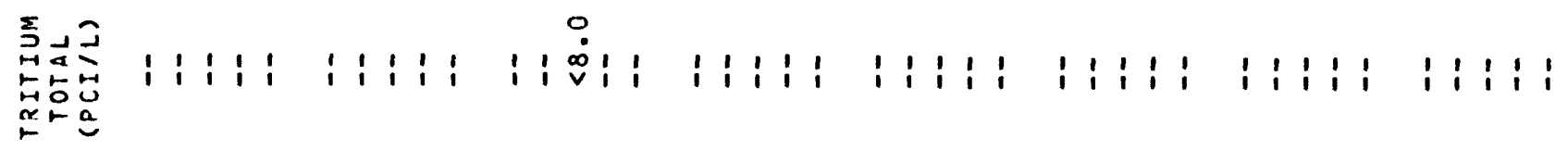

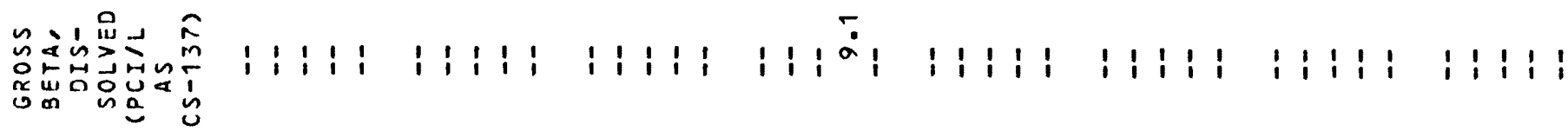

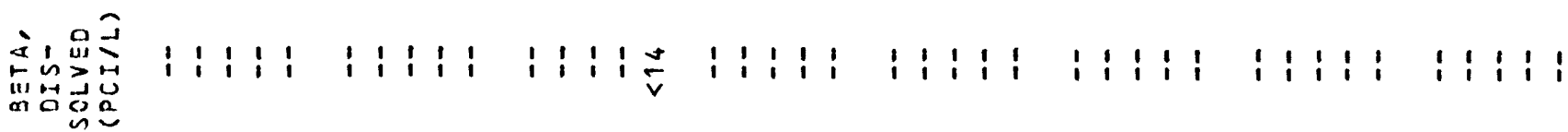

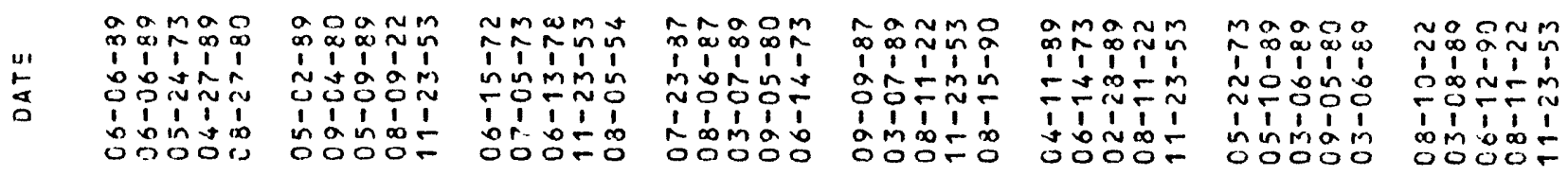




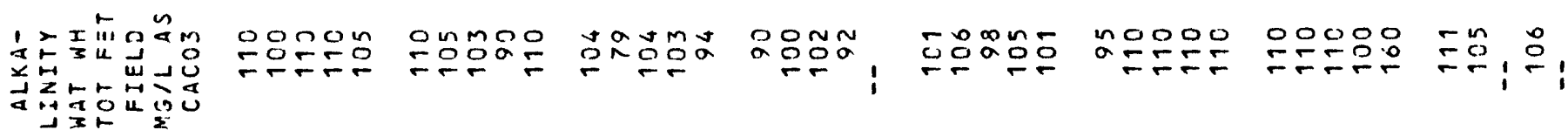

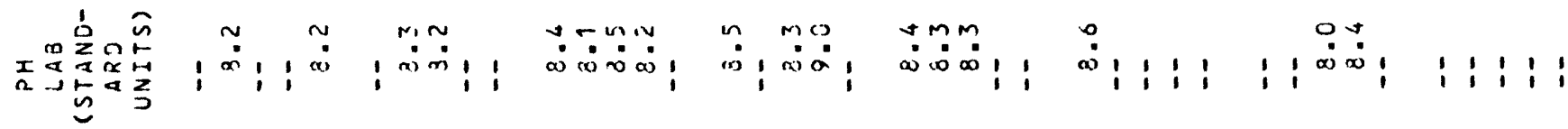

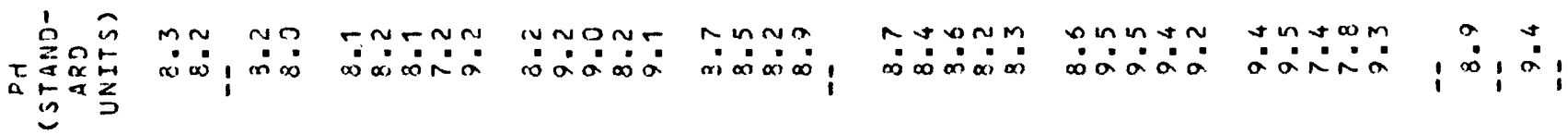

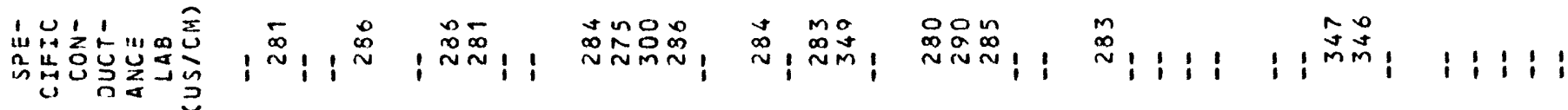

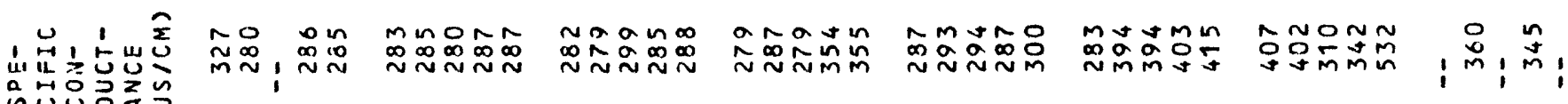

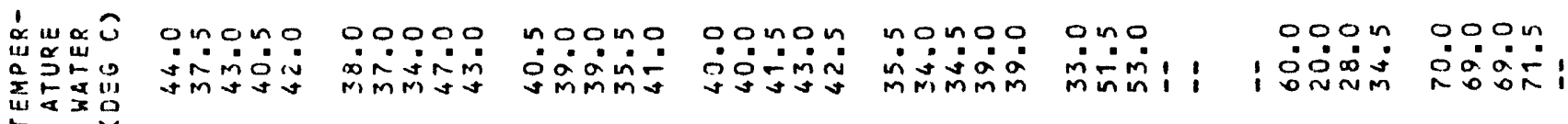

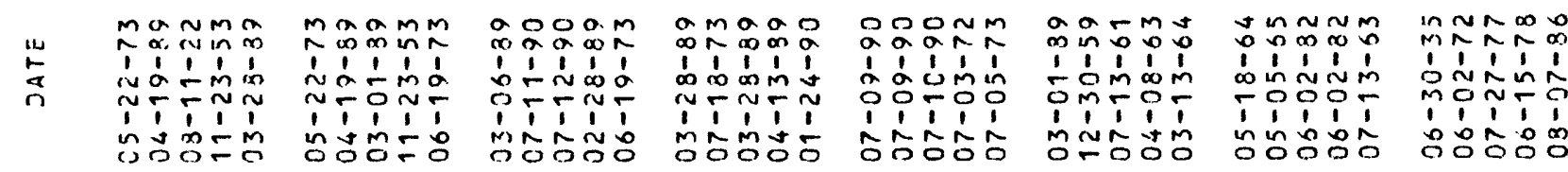
$i_{i=1}^{u}$

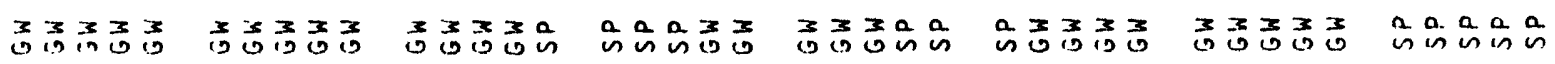
2

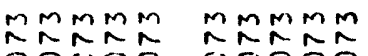

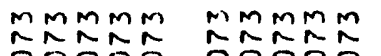

$m m m m m m m m m m m m m m m$

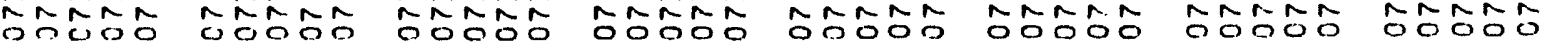

\begin{tabular}{|c|c|c|c|c|c|c|c|c|c|}
\hline 190 & 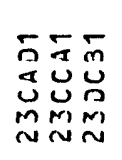 & $\begin{array}{l}a \\
a \\
0 \\
a \\
0 \\
\sim\end{array}$ & 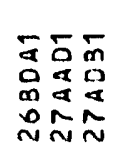 & 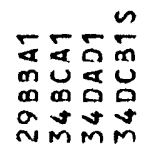 & $\begin{array}{l}n \\
n \\
\infty \\
\infty \\
\infty \\
n \\
m\end{array}$ & $\begin{array}{l}5 \\
a \\
a \\
0 \\
0\end{array}$ & 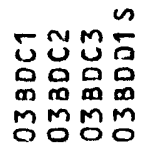 & $\begin{array}{l}5 \\
0 \\
0 \\
0 \\
0 \\
0\end{array}$ & 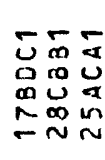 \\
\hline $\begin{array}{l}1 \\
3 \\
3\end{array}$ & 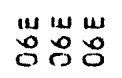 & $\begin{array}{l}\text { uI } \\
0 \\
0\end{array}$ & 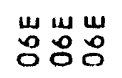 & 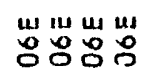 & $\begin{array}{l}\text { ü } \\
\text { o }\end{array}$ & w & 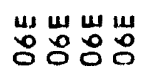 & 嵓 & $\stackrel{\text { 岃 }}{\sim} \underset{\sim}{\sim}$ \\
\hline & $\begin{array}{l}\approx \sim \sim \\
\tilde{c} \tilde{O} \tilde{O}\end{array}$ & $\tilde{0}$ & $\begin{array}{l}\sim \sim n \\
\tilde{0} \tilde{0} \hat{0}\end{array}$ & $\begin{array}{l}\approx \sim \sim n \\
\tilde{C} \tilde{0} \tilde{0}\end{array}$ & $\tilde{n}$ & $\underset{\infty}{\infty}$ & $\begin{array}{l}n \sim n \\
\infty \\
\infty\end{array} \infty$ & $\stackrel{n}{0}$ & $\begin{array}{l}n \sim n \\
0 \% a \\
0\end{array}$ \\
\hline
\end{tabular}




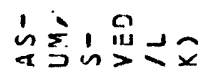

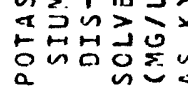

$\sum_{i=1}, \quad 0 \frac{\pi}{2}$

$i+$ in $>19$

on 品要

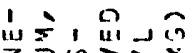

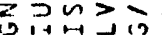

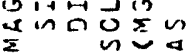

mo:

rn:

a) on a

mingm

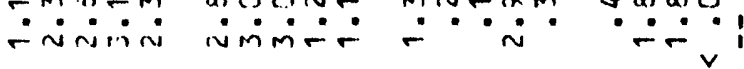

m?

$\sum_{i=1} \prod_{i=0}^{\prime \prime}$

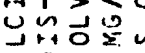

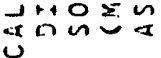

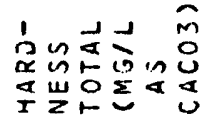

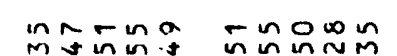

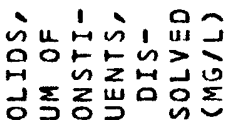
일일

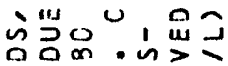

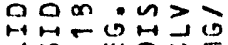

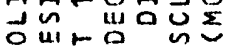
ก̃ $\stackrel{N}{N}, \stackrel{n}{\sim}$

miñma

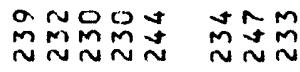
$\sim \sim \sim$ ! $\hat{n} \tilde{n} M \cong$ ÑÑÑ moำำ

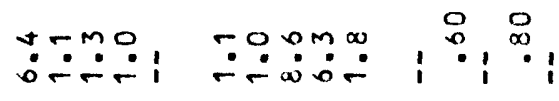

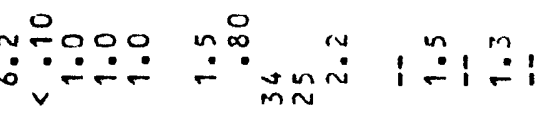
뚬ำ

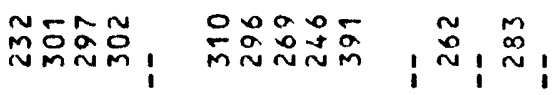
i i iัn! i i i i i 1 i i

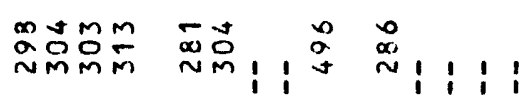

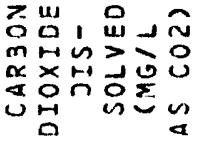

maton

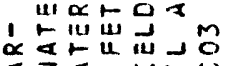

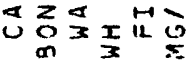

I $41 \alpha \leftarrow 0 \stackrel{n}{a}$

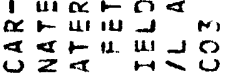

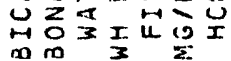

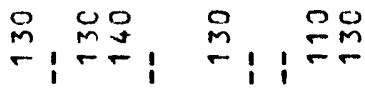

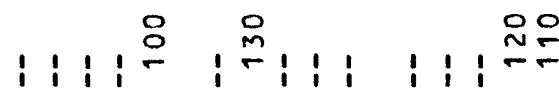

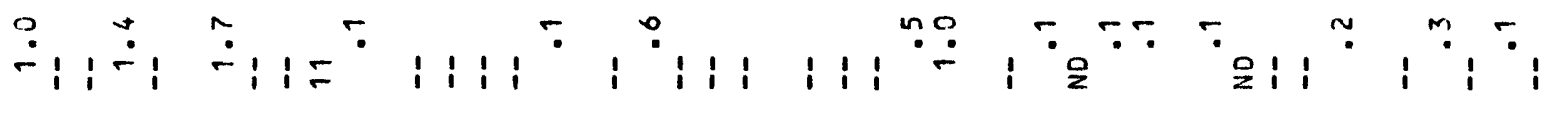

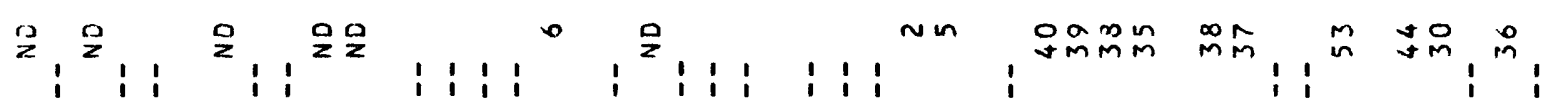

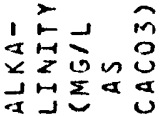

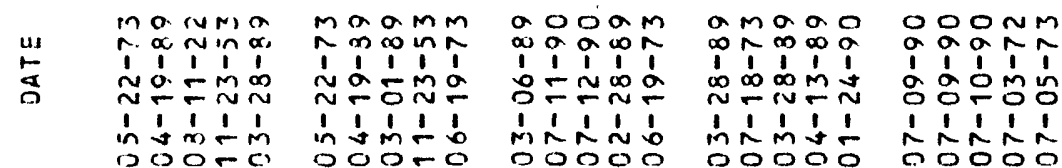
ogonm aormu

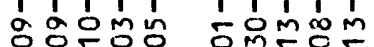

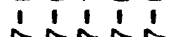
ḿ! 1 snm 1: $1: 0$ ๓ำำต in in: 1 man

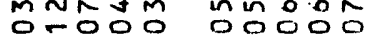
웡 in b́t่́n 00000 


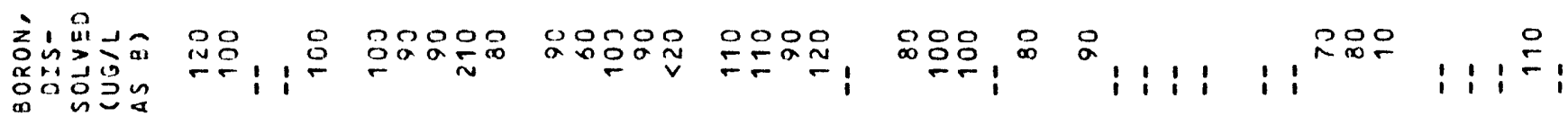

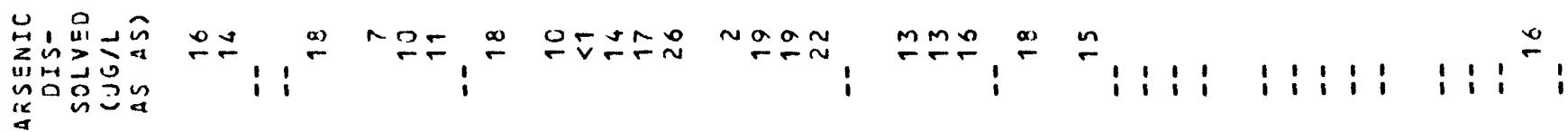

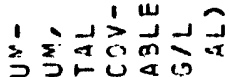

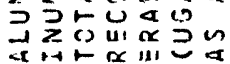

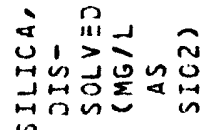

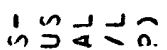

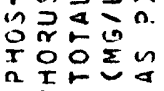

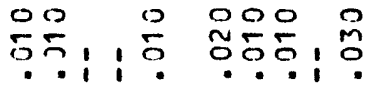

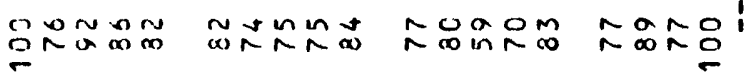
.

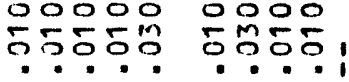
$\vee \vee \vee \vee$

$\checkmark v v$

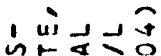
पूत्व

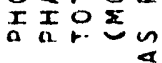

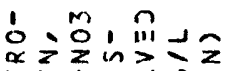

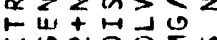

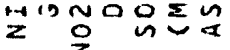

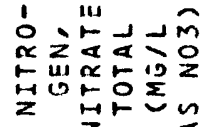

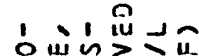

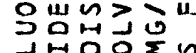

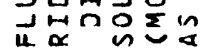
किं ij mimmon

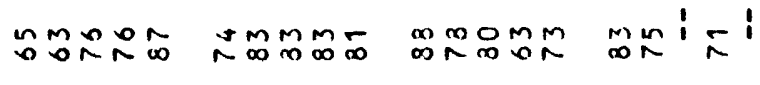

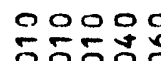
․․․․․․

$\vee \vee v$

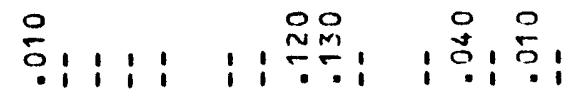

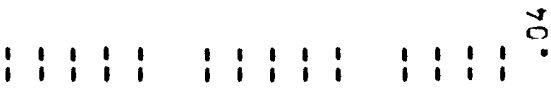

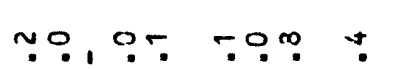

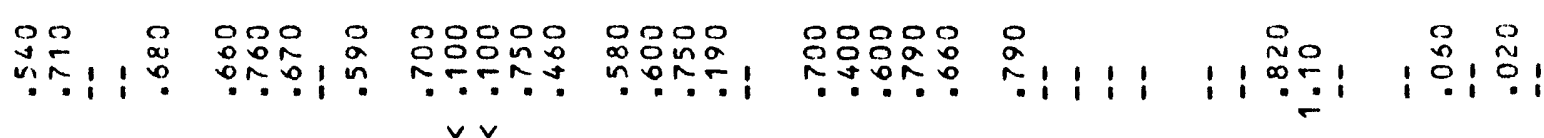

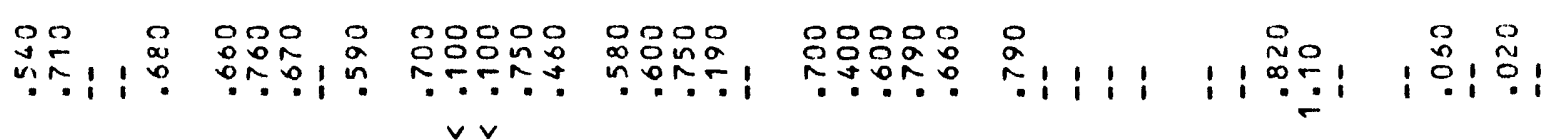

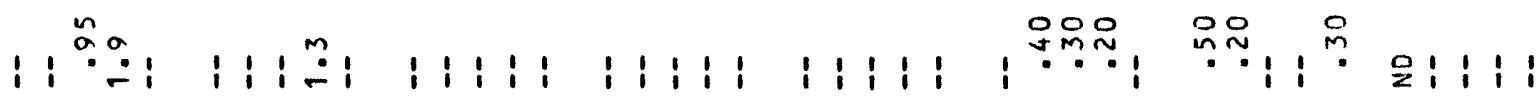

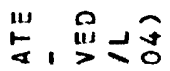

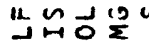
근명은

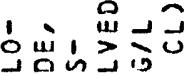

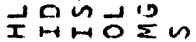

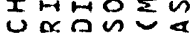

n Noon $\because \because \because 9 \% ? \% 90 \%$ $m \infty ? ?$ $\infty \operatorname{\infty in} \theta$ $\infty 000 \infty$ कo

inos: MaNma maOMm aOOAM Tîn

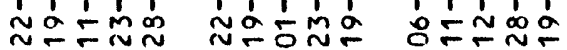

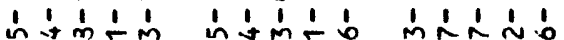
oO등
$0 m a 00$

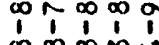

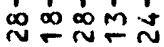

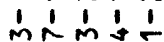
ốmó
000 n iogî́ ogógín

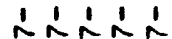
oóogo

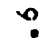

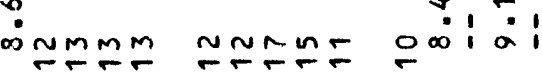

inmoñ 윰ำ

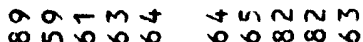
1

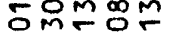
mंñ் ๓กิ์ธิธี in'向向 oño응 $\operatorname{mnn} \infty$ TNÑ̂́

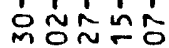

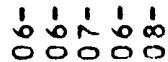




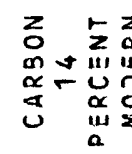

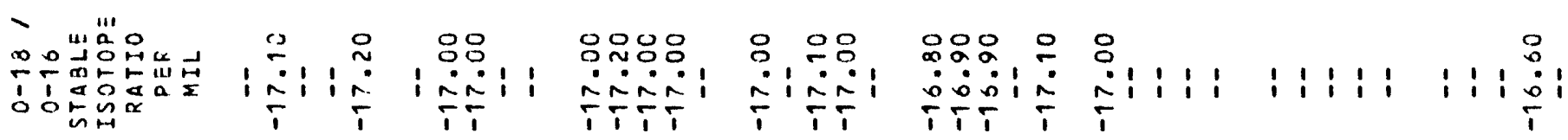

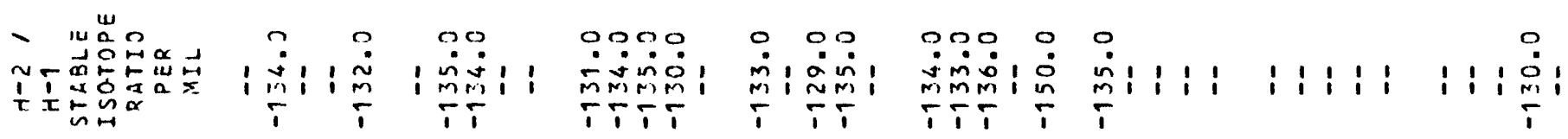

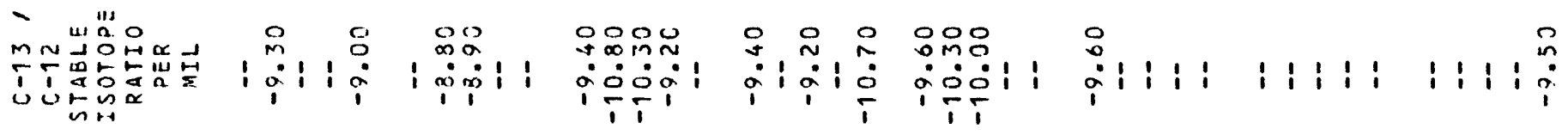

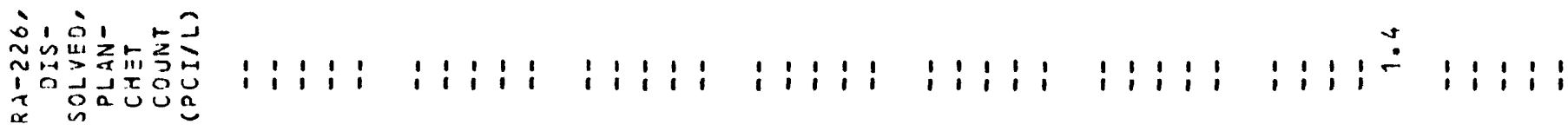

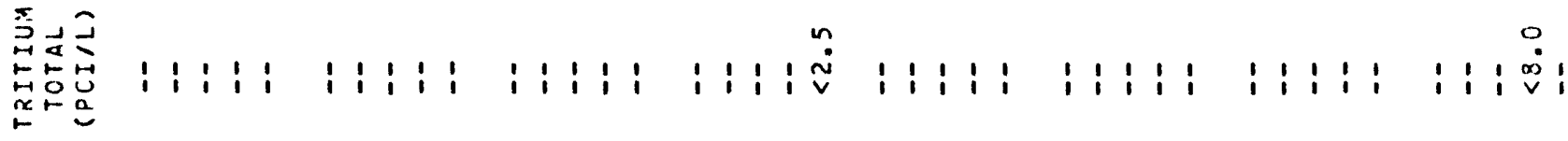

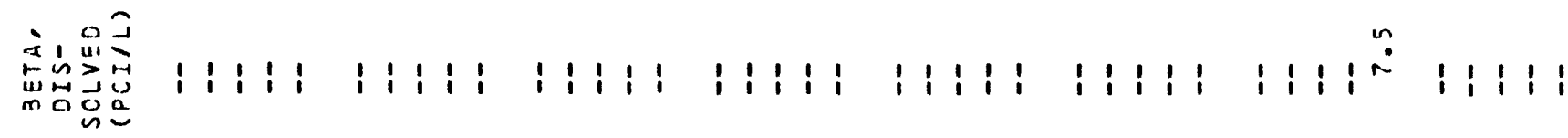

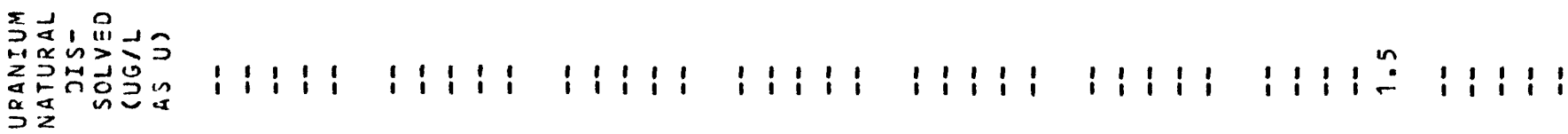

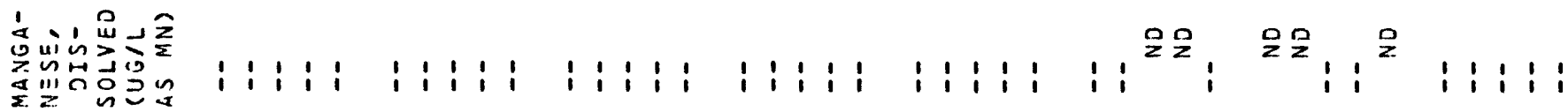

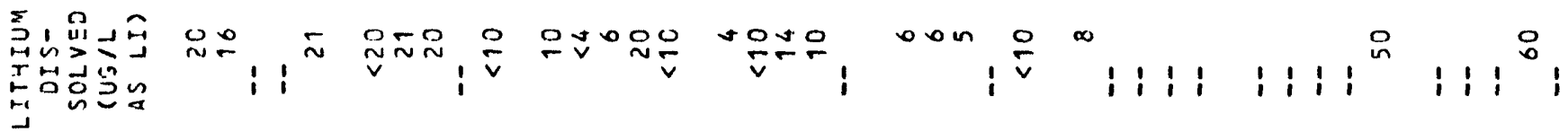

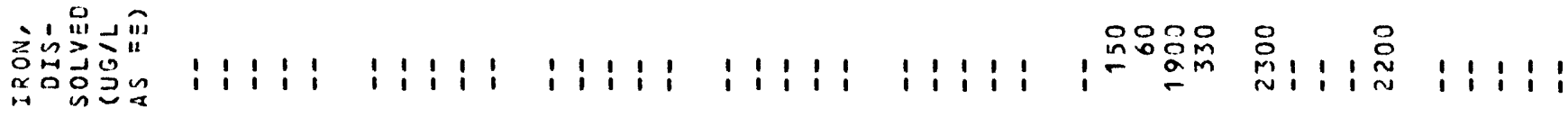

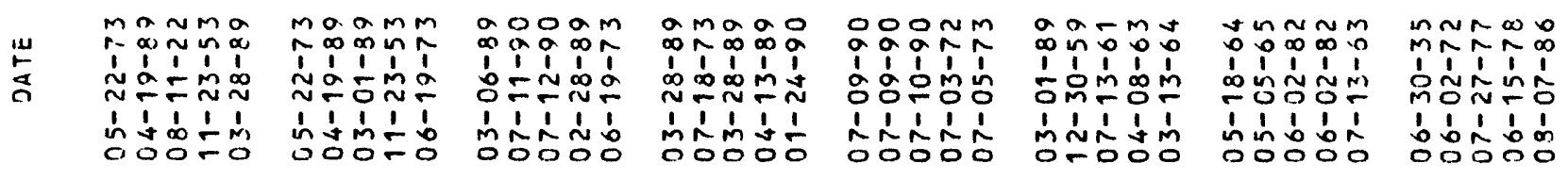




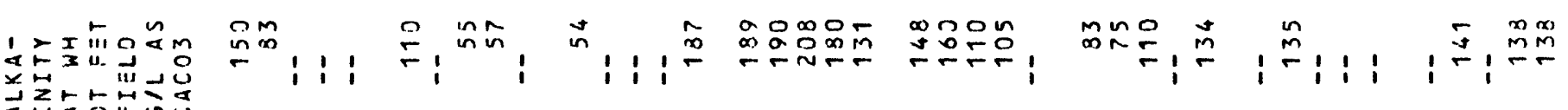

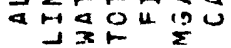

茫出品 艾出可焉 希 品 i i i

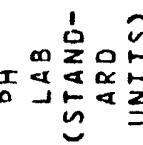
$\stackrel{0}{\infty} 11$

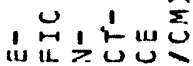

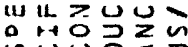

' us $\propto \hat{U}$

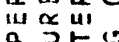

$\sum t x$ แ⿺

id

$\stackrel{u}{\leftarrow}$

maoun

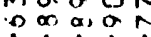
$1 \frac{1}{2} \frac{1}{m}$

OM

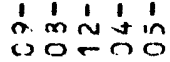

nos on

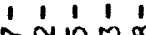

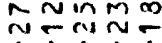

1 1 i

b'óñ

$\begin{array}{lllll}\infty & 0 & 0 & 0 & n \\ 0 & 0 & \alpha & 0 & 1 \\ 1 & 1 & 1 & 1 & 1 \\ 0 & \sim & m & 0 & 0 \\ \sim & 1 & 0 & 1 \\ 1 & 1 & 1 & 1 \\ 0 & \infty & 0 & 1 & 1 \\ 0 & 0 & 0 & - & 0\end{array}$

ingNan

순

ำññ

ำ 11

กํํㅇํㅇ

a in

in

ming 11

우웅

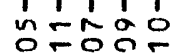

$\stackrel{a 1}{5}$

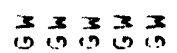

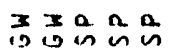

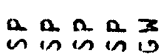

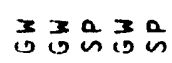

a a. $x=$ $15 \sin \frac{1}{10}$

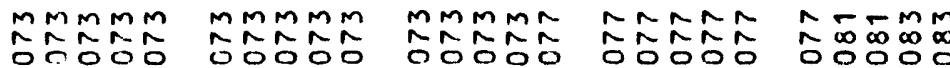

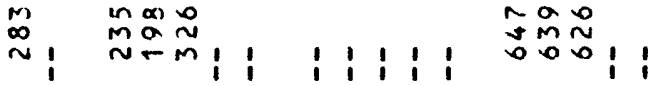

$\ln n$

$\therefore 0 \infty i$ i i i i i

$\infty 00$

$\therefore \dot{\infty} ;$ i

mixia mámo

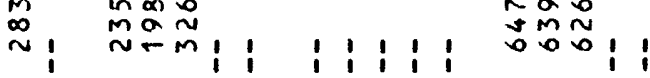

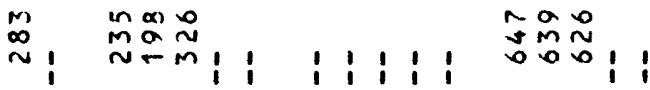

$\underset{\infty}{\infty} \sim \infty \sim \infty$

1111

$\sim \sim 0-\infty$

$\begin{array}{lll}1 & 1 \\ 0 & 1 & 0\end{array}$

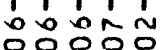

a $0 N N N$

$\hat{1} \hat{1}$

io in s is

i 1515

1

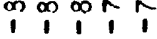

¿mo 0 in

1.11

กับm

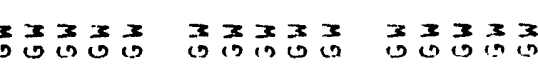




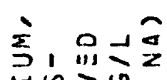
in $\ln ^{\prime \prime}$

으원

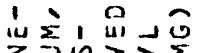

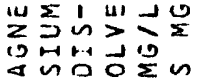

ㅍำ

oi

of: $i^{2}$

ะก๊ำำ

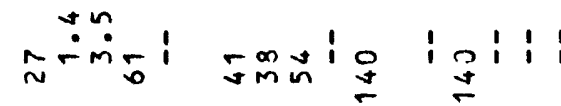

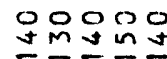

?용․

$\dot{9}: 1:$

이눈

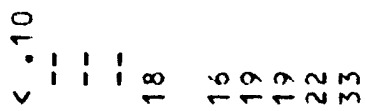

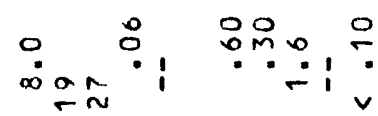

i $\frac{0}{1}: 1:$

जo 000

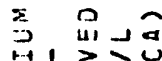

تnٔ

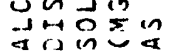

is

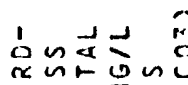

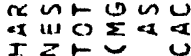

的的的嵒

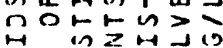

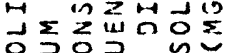

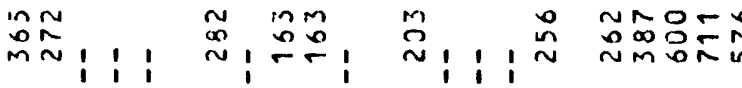

$\stackrel{n}{n} \approx$

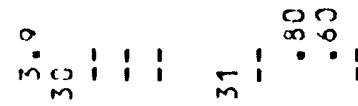

in : i :

$\sin \sin ^{2} \sin$

$\hat{m} \rightarrow \infty$

m!

$\because \because=1 \div$

2

0000

$\stackrel{0}{\sim}$,

$\sim 1 ; i^{\circ}$

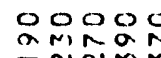

m曹

$\tilde{n}^{m} \dot{m}$

i 1 i

$m \sim$

$v v$

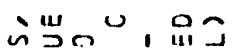

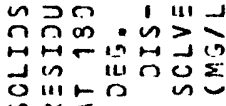

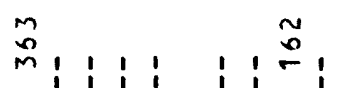

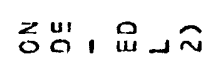

$m \omega n>=0$

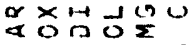

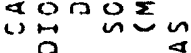

$\because$

i i i

$\infty m$

$\stackrel{m}{\sim}$

눈

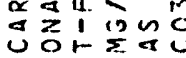

o난

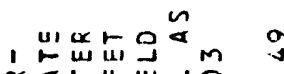

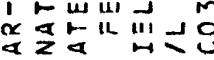

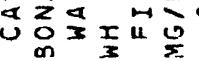

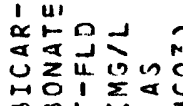

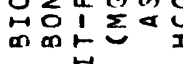

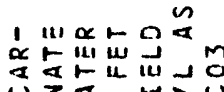

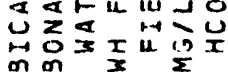

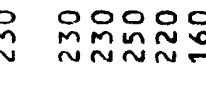

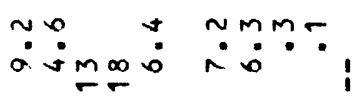

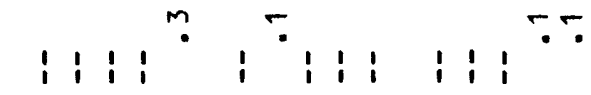

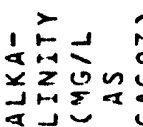

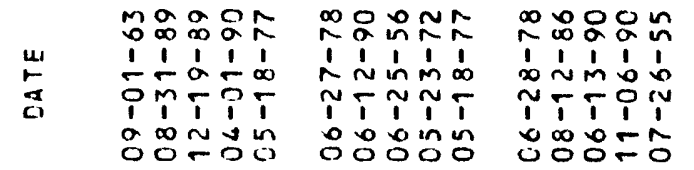

के

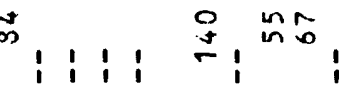

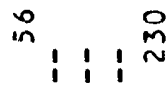

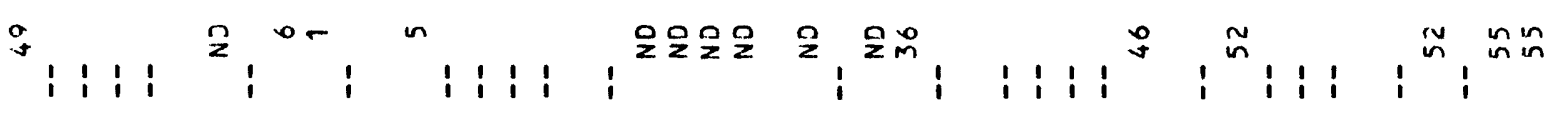

$1: 1:$

i : i i

i: $: 1:$ i: in $\tilde{n}$

$11: 1$ $1: 1: 1: 1: 1:$ : $1: 11$

$1: 11$ : 1 1 $1:$

: : : : : : : : $\stackrel{\infty}{=} \stackrel{1}{\simeq}$ 1) 11

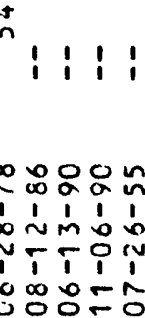
$1111+11111111^{2}$

용 운 $\stackrel{\circ}{m}: 1$

inaaN b́míni זTNT

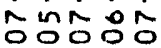

జ $\sim \tilde{\infty}_{\infty}^{\infty}$

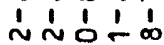

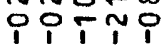

ㅇํㅇํํㅇ

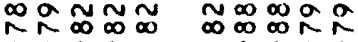

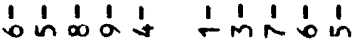
NTT TT TTIN

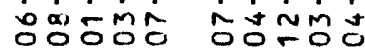




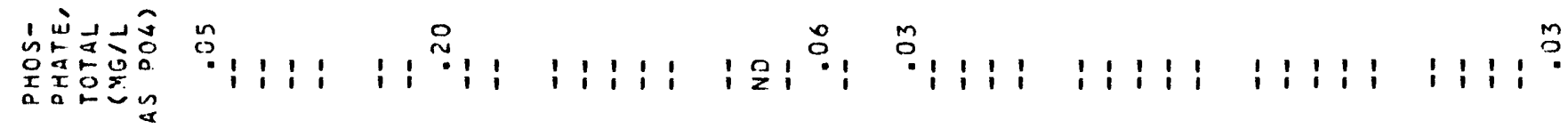

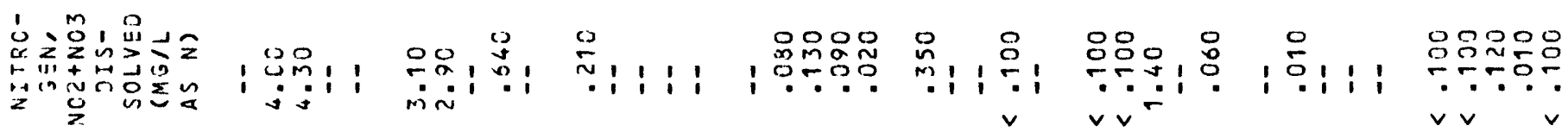

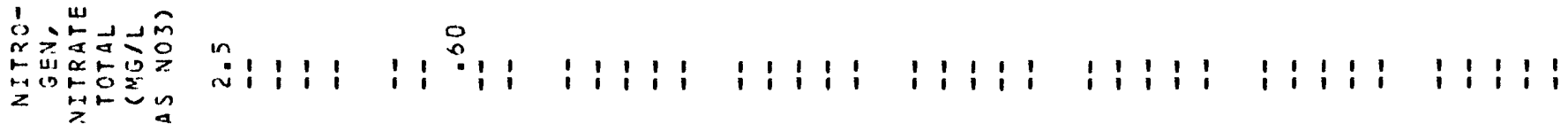

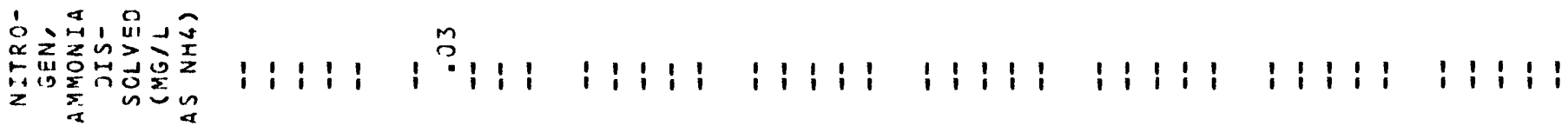

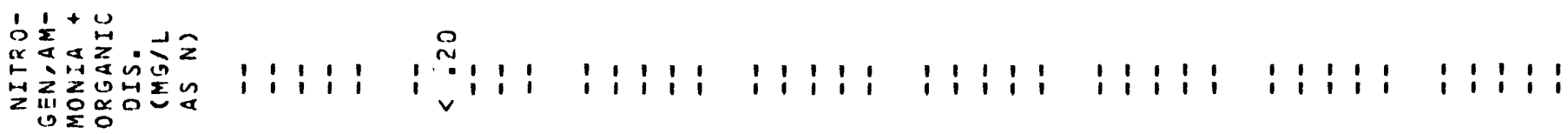

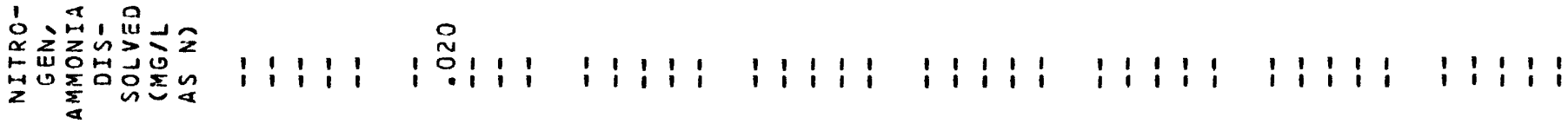

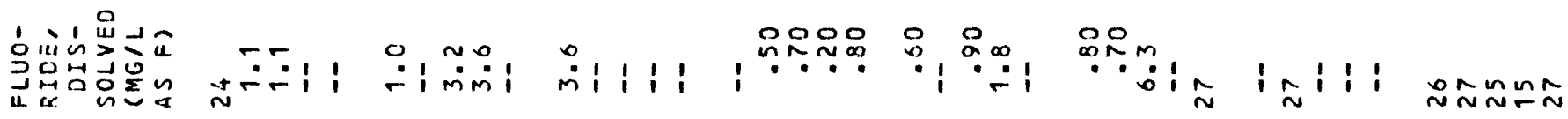

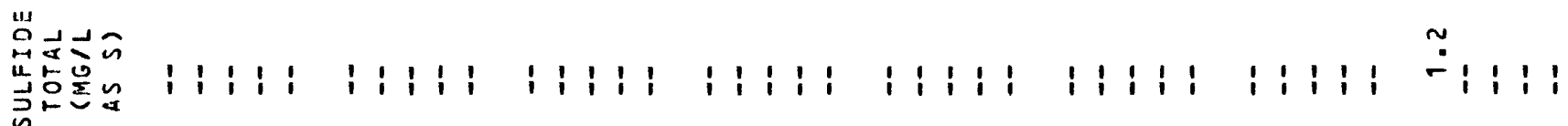

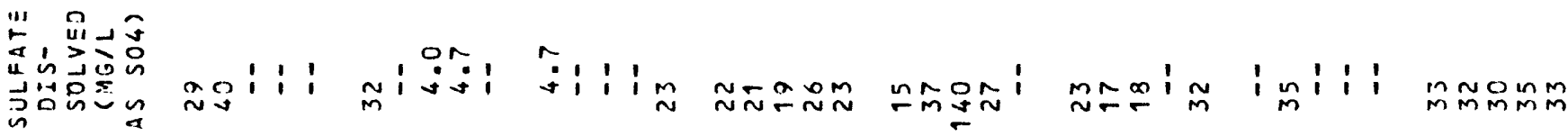

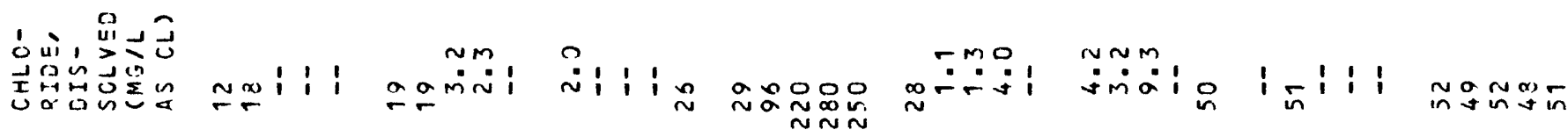

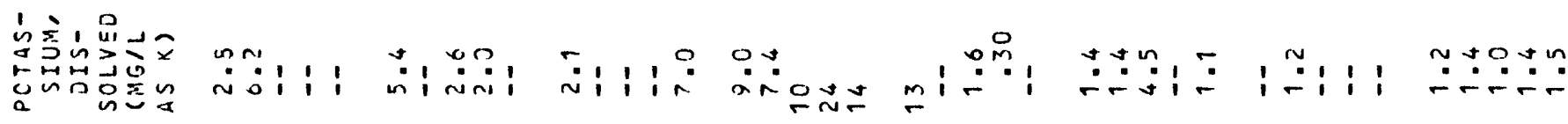

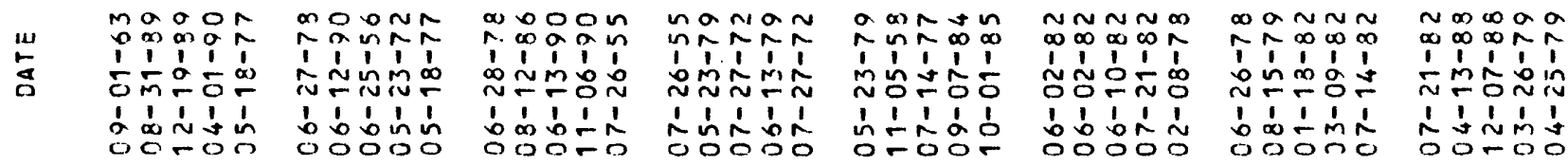




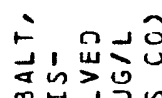

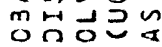

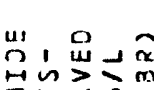

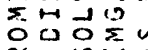

a

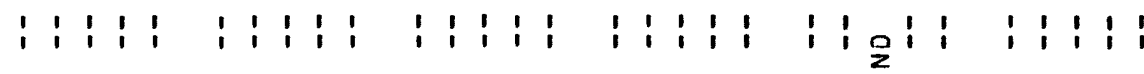

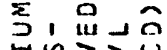

$\because n>>0$

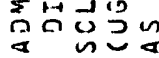

i $1: i:$ i i i i

证出さた

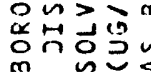

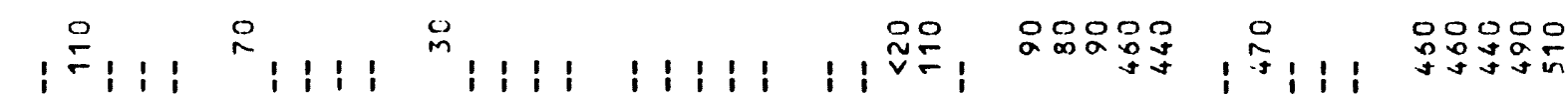

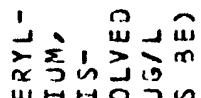

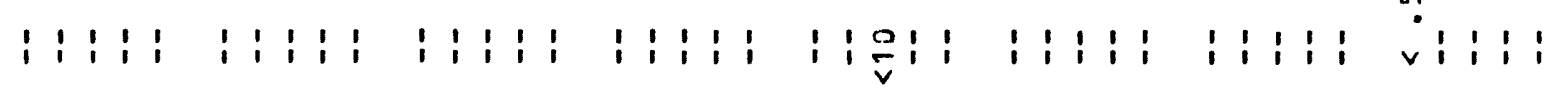

产, 品余

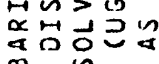

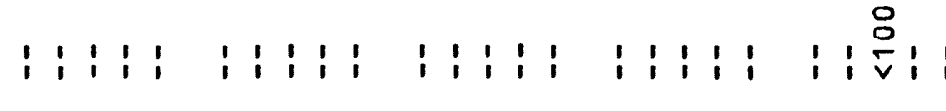

$i: i:$

$i: i: i$

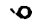

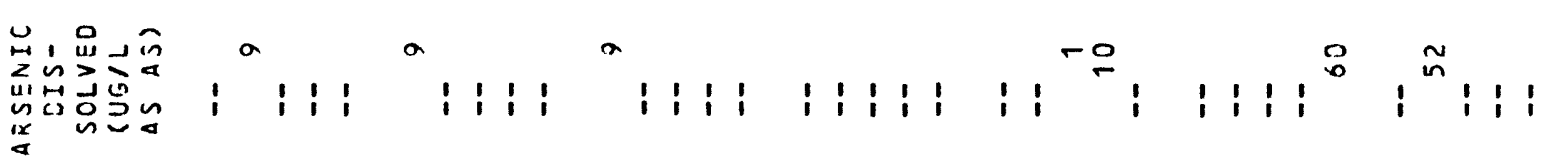

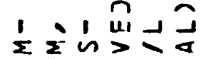

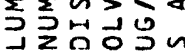

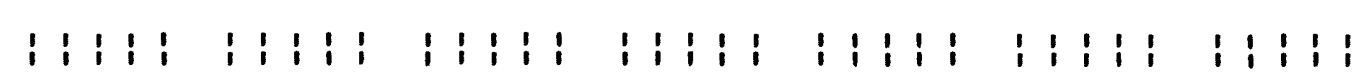

웅

$\sin 8$ 영ำ

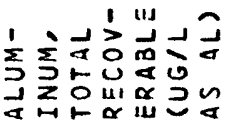

$\stackrel{m}{2}: i$

: $: i: 1$

$i: i: i$

$1: 1: 1: 1: 11$

i: $1: 1:$

i:ili i i i i

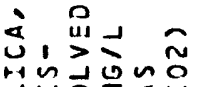

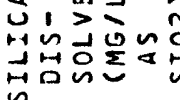

$201: 1$

$\sum_{\substack{i \\ \infty}}^{-i m}$

$\stackrel{2}{\simeq}: 1:$

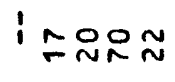

$0_{i} \simeq \underbrace{\prime}$

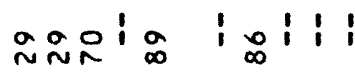

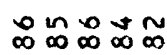

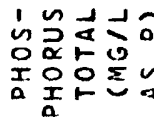

1 ํํㅁ111

$9: 1: 1:$

오ำกัญิั

ํำ 우ํํำ:

웅ํํㅇ응 움

iํำi i :

이ำ

$\checkmark$

$v$

$\checkmark$

$\vee \vee v$

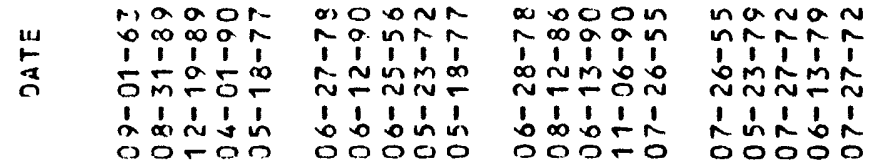

asonton

nnnme nannen

o dis

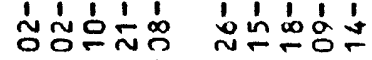

íniti

Nuำ

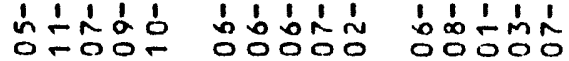

Noma

$\infty i_{1}^{\infty}$

西的然

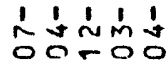




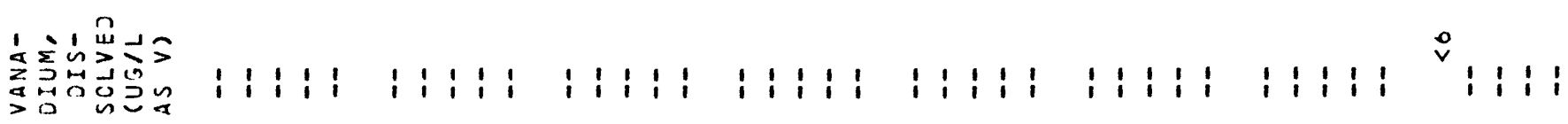

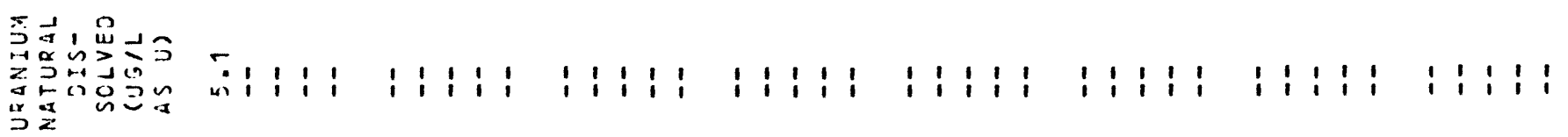

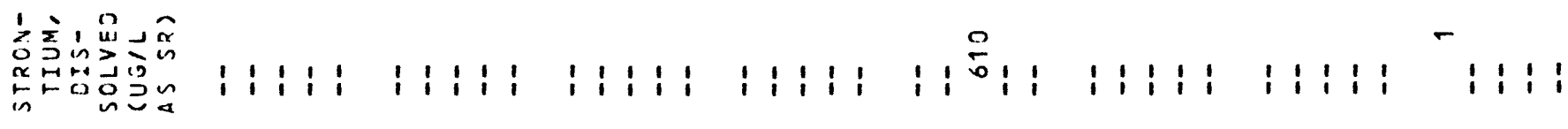

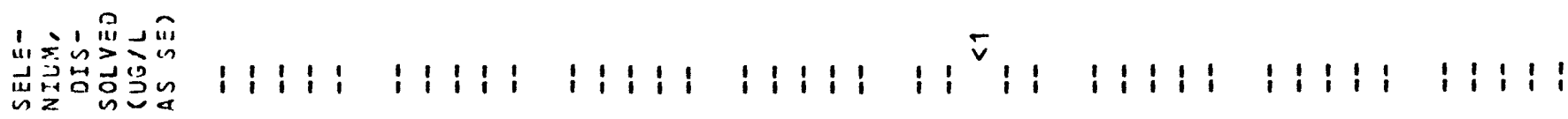

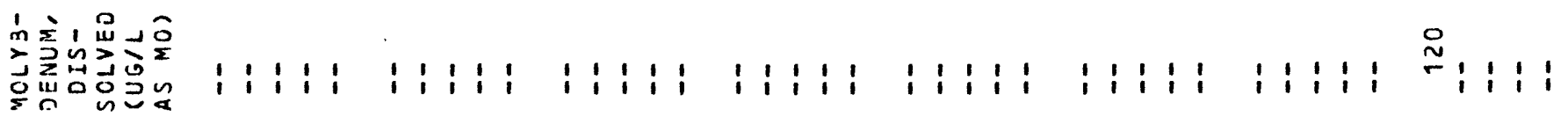

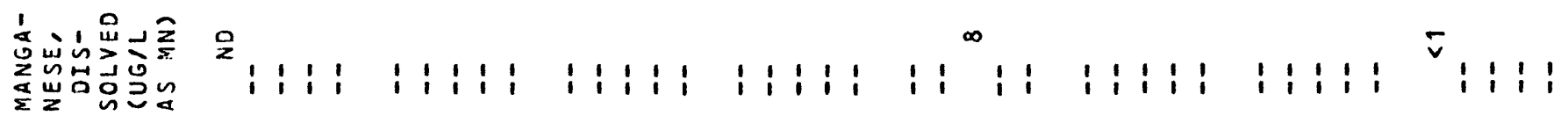

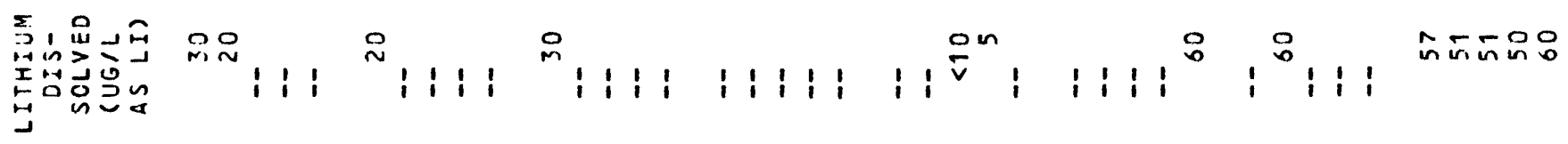

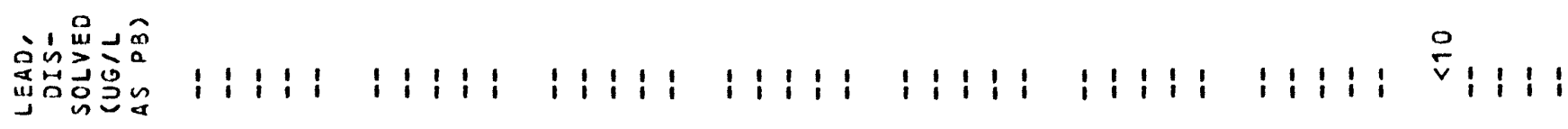

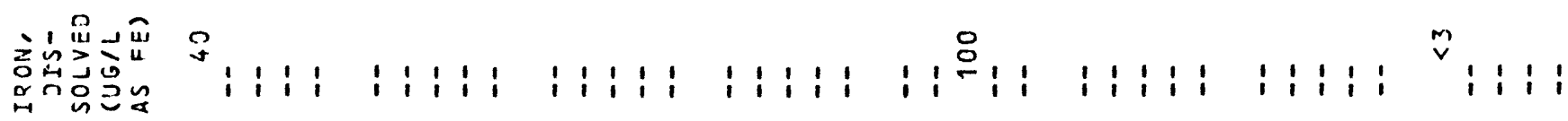

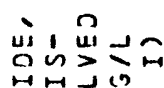

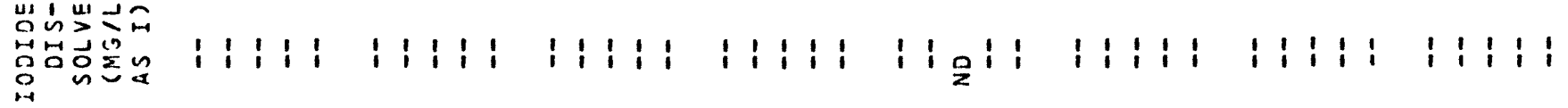

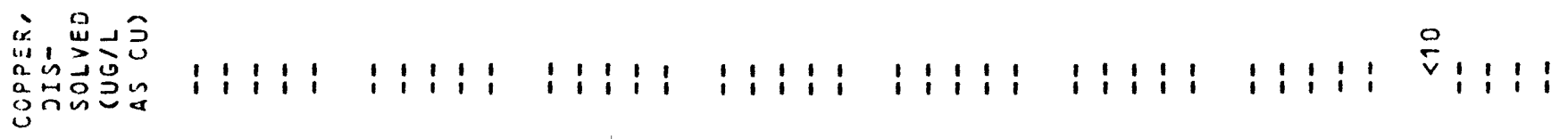

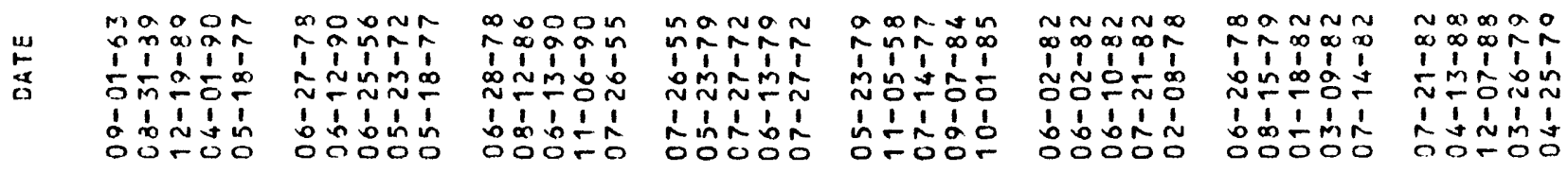




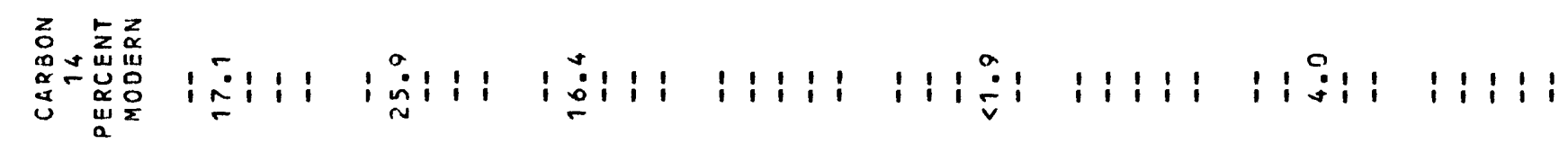

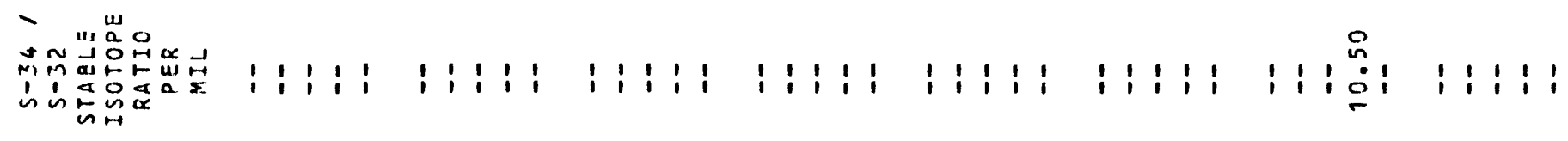

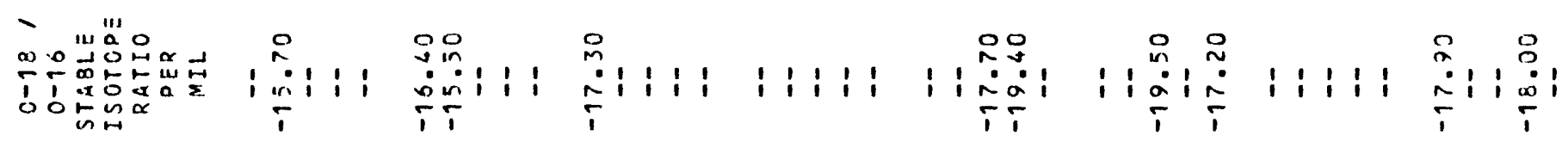

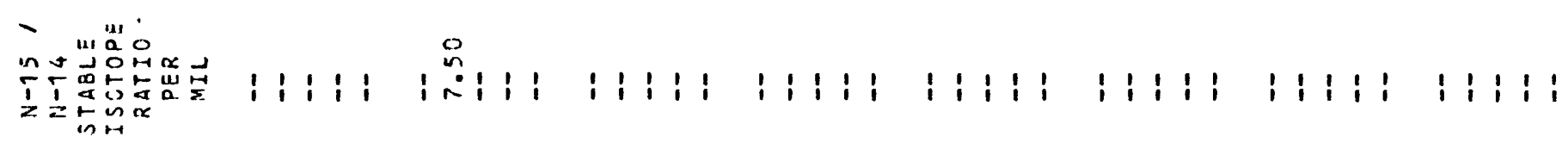

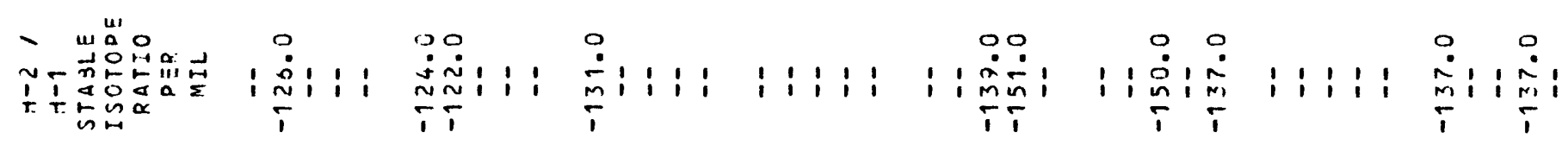

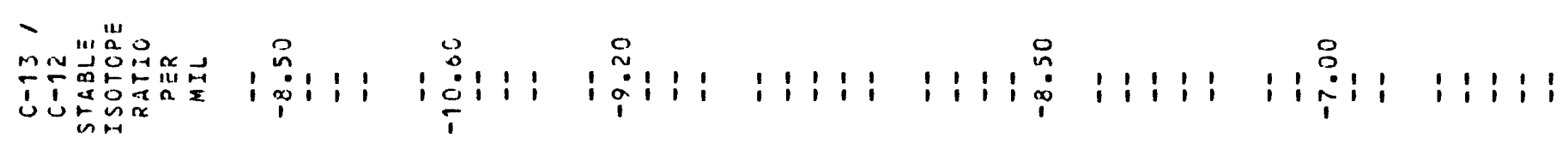

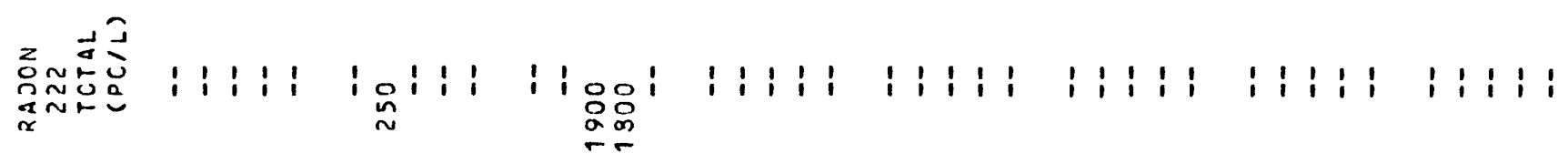

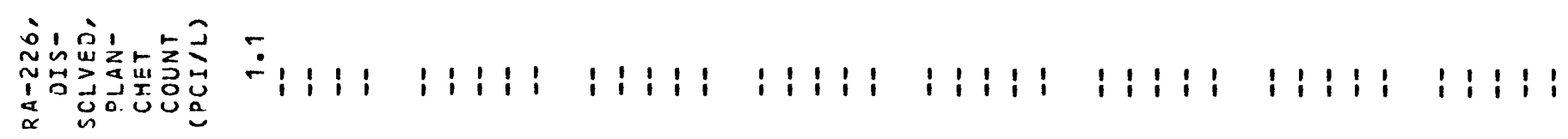

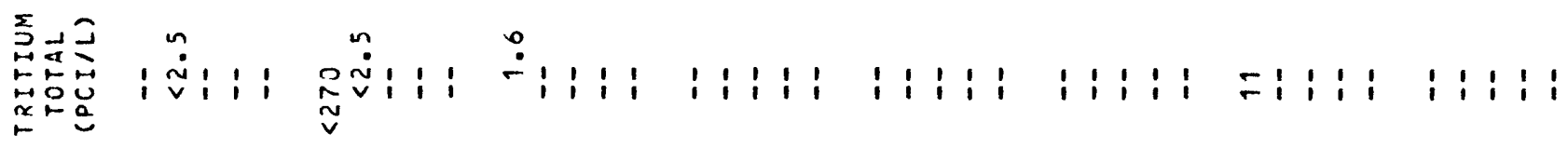

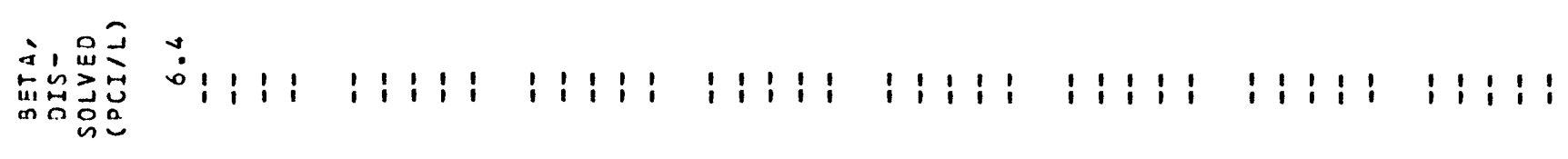

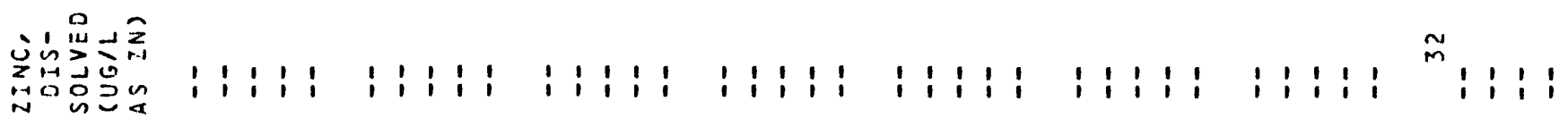

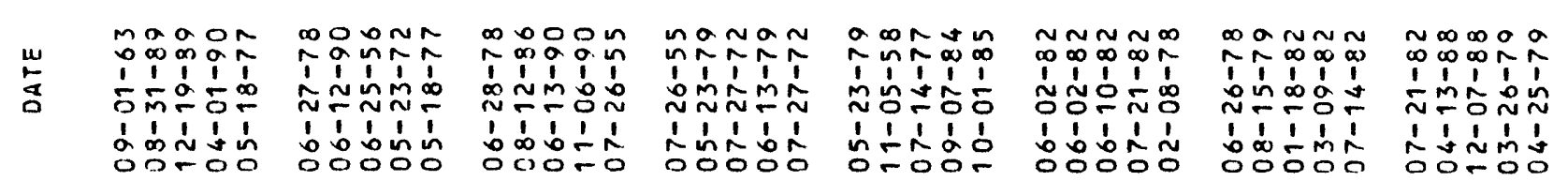




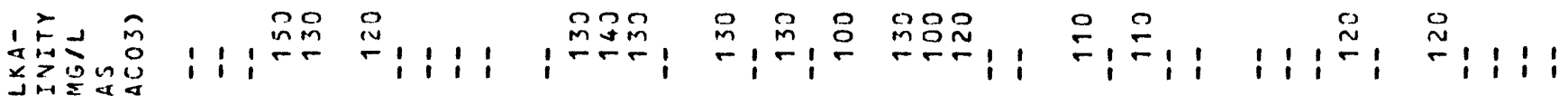

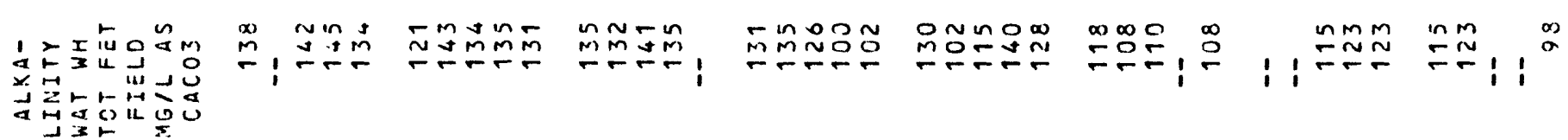

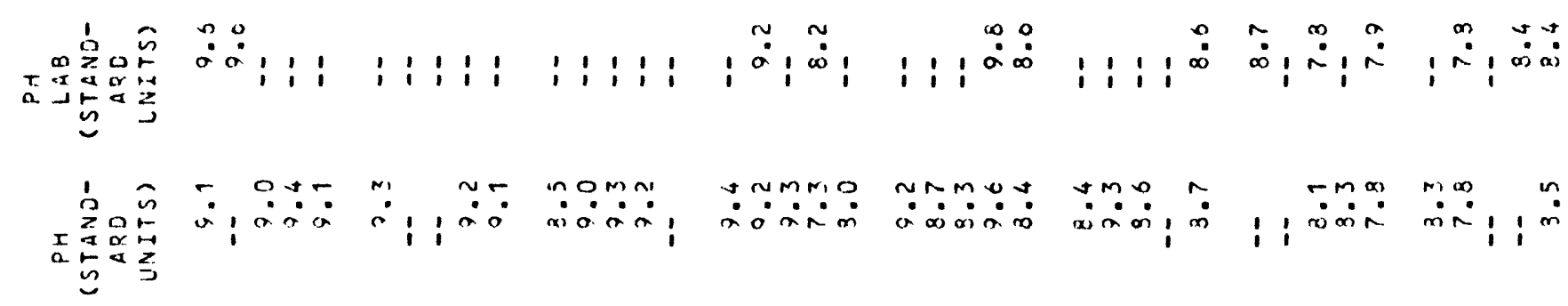

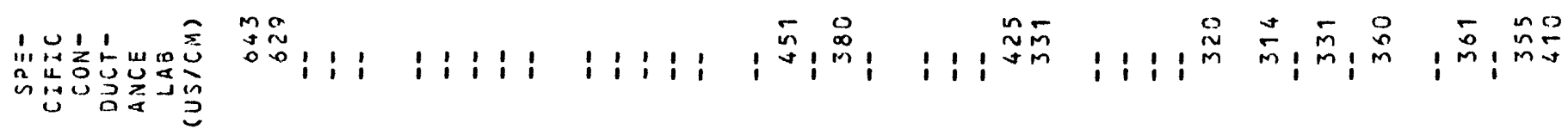

1.

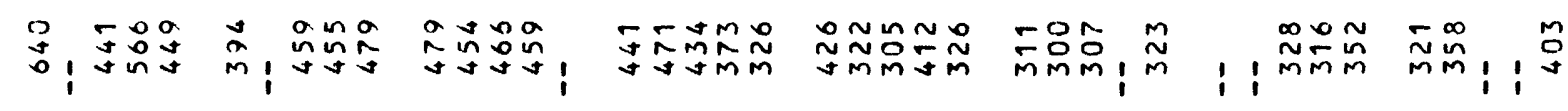

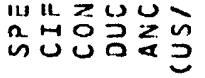

$\stackrel{\sim}{\propto} \propto \widehat{U}$ 岁品比

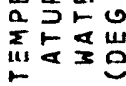

omoon minom in

0000 ก0.0\% no. ? oo in 0 o 0 in

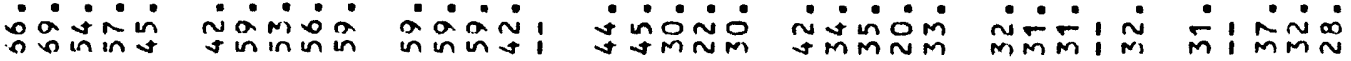

. in 0 in

\section{$\underset{1}{\infty}$}

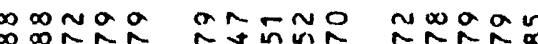

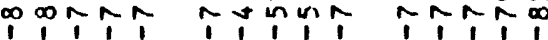

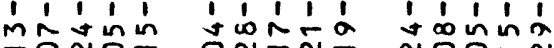

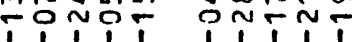

1111

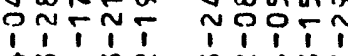

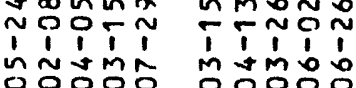

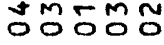

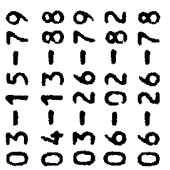

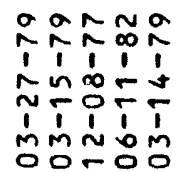

a)

$\hat{i} \hat{i}, \infty$

$+\infty$ na

i

momñ

$\infty 0-a-$

$\infty \infty \infty \pi \infty \quad N \infty \infty \infty \infty$

in 0 is

$0 \div 055$

Nonm

11111

N⿴囗十心O

m ñ 11

$\sum_{11}^{10}$

$x_{15} x_{10}$ in $\frac{a}{10}$

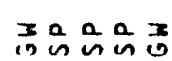

$\frac{3}{6}=\frac{7}{6} \frac{3}{6} \frac{3}{6}$

$\frac{3}{19} \frac{7}{6} \frac{x}{60}$

$\frac{x}{6}=\frac{3}{0} \frac{x}{0}$

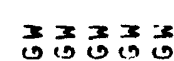

$\frac{x}{19}=3 \frac{7}{6}=$

$\frac{7}{19} \frac{3}{15} \frac{3}{19}$

2
2
0
0

$m m m m m$

$m m m m m$

$m m m m m$

$m m m m m \quad m m m m m$ O

$\begin{array}{llll}\infty & \infty & \infty & \infty\end{array}$

$m m m m m$

$m m_{\infty} m m \quad m m m m m$

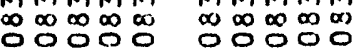

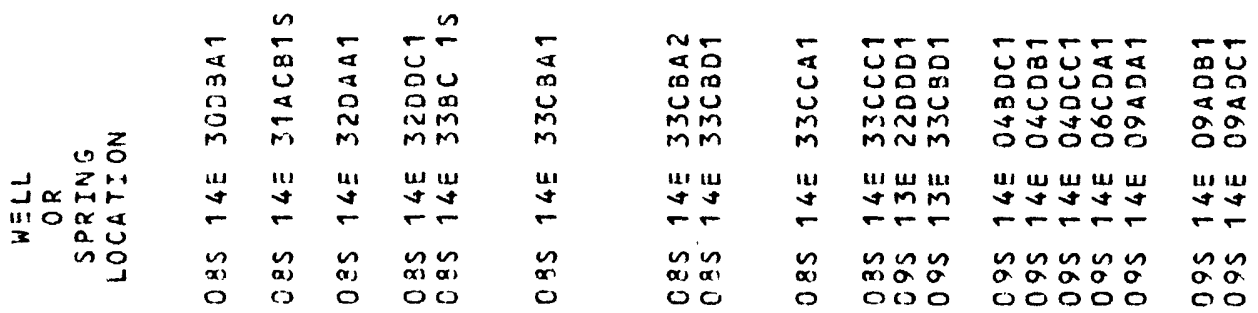

\begin{tabular}{|c|c|}
\hline 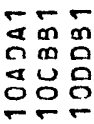 & $\begin{array}{ll}5 & 5 \\
0 & 0 \\
0 & 0 \\
0 & m \\
m & =\end{array}$ \\
\hline $\begin{array}{ll}\omega \mu \\
+\end{array}$ & $\begin{array}{l}19 \\
\square \\
\square\end{array}$ \\
\hline 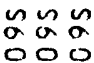 & $\begin{array}{ll}\sim & n \\
0 & a \\
0 & 0\end{array}$ \\
\hline
\end{tabular}




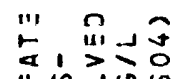

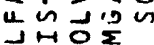

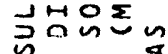

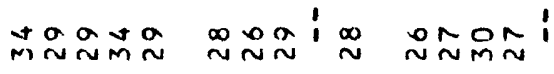

c, is ,

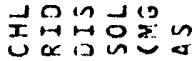

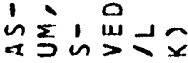

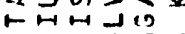
의의

$\because \because \because \because \because \div 11:$ $\because \because \because \div$ :

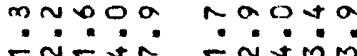

กNONG $9 m ! m i n$ $\sum_{i=1}, D \geq \frac{5 \pi}{2}$ $\ddot{n} \rightarrow \pm 19$

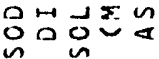

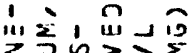

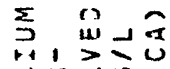

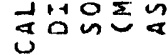

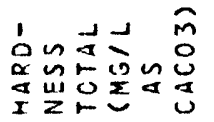

inus is is

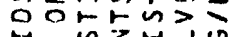

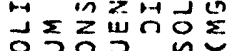

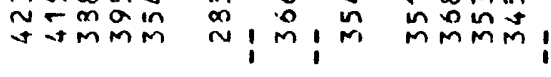

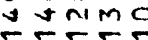

2:1:

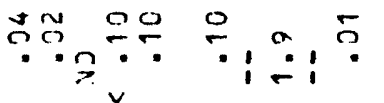

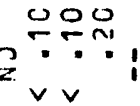

tanga

N M I I M

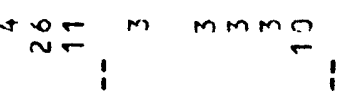

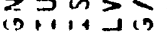

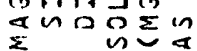
Uin

$m \sim m n t$ in $\because \ddot{n}-\infty m i n$ $\sim \sim N \infty m$ M

nom

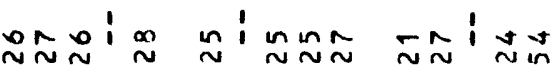
응으음은

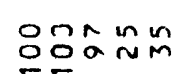

momar N ovin in $\because \div \div 09$ 운은? ํㅗㅇ

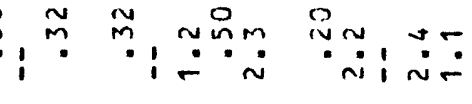
mะกำ $\div 1 \div 1 \div \div \div \div \div !$ $n m \sim \underset{\sim}{\infty}$

*ேั omin:

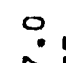

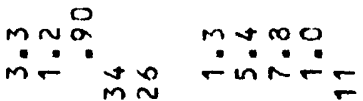

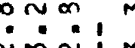

$m, \because n \infty$

wo on

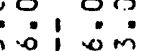
낸용

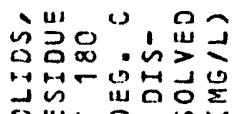
c) 프

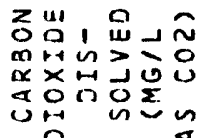

$11^{m-n} \div, \div$

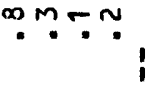

$m \pm 5 n ̃$ MMMN
mâOON

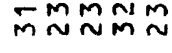

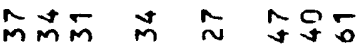
$\sim N i, \stackrel{m}{N}, \stackrel{N}{N}$ $m o$ in $\sim \sim \sim N$

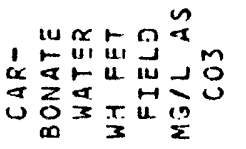

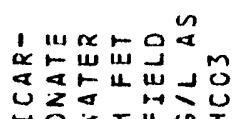

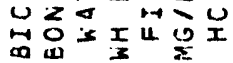

$\stackrel{u}{a}$

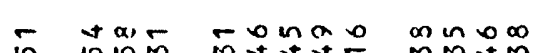

in, inging moing

maso

m, mơ요

$\infty 00$

$\infty$

$\min _{\infty}^{\infty}, \stackrel{0}{\sim}$

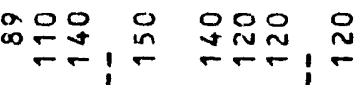

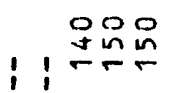

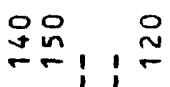

$\infty_{\infty}^{\infty} \sim \alpha \propto$ ำ

m1! ini

-

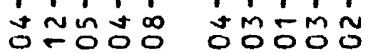

Noan in tón in i

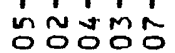

$a^{\infty} \infty \underset{\infty}{\infty} \sim \infty$

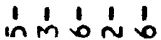
Tin Món
ลำก 1 in 1 ! ! ! 눈다 MNㄷㅇ응
ำกำ

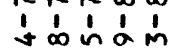
$\div 0 \div \frac{1}{1}$ moํํㅇ웡 $\infty_{\infty}^{\infty} \underset{\infty}{\infty} \frac{\alpha}{1}$ 뇌의 단다 녕ํํㅇํㅇ

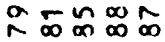
12111 논 mㄷํㅇ응 


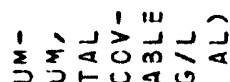

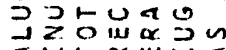
元, 至” $\rightarrow$ in $\rightarrow 3$ is

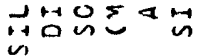

in $\begin{aligned} & \mathrm{i} \\ & \mathrm{a}\end{aligned}$ o or 5 $\begin{array}{lll}0 & 0 \\ 0 & 0 & 0\end{array}$

11, $11+1,11^{2}$ $\stackrel{2}{2}$

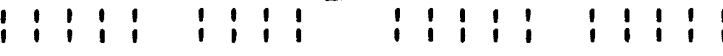

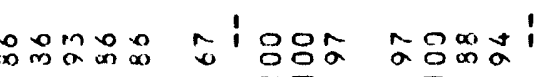

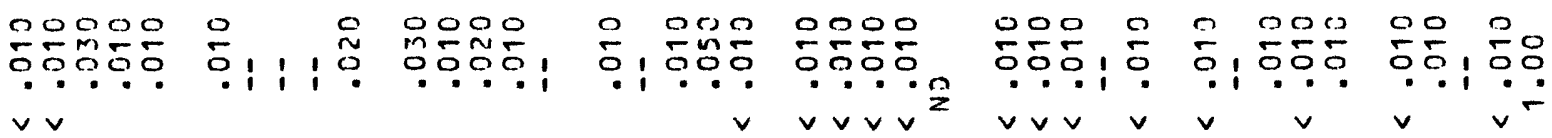
$\checkmark v$

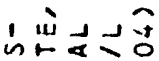

205

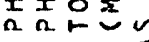

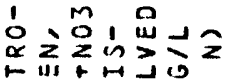

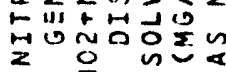

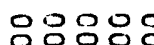

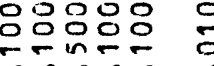

$\because \because \div$

$\frac{0}{1}: 11:$

욱응요

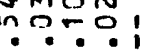

웅응응요 웅응웅응

vvir vir vo

$\checkmark$

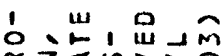

$\alpha \sum_{x \rightarrow 10}$

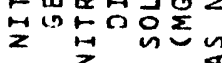

으노ำ

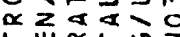

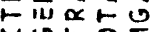

늘을

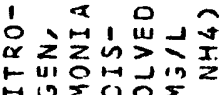

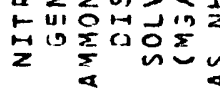

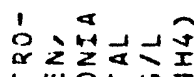

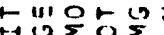

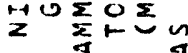

b 奖, 品

$\alpha z \alpha n>\geq 2$

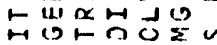

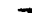

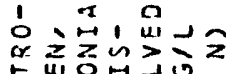

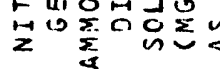

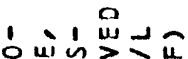

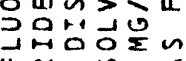

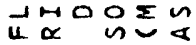

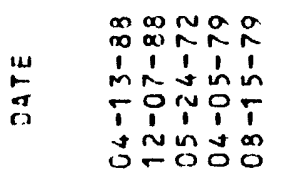

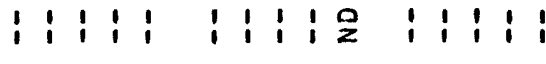

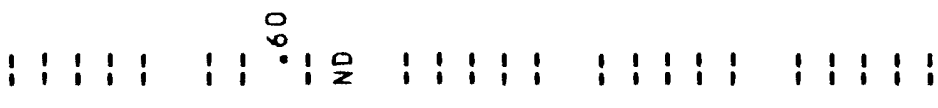

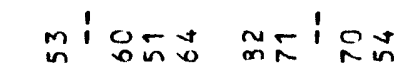

응웅응 응

i i i $1: i d:$

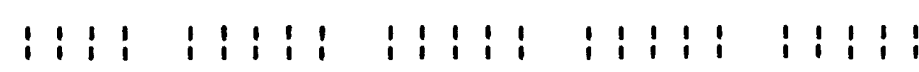

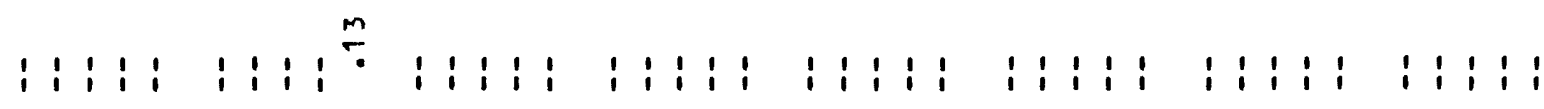

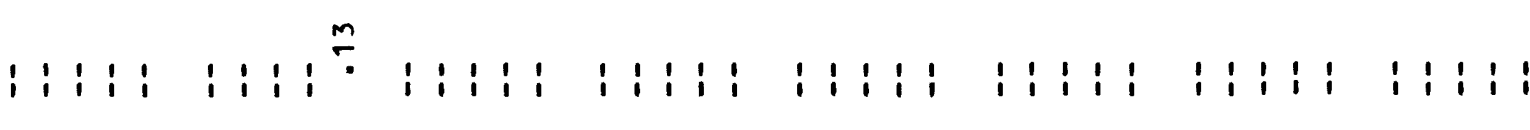

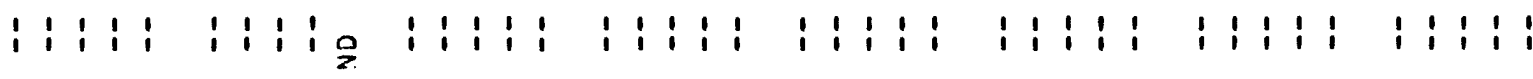

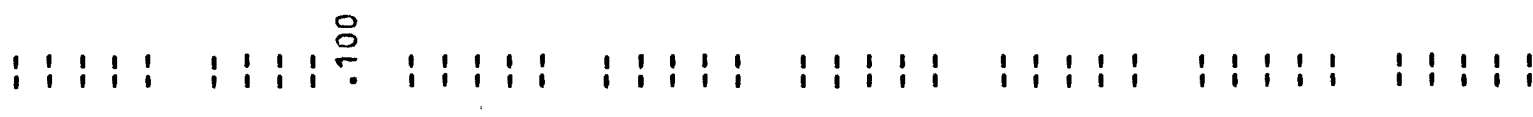
NNNัN

ำ

ín

iTtT Dmoma minoro
수숭

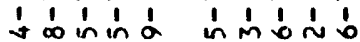
Nop-n ñ⿻ำ
TTitis

mimo $\because \infty$

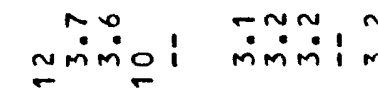

$\ddot{\sim} \dot{m} \ddot{\sim} \dot{\sim} \sim$

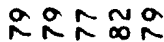

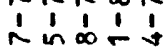
TTíT mmńm
ำํำ J́ńnám Fit momn:
$191: 19119$ 


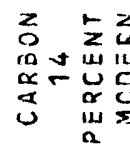

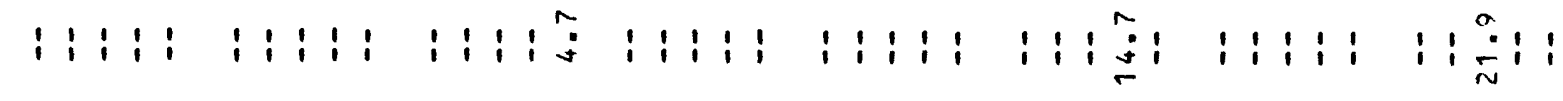

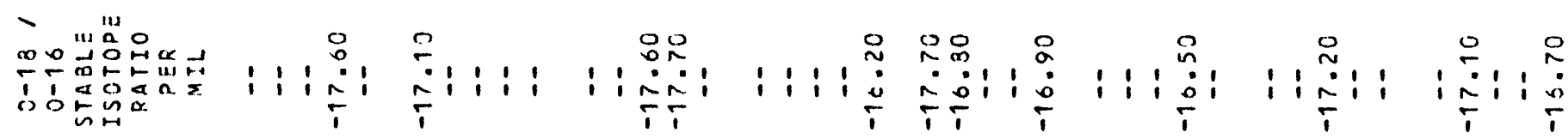

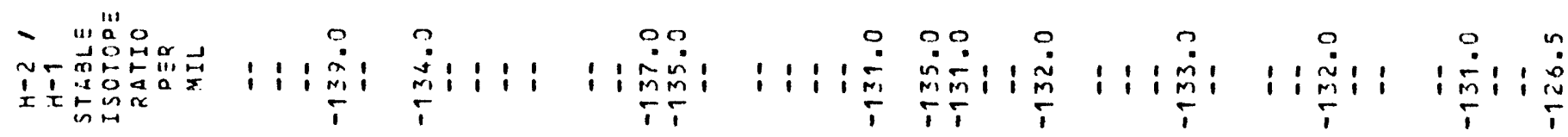

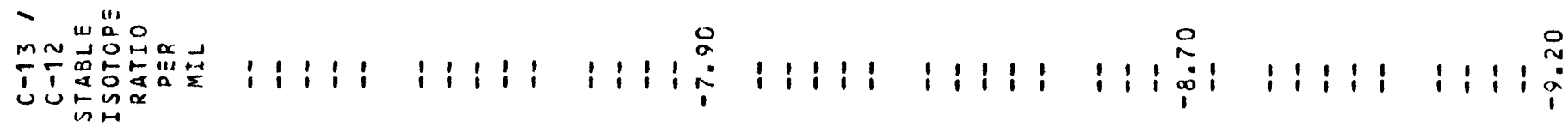

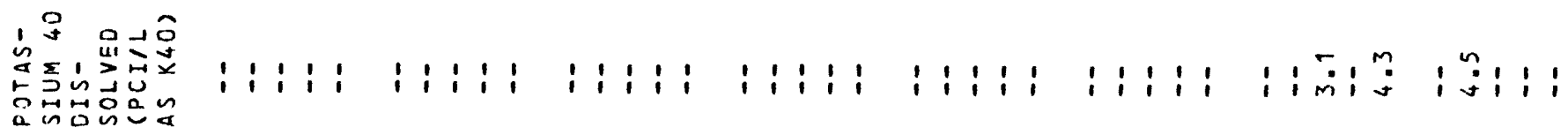

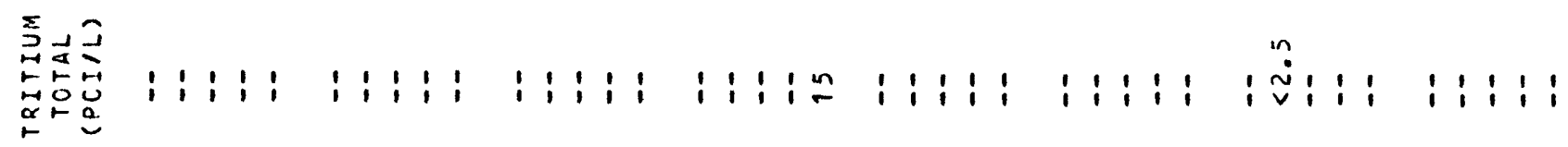

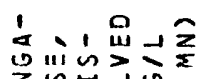

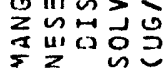

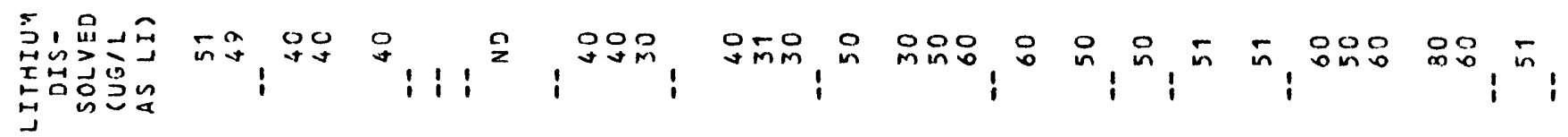

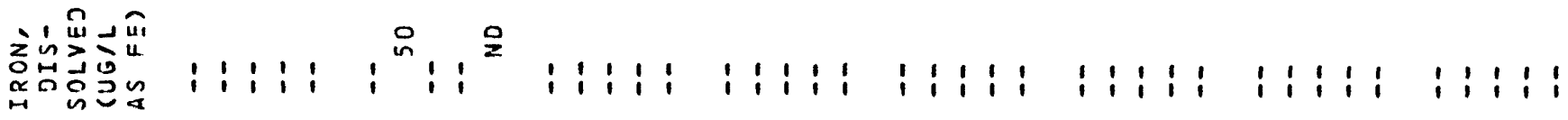

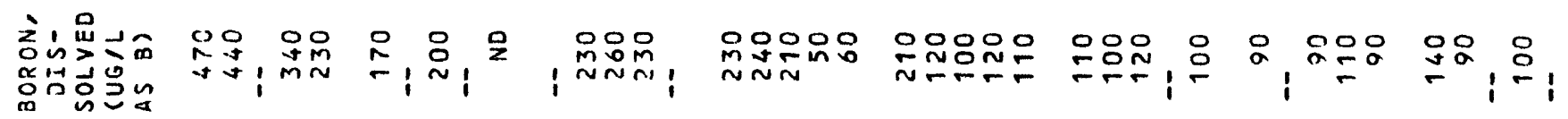

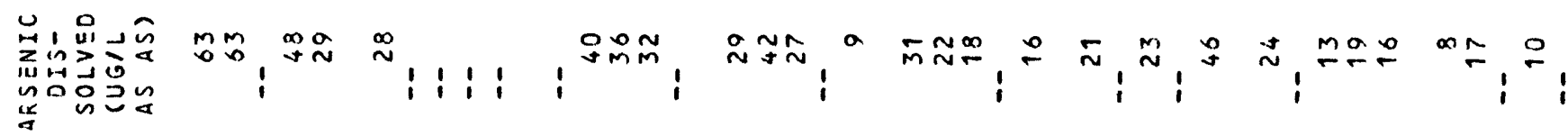

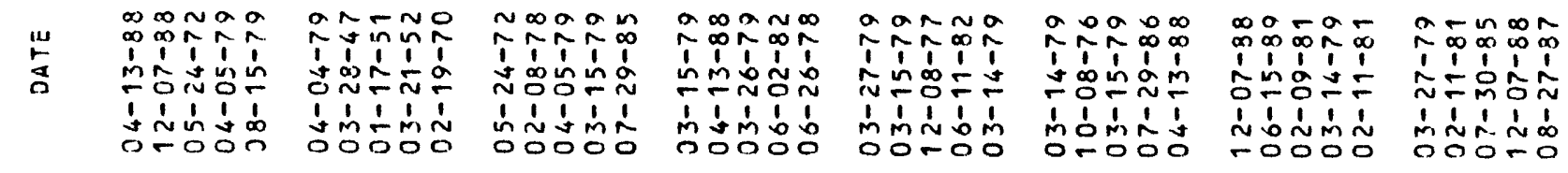




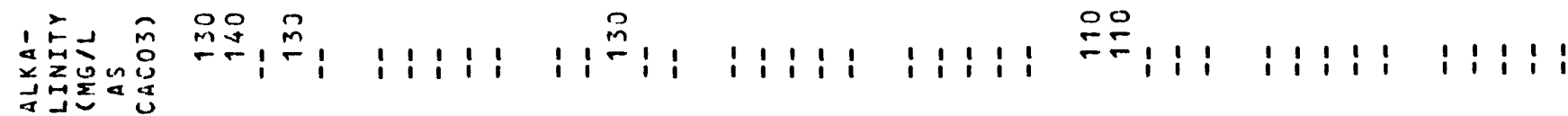

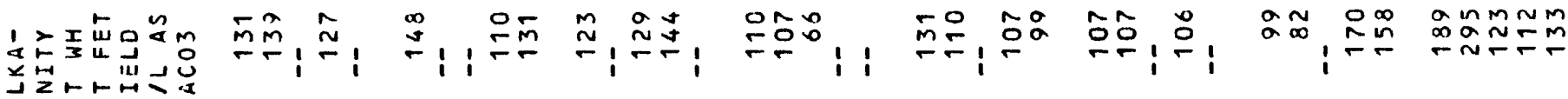

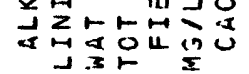

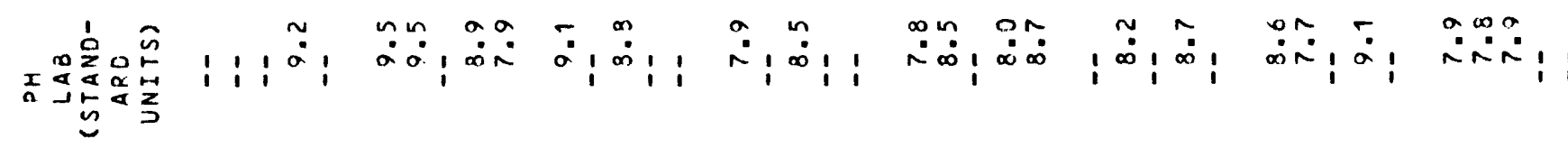

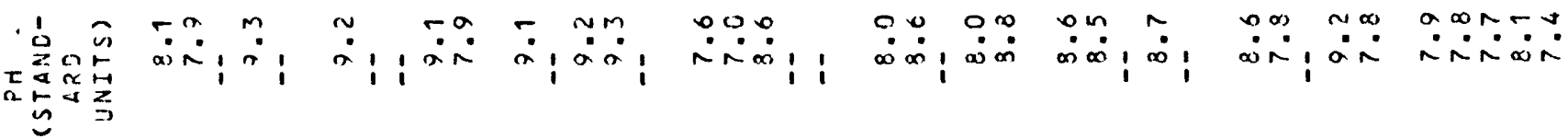

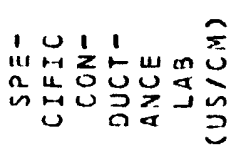

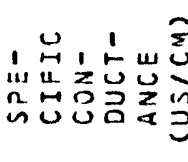

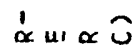

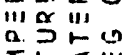
$\sum_{i=1}^{2} \leq 0$

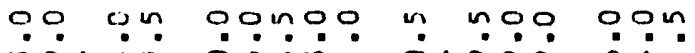

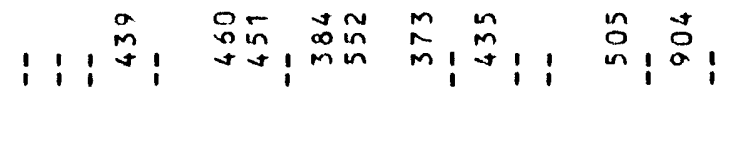

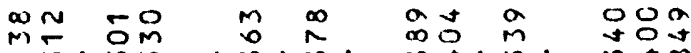

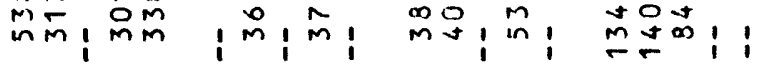

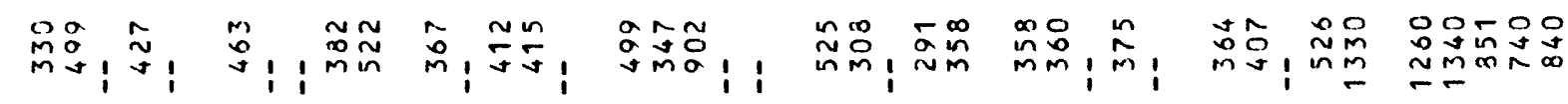

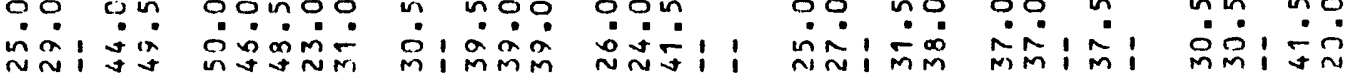

0 in 0.0 in

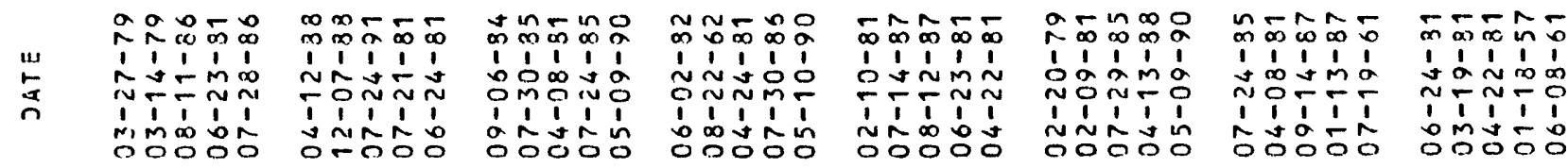

芒年

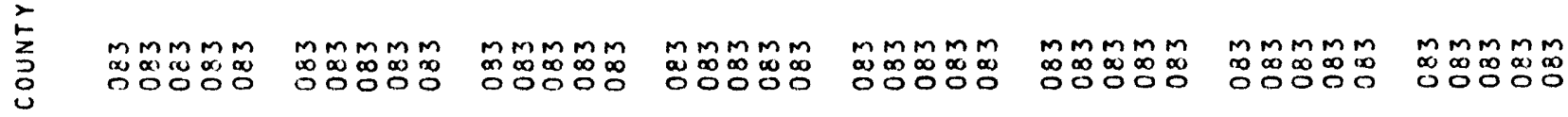

\begin{tabular}{|c|c|c|c|c|c|c|c|c|c|c|c|c|}
\hline & 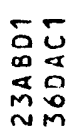 & $\begin{array}{l}\vdots \\
\vdots \\
u \\
\vdots\end{array}$ & $\begin{array}{l}\overline{0} \\
0 \\
0 \\
0 \\
0 \\
m \\
-1 \\
m\end{array}$ & $\begin{array}{l}5 \\
0 \\
0 \\
0 \\
0\end{array}$ & 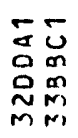 & $\begin{array}{l}\bar{u} \\
0 \\
0 \\
0 \\
0 \\
\sigma \\
0 \\
0\end{array}$ & 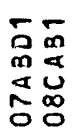 & 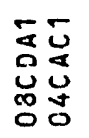 & $\begin{array}{l}5 \\
\vdots \\
0 \\
0 \\
0\end{array}$ & 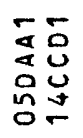 & $\begin{array}{ll}5 & \bar{a} \\
\infty & 0 \\
0 & 0 \\
0 & 0 \\
0 & 0 \\
0 & 0\end{array}$ & 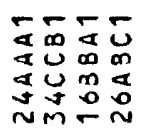 \\
\hline$\exists \propto \sum_{\alpha=1}$ & 岂 & $\stackrel{u s}{n}$ & 苫出 & 虫 & 岕崩 & $\underset{\sim}{\stackrel{u}{\sim} \underset{m}{M}}$ & 虫皆 & 㟧 & $\stackrel{w}{N}$ & 㟧 & 虫 & 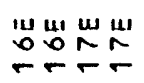 \\
\hline$\sim 0$ & ڤa & 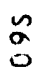 & $\begin{array}{l}n a \\
\alpha \sigma a\end{array}$ & 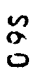 & صa & 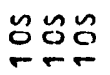 & $\dddot{2} \mathscr{0}$ & ¿ू & $\stackrel{0}{0}$ & 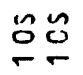 & $\tilde{0} \approx$ & 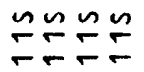 \\
\hline
\end{tabular}




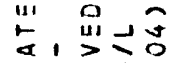

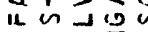

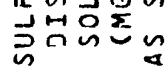

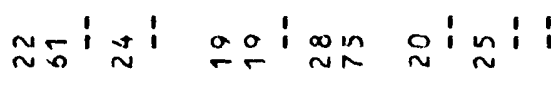

șmi

åi

요리

은 1 mo

잉오음

它, 省出

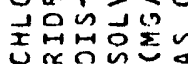

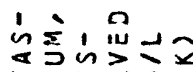

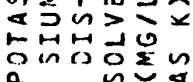

$\stackrel{m}{m} \div$

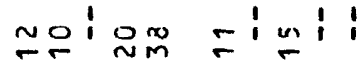

$m \stackrel{x}{m}$

mEI

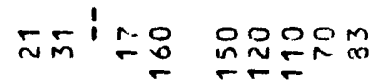

空,

In $\begin{aligned} & 0 \\ & \text { in }\end{aligned}$

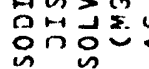

$m 5 ! 2$

o인

$n_{\infty}: a !$

Mm̃ i

$m \sigma i n$

$\ln _{n} i n$

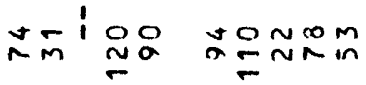

紧品品

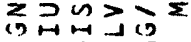

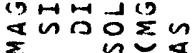

$\because, \because, 90, \div 0 \% 0, \%$

añ

$\because \frac{\pi}{2}$,

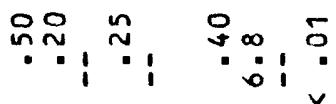

vi

$\sum_{n, 1}, \stackrel{0}{>} \pm \underline{u}$

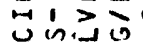

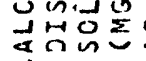

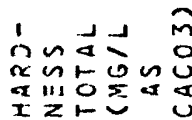

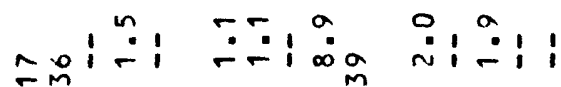

non i i

mílom

$\because m, n$,

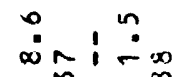

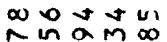

斻出的, 然

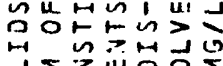

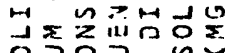

$\infty m$ in $\sim \sim m m \sim[n \infty$

No!

$+\infty \infty$

m $m$ in i

na oa

Mñ $\stackrel{2}{\sim} \stackrel{0}{N}$

$\stackrel{a}{n} n, \hat{n} ;$

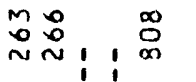

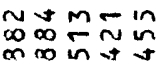

药 $v$, it

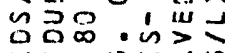

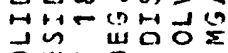

$\overrightarrow{0}$ iี

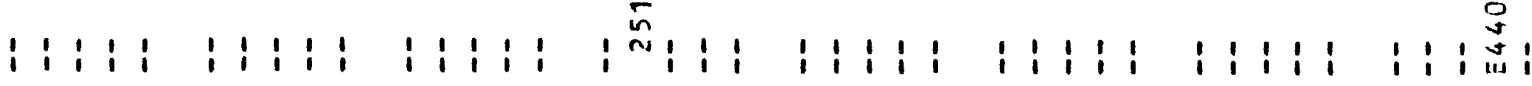

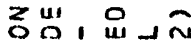

ant

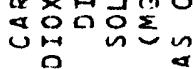

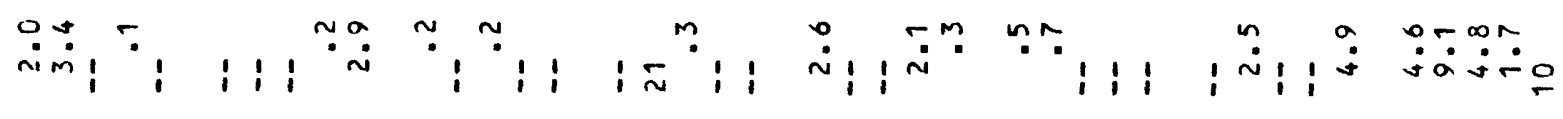

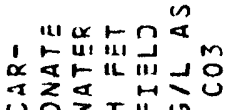

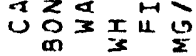

1"

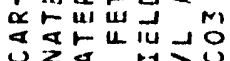

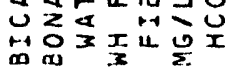

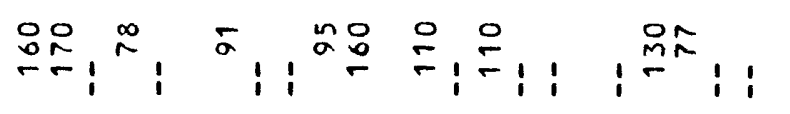

웅음음

온, 울은

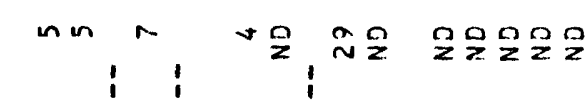

$\operatorname{coc}_{\infty}^{\infty} \infty_{\infty}^{\infty}$

$\infty_{\infty}^{\infty} \sigma r-$

is 1 is

$\infty \infty \propto \infty \infty$

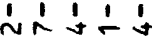

-

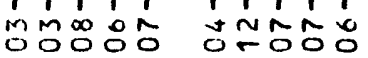

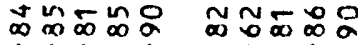

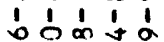

i M

oncosion

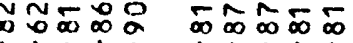

'ñ'bl

o

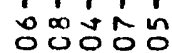

$1-i \frac{1}{1}$

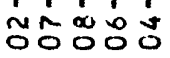

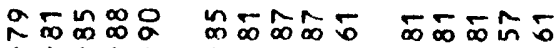

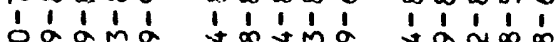

Nỗ⿺辶

Nỗó

is $0=-\frac{1}{10}$

$1,1,1$

Titi

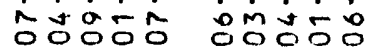




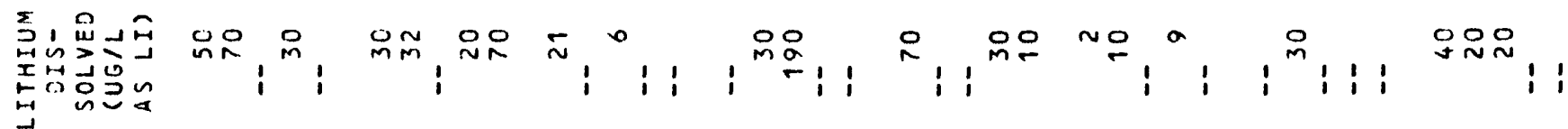

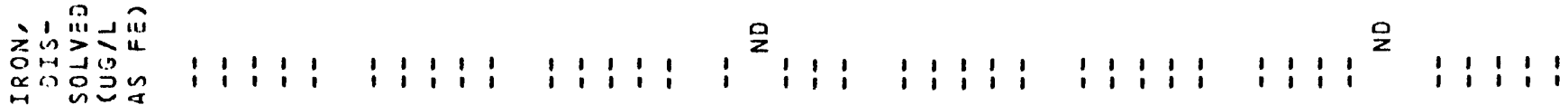

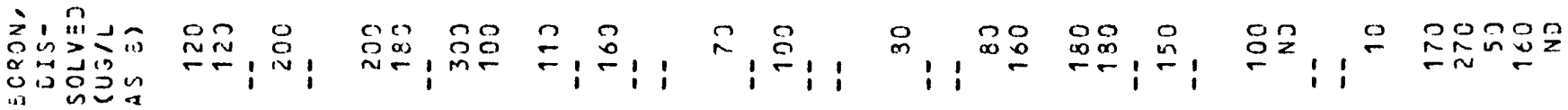

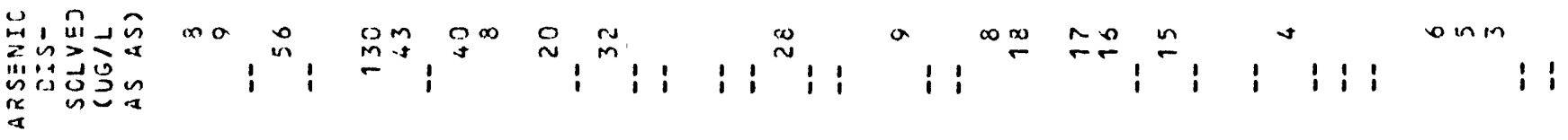

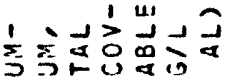

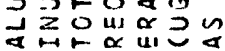

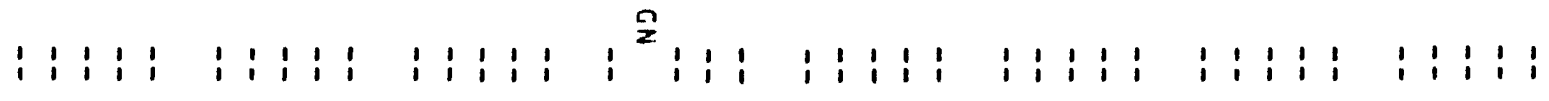

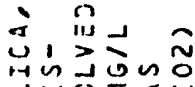
$\rightarrow$ in 0 过

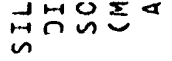

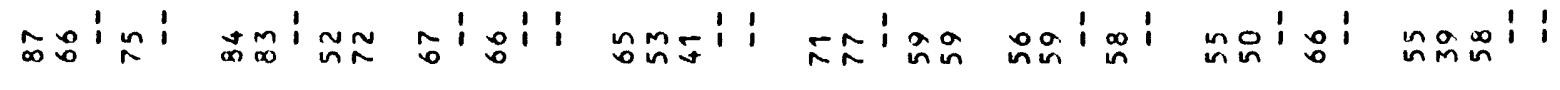

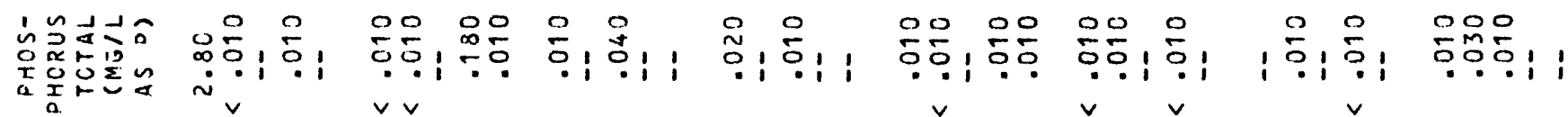

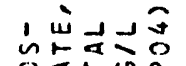

c) 4 เ

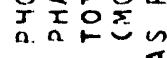

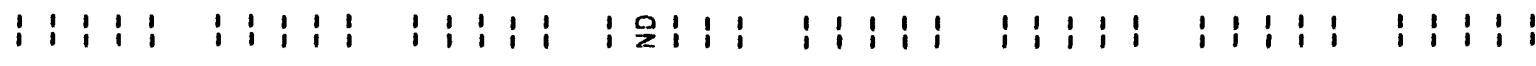

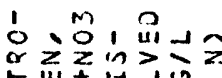
a

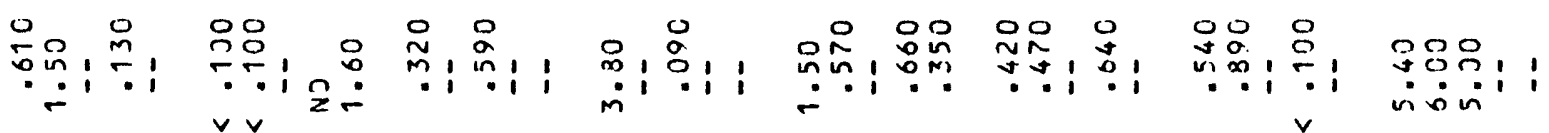
$\stackrel{1}{\alpha} \sum \stackrel{\prime \prime}{a}=\hat{m}$

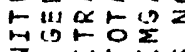

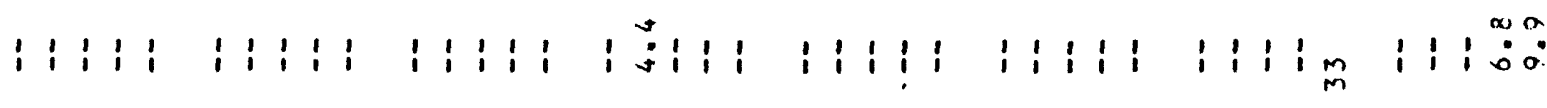

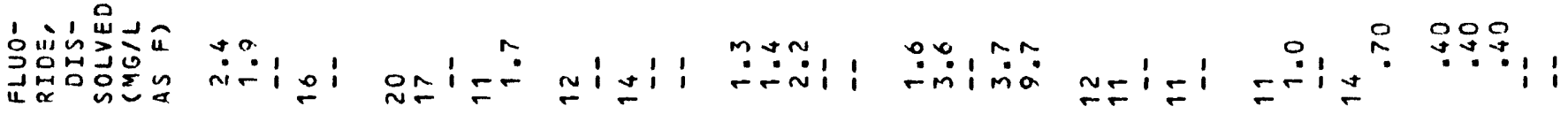

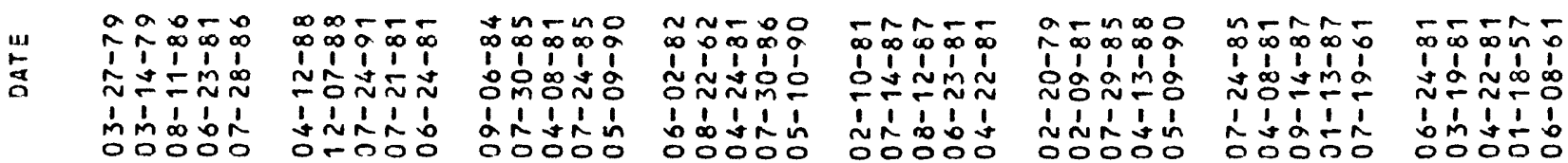




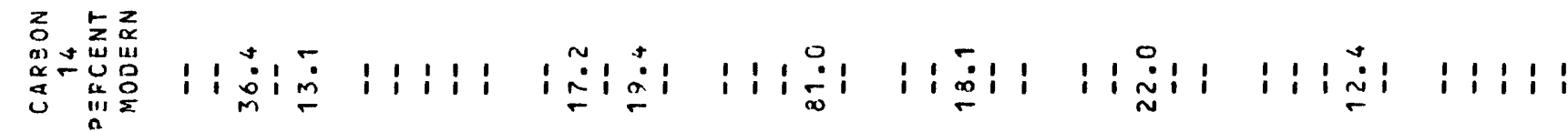

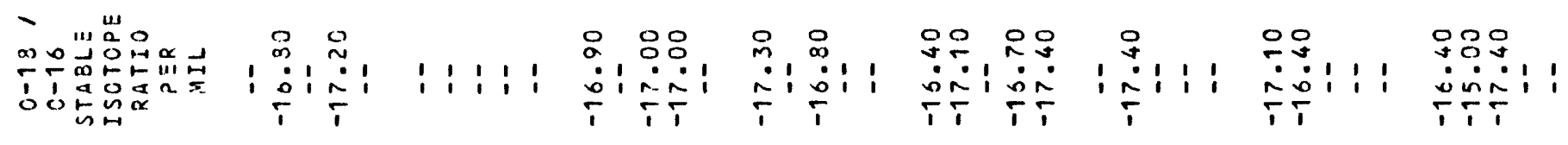

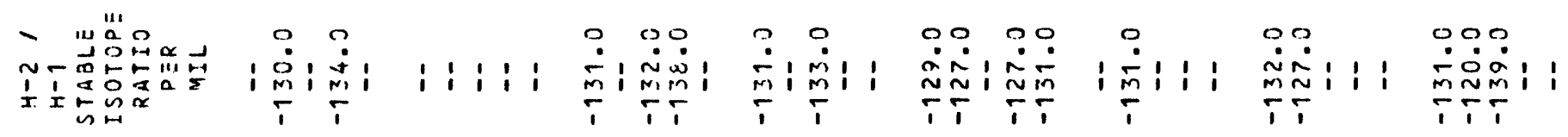

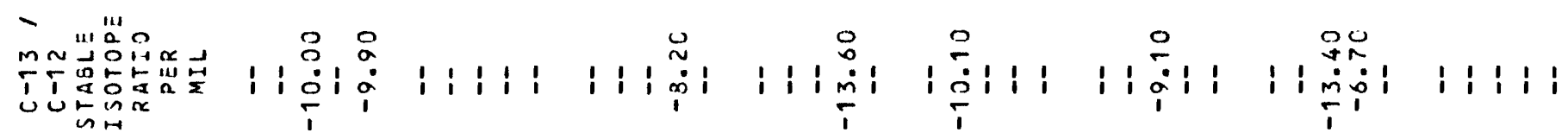

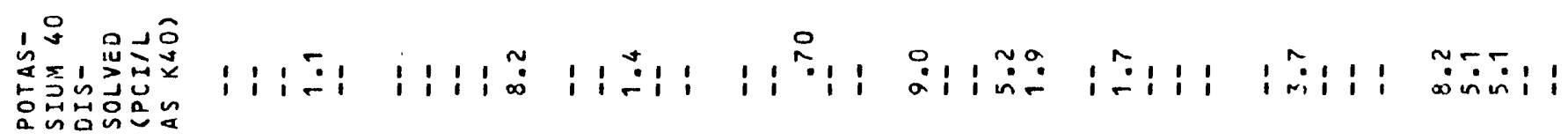

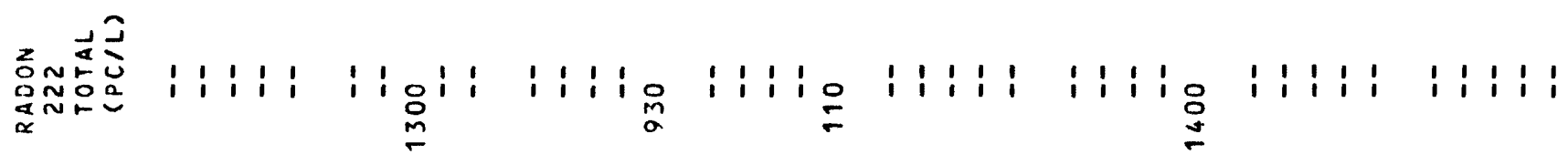

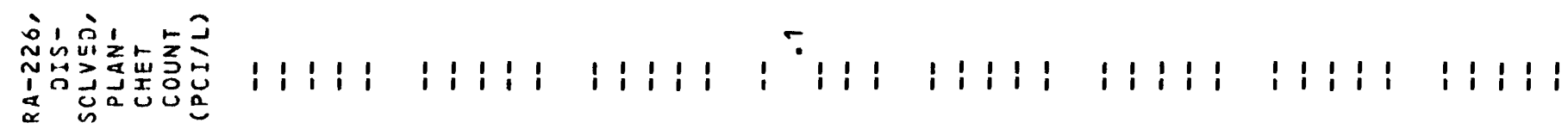

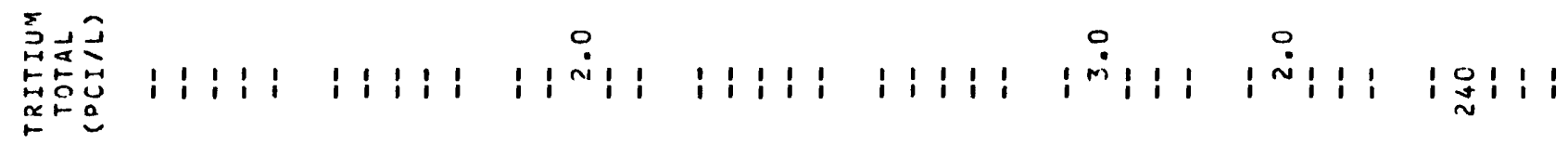

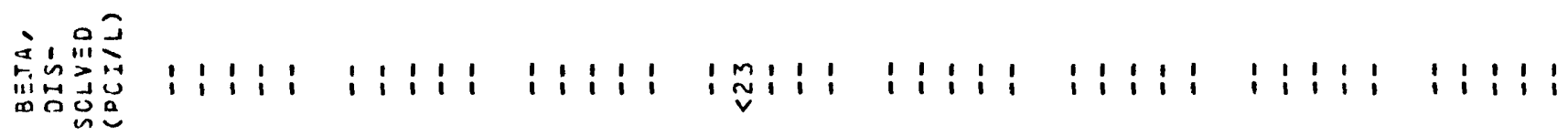

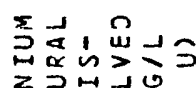

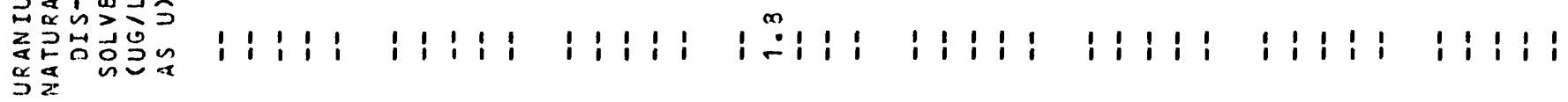

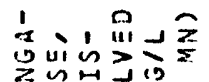

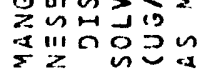

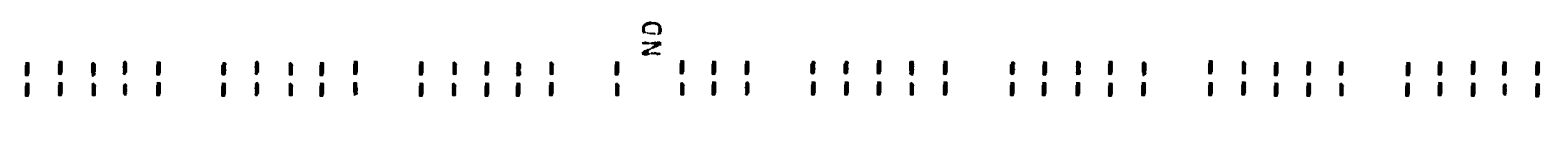

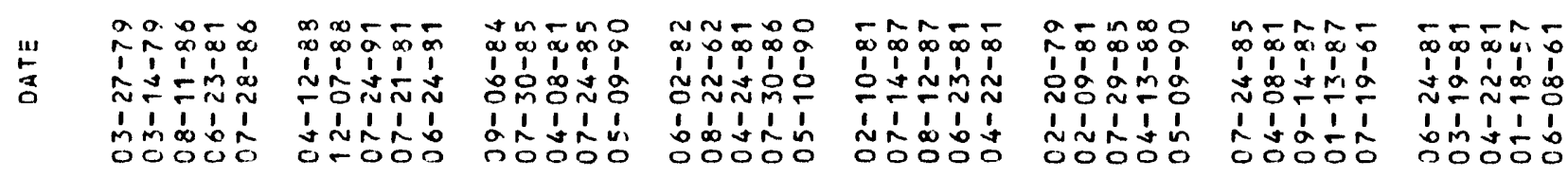




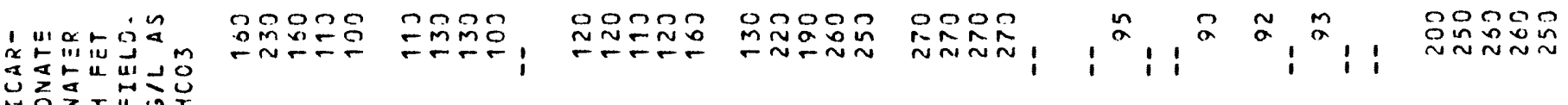
$\lim _{i} x^{x} 4$ is $x$

1)

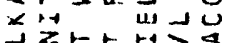

moda a d o ono nNamo

mam $\infty \infty_{\infty}$

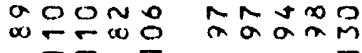

Commo $20-0$

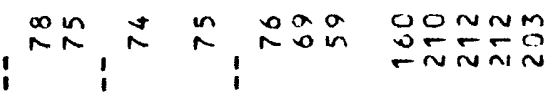

$x+1=01120$

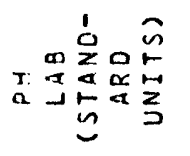

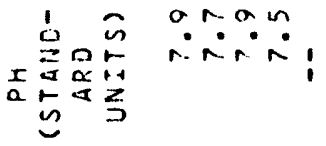

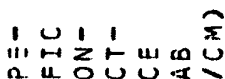

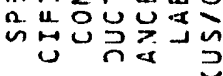

1 U, 1 出

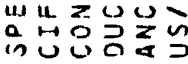

í

ut $\alpha$ u

$\sum$ 在

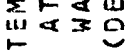

$\stackrel{a}{a}$

$-\sim \sim \sim n$

a) $\alpha_{1}^{\infty}, 1$

v $=0 \sim 0$

1 1 i i

ㄴํㅇำ

- ․․?

ióni

in 0000

inivin

oginoo mimiñ

in o o in

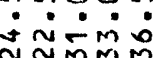

000 in ínin.

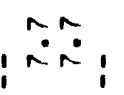

uninod ontoin mumbm $\stackrel{n}{\sim}, \stackrel{0}{n}$ $i: i^{m} !$ i i i i

$-\pi \sim-n$

$? \% \infty \% \infty$

mogno

$\begin{array}{lllll}1 & 1 & 1 & 1 \\ 0 & 0 & 2 & \infty\end{array}$

$\sim N \sim \sim n$

in 110

ำ

on $\cos \pi$

क ⿻ ㇒ : 1

잉에 에 운

1 1 1 1'

bogá

m旅品

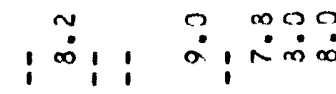

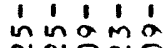

iñono

$\because \approx-\infty \approx$

in

1 1 1 1

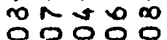

añon

in m

돈

Ti⿱ 151

O등응

$-\ln _{\infty}-\infty a$ $\infty \infty \infty \infty$

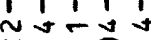
i $250 \div$

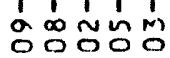

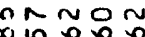

1110 에의 i 1 i

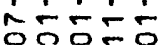

$\underset{n}{a 1}$

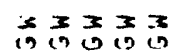

$3 \frac{3}{10} 33$

3 $3=30$

$\frac{3}{6} \div \frac{3}{6} \frac{3}{19}$

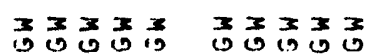

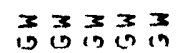

$\frac{3}{6}=\frac{3}{6}=19$

ż

$m m m m m$

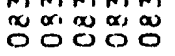

$m_{\infty} m m_{\infty} m$

$m m m m$

mMmm món $M M M m M$

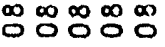

MMMMm

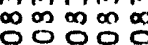

$m m m m m$

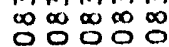

$m m m m m$ कo⿱

\begin{tabular}{|c|c|c|c|c|c|c|c|c|c|c|c|c|}
\hline & 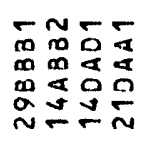 & 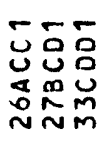 & $\begin{array}{l}\bar{a} \\
0 \\
0 \\
m \\
m\end{array}$ & 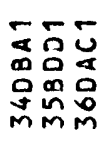 & 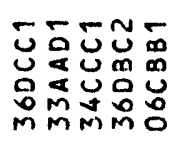 & 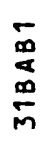 & $\begin{array}{l}n \\
\infty \\
\infty \\
\infty \\
m \\
m\end{array}$ & $\begin{array}{l}\frac{5}{\alpha} \\
0 \\
0 \\
0 \\
0\end{array}$ & 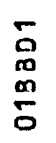 & $\begin{array}{l}5 \\
0 \\
0 \\
\alpha \\
\vdots \\
0\end{array}$ & 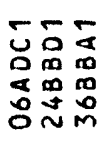 & $\begin{array}{l}55 \\
0 y \\
00 \\
00 \\
0\end{array}$ \\
\hline 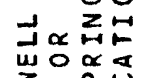 & 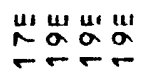 & 㟧㟧㟧 & $\stackrel{m}{\sigma}$ & 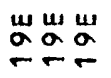 & 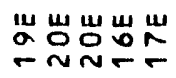 & $\stackrel{M}{\sim}$ & $\stackrel{M}{\sim}$ & $\underset{\infty}{m}$ & $\stackrel{111}{\infty}$ & "1' & 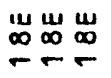 & 虫 \\
\hline & $\because \approx 2 \frac{1}{2}$ & 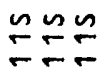 & $\check{n}$ & $\because \frac{2}{\square}$ & 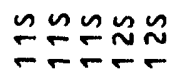 & $\tilde{\sim}$ & $\approx$ & $\stackrel{\sim}{\sim}$ & $\stackrel{\sim}{\sim}$ & $\stackrel{n}{\sim}$ & $\cong \sim$ & $\tilde{m} m$ \\
\hline
\end{tabular}




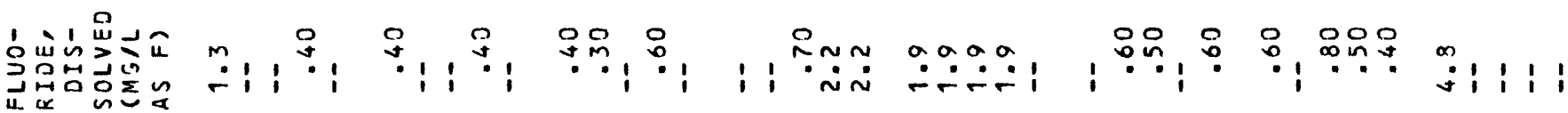
崖, 品过守

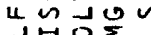

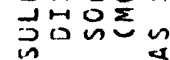

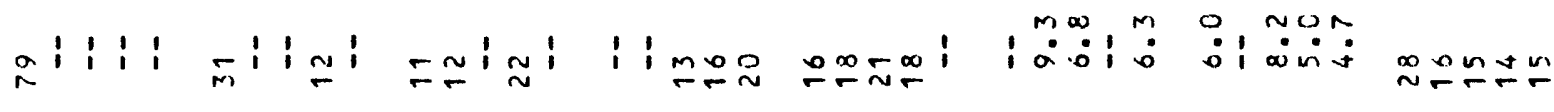

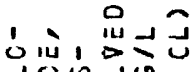

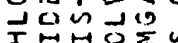

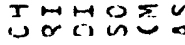

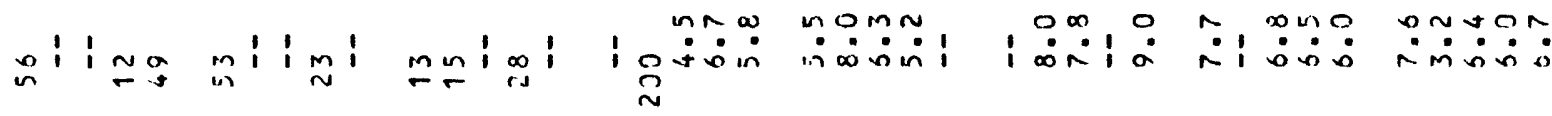
in \&

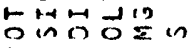

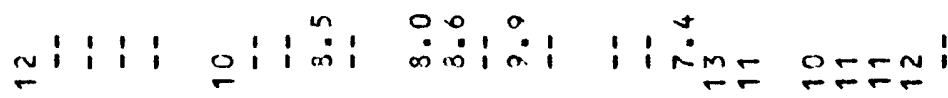

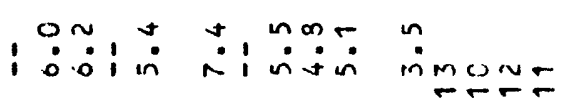

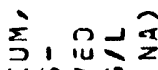
$\sum_{i \rightarrow 10}$

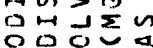
gili a

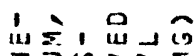

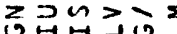

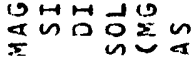

西

ร

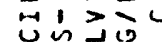

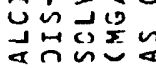
$\pm 1 ; i \geq 1 ;$ $\stackrel{\sim}{\sim} i_{m}$ I'

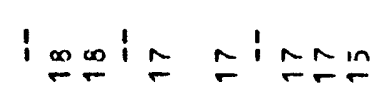
व̊mm̃

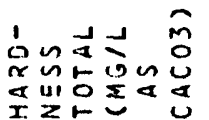

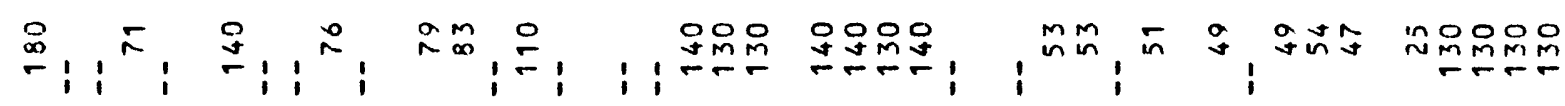

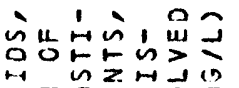

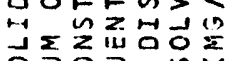

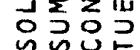

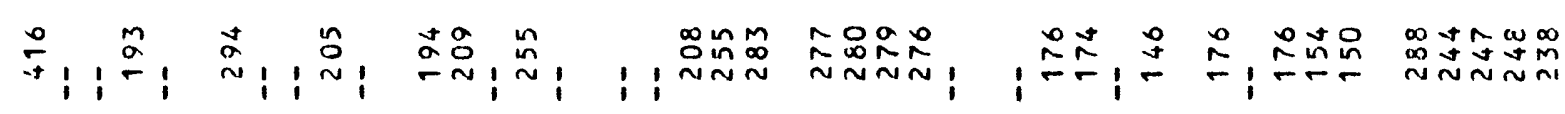

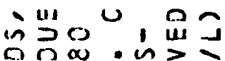

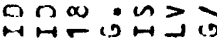

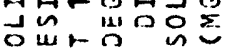
$\therefore i: \dot{m}: \dot{0}: i \dot{m}$ $\because \dot{0}:$ :

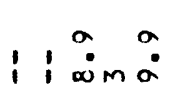
穴穴 in ax

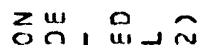
ming

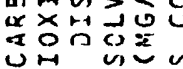

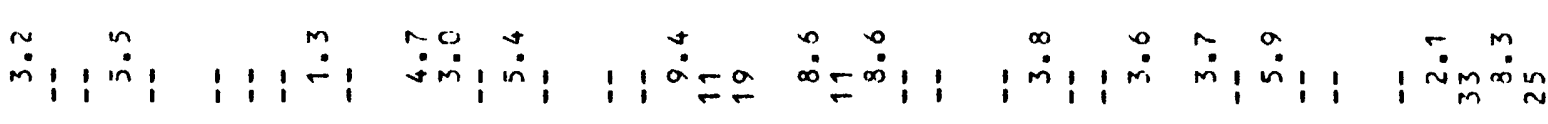

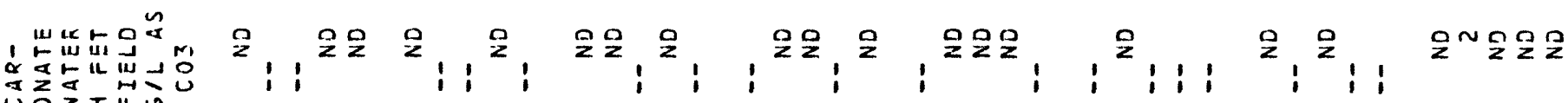

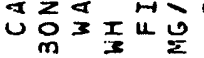

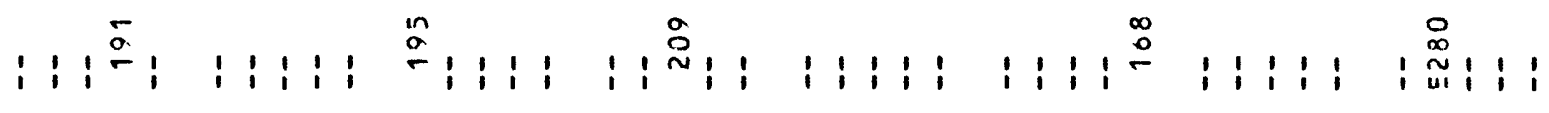

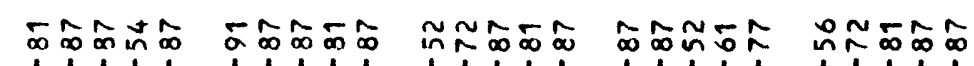

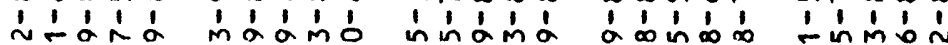
TTPT TOPNT

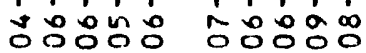




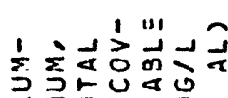

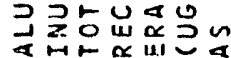

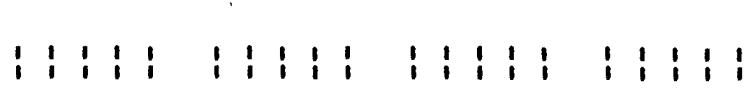

옹

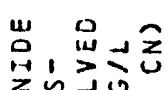

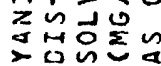

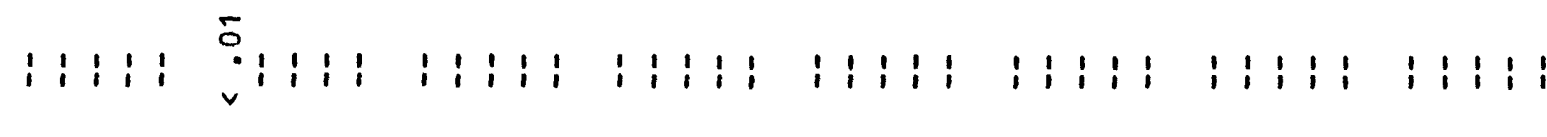

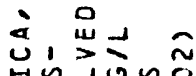

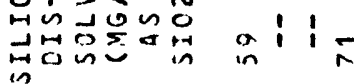

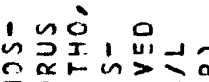

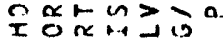

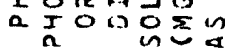

1:il

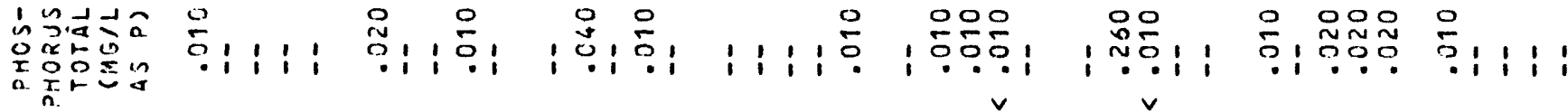

iii高出出

ñtin

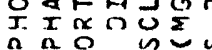

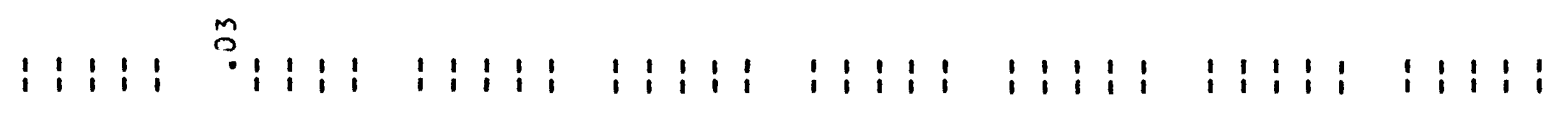

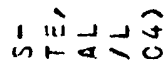

어의

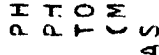

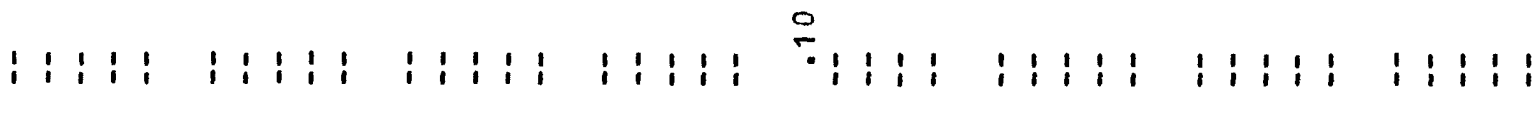

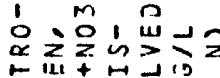

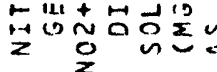

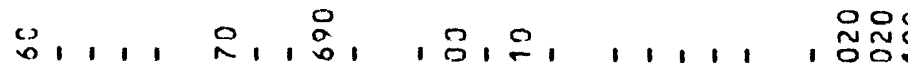

웅ํำ

웅용

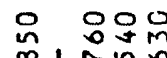

$\stackrel{\circ}{\circ}: 1$ i : $1:$

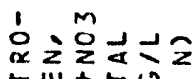

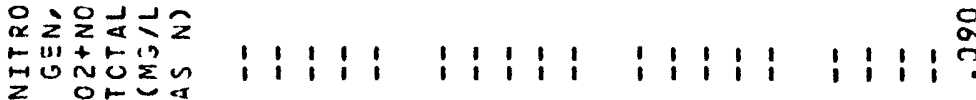

$\stackrel{\circ}{\circ}$

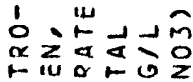

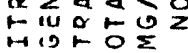

벌난ㄹ⿺

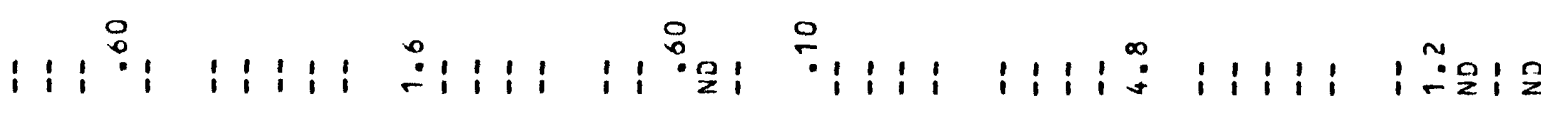

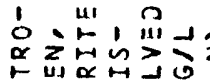

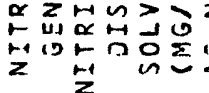

$\frac{0}{0}$

运出出士

$\alpha z z n>2$

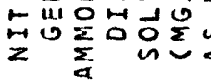

(2)

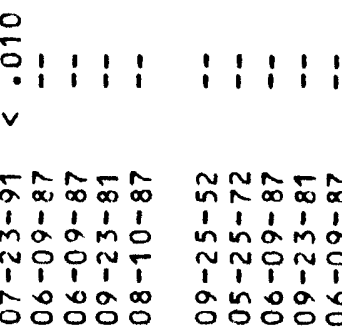

1 1 i i

1:1 1

$11 ! 11$

i i i

$\checkmark$

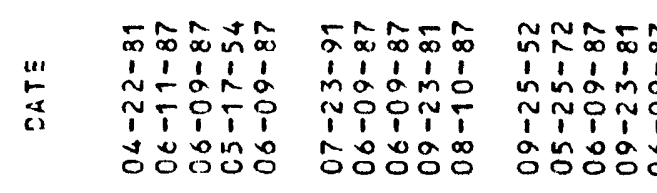

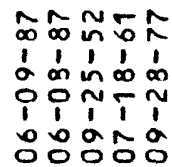

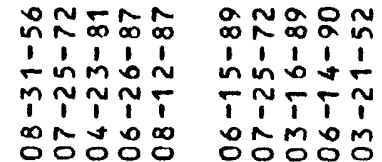

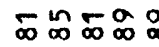

$\infty$ in 600

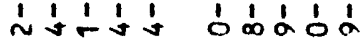

íti itimi

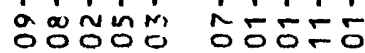




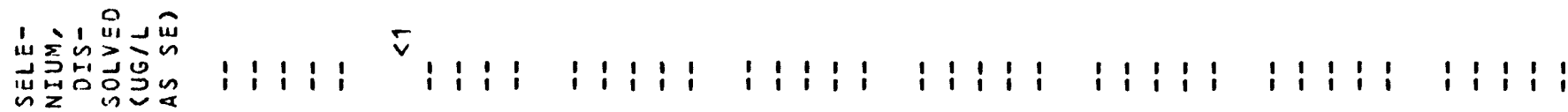

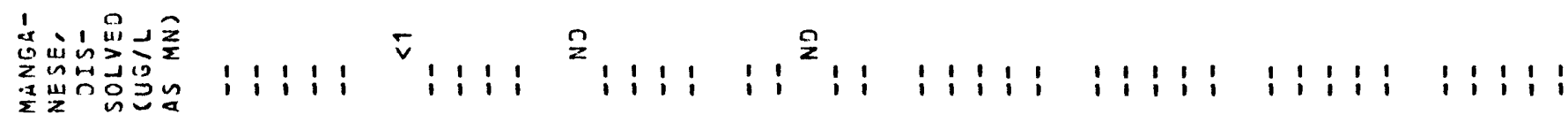

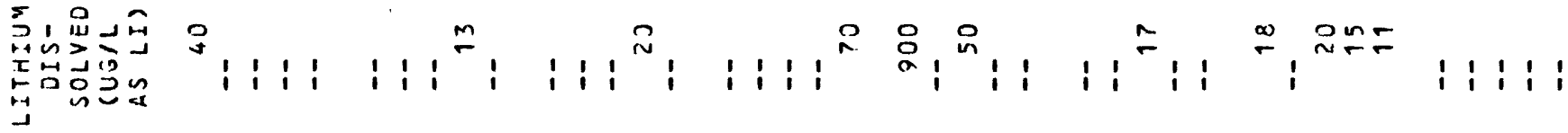

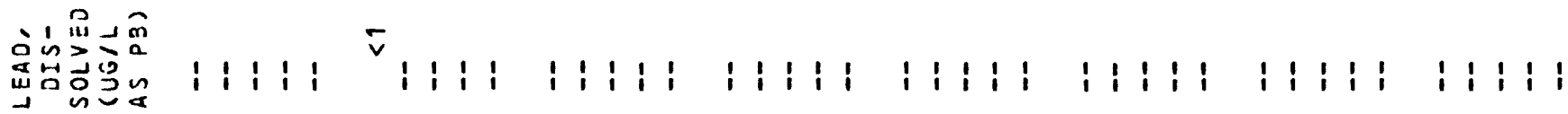

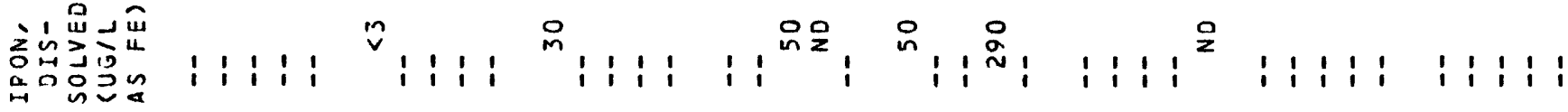

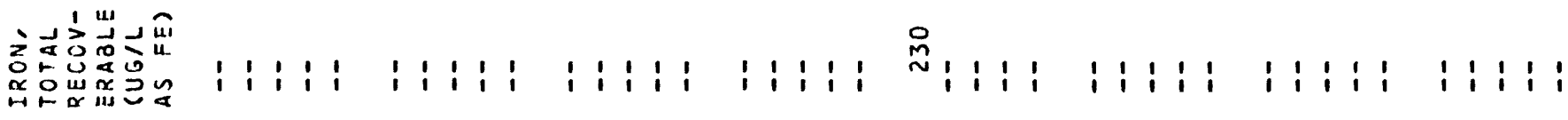

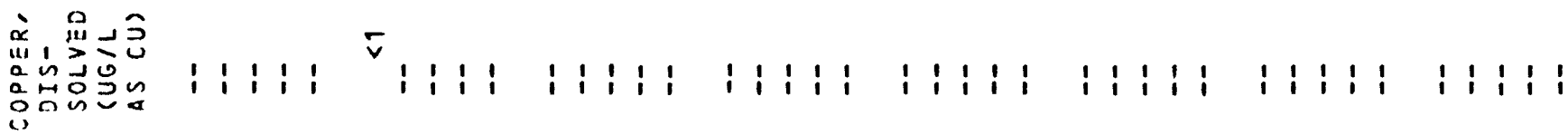

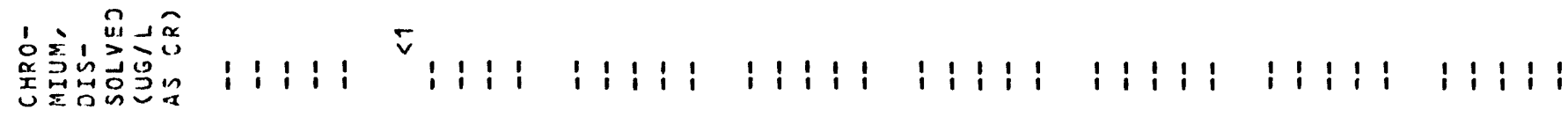

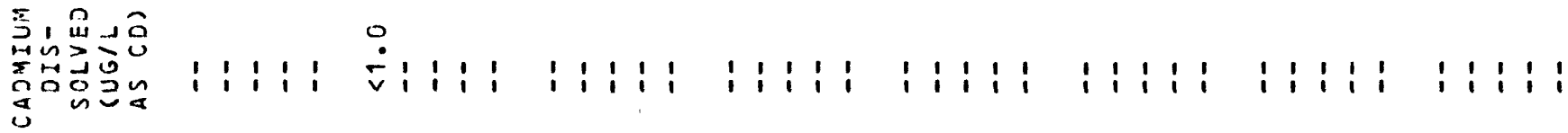

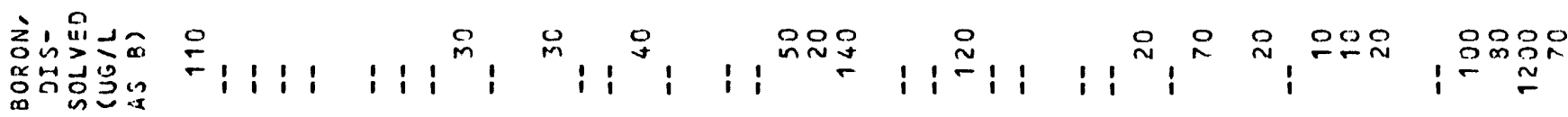

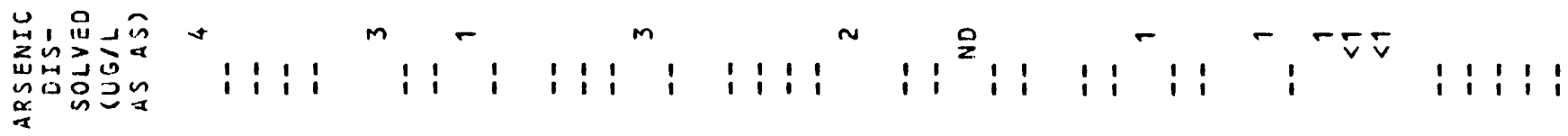

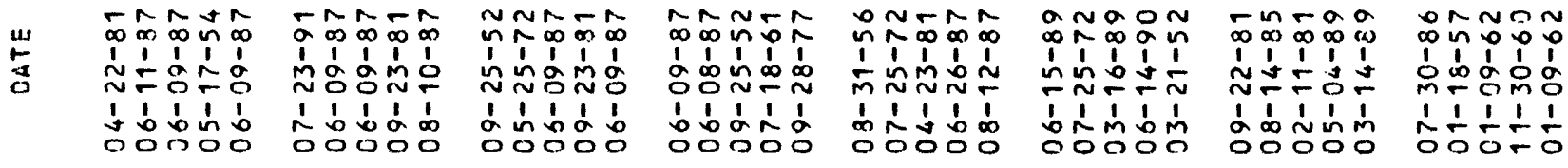




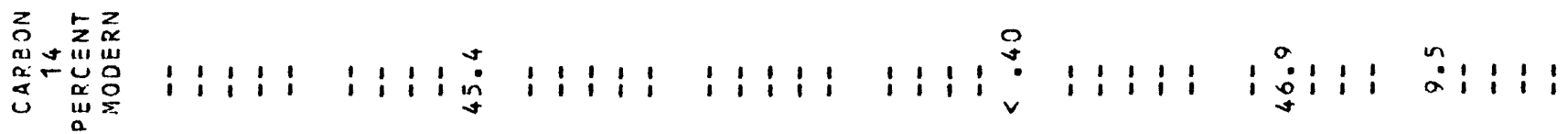

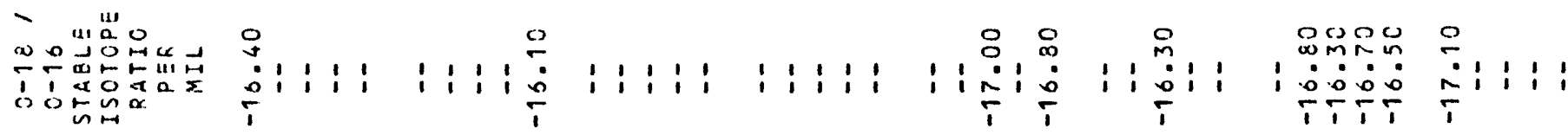

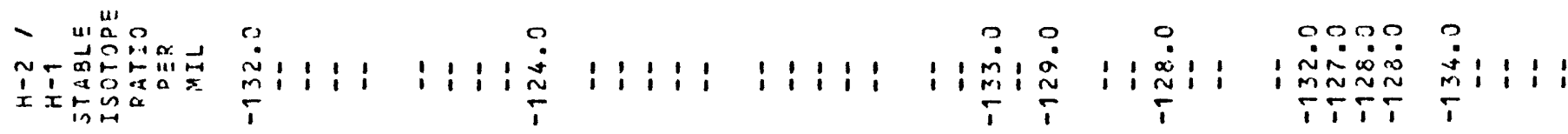

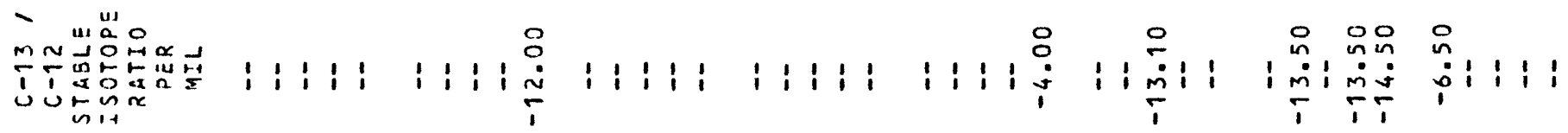

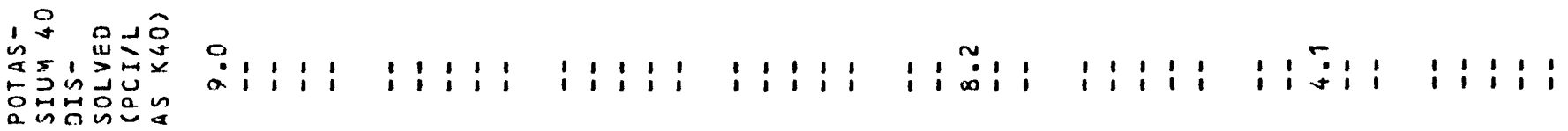

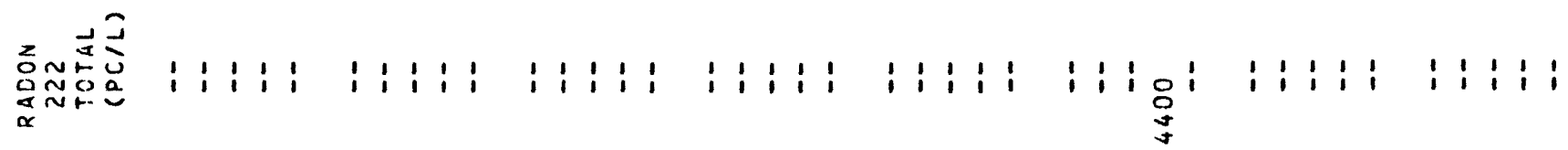

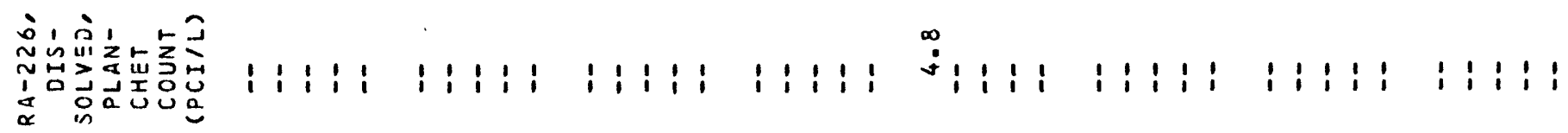

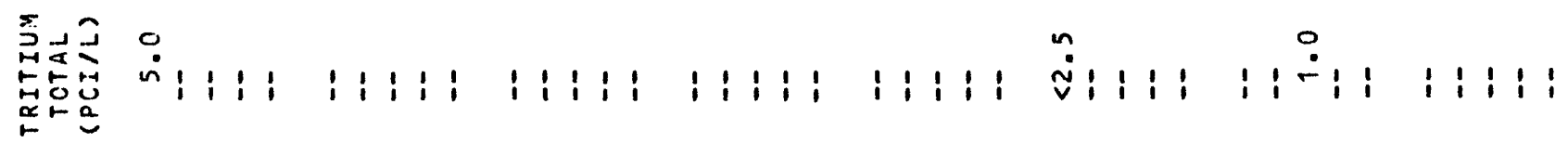

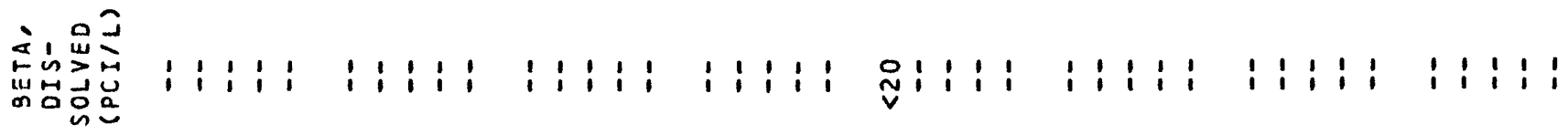

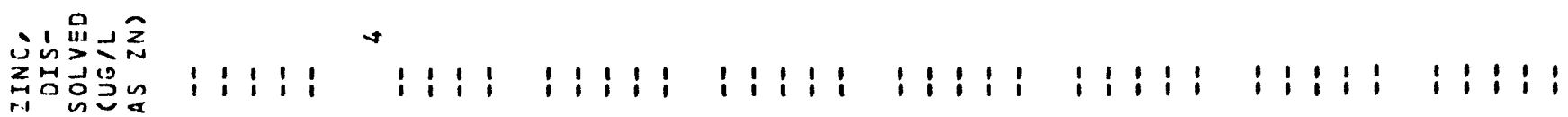

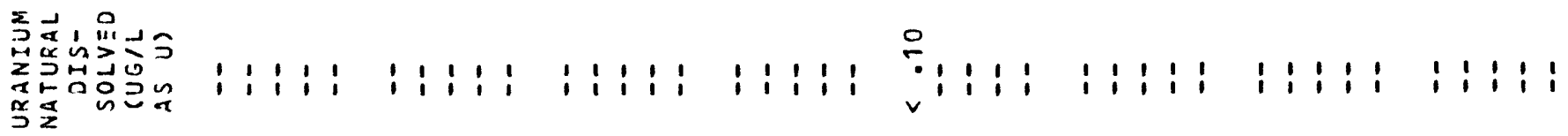

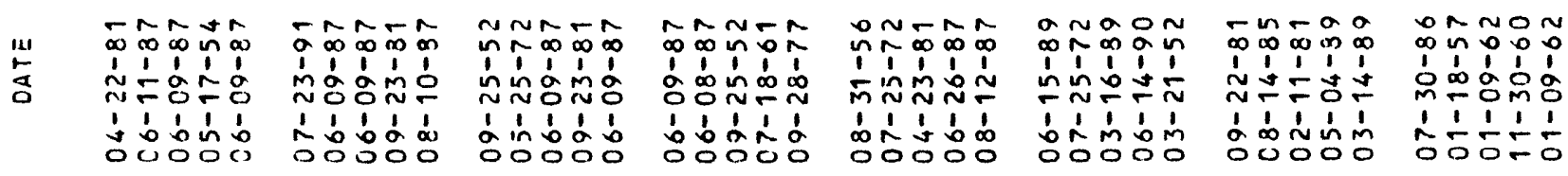




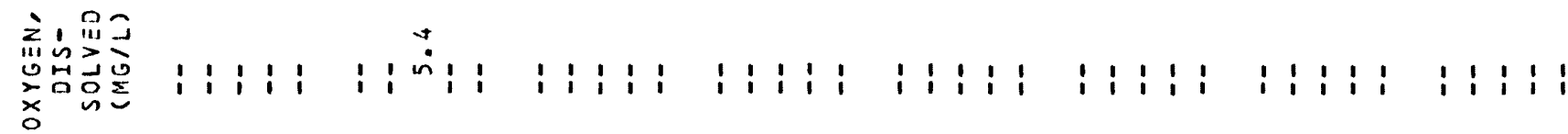

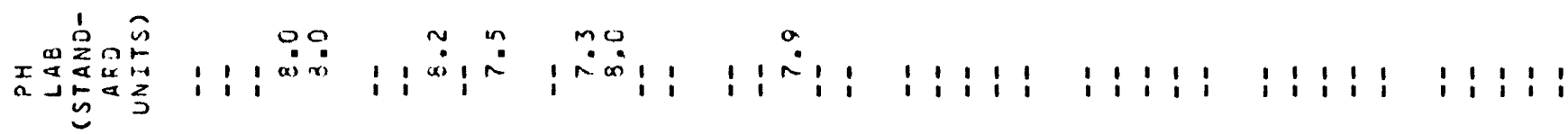

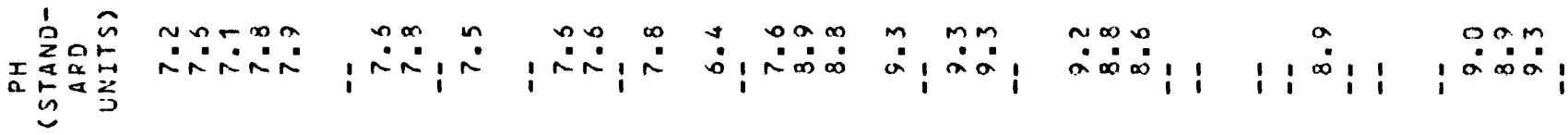

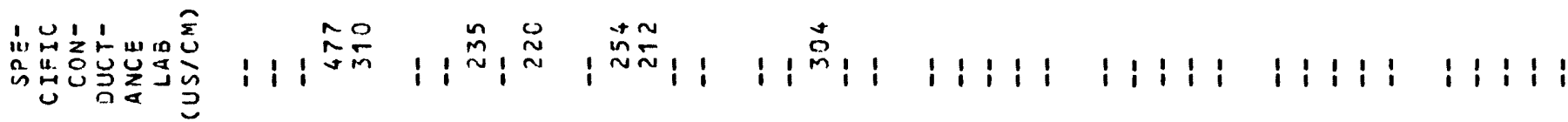

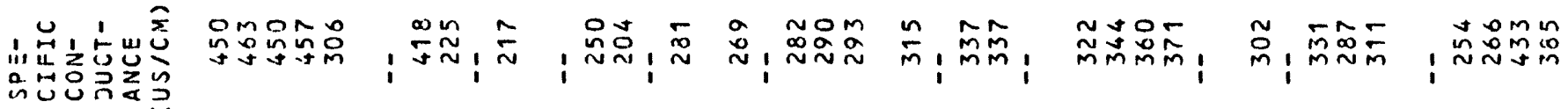
\&u

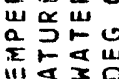

ininininu nino o ino o n nog

onoon noonn 00000

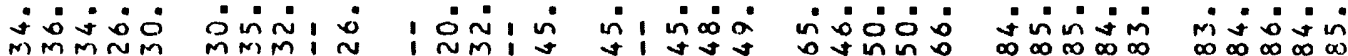

000 in a

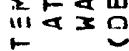

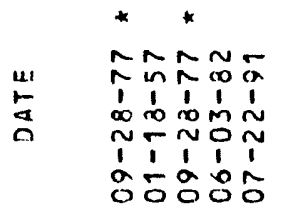

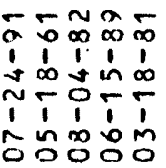
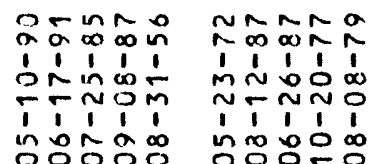

arãán

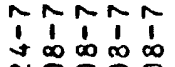
$\sim 20$ gूo요용

กิํํํํำ

n๓o용

astróo

in $N \sim \infty \infty$

กิกิโลิ

이묘

$\begin{array}{llllll}1 & 1 & 1 & 1 & 1 \\ 0 & \infty & 0 & 0 & 1\end{array}$

araag

thãa

on in ${ }^{1}$ i

i. 1 i

1.

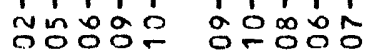

$\stackrel{H}{\stackrel{L}{n}}$

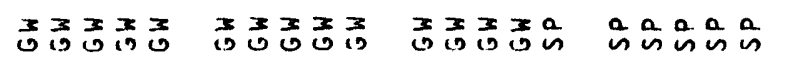

a. a a a

a a a a

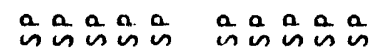

$\sum_{0}^{2}$

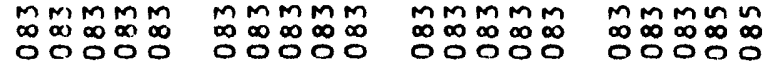

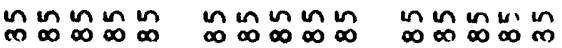

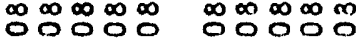

min $n$ in in

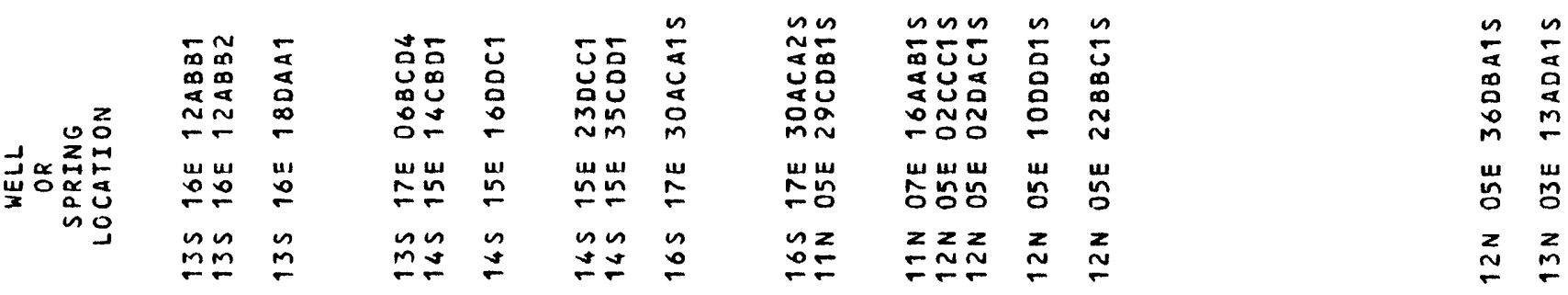




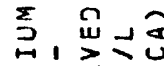

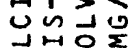

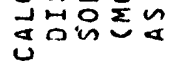

ㄴ.

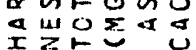

$\Delta-t m \sim$

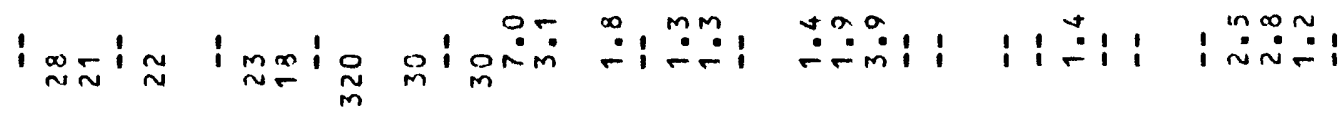

\section{oogon} mogniñ

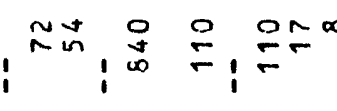

的的品

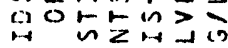

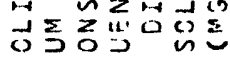

$\infty 0 \infty \pi 0$ ํํㄴำ

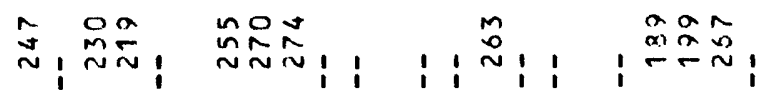

的。”等

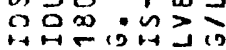

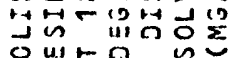
Ouㅏㅁ

z整品

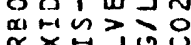
和品

$\omega_{i=1}$ in

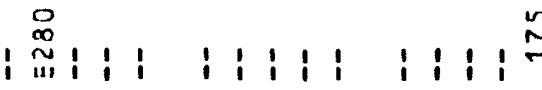
$\stackrel{n}{\approx}$

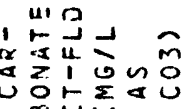

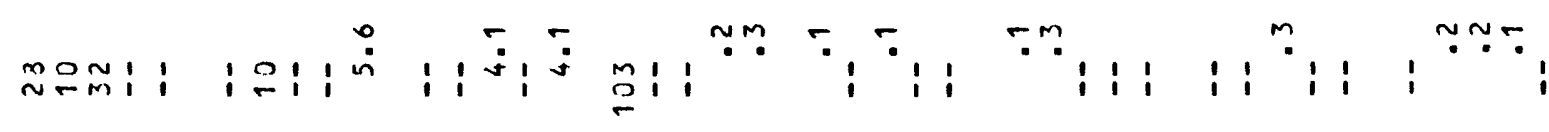

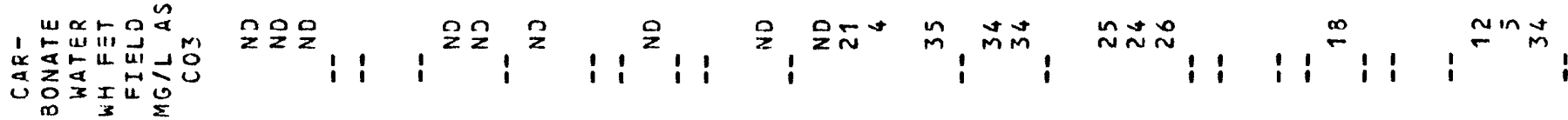

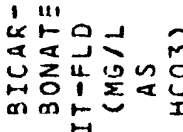
1 $w \alpha x+0 \tilde{x}$

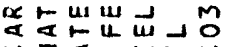

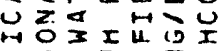

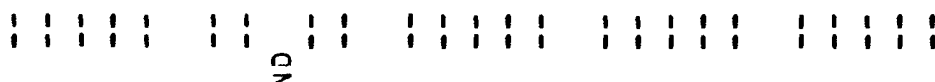

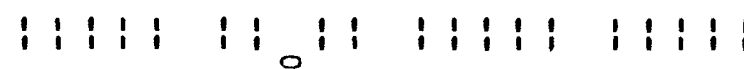
! 1 : : : 1:1:1 1 1:1:1:1:1 $\stackrel{\circ}{\stackrel{1}{ }}$

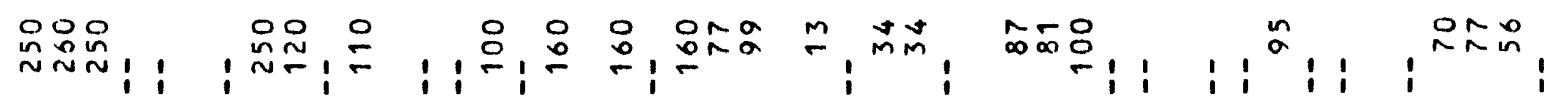

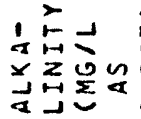

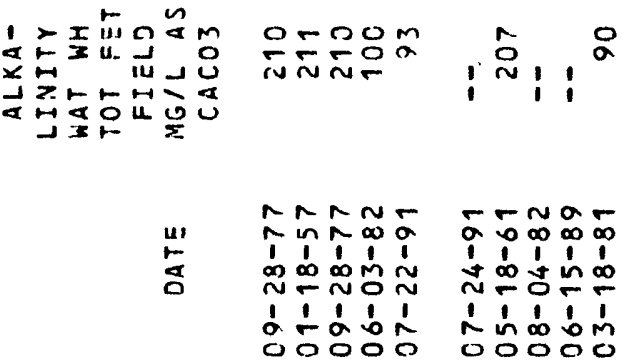
$\stackrel{\circ}{M} \stackrel{m}{m} \stackrel{m}{m} \stackrel{m}{m}^{\infty}$ $\stackrel{0}{\infty}, \stackrel{\infty}{\infty}$

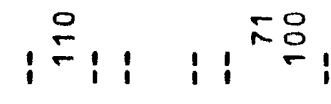

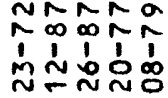
iń。 约文ㅇํㅇ웅

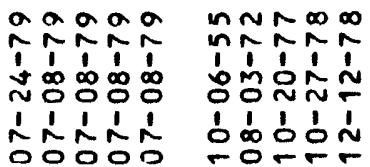

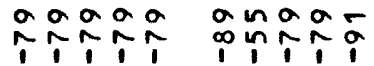

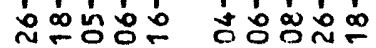

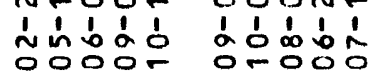




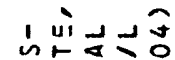
단도

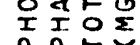

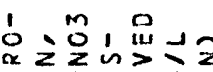

$x \sum_{i=1} \sum_{n \rightarrow 0}$

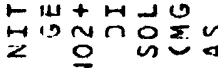

'. $m$

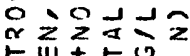

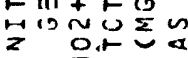

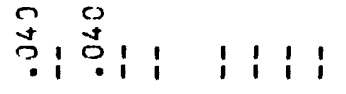

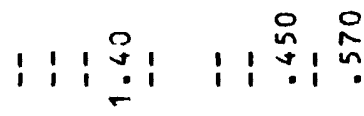

$\stackrel{0}{n} !$

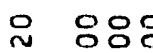

옹응

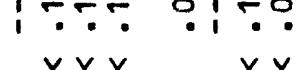

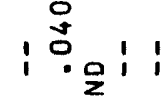

!

i i i i i

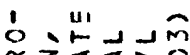

i11 $x=0$

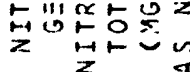

i־i i isi i i i i

' 证出

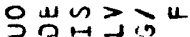

u. $x$, o 0 is

numos

บะก?

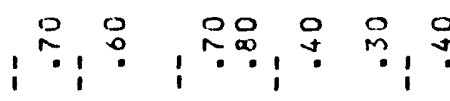

$\simeq m$

$n i \infty n i \quad a m n i$

$\because$ i i i i i i i i i

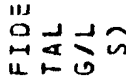

공요

i i i i i ivi

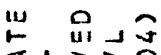

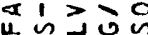

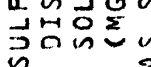

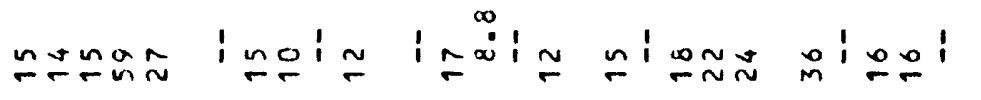

ㄸํ' I I I

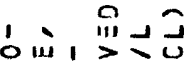

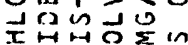

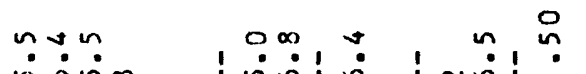

\section{$\infty$}

Ua. o is

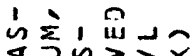

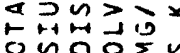

a in on

产, 出上交

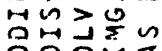

䗆品

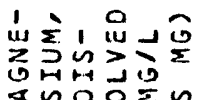

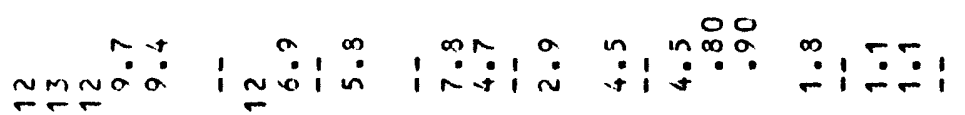

$\infty \sim \infty$

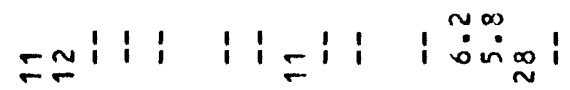

的喝采

둥ำ

$\lim _{\tan }$

$10 m ! m$

m!

$\sim \widetilde{n} \mid$

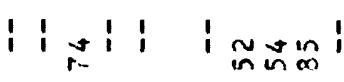

imi:

$\because \div \div \div !$

$\stackrel{0}{\circ} \frac{0}{2}$

i :

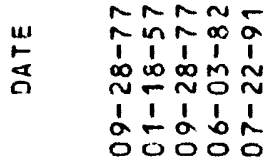

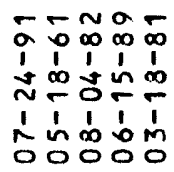

2.

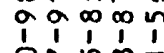

은

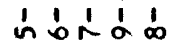

nNara aagaa

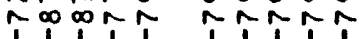

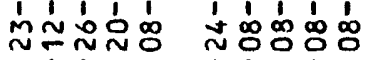

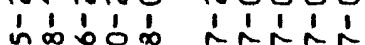

$\sim \sim \sim \infty \infty$

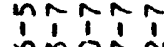

om识㾞

b́bón

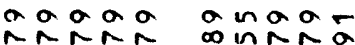
$\hat{1} \hat{1} \hat{1} \hat{1}$ i

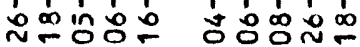

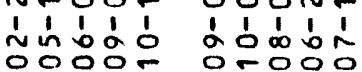


这, 品出 a

$\cup$

吉的品过

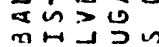

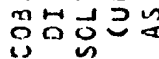

1:1:1 1:

$\sum$ ำ 为然至

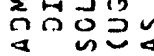

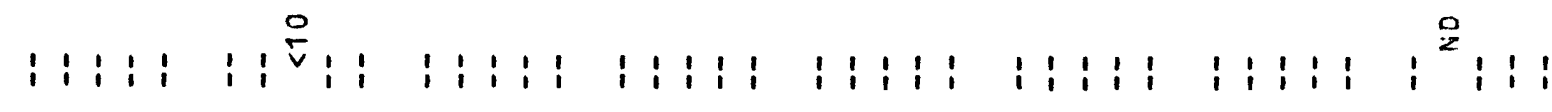

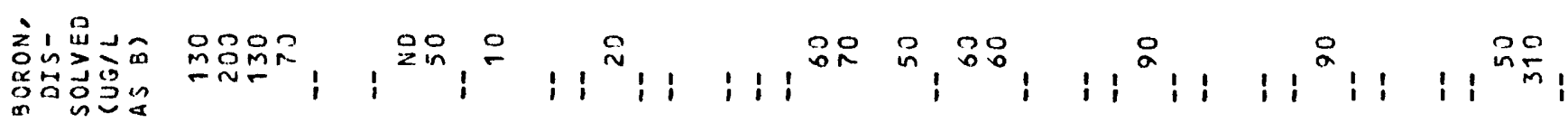

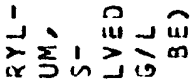

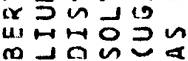

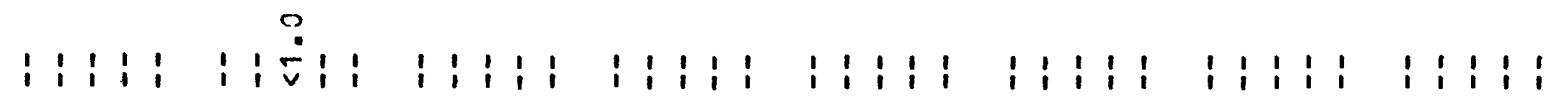

产, 品衣主

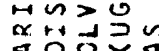

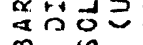

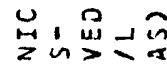

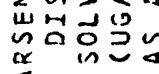

$\bar{v}, \bar{v}: 1: i^{n}$

$1111111^{\infty}$

i $1:$ i :

i: $1: 1$

$1: 1 ; 1$

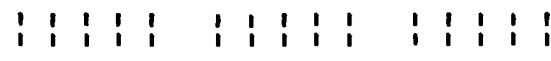

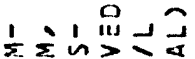

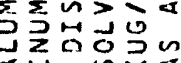

$\begin{array}{lllll:}1 & 1 & 1 & 1\end{array}$

$1: 1411911:$

: 1:1

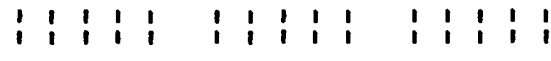
n

$11: 11: 11^{\circ}$ i i i i i

1111

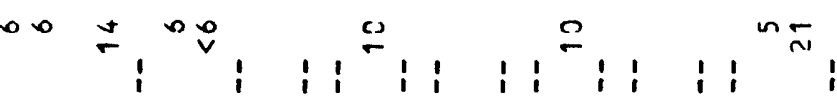

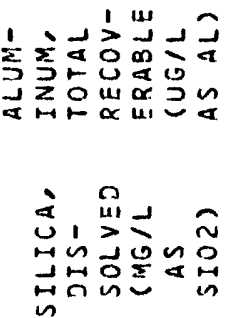
$i^{2} i$ $1: 19: 1: 11:$ $1: 1: 1:$ i i i :

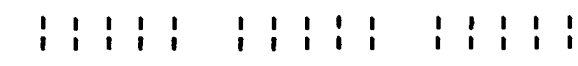

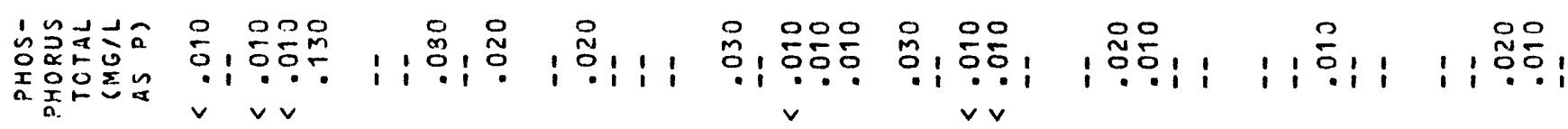

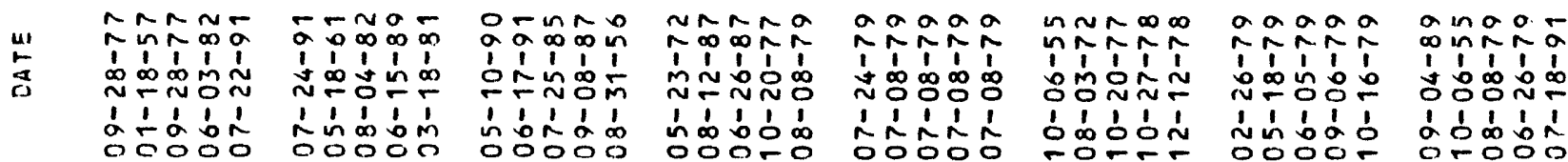




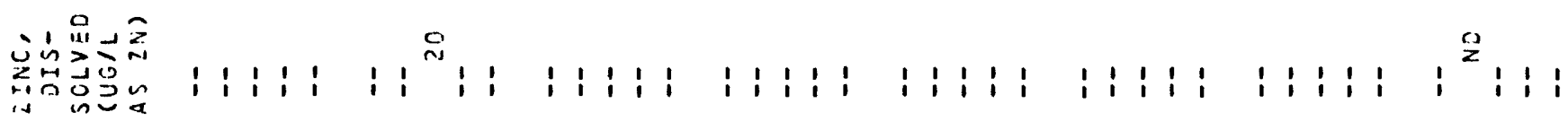

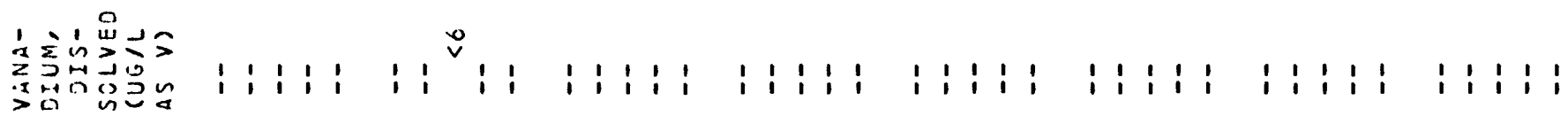

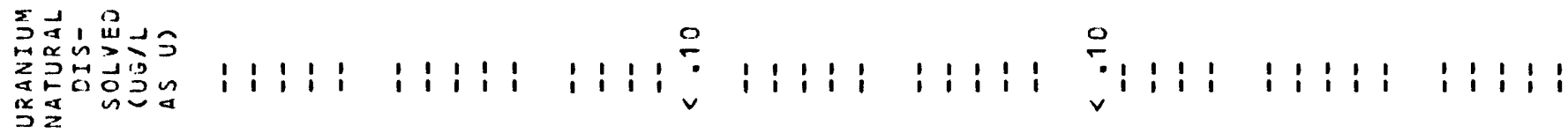

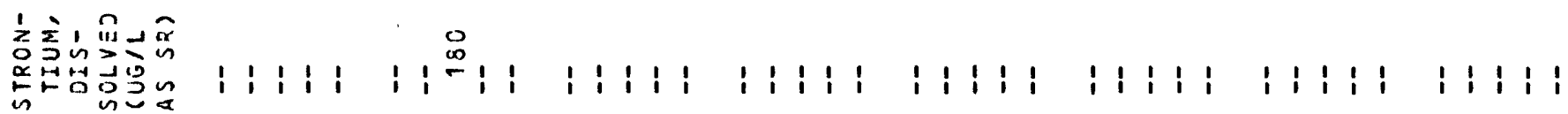

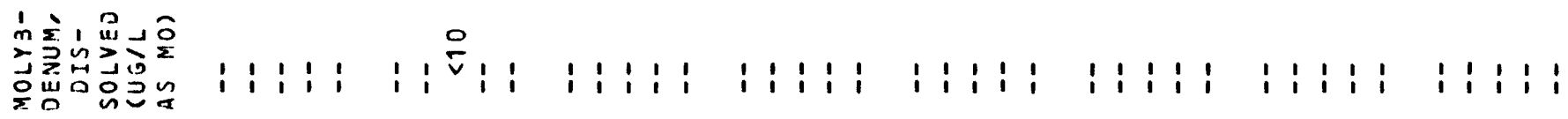

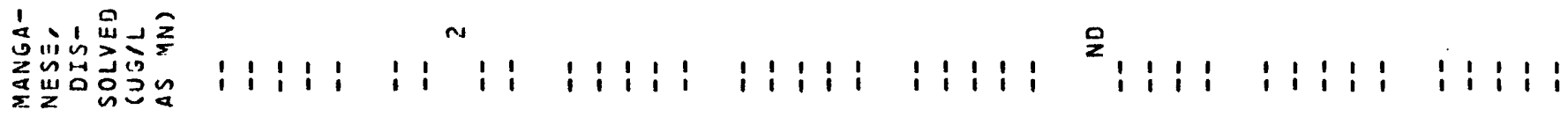

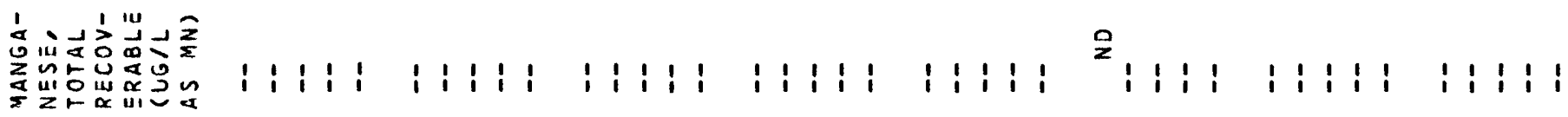

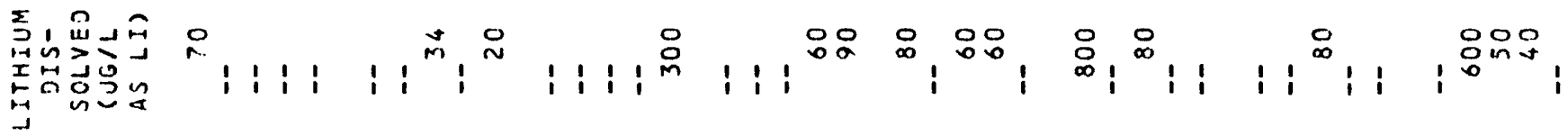

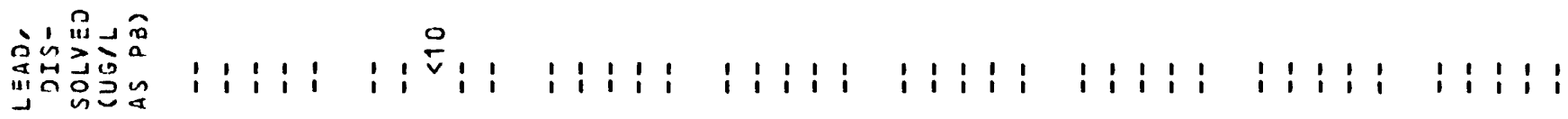

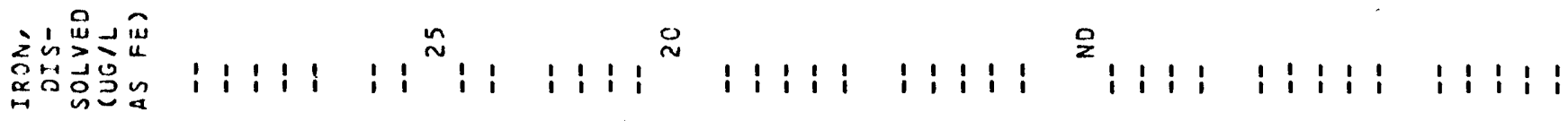

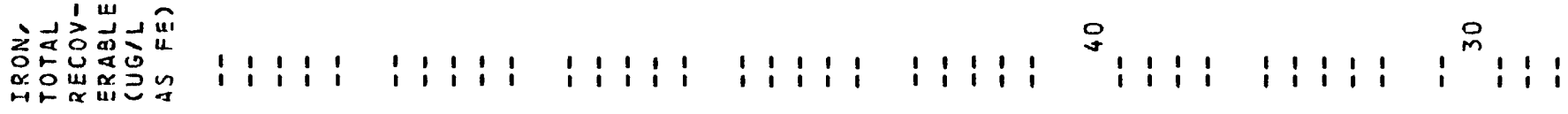

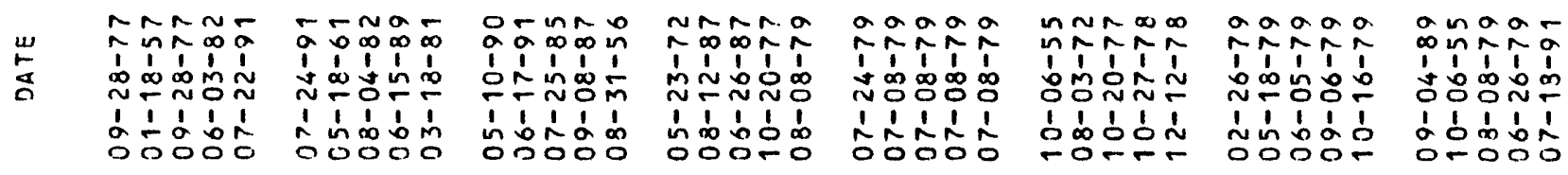




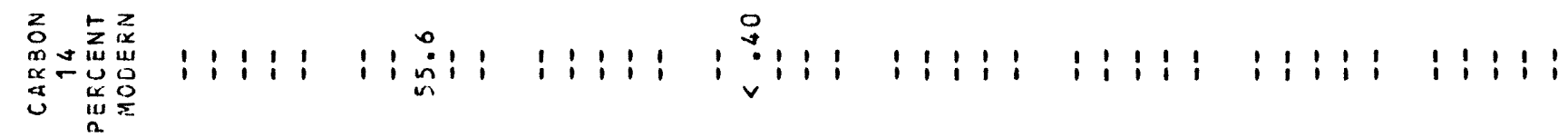

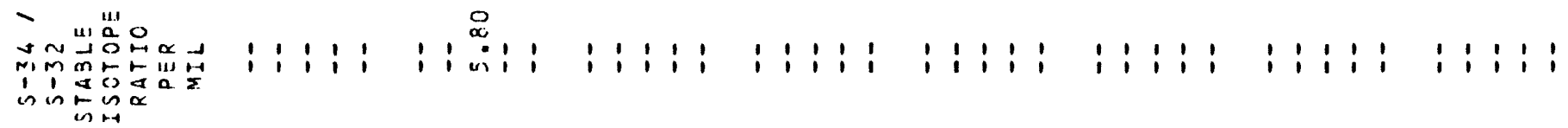

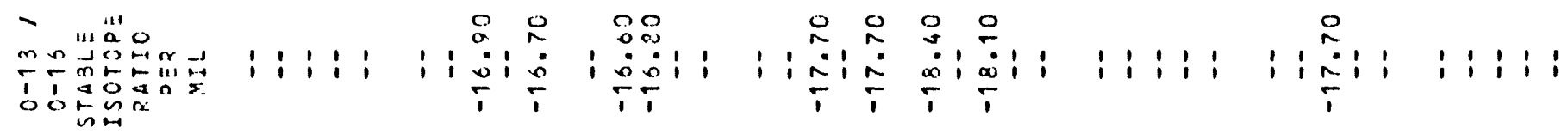

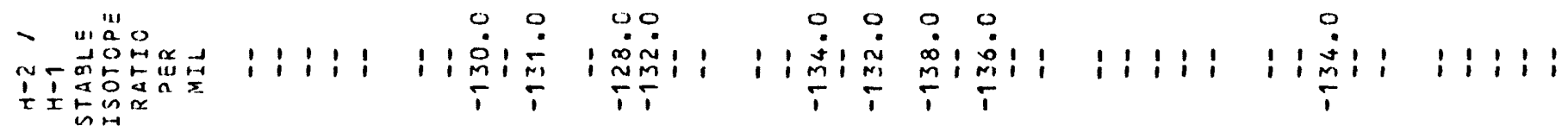

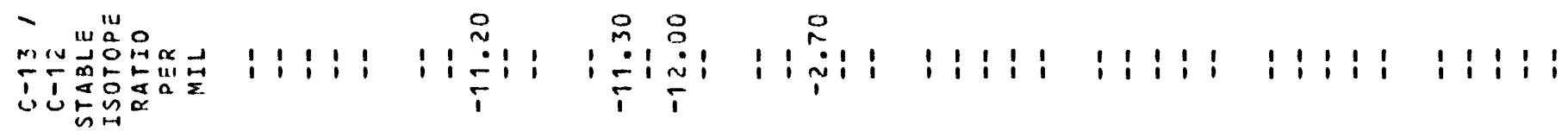

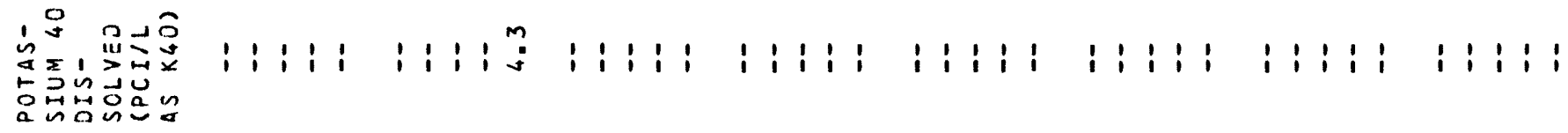

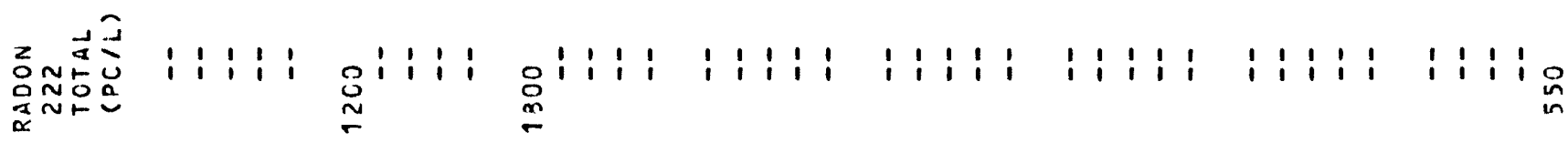

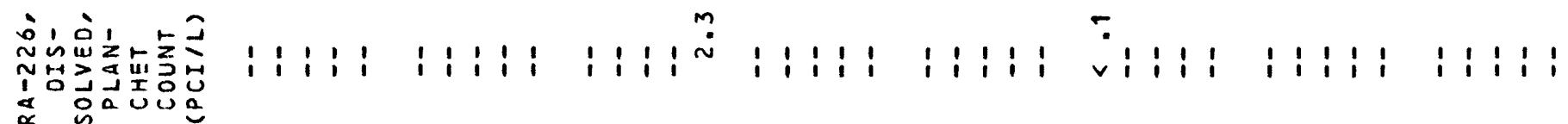

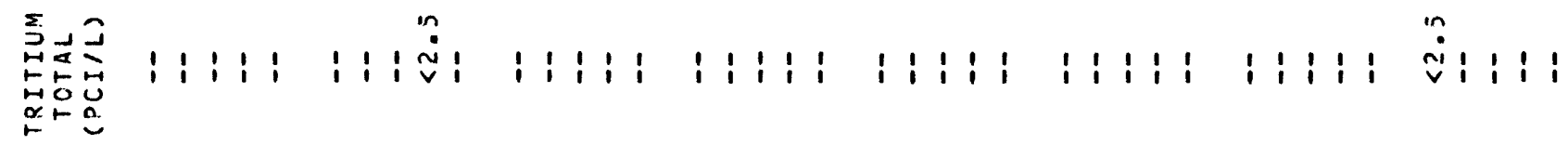

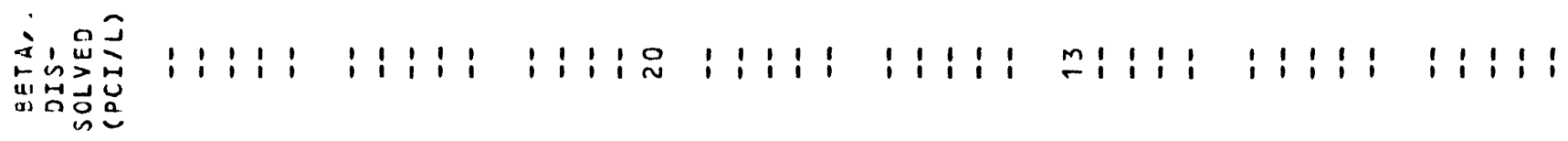

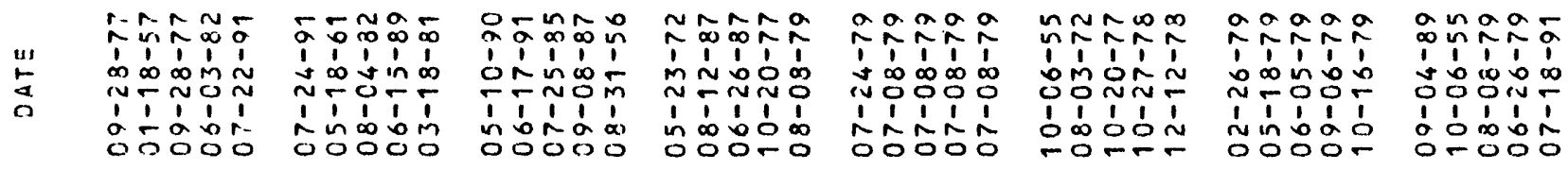




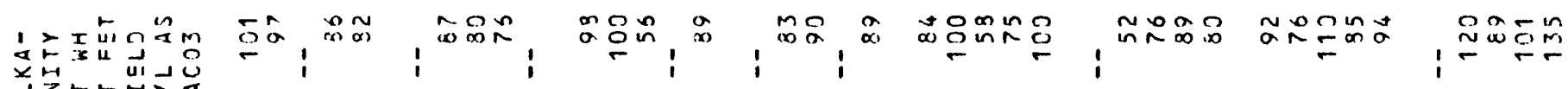

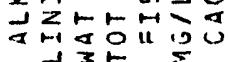

$\sum_{i 11}$ in

0

x

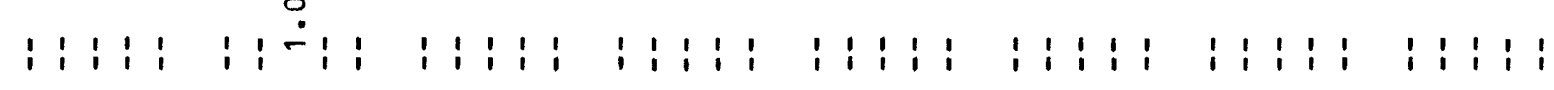

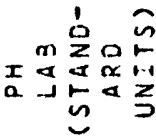

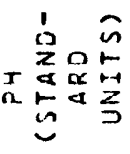

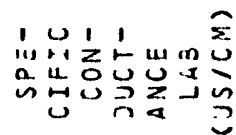

,

$m \pm 0$ t

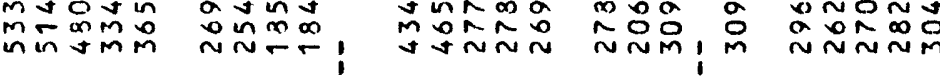

in

\& ui $<\hat{U}$

ul $\stackrel{\alpha}{\supset} \amalg$

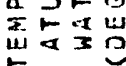

0 in 0 in

ímini

nici,

$\because 00 \%$

. 0000

Ono ?

0000? 0000

mmin in in

유유:

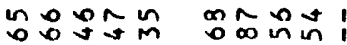

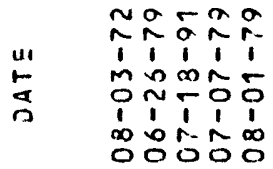

anm

$\begin{array}{llll}0 & m & 0 \\ 1 & 1 & 1 & 1 \\ 0 & 1 & 1\end{array}$

$0 m n=n$

iti 15

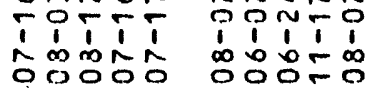

करำ

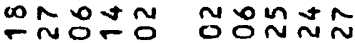

$\begin{array}{lllll}1 & 1 & 1 & 1\end{array}$

11111

No 0000

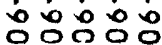

이요

$1 ; 1\}$

엉ํำ

11111

NOONO

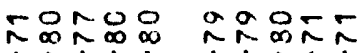

1111111111

Mnwoin mmNot

PNTPO MMNOT

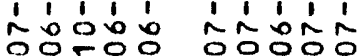

$\underset{5}{m}$

a a a a

$\frac{x}{0}=\frac{x}{10} \times \frac{x}{10}$

a $a$ a a

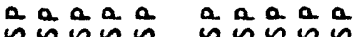

aaaㅇa

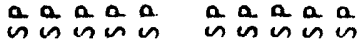

nnmmn $\infty \infty \infty \infty$

nnmmn nminn 00000

00000

舟

$\infty$ $\min n \min$ $\infty \infty \infty \infty \infty$ mnnminnmin $n$

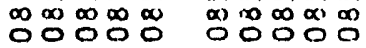

\begin{tabular}{|c|c|c|c|c|c|c|c|c|c|c|c|c|}
\hline 'z ט & $\begin{array}{l}n \\
\frac{n}{n} \\
\frac{1}{n} \\
m\end{array}$ & $\begin{array}{l}n \\
\frac{n}{n} \\
a \\
0 \\
0 \\
n\end{array}$ & 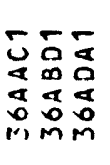 & $\begin{array}{l}n \\
\frac{1}{a} \\
0 \\
0 \\
2\end{array}$ & 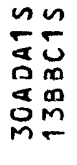 & 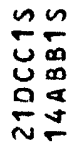 & $\begin{array}{l}n \\
\vdots \\
\vdots \\
\vdots \\
5\end{array}$ & 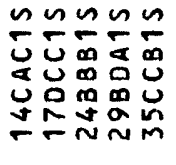 & 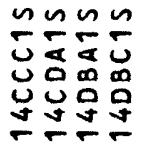 & 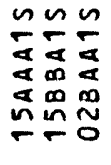 & $\begin{array}{l}n \\
\infty \\
0 \\
m \\
m \\
m\end{array}$ & 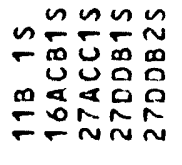 \\
\hline 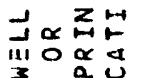 & $\begin{array}{l}\text { ü } \\
\text { c }\end{array}$ & ü & 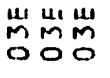 & $\begin{array}{l}\text { ü } \\
0 \\
0\end{array}$ & 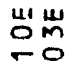 & 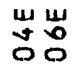 & $\begin{array}{l}\text { w } \\
\text { o }\end{array}$ & 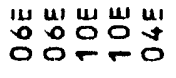 & 岩岁㟧㟔 & 茪苝崩 & $\stackrel{H}{O}$ & 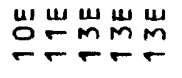 \\
\hline - ng & $\frac{2}{N 1}$ & $z_{m} z$ & $\begin{array}{l}z z z \\
y \\
r-r\end{array}$ & $\begin{array}{l}z \\
4 \\
5\end{array}$ & $\begin{array}{l}z_{2} \\
\vdots 2 \\
-\pi\end{array}$ & $z_{n}^{z}$ & in & 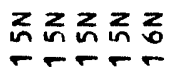 & $\begin{array}{l}z z z \\
0 \\
0\end{array}$ & $\begin{array}{l}z z z \\
0 \\
0\end{array}$ & $\underset{n}{n}$ & 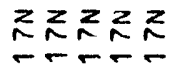 \\
\hline
\end{tabular}




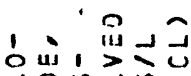
卉品出古要 可我员品

'ं 的的分

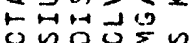

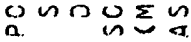

armo gog $\because \div ! \div \div \quad 1 \div \div$

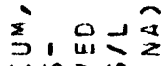

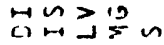
on่

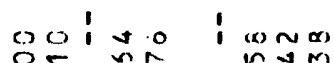

कः

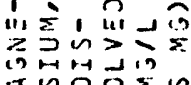
$\frac{0}{2 !} \div \frac{0}{2}$ $10 \div$

$\left.\stackrel{\sum}{\supset}, a\right)$ 式的 aํํ의 $\because \because 1 \because 9$ : $\because \because \dot{m}$ DNTnM $\ddot{-\dot{n} \dot{r}}$

m?

(1)

$\because \because \because \frac{2}{4}$

$\div$

: :

$n+n m$

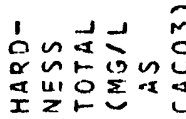

的出社, 只

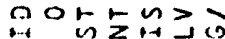

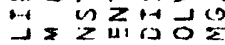

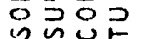

in ${ }^{\prime \prime} 0,0$,

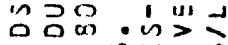
Hin

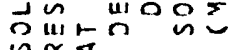

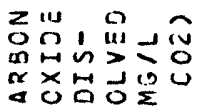

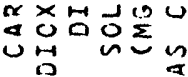

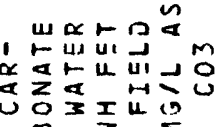

$\because \simeq \because \div, \because \simeq ! !$

$\because \simeq$

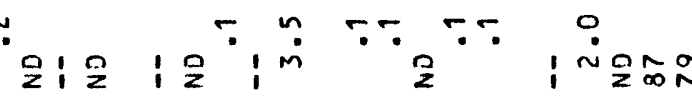

$m m m r$

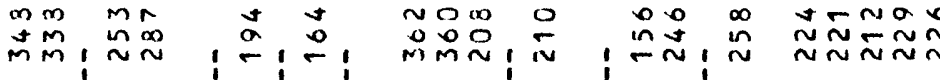

ind i i

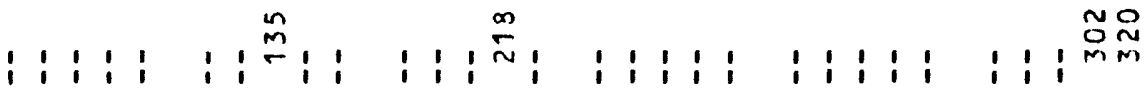

$\operatorname{los}$

oง ovo mं - :

$\operatorname{lin} \sin n$ $\because \frac{2}{2}$

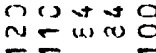
r $2 \sum^{0} \geq 2$

mosom $\ddot{\sim} \dot{\sim} \dot{-} \dot{-}$

$n^{\sin t}$ :

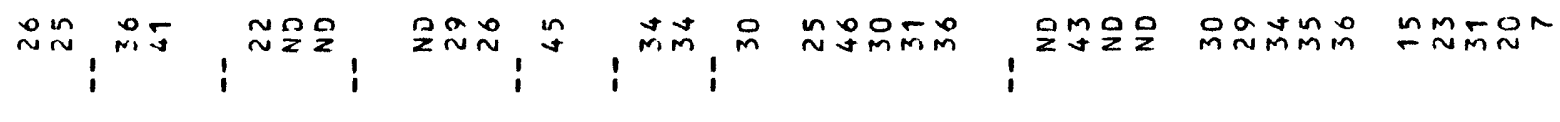

(2)

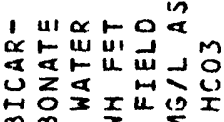

\section{man}

$\operatorname{mon}$ o a

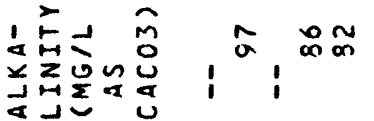

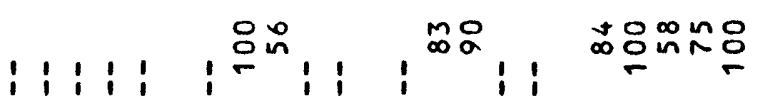

in 음요 mon

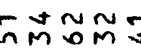

Naรå

$\stackrel{4}{a}$

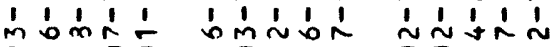

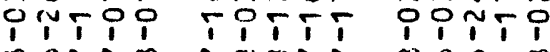
nỗón กั0ญ $\begin{array}{llll}1 & 1 & 1 & 1 \\ 0 & 0 & 0 & 0\end{array}$

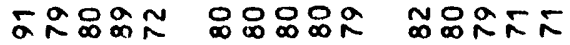

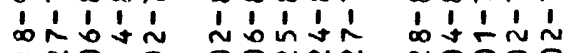
in

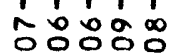

o 10 00000 i⿻ำ

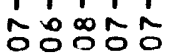

anom

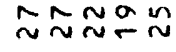

$\operatorname{con} \alpha$

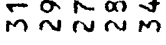

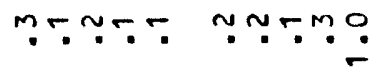

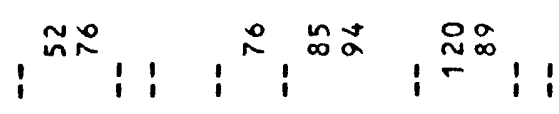

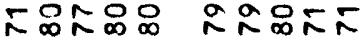

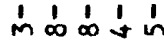
1: 190

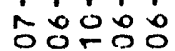
11 m! s MMNO

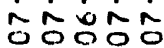




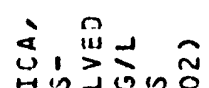

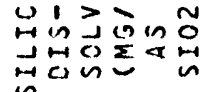

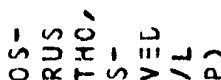

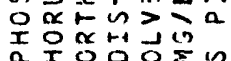
a

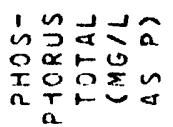

$\stackrel{0}{0} \frac{0}{0}, \frac{0}{0}$

11:11 1:1 :

1:111

191111

i i i i i ios ?

0,111 - i : i ?

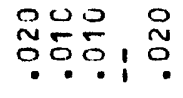

$1000, \frac{0}{0}$

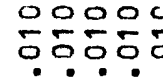

$\checkmark \vee \vee v$

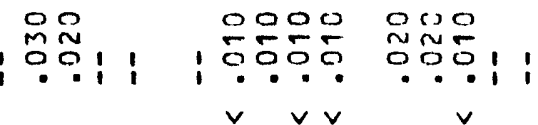

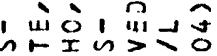

थ下

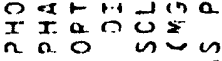

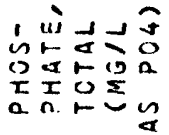

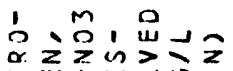

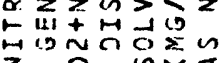

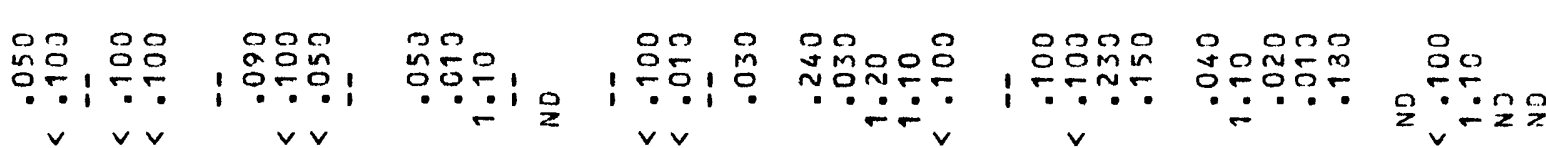

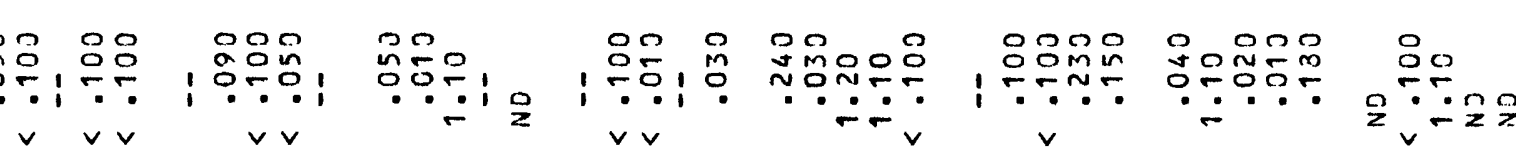

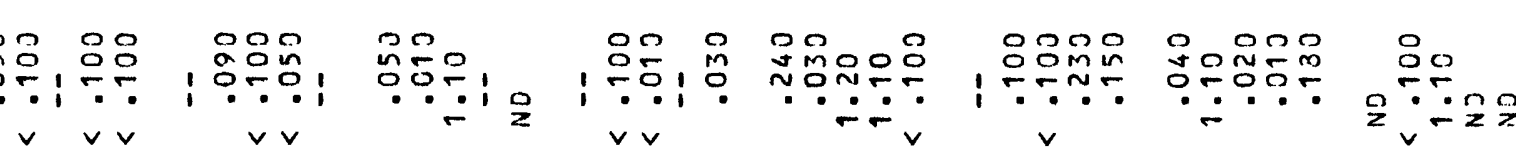

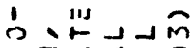

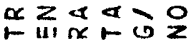

牙"

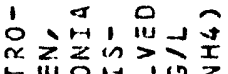

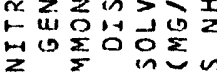

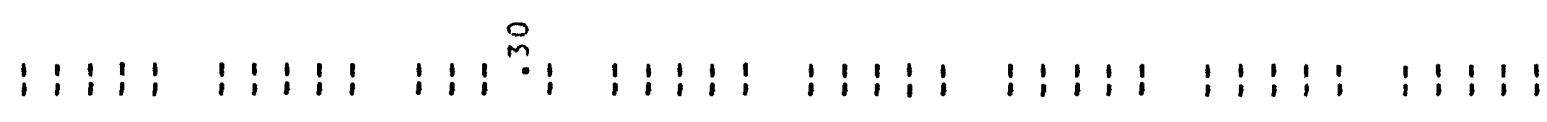

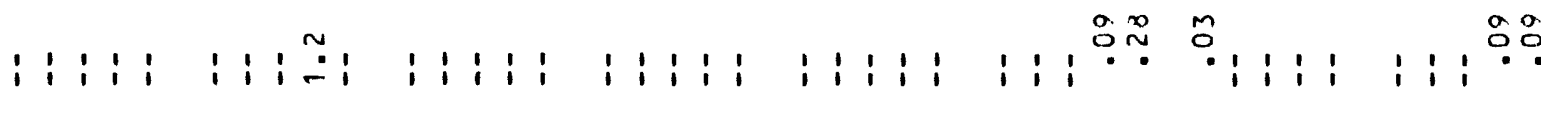

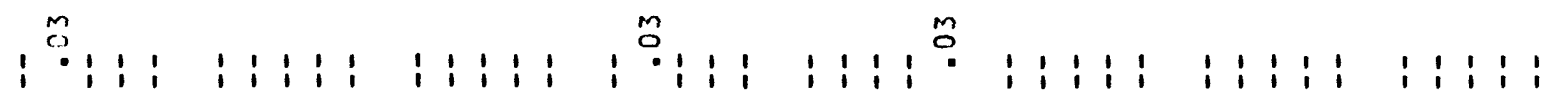
a in

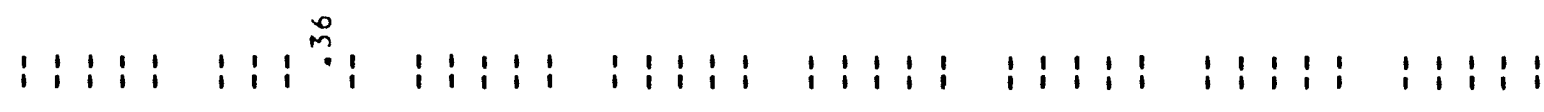

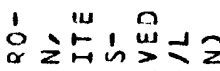

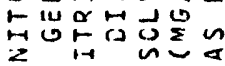

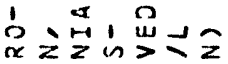

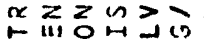

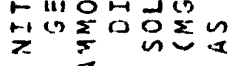

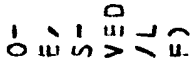

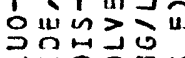

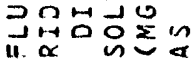

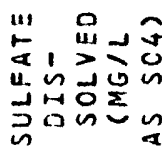

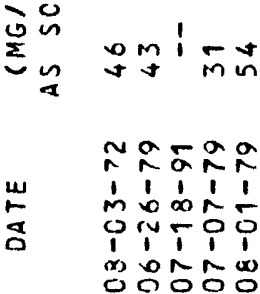

min

nomir

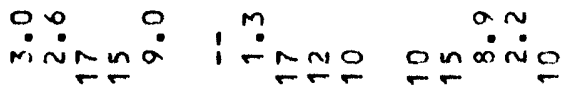

$\stackrel{\sim}{\sim} \min m$

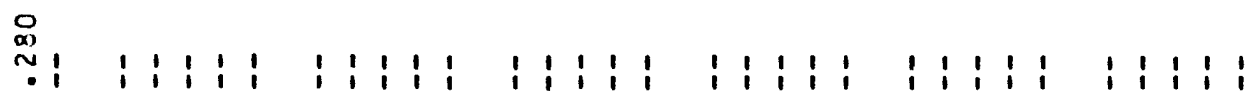

On

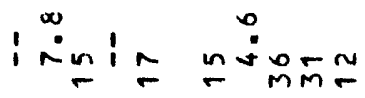

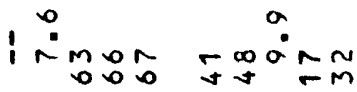

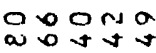

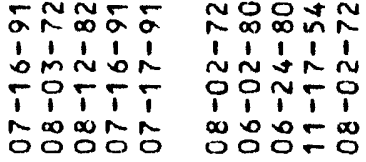

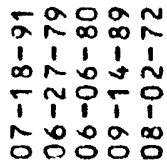

옹용요 잉

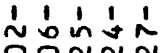

i. 1

ㅇํㅇㅇํㅇ

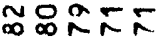

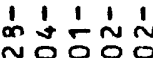
T1 1000

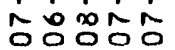

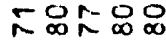

mon: 1 '

웅ํㅇ

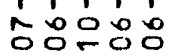

오은

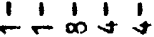
M M 승요응 


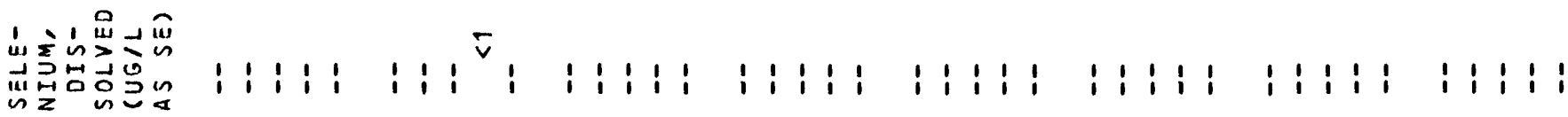

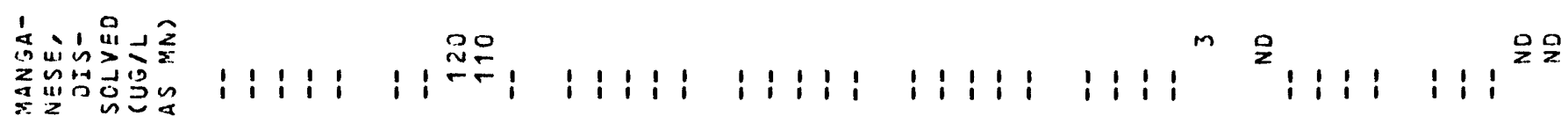

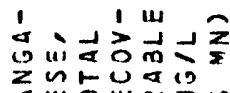

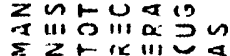

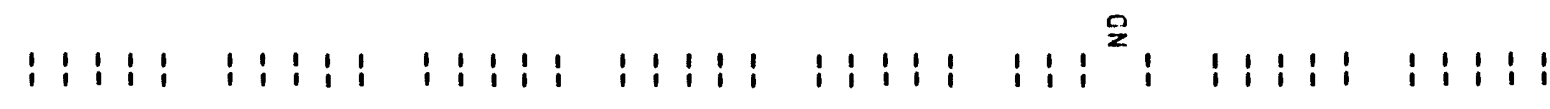

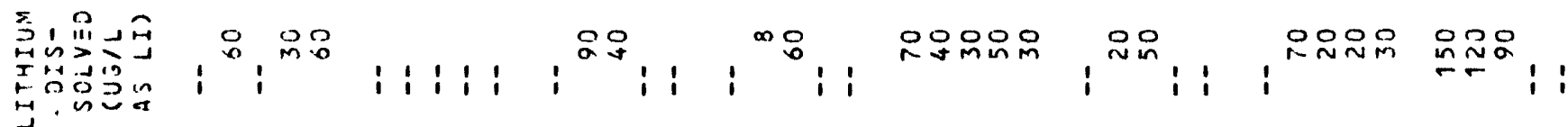

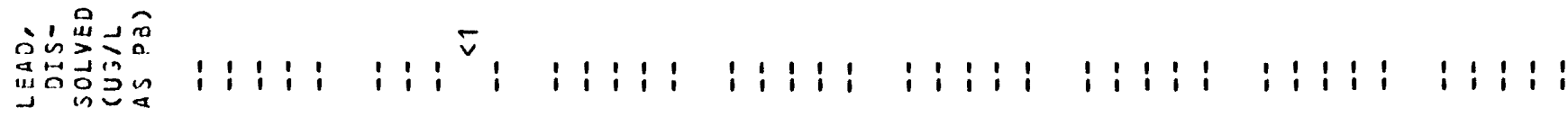

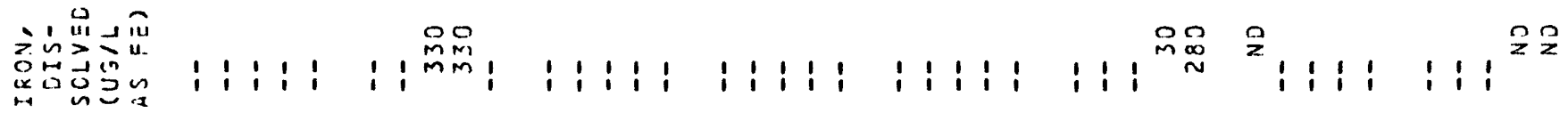
in,$\stackrel{n}{>} \pm \hat{\jmath}$ and

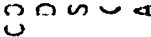

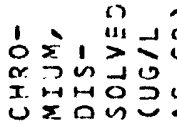
: : : : : : : ${ }^{5}:$ : : : : : : : : : : : : : : : : : : : : : : : : : : :

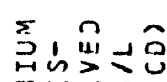

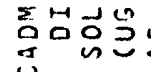

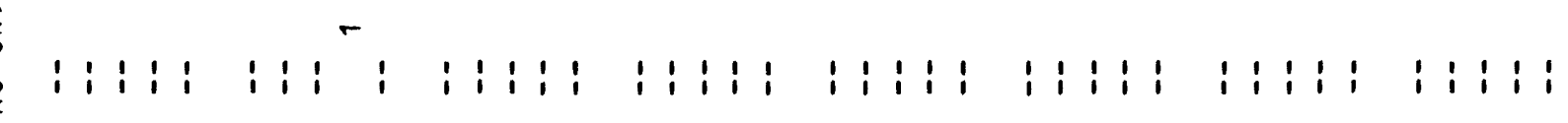

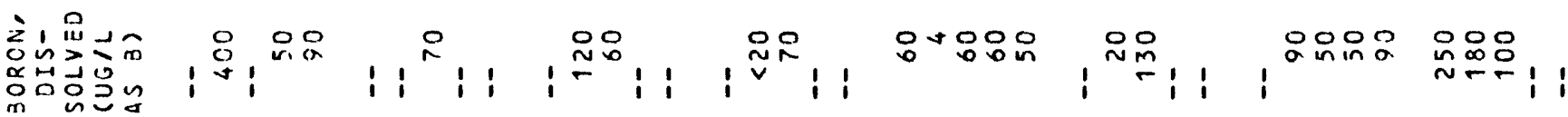

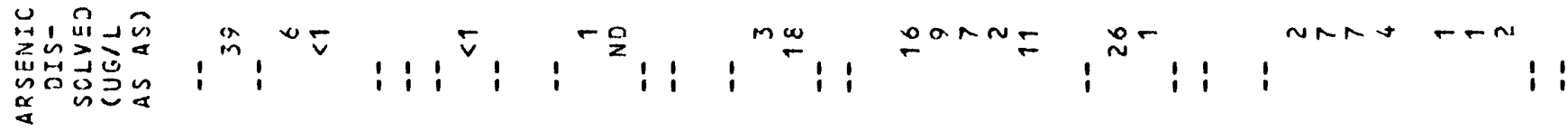

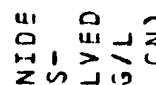

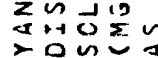

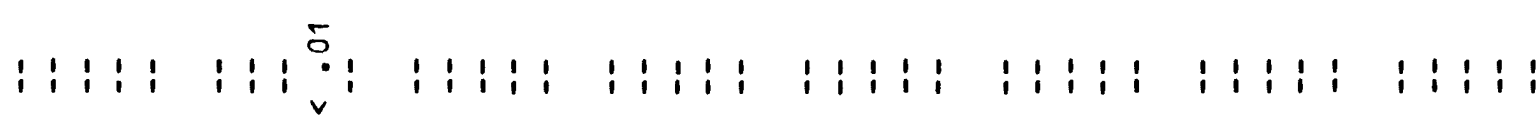

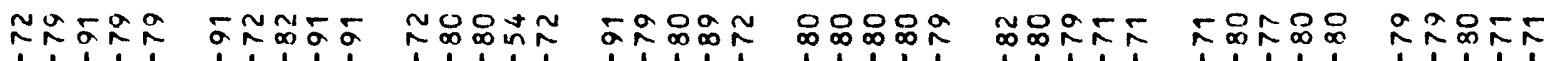
m.

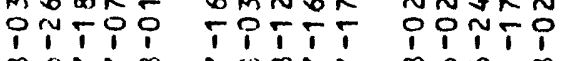

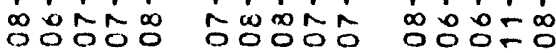

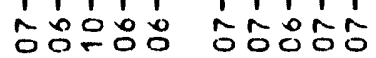




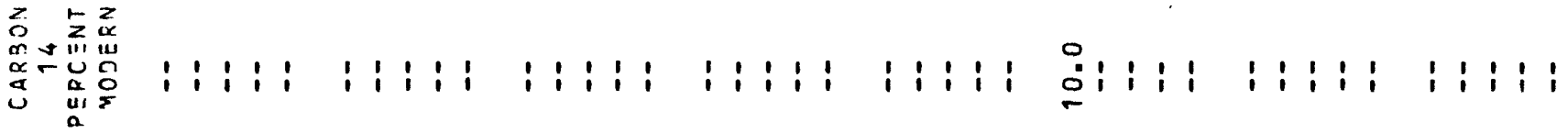

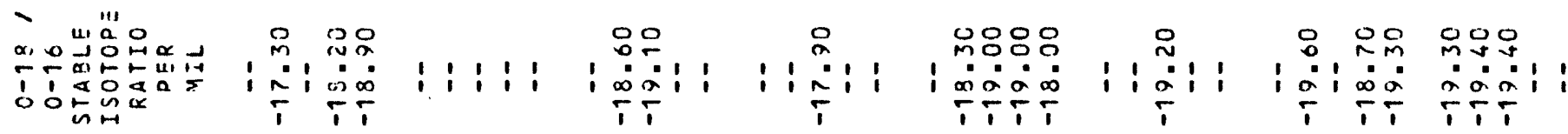

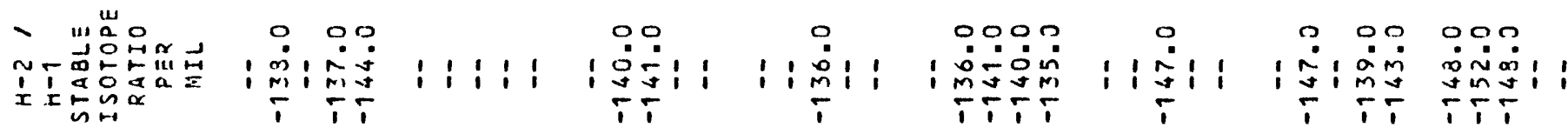

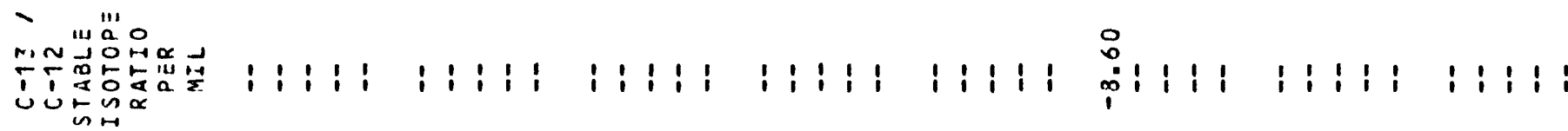

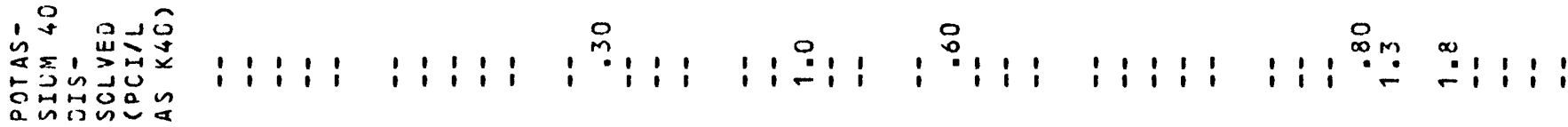

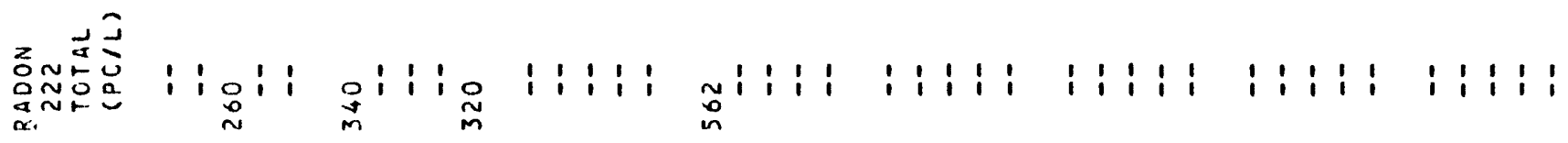

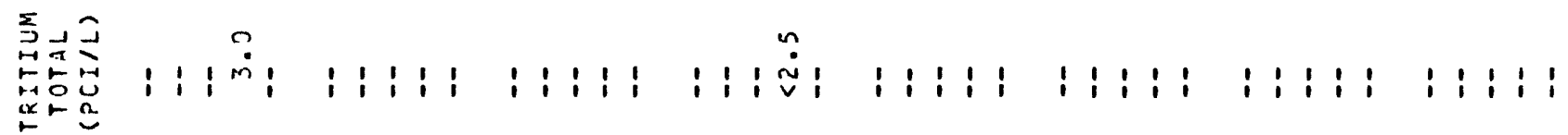

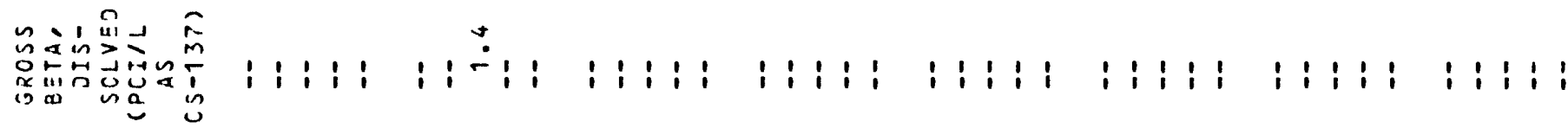

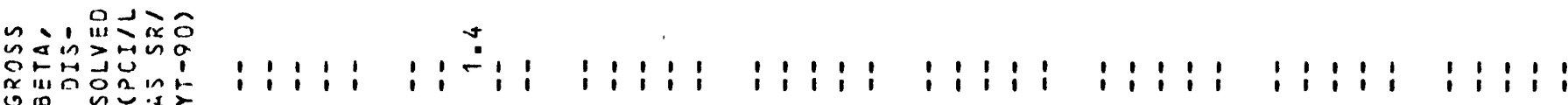

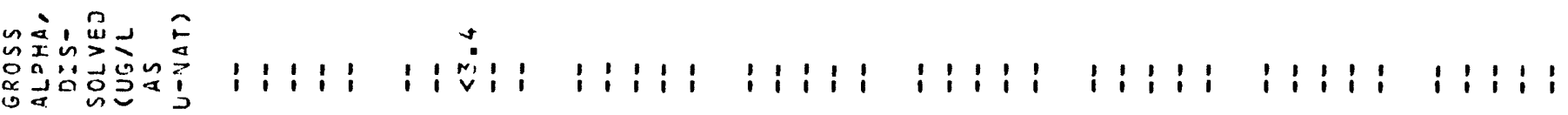

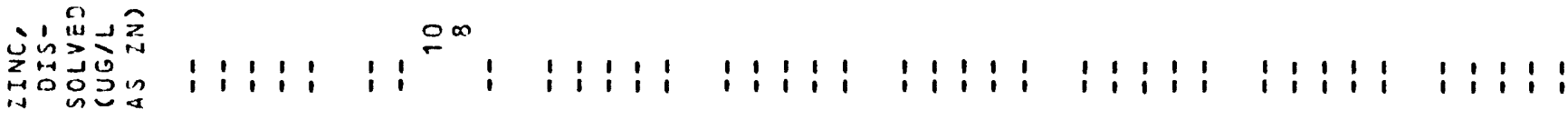

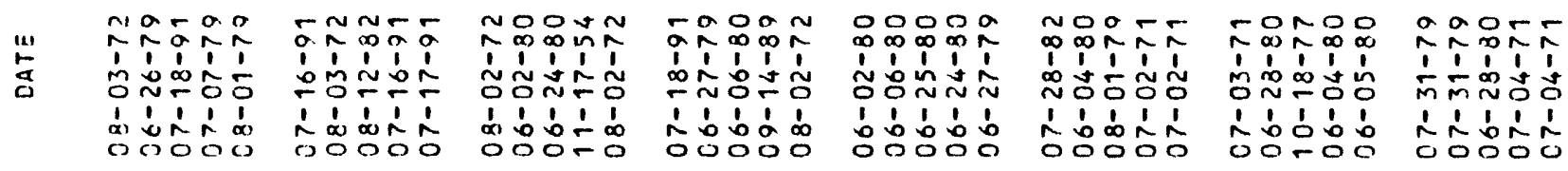




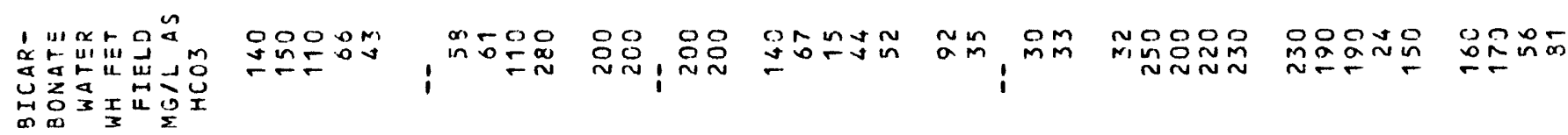

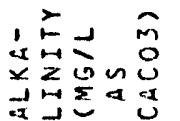
$i: \stackrel{0}{2}, \hat{n}: \stackrel{\infty}{i}:$

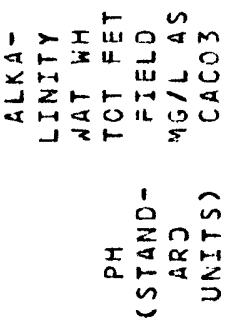

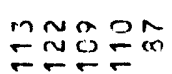
mnmn nen on I

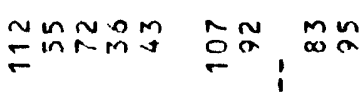

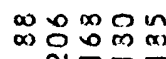
$\stackrel{\sim}{\sim} \stackrel{m}{\sim}$

nmama $n \pi \cdot a \alpha$ manm wmin

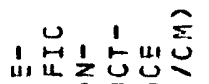

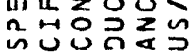

$\stackrel{1}{\alpha} \propto \mathcal{O}$

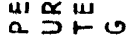
部吉出

0000
000 a.am |

$m a c a s o$ in in $\dot{0}$ $\stackrel{0}{\circ}:$ $\because \because \because \because \ddot{\infty}$ m: $\ddot{a r a ́ m}$

ก $\infty$ r prumen

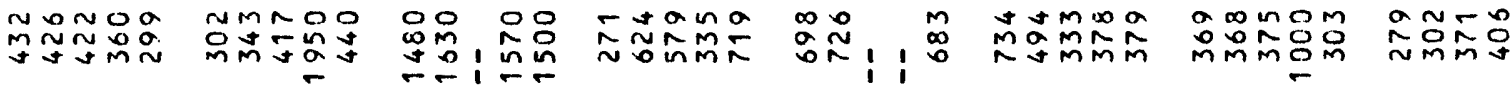

o. in on Nimmio

0.0000 $\therefore \sim i n i$

o m o m o -min-m

onooo ல்றோ

o in 000 nooom mooo inio: $\dot{n} \dot{0} \dot{0}$ N N N 1,200

$\infty a \infty$ in $\pi$

Rnor

nis

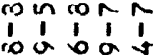
nond

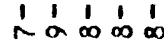

Nmamm

b's mơ $\begin{array}{lllll}1 & 1 & 1 & 1 & 1 \\ 0 & \infty & 0 & 0 & 0\end{array}$ 잉요

$m m m M \infty$ a. åño 11111

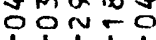
กิ่00\% 응ㅇㅇㅇ

wa

은으는

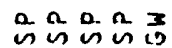

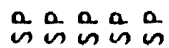

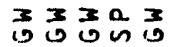

Nmonm

1 1 c

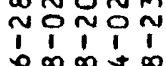
我
ก๊กัก๊

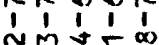
ำ 눙 11111

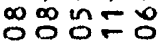

in in in in in min

$\operatorname{nin} \operatorname{in} N$ $\infty \infty \infty \infty$

กิ人ヘ

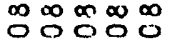

กิ

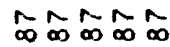

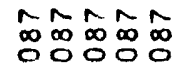

$\sim \sim \sim \sim N$ $\infty \infty \infty \infty$

$\hat{\infty} \sim \sim \sim$ $\infty \infty \infty \infty$

\begin{tabular}{|c|c|c|c|c|c|c|c|c|c|c|}
\hline & 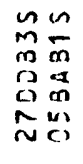 & 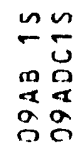 & 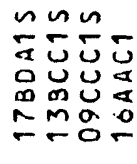 & $\begin{array}{l}n \\
o \\
0 \\
0 \\
0 \\
n \\
0\end{array}$ & 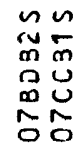 & 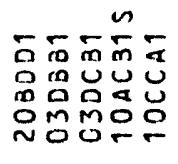 & $\begin{array}{l}\tilde{3} \\
\text { ㅁ }\end{array}$ & 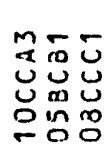 & $\begin{array}{l}5 \\
a \\
a \\
m \\
m\end{array}$ & 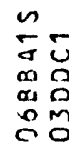 \\
\hline 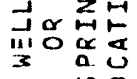 & Mu & 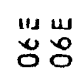 & 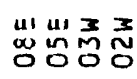 & m & 勇齐 & 录 & $\frac{3}{8}$ & 预予蛋 & $\frac{3}{0}$ & 沓录 \\
\hline & $\begin{array}{l}2 \geq \\
\leq 1\end{array}$ & $z_{\infty} z$ & $\begin{array}{l}z \\
z \\
x \\
\sim\end{array}$ & $\frac{z}{\square}$ & $z \geq$ & $\begin{array}{l}z z z \\
=\frac{2}{\prime}\end{array}$ & $\frac{z}{z}$ & $\begin{array}{l}z Z Z \\
=m M\end{array}$ & $\underset{m}{z}$ & $\begin{array}{l}23 \\
01\end{array}$ \\
\hline
\end{tabular}




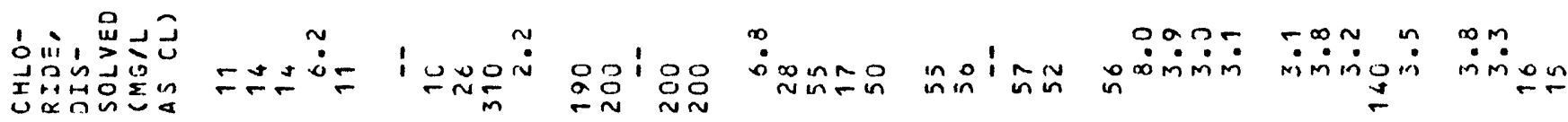

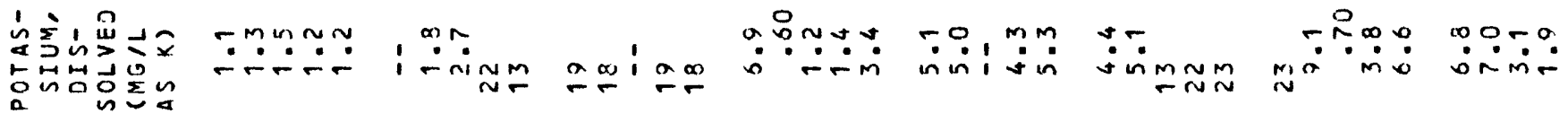

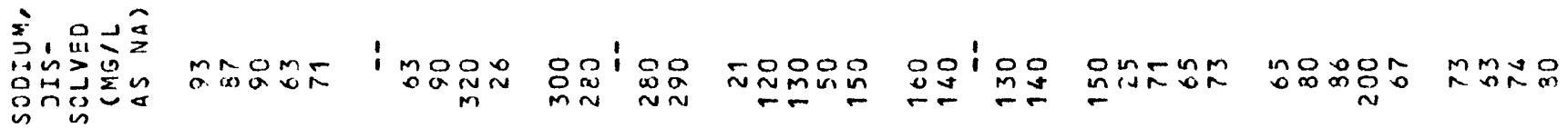

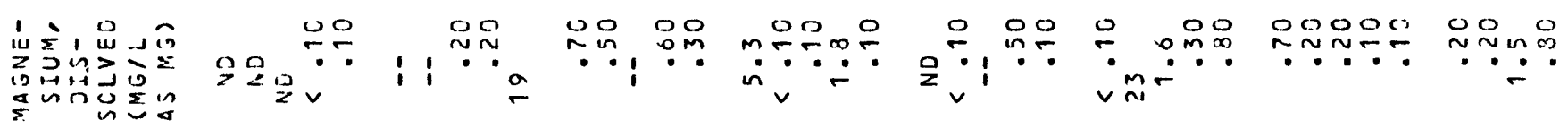

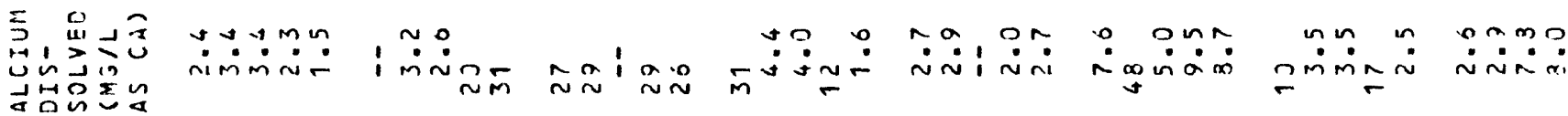
ô x $z$ at $0=0$

in 1 in, 品

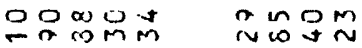

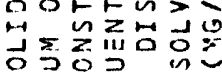
(1) (x) 12 $6-1$

$$
\text { i wor }
$$

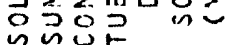

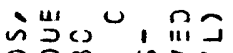
解: in in Oैw

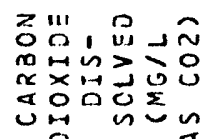
waro⿺

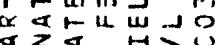

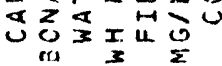

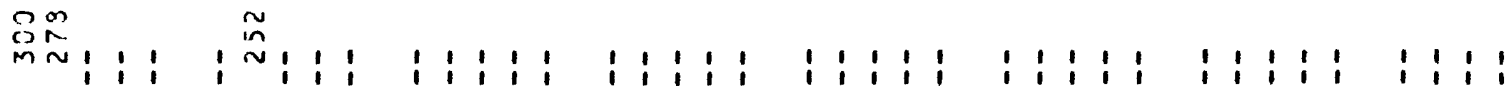

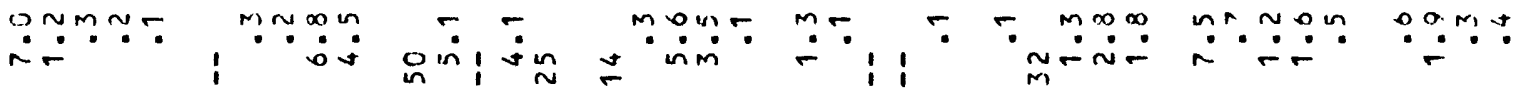

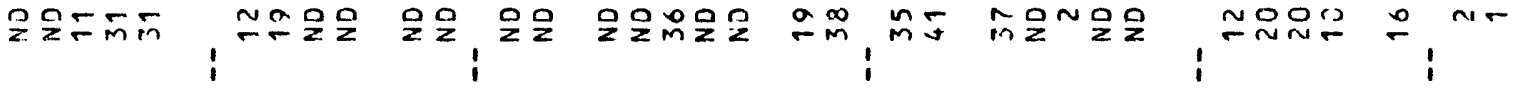
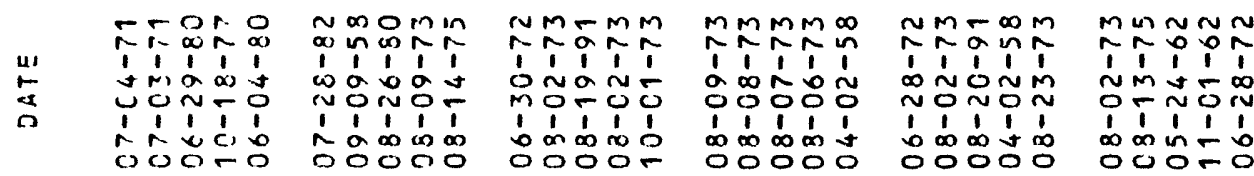

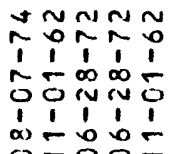

re turn $\pi \hat{1}$ $\infty \pi n$ Non a) 1 - 


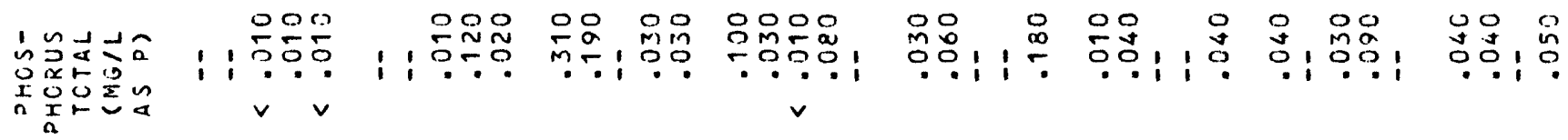

的就呈守

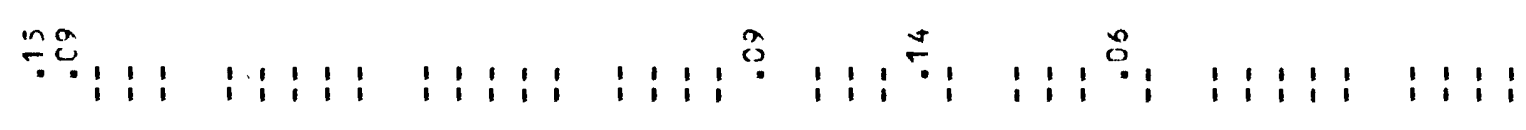

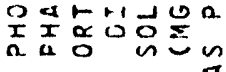

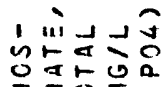

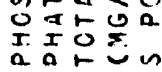

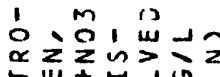

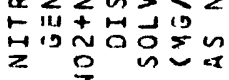
i i i

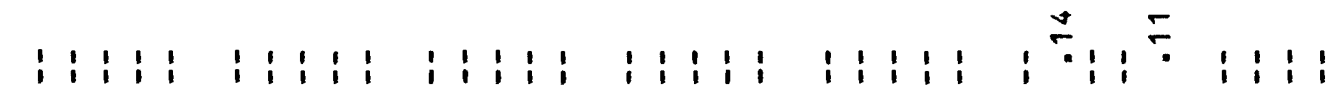

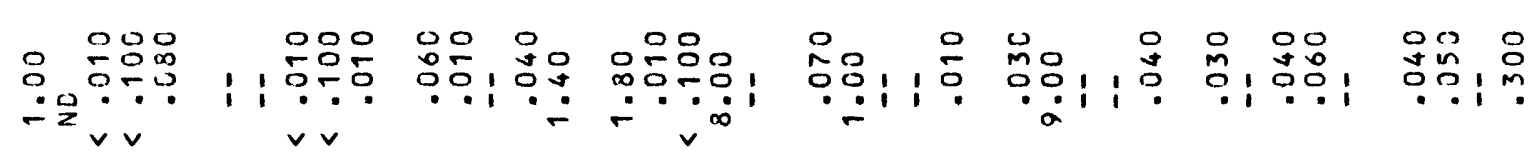

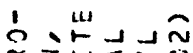
$\propto \geq \omega \alpha \geq 0$

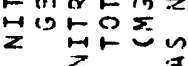

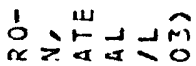
ㄴiा or 5 in

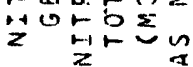

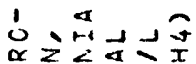
$\alpha \sum_{111} \leq x=\pi$

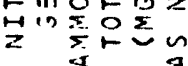

'站晏士正

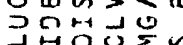
แ1

: : : :

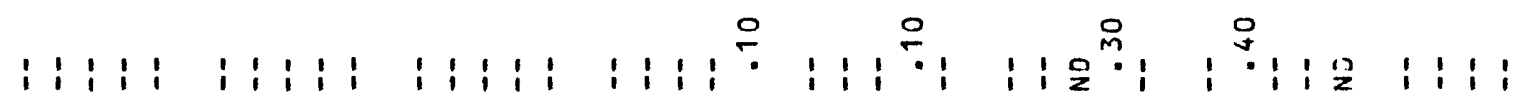

$\stackrel{11}{0}, 0$

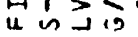

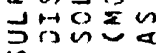

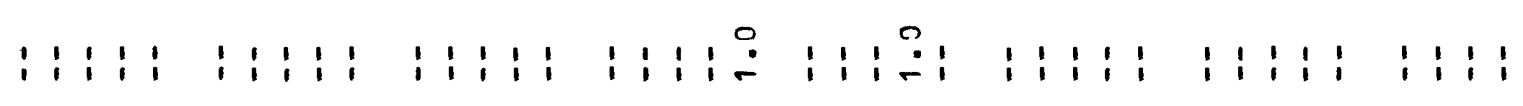
"21,

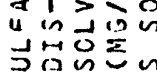

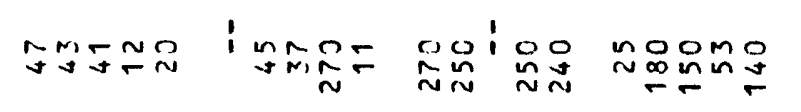

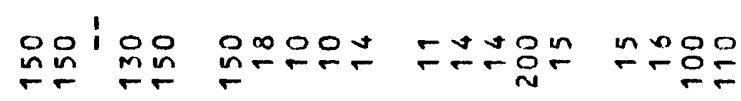

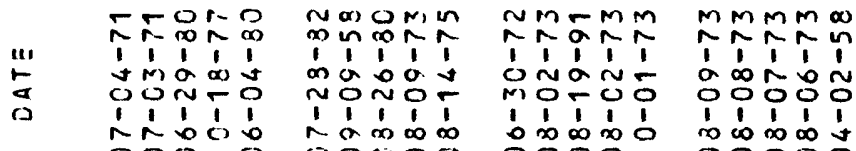

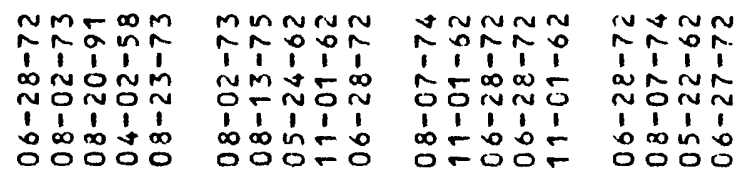




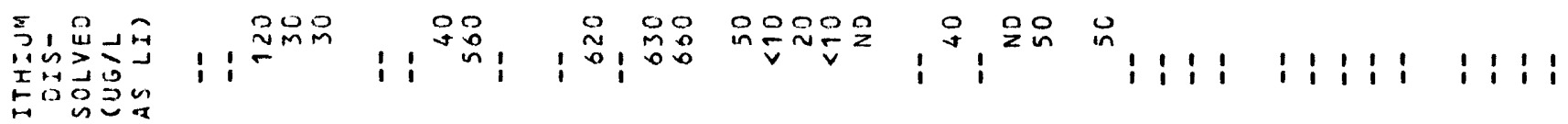

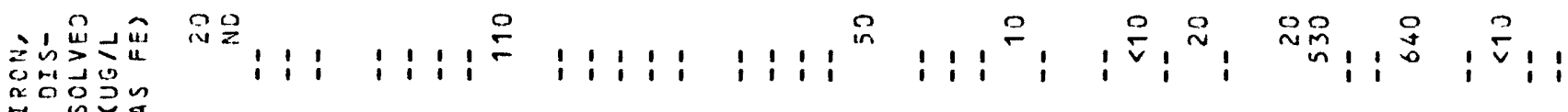

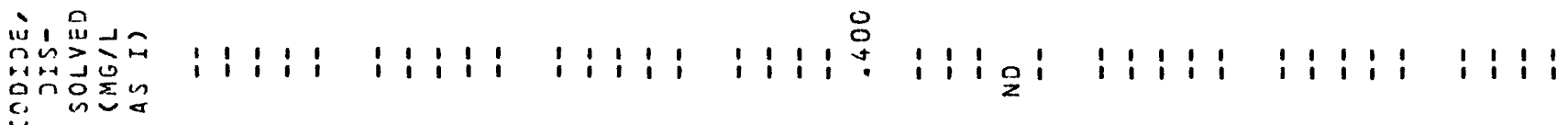

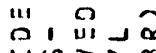

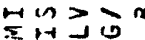

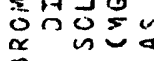

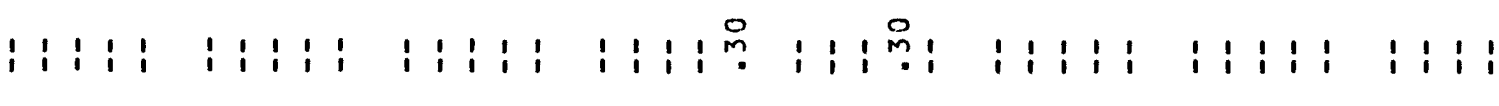

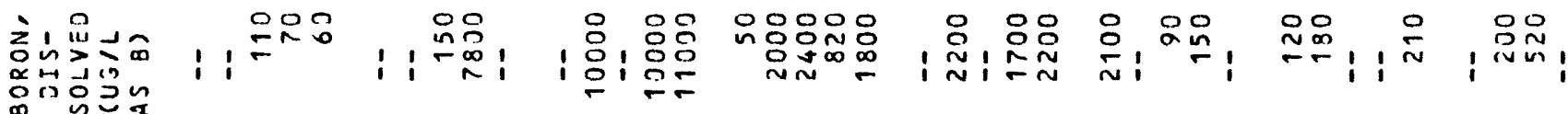

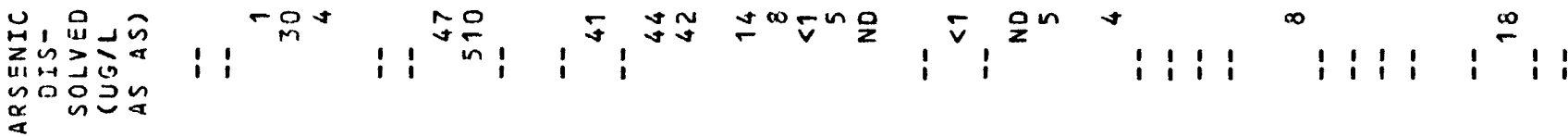
产部落寻

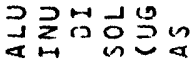

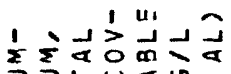
रे

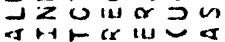

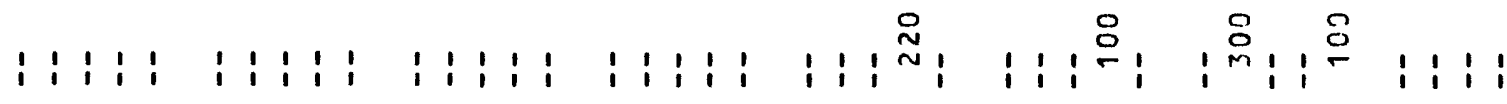

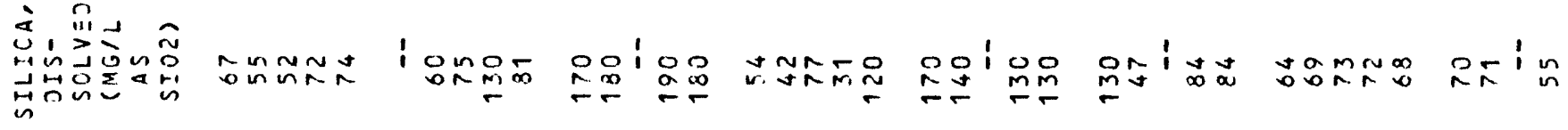

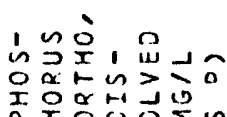
우우 政:

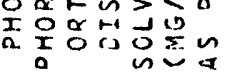

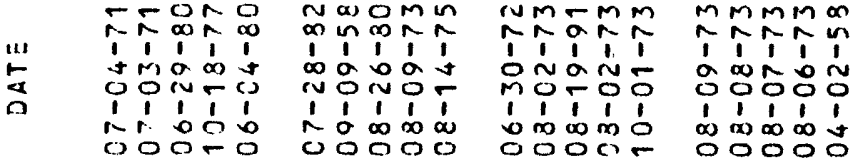

NmT̃în N

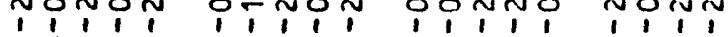
ำ 


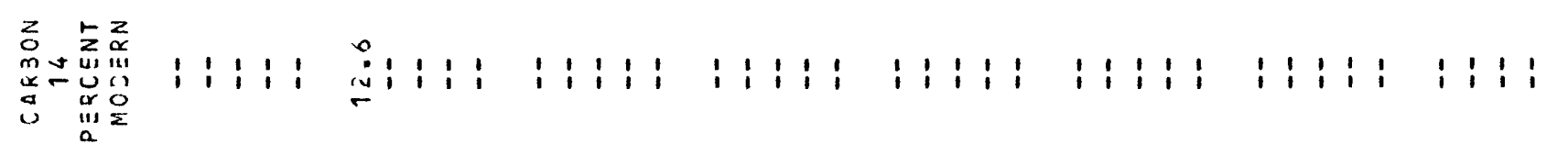

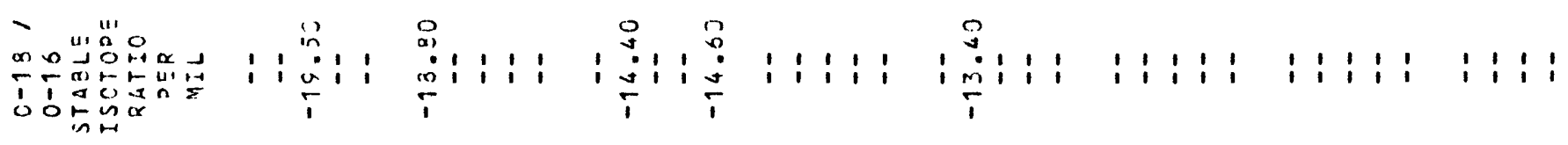

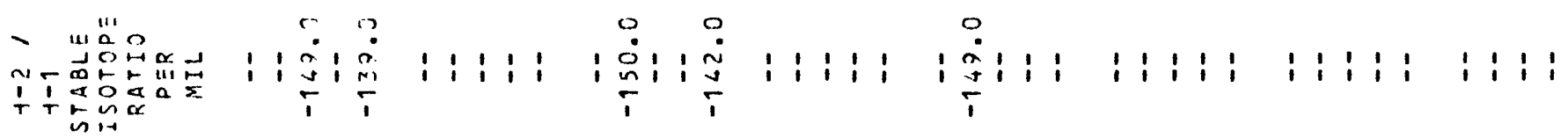

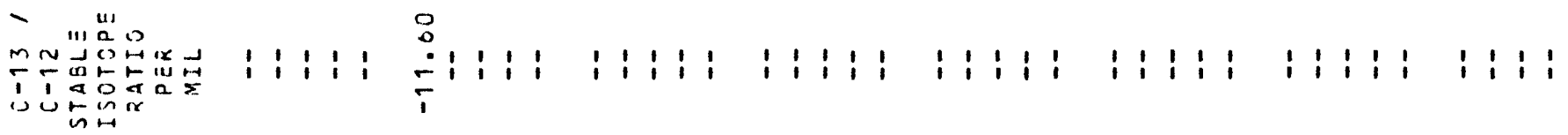

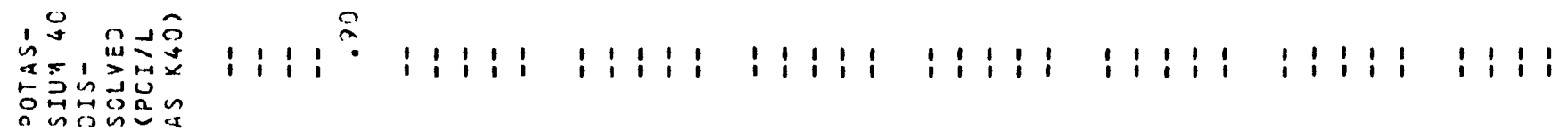

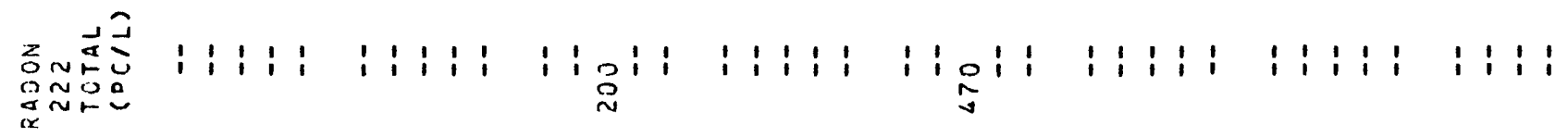

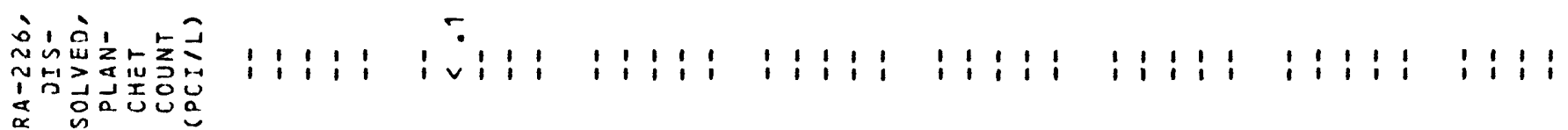

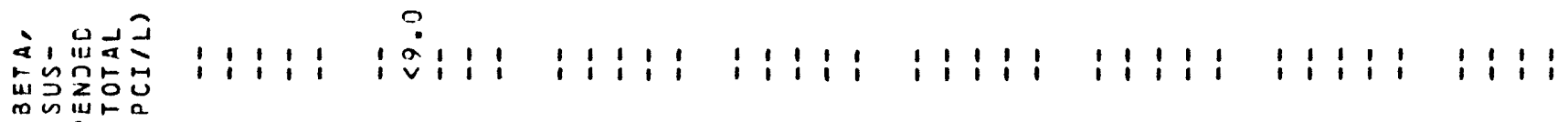

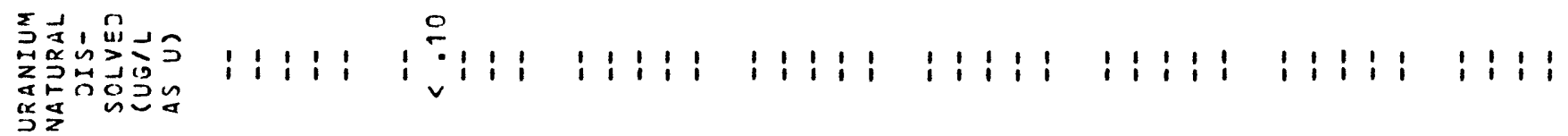

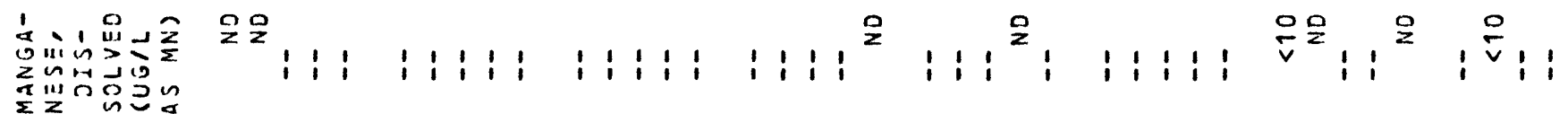

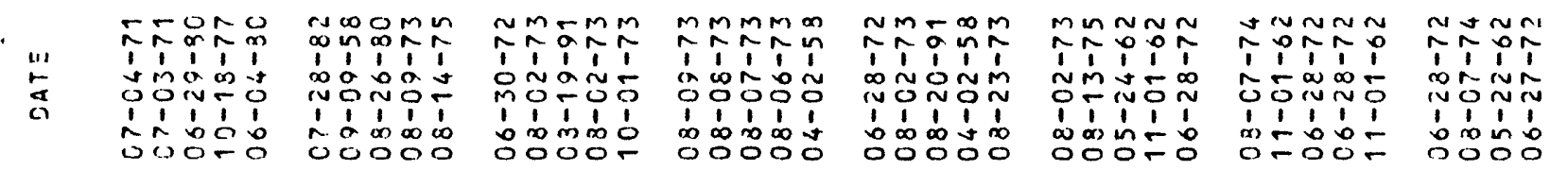

